Old Dominion University

ODU Digital Commons

Summer 1997

\title{
Systematics of the Freshwater Amphipod Genus Crangonyx (Crangonyctidae) in North America
}

Jun Zhang

Old Dominion University

Follow this and additional works at: https://digitalcommons.odu.edu/biology_etds

Part of the Fresh Water Studies Commons, and the Zoology Commons

\section{Recommended Citation}

Zhang, Jun. "Systematics of the Freshwater Amphipod Genus Crangonyx (Crangonyctidae) in North America" (1997). Doctor of Philosophy (PhD), Dissertation, Biological Sciences, Old Dominion University, DOI: $10.25777 / \mathrm{s} 8 \mathrm{tw}-2 \mathrm{q} 09$

https://digitalcommons.odu.edu/biology_etds/75

This Dissertation is brought to you for free and open access by the Biological Sciences at ODU Digital Commons. It has been accepted for inclusion in Biological Sciences Theses \& Dissertations by an authorized administrator of ODU Digital Commons. For more information, please contact digitalcommons@odu.edu. 


\section{SYSTEMATICS OF THE FRESHWATER AMPHIPOD GENUS CRANGONYX \\ (CRANGONYCTIDAE) IN NORTH AMERICA}

by

Jun Zhang

B.S. 1984, Sichun University

M.S. 1987, Nanjing Normal University

A Dissertation submitted to the Faculty of Old Dominion University in Partial Fulfillment of the Requirement for the Degree of

DOCTOR OF PHILOSOPHY

ECOLOGICAL SCIENCES

OLD DOMINION UNIVERSITY

August 1997

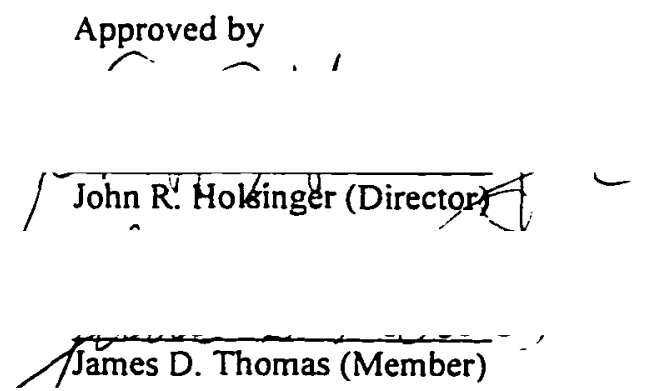

$\overline{\text { Kent E. Carpenter (Member) }}$ 


\section{ABSTRACT \\ SYSTEMATICS OF THE FRESHWATER AMPHIPOD GENUS CRANGONYX \\ (CRANGONYCTIDAE) IN NORTH AMERICA.}

Jun Zhang

Old Dominion University, 1997

Director: Dr. John R. Holsinger

The systematics of the amphipod genus Crangomx of North America (north of Mexico) is revised. based on available collections (ca. 2240) and literature dealing with the genus. A grand total of 42 North America species of Crangonyx are recognized in the present study. 24 of them new to science. All species are described or redescribed and figured, utilizing external morphological features. Keys to both species groups and individual species are given. Phylogenetic trees are built using computer programs (PAUP. Hennig86. MacClade) based on 26 characters. Wagner parsimony produced 18 parsimonious trees and Fitch parsimony produced 45 trees. The consensus tree of both methods and a hypothesis phylogenetic tree of the North America species of Crangonyx are developed along with a discussion of Crangonyx phylogeny and character evolution. Six monophyletic species groups are recognized.

With respect to the origin of Crangonvx. putative ancestors of the genus probably originated as very old freshwater lineage dating back at least to the Mesozoic and possibly earlier. The ancestor of modern Crangonyx species possibly resembled $C$. forbesi and separated from the common ancestor of Synurella and Crangonyx somewhere on Laurasia. Species of Crangonyx have exploited a wide variety of surface and groundwater habitats in North America. Many of the spring/seep dwellers are apparently predated to life in subterranean waters. A detailed distribution map is given for each species with an interpretation of its biogeography. Almost all species of the genus occur in eastern North America. east of the Rock Mountains. The species of Crangonyx with distribution in glaciated areas also occur south of the southern extent of Pleistocene glaciation. Distribution patterns are analyzed and compared with phylogeny in an attempt to evaluate extrinsic barriers. dispersal limits and other geographic and geological considerations that might have a bearing on species ranges.

Eleven species of Crangonvx in North America are troglobites. many of which are found in karst areas in the Appalachians and Interior Low Plateaus regions. The origin of troglobitic members of the genus is attributed to active or passive invasion and colonization of subterranean waters by preadapted epigean ancesters. sometimes under climatic constraint and sometimes by adaptive shifts into new niches. Perhaps a few troglobitics species originated through peripheral isolation in subterranean waters from previously established troglobites.

Human introduction of $C$. pseudogracilis in Europe and $C$. floridanus in Japan is explained by the ballast water theory, which suggests that small aquatic organisms, such as certain species of amphipods. are transported in the baliast water of ocean-going ships. 


\section{ACKNOWLEDGMENTS}

I am especially grateful to my major professor Dr. John R. Holsinger. for his continued direction. support and advice. I thank my graduate committee members. Dr. James D. Thomas and Dr. Kent E. Carpenter. for their helpful advice and support.

I am grateful to the persons and institutions listed below for making specimens available for study: Charles K. Biernbaum. College of Charleston: Joan Jass. Milwaukee Public Museum: Diana R. Laubitz. Canadian Museum of Nature: R. \& M. Lindeman: Sara LeCroy. University of Southern Mississippi: Jerry Lewis: Scott Harden; Hiroshi Morino, Ibaraki University: National Museum of Natural History (Smithsonian Institution): Museum of Comparative Zoology. Harvard University: and Steven Roble and Christopher Hobson from the Virginia Natural Heritage Program. I am also grateful to the Office of Publications and Graphics at Old Dominion University for assistance with preparation of the maps and figures.

Most of this study was supported by research assistantships and special doctorate fellowships from Old Dominion University and the Department of Biological Sciences. Special thanks to Dr. Frank P. Day, the director of Ecological Sciences Program. for granting me these assistantships. In addition. a part of this study was supported by a grant from the National Science Foundation (DEB-9521752-PEET) to Dr. John R. Holsinger.

Thanks to many professors and students in the Department of Biological Sciences who helped and supported me during my study and stay there. Special thanks to Professor Emeritus. Dr. Donald Messersmith. University of Maryland and his wife and others who helped me come to United States to study and their great support.

Finally. my wife. Zhenhong Sun is especially thanked for her tolerance. sacrifice, and support. 


\section{TABLE OF CONTENTS}

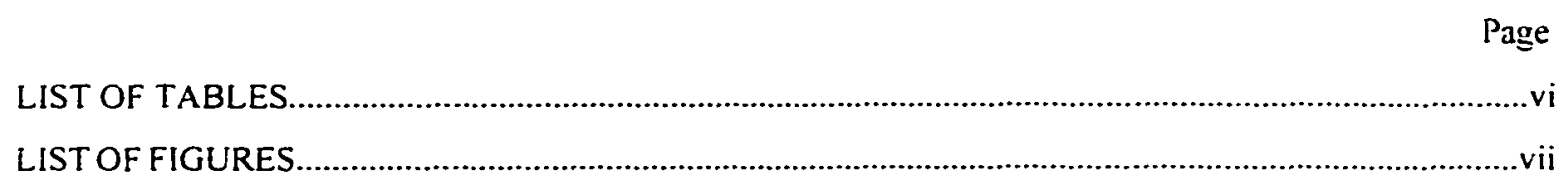

\section{Chapter}

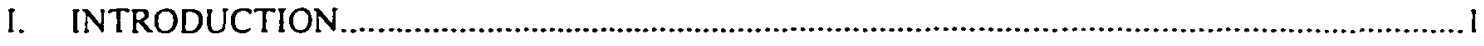

REVIEW AND HISTORICAL PERSPECTIVE ....................................................?

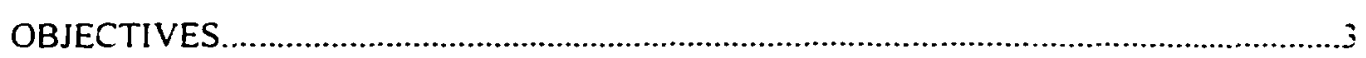

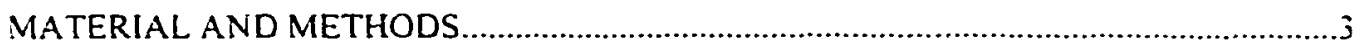

II. MORPHOLOGICAL ANALYSIS OF CR.ANGONYY CHARACTERS.................................8

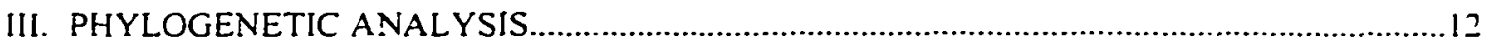

CHARACTERS AND CHOICE OF OUTGROUP ..................................................12

RESULTS OF PHYLOGENETIC ANALYSIS AND DISCUSSION .............................17

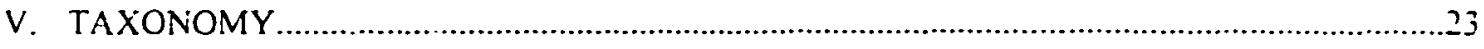

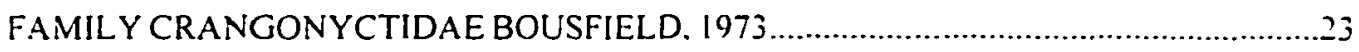

Genus Crangonyx Bate. 1859........................................................................................

Key to the Species Groups and Ungrouped Species of Crangonyx in North America....25

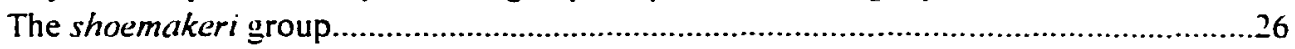

Key to the Species of the shoemakeri group......................................................... 8

Crangonyx shoemakeri (Hubricht and Mackin ).......................................................28

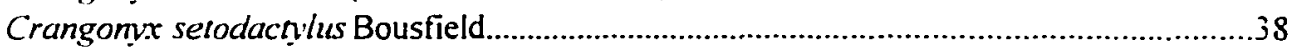

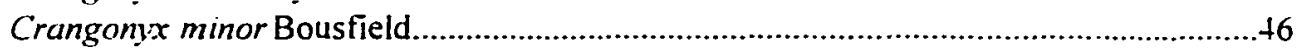

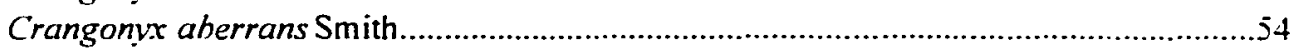

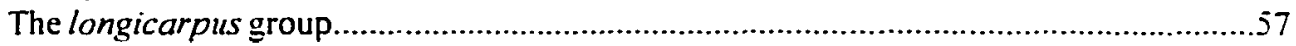

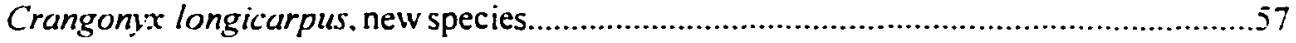

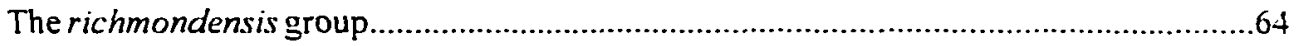

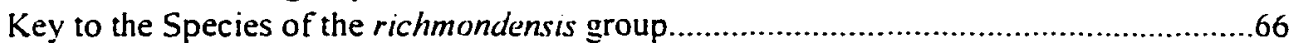

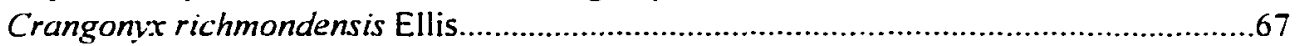

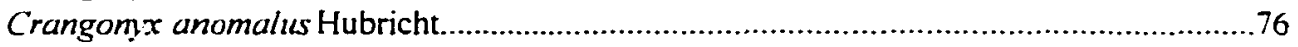

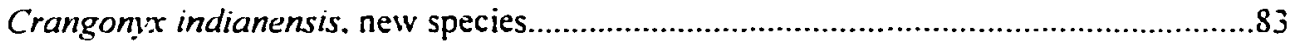

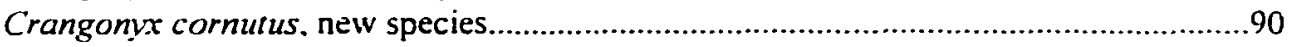

Crangonyx obliquus (Hubricht and Mackin) ..............................................................96

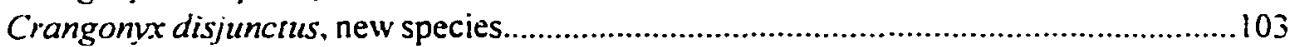

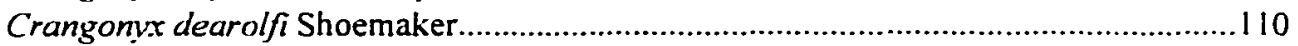

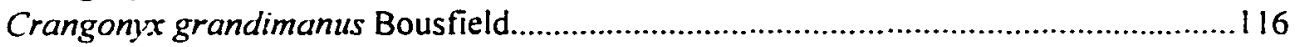

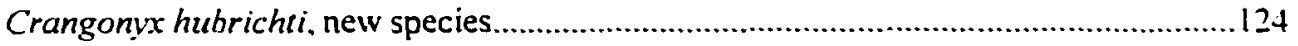

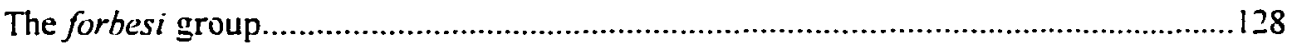

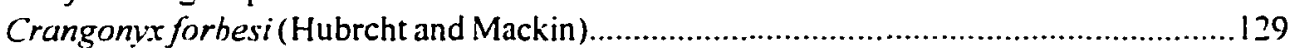

The insolitus group.........................................................................................

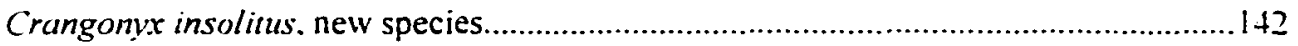

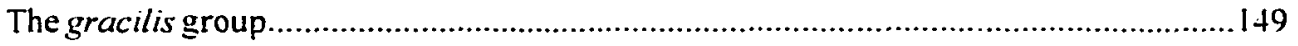

Key to the species of the gracilis group............................................................... 149

Species of the gracilis group unassigned to subgroups............................................ 1 1 
Chapter

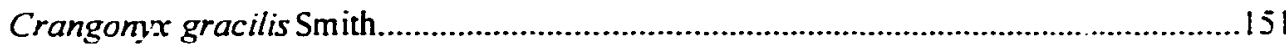

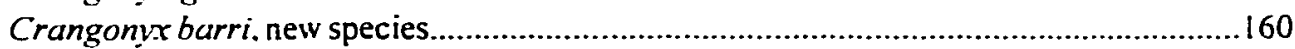

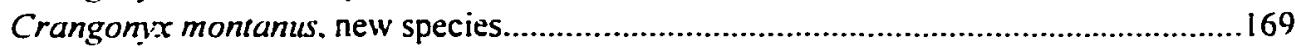

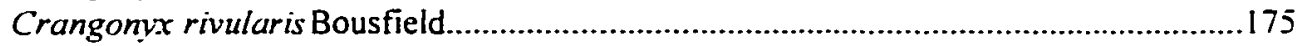

Crangonx packardi Smith................................................................................183

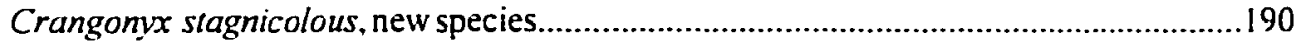

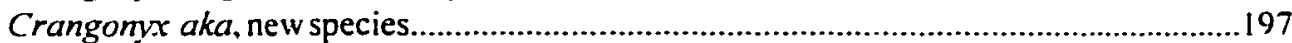

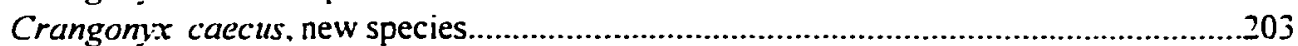

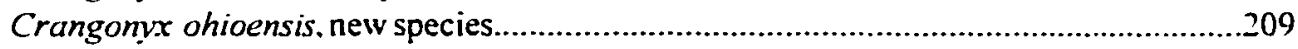

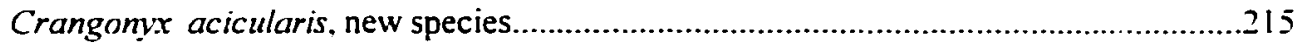

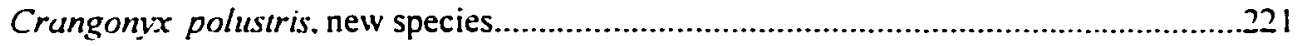

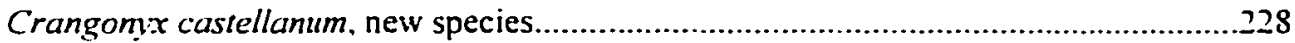

The hobbsi subgroup.

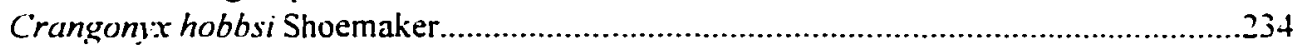

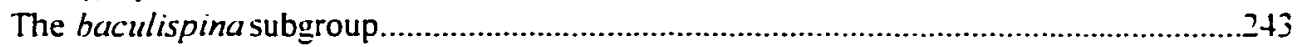

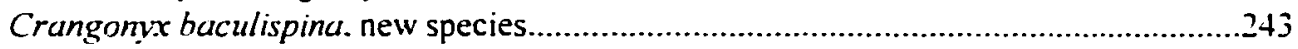

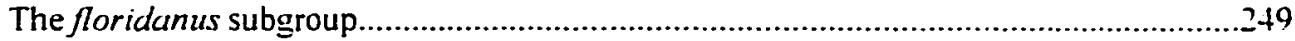

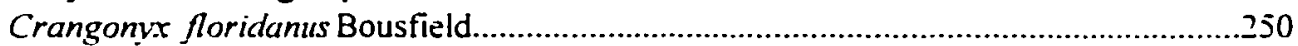

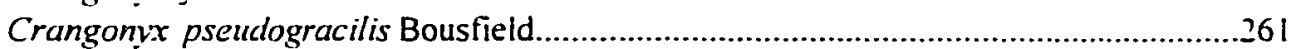

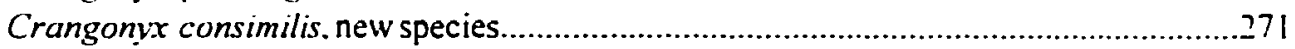

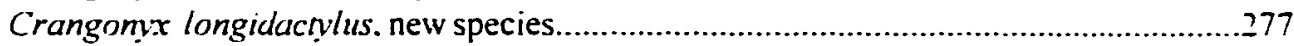

Species Unassigned to Groups..............................................................................283

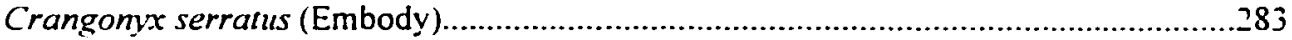

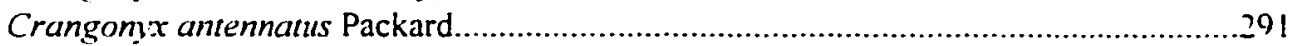

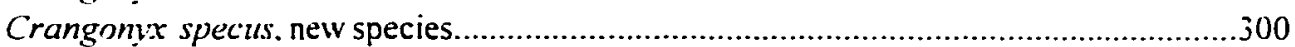

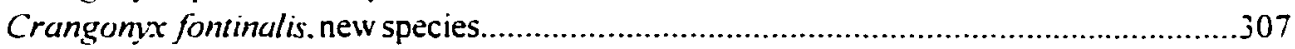

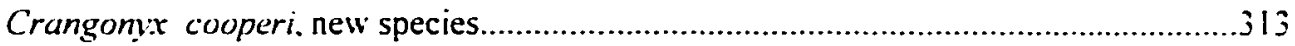

Crangonix lewisi, new species...........................................................................

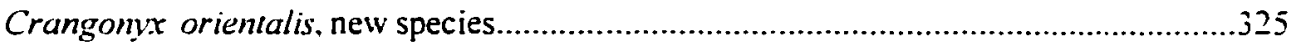

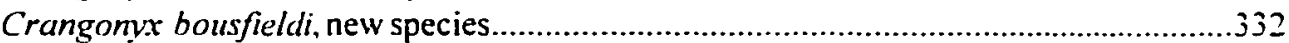

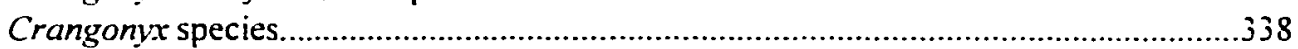

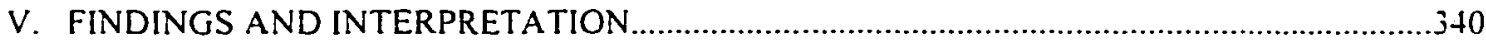

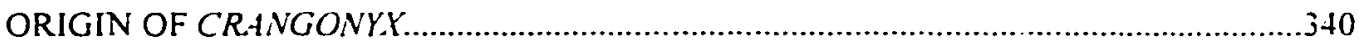

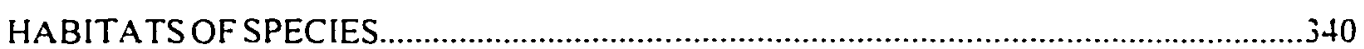

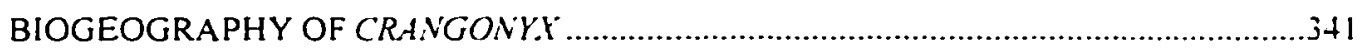

ORIGIN OF TROGLOBITES.............................................................................................349

INTRODUCTION OF C. PSEUDOGRACILIS AND C. FLORIDANUS TO AREAS

OUTSIDE THEIR ESTABLISHED RANGES.....................................................................

LITERA TURE CITED

VITA 


\section{LIST OF TABLES}

TABLE

1. Characters and character states used in the phylogenetic analysis of Crangonyx species from North America...

2. Character matrix for the species of Crangonyx from North America.. 16

3. Records of species of Crangonyx from five kinds of generalized habitats in North America. .342 


\section{LIST OF FIGURES}

FIGURE

1. Consensus tree from cladistic analysis of North America Crangonyx using Wagner parsimony.............18

2. Consensus tree from cladistic analysis of North America Crangomx using Fitch parsimony.

3. The phylogenetic hypothesis of North America Crangomx based on the two consensus trees (Figs. 1-2) and the observations Crangonyx species...........................................................20

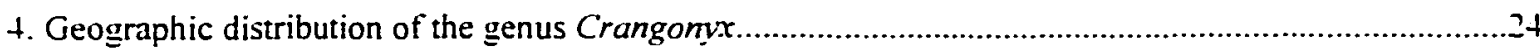

5. Geographic distribution of the shoemakeri group in North America...........................................27

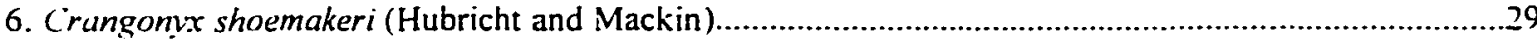

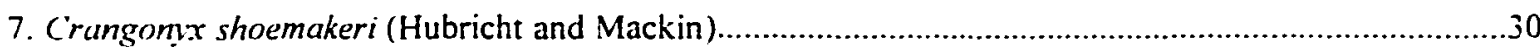

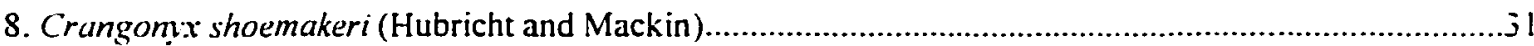

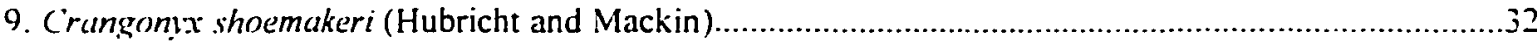

10. Distribution of Crangony shoemakeri in North America.........................................................33

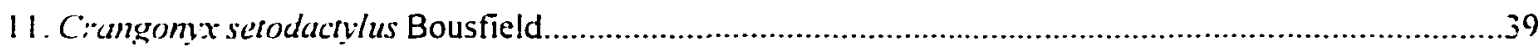

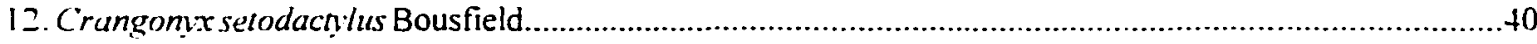

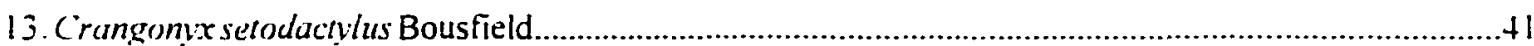

14. Distribution of Crangonyx setodactylus in North America...........................................................?

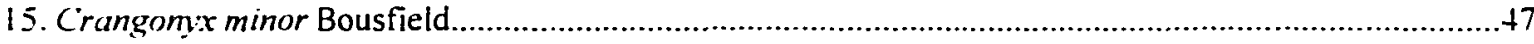

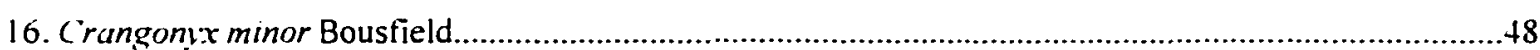

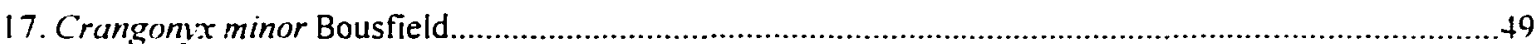

18. Distribution of Crangony:x minor in North America............................................................

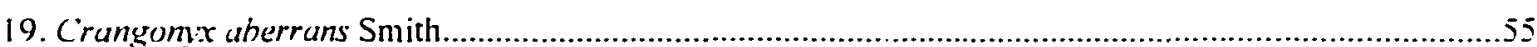

20. Distribution of Crangonyx aberrans in the North America.......................................................56

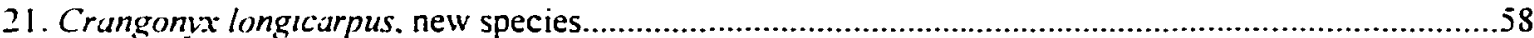

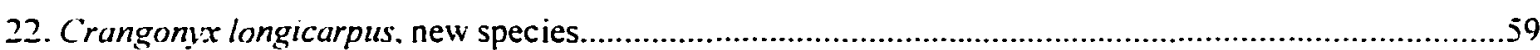

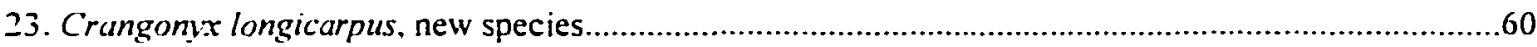

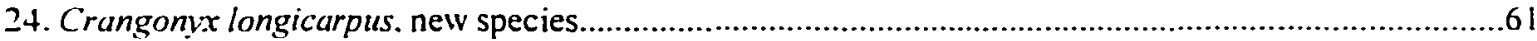

25. Distribution of Crangonvx longicarpus in North America...........................................................62

26. Geographic distribution of the richmondensis group in North America......................................65

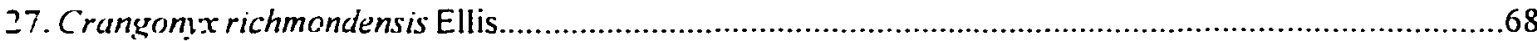

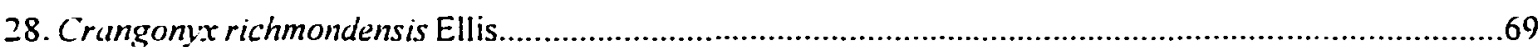

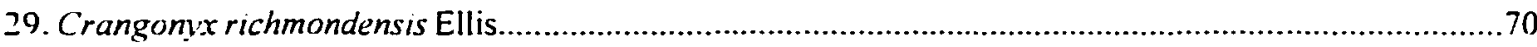

30. Distribution of Crangonux richmondensis in North America....................................................

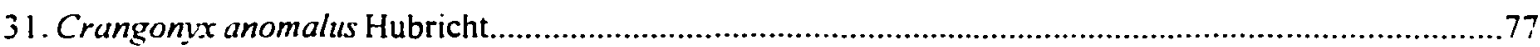

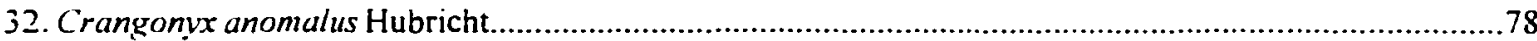


LIST OF FIGURES. continued

FIGURE

PAGE

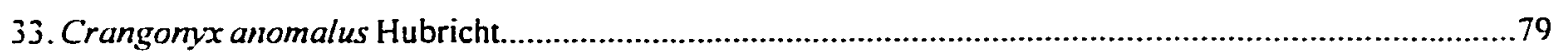

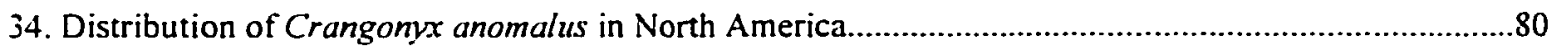

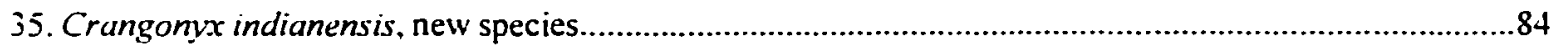

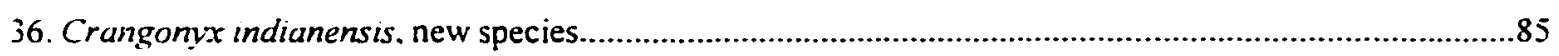

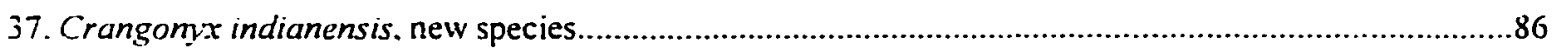

38. Distribution of Crangonvx indianensis in North America.......................................................................

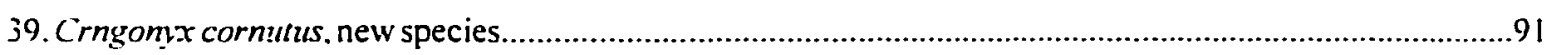

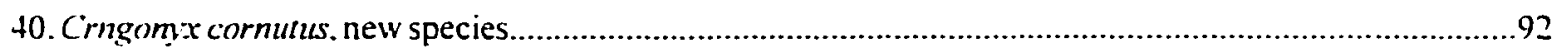

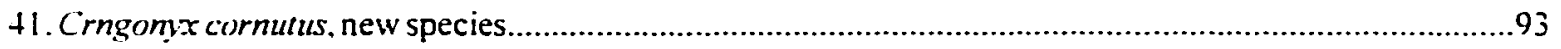

42. Distribution of Crangonx cornutus in North America.................................................................94

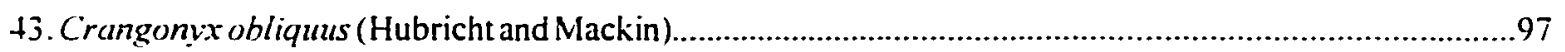

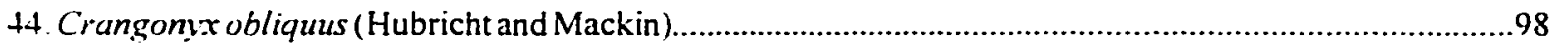

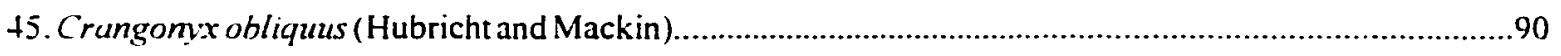

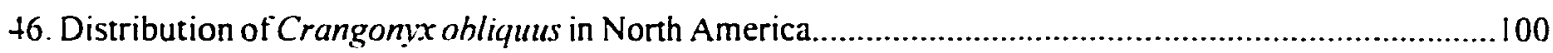

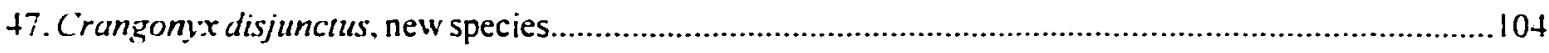

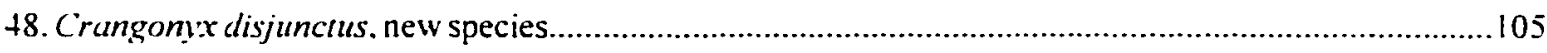

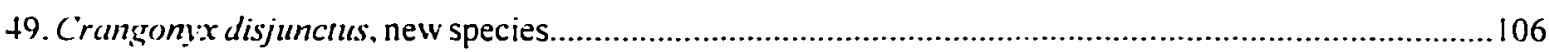

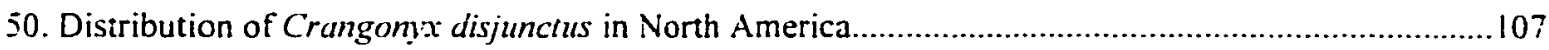

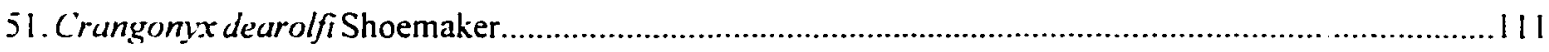

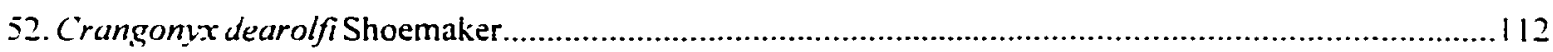

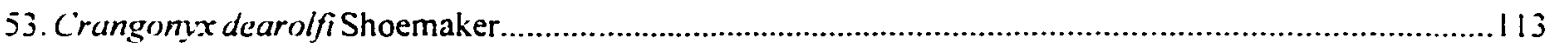

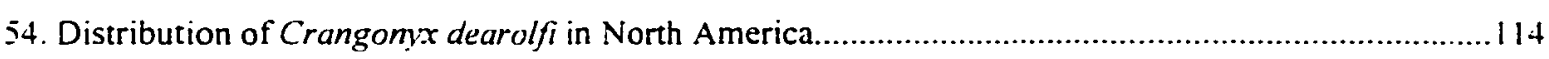

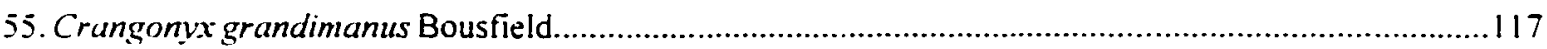

56. Crangonyx grandimanus Bousfield......................................................................................118

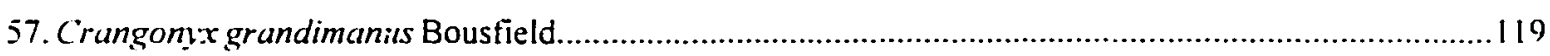

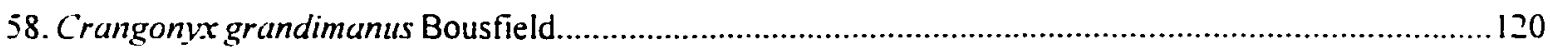

59. Distribution of Crangonyx grandimanus in North America.......................................................121

60. Crangonvx hubrichti, new species............................................................................................ 125

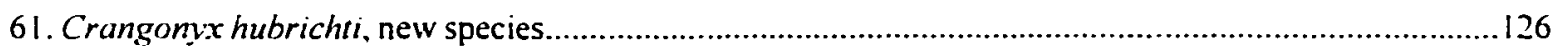

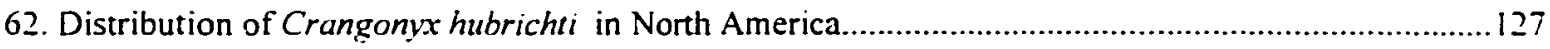

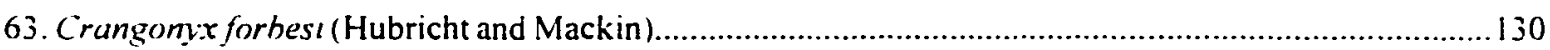

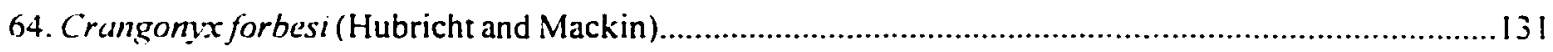

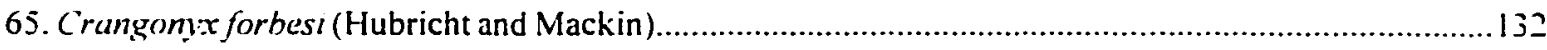




\section{LIST OF FIGURES. continued}

FIGURE

66. Crangomx forbesi (Hubricht and Mackin).

67. Distribution of Crangomx forbesi in North America.

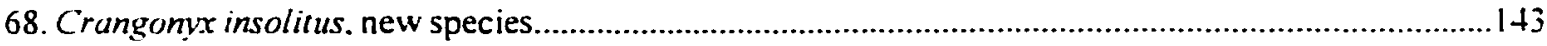

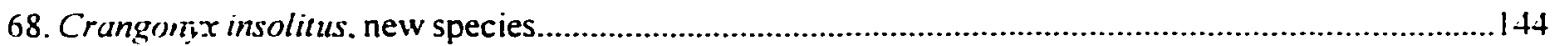

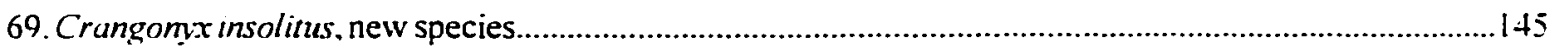

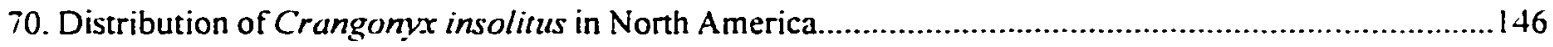

72. Geographic distribution of the gracilis group in North America.............................................. 48

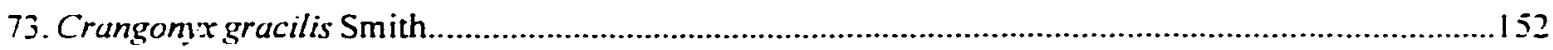

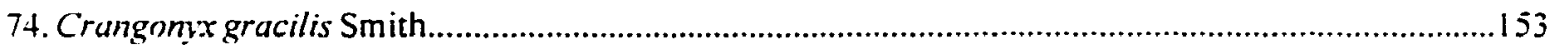

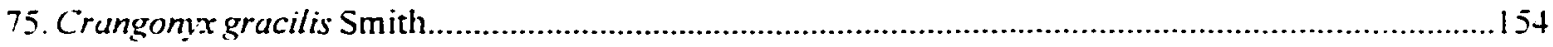

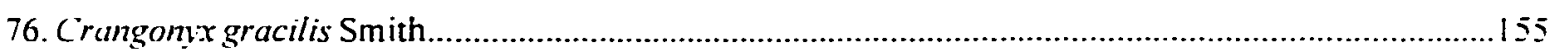

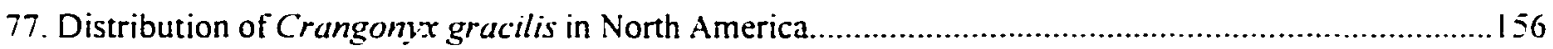

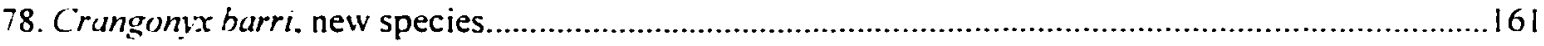

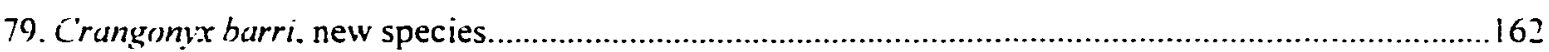

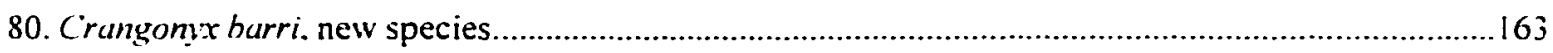

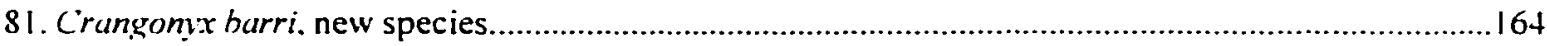

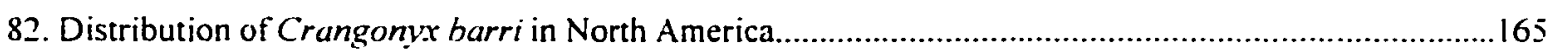

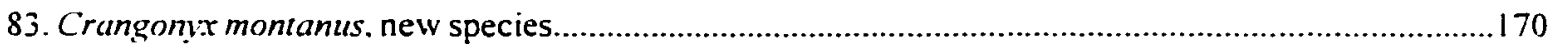

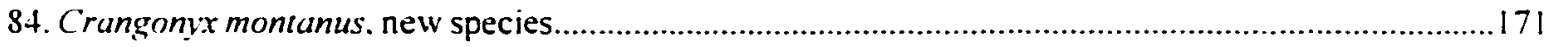

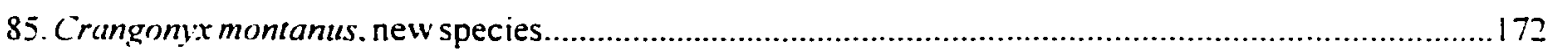

86. Distribution of Crangonyx montunus in North America......................................................173

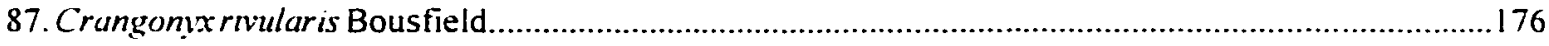

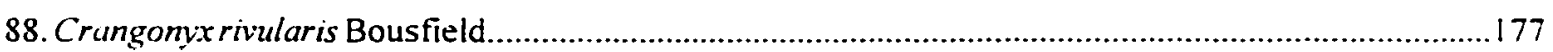

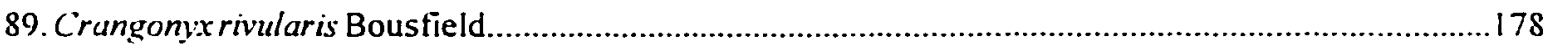

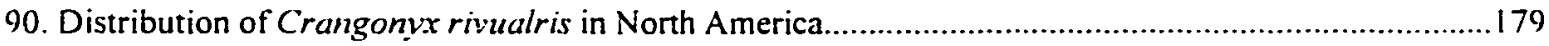

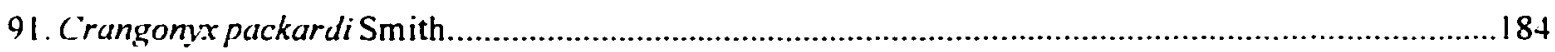

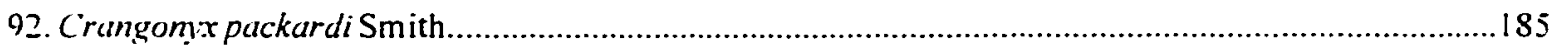

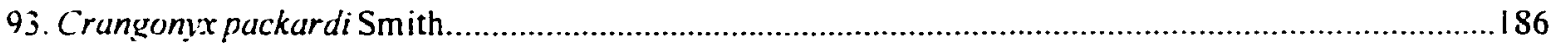

94. Distribution of Crangonlx packardi in North America............................................................187

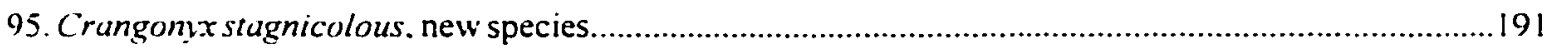

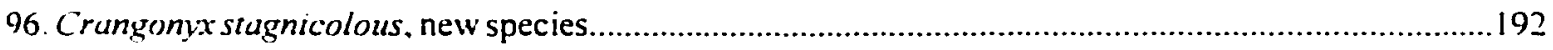

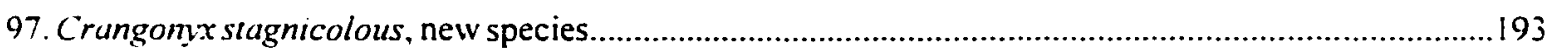

98. Distribution of Crangonyx stagnicolous in North America.................................................... 194 
LIST OF FIGURES. continued

FIGURE

PAGE

99. Crangomx aka, new species. 198

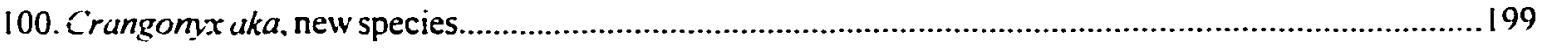

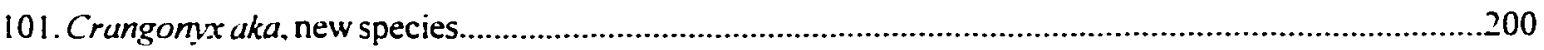

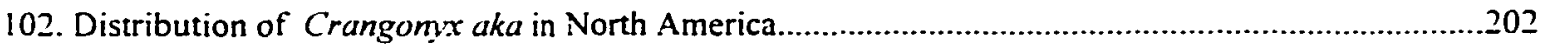

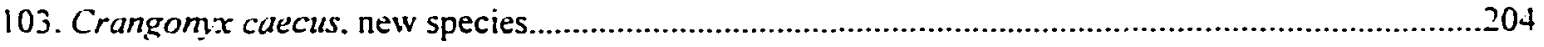

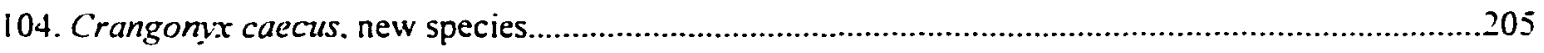

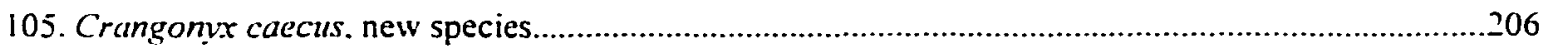

106. Distribution of Crangomx caecus in North America.....................................................207

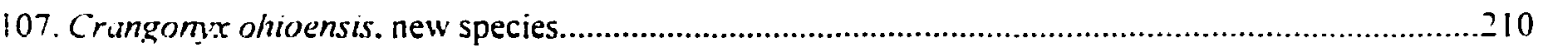

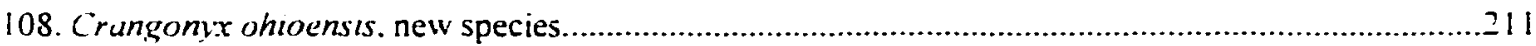

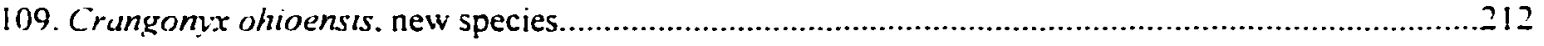

110. Distribution of Crangomx ohioensts in North America ...........................................................213

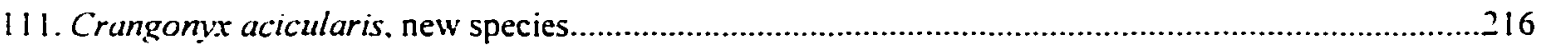

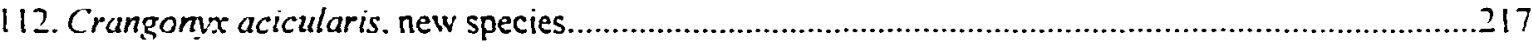

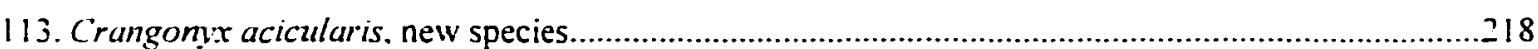

114. Distribution of Crangonlx acicularis in North America...........................................................213

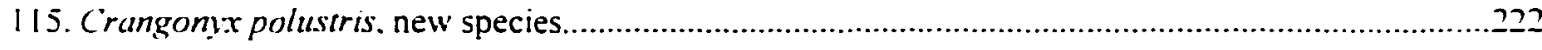

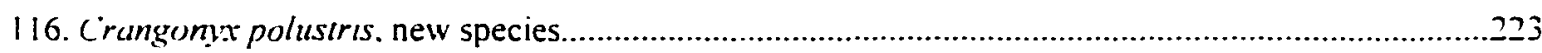

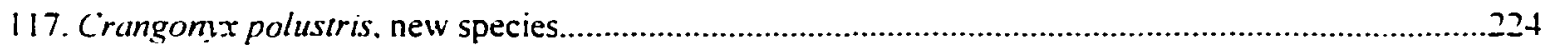

118. Distribution of Crangonx polustris in North America..........................................................213

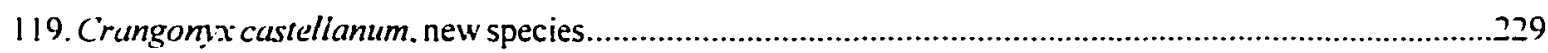

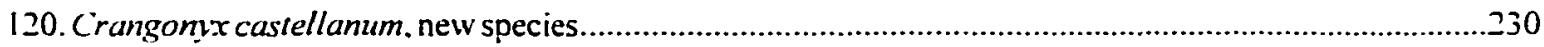

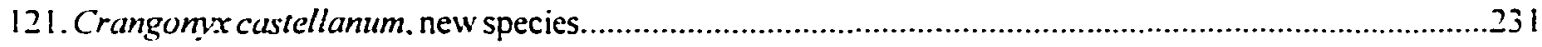

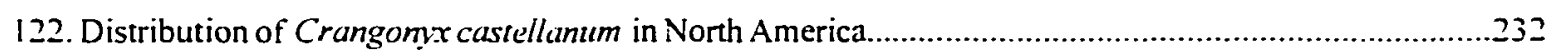

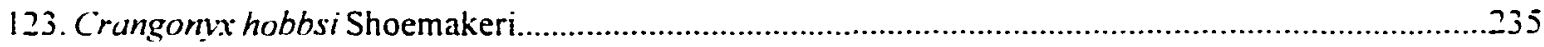

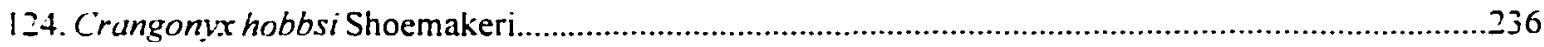

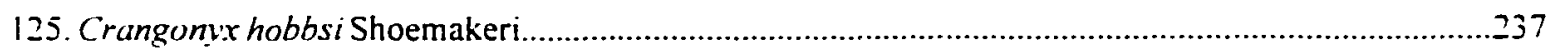

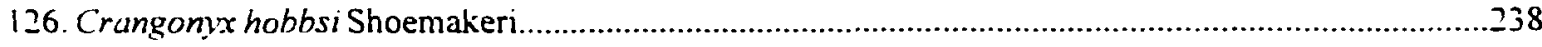

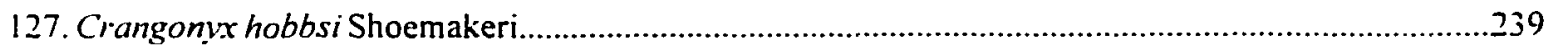

128. Distribution of Crangonyx hobbsi in North America...............................................................240

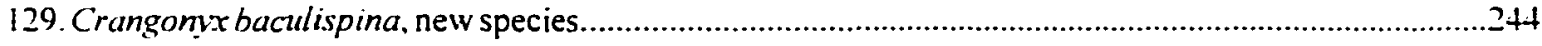

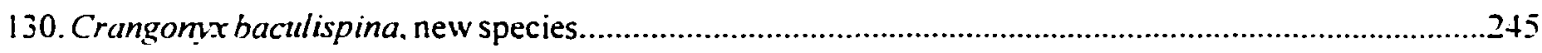

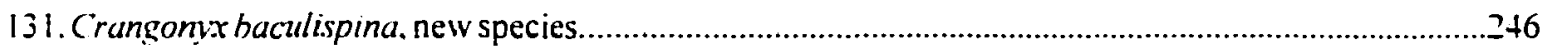


LIST OF FIGURES, continued

FIGURE

PAGE

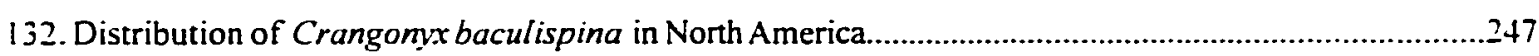

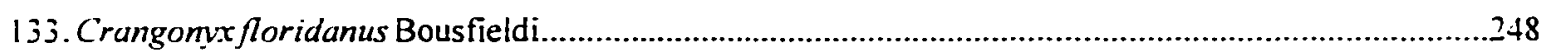

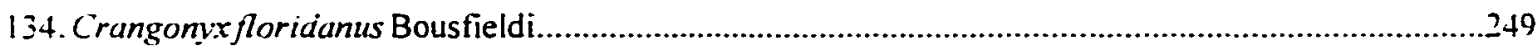

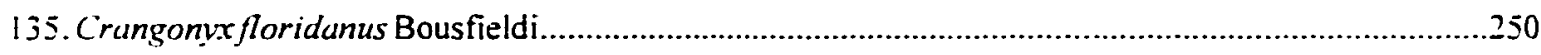

136. Distribution of Crangonvx floridanus in North America...........................................................247

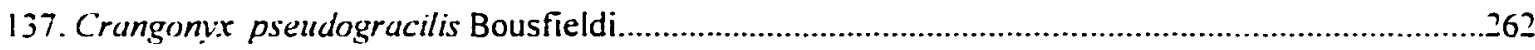

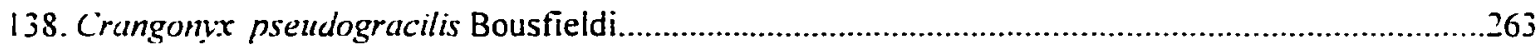

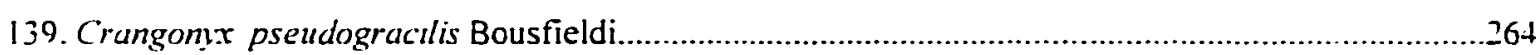

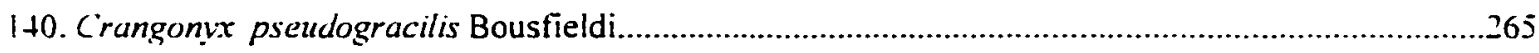

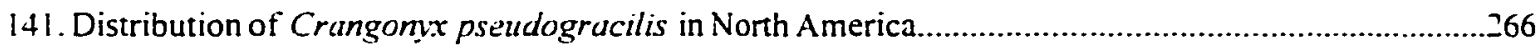

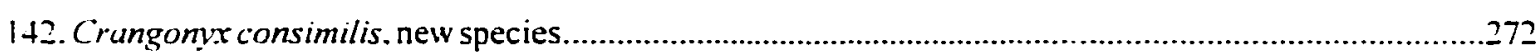

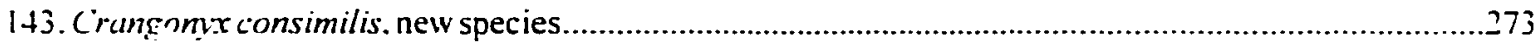

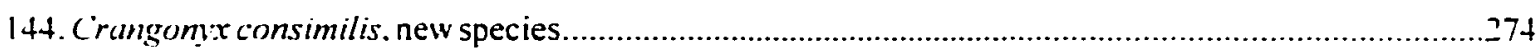

145. Distribution of Crangonvx consimilis in North America.............................................................. 75

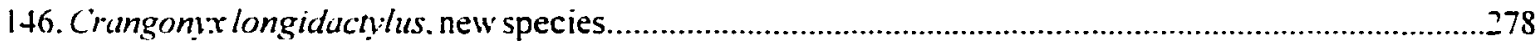

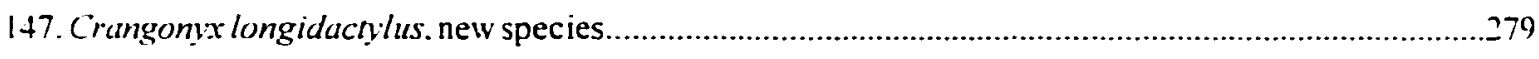

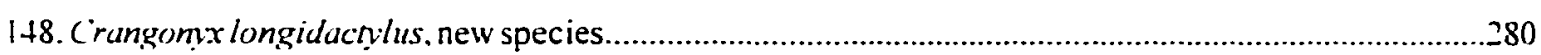

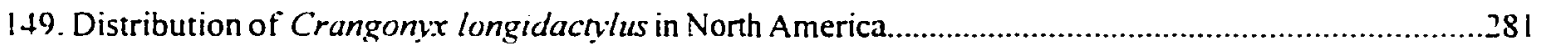

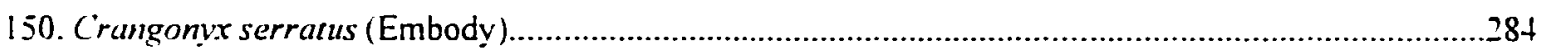

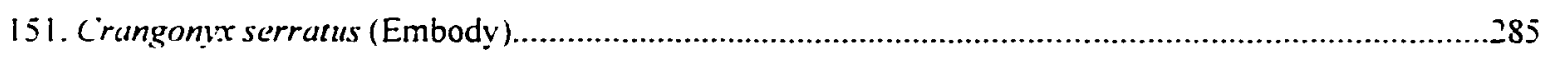

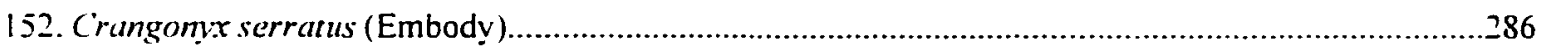

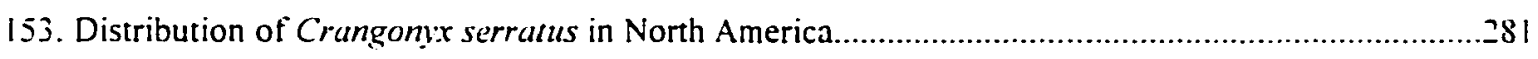

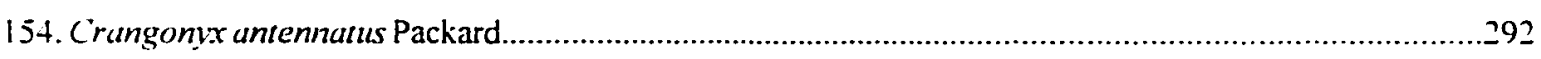

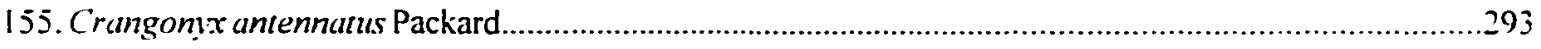

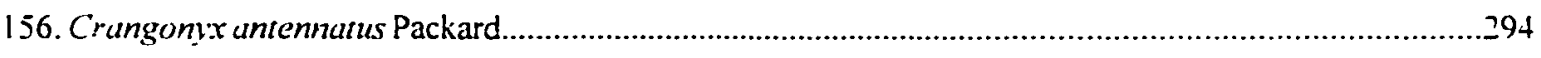

157. Distribution of Crungonvx antennatus in North America...............................................................281

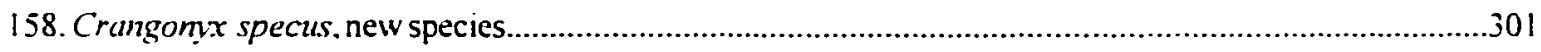

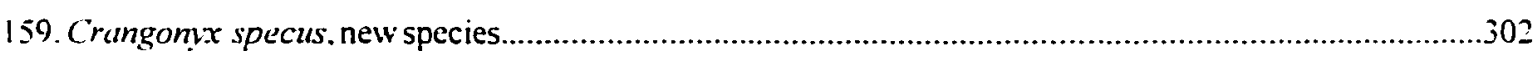

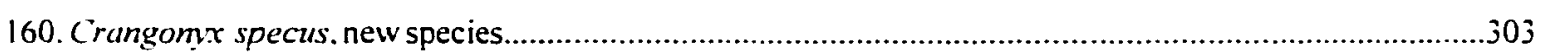

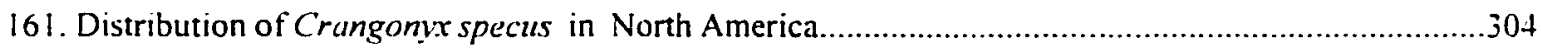

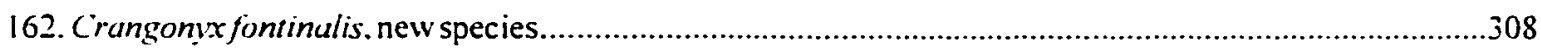

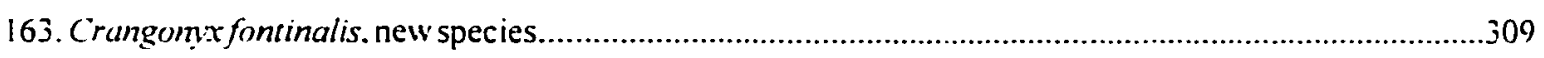

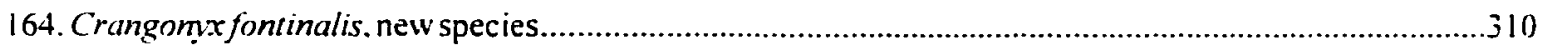




\section{LIST OF FIGURES, continued}

\section{FIGURE}

PAGE

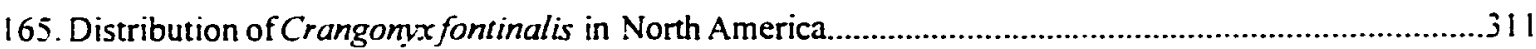

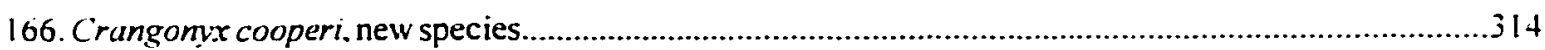

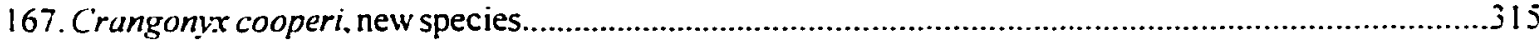

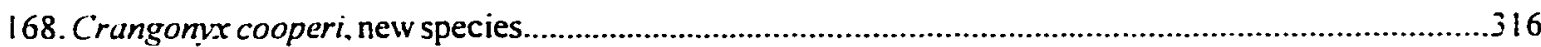

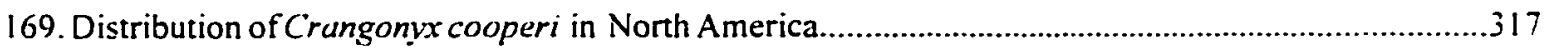

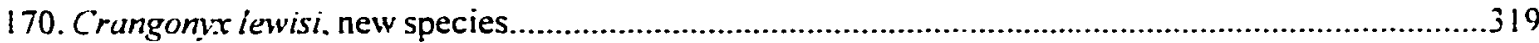

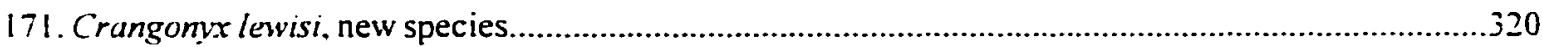

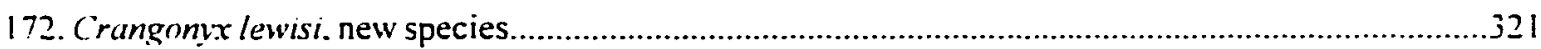

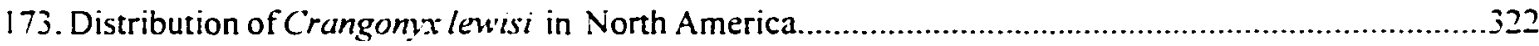

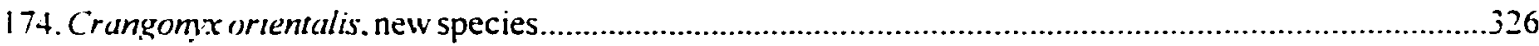

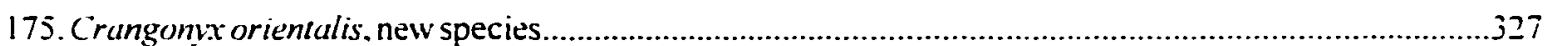

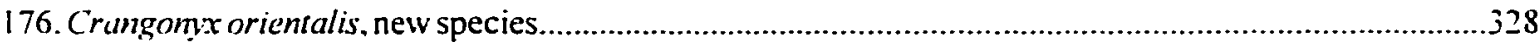

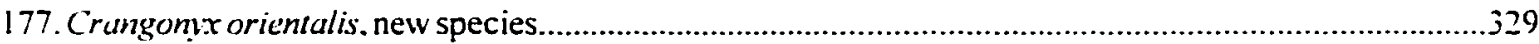

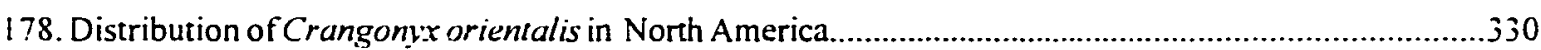

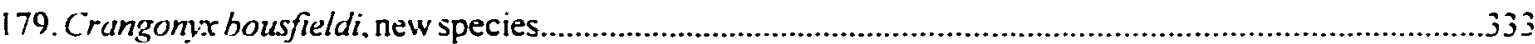

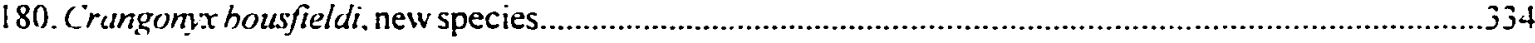

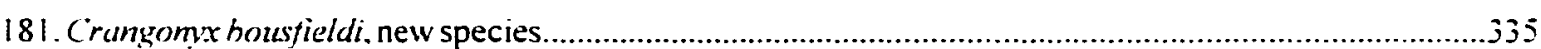

182. Distribution of Crungonyx housfieldi in North America.................................................................330 


\section{CHAPTER I}

\section{INTRODUCTION}

Amphipod crustaceans are one of the largest groups of the freshwater invertebrates with respect to number of species and overall biodiversity. Eleven families and 19 genera are represented in the freshwaters of North America. This study revises the systematics of the freshwater amphipod genus Crangonx according to modern standards.

Crangomyx belongs to the family Crangonyctidae, the largest family of freshwater amphipods in North America. Of the two largest genera of Crangonyctidae. Stygobromus and Crangony, Crangonx is the least studied. Holsinger (1972) first reviewed the taxonomy of this genus. with a key to the North America species and detailed geographic distributions for each species. He established several species groups based on morphological similarity and also identified important taxonomic problems. He found there were about 20-25 undescribed species and that the taxonomic status of some of the described species was not clear. Many species were poorly described and difficult to identify. Knowledge of individual and population variability was incomplete. Several years later. Holsinger (1977) gave a much more detailed diagnosis of this genus, listed all known species of Crangomy and discussed its distribution and ecology. Many problems and questions about Crangonyx still had not been solved. For example: How many species of Crangonyx occur in North America? What is the phylogenetic relationship among Crangomx species?

A complete taxonomic revision of Crangonxx addresses the remaining questions regarding their taxonomy, phylogeny and biogeography. There are other cogent reasons for this study. Crangonux has more epigean species than any other freshwater amphipod genus in North America. and occurs in a wide variety of freshwater habitats. This genus may be important for environmental monitoring. and gaining a better understanding of the ecology of freshwater communities. The study of biodiversity in Crangonvx is also important for biospeleology, because seven described species occur in caves and several newly recognized species also inhabit subterranean groundwater. This study will also try to answer the following biospeleological questions: What are the relationships among surface and subterranean species? Did the subterranean species evolve, under climatic stress. by exploiting newly available environments. or by some other mechanism? The rapid deterioration of freshwater habitats and concomitant loss of biodiversity requires us to describe the invertebrate fauna of North America as quickly as possible. This study. combined with the works by Holsinger on Stygobromus, will give us a much more complete understanding of the systematics, zoogeography and phylogenetics of crangonyctid amphipods.

Forty-two North American (north of Mexico) species of Crangomx are recognized and covered in this study: 24 of them are new to science. All species are described or redescribed. utilizing external morphological features. Phylogenetic trees are built using computer programs (PAUP. Hennig86. MacClade) with a discussion of Crangonvx phylogeny and character evolution. A detailed distribution 
map is given for each species with an interpretation of biogeography. The evolution of subterranean species is discussed in some detail.

\section{REVIEW AND HISTORICAL PERSPECTIVE}

The genus Crangomyx was established by Bate in 1859 for the subterranean species Crangonx subterranezs. which was found in a well at Ringweed. Hampshire, England. Bate (1859) gave the following generic diagnosis: "Like Gammarus, but, not having fasciculi of spines upon the posterior segments of the pleon, and having the posterior pair of pleopoda unibranched. Telson single." Forty years later. Stebbing (1899) thought Crangomyx did not have a small inner ramus on the third uropod according to Bate's description of the type species, so he erected Eucrangonyx for species with a small inner ramus on the third uropod and assigned to it those species of Crangonyx with this structure. Schellenberg ( 1934$)$ solved the problem of Crangomx vs. Eucrangonvx, when he found that the specimens of English Crangonyx subterraneus. contrary to the claim of Bate, possess a rudimentary inner branch of the third uropod. Thus. Stebbing's genus Eucrangomy is a junior objective synonym of Crangony. Because Bate gave just two diagnostic morphological characters of Crangonyx (one of them actually wrong), many nonCrangonyx subterranean freshwater species were assigned to Crangonx but later removed to other genera.

In his comprehensive review of the family Crangonyctidae. Holsinger (1977) summarized Crangonix and gave a thorough generic diagnosis. Although he listed 25 species of Crangonx. he pointed out that the four Asiatic species are very questionable members of the genus. Three of them $(C$. schizurus. $C$. setiferis. and $C$. shimizul) are now assigned to other genera, while the status of $C$. arsenjevi. although probably a species of Crangonyx. cannot be definitively determined (see Holsinger. 1977 for more detailed explanation). The following paragraphs give a brief history of the description of Crangomix species and their discovery (although some species were first assigned to Eucrangomx).

The first described species of Crangonyx was C. subterraneus. described by Bate for specimens from a well in England. Smith (1871) described the second species C. gracilis from Lake Superior. Packard (1881) described $C$. antennatus from Nickajack Cave, Marion County. Tennessee. Smith (1888) described C. packardi from a well in Orleans. Indiana. Embody (1911) described Eucrangonvx serratus from a site near Ashland. Virginia. Derzhavin (1927) described E. arsenjevi from springs in the Ussury River basin of extreme southeastern Russia, but as noted above its taxonomic status is unclear. Borutzky (1928) described C. chlebnikovi from a cave in the Ural Mountains of Russia. Schellenberg (1935) described C. paxi from an artificial gallery (mine shaft) in the Klesnica Vally near Kletno in the Sudeten Mountains of southwestern Poland (then in Germany). Hubricht and Mackin ( 1940) described three new species of Eucrangomy, and Hubricht (1943) later moved them to Crangomx. These species include C. forbesi from Missouri. C. obliquus from Arkansas, and C. shoemakeri from Washington. DC. Ellis (1940) described C. ric/mmondensis from a swamp in South Carolina. Shoemaker (1941) described C. hobbsi from caves in 
Florida and later (1942) described $C$. dearolfi from caves in Pennsylvania. Hubricht and Harrison (1941) described $C$. occidentalis from the state of Washington. but Bousfield subsequently (1958) suggested that it was a subspecies of $C$. richmondensis. Hubricht (1943) described $C$. anomalus from springs in southern Ohio and northern Kentucky. Bousfield (1958) described C. minor. C. pseuodgracilis. C. rivularis. and $C$. setodactylus from eastern North America. He described C. alpinus from Oregon (1963a) and C. floridanus and C. grandimanus from Florida (1963b). The most recent description of a species was of Crangonix aherrans by Smith (1983) from Massachusetts.

Of the 23 species presently assigned to the genus Crangonvx. 19 are found in North America. three species (all subterranean) are found in Europe. and only one species. C. arsenjevi. is found in Asiatic Russia (poorly described. although the drawing of uropod 3 is diagnostic for Crangomx [see Derzhavin. 1927]). I recognized 18 of the 19 described species from North America in the present study. Crungomx aipinus proved to be a synonym of $C$. richmondensis. Moreover no subspecies of $C$. richmondensis are recognized by me (see remarks section of C. richmondensis description). A total +2 species are treated in this study, 24 of which are newly described and 18 are completely redescribed and figured except $C$. aberrans.

\section{OBJECTIVES}

There are three objectives in this study:

(1) Alpha-level taxonomy: description of new species and redescription of the old species to revise the taxonomy of Crangony in North America based on all available collections and all pertinent literature.

(2) Phylogenetic analysis: utilization of the taxonomic data in cladistic analyses to construct a phylogeny of Crangonyx:

(3) Biogeographic analysis: clearly define the patterns of species distribution. correlate these patterns with phylogeny, and evaluate extrinsic barriers. dispersal limits and other geographic and geological considerations.

MATERIAL AND METHODS

Material

Specimens came primarily from the research collections of J. R. Holsinger and by loan from the Vational Museum of National History (Smithsonian Institution). Several other institutions with smaller collections also made their specimens available. Almost all Crangonvx collections reported in the literature from North America were examined. There included about 2240 collections. each containing 1-100 (or more) specimens. Types of all known species from North America are deposited in one of three places: 
nine in the Nation Museum of Natural History under the catalog numbers of the former United States National Museum (USNM); eight in the Canadian Museum of Nature (except the holotype of $C$. pseudogracilis, which cannot be found in their collections and is possibly lost): two in the Museum of Comparative Zoology at Harvard University. although only one could be found and examined (the holotype of C. alpinus cannot be found and is possibly lost).

The following is a list of the institutions or research collections where specimens of Crangomx are deposited. The abbreviations that follow each listing are used throughout the text.

American Museum of Natural History (AMNH)

Canadian Museum of Nature (CMN)

Florida State Museum (FSM)

Holsinger. J. R.. Department of Biological Sciences. Old Dominion University (JRH)

Vilwaukee Public Museum (MPM)

Museum of Comparative Zoology. Harvard University (MCZ)

National Museum of Natural History of the Smithsonian Institution (USNM):

North Carolina State Museum of Natural History (NCMNH).

All holotypes and many paratypes of new species designated in this paper will be deposited in the National Museum of Natural History. Selected paratypes for new species described from Virginia will be deposited in the Virginia Museum of Natural History in Martinsville, VA. Remaining paratypes are deposited in Dr. Holsinger's research collection. The entire Holsinger research collection will eventually be deposited in the National Museum of Natural History. Under the material examined section for each species. if the deposition of a collection is not indicated for a museum by an abbreviation in parentheses. it is in the Holsinger research collection.

Five species are recorded from municipalities in southeastern Virginia that have undergone name changes because of the merger of cities and counties into larger units with city names. In the "material examined" section for these species. the original county place names are used for all collections made prior to the mergers. These localities are: Norfolk County (now the city of Chesapeake): Elizabeth City County (now the city of Hampton): Warwick County (now the city of Newport News): Nansemond Country (now the city of Suffolk): and Princess Anne County (now the city of Virginia Beach). For collections made after the mergers. the current city name is given. with the original county name in parentheses.

Methods

Morphological analysis and species identification

All specimens in available collections were examined and where necessary compared with the type 
specimens. Specimens were first examined under a dissecting microscope. and then under a compound microscope after slide mounted appendages were prepared if needed. Routine determination can be made with the aid of a dissecting microscope for some species. However, slides must be prepared for accurate identification of most species, as well as for morphological analysis for all species. Prepared slides were examined with the aid of a compound microscope for smaller sized species and for purposes of illustration. Illustrations of important detailed external morphological structures of species of the Crangonytidae are found in Hoisinger's (1972) handbook. Dissecting and slide making techniques were adopted from Holsinger( 1967) and Barnard (1969). I follow Holsinger's (1972) terminology and definitions of external morphological structures.

The results of the morphological analyses will also be used to determine geographic variation. as well as to distinguish individual species, and to establish morphological difference among species. Based on morphological analysis. each species is given a unique morphological diagnosis to establish its specific status. Some species proved to be synonyms of others. whereas some collections proved to contain more than one species. Also new species were found in both new and old collections. Every collection was determined to species level and either assigned to species already described or. if found to be undescribed. to a new species. Keys to all species were constructed.

In comparison with many arthropod groups. amphipods are taxonomically difficult because the genitalia are soft finger-like processes that cannot be used for identification. However. every external body part has some diagnostic value and $\mathrm{I}$ used a combination of characters to identify species. Although this procedure generally works well. it may also cause some problems as pointed out below.

As stated by Holsinger (1967), there are two major problems inherent in amphipod taxonom:: (1) continuous growth and development through successive instars. and (2) variation in size and proportion of a number of taxonomically important characters in older animals. Another problem is the seasonal changes of selected diagnostic characters. Geographic variation is also a problem. I used sexually mature males and females whenever possible as a basis for morphological analysis. description and illustration.

It is easy to distinguish between mature females and males of species of ('rangonx. Mature males have a pair of soft finger-like penes on the ventrum of the 7 th pereonite. calceoli on antenna 2. and a curled or laterally deflected outer ramus of uropod 2, which is often reduced in size. Mature females have brood plates (with long marginal setae when fully developed). In some species. however. immature females may have penes and/or calceoli and it is sometimes difficult to distinguish immature individuals. I used the following criteria: if a specimen has penes but also brood plates (in most cases the brood plates have not completely developed), it is assigned as a female. All specimens lacking the secondary sex characters described above are considered juveniles. 
Description and illustration

Every species is given a detailed description and drawn. The format is based on Holsinger's (1978) monograph on Stygobromus. The drawings were made with the aid of a drawing tube (camera lucida) mounted on a Leitz compound microscope. For each species, a specimen of each sex was dissected and the appendages mounted on slides according to the methods described by Holsinger (1967).

The following appendages of the female were drawn: antenna $I$ and 2. the gnathopods ( $I$ and 2 ). part of pereopod 3. pereopod 4. part of pereopods 5-6. pereopod 7. all pleonal plates, all uropods. telson. and the mouthparts. The antenna 2. gnathopods ( 1 and 2), pereopod 7. uropods. and telson were drawn for males.

Phylogenetic analysis

The purpose of this aspect of the research was to explore the phylogenetic relationships of species of Crungonyx using cladistic methods in accordance with the principle of maximum parsimony. Maximum parsimony methods search for minimum-length trees--trees that minimize the amount of evolutionar: change needed to explain the available data under a prespecified set of constraints upon permissible character change (Swofford. 1991). Another important phylogenetic criterion is that monophyletic groups can only be defined on the basis of synapomorphies (Hennig. 1966).

Many crustacean studies including this one have shown that at the species level. cladistic studies cannot achieve well corroborated phylogenies. Three main problems may prevent reliable results: (1) the abundance of homoplasies. (2) difficulty in finding useful outgroups. and (3) the uncertainties of polarities.

Holsinger ( 1986) first gave a hypothesized phylogenetic relationship for the extant genera of Crangonyctidae and later refined it (Holsinger, 1994) using cladistic methodology. The resulting cladogram of Holsinger (1994) was used as a reference in this study. The method of outgroup comparison for polarizing characters given by Maddison et al.(1984) was followed. Based on Holsinger's ( 1994) result. Synurella was used as the sister group of Crangonyx. Further removed outgroups consisted of Strgonyx. Stigohromus and Bactrurus.

Cladistic analysis results in assumptions about synapomorphs. which in turn define nested subsets of species within the genus. Characters used in the analysis were chosen on the basis of morphological analysis of species. Autapomorphies were used to establish (anagenetic) lineages but deleted from the cladogram. A character data matrix of Crangonyx was then established and used in the computer programs to construct cladograms. Phylogeny was generated using PAUP 3.0 (Swofford, 1991) and also tested using Hennig86 (Farris, 1988). The branch-and-bound algorithm in PAUP 3.0 is too slow to be useful with my data set. Instead. I used the heuristic search option with TBR branch swapping and random (50 replicates in the final analysis) addition of taxa. Two variations of parsimony methods were tried: Wagner parsimony. 
which permits characters to revert freely to the plesiomorphic state. and Fitch parsimony. which permits multistate characters unordered and any character state can transform directly into any other state.

MacClade 3.0 (Maddison and Maddison. 1992) was used for data and tree handing. and for computation of various tree statistics.

The most parsimonious cladogram was selected on the basis of parsimony principles. After the cladistic analysis. several monophyletic subgroups of Crangonvx were established and a clear picture of species relationship within Crangonvx emerged. Hypotheses about speciation and biogeographic pattems were developed by combining phylogenetic data and geographic distributions of species.

\section{Biogeography}

All locality records for each species were plotted on distribution maps. Interpretation of these results was utilized to explain the origin and distribution patterns of species of (rangonx. A attempt was made to determine the relationship between species and the limits of their geographic distribution. As a result. I was able to determine that some species are endemic to a given area. whereas others have much broad distributions which do not correspond to a specific area. Based on these analysis. I attempted to determine if present distributions are the result of vacariance, or dispersal, or a combination of both (Humphries and Parenti. 1986. Myers and Giller. 1988). In some instances. attempts were made to relate splits on the cladogram to certain climatic and/or geological changes that might have influenced geographic isolation. 


\section{CHAPTER II}

\section{MORPHOLOGICAL ANALYSIS OF CRANGONYY CHARACTERS}

The following is a list of characters that resulted from morphological analysis. They are used in species identification. description. and cladistic analysis.

Female:

Body length.--This is a measurement from the front of the head to the end of uronite 3 (excluding antennae. telson and uropod 3). Since intraspecific variation in size occurs between generations and geographically widespread populations. differences in this character. especially minor ones. are of limited diagnostic value. However. it is important for comparative purposes.

Head region.--For most species, the lateral lobe of head is broadly rounded anteriorly without an inferior sinus. For a few species. especially $C$. hobbsi and $C$. grandimanus, the lateral lobe is slightly concave with a distinct inferior sinus as seen in typical Stygobromus species.

Eyes can be very large and ovate or irregular in shape to very small and rounded for surface species. or reduced to a few specks or totally absent for stygobiont species. The presence or absence of eyes and their shape and size are very good diagnostic characters for some species. but they are also variable in others. especially for species with both surface and subterranean populations. The eyes of subterranean populations are reduced in different degrees. This can be easily observed in $C$. setocluctrlus and $C$. floridanus.

Antenna 1.--Peduncular segment $\mathrm{l}$ is always stout and slightly longer or subequal to peduncular segment 2. which is about 1.5 times longer than peduncular segment 3 . However in $C$. grandimamus peduncular segment 2 seems elongated and about 2 times longer than peduncle 2 . The number of segments of the tlagellum varies between large and small specimens and different populations and hence has little diagnostic value. But it is interesting to note that for most species the range of the number of flage:llar segments is from 20-27. For a few species. such as $C$. dearolfi. $C$. grandimanus. $C$. forbesi the number of segments may reach 36 . For them the longer flagellum is a diagnostic character.

Antenna 2.--The length and distribution of marginal clusters of setae on peduncie segments 4 and 5 has no diagnostic value for most species. However, they are can be very densely distributed and have little variation for a few species such as $C$. dearofi and $C$. forbesi. Peduncular segment + is slightly longer than peduncle 5 except in $C$. grandimanus, in which it becomes much longer and is a diagnostic character for that species.

Mandibles.--The terminology used to describe the setae configuration of segment 3 of paip is according to Stock (1974). The presence (or absence) and number of A-setae and B-setae is diagnostic of species groups. Three states can be recognized: with several A and several B setae. with several A and $1-2$ 
$B$ setae and with only $1-2$ A setae.

Maxilla 1.--The number of apical plumose setae of inner plate is a diagnostic character of species group and species. Although the number can vary between specimens, the range of the variation was found to be very small: $1-2$ less or more setae. The number is from 3 in some species to more than 10 in others.

Maxilla 2.--The number of plumose setae on the inner margin of inner plate is a very good diagnostic character of species groups and species. Like the apical plumose setae of the inner plate of maxilla I. the intraspecific variation is very small. The number is from $I$ in some species to more than 10 in others. In many species the plumose setae are in an oblique row.

Maxilliped.--The outer lobe of maxilliped has a row of small slender spines on the inner margin but the number is variable. For most species these spines are pectinate but for a few species $(C$. dearofi. $C$. hubrichti and $C$. serratus) they are not. Inner lobe apical setae and spine shape and number are also variable. The outer lobe is distinctly higher than the inner lobe. except in $C^{\circ}$.hohbsi. in which it is just slightly higher than the inner lobe.

The ratio of the nail length versus the dactyl length of the palp is diagnostic for species groups. The range of the ratio is from 0.17 to 0.50 . but for most species it is about 0.33 . The number of setae on the inner margin is highly variable in some species. although it is about $2-3$ for most species. or $4-5$ for others. In $C$. forbest it is always 6-9. Besides setae. $3-4$ spine teeth are also on the inner margin of the dactyl in $C$. insolitus. The number of setae on the outer margin of the dactyl is always 1 . except for $C$. dearolfi which has about 4.

Gnathopods I and 2.--The shape and size of the propod and carpus. shape of the spine teeth. and the arrangement of superior medial setae are very good diagnostic characters of species groups and species. Three states are easily recognized: (1) The propod is broader and longer and 2 or more times larger than carpus. the palmar margin is strongly oblique and longer than the posterior margin and armed with "normal" notched spine teeth. the anterior margin is always more than 2 times longer than posterior margin. and most superior medial setae are inserted in groups of setae 2-5. The posterior margin of the carpus is short and always less than half as long as the anterior margin. (2) The propod is slightly broader than the carpus. which is $1 / 2$ to $3 / 4$ the length of the propod, the palmar margin is always slightly oblique and shorter than the posterior margin and armed with "normal" notched spine teeth or simple spine teeth. the anterior margin is not significantly longer than the posterior margin. and the superior medial setae are singly inserted. The posterior margin of the carpus is always longer than half the length of the anterior margin. (3) The propod and carpus are both long and slender, the same width as the other segments of gnathopods. the carpus may even be longer than the propod. The palmar margin is much shorter than the posterior margin and armed with simple spine teeth or symmetrically notched. slender spine teeth. The posterior margin of both propod and carpus are armed with many groups of very long setae.

The kind of spine teeth and their arrangement on the defining angle are strongly diagnostic for species groups and species but also very difficult to observe. The notched-serrate spine tooth is found there for 
many species but absent for others. For some species at the end of the outside of the defining angle there is a large strongly notched or simple spine tooth.

The palmar margin of the propod is straight. convex or concave. The posterior margin of the propod always bears several groups (3-7) of setae, but in $C$. forbesi the number of groups is about 10 .

The dactyl has one tooth at the base of the nail but it may have more teeth along the inner margin. a diagnostic character of the shoemakeri groups. The number of setae on the outer margin of the dactyl is diagnostic for species.

Pereopods 3-7.--The number of setae along the coxal margins are described and are good characters for some species. The posterior margin of the bases of pereopod 5-7 have serrations that range from very shallow (e.g. C. anomalus) to deep (e.g. C. richmondensis) to very deep (e.g. C. serratus) and is characteristic for many species. The number of serrations is diagnostic for some species.

The number of setae on the anteroproximal margin of the bases of pereopod 5-7 is highly variable but in a few species there are no setae here. which is a characteristic for them. The posterior margins of the bases of pereopods $5-7$ in C. anomalus are greatly expanded. which easily distinguishes it from other species.

The coxal gills of pereopods $3-6$ are attached to the inside of the coxae. However. that of pereopod 7 actually originates from the thin membrane between the coxa and the basis, thus it is not a true coxal gill as the older literature suggests.

Pleonal plates $\mid-3 .--$ The presence or absence of subventral spines on pleonal plate $I$ and the total number of these spines on pleonal plate 2 and 3 are important characters of species groups and species. The distoposterior comer can be produced and acute or not produced and round (although in some cases the precise condition is very difficult to determine). In some species the corner varies from slightly produced to very produced. The number and arrangement of the setae on the posterior margins are very important characteristics for species. Ventral spines and setae are found in C. insolitus.

Pleopod 1 .--The presence or absence of setae along the posterior margin of peduncle is characteristics of species groups. The number of retinaculae for most species is 2 but it can up to 6 and is a good characteristic of species groups.

Uropods 1-2.--The total number of spines on the peduncle and inner and outer rami are described. The number of spines on the penduncle of uropod $I$ is diagnostic for some species. Tiny basofacial-like spines are found on the peduncie of uropod $I$ in $C$. insolitus. Although this is the first recorded occurrence of this structure in the Crangonyctidae. these spines are much smaller and situated lower on the peduncle than in other groups of amphipods (such as the hadziids). However, because they are unique in Crangontx. they are a useful diagnostic character for this species.

Lropod 3.--The ratio of outer ramus length versus peduncle length is diagnostic for species groups. The number of spine groups on the inner ana outer margin of the outer ramus is characteristic of some species. 
Telson.--The shape of the telson and the number of apical spines are diagnostic for species groups and individual species. The depth of the apical notch or cleft is highly variable in some species but also more constant in others. The presence of dorsal and lateral spines is a characteristic of $C$. aberrans. In $C$. serratus. dorsal spines are found in the middle of the long, deeply cleft telson.

Male:

Body length.- When mature. males are almost always smaller and slenderer than females. This may be due to the fact that the male does not need to carry eggs for development. However in $C^{\prime}$. anomulus some males are longer than the longest females and have the same body shape.

Antenna 2.--Calceoli are present on peduncle segments 4 and 5 and the first several flagellar segments. excepting males of $C$. hobhsi and $C$. caecus. The absence of calceoli is characteristic of these two species.

Gnathopods 1 and 2.--The propod is always much broader and more than 2 times longer and larger than the carpus. but in $C$. hobbsi and $C$. longicarpus the propod is not broader and just slightly longer and larger than carpus, which becomes longer. The palmar margin of the propod is armed with notched spines. except in $C$. haculispina in which the spines are rod-like. The sexual dimorphism of gnathopods is an important diagnostic character for species groups.

Uropod 2.-- Along with comparatively small body size. calceoli on antenna 2 and proportionately larger gnathopods. a sexually dimorphic uropod 2 has proven to be the most important diagnostic character for species. The outer ramus is typically curved laterally to backward in mature males and also often reduced in size. Significant interspecific variation is found in the arrangement of spine shape and number of spines on the outer ramus. For many species it proved to be the only good character for accurate identification. 


\section{CHAPTER III}

\section{PHYLOGENETIC ANALYSIS}

\section{CHARACTERS AND CHOICE OF OUTGROUP}

The cladistic analysis of Crangomx is based on 26 characters (Tables 1. 2). The characters chosen are based on morphological analysis and comparison with outgroup species. which has shown them to have phỵlogenetic significance.

The phylogeny of Crangonyctidae was first investigated by Holsinger (1986) and amended by him in 1994 using cladistic analysis. According to Holsinger's (1994) phylogenetic hypothesis. among the 6 extant genera. Crangonyx. Synurella and Lvurella have a common ancestor by virtue of a single sýnapomorphy: lateral lobe of head broadly rounded with inferior sinus indistinct. Crangonyx is in turn separated from the common ancestor of Lyurella and Synurella by having an autapomorphy: uropod 3 has a very small inner ramus and the outer ramus is longer than the peduncle. On the other branch are three subterranean genera: Bactrurus. Stygobromus and Stygonyx. They are united by five synapomorphies. Pseudocrangonyctidae. which consists of Procrangomix and Pseudocrangonyx and is apparently closely related to Crangonyctidae. is the outgroup in the Holsinger (1994) study. I accept Holsinger's (1994) phylogenetic hypothesis about Crangonyctidae and chose the outgroup of Crangomx based on it. The first outgroup of Crangonlx is Sinurella and the second outgroup consists of Stygobromus and Stygonyx.

Sy:nurella includes 18 species (Holsinger, 1977), and like Crangonyx it occurs in both Eurasia and North America. Three epigean species occur in the eastern United States and specimens of these species were examined. The morphology of Symurella is very similar to Crangonyx. Liturella contains only one species. L. hrrcana. which is known only from two freshwater springs near the southwestern end of the Caspian Sea in the Talish region of Russia.

Since the phylogeny of Symurella and Lyurella was not known at the present time. character states must be extracted from literature for these two genera. Many species of Sinurella and Lizurella are poorly described. and considerable variation of character states exists. The polarity of character states cannot be completely determined by the rules set by Maddison and Maddison (1984). This is not a rare case. The polarity of only a few characters can be positively determined. For other characters, they can be polarized by a program according to the most parsimonious solution.

In my analysis. all 26 characters were initially left unordered. The most reliable criterion for the transformation series is the cladogram itself and the congruence with other characters. The character state transformations are the outcome of the analysis , and not a prior assumption (Swotford. 1991).

Qualitative, discontinuous quantitative and continuous quantitative characters are used in this analysis. Qualitative characters are based on presence or absence. or on differences in shape. Discontinuous 
Table 1. Characters and character states used in the phylogenetic analysis of Crangomx species from North America. Based on mature males and females.

1. Mandible: segment 3 of palp

0 : with more than $2 \mathrm{~A}$ and $2 \mathrm{~B}$ setae

1: with 1-2 A and/or 1-2 B setae only

2. Maxilla $\mathrm{I}$ : number of apical plumose setae on inner plate

$0:>3$

$1: 2-3$

3. Maxilla 2: number of plumose setae on inner face of inner plate

$0:>3$

$1: 1-3$

4. Maxilliped: ratio of nail length: dactyl length of palp

$0: 0.33-0.50$

1: $0.20-0.25$

5. Gnathopod I of female: spine teeth on palmar margin of propod

0 : notched

I: symmetrically notched

2: simple

6. Gnathopod I of female: defining angle of propod with strongly notched-serrate and/or serrate spine teeth 0: present

1: absent

7. Gnathopod I of female: teeth on inner margin of dactyl of propod and I tooth near the nail 0 : with no other teeth

1 : with a row of teeth or I to several teeth

8. Gnathopod 1 of female: propod 2 times or more larger than carpus

$0:$ no

1: ves

9. Gnathopod 1 of female: number of superior medial setae per set

0 : more than 2 setae

I: typically I seta only

10. Male and female with sexually dimorphic gnathopod I

0 : present

1: absent

II. Gnathopod 2 of female: spine teeth on palmar margin of propod

0 : notched

1: symmetrically notched

2: simple

12. Gnathopod 2 of female: propod 2 times or more larger than carpus

0 : no

$1:$ yes 
Table I. continued

13. Gnathopod 2 of female: defining angle of propod with very distinctive notched-serrate and or serrate spine teeth

0 : absent

I: present

14. Gnathopod 2 of female: teeth on inner margin of dactyl of propod and I tooth near the nail 0 : with no other teeth

I: with a row of teeth or 1 to several teeth

15. Male and female with sexually dimorphic gnathopod 2

0 : present

1: absent

16. Gnathopod 2 of female: number of superior medial setae per set

0 : consists more than 2 setae

1: mostly consists I seta only

17. Gnathopod 2 of female: palmar margin with 2 or more rows of spine teeth per side or with more than 20 notched spine teeth per side

0 : absent

I: present

18. Gnathopods of female: carpus slender. subequal or longer than propod

$0:$ no

l: yes

19. Pleopod I of female: many setae along posterior margin of peduncle

0 : present

I: absent

20. Pleopod I of female: number of retinaculae

$0:=2$

$1:>2$

21. Telson of female: number of spines on each lobe

$0:-4$

$1:-4$

22. Gnathopod I of male: defining angle of propod with notched-serrate and or serrate spine teeth

0 : present

1: absent

23. Gnathopod 2 of male: defining angle and palmar margin of propod with the similar spine teeth

0 : yes

1: no

24. Gnathopods of male: spine teeth on palmar margin of propod

0 : notched

1: mostly simple

2. rod like and unnotched 
Table I. continued

25. Outer ramus of male uropod 2 with comb spines

0 : absent

1: present

26. Gnathopod $I$ of male and female: defining angle with I row of spines on inside and 2 rows of non-serrate spine teeth on outside

0 : absent

1: present 
Table 2. Character matrix for the species of Crangonyx from North America.

\begin{tabular}{|c|c|}
\hline Taxa & $\begin{array}{l}\text { Character } \\
00000000011111111112222222 \\
12345678901234567890123456\end{array}$ \\
\hline Sỵnurella & 00000000000000000000000000 \\
\hline aberrans & 10000010100011010000110010 \\
\hline açicularis & 10002000102010010000111000 \\
\hline aka & 10002000102010010000110000 \\
\hline anomalus & 00010101010110101011000001 \\
\hline antennatus & 10000001110100110000100000 \\
\hline baculispina & 10002000102000010100101200 \\
\hline barri & 10002000102010010000111000 \\
\hline bousfieldi & 10000000100010010000100000 \\
\hline castellanum & 10002000102010010000110000 \\
\hline caecus & 10002000102010010000111000 \\
\hline consimilis & 10002000102010010000111010 \\
\hline cooperi & 10000000100010010000111000 \\
\hline cornutus & 00010101010100100011100010 \\
\hline dearolfi & 01110101010100101011000001 \\
\hline disjunctus & 00110101010100101011100001 \\
\hline tloridanus & 10002000102010010000111010 \\
\hline fontinalis & 10000000100010010000100000 \\
\hline forbesi & 00000001010100101011010000 \\
\hline gracilis & 10002000102010010000111000 \\
\hline grandimanus & 01110101010100101011100001 \\
\hline hobbsi & 10002000102010010100111100 \\
\hline hubricliti & $011101010 ? 0100 ? 010111 ? ? ? ? ?$ \\
\hline indianensis & 00010101010100101011111001 \\
\hline insolitus & 00000000110000100011010000 \\
\hline lewisi & 10000000100010010000110000 \\
\hline longicarpus & 10001100101000010110100000 \\
\hline longidactylus & 10002000102010010000111010 \\
\hline minor & 10000010100011010000110000 \\
\hline montanus & 10002000102010010000111000 \\
\hline obiiquus & 11110101000100101011100001 \\
\hline ohioensis & 10002000102010010000111000 \\
\hline orientalis & $10000000100010010000 \backslash 10010$ \\
\hline packardi & 10002100102000010000100000 \\
\hline polustris & 10002000102010010000111000 \\
\hline pseudogracilis & 10002000102010010000111010 \\
\hline richmondensis & 11110101010100101011100001 \\
\hline rivularis & 10002000102010010000111000 \\
\hline serratus & 00000001010100110000100000 \\
\hline setodactylus & 10000010100011010000110000 \\
\hline shoemakeri & 10000010100011010000110010 \\
\hline specus & 10000001010110110000111000 \\
\hline stagnnicolous & 10002000102010010000111000 \\
\hline
\end{tabular}


quantitative characters are based on counts of spines or setae. Continuous quantitative characters are based on relative length of appendages. As in the Notenboom (1988) and Stewart and Griffiths (1995) cladistic studies of freshwater amphipods. ratios and counts are included in this study. As Stewart and Griffiths (1995) stated: "These characters avoided the use of subjective character state definitions. and were needed because of the shortage of usable characters." Like the above two studies. when coding these data. care was taken to identify a clear gap.

\section{RESULTS OF PHYLOGENETIC ANALYSIS AND DISCUSSION}

The Wagner parsimony produced 18 most parsimonious trees and the Fitch parsimony produced 45 trees. The consensus tree of both methods are given (Figs. 1-2). Using MacClade to analyze the character transformation of these two consensus trees reveals only three differences. In Wagner parsimony. for characters 5. 11 and 24. state 0 can only transform to either state I or 2. and states 1 and 2 can transform to each other. In Fitch parsimony. for characters 5 and 11 . state 0 can only transform to 2. and 2 can only transform to 1. for character 24, state 0 can either transform to I or 2 and no other changes are allowed.

For character 5 and 11 . my hypothesis is that the state 0 can transform to 1 and 2 . and no other transformations are allowed. It is similar to that of Wagner parsimony but excludes the possibility that state I can transform to 2 or vice versa. State 2 of character 5 and 11 is palmar margin of female gnathopod propod with slender symmetrical spine teeth. Since only one species. C. longicarpus, which is found only on the Coastal Plain of Virginia and North Carolina. has this character, it is probable that this character is not in the evolutionary intermediate stage between notched spine teeth and simple spine teeth. If this is the case. as Fitch parsimony suggests, many species with this character should be found further inland.

For character 24, the transformation of Fitch parsimony seems reasonable, since both states 1 and 2 are believed to have evolved directly from state 0 . and the species with this state may very well be separated from each other.

Because of these three character transformation differences. we get two different consensus trees (Figs 1-2). Despite the difference, both trees bear many similarities, with several of the same species groups wall defined in both trees. Both trees define the shoemakeri group by characters 7 and 14, the richmondensis group by characters 4.6.8 and 10. and the gracilis group by characters 5 and 11 . Although we do get some well defined species groups, we can see from both consensus trees that phylogenetic relationship between some of the groups and species in these groups still cannot be solved by this analysis. This is the case because of the many difficulties mentioned earlier as being inherent in species level phylogenetics of Crangonyctidae.

Based on the two consensus trees and a prior assumption based on observation of species of Crungomx species. I selected the hypothesis phylogenetic tree of Crungonx species of North America shown in figure 3. The hypothesis tree is one step more than the consensus tree of Wagner parsimony and 


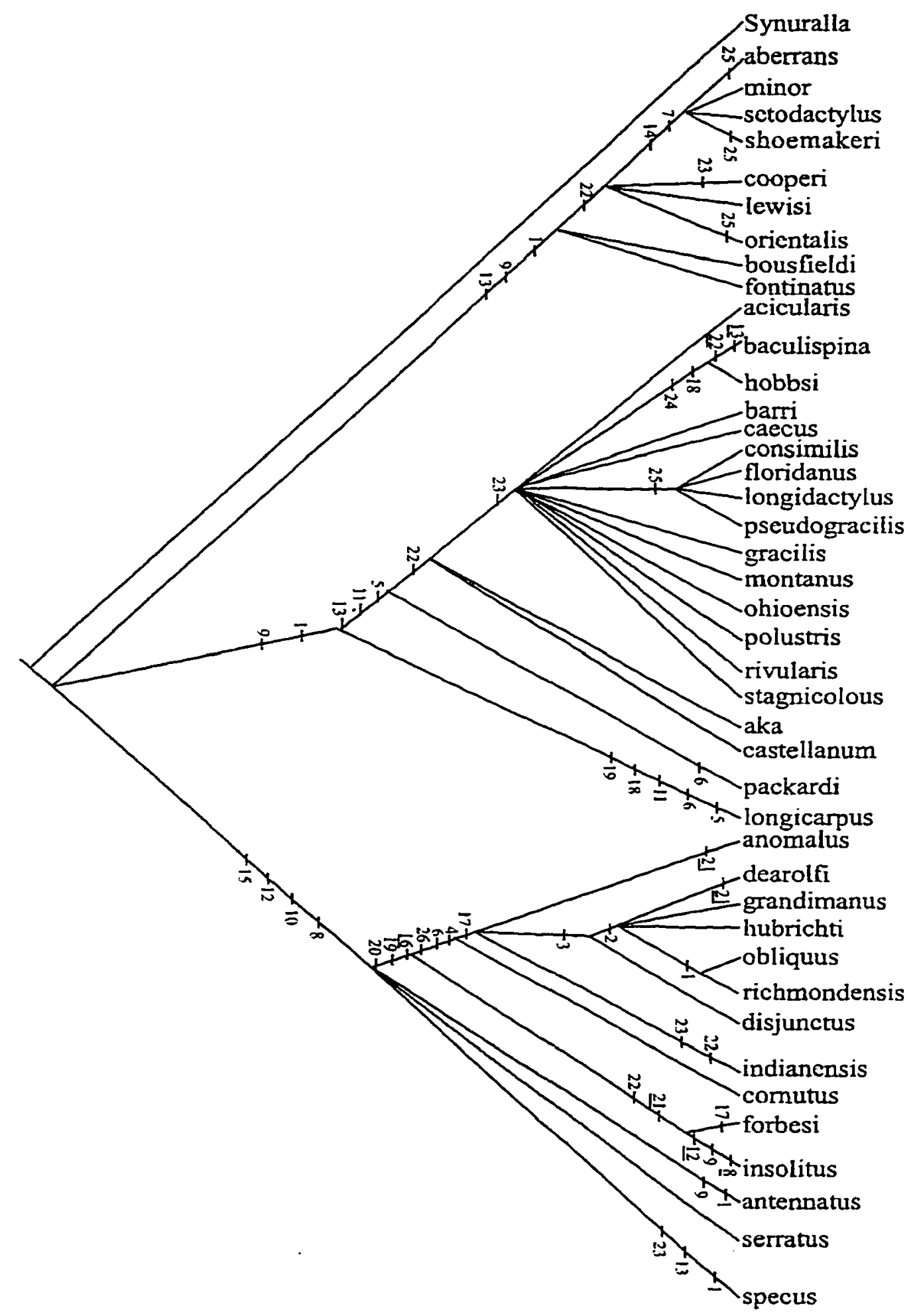

Figure 1.--Consensus tree from cladistic analysis of North America Crangomx using Wagner parsimony. Tree length $(T L)=57: \mathrm{CI}=0.51$. Underlined characters indicate a reversal of that character. 


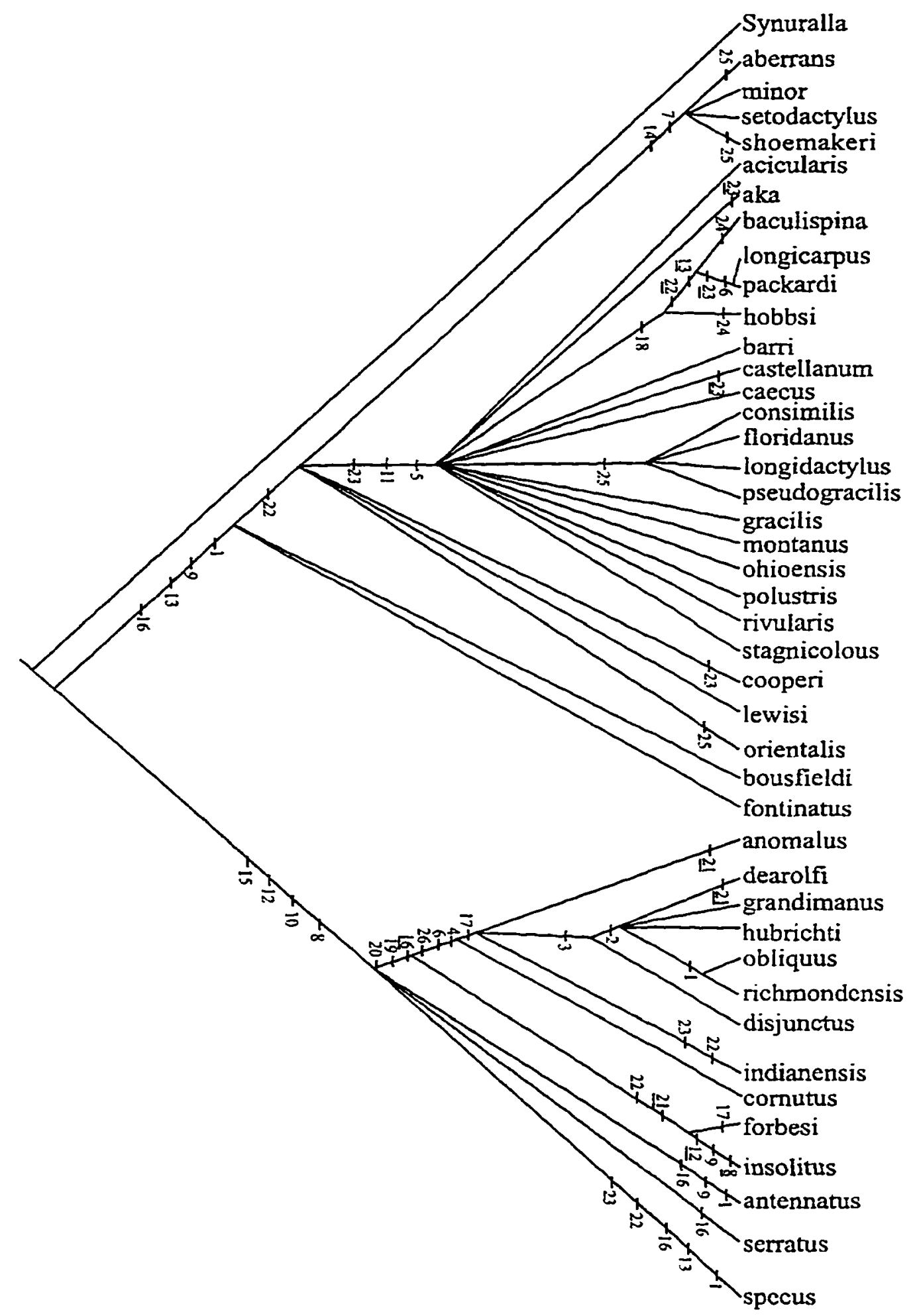

Figure 2.--Consensus tree from cladistic analysis of North America Crangonyx using Fitch parsimony. $\mathrm{TL}=55: \mathrm{CI}=0.53$. Underlined characters indicate a reversal of that character. 


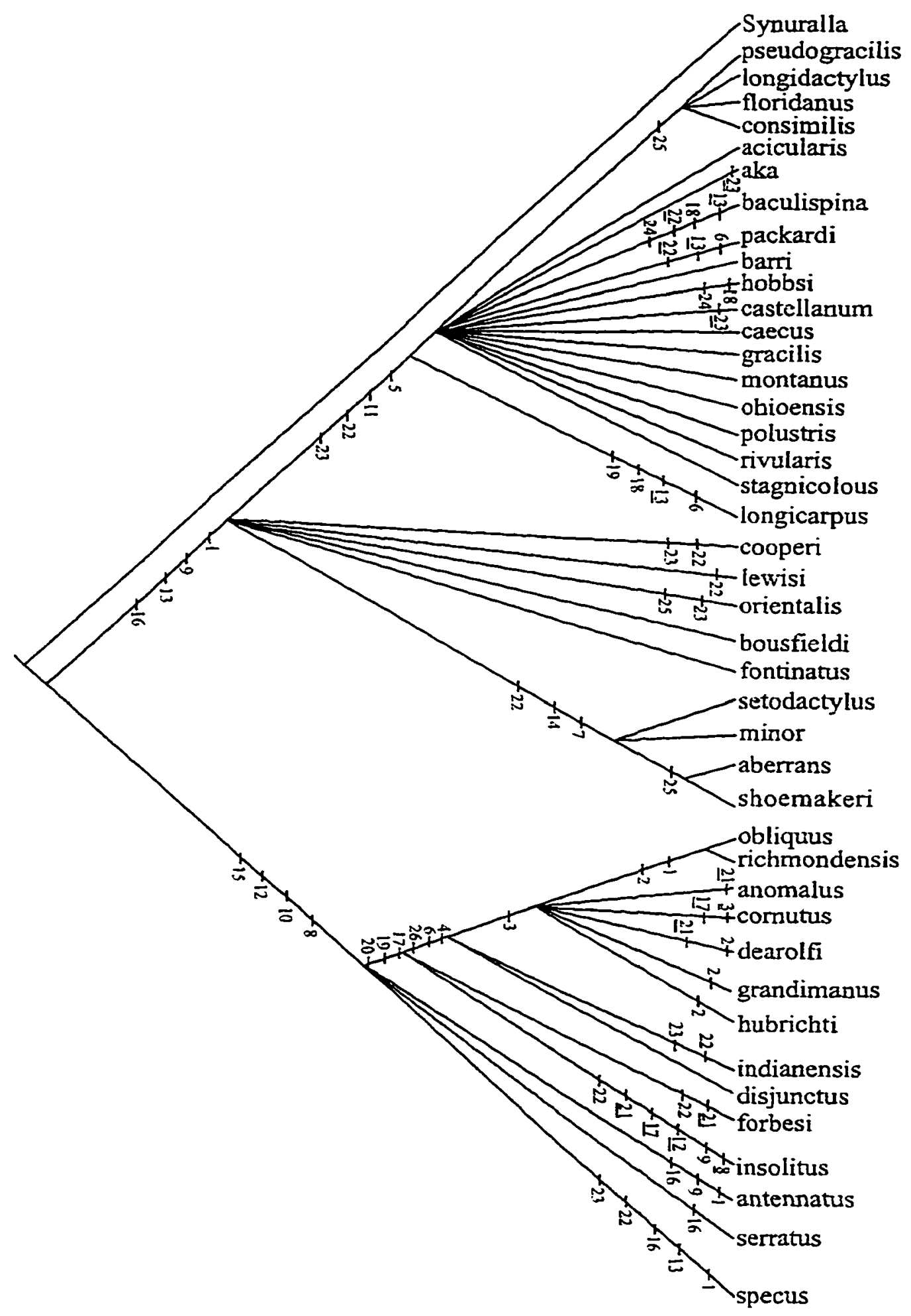

Figure 3.--The phylogenetic hypothesis of North America Crangomy based on the two consensus trees (Figs. 1-2) and the observations of Crangomx species. $\mathrm{TL}=58: \mathrm{Cl}=0.53$. Underlined characters indicate a reversal of that character. 
3 steps more than that of Fitch parsimony. As in the above two consensus trees. only species groups or subgroups can be recognized and the phylogenetic relationships of some groups are not clear.

The first branch. which is defined by characters 8, 10. 12 and 15. includes 3 species groups (insolitus. forhest and richmondensis) and 3 ungrouped species(Crangomx antennatus, C. serratus, and C. specus). These three species group composes a clade. Characters 8 and 12 in $C$. insolitus. which are in the plesiomorphic state. may indicate this species is nearer to the ancestor species of the branch than the hypothesis suggests. However characters 16, 19 and 20 place it near the forhesi and richmondensis groups.

The richmondensis group. which is defined by characters 4 . and 26. is the most advanced and has the second greatest species diversity of all species groups. It contains 9 species and is represented in a wide variety of freshwater environments: it is the most widely distributed group in North America.

The second branch is detined by character 9 and seems to take an opposite evolutionary path. It includes 3 species groups( shoemakeri. longicarpus and gracilis). and 5 ungrouped species( (rangon!x houspicldi. C. cooperi. C. fontinalis. C. lewisi and C. orientulis). The shocmakeri group and the 5 ungrouped species are at the bottom of this branch. They all have notched spine teeth on palmar margins of gnathopods of the female. This is an ancestral character. Morphologicaily they are closely similar and the phylogenetic analysis will not separate them into different higher branches. Instead they all come from the same node.

The shoemakeri group is defined by characters 7 and 14 in both Wagner and Fitch parsimony trees but there is a probability that these two characters may also be independently derived. The distribution of four species of shoemakeri group are not continuous and the species differences are greater than with the five ungrouped species. If future studies indicate that this species group is paraphyletic. we may conclude that parsimony methods may not be the best criterion to reveal the phylogenetic relationship among Crangomx.

The longicarpus species group contains only one known species. C. longicarpus. and is detined by characters 5.6.9. II and 19. It is an unusual species with very slender. symmetrically notched spine teeth on the gnathopods of the female. It is known only from the Coastal Plain of Virginia and North Carolina. It seems to be an evolutionary end point. derived earlier from the ancestor of the gracilis group prior to the wide distribution of the gracilis group species. As more species of gracilis group evolved. it is possible that $C$. longicarpus became extinct in many places and is now found only on the Coastal Plain.

The gracilis group is defined by characters 5.9 and 11 and is the largest species group of (rangomx: it contains 18 species. The species of the gracilis group are all small in size and the palmar margins of the female gnathopod propods have unnotched spine teeth. These species inhabit a wide variety of freshwater habitats and are widely distributed in North America. The morphological difference between many of the species is very small and species identification depends primarily on the structure of male uropod 2. Females of many of these species are almost always alike. The cladistic analysis did not reveal any phylogenetic relationship among the gracilis group species. except for a floridanus subgroup that is defined by character 25. Small morphological differences and the lack of a clear phylogenetic relationship 
may suggest that all species evolved from a widely distributed. common ancestor following geographic isolation. However. an alternative hypothesis is that some of the species may not. by strict detinition. be truly biological species. 


\section{CHAPTER IV}

\section{TAXONOMY}

FAIVILY CRANGONYCTIDAE BOUSFIELD. 1973

Schellenberg (1936) recognized the Crangonyx group of the family Gammaridae. which included 15 genera of freshwater amphipods. Following the work of Schellenberg(1936). several workers discussed this group concept and accumulated more knowledge about it (see Holsinger. 1967 and Holsinger. 1977 for more details). Bousfield ( 1973 ) erected the family Crangonyctidae to encompass many of the genera which had been assigned previously to the Crangonx group. Holsinger (1977) summarized this family and all genera. including a list of species, distribution and ecology of genera and a key to the genera. Holsinger (1977) recognized 6 genera in the tamily Crangonyctidae: Bactrurus. Crangonıx. Lyurella. Palacogammarus. Stygobromus, and Sinurella. Subsequently. Bousfield and Holsinger (1989) described the new genus Stygonx and included a new key to the genera of Crangonyctidae.

Genus Crangonyx Bate. 1859

Crangempx Bate. 1859

Eucrangomx Stebbing. 1899

Diagnosis.--Uronites free: lateral lobe of head broadly rounded anteriorly without inferior sinus or rarely narrowly rounded with weak inferior antennal sinus; eye relatively large. small or absent. First peduncular segment of antenna I subequal in length to second. Antenna 2 of mature male with calceoli except in 2 subterranean species. Outer plate of maxilliped with stiff setae and small pectinate spines along the inner margin. Lower lip with well developed outer lobes and small, typically indistinct inner lobes. Gnathopods: propod of second larger than first: thin and long rastellate setae present on posterior margin of propod. segment 5 and segment 4 (except 1 species with short thick rastellate setae on posterior margin of segment 5). Coxal plates $1-4$ deep. deeper than corresponding body segments. longer than broad. Pereopod 6 longer than 7. Coxal gills rather large, subcvate, present on pereopods 3-7. although modified on 7. Single. median sternal gills present on pereonites 2 and 3. simple paired sternal gills on pereonites 6 and 7 and usually on pleonite 1 . Brood plates of sexually mature female typically rather large in size and expanded distally. Pleonal plates: distoposterior corners usually distinct. often produced and acute: posterior margins typically with I short seta each. but sometimes up to 3 setae. Uropod 2 sexually dimorphic in mature males with outer ramus reduced and deflected laterally and sometimes curled backward. Lropod 3 biramous: outer ramus 1 -segmented, up to 2.5 times length of peduncle. armed 


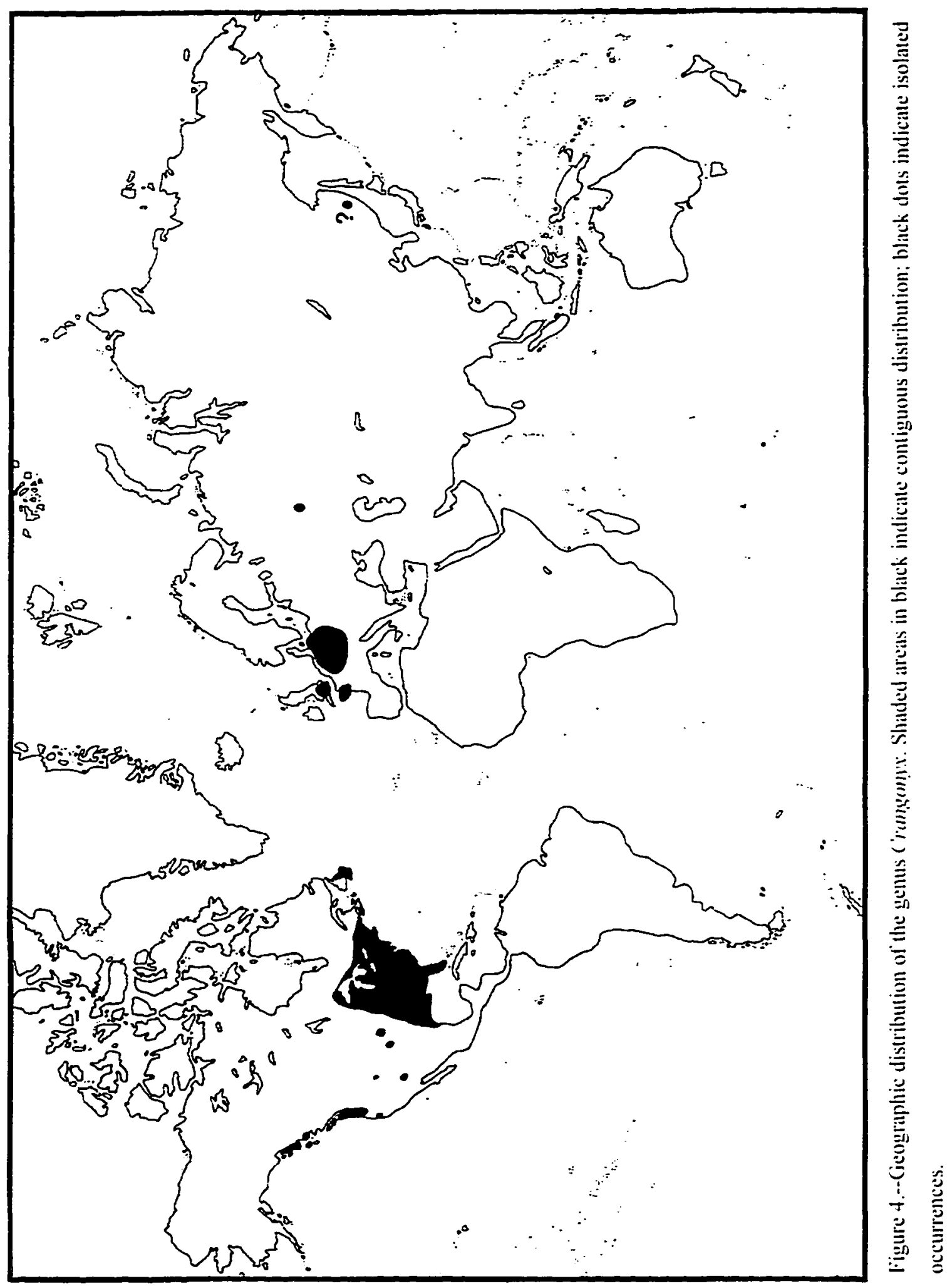


laterally and apically with spines: inner ramus small and scale-like. Telson typically rather short. usually broader than long: apical margin incised from $1 / 4$ to $2 / 3$ distance to base (but seldom more than $1 / 2$ ): apical lobes with few spines: lateral and dorsal margins typically without spines.

Type-species: Crangomx subterraneus Bate. 1859 by monotypy.

Distribution and ecology. --The global distribution of Crangonvx is shown in figure 4 .

Remarks.-- Based on the hypothesized phylogenetic relationship given by Holsinger (1994).

Crungonyx has a close relationship with Lyurello and Synurella: united by a single synapomorphy: lateral lobe of head usually broadly round and with inferior antennal sinus usually indistinct. However. the lateral lobe is more narrowly rounded and there is an inferior antennal sinus in at least a few Crangonx species. This is thought to be an apomorphic character that was derived from the ancestral species with broadly rounded lateral lobe. Crangonvx can be easily distinguished from Lịrella and S!rnurella and other genera of the family Crangonyctidae by the outer ramus of 3rd uropod. which is longer than the peduncle and by the presence of a scale-like inner ramus.

Key to the Species Groups and Ungrouped Species of Crangonıx in North America

(based on mature males and females)

1. Palmar margin of gnathopodal propods of female lined with small. weak simple spine teeth: superior medial setae of gnathopodal propod 2 singly inserted gracilis group

Palmar margin of gnathopodal propods of female lined with distally notched spine teeth: superior medial

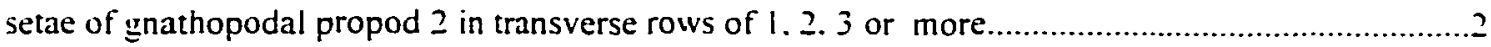

2. Inner margin of gnathopodal dactyls of female armed with blade-like teeth. shosemakeri group Inner margin of gnathopodal dactyls of female without blade-like teeth except one near the nail..............

3. Carpus and propod of gnathopods of female very long: carpus equal to or longer than propod paimar margin of gnathopodal propods of female lined with slender. symmetrically notched spineteeth longicurpus group

Carpus and propod not elongate: carpus shorter than propod: palmar margin lined with thick. non-symmetrically notched spine teeth. 4

4. Bases of pereopods 5-7. posterior margins deeply serrate: telson of female much longer than broad. notched up to $2 / 3$ distance to base. with dorsal spines C'serratus

Bases of pereopods 5-7. posterior margins not deeply serrate: telson longer than broad or border than

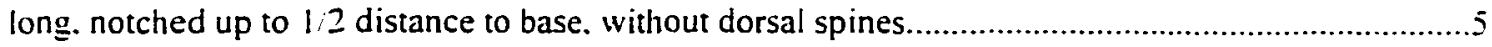

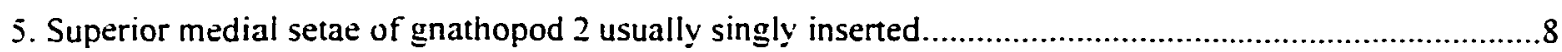

Superior medial setae usually inserted in groups of 2 or more setae..........................................6

6. Gnathopods I and 2: propod more than 2 times larger than carpus. lacking notched-serrate and or serrate 
spine teeth on defining angle.

richmondensis group

Gnathopod $I$ and 2: propod up to 2 times larger than carpus: with notched-serrate and'or serrate spine teeth on defining angle

7. Ventral margin of female pleonal plates with spines and setae: peduncles of female uropods $I$ and 2 of with small spines on ventral side. insolitus group

Ventral margin of female pleonal plates lacking spines and setae: spines absent from the ventral side of

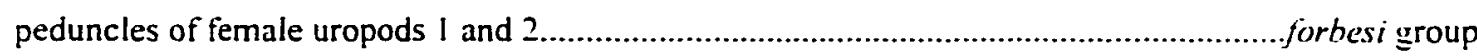

8. Outer ramus of male uropod 2 with comb spines.. C. oricnialis

Outer ramus of male uropod 2 without comb spines 9

9. Posterior margins of pleonal plates with 2-3 setae. and I seta barely above distoposterior corner C. housfieldi

Posterior margins of pleonal plates with I seta located well above distoposterior corner. 10

10. Propod of female gnathopod 2 with I very large spine on outer margin at end of defini angle

Propod of female gnathopod 2 with 1 large and I or more small spines on outer margin at end of detining angle.

11. Outer ramus of male uropod 2 with $3-5$ spines clumped near distomedially. torming a comb spine row: palmar margin of propods of female gnathopods strongly oblique. C. antennatus

Outer ramus of male uropod 2 with spines not distomedial: palmar margin of propods of female gnathopods not very oblique.

12. Outer ramus of male uropod 2 with 5 evenly distributed spines medially. lateral margin with more than 5 spines

C. fontinalis

Outer ramus of uropod 2 of male with $1-2$ spines.

C. specus

13. Outer ramus of male uropod 2 with $1-2$ spines distomedial. lateral margin with more than 5

spines evenly distributed

C. lewisi

Outer ramus of male uropod 2 of male with very thin spines (setal-like)..

C. coopert

\section{The shoemakeri Group}

Diagnosis.--Adult size range ca. 10.0-17.0 mm. Palm of female gnathopod propods armed with large notched spine teeth, superior medial setae singly inserted. Gnathopods 1 and 2 sexually dimorphic: propod of gnathopods 1 and 2 of female not 2 times larger or longer than carpus, inside of defining angle with notched-serrate spine teeth. outside with serrate spine teeth. inside margin of dactyl armed with blade-like teeth forming a row. length of palm shorter than posterior margin. Male gnathopod propods larger and wider. carpus smaller and narrower. propod more than 2 times larger than carpus and much broader. detining angle of gnathopod 1 of male with notched-serrate spine teeth. defining angle of propod of 
gnathopod 2 with same type spine teeth as on palm. palm length longer than posterior margin. inner margin of dactyl lacking teeth. Uropod 3 of female shorter.

Remarks.--The shoemakeri group is composed of four species. with disjunct distributions in New England. Maryland-Virginia. and the east-central US (fig. 5 ).

Key to the Species of the shoemakeri group

1. Inner margin of dactyls of pereopods 3-7 with 2-3 setae: palm of female gnathopod propods not concave C. setoderturis

Inner margin of dactyl of pereopods $3-7$ with I seta: palm of female gnathopod propods siighlty. to distinctly concave.

2. Inner margin of dactyl of female gnathopods with a row of blade-like teeth: telson with apical spines only

Inner margin of dactyl of female gnathopods with few teeth. not forming a row: telson with dorsal andior lateral spines. C. aherrans

3. Outer ramus of male uropod 2 with comb spines C. shoemakeri

Outer ramus of male uropod 2 without comb spines. bearing several short spines only. C. minor Crangonx shoemakeri (Hubricht and Mackin)

Figures 6-10

Eucrangonyx shoemakeri Hubricht \& Mackin, 1940:198, fig. 6 [in part] [type-locality: pools along the Potomac River. $2 \mathrm{mi}$ W of Georgetown. Washington. D. C.]. [Not Mackin. 1941:29]. Crangomx shoemakeri (Hubricht \& Mackin).--Bousfield. 1958:95-96.--Holsinger. 1972:47. figs. 12a. I2c.

19: 1977:252.--Barnard \& Barnard. 1983:435. map 12.--Fitzpatrick. 983:145.--Pennak. 1989:483. [Not Hubricht. 1943:690-691.--Kenk. 1949:48.--Cole. 1957:36. fig. 2f]

Material examined.--WASHINGTON D.C.: Burleith Woods in Georgetown. numerous specimens in approximately 23 collections (USNM) from small streams, springs and bogs. W. H. Ball. N. Blandford. D. Padgett, and A. Pizzini (the majority). 1934-1939: Chain Bridge area near Potomac River. numerous specimens in approximately 20 collections (USNM) from mostly pools and ponds. W. D. Appel. W. H. Ball. G. E. Barnes. G. W. Gill. W. P. Hay. L. Hubricht. A. Pizzini. E. D. Reid. C. R. Shoemaker, and H. Wood. 1894-1941: Wetzels Spring and immediate vicinity (Georgetown), numerous specimens in approximately 34 collections (USNM). L. Hubricht. A. Pizzini (the majority). and A. Shansey: other D. C. localities. including Georgetown. Kenilworth. Montrose Park. National Zoological Park, and Oxon Run. 


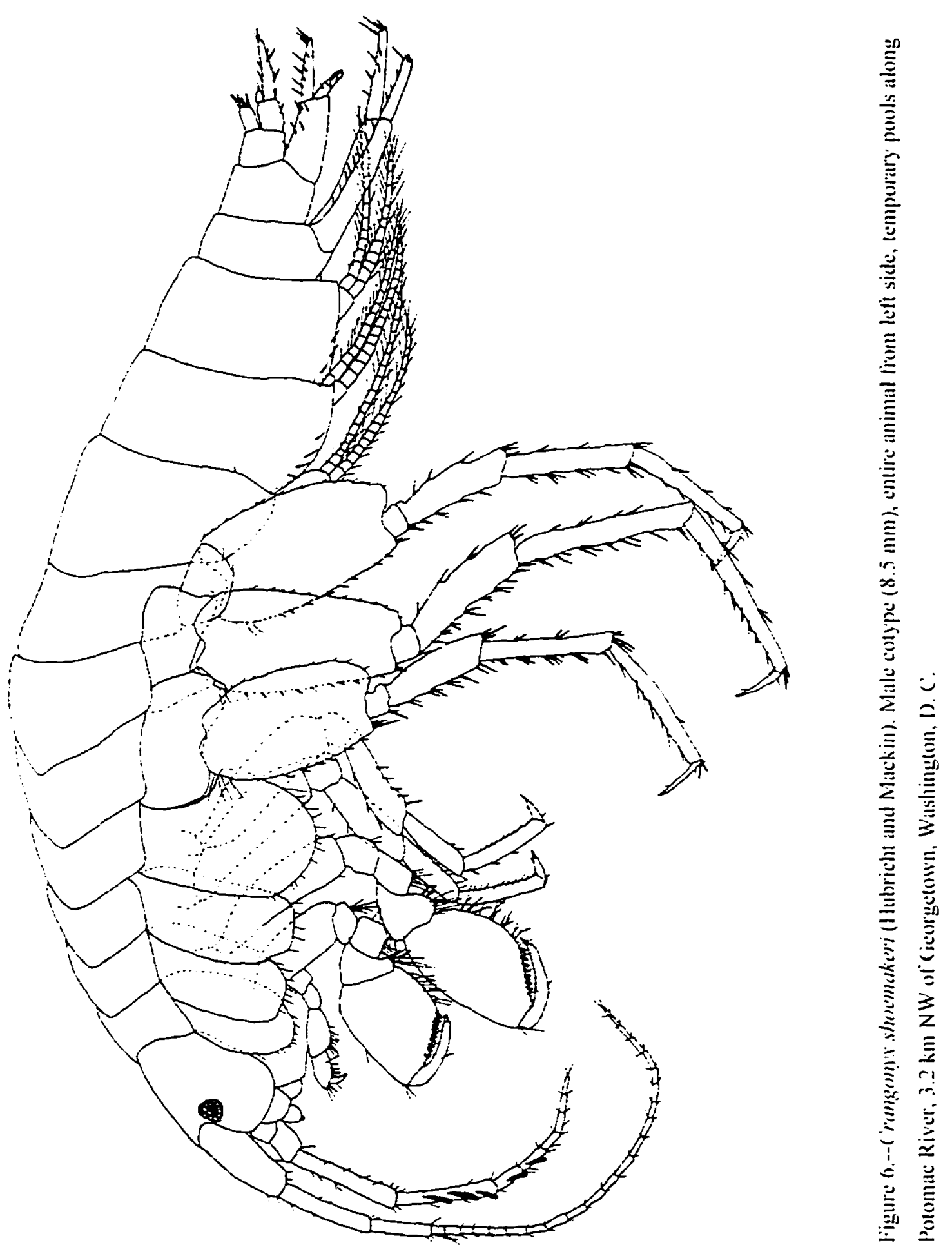




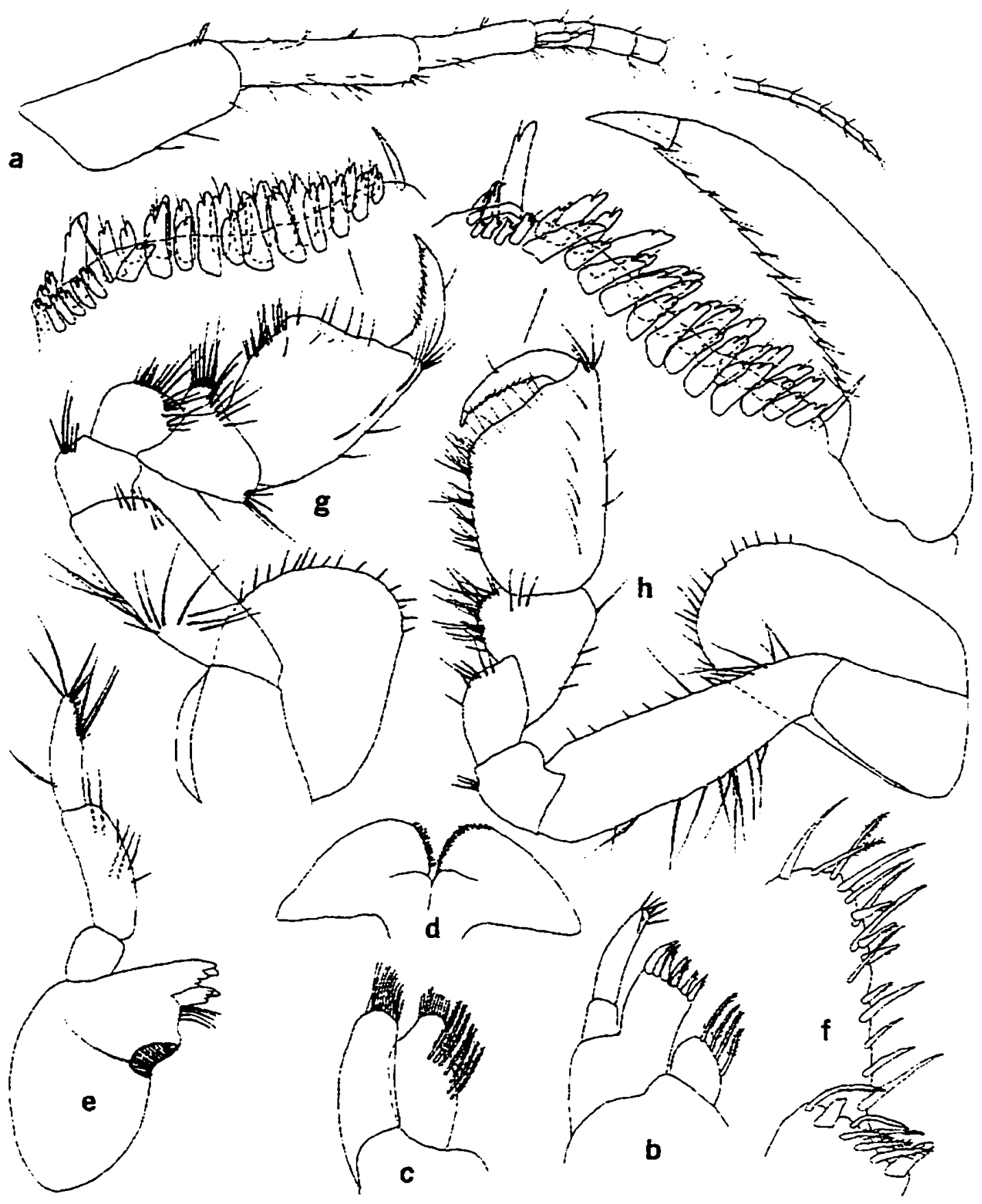

Figure 7.--Crangonux shoemakeri (Hubricht and Mackin). Female cotype (12.5 mm), temporary pools along Potomac River, $3.2 \mathrm{~km} \mathrm{NW}$ of Georgetown. Washington. D. C.: a. antenna 1: b. c. maxillae 1. 2: d. lower lip: e. left mandible: $f$, inner and outer plates of maxilliped (greatly enlarged): g. $h$. gnathopods 1. 2 (palmar margins enlarged). 


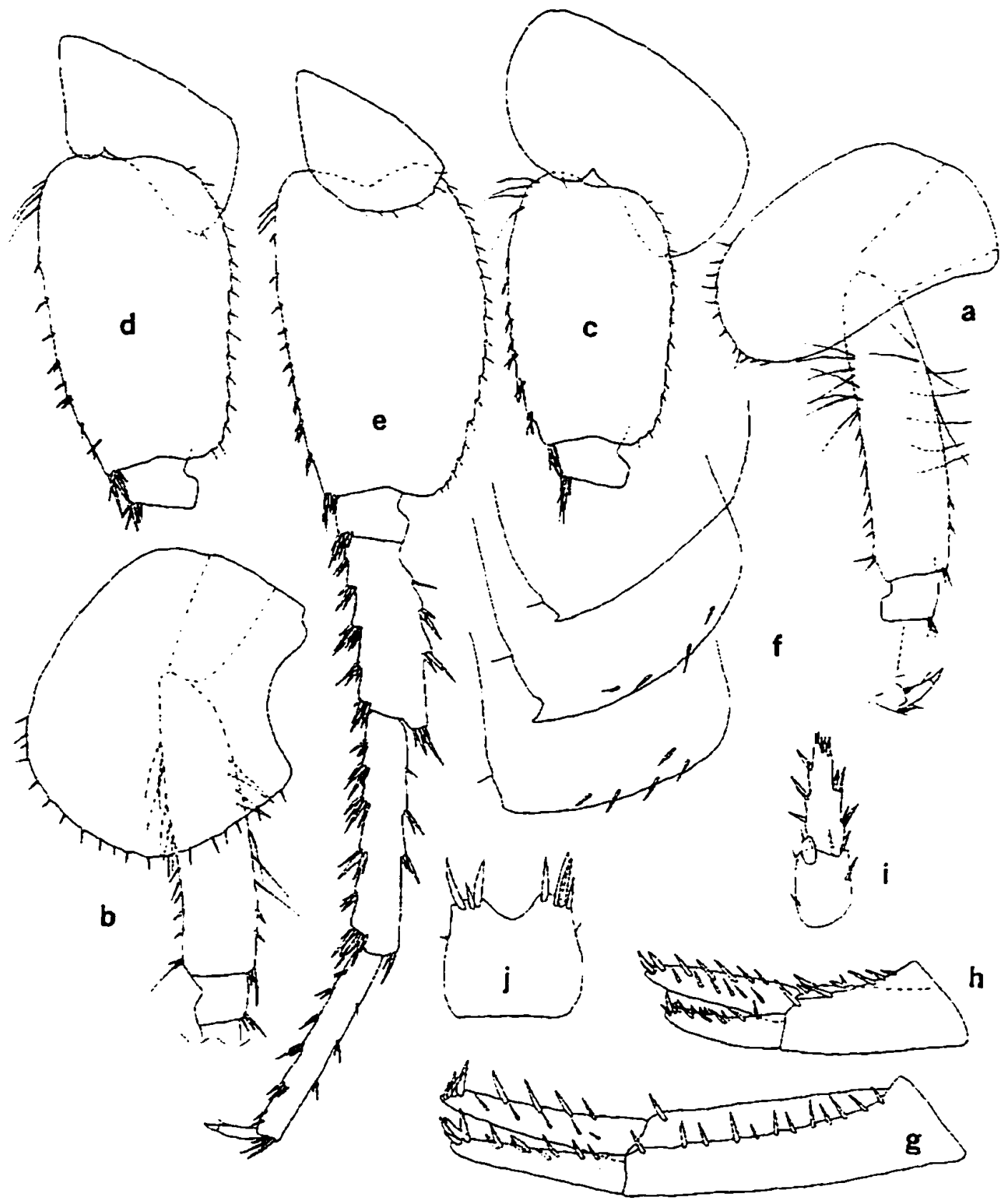

Figure 8.--Crangonvx shoemukeri (Hubricht and Mackin). Female cotype (12.5 mm). temporary pools along Potomac River. $3.2 \mathrm{~km}$ NW of Georgetown. Washington. D. C.: a. b. c. d. pereopods 3. 4. 5.6 (in part): e. pereopod 7: f, pleonal plates; g, h. i. uropods 1. 2. 3: j. telson. 


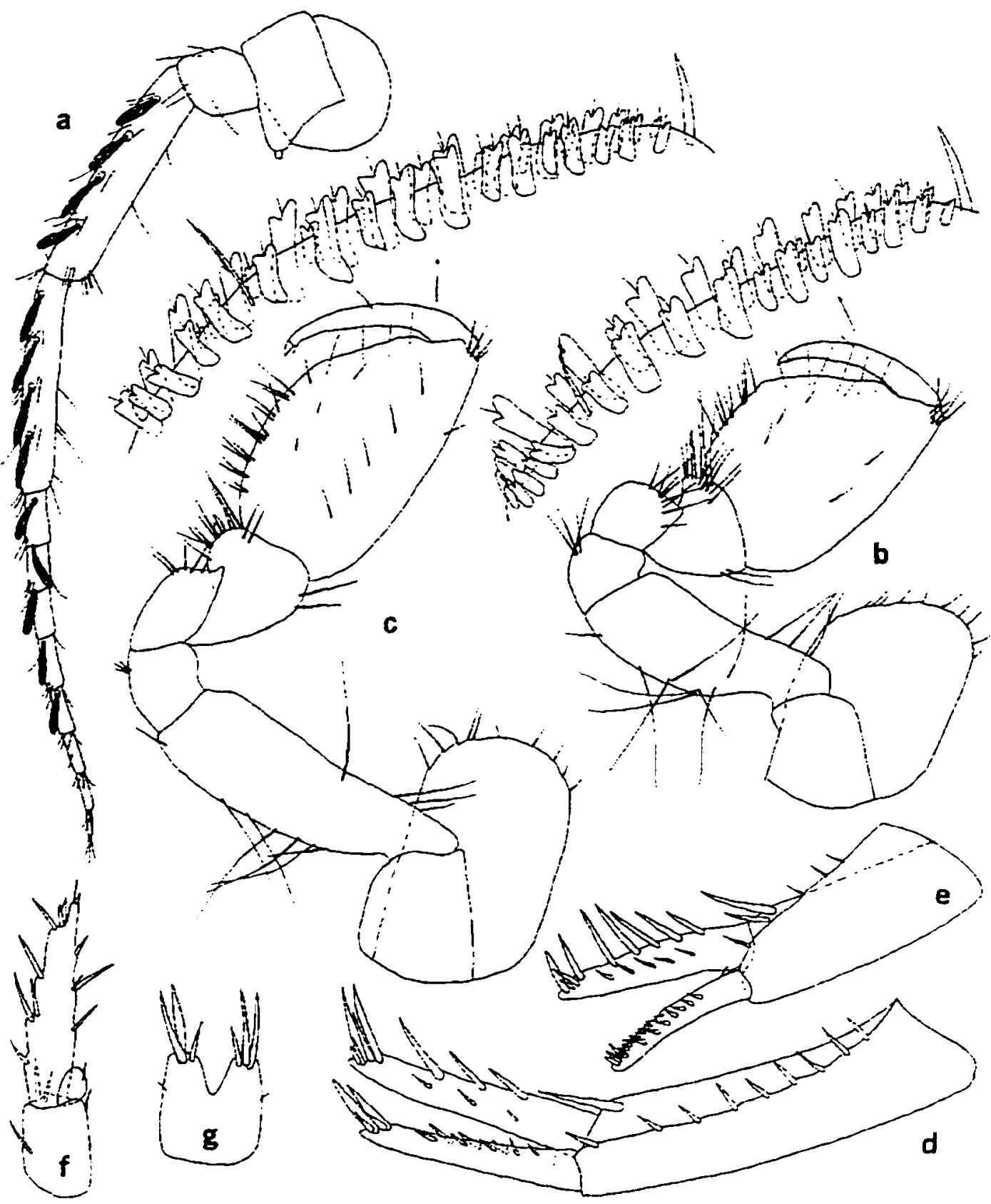

Figure 9.--Crangonvx shoemakeri (Hubricht and Mackin). Male cotype $(6.4 \mathrm{~mm})$. temporary pools along Potomac River, $3.2 \mathrm{~km} \mathrm{NW}$ of Georgetown. Washington. D. C.: a. antenna 2: b. c. gnathopods 1. 2 (palmar margins enlarged): d. e. f. uropods 1, 2. 3: g. telson. 


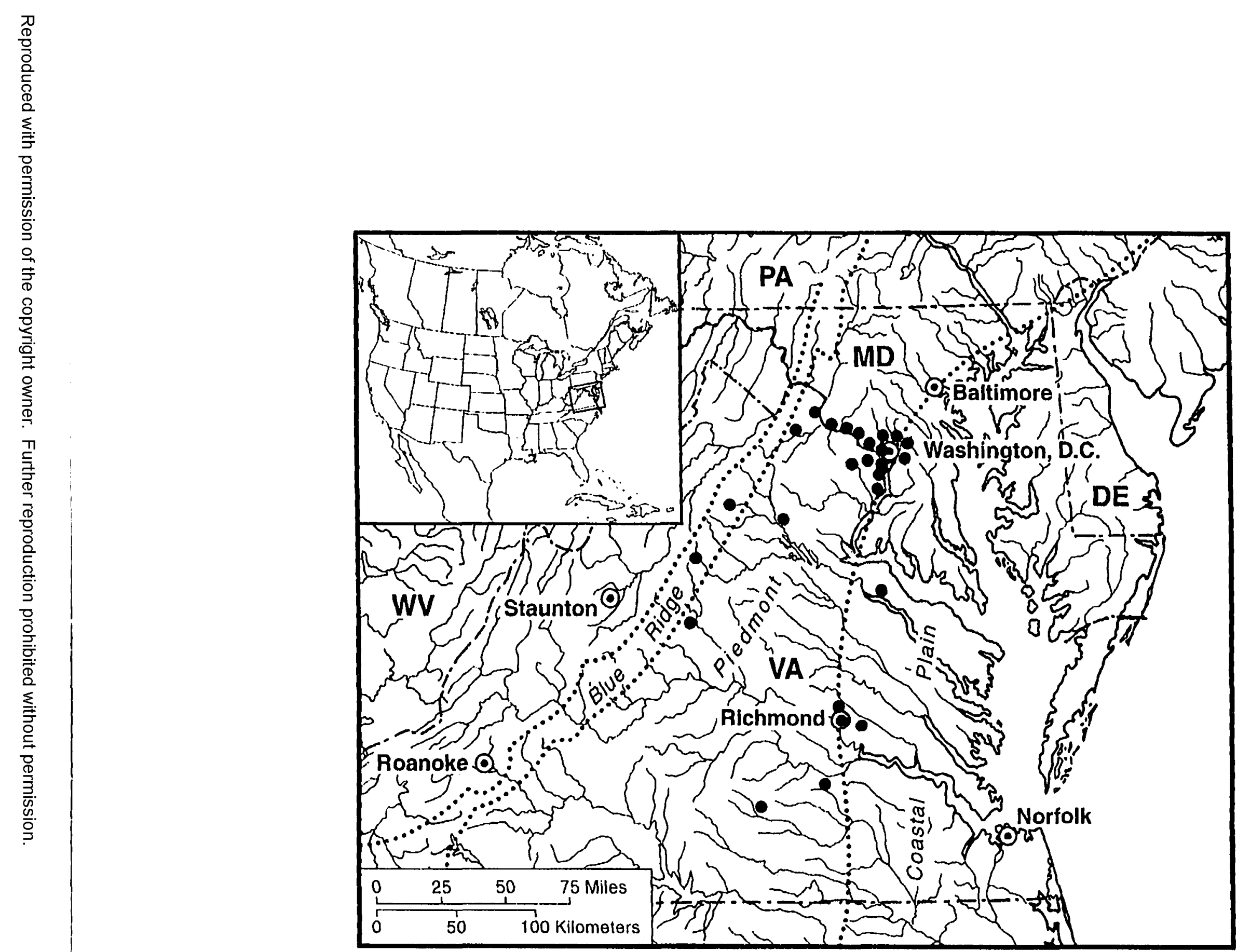

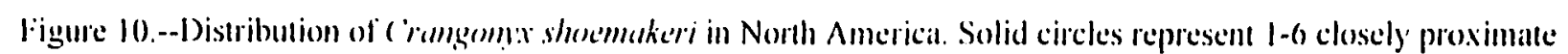
locilities. 
1926-1941. numerous specimens in approximately 40 collections (USNM) from mostly small streams. springs and bogs, W. H. Ball. L. Hubricht. A. Pizzini (the majority), J. W. Price. J. Scofield, and F. Thompson. 1926-1941. and collection in JRH from Rock Creek Drive Spring. D. Feller. 31 Mar 1995. MARYLAND. Allegany Co.: seep-fed pool, $3 \mathrm{~km}$ SE of intersection of Uhl highway and Wilson Rd. 1 . I : . D. Feller. 15 Mar 1995: Montgomery Co.: Bear Island. 4 collections (USNM) from ponds. R. Bray. J. Fowler and T. Ulke. 1935-1941: small pond on E. W. Birgfeld farm. 4 ₹. 1 \&. J. E. Benedict. Jr.. 10 Feb 1926 (USNM): pool near NW Branch at Burnt Mills. 1 ?. W. H. Ball. 24 Mar 1935 (USNM): Chain Bridge. 70 specs ( $\left.ๆ . z^{*}\right)$. G. E. Barnes. 9 Apr 1915 (USNM): grassy flood plain ponds. upper Rock Creek. $N$ of Chevy Chase. $10 £ .6$. J. Fowler. 10 Mar 1941 (USNM): woodland pond near Dalecarlia Resevoir at B \& O tracks. 4 . I $d$, collector not given, i Jan 1941 (USNM): pond on Indian Spring golf course at Four Corners. $1 \fallingdotseq .5 \approx, 18$ juvs. A. Pizzini. 13 Jan 1938 (USNM): small stream on Dean's property. Kensington. 1 ‡. W. H. Ball. 4 May 1934 (USNM): brook on Leiter estate. on Potomac River. $10 \fallingdotseq .8=$ C. R. Shoemaker. 20 Apr 1915 (USNM): damp leaves at Linden. 1 . $1:$ K. Hobbs. 3 Jun 1924 (USNM): ice pond near Rock Creek. Linden. 30 specs ( $\subsetneq$. $\left.\Xi^{*}\right)$. J. E. Benedict. 28 Feb 1926 (USNM): Martinsburg: 3 collections (USNM) from ponds. R. Bray and J. Fowler, 1940-1941: Plummers Island: 5 collections (USNM) from ponds and springs, W. D. Appel. C. R. Shoemaker and W. P. Webb. 1917-1935: Takoma Park (habitat not given), 4 \%. H. Ball, 16 Mar 1930 (USNM): Seneca: 2 collections from stream and pond in grassy meadow. J. Fowler. March 1941: Sligo Branch. $2 \supseteq .3 \approx$, collector not given. Feb 1916 (USNM): seeps and springs on Watts Branch at Potomac. 2 ?. 1 juv.. E. Yuster. 17 Apr 1994: seep/spring near Old Anglers Inn. $1 \bar{z} .3 \approx$. D. Fong. 16 Mar 1995: Monocacy Spring in C \& O Canal Nat. Hist. Pk.. I $\therefore$ D. Feller. I Apr 1994: Gold Mine Tract Spring $5.1 .1 \mathrm{~km} \mathrm{~W}$ of intersect. of Falls Rd and MacArthur Blvd. 2 . D. Feller. 7 Mar 1995: Great Falls North Spring 6.0.85 km NE of mouth of Cool Spring Bridge.

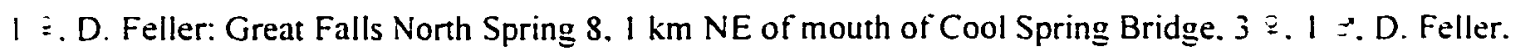
30 Mar 1995: Gum Spring. Brunswick Town Park. Brunswick. 6 . D. Feller. 30 Mar 1995: Limkiln Branch Spring. $0.1 \mathrm{~km}$ E of mouth of branch C \& O Canal Nat. Hist. Pk.. I $\Xi$. D. Feller. 23 March 1995: seeps. MacArthur Blvd, $0.8 \mathrm{~km}$ SE of mouth of Limkiln Branch C \& O Canal Nat. Hist. Pk.. 2 . D. Feller. 30 Mar 1995: Swains Lock Spring 2. $0.3 \mathrm{~km} \mathrm{NW}$ of Swains Lock. 2 @. D. Feller. 13 Apr 1995: Violets Lock Spr.. $0.8 \mathrm{~km}$ SE of intersect of River Rd and Violets Lock Rd. 2 2. D. Feller. 13 Apr 1995: Prince Georges Co.: small brook near Hyattsville. 30 specs (q. c゙). A. Pizzini. 10 Feb 1929 (USNM): creek in meadow ca. $1.6 \mathrm{~km}$ S of Riggs Mill. NW of Hyattsvills. 50 specs. R. Greenfield. 10 Feb 1929 (USNM): bog behind Oakcrest Towers just $N$ of Parkland. 44 specs ( $1.6 \mathrm{~km}$ Hyattsville. 2 ₹. 7 ॰. R. Greenfield, 18 Feb 1928 (USNM): stream in Takoma Park tlowing into Sligo Branch. 4 ₹. $2 \approx$ W. H. Ball. 5 Apr 1936 (USNM). VIRGINIA. Albemarle Co.: Charlottesville (habitat not given). $99.3 c^{*}$. R. L. Hoffman. 20 Nov 1947 (USNM): Alexandria. small seep-fed stream in woods behind Hamlet West Apartments, 19 \&. 30 o. J. R. Holsinger. 11 Mar 1973: Arlington Co.: spring at Glencarlyn. 4 Є. $2 \approx 1$ juv. C. R. Shoemaker. 7 Jul 1918 (USNM): Caroline Co.: Fort A. P. Hill. II 
collections (JRH) from seeps and small springs. P. H. Stevenson. April-May 1993: Culpepper Co.: flood pool. Rappahannock River. 5 ₹. 1 c. A. Weaver. 28 Mar 1967: small swamp $8.0 \mathrm{~km}$ E of Amissville. 28 specs ( $\subsetneq$. $)$ ). collector not given. 10 May 1940 (USNM): Dinwiddie Co.: old well. $20 \mathrm{~km}$ WSW of Darvils. $1 \cong$. P. H. Stevenson. 29 Mar 1993: Faifolx Co.: Dyke (habitat not given). 2 ?. W. D. Appel. 18 Feb 1911 (USNM): swamp near Mount Vernon. $4 \cong .10$. W. S. Webb. 22 Apr 1917 (USNM): tiny spring and run $3.2 \mathrm{~km}$ ESE of Fairfax. $2 \cong .10 \approx .6$ juvs. J. R. Hoisinger et al.. $13 \mathrm{Jul}$ 1967: spring on Dogue Creek. 29 specs $\left(\Xi . \sigma^{2}\right)$. P. Opler. 23 Nov 1978: spring in Fairfax Co. (specific location not given). I2 ₹. A. Pizzini. 12 Nov 1949 (USNM): pool below spring. Annandale, 1 . W. H. Ball. 19 Nov 1940 (USNM): stagnant pond between Aqueduct Bridge and Chain Bridge. 25 specs ( $\Xi$. ). G. W. Gill. 3 Apr 1913 (USNM): smail pools between Belle Haven and Dyke. 2 ․ 3 \&.W. H. Ball. 14 Apr 1935 (USNM): Black Pond area. 3 collections (USNM) from ponds. H. S. Barber and C. R. Shoemaker. 1915 and 1929: Capt. Hickory CreekForestville area. + collections (USNM) from spring and streams. A. Pizzini. Feb.-iMar. 1947: muck hole 1.6 $\mathrm{km} \mathrm{W}$ of Centreville. $2 \approx$ collector not given. 10 May 1940 (USNM): small pool $1.6 \mathrm{~km}$ below Chain Bridge. 10 : 2 :. A. Pizzini. 4 Nov 1934 (USNM): Chopawamsic Park near Quantico. 3 collections (USNM) from spring and bog. A. Pizzini, Mar and May 1947: small stream in Colchesler. $4 \equiv .1=$ W. H. Ball. 24 Dec 1934 (USNM): Difficult Run between Leesburg Rd and Potomac River. $3 \equiv .3: 2$ juvs. W. H. Ball. 4 Feb 1940 (USNM): small stream on hillside in open woods near Difficult Run. $6: 3 \approx * 1$ juv. W. H. Ball. 30 Dec 1934 (USNM): small pond near Difficult Run. I . C. R. Shoemaker and W. D. Appel. 10 Dec I91 I (USNM): pool in woods at Dyke, I juv. W. H. Ball. 7 Apr 1934 (USNM): stream source 1.6 $\mathrm{km} \mathrm{S}$ of Forestville. $8 \subsetneq$. A. Pizzini. 8 Mar 1947 (USNM): spring seepage tributary oft W side of swamp trail. $0.5 \mathrm{~km}$ NNE of Fairview. 20 specs ( $\$$. $₫$ juvs). C. S. Hobson. 8 Apr 1994: Great Falls area. 4 collections (USNM) from seeps. ponds and pools. H. S. Barber and C. R. Shoemaker. 1924-1934, and 3

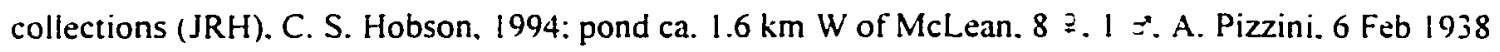
(USNM): Packard Creek on R. S. Bray property. Fairfax City. $6 \mp .1 \approx .7$ juvs. R. S. Bray. 12 Jul 1941 (USNM): Scott Run and immediate vicinity. numerous specimens in approximately 15 collections (USNM) from springs and small streams, mostly by A. Pizzini. 1935-1939. and 2 collections (JRH) from springs. J. R. Hoisinger. A. Pizzini and M. Straskraba. 1965-1967: stream near Trammel's Landing above Great Falls.

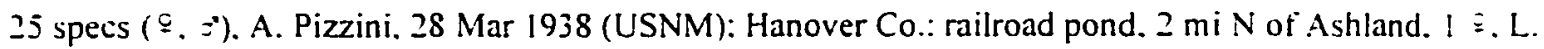
Hubricht. I Apr 1950 (USNM): Henrico Co.: small stream $1.6 \mathrm{~km} N$ of Bryan Park. Richmond. $10 \fallingdotseq .3 \approx$ L. Hubricht. 8 May 1944 (USNM): Loudon Co.: spring $1.6 \mathrm{~km} \mathrm{~N}$ of Leesburg. $1 \subsetneq .2 \geq$ J. C. Bridewell. 20 Apr 1939 (USNM): Middleburg: 2 collections (USNM) trom stream and spring bog. A. Pizzini. 19381939. and I collection (JRH) from bog. J. R. Holsinger. May 1965: Madison Co.: spring on Double Top Mountain. Syria. $15 \equiv .3 \star^{*}$. T. Ulke. 25 Aug 1935 (USNM): spring at Syria. $11 \varsubsetneqq .2 \approx .2$ juvs. A. Pizzini. + Sep 1938 (USNM): New Kent Co.: seep and small stream in Crump Swamp. $24 \mathrm{~km}$ E of Richmond, 12 E. $+\neq 0.1$ juv. J. R. Holsinger. $23 \mathrm{Apr} 1970$ and $21 \& .8 \approx .2$ juvs. 19 Mar 1972: Nottoway Co.: small pond $1.8 \mathrm{~km}$ NE of Birchen Lake and 3 ₹.P. H. Stevenson. 29 Mar 1993: Prince William Co.: stream along 
Occoquan Creek near Hoadly P.O.. just below mouth of Bull Run. $10 \subsetneq .2 c$. 1 juvs. W. H. Ball. 30 Nov 1940 (USNM): Rappahannock Co.: small swamp. $4.8 \mathrm{~km} \mathrm{~W}$ of Washington. $6 \cong .1:$ T. K. Ellis. 11 May 1940 (USNM).

Diagnosis.--Distinguished from $C$. setodactylus and $C$. minor by having comb spines on the outer ramus of uropod 2 of male and from $C$. aherrans by lacking dorsal and lateral spines on telson. having more plumose setae on inner surface of inner plate of maxilla 2. more setae on inner margin of dacty.l of maxilliped palp and on ventral margin of coxa of pereopod 7. Largest male. $9.5 \mathrm{~mm}$ : largest female. 13.5 $\mathrm{mm}$.

Female.--Eye small and round. Antenna 1, 55-57 percent length of body. about I.8 times longer than antenna 2: primary flagellum with up to 27 segments. Antenna 2. flagellum with up to 9 segments.

Mandibles subequal. spine row with about 5 spines: segment 2 of palp with about 7 long setae. segment 3 with $1 \mathrm{~A}$ seta. $3 \mathrm{C}$ setae. $4 \mathrm{E}$ setae and a row of $\mathrm{D}$ setae. lacking B-seta. Maxilla I: inner plate with 4-5 apical plumose setae: palp with 5-6 slender spines on apex. Maxilla 2. inner plate with oblique row of 5-7 plumose setae on inner margin. Maxilliped: inner plate apically with 2 bladelike spines. 3 naked spines. and 5-6 plumose setae extending from inner margin to apex: outer plate with row of naked setae and 3-t slender pectinate spines on inner margin and I apical plumose seta: dactyl with $4-5$ setae along inner margin: dactyl nail very long. about $1 / 3$ length of dactyl.

Propod of gnathopod I slightly less 2 times longer than carpus: palm concave in middle. subequal in length to posterior margin. with 11 spine teeth on inside. 11 on outside: detining angle with 5 notchedserrate spine teeth on inside. I large notched and 3 serrate spine teeth on outside: posterior margin with about 4 sets of setae inserted in groups of 2-5. Dactyl of gnathopod I with row of about 9 short setae and $1+$ blade-like teeth along inferior margin: nail shor. Ventral margin of coxa 1 with 16 setae.

Propod of gnathopod 2 about 2 times longer than carpus: palm concave. shorter than posterior margin. with 13 spine teeth on inside. 4 on outside: defining angle with 1 large notched spine tooth and 2 serrate spine teeth on outside. 5 notched-serrate spine teeth on inside; superior medial setae singly inserted: posterior margin with 5 sets of setae in groups of 2-6. Dactyl of gnathopod 2 with row of 9 short setae and 12 blade-like teeth along inferior margin: dactyl nail short. Ventral margin of coxa 2 with 15 setae.

Coxa of pereopod 3 with 12 marginal setae. Coxa of pereopod 4 with 19 marginal setae: dactyl of pereopod 4 about 35 percent length of propod. Pereopod 5: basis with 15 shallow serrations along posterior margin. 9 sets of short spines in groups of $1-3$ on anterior margin. 3 long setae on anteroproximal margin. Pereopod 6: basis with 16 shallow serrations along posterior margin. 8 sets of short spines on anterior margin. 4 setae on anteroproximal margin. Pereopod 7: coxa with 5 setae along the posterior margin: basis with 20 shallow serrations along posterior margin, 10 sets of short spines on anterior margin: dactyl about 30 precent length of propod.

Pleonal plates: posterior margin of plate I slightly convex. with I seta. distoposterior corner acute but not produced: posterior margin of plate 2 nearly straight. with 1 seta. distoposterior corner produced and 
acute. ventral margin with 4 (subventral) spines: posterior margin of plate 3 nearly straight. with 1 seta. distoposterior comer neither produced nor acute, ventral margin with 5 (subventral) spines. Peduncle of pleopod I with 2 retinaculae. lacking setae on outer margin.

Uropod 1: inner ramus about 65 percent length of peduncle. armed with about 13 spines: outer ramus with about 12 spines; peduncle with row of 12 outer and 1 inner spines. Uropod 2: inner ramus subequal in length to peduncle, armed with about 15 spines: outer ramus with about 13 spines: peduncle with 6 outer and 3 inner spines. Uropod 3 : inner ramus with I spine: outer ramus about 1.5 times longer than peduncle. inner and outer margins each with $3-4$ sets of spines in groups of 1-3. Telson slightly broader than long. notched about $1 / 5$ distance to base: each lobe with $3-4$ apical spines. which are less than $1: 2$ length of telson.

Male.--Differing from female as follows: smaller size. but with approximately same body shape. Antenna 2: calceoli present on peduncular segments 4 and 5 and first 2-5 segments of tlagellum.

Propod of gnathopod I proportionately much broader than and 2 times longer than carpus: palm slightly concave. shorter than posterior margin. armed with 12 spine teeth on inside and 12 on outside: defining angle with 2 notched and 4 notched-serrate spine teeth on inside. 3 notched and 2 serrate spine teeth on outside.

Propod of gnathopod 2 more than 2 times longer than carpus: palm strongly oblique. with 9-12 spine teeth on inside. 17 on outside: defining angle with 4 spine teeth on inside and 7 on outside.

Liropod 2: inner ramus with about 14 spines: outer ramus with inner row of comb spines on distal half. about 7 short spines on outer margin. apex with 3 spines: peduncle with 4 thin outer spines. and 2 inner spines. Uropod 3: outer ramus about 2 times longer than peduncle. Telson little longer than broad. notched I 3-1 2 distance to base: each lobe with 3 apical spines. which are about 23 length of telson.

Distribution and ecology.--The range of $C$. shoemakeri extends from south-central Maryland south and southwest to south-central Virginia (fig 10). This species is an inhabitant of temporary pools and ponds (often with grassy bottoms), springs. small streams, and bogs. Ovigerous females occur from February to May. followed by immatures during the summer.

Remarks.--Hubricht and Mackin (1940) described C. shoemakeri from cotype material collected in Washington. D.C., but they also assigned specimens from Illinois and Oklahoma to this species (which proved to be $C$. minor). in which males lacked comb spines on uropod 2. Hubricht (1943) also later reported this species from numerous localities in Kentucky. Illinios. Indiana. Michigan. Missouri and Ohio. However. Bousfield (1958) was correct in doubting that these collections were $C$. shoemakeri. and Holsinger (1972) and the present study have shown these collections to contain three different species. including $C$. setodactylus, $C$. minor and $C$. forbesi. 
Crangonux setodactylus Bousfield

Figures $11-14$

Crangonix setodactylus Bousfield. 1958:96-98. fig. 14 [type-locality: Spitler Creek. 4 mi W of Norwich.

Oxford Co.. Ontario. Canada].--Holsinger. 1972:46. figs. 11j. 19: 1977:252.--Williams \& Hynes.

1976:771: 1977:59.--Barnard \& Barnard, 1983:435. map 12.--Fitzpatrick. 1983:145.--Pennak.

1989:482.

Crangonyx shoemakeri (Hubricht and Mackin).--Hubricht. 1943:690 [in part].

Material examined.--ONTARIO. Oxford Co.: Spitler Creek. 4 miles W of Norwich. holotype allotype $z .4 \equiv$ paratypes. L. Boustield. 29 May 1957 (CMN): Stony Creek. $6.4 \mathrm{~km} \mathrm{~W}$ of Tillsonburg. $5 \cong$. E. L. Bousfield. 29 May 1957 (USNM). INDIANA. DeKalb Co.: outlet of drain. $9.1 \mathrm{~km} \mathrm{E} \mathrm{of} \mathrm{Corunna.} 10$ specs. L. Hubricht. 19 Apr 1942 (USNM): Hendricks Co.: spring. $4.5 \mathrm{~km}$ ENE of Plaintield. $6 \vdots . \mathrm{L}$. Hubricht. 17 Apr 1942 (USNM): Henry Co.: small stream. $2.1 \mathrm{~km} \mathrm{NW}$ of Blountsville. 100 specs $1 .$. juvs). L. Hubricht. 27 Apr 1941 (USNM): Huntington Co.: slough. $5.3 \mathrm{~km}$ NE of Huntington. 100 specs. L. Hubricht. 27 Apr 1941 (USNM): Noble Co.: outlet of drain. $0.9 \mathrm{~km}$ SE of Kendallville. 38 specs. L. Hubricht. 19 Apr 1942 (USNM): Putnam Co.: temporary stream. $8.5 \mathrm{~km}$ ENE of Harmony. $39 \sqsubseteq .46 \mathrm{z}$. Hubricht. 16 Apr 1942 (USNM): county not given: small tributary of Richland Creek on indiana Rt 48.1 Z. N. Hynes. 5 Jan 1963 (USNM). KENTUCKY. Bourbon Co.: small spring. $4.5 \mathrm{~km}$ SW of Millersburg. 8 $\approx$. L. Hubricht. $25 \mathrm{Apr} 1941$ (USNM): spring, $9.1 \mathrm{~km} \mathrm{SW}$ of Paris. 20 specs. L. Hubricht. $25 \mathrm{Apr} 1941$ (USNM): Boyle Co.: Salt River Drainage near Atoka. 2 \&. S. Lrider. 6 Mar 1971: Bullitt Co.: stream W of Mit. Carmel Church. $3 \Xi .1 \%$. N. Hynes. 9 Jan 1985 (USNM): creek at T-junction W of Crutchmere Hollow. $5 \Xi .5 \therefore$ N. Hynes. 9 Jan 1985 (USNM): small stream just E of Mt Carmel Church. $19 \Xi .1 \therefore \mathrm{N}$ Hynes. 9 Jan 1985 (USNM): Carroll Co.: tributary to Ky. River. $6.2 \mathrm{~km}$ SSW of Carrollton. $6 \mp .1 \geq$ J. E. and M. R. Cooper. 26 Mar 1967: Clark Co.: Big Stoner Creek at SR Rt 15. 24 specs. R. Fox. 6 Feb 1971: spring. $9.6 \mathrm{~km}$ NE of Winchester. L. Hubricht. 22 Apr 1949 (USNM): stream ca. $11.2 \mathrm{~km}$ W of Clark County courthouse on Colby Pike. $89.5 \approx *$, R. Fox. I Jan 1971: Fayette Co.: Bryan Station Spring. 3 氵 2 $\therefore$ J. R. Holsinger. I Feb 1964 and $9 \subsetneq .6 c^{\circ}$. R. Fox et al.. 3 Jan |971: lake on US $421-25 \mathrm{ca} .100 \mathrm{~m} \mathrm{NW}$ of State Road 4. SE of Lexington. I \&. R. Fox. 1 Jan 1971: Maedico Creek, ca. $8.0 \mathrm{~km}$ W of Clark-Fayette Co. line on Todds Road. 3 q. 18 ?. R. Fox, 1 Jan 1971: Pompoon Creek. ca. $11.2 \mathrm{~km}$ W of Clark-Fayette co. line on Todds Road. $5 ₹ .70^{\circ}$. R. Fox. I Jan 1971: spring stream entering $N$ of Elkhorn Creek. 8 \&. $9:$ R. Fox et al.. 3 Jan 1971: unmarked stream beside Bryan Station Rd. ca. $1.6 \mathrm{~km} \mathrm{NE}$ of St Rt 4 . NE of Lexington, 20 specs. R. Fox et al.. 3 Jan 1971: Bryan Station Rd (habitat not given). $0.5 \mathrm{~km} \mathrm{NE}$ of Eastin Rd. 0.5 km NE of Lexington. 300 specs. L. Hubricht. 25 Apr 1941 (USNM): Coldstream Farm Stream. 3 $\Xi . J$. R. Holsinger. Apr 1965 and $9 \approx .11$ Apr 1965: pond on Coldstream Farm. $+\bar{F}, 3 \approx *$ J. R. Holsinger. 


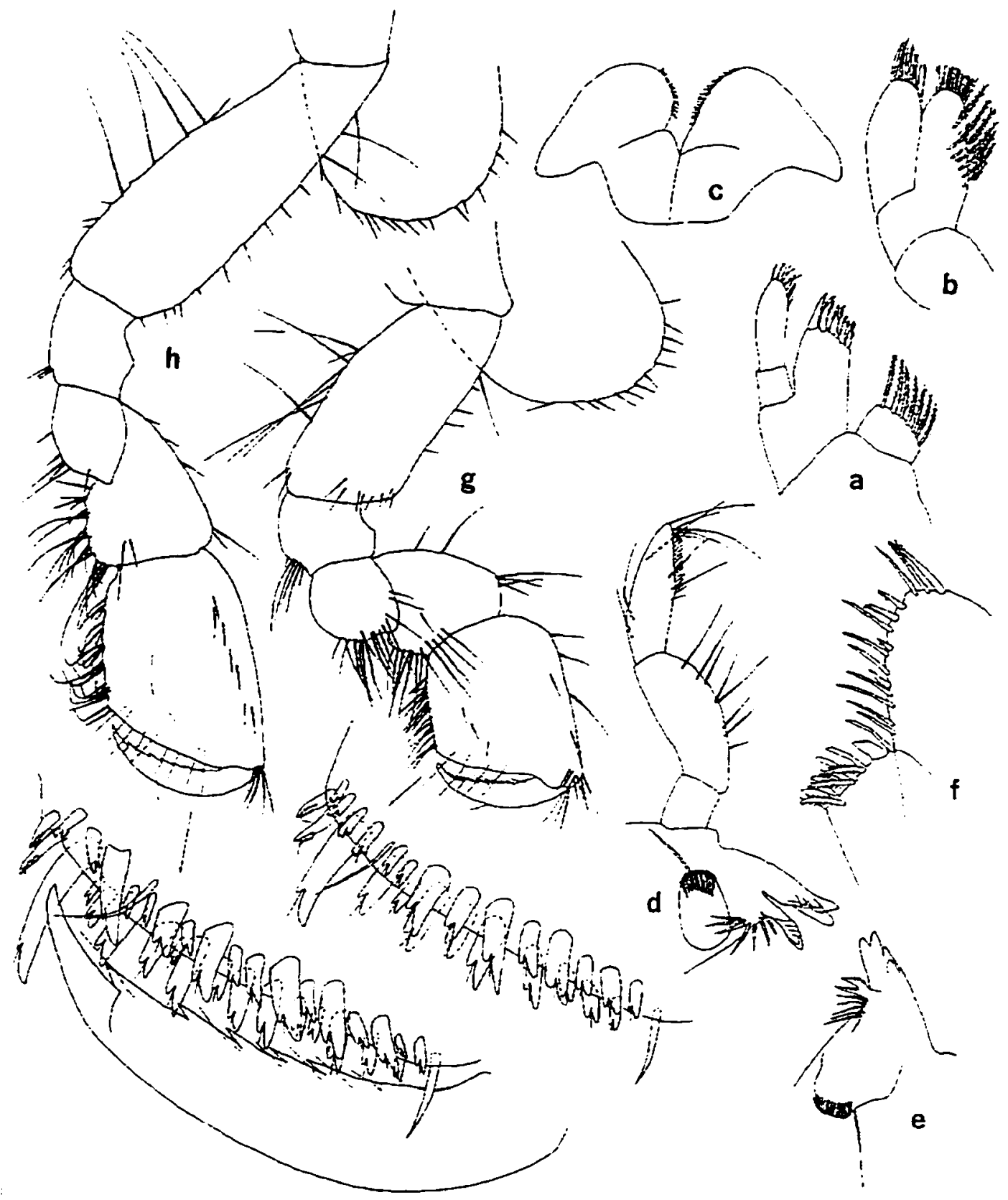

Figure $11 .--$ Crungonyx setodactylus Bousfield. Female $(10.8 \mathrm{~mm})$. Brỵn Station Springs. NE of Lexington. Fayette Co.. Kentucky: a. b. maxillae 1. 2: c. lower lip; d. left mandible: e. dentate part of right mandible: f. inner and outer plates of maxilliped (greatly enlarged): g. h. gnathopods I. 2 (palmar margins enlarged). 


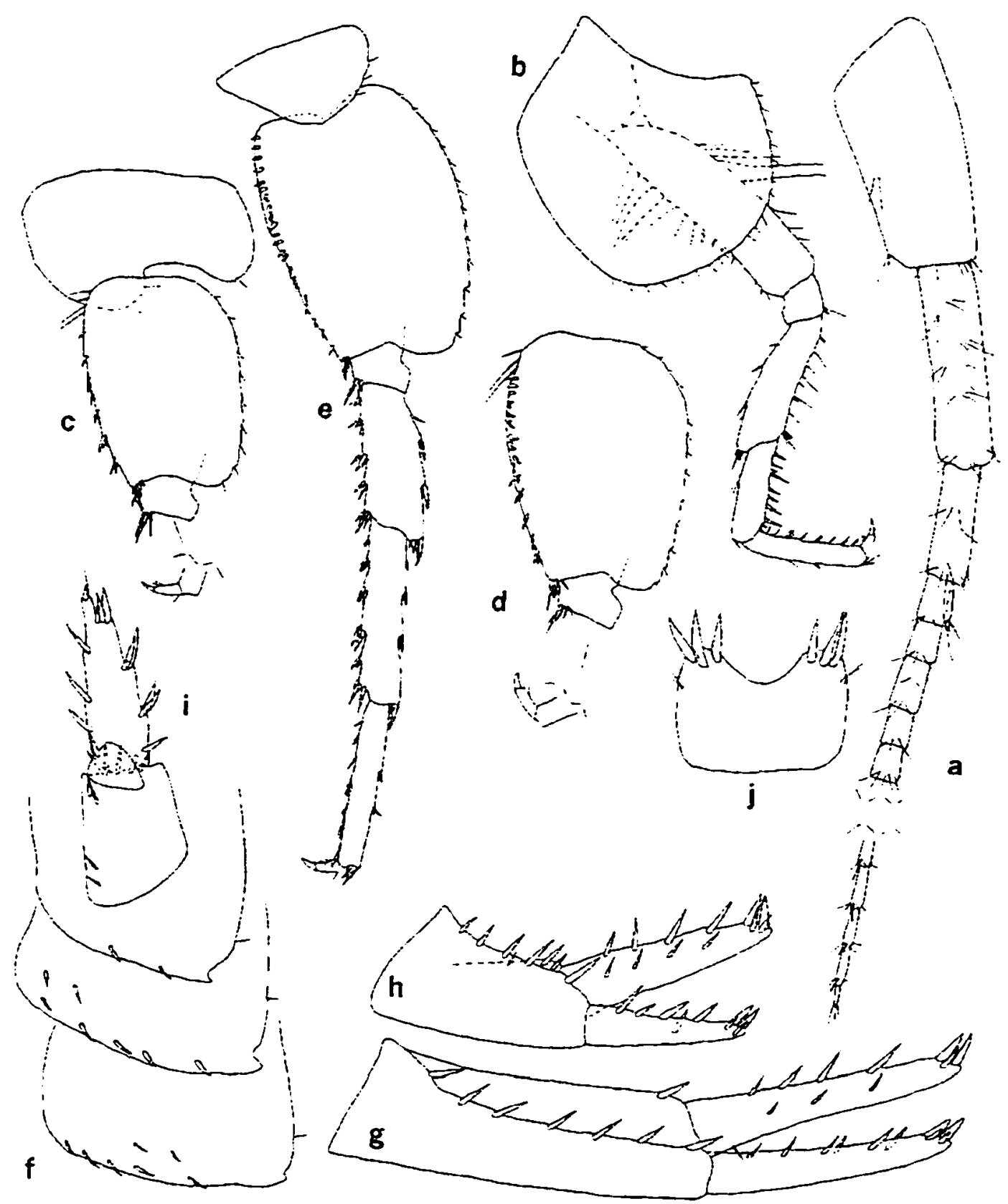

Figure 12.--Crangonyx setodactylus Bousfield. Female (10.8 mm). Bryan Station Springs. NE of Lexington. Fayette Co.. Kentucky: a. antenna I: b. e. pereopods 4. 7: c. d. pereopods 5.6 (in part): f. pleonal plates: g. h. i. uropods $1.2,3: \mathrm{j}$, telson. 


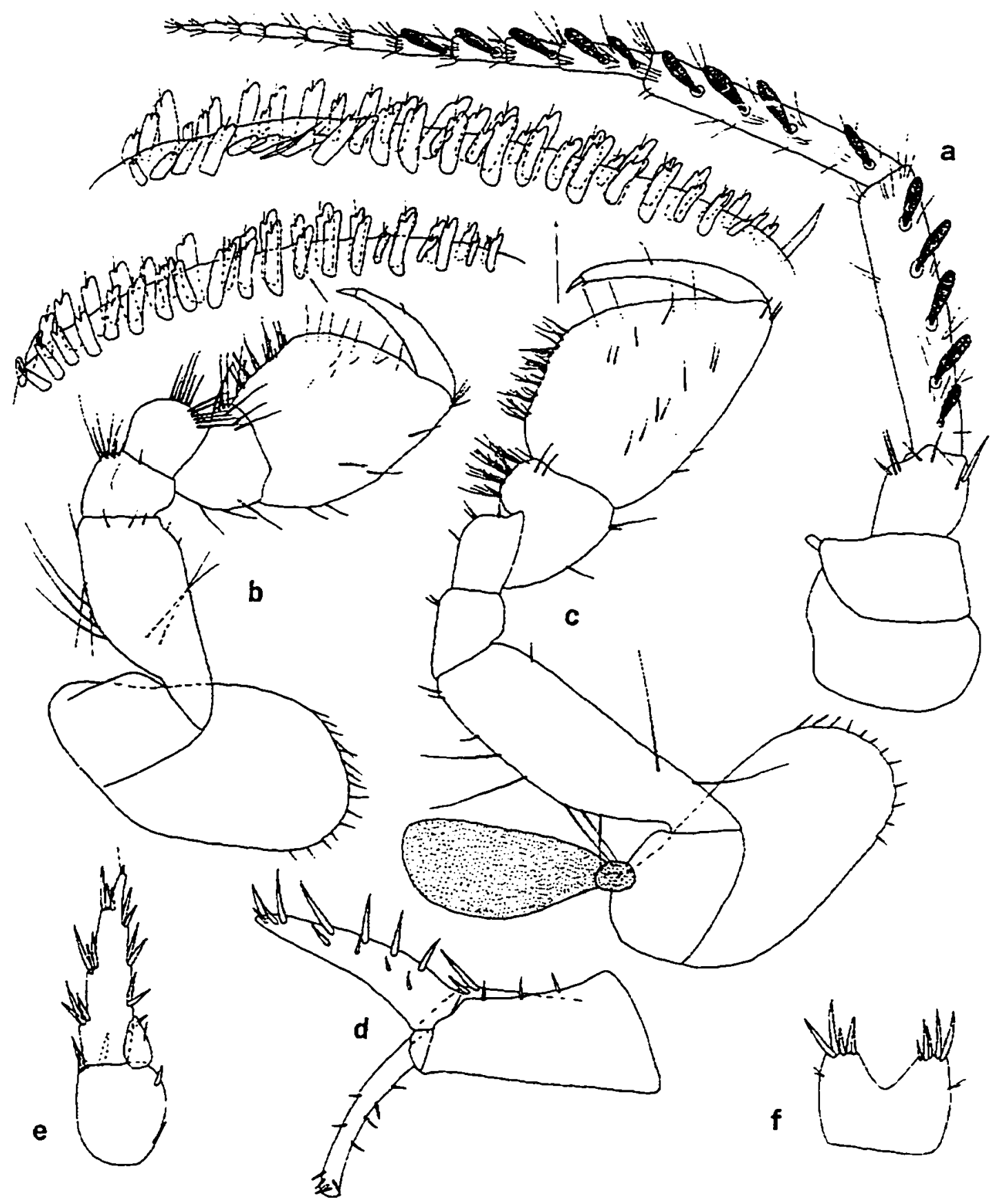

Figure 13.--Crangonyx setodactylus Bousfield. Male $(9.7 \mathrm{~mm})$. Bryan Station Springs. NE of Lexington. Fayette Co.. Kentucky: a. antenna 2: b. c. gnathopods I. 2 (palmar margins enlarged): d. e. uropods 2. 3: f. telson. 


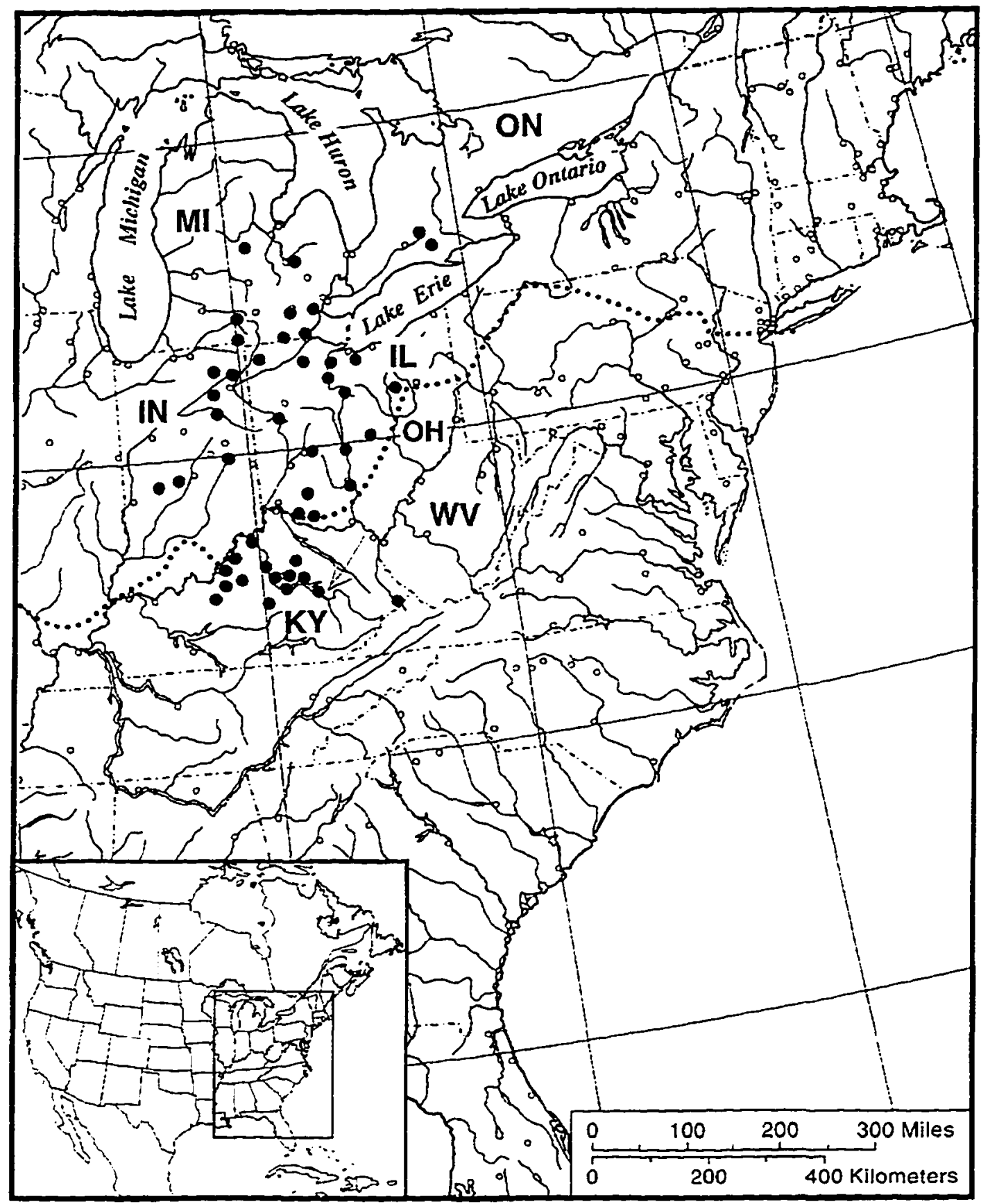

Figure 14.--Distribution of Crangomyx setodactylus in North America. Solid circles represent 1-20 closely proximate localities. The dotted line indicates the approximate southern extent of glaciation during the Pleistocene 
II Apr 1965: Crasmere Farm No.I spring, Higbee Mill Road. $4 \cong .3$ ๔. J. R. Holsinger. 7 Mar 1964: Idle Hour Spring No.1 on Rt 25.4 $9.2 \approx$ J. R. Holsinger and R. M. Norton. 11 Apr 1964: Idle Hour Spring No.2 near Rt 25. 11 @. $6 \sigma^{\circ}$. J. R. Hoisinger and R. M. Norton. I1 Apr 1965: stream near Lansdowne Tates Creek Rd. Lexington. 25 specs. J. R. Holsinger and R. Carpenter. 5 Apr 1965: seep-fed swamp off Tates Creek Rd. Lexington. $2 \Xi_{.}+c^{*}$. J. R. Holsinger. I Mar 1965: Russell Cave Spring. 20 specs. L. Hubricht. 22 Nov 1940 (USNM) and I \. 2 . J. R. Holsinger. 16 Nov 1963 and 19.7 juvs. W. Andrews. 22 Oct 1966: spring. $1.3 \mathrm{~km}$ E of Fort Spring, 50 specs. L. Hubricht. 22 Nov 1940 (USNM): spring near Idle Hour Golf Course. Lexington. $11 \cong .6 \%$. R. M. Norton. 18 Jan 1964: spring on Keene Rd just S of JessamineFayette Co. line. $1 \supsetneq .6 \approx$. J. R. Holsinger. 7 Mar 1964: small spring. $4.6 \mathrm{~km} \mathrm{SSW}$ of Jimtown. L. Hubricht. 22 Nov 1940 (USNM): spring. $1.6 \mathrm{~km}$ E of Warrentown, 50 specs. L. Hubricht. 22 Nov 1940 (USNM): spring on Waveland Farm near Lexington. 27 specs, J. R. Holsinger. 7 : Mar 1964: spring-fed stream on Waveland Farm near Lexington. $4 \cong .6 c^{\prime}$ I juv. J. R. Holsinger. 23 Jan 1965 and 75 specs. 11 Apr 1965: Franklin Co.: small spring just E of Bridgeport. 25 specs. L. Hubricht. 22 Nov 1940 (USNM): Davis Branch near Harvieland (tributary to ky. river). $3 \equiv$. J. E. and M. R. Cooper. 26 Mar 1967: Hardin Co.: slough. just $W$ of West Point. $2 \leqq$. L. Hubricht. 21 Apr 1956 (USNM): Jefferson Co.: brook in Audubon Park Golf Course. 37 specs. G. A. Cole. 8 Apr 1955: Cave Run Creek SE of Fisherville. $1 \therefore$ G. A. Cole. 4 Apr 1954: Middle fork of Beargrass Creek in Seneca Park. Louisville. 1 . $1 \approx$. G. A. Cole. 1 Mar 1954 (JRH), 2 ․ G. A. Cole. 14 Mar 1954 (USNM) and 1 ฉ. 1 ะ.. 25 Apr 1954:: Caperton Swamp. Indian Hills Rd W, 20 specs. Ilaria. 26 Mar 1954 and 20 specs. G. A. Cole. 26 Mar 1954 (USNM): Caperton Swamp. temporary pond east of Indian Hill Trail. $2 \nsubseteq .3 \approx$. G. A. Cole, collection date not given: middee Fork of Beargrass creek. crossing County Club Rd. $5 \Xi .3 ;$. G. A. Cole. 2 May 1954:

Pennsylvania Run at Mt. Washington Road crossing it. $2 \approx$. G. A. Cole. 21 Mar 195+(JRH) and $2 \equiv .1:$ G. A. Cole. 21 Mar 1954 (USNM); Pennsylvania Run where Manslick Rd crosses. 5 E. G. A. Cole. 21 Mar 1954: smail intermittent ditch in Audubon Park golf course. 60 specs. G. A. Cole. 20 Nov 1954 and 13 $\Xi .5 \iota^{*}$.G. A. Cole. 12 Mar 1955: small temporary pond off US +2 on Poplar Lane. $N$ of Church. $1 \equiv . G$. A. Cole. 29 Mar 1954: small tributary of Harrods Creek. 5 ९. $1 \approx$. R. Fox. 21 Nov 1970: Beargrass Creek below bridge no. 7. Cherokee Park. Louisville. 4 ९. L. Hubricht. 6 May 1956 (USNM): intermittent brook at Poplar Level Road and Audubon Dr.. Louisville. 8 ₹ $10 c^{\circ}$. G. A. Cole. 13 Mar 1954 (USNM): Jessamine Co.: Marble Creek. $3 \subsetneq .2 \approx$. T. G. Marsh. et al.. 22 Jan 1967: spring. $4.3 \mathrm{~km} N$ of Nicholasville. 25 specs. L. Hubricht. 24 Apr 1941 (USNM): spring. $9.8 \mathrm{~km} \mathrm{~N}$ of Nicholasville. 25 specs. 24 Apr 1941 (USNM): Oldham Co.: stream beside St Rd 329. between Harrods Creek and US $+2.1 \dot{\Xi} .3 \approx$. R. Fox. 3 Apr 1971: tributary to Harrods Creek. $6 \sqsubseteq .2 \approx$. R. Fox. 21 Nov 1970: Pike Co.: Mouth of Spring. S of Elkhorn. $17 £ .6$ o. H. Garwan. 22 Apr 1930 (USNM): Spencer Co.: Brashears Creek. Rivals. $2 \equiv$. N. Hynes. 13 Jan 1985 (USNM): Woodford Co.: Sunnyside Farm spring. 7.2 km E of Versailles. 25 specs. L. Hubricht. 22 Nov 1940 (USNM); small spring, $4.8 \mathrm{~km} \mathrm{~N}$ of Versailles. 50 specs, L. Hubricht. 22 Nov 1940 (LSNiM). MICHIGAN. Branch Co.: ditch, $4.9 \mathrm{~km}$ S of Coldwater, 50 specs. L. Hubricht. 19 Apr 1942 
(USNM): Calhoun Co.: stream. $5.3 \mathrm{~km}$ E of Burlington. 1 . L. Hubricht. 1 May 1941 (USNM): Genesee Co.: outlet of drain. $5.8 \mathrm{~km}$ N of Fenton. $9 ₹ .27 \approx$. L. Hubricht. 19 Apr 1942 (USNM): Gratiot Co.: pond in woods. $4.8 \mathrm{~km} \mathrm{NW}$ of Alma. 4 \&. E. P. Creaser. 6 May 1935 (USNM): Lenawee Co.: slough. $0.8 \mathrm{~km} \mathrm{~W}$ of Hudson, 100 specs. L. Hubricht. 29 Apr 1941 (USNM): Monroe Co.: outlet of drain. $4.5 \mathrm{~km} \mathrm{~N}$ of Petersburg. $29 \equiv .12 \mathrm{c}$. L Hubricht. 18 Apr 1942 (USNM): Washtenaw Co.: temporary pond W side of Milford Rd. 16 km from Ann arbor. 1 \%. R. Kenk. 9 Apr 1941 (USNM): Wayne Co.: creek in woods on Redf Golf Course. 7 \%. R. and K. E. Goellner, 25 Dec 1940 (USNM) and 18 ₹. $5 \approx ⿱$. I Jan I941 (USNM): woodland pools. Southfield Road and Oakwood Blvd.. Dearborn. 4 ๑. L. Hubricht. 7 Apr 1946 (USNM): ditch. $0.8 \mathrm{~km}$ E of Melvindale. 2 \&. L. Hubricht. 24 Mar 1946 (USNM); County, habitat and collector not given: East Harbor. 1 \% 1 juv. 28 Sep 1930 (USNM). OHIO. Auglaize Co.: 14 ミ. habitat. collection date and collector not given (USNM); Brown Co.: spring rill. $2.9 \mathrm{~km}$ S of Georgetown. $2 \cong$. L. Hubricht. 25 Apr 1941 (USNM): Clark Co.: small stream. $2.1 \mathrm{~km} \mathrm{E} \mathrm{of} \mathrm{Donnellsville.} 3 \equiv .4 \approx$. L. Hubricht. 17 Apr 1942 (USNM): Clermont Co.: Shayler Run. 87 specs. E. L. Robinson. 25 Feb 1970. 100 specs. 9 Mar 1970 and 85 specs. 12 Mar 1970: Mt. Carmel (habitat not given). $2 ?$. E. L. Surber. 26 Mar 1962 (USNMI): Clinton Co.: small spring, $1.1 \mathrm{~km}$ SE of Westboro. 3 ?. L. Hubricht, 25 Apr 194I (USNM): Crawtord Co.: outlet of drain. $0.8 \mathrm{~km}$ SSW of Chatfield. 35 specs. L. Hubricht. 18 Apr 1942 (USNM): Erie Co.: Millers Blue Hole. US Rt $6.16 \mathrm{~km}$ W of Sandusky. $8 \cong .6 \approx$ H. P. Clemens. 8 Apr I948 (USNM): Franklin Co.: pond in woods. Columbus. 2 ₹. 2 c. N. C. Furton. 25 Feb 1930 (USNM): Licking Co.: Cold Spring Run. $6.2 \mathrm{~km}$ from Newark. I $\approx$. N. C. Furton. 11 Apr 1933 (USNM): Ross Co.: brook. $1.6 \mathrm{~km}$ W of Chillicothe. $16:$ collector not given. 8 Apr 1931 (USNM): Sandusky Co.: Fremont. 9 . J. Norrocky. 13 Feb 1984 (USNM): Seneca Co.: outlet of drain. $1.6 \mathrm{~km}$ E of Frank. 100 specs. L. Hubricht. 18 Apr 1942 (USNM): Seneca Caverns, $10 \cong$. D. A. Hubbard. 30 Jul 1992. $2 \subsetneq .3 \approx$. D. A. Hubbard. Jr.. 29 Dec 1993: Wayne Co.: Shreve (habitat not given). 3 ₹.W. Shear, 23 Mar 1962: pools. Shreve. 4 §. 1 ×. MLK. 4 May 1962: Brown's pools. Shreve. $2 £$. A. Weaver. 25 Apr 1960 and 29 . MLK. 4 May 1962: ditch. Blatchleyville. 3 $\fallingdotseq .2:$ MLK, 9 Apr 1962 and 24 specs. MLK. 20 Apr 1962: Killbuck flood pools. $11 \sqsubseteq .2 \approx$. A. Weaver. 27 Mar 1962 and 3 ․ 1 c. A. Weaver. 26 Apr 1960; Brown's Lake flood pools. 11 specs. A. Weaver. 22 Mar 1962: Miller's Blue Hole near Castolia. 4 . A. Weaver. 3 Jul 1961 and $+\equiv .1$. A. Weaver. 6 Jul 1964: Williams Co.: temporary pools. $3.2 \mathrm{~km} \mathrm{SW}$ of Bryan. 50 specs. L. Hubricht. 28 Apr 1941 (USNM): temporary pool. $3.2 \mathrm{~km} \mathrm{~N}$ of Hamer. 20 specs. L. Hubricht. 28 Apr 194 I (USNM): Wood Co.: outlet of drain. $2.1 \mathrm{~km}$ SE of Perrysburg. 50 specs. L. Hubricht. 18 Apr 1942 (USNM).

Diagnosis.--Distinguished from other members of the shoemakeri group by having 2-3 setae on inner margins of dactyl of pereopods 3-7: straight palms of gnathopod propods in female: pleonal plate 1 with subventral spines: and with many subventral spines on pleonal plates 2-3. Largest male. $12.0 \mathrm{~mm}$ : largest temale. $17.0 \mathrm{~mm}$.

Female.--Eye small and round. Antenna 1. about 50 percent length of body, about 1.7 times longer than antenna 2: primary flagellum with up to 28 segments. Antenna 2. flagellum with 10-1 I segments. 
Mandibles subequal. spine row with 5-7 spines: segment 2 of palp with about 8 long setae. segment 3 with 2 A setae. I B seta. 2 C setae. 4-5 E setae and row of D-setae. Maxilla 1: inner plate with 7-8 apical plumose setae; palp with 7-8 slender spines on apex. Maxilla 2. inner plate with oblique row of 7-9 plumose setae on inner margin. Maxilliped: inner plate apically with 3 bladelike spines. 2 naked spines. and $3-5$ plumose setae extending from inner margin to apex: outer plate with row of naked setae and $j-4$ slender pectinate spines on inner margin and I apical plumose seta: dactyl with 4 setae along inner side: dactyl nail very long. about $1 / 3$ length of dactyl.

Propod of gnathopod 1 less than 2 times longer than carpus: paim almost straight. shorter in length than posterior margin. with 10 spine teeth on inside. 9 on outside: defining angle with 4 notched-serrate spine teeth on inside. 1 large notched and 2 serrate spine teeth on outside: posterior margin with about 5-6 sets of setae inserted in groups of 2-4. Dactyl of gnathopod 1 with row of 7 short setae and 5-7 blade-like teeth along the inner margin. nail long. Ventral margin of coxa 1 with 13 setae.

Propod of gnathopod 2 less than 2 times longer than carpus: palm almost straight. shorter than posterior margin. with 13 spine teeth on inside. 12 on outside: defining angle with 3 notched (one very large) and 2 serrate spine teeth on outside. 2 notched and 2 notched-serrate spine teeth on inside: inferior medial setae singly or doubly inserted: superior medial setae singly inserted: posterior marginal with 5-6 sets of setae in groups of 3-7. Dactyl of gnathopod 2 with row of 6 short setae and about 5 blade-like teeth along inner margin: dactyl nail long. Ventral margin of coxa 2 with 16 setae.

Coxa of pereopod 3 with 12 marginal setae. Coxa of pereopod 4 with 20-22 marginal setae: dactyl of pereopod 4 about 33 percent of propod. Pereopod 5: basis with 10 shallow serrations along posterior margin: 7 sets of short spines in groups of $1-3$ on anterior margin: $3-4$ long setae on the anteroproximal margin. Pereopod 6: basis with 14 shallow serrations along posterior margin: 15 sets of short spines on anterior margin: 3 long setae on anteroproximal margin. Pereopod 7: coxa with about 3 setae along posterior margin: basis with 14 shallow serrations along posterior margin: about 19 sets of short spines on anterior margin: dactyl about 30 percent length of propod. Dactyl of pereopods $3-7$ with $2-3$ setae on inner margin.

Pleonal plates: posterior margin of plate I slightly convex. with I seta. distoposterior corner acute but not produced, ventral margin with $1-2$ (subventral) setae: posterior margin of plate 2 nearly straight with 1 2 setae. distoposterior corner slightly produced and acute. ventral margin with 7 (subventral) spines: posterior margin of plate 3 nearly straight with I seta. distoposterior corner neither produced nor acute. ventral margin with 7 (subventral) spines. Peduncle of pleopod 1 with 2 retinaculae. lacking setae on outer margin.

Uropod 1 : inner ramus 70 percent length of peduncle. armed with about 10 spines: outer ramus with about 10 spines: peduncle with row of 7 outer and 1 inner spines. Uropod 2: inner ramus armed with about 12 spines: outer ramus with 9 spines: peduncle with 5 outer and 3 inner spines. Uropod 3 : inner ramus with I spine. outer ramus about 1.5 times longer than peduncle. inner and outer margins each with $3-4$ set of 
spines in groups of $1-3$. Telson broader than long, notched about 1/4 distance to base: each lobe with 3 apical spines and 1-2 subapical plumose setae. spines a little shorter than 1/2 length of telson.

Male.--Differing from female as follows: smaller with more slender. elongate body. Antenna 2: calceoli present on peduncular segments 4 and 5 and first 4 segments of flagellum.

Propod of gnathopod I more than 2 times larger than carpus. palm longer than posterior margin. armed with about 10 spine teeth on inside. 17 spine teeth on outside; defining angle with 3 notched and 1 notched-serrate spine teeth on inside and 3 notched and 2 serrate on outside. Propod of gnathopod 2 more than 2 times larger than carpus, palm margin strongly oblique, longer than posterior margin. with 17 spine teeth on inside, 20 spine teeth on outside: defining angle with 6 spine teeth on inside and 9 on outside.

Uropod 2: inner ramus with about 12 spines: outer ramus strongly curved laterally. with about 11 spines: peduncle with 4 outer spines and 2 inner spines. Lropod 3 : outer ramus about 2 times longer than peduncle.

Distribution and ecology.--The range $C$. setoduct!lus extends from southeastern Ontario south and west across southern Michigan through much of Ohio. eastern and central Indiana and into northcentral Kentucky (fig. 14 ): it also found in the most eastern part of Kentucky. In Ontario and Ohio. its range overlaps with that of $C$. minor. with which it is closed related.

This species is commonly found in a variety of cold-water habitats. including small streams. outlets of drains. springs. sloughs and temporary pools and very rarely in cave streams. The cave populations may have greatly reduced eves but the eves are never completely lost.

Remarks.--Almost all collections reported by Hubricht (1943) as C. shoemukeri from Ohio. Michigan and kentucky were examined by me and found to belong to $C$. setodactrilus.

Crangonix minor Bousfield

Figures 15-18

Crungonyx minor Bousfieid. 1958:98-100. fig. 15 [type-locality: Stony Creek. + mi W of Tillsonburg.

Oxtord Co.. Ontario. Canada].--Holsinger. 1972:4 I [in part]: 1977:252.--Page. 1974:94.--Williams \&

Hynes, 1976:771: 1977:59.--Barnard \& Barnard. 1983:434. map 12 [in part].--Fitzpatrick. 1983:145.--

Pennak. 1989:483 [in part].--Jass \& Klausmeier, 1995:8.

Eucrangonxx shoemakeri Hubricht and Mackin, 1940:198 [in part].

Crangonyx shoemakeri (Hubricht and Mackin).--Hubricht. 1943:690- 691 [in part].

Material examined.-- ONTARIO. Oxford Co.: Stony Creek, $6.4 \mathrm{~km} \mathrm{~W}$ of Tillsonburg. holotype $\cong$. allotype $\approx$ (NMC), paratopotypes $4 \subsetneq$ and $2 \approx *$ E. L. Bousfield. 29 May 1957 (USNM): ARKANSAS. Greene Co.: seep $8.0 \mathrm{~km} \mathrm{~N}$ of Brookland, $2 \subsetneq .1$ juv, D. Barnett, 16 Feb 1970. ILLINOIS. Bond Co.: 


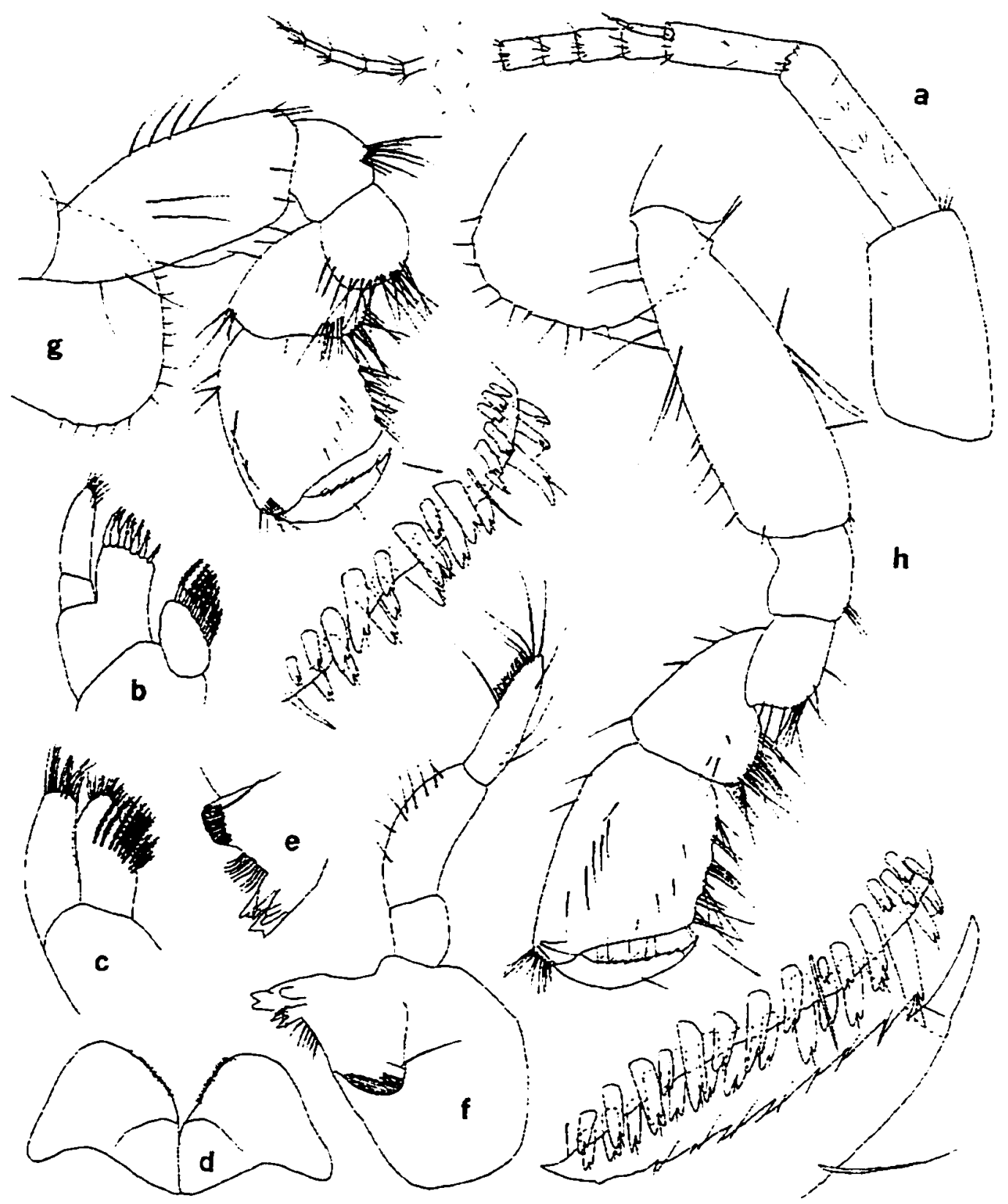

Figure 15.--Crangonix minor Boustield. Female $(11.8 \mathrm{~mm})$. Outlet of drain. $2.4 \mathrm{~km} \mathrm{SW}$ of Galesburg. Knox Co.. Illinois: a. antenna 1: b. c. maxillae 1. 2: d. lower lip: e. dentate part of left mandible: f. right mandible: g. h. gnathopods 1,2 (palmar margins enlarged). 


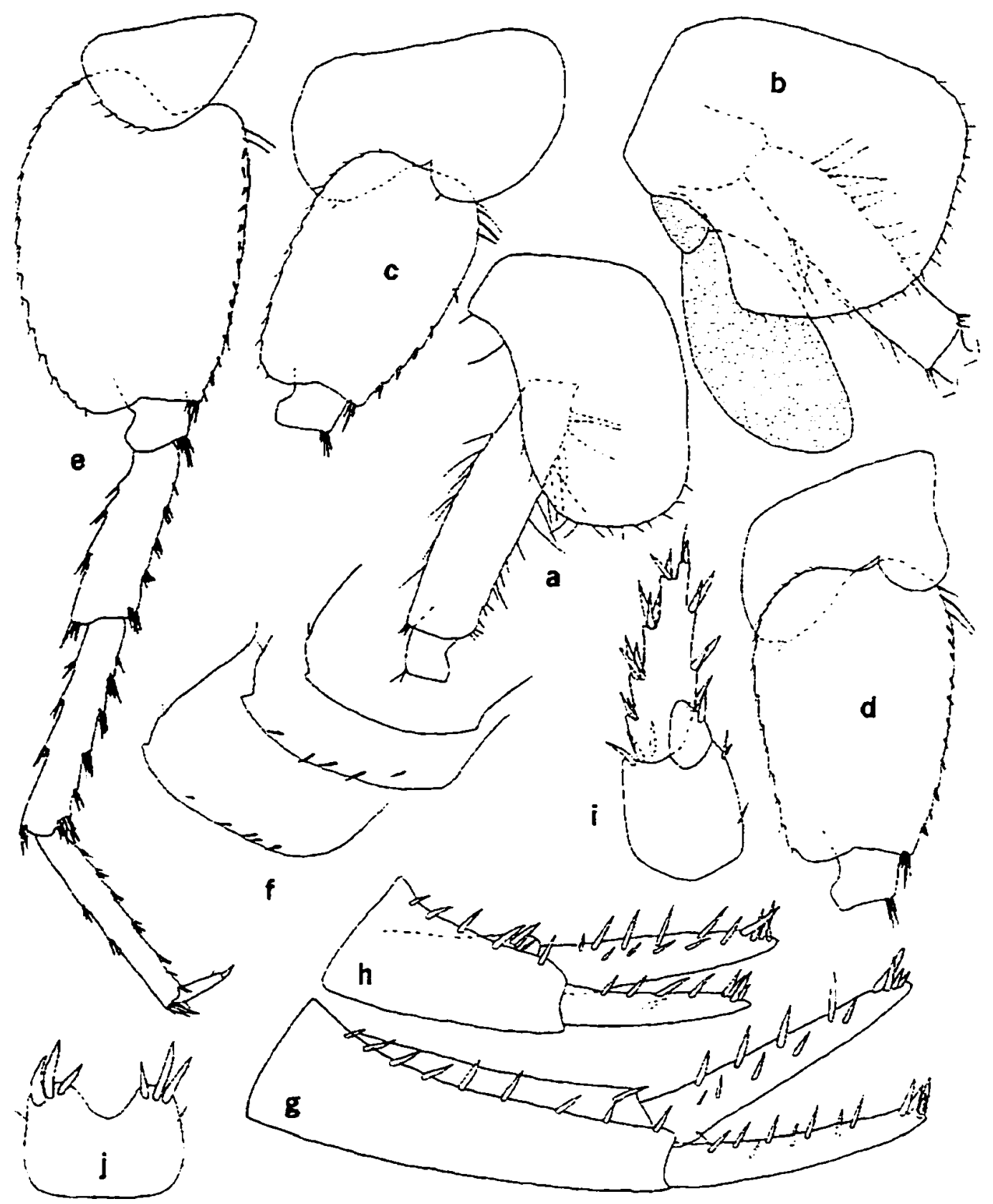

Figure 16.--Crangonyx minor Bousfield. Female $(11.8 \mathrm{~mm})$. Outlet of drain. $2.4 \mathrm{~km} \mathrm{SW}$ of Galesburg. Knox Co.. Illinois: a. b. c. d. pereopods 3.4.5.6 (in part): e. pereopod 7: f. pleonal plates: g. h. i. uropods $1,2,3:$ j, telson. 


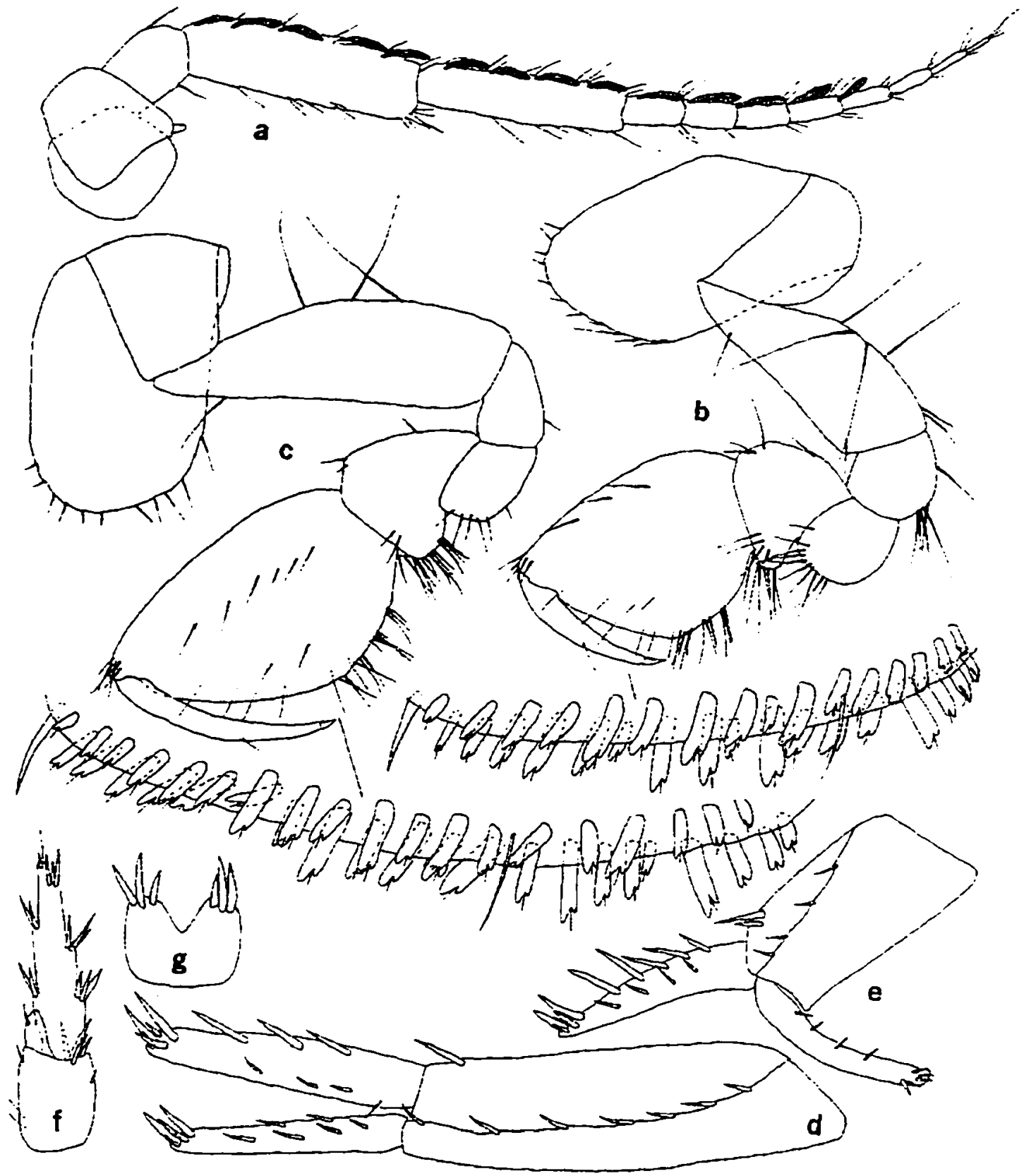

Figure 17.--Crangomx minor Bousfield. Male $(6.3 \mathrm{~mm})$. Outlet of drain. 2.4 $\mathrm{km} \mathrm{SW}$ of Galesburg. Knox Co.. Illinois: a. antenna 2: b. c. gnathopods 1, 2 (palmar margins enlarged); d. e. f. uropod 1. 2. 3: g. telson. 


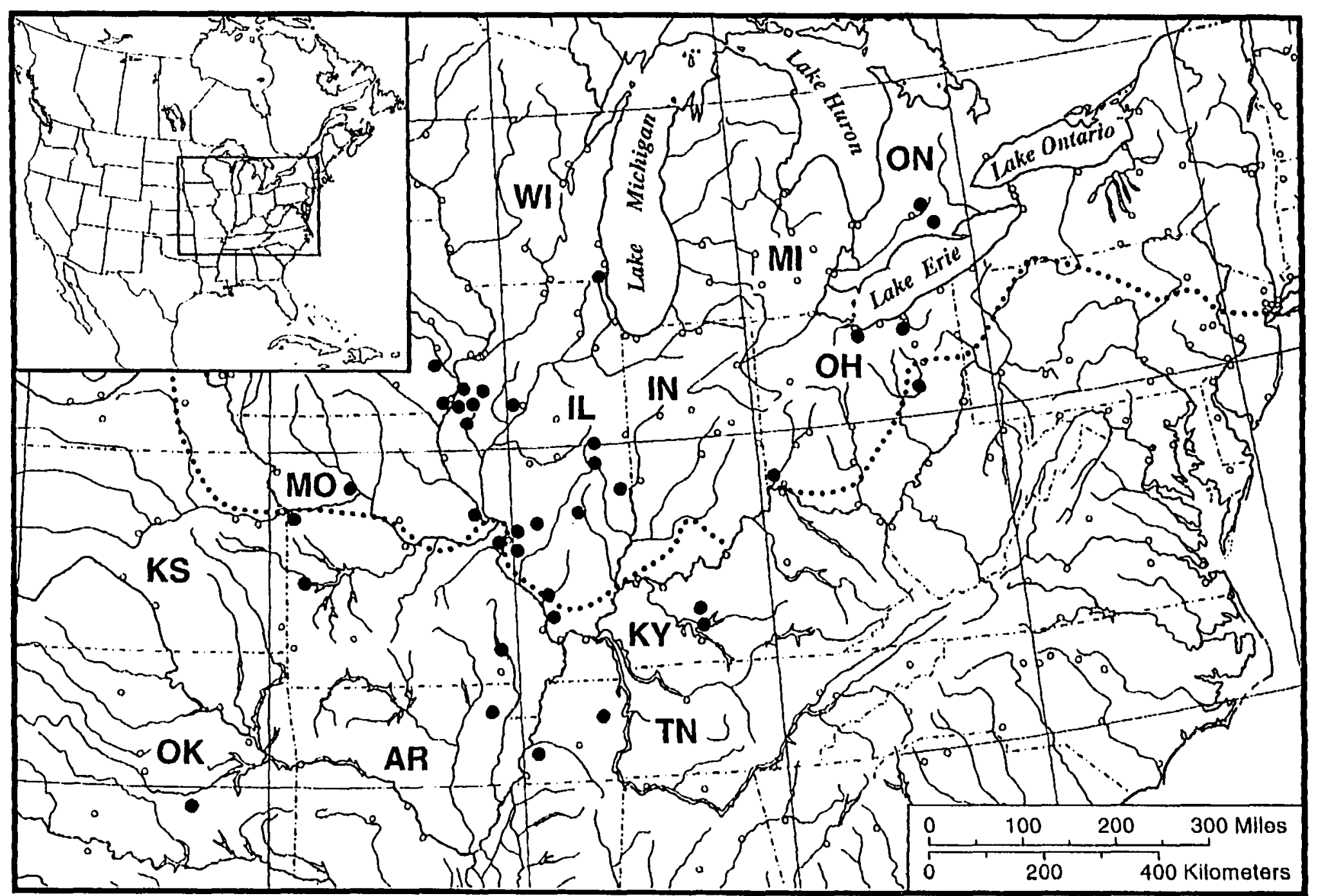

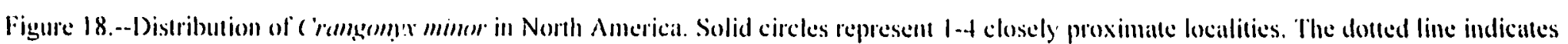
the approximate southern extent of glaciation during the Plesistocene. 
temporary stream $0.8 \mathrm{~km}$ W of Beavercreek. $59.10 \approx$. L. Hubricht. 16 Apr 1942 (USNM): Clark Co.: spring $3.0 \mathrm{~km}$ ENE of Marshall. 7 : L. Hubricht. $16 \mathrm{Apr} 1942$ (USNM): Champaign Co.: Oxbow.

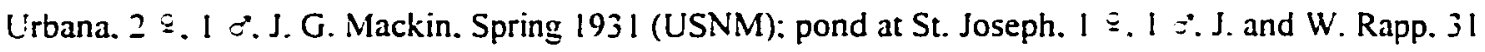
Mar 1946 (USNM): Douglas Co.: pitfall trap in wetland. $3.2 \mathrm{~km} \mathrm{~N}$ of Arcola Prairie. 25 . E. A. Lisowski. 8 Nov - 6 Dec 1993: Effingham Co.: temporary stream $1.6 \mathrm{~km}$ SW of Funkhouser. $100 \operatorname{specs}\left(\bar{z}_{.} z^{*}\right)$. L. Hubricht. 16 Apr 1942 (USNM): ditch $3.0 \mathrm{~km}$ WSW of Altamont. $35 \varepsilon .18$ c. L. Hubricht. 16 Apr 1942 (USNM): Henderson Co.: outlet of drain $2.7 \mathrm{~km}$ W of Biggsville. 1 \%. L. Hubricht. 25 Apr 1942 (USNM): Jackson Co.: seep in sandstone in Giant City State Park. $1 \Xi^{*}$. J. J. Letwis. 5 May 1974: Knox Co.: Arbingdon. $15:$ W. P. Hay, collection date not given (USNM): outlet of drain. $3.2 \mathrm{~km} \mathrm{~N}$ of Abingdon. 55 specs $(§$. :). L. Hubricht. 4 May 1941 (USNM): outlet of drain $2.6 \mathrm{~km} \mathrm{SW}$ of Galesburg. 100 specs ( $\because$ ). L. Hubricht. + May 1941 (USNM): Madison Co.: spring $0.6 \mathrm{~km} \mathrm{SW}$ of Troy. $29 \Xi .15 \approx$. L. Hubricht. 16 Apr 1942 (USNM): temporary pool near Silver Creek. $3.6 \mathrm{mi}$ W of St. Jacab. $2 \fallingdotseq .2 \div$. L. Hubricht. 16 Apr 1942 (USNM): Mcdonough Co.: outlet of drain. $3.2 \mathrm{~km}$ E of Good Hope. $18 \Xi .+\approx *$. L. Hubricht. 4 May 1941 (USNM): outlet of drain. $4.8 \mathrm{~km} \mathrm{~S}$ of Colmar. 5 Z. $44 \approx$. L. Hubricht. + May 1941 (USNM): Peoria Co.: spring $5.0 \mathrm{~km} \mathrm{~W}$ of Princeville. $179.40 \approx$. L. Hubricht. 4 May 1941 (USNM): St. Clair Co.: temporary pool. ditch $1.6 \mathrm{~km} \mathrm{~S}$ of Falling Spring. $4 \Xi .1 \approx$. L. Hubricht. 24 Apr 1938 (USNM): temporary pond. roadside ditch. $N$ of Dupo. I $\approx$. L. Hubricht. 24 Apr 1938 (USNM): Union Co.: temporary pool near McCann School. 2.5 mi NE of Aldridge. $10 \cong .10 \approx$. L. Hubricht. 14 Apr 1940 (USiviv): small spring-fed marsh. $4.0 \mathrm{~km}$ E of Ware. 50 specs ( $\because$ ). L. Hubricht. 27 Apr 1940 (USNM). IOWA. Des Moines Co.: outlet of drain $0.3 \mathrm{~km} \mathrm{NW}$ of Danville, 46 specs ( $\left(z^{*}\right)$. L. Hubricht. 24 Apr 1942 (LSNM): outlet of drain $5.8 \mathrm{~km}$ E of Middletown. $13 \cong .20 \mathrm{z}$. L. Hubricht. 25 Apr 1942 (USNM): Washington Co.: stough of English River $4.0 \mathrm{~km}$ E of Riverside. $15 \Xi .17 \approx$. L. Hubricht. 24 Apr 1942 (USNM): outlet of drain. 1.6 $\mathrm{km}$ S of Haskins. 51 इ. 10 o*. L. Hubricht. 24 Apr 1942 (USNM). KENTUKEY. Edmonson Co.: seep 1.6 km NW of Mammoth Cave. $20 \cong$. L. Hubricht. 13 Apr 1957 (USNM): Gravson Co.: rill $7.2 \mathrm{~km} \mathrm{SW} \mathrm{of}$ Leitchfield. $29.1 \approx 0$. L. Hubricht. 4 May 1958 (USNM). MISSOURI. Carroll Co.: tile drain in Bosworth. 1 ૬. J. Craig. 29 Apr 1974: Jackson Co: 1 mi W of Lake Tapawingo (habitat not given). 13 ¡. 3 . P. J. Spangler. $30 \mathrm{Mar} 1952$ (USNM): Lincoln Co.: marsh $3.2 \mathrm{~km} \mathrm{~S}$ of Apex. $14 \Xi .2 \approx$. L. Hubricht. $22 \mathrm{Mar}$ 1941 (USNM): St. Charles Co.: temporary pool $0.5 \mathrm{~km}$ S of St. Peters. $26 \approx .40 \approx$. L. Hubricht. 22 Mar 1941 (USNM): St.. Louis Co.: Marsh. N end of Creve Coeur Lake. $3 \div .1 \approx$. 1 juv. L. Hubricht. 8 Mar 1936 (USNM): slough of Mississipi River $1.6 \mathrm{~km} \mathrm{~S}$ of Grimsby. $15 \cong .1 \approx$. L. Hubricht. 29 Feb 1936 (USNM) and $15 \cong .4 \approx .25$ Apr 1938 (USNM): Wayne Co.: temporary pond. just E of Greenville. $1 \cong .1$ juv. L. Hubricht. 5 Apr 1941 (USNM). OHIO. Butler Co.: dug well on Francis Farm at Sandow. $2 \leftleftarrows .1$ : S. R. Williams. collection date not given (USNM): Cuyahoga Co.: small shallow pool in woods near Gates Mills. 2 . 22 . N. C. Furtos. 8 Apr 1933 (USNM): Erie Co.: stream near hatchery. Blue Hole. Castalia. 2 ¿. N. C. Furtos. 22 Jul 1931 (USNM): Tuscarawas Co.: New Philadelphia (habitat not given). $139.5 \approx$ V. Sterki. collection date not given (USNM). OKLAHOMA. Pontotoc Co.: temporary stream E of Ada. 25 
specs ( Carroll-Madison County Line. $12 £ .13 c^{*}$. R. Fox et al.. 30 Dec 1970: Tipton Co.: seep $7.0 \mathrm{~km} \mathrm{SW}$ of Crosstown. $3 \varsubsetneqq .2 \approx$ L. Hubricht. 26 Feb 196 I (USNM). WISCONSIN. Kenosha Co.: unnamed stream at County Trunk Highway AH $3.5 \mathrm{~km}$ E jct Hwy 83. 2 \&. J. P. Jass \& B. Klausmeier. 6 May 1994 (MPM): N side County Trunk Highway $K$ just $W$ of 194.0 .5 km E of County Trunk Highway MB. $2 \equiv .5:$. J. P. Jass and B. Klausmeier (MPM).

Diagnosis.--A member of shoemakeri group easily distinguished from $C$. shoemakeri and $C$. aberrans by absence of comb spines on uropod 2 of male; from $C$. setoductvlus by having only I seta on inner margin of dactyl of pereopods 3-7. more setae on coxa ventral margin of pereopod 7. unproduced distoposterior comers of pleonal plates. and outer ramus of uropod 2 of male without inner spines (with about 4 short outer spines). Largest male. $8.5 \mathrm{~mm}$ : largest female. $12.5 \mathrm{~mm}$.

Female.--Eye large and ovate. Antenna 1 . about 52 percent length of body. about 1.8 times longer than antenna 2: primary fiagellum with up to 24 segments. Antenna 2. flagellum with 8-10 seyments. Mandibles subequal. spine row with 5-7 spines: segment 2 of palp with 7 long setae. segment 3 with 2 A setae. I B seta. I C seta. 5 E setae and row of 14 D setae. Maxilla 1: inner plate with 7-8 apical plumose setae: palp with 5-6 slender spines on apex. Maxilla 2. inner plate with oblique row of 8 plumose setae on inner margin. Maxilliped: inner plate apically with $3-4$ bladelike spines. 3 naked setae. and about 9 plumose setae extending from inner margin to apex: outer plate with 4-5 slender pectinate spines on inner margin and I plumose seta on apex: dactyl with 5 setae along inner margin: dactyl nail long, about 13 length of dactyl.

Propod of gnathopod 1 less than 2 times longer than carpus: palm shorter than posterior margin. concave. with 10 spine teeth on inside. 11 on outside: defining angle with 5 notched-serrate spine teeth on inside. I large notched and 2-3 serrate spine teeth on outside: superior medial setae in 3 sets. singly or Joubly inserted: posterior margin with 5 sets of setae in groups of 1-4. Dactyl of gnathopod I with row of short setae and blade-like teeth along inner margin: dactyl nail long. Ventral margin of coxa 1 with 15 setae.

Propod of gnathopod 2 less than 2 times longer than carpus: palm shorter than posterior margin. concave, with about 9 spine teeth on inside. II on outside; defining angle with 2-3 notched and 4 notchedserrate spine teeth on inside. 3 notched and $1-j$ serrate spine teeth on outside: about 6 superior medial setae. singly inserted: posterior margin with 5 sets of setae in groups of 2-5. Dactyl of gnathopod 2 with row of short setae and blade-like teeth along inner margin. Ventral margin of coxa 2 with 13 setae.

Coxa of pereopod 3 with 12 marginal setae. Coxa of pereopod 4 with 23 marginal setae. dactyl of pereopod 4 about 33 percent length of corresponding propod. Pereopod 5: basis with 15 shallow serrations along posterior margin: 5 short spines on anterior margin: $3-4$ long setae on the anteroproximal margin. Pereopod 6: basis with 18 shallow serrations along posterior margin. 9 sets short spines in groups of $1-2$ on anterior margin: 3-4 long setae on anteroproximal margin. Pereopod 7: coxa with 4-6 setae along posterior margin: basis with $17-19$ serrations along posterior margin. 15 short spines on anterior margin: 2 long setae 
on anteroproximal margin.

Pleonal plates: posterior margin of plate I slightly convex. with I seta. distoposterior corner not produced: postertor margin of plate 2 nearly straight with I seta. distoposterior corner acute but not produced. ventral margin with 5 (subventral) spines: posterior margin of plate 3 nearly straight with I seta. distoposterior comer not produced, ventral margin 5 (subventral) spines. Peduncle of pleopod I with 2 retinaculae. lacking setae on outer margin.

Uropod 1: inner ramus 70 percent length of peduncle. armed with about 12 spines: outer ramus with 14 spines: peduncle with 9 outer and 1 inner spines. Uropod 2: inner ramus armed with 10 spines: outer ramus with 16 spines: peduncle with 2 outer spines. 3 inner spines. Uropod 3 : inner ramus with 1 spine. outer ramus about 1.5 times longer than peduncle. inner and outer margin each with $3-4$ sets of spines in groups of $1-3$. Telson broader than long. notched about 1.3 distance to base. each lobe with $2-3$ apical spines. spines about $1 / 2$ length of telson.

Male.--Differing from female as follows: smaller size with slender. elongate body. Antenna 2: flagellum with 8 segments, calceoli present or peduncular segments $3-j$ and first 3 segments of flagellum.

Propod of gnathopod I more than 2 times longer than carpus: palm slighlty longer than posterior margin. oblique and convex. with 11 spine teeth on inside. 12 on outside: detining angle with I notched and 4 notched-serrate spine teeth on inside. 4 notched and 2 serrate spine teeth on outside. Propod of gnathopod 2 more than 2 times longer than carpus: palm longer than posterior margin. oblique and convex. with 13 spine teeth on inside. 13 on outside: defining angle with 6 spine teeth on inside and 8 on outside.

Liropod 2: inner ramus with 12 spines: outer ramus strongly deflected and curved, with 4 short outer spines and 4 spines on apex, lacking inner spines: peduncle with 5 outer spines. and 2 inner spines.

Variation.--Significant variation was found in the number of blade-like teeth along the inner margin of dactyl of gnathopods in female. Normally the teeth are arranged one by one on the margin. but in some specimens blade-like teeth may occur as just several or one in the middle of the margin.

Distribution and ecology.--This species is recorded from southeastern lowa. central and southern Illinois. northwestern Missouri. central Kentucky. northeastern Arkansan. western Tennessee. southern Oklahoma and southern Ontario (fig. 18). In Ontario and Ohio. its range overlaps with that of $C^{\circ}$. setodactilus.

Crangonyx minor inhabits a variety of aquatic habitats. including small streams. sloughs. ditches. drains. springs. and ponds.

Remarks.--Almost all collections reported by Hubricht (1943) as Crangonix shoemakeri from Illinois. lowa and Missouri and examined by me in this study belong to $C$. minor. The type localities for $C$. minor and $C$. setodactilus are only a few kilometers apart.

This species has not been found in Indiana to date. The previous records for Indiana (see Holsinger. 1972. map 19: Barnard \& Barnard. 1983. map 11: and Pennak. 1989. page 483) are incorrect: they are actually records for C. setodactylus. 
Crangomx aberrans Smith

Figures $19-20$

Crangonyx pseudogracilis Bousfield.--Smith. 1981:11. fig. 6. [in part].

Crangmmix uberrans Smith, 1983:356-363. figs. 1-5. [type-locality: Spot Pond Brook. Stoneham.

Middlesex Co.. Massachusetts].--Smith. 1988:46, figs. 35. $43 \mathrm{c} .+4 \mathrm{c} .+5 \mathrm{~b}$.

Material examined.--CONNECTICUT. Windham Co.: swamp. $2.9 \mathrm{~km}$ NE of Thompson. 25 specs. $\mathrm{L}$. Hubricht. 10 Jun 1965 (USNM): MASSACHUSETTS. Middlesex Co.: Inch Brook. Acton. 4 . $3 z$. D. G. Smith. 22 May 1982: Spot Pond Brook. Stoneham, holotype $\bar{~}$ allotype $\geq .4 \geq .2$ z. 9 juvs (paratypes). D. G. Smith. 5 Aug 1982 (MCZ). RHODE ISLAND. Providence Co.: 6 ₹. F. P. Drowne. collection date not given. (USNM).

Diagnosis.--A small species of shoemakeri group distinguished from all other species of Crungrmx by presence of dorsal, lateral. and ventrolateral spines on the telson: and from other members of the shocmakeri group by having just a few teeth on inner margin of dactyls of gnathopods of female: and specifically from $C$. setoductrlus. and $C$. minor by having comb spines on uropod 2 of male. Largest male. $6.5 \mathrm{~mm}$ : largest female. $10.0 \mathrm{~mm}$.

Description.--Smith (1983) gave a very accurate description and good illustrations of this rather unusual species. The following is a brief supplement to Smith's description.

Female: Antenna 1. primary flagellum with up to 20 segments: inner margin of outer lobe of maxilliped with 4-6 slender pectinate spines: posterior margin of propod and carpus of gnathopods armed with weakly rastellate setae: pleonal plates $1-3$. each with I seta on posterior margin: distoposterior corner of pleonal plate 3 weakly acuminate with 2 submarginal spines: for most specimens outer margins of dactyl of pereopods 6-7 have only I setae.

Distribution and ecology.--This species was originally found in northeastern Massachusetts. but new locality records have extended its range south to Connecticut and Rhode Island (fig. 20). It is an inhabitant of small rivers. brooks, and swamps.

Remarks.--The presence of notched spine teeth on the palms of gnathopodal propods of $C^{\circ}$. uberrans distinguishes it from all species of the gracilis group. It is assigned to the shoemakeri group because of the following two characters: (1) spine teeth of defining angle of gnathopods like those of other three species of the shoemukeri group: (2) inner margin of gnathopodal dactyl with blade-like teeth in female as in other species of shoemakeri group (although just a few in number).

Crangonyx aherrans is unique among Crangonyx by having lateral spines on telson. However dorsal spines are found on the telson of $C$. serratus. and in some specimens of $C$. dearolfi. 


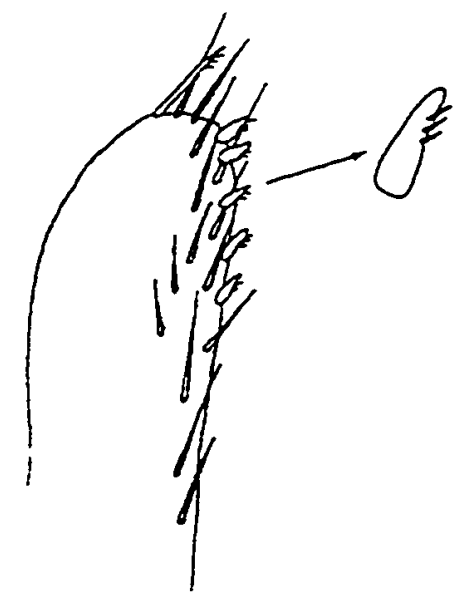

Figure 19.--Crangonyx aberrans Smith. Female holotype $(7.0 \mathrm{~mm})$. Spot Pond Brook. Stoneham. Massachusetts: outer plate of maxilliped (greatly enlarged). 


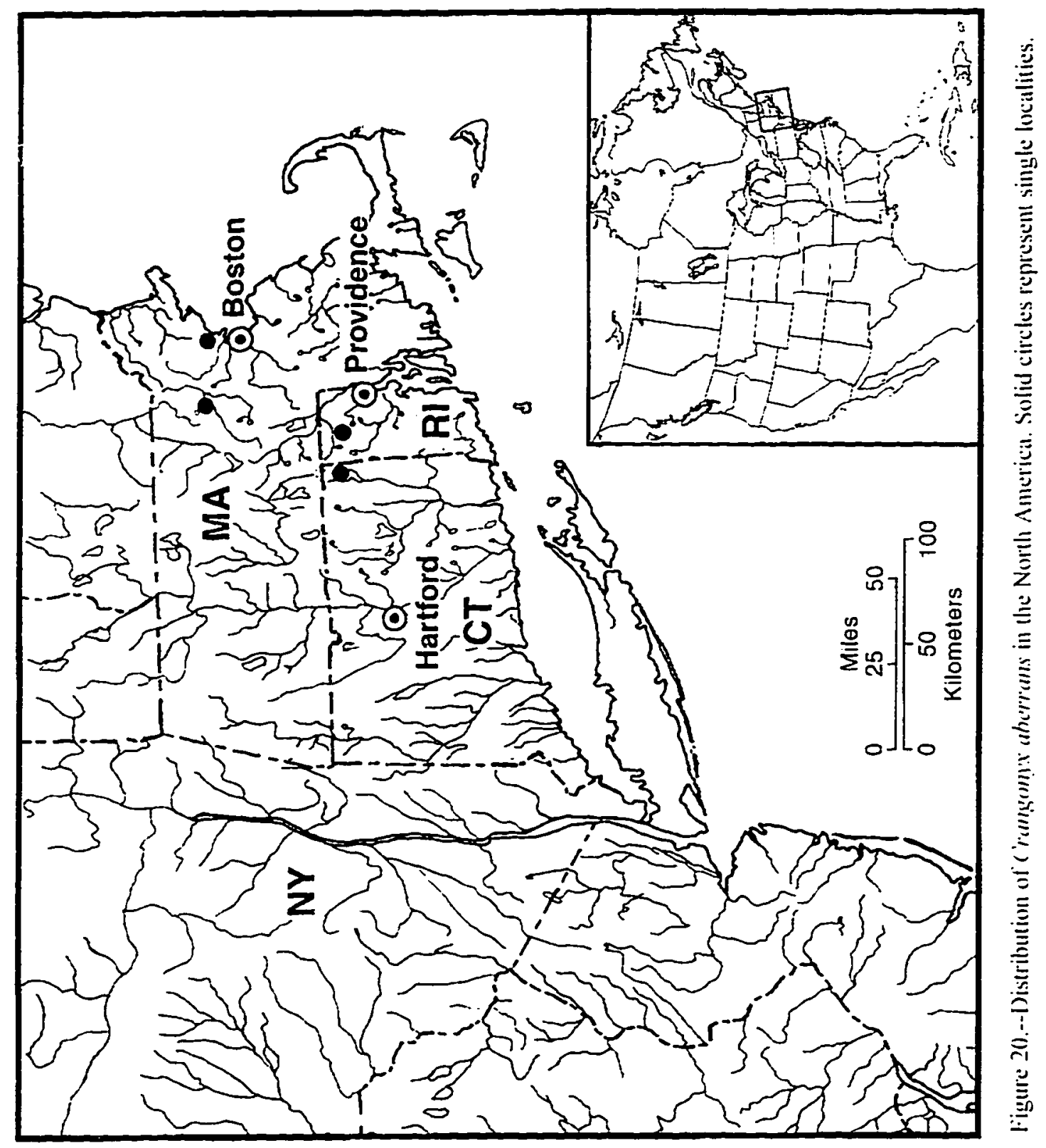




\section{The longicarpus group}

Diagnosis.--Adult size range, 5.5-9.4 mm. Segment 3 of palp of mandibles with 2 A setae and I B seta. Maxilla 1: inner plate with 8 apical plumose setae. Maxilla 2, inner plate with oblique row of 8 plumose setae on inner margin. Gnathopods of female: carpus slightly longer or subequal to propod. posterior margin armed with many sets of long setae: propod palm armed with symmetrically-notched spine teeth: superior medial setae singly inserted: defining angle of propod of gnathopod I with several notched spine teeth on inside (direction of notch is opposite the dactyl). defining angle of propod of gnathopod 2 with "normal" notched spine teeth. Gnathopods of male: carpus subequal in length to propod in gnathopod I and shorter and slightly narrower in gnathopod 2: palm armed with (normal) spine teeth: the defining angle like that of female. Peduncular segment of pleopod I with only 2 retinaculae. but with a few setae on outer margin.

Remarks.--This group is composed of a single species that inhabits the Coastal Plain of southeastern Virginia and northeastern North Carolina. The symmetrically-notched spine teeth on the propod palms of gnathopods of the female may represent an evolutionary intermediate stage between larger notched spine teeth and small simple spine teeth. It is likely that additional species with this distinct character will be lound in the future.

\section{Crungomx longicarpus, new species}

Figures 21.25

Material examined (paratypes unless designated otherwise).--NORTH CAROLINA. Hertford Co.: Winton. 1 (not paratype). collector unknown. 23 Mar 1940 (USNM). VIRGINIA. Henrico Co.: Brook Hill. N of Richmond. 39 . A. Pizzini, 30 Apr 1939 (USNM); Isle of Wight Co.: small stream. $1.6 \mathrm{~km} \mathrm{~S} \mathrm{of}$ Rescue. $29.1 \approx$. L. Hubricht, 12 Mar 1944 (USNM); outlet of drain on Taylor Farm. $5.6 \mathrm{~km} \mathrm{~N} \mathrm{of}$ Chuckatuck. 1 ๖. J. R. Holsinger. 27 Mar 1983: James City Co.: Taskinas Creek tributary headwaters. about $2.5 \mathrm{~km}$ NE of Christensons Corners. I $\Xi$ (not paratype). C. S. Hobson and D. J. Stevenson 29 Dec 1994: Nansemond Co.: Whaleyville. $2 \mp .1 \propto$. R. Bray. 22 Mar 1940 (USNM): springs. $3.2 \mathrm{~km} \mathrm{~W} \mathrm{of}$ Driver. 6 ¡. L. Hubricht. 26 Mar 1944 (USNM): Norfolk Co.: small stream. $0.8 \mathrm{~km} \mathrm{~W}$ of Churchland. $2 \equiv$. L. Hubricht. 30 Jan 1944 (USNM): Northampton Co.: Crayfish burrows along Rt. 600 at Seaview. holotype ₹. $18 \Xi .25$ \%.F. W. Grimm and J. M. Odell. 6 Dec 1964 (USNM): Holly Grove Creek. Rt. 606 crossing. 1 ₹.P. H. Stevenson. 27 Oct 1992: Suffolk (Nansemend County), shallow well opening to drain tiles. Barlow Farm, 3.2 km NNE of Chuckatuck. $3 \cong .1$, J. R. Holsinger. 27 Mar 1983: Warwick Co.: pools in woods. $4.0 \mathrm{~km} \mathrm{NW}$ of Newport News, II $9.9 \mathrm{c}$. L. Hubricht. 23 Jan 1944 (USNM): York Co.: hillside seepage $\mathrm{N}$ of Colonial Parkway Bridge. 6 Z. 2 \&. S. M. Roble. 22 Aug 1994. 


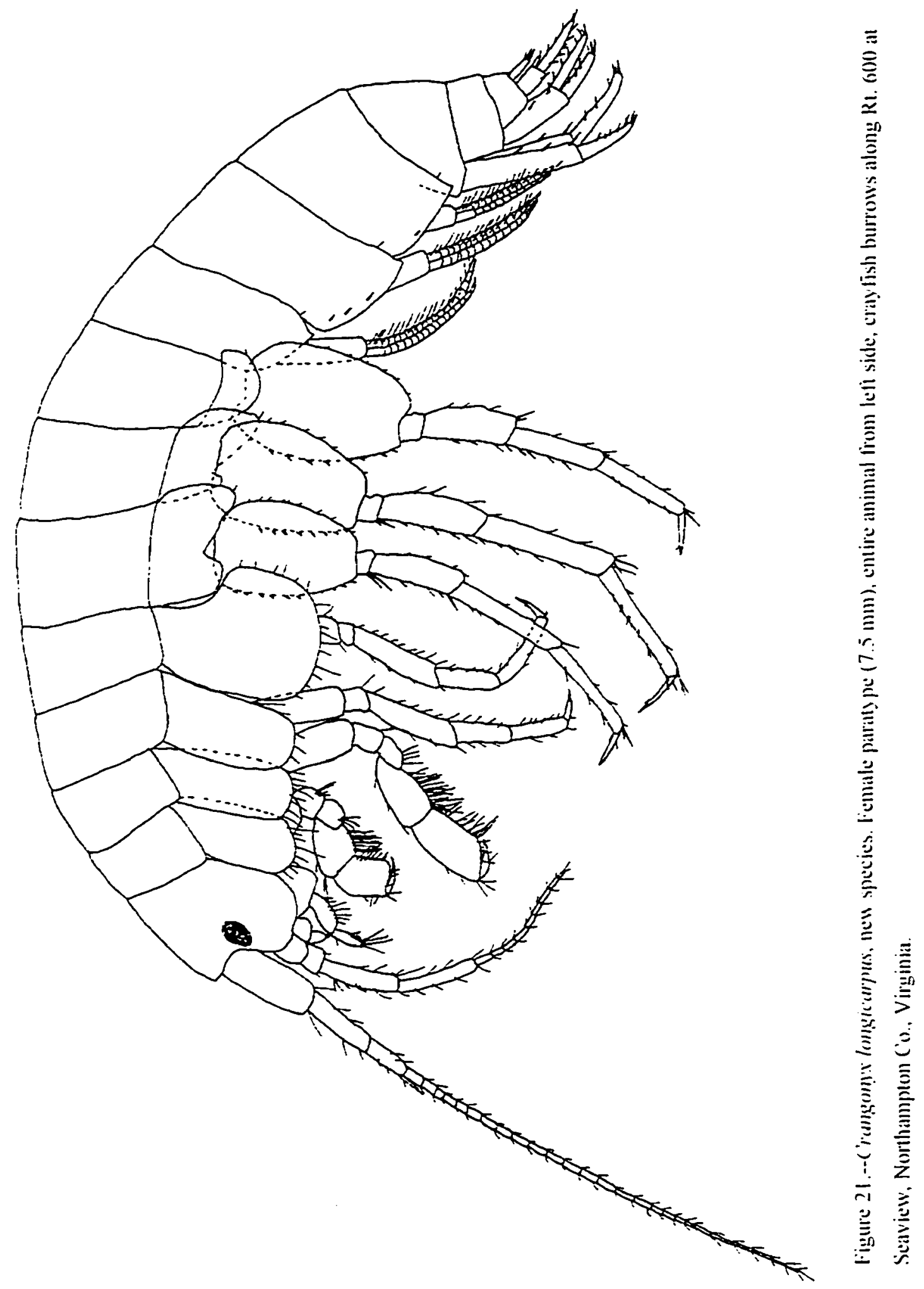




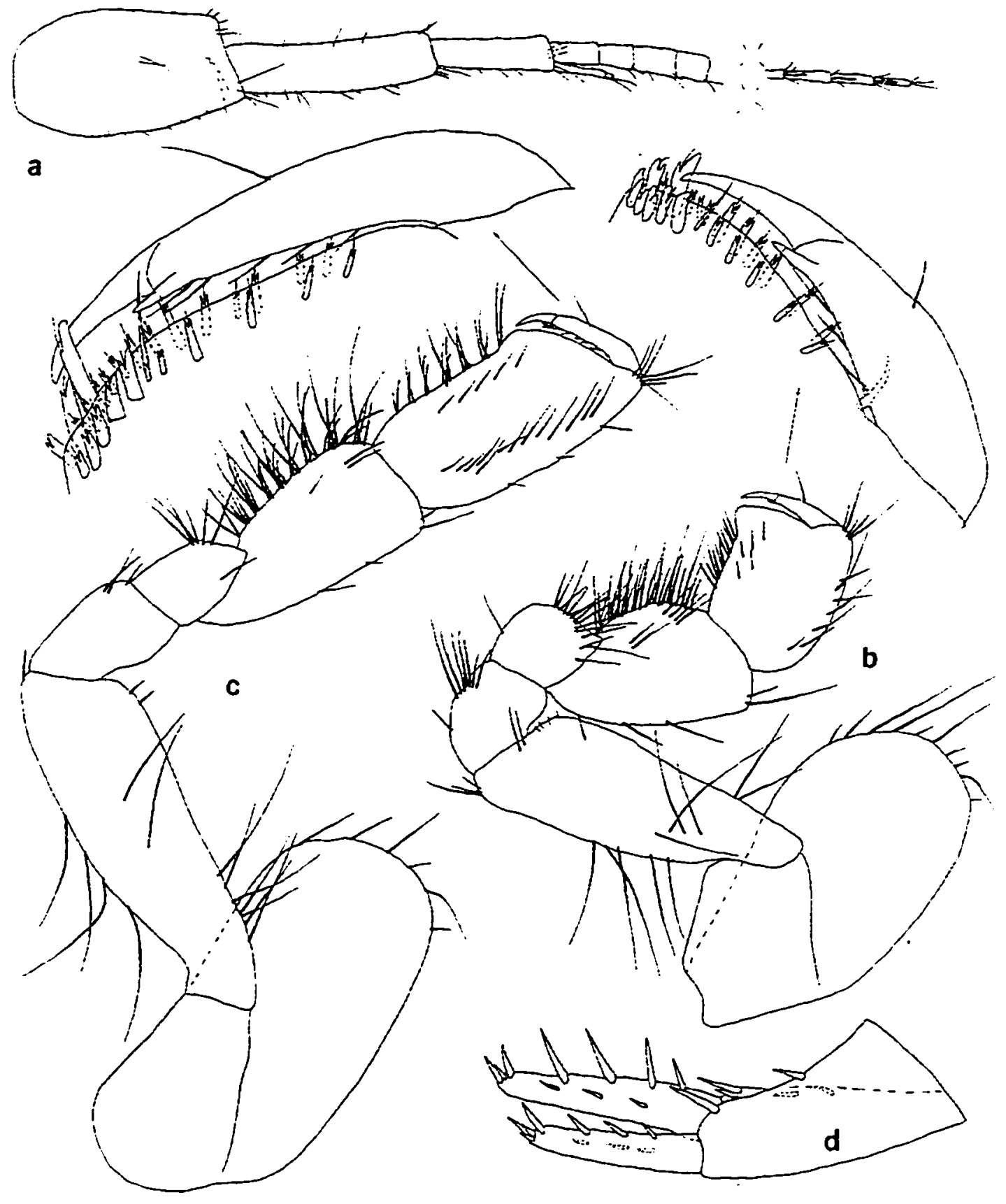

Figure 22.--Crangonvx longicarpus, new species. Female paratype $(8.5 \mathrm{~mm})$. craytish burrows along Rt. 600 at Seaview. Northampton Co., Virginia: a. antenna 1: b. c. gnathopods I. 2 (palmar margins and dactyls enlarged): d. uropod 2. 


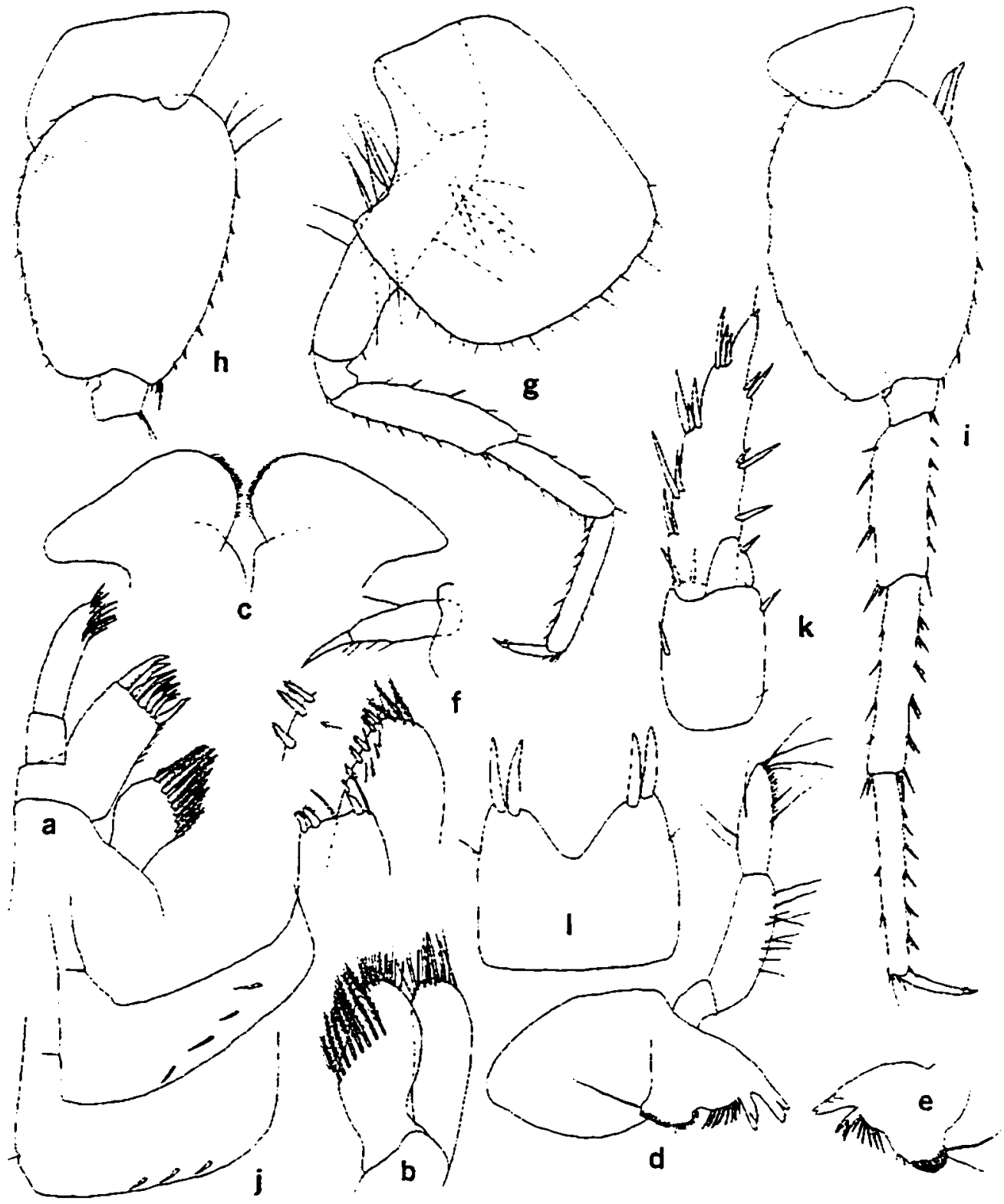

Figure 23.--Crangonvx longicarpus. new species. Female paratype $(8.5 \mathrm{~mm})$. craytish burrows along Rt. 600 at Seaview. Northampton Co.. Virginia: a. b. maxillae I. 2: c. lower lip: d. left mandible: e. dentate part of right mandible: $f$. inner and outer plates and dactyl of palp of maxilliped (greatly enlarged): g, pereopod 4: h. pereopod 6 (in part): i. pereopod 7: j. pleonal plates: $k$. uropod $3: I$. telson. 


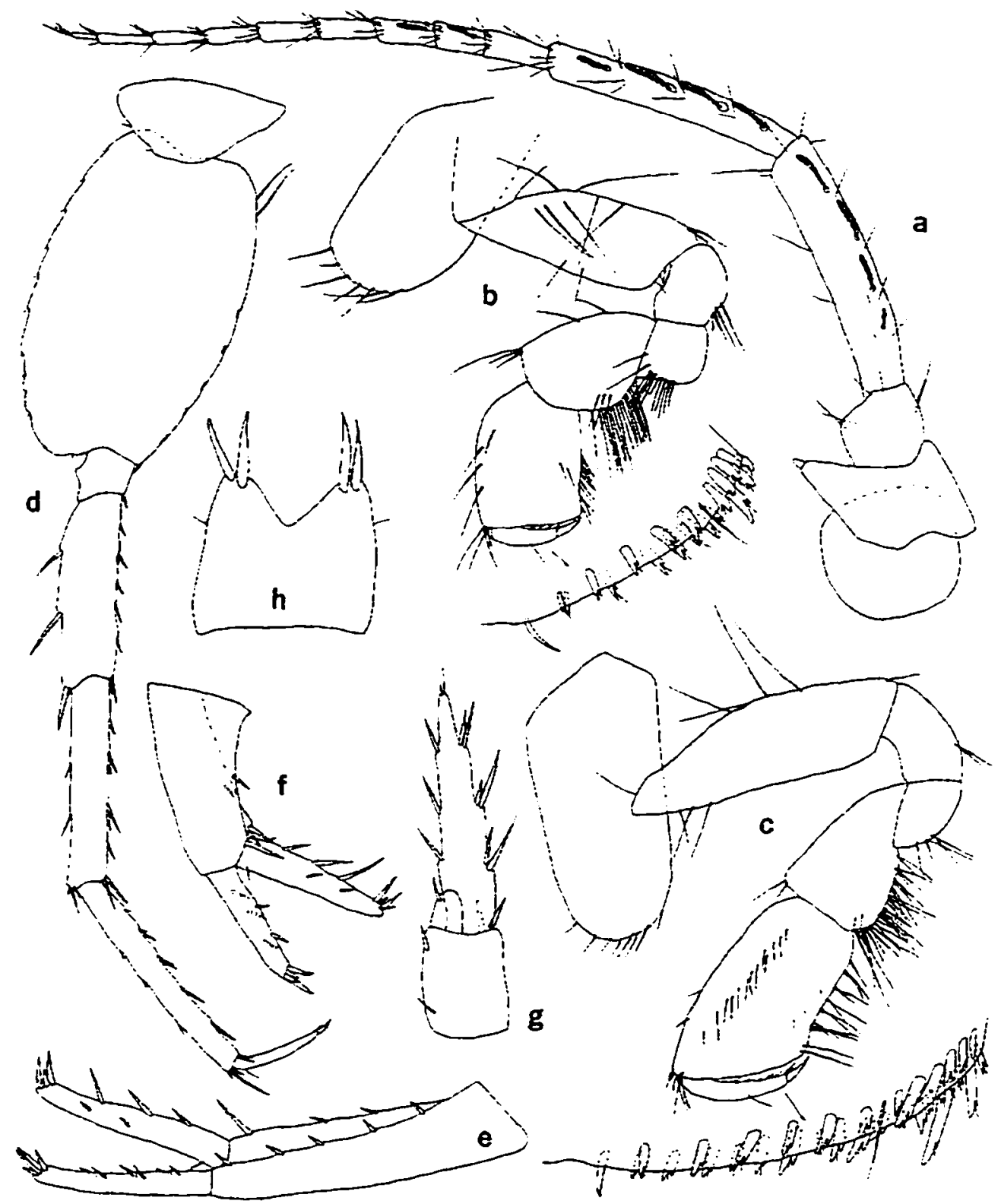

Figure 24.--Crangonvx longicarpus, new species. Male paratype $(6.2 \mathrm{~mm})$, cray tish burrows along Rt. 600 at Seaview. Northampton Co.. Virginia: a. antenna 2: b. c. gnathopods 1. 2 (palmar margins enlarged): d. pereopod 7: e. f. g. uropods 1. 2. 3: h. telson. 


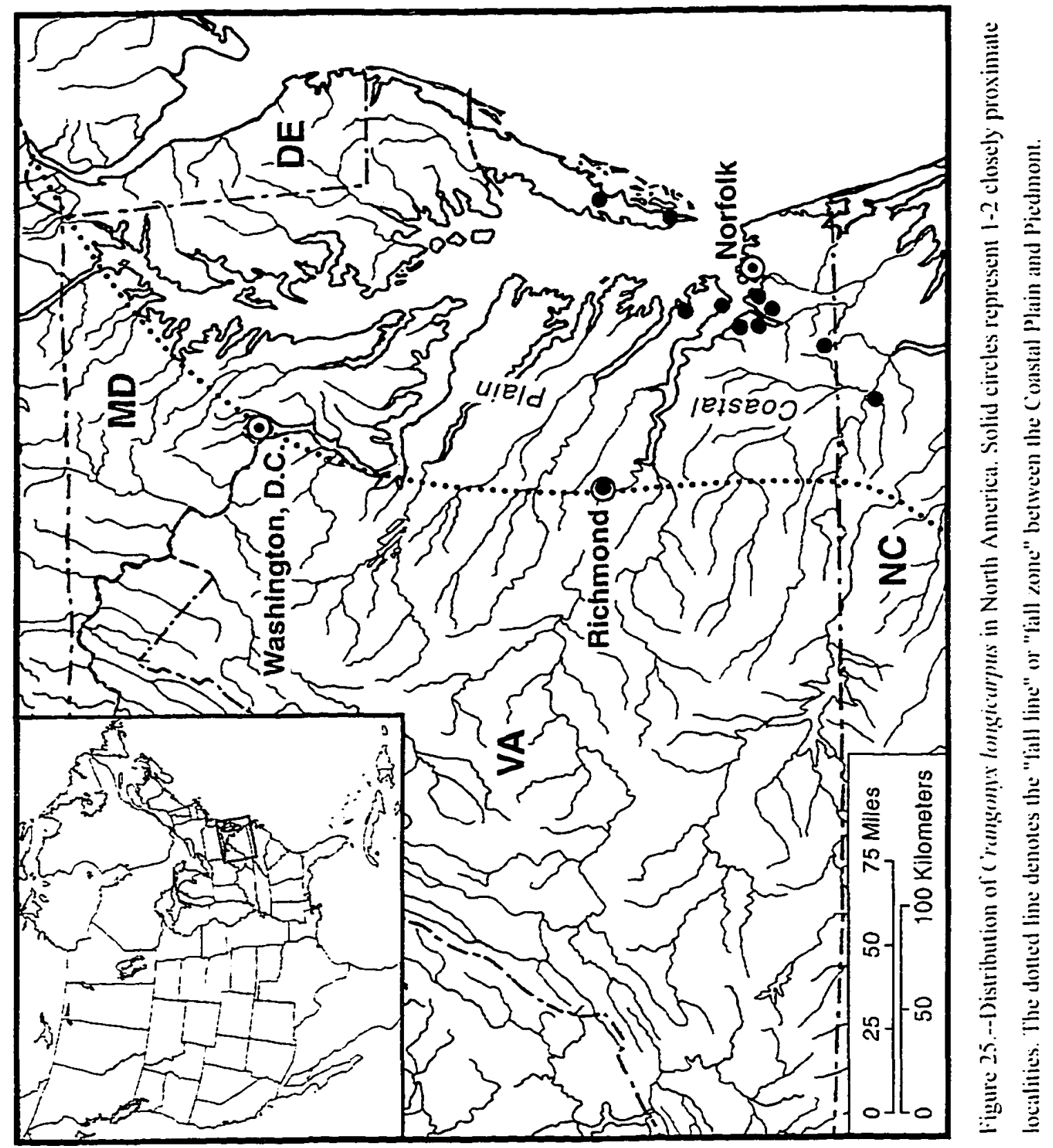


Diagnosis.--A small species distinguished from all other species of Crangonyx by having unique slender symmetrically-notched spine teeth on gnathopods of female. Superficially resembling $C^{\circ}$. hobbsi by the with very long carpus and propod of gnathopods in both sexes. In female gnathopods. the carpus is longer than propod. Largest male, $6.2 \mathrm{~mm}$ : largest fernale. $9.4 \mathrm{~mm}$.

Female.--Eye large and round. Antenna 1, about 50 percent length of body. about 1.8 times longer than antenna 2: primary flagellum with about 24 segments. Antenna 2: flagellum with 10 segments. peduncular segments 4 and 5 each with 3-4 sets of marginal long setae. Mandibles subequal. spine row with 6-8 spines: segment 2 of palp with about 10 setae. segment 3 with 2 A setae. I B seta. 2 C setae. $+E$ setae and a row of D setae. Maxilla 1: inner plate with 8 apical plumose setae: plap with 13 slender spines on apex. Maxilla 2. inner plate with oblique row of 8 plumose setae on inner margin. Maxilliped: inner plate apically with 3 bladelike spines. 2 naked spines. and 5-6 plumose setae extending from inner margin to apex: outer plate with row of naked setae and about 6 pectinate spines on inner margin and 3 apical plumose setae: dactyl with 4-5 setae on inner margin, dactyl nail very long, about $1 / 3$ length of dact.l.

Gnathopod I: carpus longer than propod, posterior maryin very long. with many very long setae: propod palm much shorter than posterior margin. litt!e convex, not oblique. with about 5 symmetrically notched slender spine teeth on inside. 7-9 on the outside: defining angle with five (normal) spine teeth on inside. 3 on outside: superior medial setae few in number and mostly singly inserted: posterior margin with $4-5$ sets of setae in groups of $1-3$. Dactyl with about 3 short setae on inner margin. nail very long. about 13 length of dactyl. Ventral margin of coxa I with 9-1 I setae. some very long.

Gnathopod 2: carpus equal or slightly longer than propod, posterior margin very long. with 9-10 sets of very long setae: propod palm much shorter than posterior margin. little convex and oblique, with about 5 symmetrically notched. slender spine teeth on inside, up to 13 on outside: detining angle with 5 (normal) spine teeth on inside. 3 on outside. 1 very large: superior medial setae about 13 in number. singly inserted: posterior marginal with 7 sets of long setae in groups of $2-4$. Dactyl with 6 short setae on inner margin. nail very long, about l,4 length of dactyl. Ventral margin of coxa 1 with 6 setae.

Coxae of pereopod 3 with 6 marginal setae. Coxa of pereopod 4 with 22 marginal setae. dactyl of pereopod 4 about 20 percent length of propod. Pereopod 5 : basis with 14 shallow serrations on posterior margin: 8 short spines on anterior margin: 3 long setae on the anteroproximal margin: Pereopod 6: basis with 15 shallow serrations along posterior margin: 8 short spines on anterior margin: 3 long setae on anteroproximal margin: Pereopod 7: coxa with 2 setae along the posterior margin: basis with 12 shallow serrations along posterior margin: 6 short spines on anterior margin: 3 long setae on anteroproximal margin: dactyl more than $1 / 3$ length of propod.

Pleonal plates: posterior margin of plate I slightly convex. with I seta. distoposterior corner not produced: posterior margin of plate 2 nearly straight with I seta. distoposterior comer produced. ventral margin with 4 (subventral) spines: posterior margin of plate 3 nearly straight with 1 seta. distoposterior corner round and not produced. ventral margin with 3 (subventral) spines. Peduncle of pleopod 1 with 2 
retinaculae and less than 10 setae on distal half of outer margin.

Uropod 1: inner ramus about 80 percent length of peduncle, armed with 12 spines: outer ramus with about 11 spines; peduncle with 5 outer and 4 inner spines. Uropod 2: inner ramus armed with 10 spines: outer ramus with 10 spines: peduncle with 2 outer and 4 inner spines. Uropod 3 : inner ramus with 1 spine. outer ramus about 2 times longer than peduncle. inner and outer margin each with $3-4$ sets of spines in groups $1-3$. Telson little broader than long, notched about $1 / 3$ distance to base, each lobe with 2 apical spines.

Male.--Differing from female as follows: smaller with more slender. elongate body. Antenna 2: calceoli percent on peduncular segments 4 and 5 . and first 3 segments of tlagellum.

Gnathopod 1: carpus shape like that of female. but slightly shorter than propod. Propod palm with 5 (normal) spine teeth on inside. 7 (normal) spine teeth on outside: defining angle with + spine teeth on inside and 3 on outside: upper half of posterior margin with $4-5$ sets of long setae in groups of $1-2$. Gnathopod 2: carpus shape like that of female. smaller and shorter than propod. Propod palinar margin little convex and oblique. with 6 (normal) spine teeth on inside. 6 on outside: defining angle with 6 spine teeth on inside and 3 on outside.

Uropod 2: inner ramus with about 9 spines: outer ramus not curved. with about 3 inner and 2 outer spines. apex with 4-5 spines: peduncle with 2 outer and 3 inner spines.

Type-locality.--A ditch along VA Rt. 600 in Northampton Co.. VA. The 44 type-specimens were collected from burrows of the crayfish Cambarus diogenes. The precise location of the ditch cannot be determined.

Distribution and ecology.--This species occurs on the Coastal Plain of southeastern Virginia and northeastern North Carolina (fig. 25). Populations in Northampton County on the eastern shore of Virginia are separated from other localities by the Chesapeake Bay. Crungonvx longicurpus inhabits small streams. brooks, outlets of drain. springs. ditches. creeks. and small woodland pools. Ovigerous females were found in collections from January to March.

Etymology.--The epithet longicarpus combines "long" and "carpus". referring to the long carpus of gnathopods 1 and 2 in both male and female.

The richmondensis group

Diagnosis.--Adult size range. $14.0-20.0 \mathrm{~mm}$. Sexually mature females larger than male. except in $C^{\prime}$. anomalus in which mature males may be larger than mature females. Segment 3 of palp with 2 or more $\mathrm{A}$ setae and I or more B setae. Maxilla I: inner plate with 4-5 or sometimes fewer apical plumose setae. Maxilla 2. inner plate with oblique row of up to 7 plumose setae on inner margin in some species but only 3 or fewer in other species. Lacking distinct sexual dimorphism in gnathopods. Gnathopods 1 and 2: propod more than 2 times larger then carpus and much broader: palm of propod much longer than posterior 


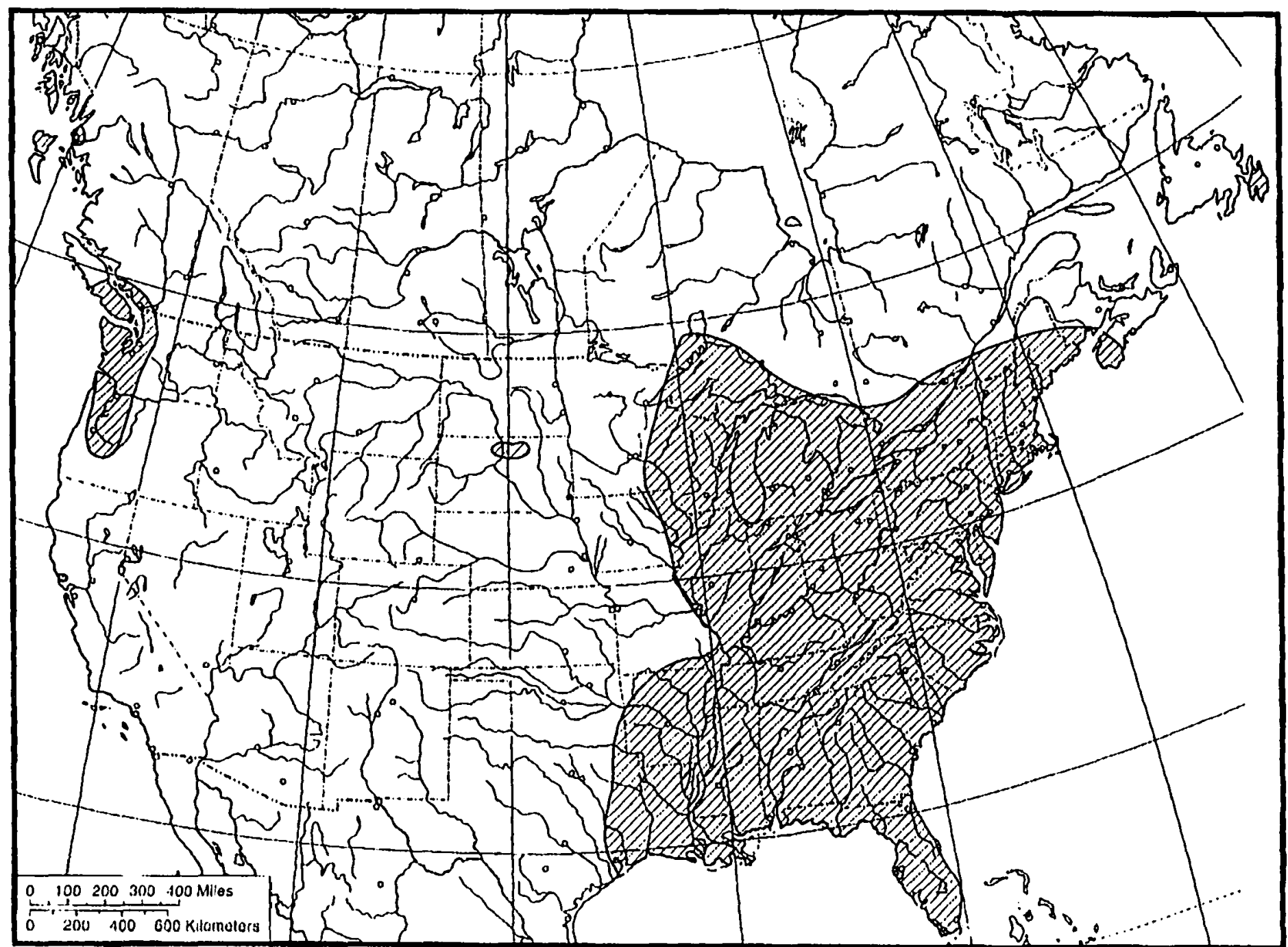

Figure 26.--(ieographic distribution of the richmendensis group in North America. Shaded areas denole areas of contiguous or near contignous distribution. Note: Sable Island distribution not shown (sect fig. 30). 
margin. armed with many notched spine teeth. always 2 rows per side: superior medial setae inserted in groups of 2-6: defining angle of propod of gnathopod 1 with I row of notched spine teeth on inside. the direction of notch being opposite the dactyl. outside with I row of small notched or simple spine teeth and I row of larger notched spine teeth. lacking serrate spine teeth or with only a few: defining angle of propod of gnathopod 2 with (normal) notched spine teeth (like those on the palm margin). Bases of pereopods 5-7 with many spines on posterior margin and many serrations on anterior margin. Peduncle of pleopod I with more than 2 retinaculae (normally 4-6) and many long setae on outer margin.

Remarks.--This group is composed of nine large species. with the widest geographic distribution of any group in North America (fig. 26). The richmondensis group is rather closed allied morphologically with the forbesi group but differs in a number of significant ways as indicated in their respective diagnoses.

Key to the Species of the richmondensis group

1. Basis of pereopods 5-7 greatly expanded posteriorly. posterior margin with numerous (more than 30 ) very weak sertations. C. unomalus

Basis of pereopods 5-7 not greatly expanded posteriorly. posterior margin without numerous weak serrations.

2. Spine teeth tri- or quadri-fid near proximal end of female gnathopodal propods: eyes absent C. dearolfi

Spine teeth typically bifid (normal): eyes present or absent. 3

3. Posterior margin of pleonal plates 2 and 3 strongly oblique and distinctly produced: eyes absent C. grandimunus

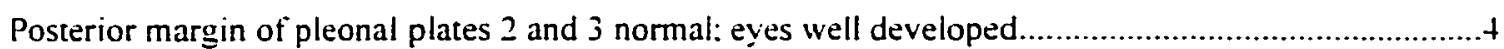

4. Posterior margins of bases of pereopods 5-7 with deep serrations. especially near distal end................5

Posterior margins of bases of pereopods 5-7 with shallow serrations..........................................6

5. Dactyl of female gnathopod 2 with 2 or more setae on outer margin C. ohlicutus

Dactyl of female gnathopod 2 with I seta on outer margin C. richmondensis

6. Telson much broader than long: corners of pleonal plates $1-3$ neither produced nor acute: inner margin of maxilla 2 with oblique row of more than 6 plumose setae C. indiunensis

Telson longer than broad or length and width subequal: comer of pleonal plate 2 produced and acute. those of pleonals plate $I$ and 3 produced or not: inner margin of maxilla 2 with $1-4$ plumose setae.....7

7. Distoposterior comers of pleonal plates 1 and 3 produced. 8 Distoposterior corners of pleonal plates $I$ and $j$ not produced. C. hubrichti

8. Outer ramus of male uropod 2 with $2-4$ thick spines on inside. C. disjunctus

Outer ramus of male uropod 2 with 1-2 large thick spines on inside and 2-3 very small spines on outside C. cornutus 


\section{Crangonvx richmondensis Ellis}

Figures 27-30

Crangonxx richmondensis Ellis. 1940:3-6, figs. 1-2 [type-locality: pond on Richmond Plantation.

Cordesville. Berkeley Co.. South Carolina],--Holsinger. 1972:43, fig. 40: 1977:252.--Smith. 1977:257-258. figs. 1-2.--Barnard \& Barnard. 1983:435. map I [ [in part].--Fitzpatrick. 1983:145.-Steele, 1983:679.--Jass \& Klausmeier, 1995:9.

Crangomx occidentalis Hubricht and Harrison. 1941:331. figs. A-L [type-locality: Echo Lake. near the Seattle-Everett Highway, just south of the King County line. Washington|.--Barnard \& Bardnard. 1983:435. map 11.

Crungonyx richmondensis richmondensis Bousfield, 1958:90-91, figs.12. 20.--Holsinger. 1972:43.--

Bousfield. 1973:69.--Smith. 1988:46.--Biernbaum. 1989:13.

Crangonvx richmondensis occtdentalis Bousfield. 1958:91-94. fig. 12: 1961:5.--Holsinger. 1972:43.

Crangomx richmondensis laurentianus Bousfield. 1958:94-95. fig. 13 [type-locality: Black Lake. Gatineau

Park near Kingsmere. Quebec].--Judd. 1963:2.--Bell. 1971:36.--Holsinger. 1972:43.--Lindeman.

Lindeman \& Lindeman. 1993:217.

Crangonvx alpinus Bousfield, !963:1-3. figs. I-2 [type-locality:Ledge Lake. Lane Co.. Oregon].--

Holsinger. 1972:33: 1977:252.--Barnard \& Barnard. 1983:434. map 1.--Fitzpatrick. 1983:145.

Material Examined.--NEWFOUNDLAND. Bog pond. $1.6 \mathrm{~km} \mathrm{~N}$ of Pouch Cave on Avalon. $3 \equiv .1 \cong$ D. H. Steele and P. E. Oshel. (collection date not given) (USNM). NOVA SCOTIA. Shelburne Co.: Pugg Lake. : . . collector not given. 13 Sep 1940 (USNM) and 20 specs. T. A. Tingley. 13 Sep $19+1$ (USNiW). ONTARIO. Berkeley's stream. Departure Bay, 8 ₹. L. G. Saunders. 27 Jun 1933 (USNM): District of Thunder bay: Caven Lake. 6 ₹.11 . R. \& M. Lindeman. 27 Oct 1991 (NMC): Edmondson Lake. $10 \equiv$. R. \& M. Lindeman. 20 Oct 1991 (NMC): Hicks Lake. 8 \& 3 s. R. \& M. Lindeman. 20 Oct I991 (NMC): Keelor Lake. $3 \varsubsetneqq .1$ \&. 3 Juvs. R. \& M. Lindeman. 5 Jul 1991 (NMC); Kekwan Zik Lake. $16 \equiv .5 \approx$ R. \& M. Lindeman. 23 Sep 1991 (NMC): Mawn lake. $13 \Xi .4 c^{\circ}$ R. \& M. Lindeman. 17 Oct 199 I (NMC): Mott Lake. $3 \doteq$. R. \& M. Lindeman. 20 Oct 1991 (NMC): Pistol Lake. $7 \doteq .2 \approx$. R. \& M. Lindeman. 25 Aug 1991 (NMC): Sparks Lake. 3 £. R. \& M. Lindeman. 4 Jun 1991: Upper Shale Lake. 4 . 7 \&. R. \& M. Lindeman. 27 Oct 1991 (NMC): Walkinshaw Lake. 2 9. 5 ક. R. \& M. Lindeman, 20 Oct 1991 (NMC):Watcomb Lake. $3 \subsetneq .3 \%$. R. \& M. Lindeman. 21 Sep 1991 (NMC): Whitfgranite Lake. 8 . R. \& M. Lindeman. 27 Oct 1991 (NMC): Creek on HWY 527 at Pumping Station. $2 \cong .1 \approx .8$ Juvs. R. \& M. Lindeman. 17 Oct 1991 (NMC); Pond on 20th side road. at Oprtrack. 5 Juvs, R. \& M. Lindeman. 20 Jun 1991 (NMC): Pond on Town Line Road off HWY 102 at City Limit North side. 8 ₹. R. \& M. Lindeman. 24 Aug 1991 (NMC). QUEBEC. Black Lake, Gatineau Park, near Kingsmere, $9 \fallingdotseq .4:$ (USNMI). $2 \Xi 12$ 


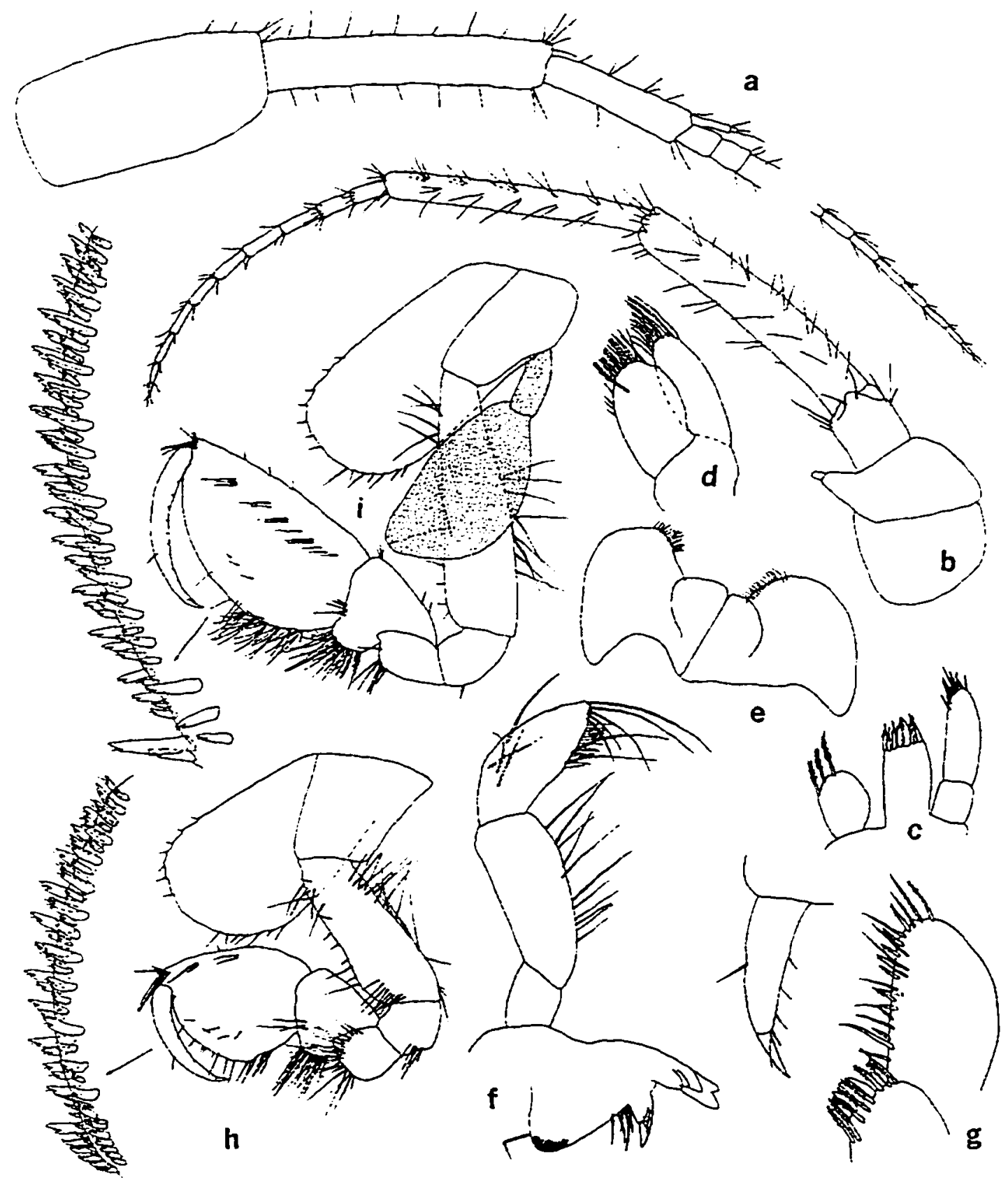

Figure 27.--Crangonlx richmondensis Ellis. Female paratype $(14.0 \mathrm{~mm})$, pond on Richmond

Plantation. Cordesville. Berkeley Co.. South Carolina: a. b. antennae 1. 2: c. d. maxillae 1. 2: e. lower lip: f. left mandible: g. inner and outer plates and palp dactyl of maxilliped (greatly enlarged): h. i. gnathopods 1. 2 (palmar margins enlarged). 


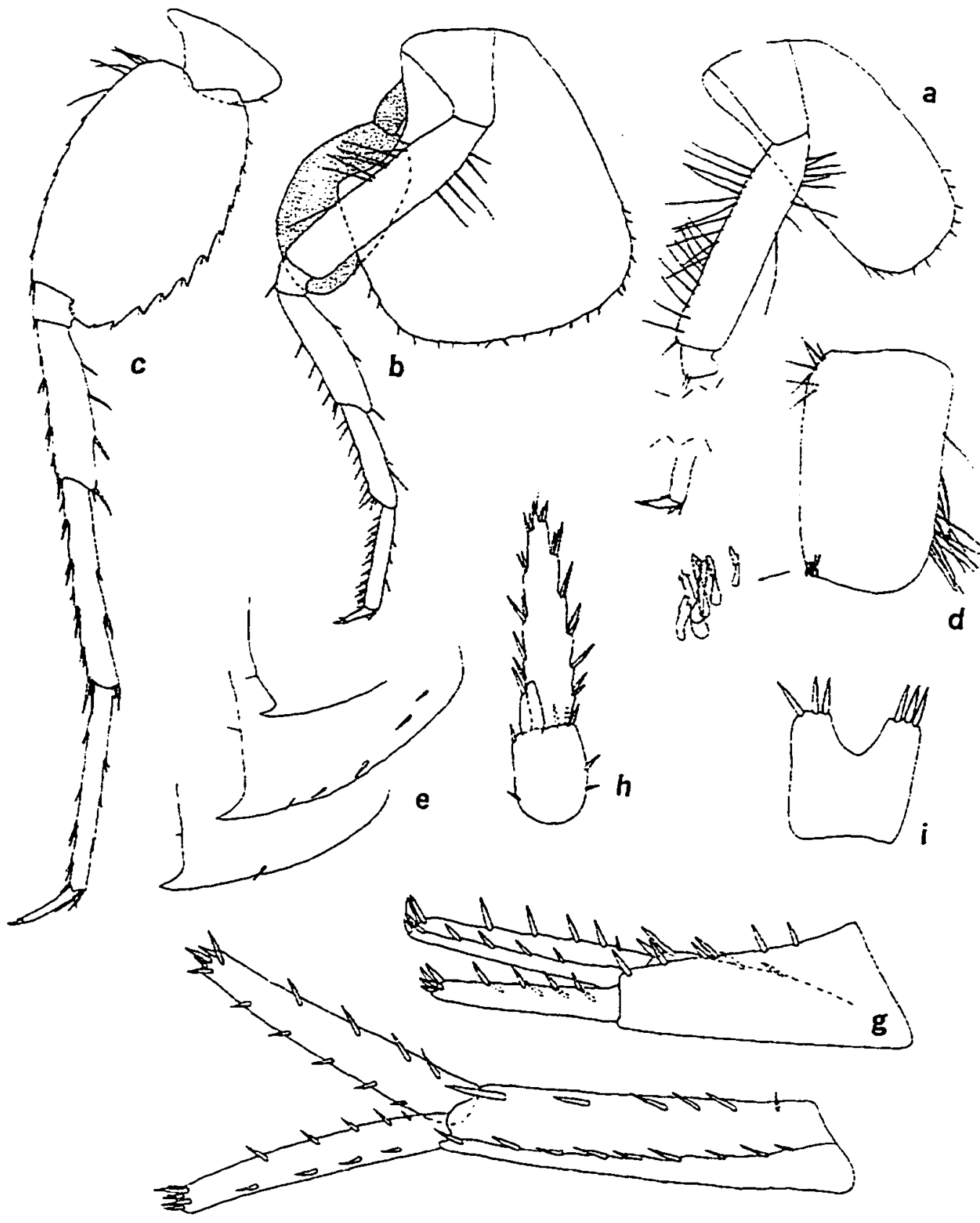

Figure 28.--Crangonyx richmondensis Ellis. Female paratype $(1+.0 \mathrm{~mm})$, pond on Richmond

Plantation. Cordesville. Berkeley Co.. South Carolina: a. pereopod 3 (in part): b. c. pereopods 4. 7: $d$. peduncle of pleopod I (retinacula enlarged): e. pleonal plates; f. g. h. uropods I. 2. 3: i. telson. 


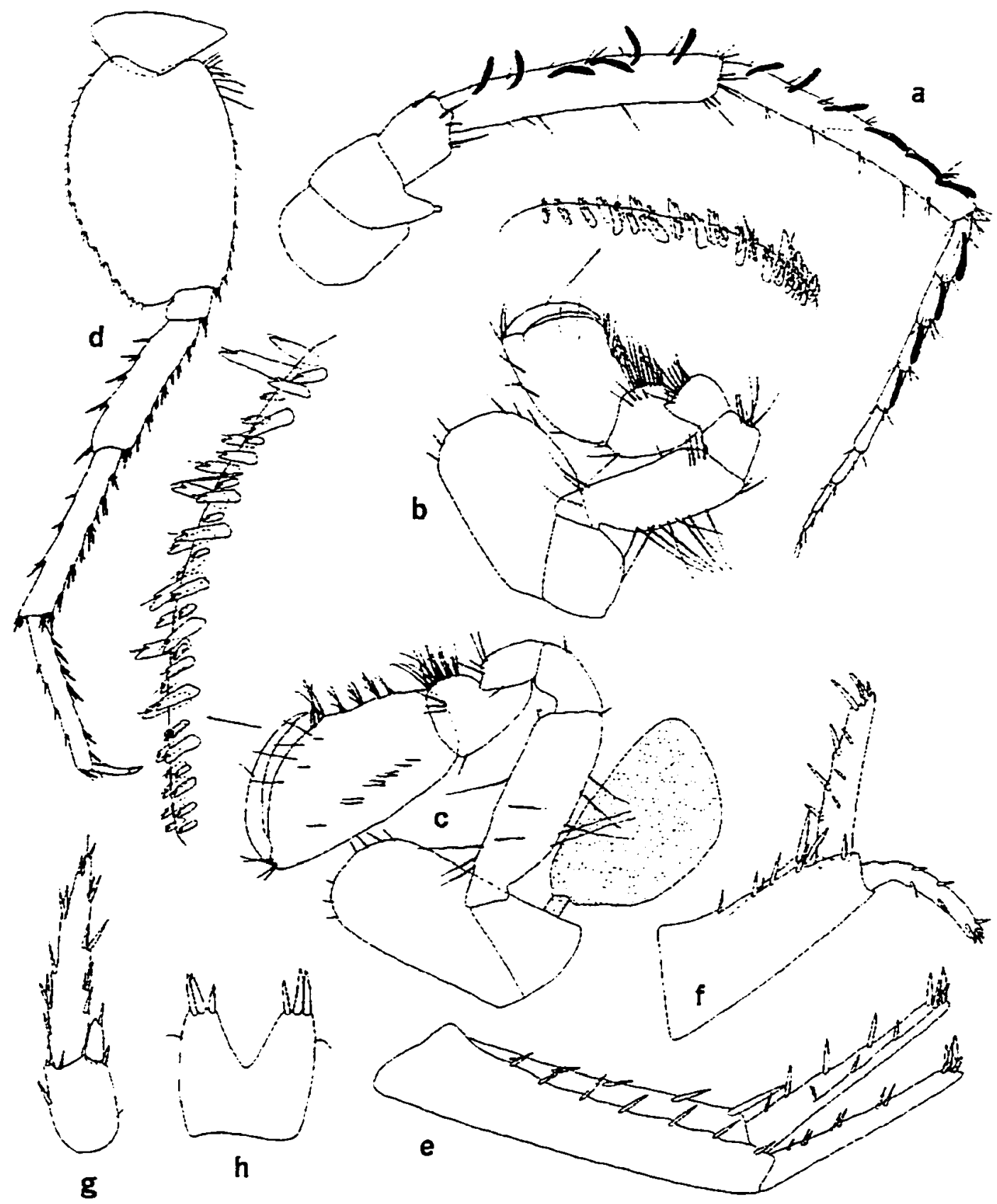

Figure 29.--Crangonyx richmondensis Ellis. Male paratype $(7.4 \mathrm{~mm})$. pond on Richmond Plantation. Cordesville. Berkeley Co.. South Carolina: a. antenna 2: b. c. gnathopods 1. 2 (palmar margins enlarged): d. pereopod 7: e. f. g. uropods 1. 2. 3: h. telson. 


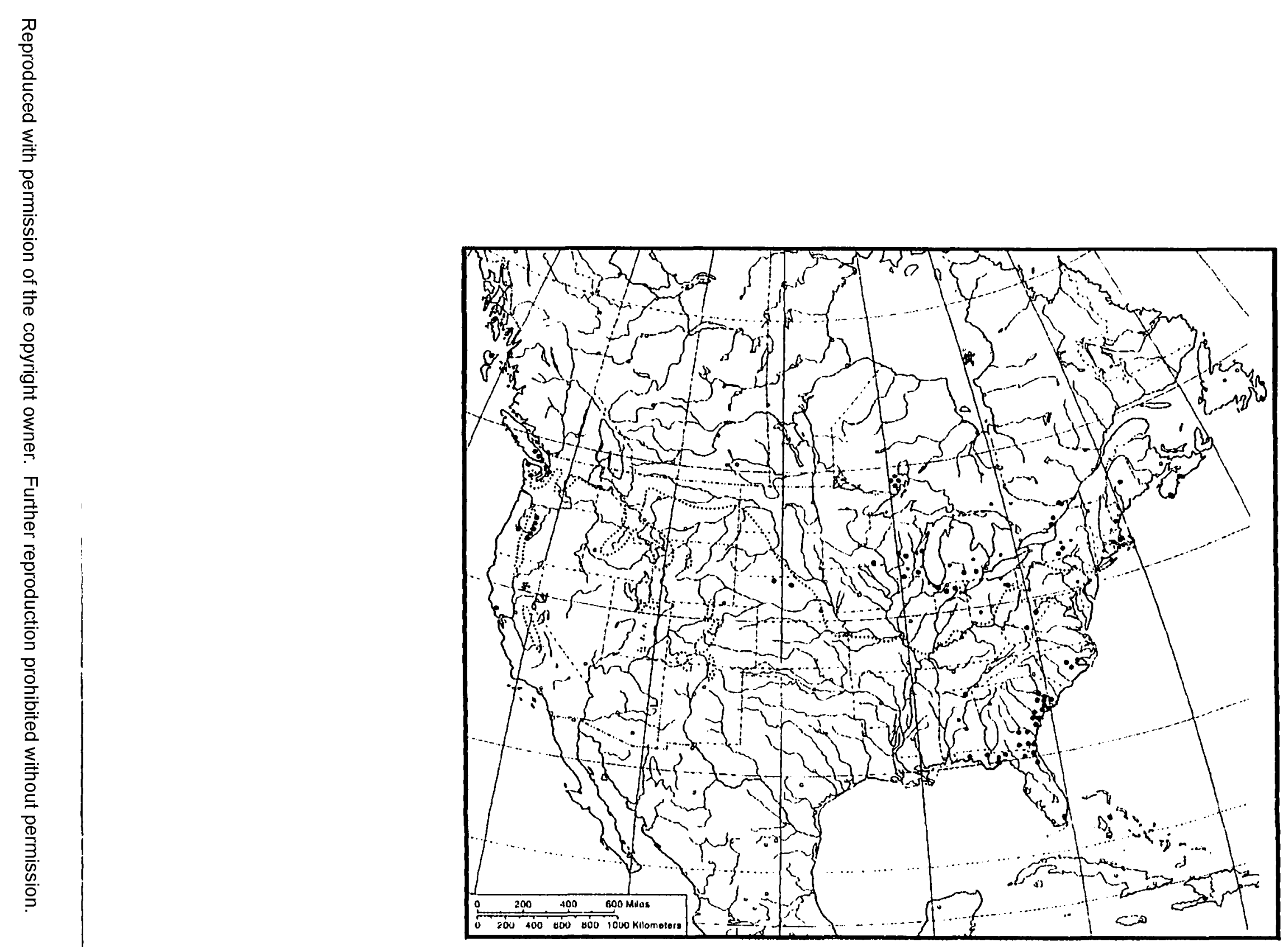

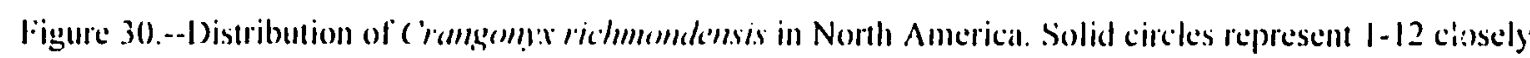
proximate localities. The donted lines indiciate the approximate southern extent of glactiation during the Pleistocene ind enclose pockets of alpine glaciation in western Nonth America. The Sable Istand locality (approximatcly $175 \mathrm{~km}$ l: of Nova Scotia) reporled by Boustickd (19706) nor shown. 
$=($ CNM). E. L. Bousfield. 27 Apr 1955 (USNM). ALABAMA. Jackson Co.: slough $2.6 \mathrm{~km}$ NW of Paint Rock. 1 ₹. $1 \approx$. L. Hubricht. 12 Apr 1941 (USNM): county not given: Alabama craytish burrows. I $\approx$ J. Martin. (collection date not given) (USNM). ALASKA. Prince of Wales-Outer Ketchikan Co.: Rivers End Cave. Prince of Wales Island. 39.5 s, K. R. Carlson. 13 Jul 1992. 20 specs. K. R. Carlson. 3 Jul 1993: outflow of sink hole lake. Prince of Wales Island. 2 9. I juv.. K. R. Carlson. 23 Jun 1995: Contact Cave. Prince of Wales Island. $8 \&$. I juv.. K. R. Carison. 27 Jun 1995: sinking stream (muskeg) upstream from Whitesocks Cave insurgence, Luck Lake area. Prince of Wales Island. 12 specs.. K. R. Carson. 4 Jul 1995: Sitka Co.: Piseco Lake=(Swan Lake). 3 \%. Bean. 3 I May I 880 (USNM). CALIFORNIA. Sonoma Co.: pond on Privot farm. 25 specs. L. L. Evg. Spring 1984 (California Dept. of Fish and Game). FLORIDA.

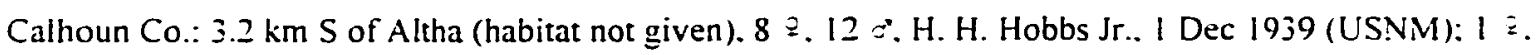
J. Martin. 17 Apr 1937 (USNM) and I $s .1$ juv. F. N. Young. 9 Jun 1938 (USNM): Columbia Co.: dried cypress pond off Double Run Road. ca. $3.2 \mathrm{~km}$ NNE of Lake City. $10 \Xi$. D. Franz 19 May 197\%: habitat not given. $44.8 \mathrm{~km} \mathrm{~N}$ of Lake City. 7 9. J. Martin. 27 Oct 1938 (USNM): Dade Co.: Little Bird Nursery Well. Miami. $1 \approx$. G. Miller. 15 Aug 1968 (USNM): Duval Co.: grassy stream W side of HWY 301.2 .4 $\mathrm{km}$ S of Nassau Co. line. I $\bar{\Xi}_{.}$J. E. and J. Cooper. 2 Jan 1965: Jefferson Co.: habitat not given. $9.3 \mathrm{~km}$ E of Capps. i $\approx$ H. H. Hobbs. Jr., 5 Feb 1938 (USNM): Leon Co.: habitat not given. Tallahasses. $1 \doteq .1 \therefore$ H. H. Hobbs. Jr.. 27 Nov 1936 (USNM): Horseshoe Bay. 1 @. $3 \approx$ H. H. Hobbs. Jr.. 27 Nov 1936 (USNM):

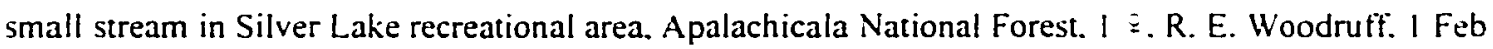
1960 (USNM): crayfish burrow. Tallahassee. $1 \cong$. H. H. Hobbs Jr.. 27 Nov 1936 (USNM): Okaloosa Co.: craytish burrows. $5.8 \mathrm{~km} \mathrm{~S}$ of Crestview. 20 specs. H. H. Hobbs. Jr.. 17 Oct 1941 (USNM): Putnam Co.: from abdomen of crayfish. $23.2 \mathrm{~km}$ E of Palatka. 2 ?. H. H. Hobbs. Jr.. 23 Oct 1936 (USNM): cray fish burrows. Orange Springs. 2 ๑. H. H. Hobbs. Jr.. 22 Nov 1936 (USNM): Sulfur Spring. near St. John's River. 1 ב. H. H. Hobbs. 23 Oct 1936 (USNM): Wakulla Co.: crayfish burrows. $3 \Xi .2 \approx$. J. Martin. 5 Jun 1938 (USNM): habitat not given. $4 \cong .1 \%$. J. Martin. collection date not given (USNM). The following USNM collections from Florida lack county and habitat data: 3 \&. 2 juvs. J. Martin. 12 Mar 1937 (USNM): 1 *. J. Martin. 9 Dec 1937 (USNM): Cypress pond. 3 ¿. J. Martin. 9 Apr 1937 (USNM): 2 juvs. H. H. Hobbs. jr.. 17 Apr 1937 (USNM): $59.4 \approx$. H. H. Hobbs, jr.. 27 Oct 1938 (USNM). GEORGIA. Appling Co.: habitat not given. $4 \cong$. Barley, collection date not given (USNM): Camden Co.: crayfish burrows. $3.5 \mathrm{~km}$ NE of Juct of Rds 40 and 110. I ․ H. H. Hobbs. Jr.. 27 Sep 1972 (USNM): Charlton Co.: crayfish burrow. $4.5 \mathrm{~km}$ E of Falkston, 5 \%. H. H. Hobbs. Jr., et al.. 27 Sep 1972 (USNM): BryanChatham Co. line. Ogeechee River at US Hwy 17.2 ฐ. R. Heard. 4 May 1970 (USNM): Clinch Co.: with craytish. 1 . J. Martin. 27 Oct 1938 (USNM) and $169.1 \approx 27$ Oct 1938 (USNM): Effingham Co.: small drainage ditch. $2.4 \mathrm{~km} \mathrm{~N}$ of Clye on St Rd I19.3 ९. 18 juvs. D. J. Peters and H. H. Hobbs. Jr.. collection date not given (USNM): Wayne Co.: swamp. $1.8 \mathrm{~km}$ NE of Jesup. 2 ¿. L. Hubricht. $20 \mathrm{Apr} 1941$ (USNM). INDIANA. Kosciusko Co.: Wawasee Lake. I . W. Scott. 22 Jun 1926 (USNM): Lake Co.: pond in Miller Woods. Indiana Dunes National Lakeshore. 4 q. $4 \approx$. R. L. Whitman. 20 Apr 1984: pond. Indiana Dunes 
National Lakeshore. 8 ฯ. R. L. Whitman. 14 Sep 1984: Marshall Co.: south shore of Outlet Bay. Lake Maxinkuckee. $30 \%$. collector not given. 17 Nov 1904 (USNM). MAINE. Piscataquis Co.: Draper Road pond, 24 . 12 c. R. Auclair. 14 Jul 1966 (USNM) and 1 juv. D. Maire. 10 Jul 1967 (USNM): York Co.: Wales Pond. Hollis. 7 9. 3 juvs. D. Mairs. II Aug 1967 (USNM) and $3 \cong 25$ Oct 1967 (USNM). MASSACHUSETTS. Norfolk Co.: Blue Hills, Milton. 4 . F. N. Young. 24 Aug 1940 (USNM): Worcester Co.: Sanctuary Pond. $N$ of Grafton. $2 \equiv .3$ C. C. E. Addy. 16 Aug 1943 (USNM). MICHIGAN. Calhoun Co.: Beadle Lake. $6.2 \mathrm{~km}$ S of Battle Creek. 4 . L. Hubricht. 20 Oct 1946 (USNM): Washtenaw Co.: permanent pond. E side of Platt Road. ca. $12 \mathrm{~km}$ SSE of Ann Arbor. numerous specimens in 14 collections (USNM), R. Kenk. 1940-1941: pond just S of Ypsilanti Airport. $8.0 \mathrm{~km}$ SE of Ann Arbor. 100 specs. L. Hubricht. 29 Apr 1941 (USNM); marsh, $12.0 \mathrm{~km}$ N of Milan. 50 juvs. L. Hubricht. 28 Jul 1946 (USNM): pond off Platt Road, $0.8 \mathrm{~km}$ N of Benis Road. 100 specs. L. Hubricht. 30 Apr 1941 (USNiM). MINNESOTA. Filimore Co.: Etna Creek. $19.2 \approx$. J. Muck and R. Newman. 18 Mar 1990. NEBRASKA. Cherry $C_{0}:$ pond. Valentine Refuge. Valentine. $3 \approx$. donated by $V$. E. Shelford. 25 Ju! I945 (USNM): Holt Co.: $3.2 \mathrm{~km} \mathrm{NW}$ of Stuart. $3 \Xi$. collector not given. 3 Jun 1937 (USNM). NEW YORK. Cortland Co.: "west pond". Chicago Bogs. 3 巨. A. M. Phillips. 25 Apr 1946 (USNM): Tompkins Co.: Dryden Lake. $13 \equiv .4 \therefore 1$ juv. D. A. Webster. 16 May 1946 (USNM). NORTH CAROLINA. Beautort Co.: upen grounds. Beaufort. $2 \bigcirc .1 こ$. A. S. Pearse. 29 Mar 1934 (USNM): Lenoir Co.: Beaverdam swamp. at US 258. ca. $0.4 \mathrm{~km}$ SSE of Jonestown. $2 \approx$. J. H. Reynolds. 23 Jan 1979 (NCMNH): Trent River at St Rd $1130.0 .8 \mathrm{~km}$ NE Nobles Crossrods. 2 9. J. H. Reynoldes. 24 Jan 1979 (NCMNH): Wilson Co.: Little Contentnea Creek at CR 1531 . ca. $6.2 \mathrm{~km}$ E of Saratoga. I P. P. S. Freed. + Feb 1979 (NCMNH): Whiteoak swamp at CR 1507. ca. $3.2 \mathrm{~km}$ NNW Saratoga. 4 E. P. S. Freed. + Feb 1979 (NCMNH). OHIO. Wayne Co.: habitat not given. Funk. 1 s. A. Weaver. 26 Mar 1958. OREGON. Benton Co.: McFoddene's

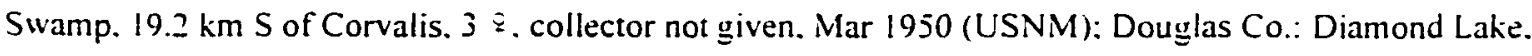
$2 \equiv .1$ : G. S. Schuytema. 18 Jun 1973; Fish Lake. 3 ¡. A. Rath. 12 Jul 1937 (USNM): Lane Co.: Gold Lake. 5 Э. F. Z. 16 Sep 1936 (USNM): Lower Marlyn Lake. 2 ‥F. Z.. 15 Sep 1936 (USNM): Upper Mariyn Lake. 19 ₹. F. Z.. 15 Sep 1936 (USNM): Linn Co.: Clear Lake. 13 ₹. $10 \approx$ Æ. 8 juvs. F. Z.. 17 Jun 1936 (USNM): Chiqiuts Lake. 2 9. F. Z.. 10 Jul 1936 (USNM): Daly Lake. 2 9. F. Z.. 2 Sep 1936

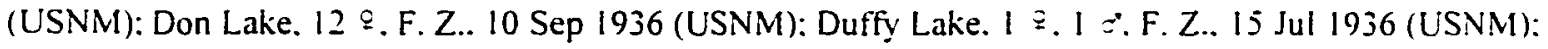
Fish Lake. 3 c. F. Z.. 15 Jun 1936 (USNM); Hunts Lake. $39.9:$ F. Z.. 31 Jul 1936 (USNM): Jorn Lake.

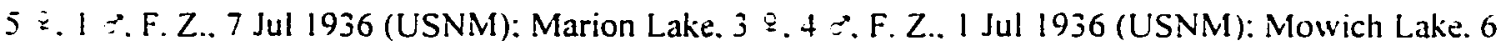
ङ.F. Z.. 16 Jul 1936 (USNM): Red Butte lake. 129.6 :. 2 juvs. F. Z.. 14 Jul 1936 (USNM): Marion Co.: Elk Lake. 30 specs. S. T. Brooks. 25 Sep 1932 (USNM), I \&. 1 o. F. Z.. 12 Jun 1936 (USNM) and 6 ¿. 4 $\approx 17$ Jun 1936 (USNM) and $9 \cong$. (collector not given): Upper Twin Lake. 1 ๑. F. Z.. 13 Aug 1936 (USNM); Russell Lake, 8 ₹. 4 o. F. Z.. 6 Aug 1936 (USNM). SOUTH CAROLINA. Berkeley Co.: slough. $4.8 \mathrm{~km} \mathrm{NW}$ of Cordesville. $1 \cong$. L. Hubricht. 21 Apr 1941 (USNM): pond. Gough. near Cordesville. 10 \&. T. K. Ellis. 11 Dec 1939 (USNM) and near Gough. 4 ?. $8:$. I Jan 1940 (USNM): 
Richmond Plantation. Gough. numerous specimens (USNM) from Alligator Pond, dammed-up area. Upper and Lower reserves. T. K. Ellis. 1939-1941: swamp. $9.6 \mathrm{~km} \mathrm{~N}$ of Pineopolis. $13 £ .1:$ collector not given. 4 Apr 1940 (USNM): Charleston Co.: Caw Caw Swamp. I Z. T. K. Ellis. 3 Apr 1941 (USNM): middle of Caw Caw Swamp. New Road. 10 \%. 4 5. T. K. Ellis. 3 Apr 1941 (LSNM): Clarendon Co.: Santee National Wildlife Refuge. 2 c. C. K. Biembaum. 15 Jan 1983 (USNM): Colleton Co.: beside Hwy 63 at Ricepatch Creek. 1 9. P. H. Carlson. 29 Nov 1982 (USNM); Hampton Co.: Cope Creek at Dirt Road. 2 ․ P. H. Carlson, 2 Oct 1983 (USNM); Jasper Co.: slough. $3.2 \mathrm{~km} \mathrm{SSW}$ of Ridgeland. $3 \cong$. L. Hubricht. 21 Apr 1941 (USNM): Orangeburg Co.: swamp. $4.0 \mathrm{~km} \mathrm{SE}$ of Bowman. $1 \Xi .15 \approx$. L. Hubricht. 22 Apr 1941 (USNM): Richland Co.: pond in Congaree River swamp on road to Wateree. 2 . collector not given. + Apr 1940 (USNM): WASHINGTON. Clallam Co.: Lake on Beawer Creek. 3.2 km $N$ of Sappho. $5 \doteq . C$. H. Harrison. 13 Jun 1939 (USNM): Gray Harbor Co.: Lake Quinault, 1 Z, C. H. Harrison. 13 Jun 1939 (LSNM): Island Co.: Cranberry Lake, Camano Island. 60 specs. C. H. Harrison. 23 Aug 1939 (USNM): Deer Lake. Whidbey Island. 25 specs. C. H. Harrison. 8 Jul 1939: Goss Lake. Whidbey Island. $9 \geqq 12$ juvs. C. H. Harrison. 8 Jul 1939 (USNM): Lone Lake. Whidbey Island. $2 \sqsubseteq$. C. H. Harrison. 8 Jul 1939 (LSNM): Lake Oliver. Whidbey Island. 30 specs. C. H. Harrison. 10 Jul 1939 (USNM): Lake Pondilla. Whidbey Island. $2 \approx$. C. H. Harrison. 20 Mar 1939 (USNM): Kristoferson's Lake. Camano Island. $t 0$ specs. C. H. Harrison. 30 Aug 1939 (USNM): pond in gravel pit, $1.6 \mathrm{~km}$ S of mud lake. Whidbey island. 50 specs. C. H. Harrison, 10 Jul 1939 (USNM): Silver Lake. Whidbey Island. 8 ミ. C. H. Harison. 5 Jul 1939 (USNM): King Co.: Echo Lake. near Seattle-Everett Hwy. (US 99). just S of King Co. Line. $25 \equiv .15$ $\therefore$ C. H. Harrison. I Jan 1940 (USNM): Echo Lake. Richmond Highlands, 100 specs. C. H. Harrison. 9 Sep 1939 (USNM): Plantation Pond. 30 specs. C. H. Harrison. 13 May 1939 (USNM): Snohomish Co.: Chase Lake. 30 specs. C. H. Harrison. 8 Oct 1939 (USNM). WEST VIRGINIA. Greenbrier Co.: Meadow River wetlands, 29 . D. C. Tarter, 19 Apr 1996: Tucker Co.: Tributary to Red Creek. Dolly Sods. $8 \equiv .1 \underbrace{\circ}$ C. Parrish. 4 May 1974. WISCONSIN. Green Co.: Allen Creek. Green and Rock counties line. I collector not given. 29 Sep 1989 (MPM): Juneau Co.: $2 \approx$. collector not given. 23 Jun 1980 (MPM): Manitowoc Co.: pond at Marken Rd. $0.3 \mathrm{~km}$ S of Hwy C. 30 specs (₹. :). B. Klausmeier. 9 Sep 1994 (MPM): Marquette Co.: large pond at County Trunk Highway E \& Y south. 15 E. J. P. Jass. 23 Aug 1994 (MPM): Waukesha Co.: just W of Eagle. 3 c. collector not given. 8 Jun I972 (MPM). $2 \approx$ collector not given. 23 Jun 1989 (MPM), 12 9. $2 \approx$ s collector not given, 3 Jun 1979 (MPM): pond near Eagle. S of Hwy $59.4 .8 \mathrm{~km} \mathrm{~W}$ of Hwy N. 3 ₹. 28 juvs. J. P. Jass \& B. Klausmeier. 3 Jun 1994 (MPM).

Diagnosis.--Nominate species of the richmondensis group distinguished from other members of the group by: having deep serrations along posterior margins of bases of pereopods 5-7: well developed distoposterior comers and few subventral spines of pleonal plates: uropod 2 of male with only 2 small inner and 2 small outer spines. Distinguished from the closely similar $C$. obliquus by having only $I$ seta on outer margin of dactyl of gnathopods. Largest male. $11.00 \mathrm{~mm}$ : largest female. $20.00 \mathrm{~mm}$.

Female.--Eye small and ovate. Antenna 1 , about 60 percent length of body, about 1.8 times longer than 
antenna 2: primary flagellum with up to 29 segments. Antenna 2, flagellum with 8-9 segments. Mandibles subequal. spine row with 5-6 spines: segment 2 of palp with 7-11 long setae. segment 3 with 3 A setae. 1 B seta. 2-3 C setae. 4-5 E setae and a row of D setae. Maxilla 1: inner plate with 3 apical plumose setae; palp with 7-8 slender spines on apex. Maxilla 2, inner plate with I plumose seta on inner margin. Maxilliped: inner plate apically with 4-5 blade-like spines. I naked spine. and up to 7 plumose setae extending from Inner margin to apex: outer plate with naked setae and 5-6 weak pectinate spines on inner margin and I plumose seta on apex: dactyl with up to 4 setae on inner margin: dactyl nail long. 144 length of dactyl.

Propod of gnathopod I more than 2 times larger and longer than carpus and much more broader: palm convex. longer than posterior margin. armed with 2 rows of about 25 spine teeth on inside. 2 rows of about 24 on outside: defining angle with 7 spine teeth on inside. outside with I row of small spine teeth and I row of 5 large spine teeth: superior medial setae in 2-3 sets. inserted singly or doubly: 2-3 sets setae on proximal half of posterior margin in groups of 2-5. Dactyl with row of up to 7 short setae on inner margin. nail very short. Ventral margin of coxa I with 14 setae.

Propod of gnathopod 2 more than 2 times larger and longer than carpus: palm strongly oblique. convex. about 2 times longer than posterior margin. armed with $2-3$ rows of about 34 spine teeth on inside. $2-3$ rows of about 35 on outside: defining angle with 2 spine teeth on inside and 2 on outside: superior medial setae in 7-8 sets inserted in groups of $1-3$. posterior margin with 6 sets of setae in groups of 3-8. Dacryl with row of short setae on inner margin. nail very short. Ventral margin of coxa 2 with $1+$ setae.

Coxa of pereopod 3 with 13 marginal setae. Coxa of pereopod 4 with 22 short marginal setae. dactyl of pereopod 4 about 35 percent length of corresponding propod. Pereopod 5: basis with 15 shallow serrations along posterior margin: 8-9 short spines on anterior margin: 10 long setae on anteroproximal margin.

Pereopod 6: basis with 13 serrations along posterior margin: 8 short spines on anterior margin: 8 long setae on anteroproximal margin. Pereopod 7: coxa with 2 setae on the posterior margin: basis with 20 serrations along posterior margin. becoming deeper distally: 7-8 short spines on anterior margin: 8-9 long setae on anteroproximal margin: dactyl of pereopod 4, 30 percent length of corresponding propod.

Pleonal plates: posterior margin of plate I slightly convex. with I seta. distoposterior corner produced and acute: posterior margin of plate 2 nearly straight with I seta. distoposterior corner produced and distinctly acute. ventral margin with 4-5 (subventral) spines: posterior margin of plate 3 nearly straight with I seta. distoposterior corner strongly produced and acute. ventral margin with |-3 (subventral) spines. Peduncle of pleopod 1 with about 6 retinaculae. posterior margin with many setae. proximal half of anterior margin with about 7-8 long setae.

Uropod 1: inner ramus 80 percent length of peduncle. armed with 14 spines: outer ramus with 14 spines: peduncle with row of 9 outer and 6 inner spines. Uropod 2: inner ramus armed with 14 spines. outer ramus with I 3 spines: peduncle with 5 outer and 5 inner spines. Uropod 3 : inner ramus with I spine. outer ramus about 2.1 times longer than peduncle. inner and outer margin each with $5-6$ sets of spines in groups of $1-3$. Telson about as broad as long, notched about $1 / 3$ distance to base, each lobe with $2-3$ apical 
spines: spine length about $1 / 3$ of telson.

Male.--Differing from temale as follows: much smaller with more slender. elongate body. Antenna 2: calceoli percent on peduncular segments 4 and 5 . and first 4 segments of flagellum.

Uropod 2: inner ramus with $\mid 1$ spines: outer ramus curved nearly to right angle with peduncle. with 2 inner and 2 outer spines. apex with 5 short spines: peduncle with 4 inner and 4 outer spines.

Variation.--The shape of pereopod 5-7 can be slightly more slender in some populations. The number of apical spines on the telson varies but is usually $2-3$ per lobe.

Distribution and ecology.--This is the most widely distributed species of Crangonyx. In western North America it occurs as far north as southeastern Alaska and in eastern North America as far north as Newfoundland: in the south it occurs in southern Florida (fig. 30). Although widely distributed across much of North America. a majority of the collections have been made from the northern Pacific cuastal region. the Great Lakes area, and on the southern Atlantic Coastal Plain.

The habitat of this species includes lakes. streams. ponds, swamps, bogs. ditches. drains and rarely caves. Sexually mature males. $7.5-11.0 \mathrm{~mm}$ : sexually mature females. 12.0 to $20.0 \mathrm{~mm}$. Newly hatched young 2.0-3.0 mm. Ovigerous females occur from about January to June. after that only immature animals are found throughout the summer and fall. The life span is probably of about I year (Holsinger. 1972).

Remarks.--After examination of all the coilections of C. richmondensis. I cannot find any good characters to separate the three previously named subspecies of $C$. richmondensts. The characters given by Bousfield (1958) to separate the subspecies were found to be variable and overlapping. Therefore. I cannot recognize them as different subspecies. Moreover, after examination of pertinent material. I could not tind any morphological differences between $C$. alpinus and $C$. richmondensis thus I consider the former a synonym of $C$. richmondensis.

Crangomyx anomalus Hubricht

Figures $31-34$

Crangonyx anomalus Hubricht. 1943:687-688. plate I [type-locality: Spring along Bryan Station road. 0.j mi NE of Eastin Road. 3 mi NE of Lexington. Fayette Co.. Kentucky].--Boustield. 1958:87.-Holsinger. 1972:33. figs. 12f. 12k. 15: 1977:25.--Barnard \& Barnard. 1983:434. map 11.--Fitzpatrick. 1983:145.--Pennak. 1989:483.

Material examined.--ILLINOIS. Pope Co.: spring $6.4 \mathrm{~km} \mathrm{NNW}$ of Golconde, $1 \approx$ L. M. Page ct al.. 6 Nov 1974. INDIANA. Jennings Co.: Cave Spring Cave. 1 \&. I $s$. R. M. Norton. If Aug 1967.

KENTIJCKY. Bourbon Co.: spring $12.8 \mathrm{~km} \mathrm{SW}$ of Millersburg, 1 . L. Hubricht. $25 \mathrm{Apr} 1941$ (USNM): spring. $9.1 \mathrm{~km}$ SW of Paris. 1 9 . L. Hubricht. 25 Apr 1941 (USNM): Clark Co.: stream about $11.2 \mathrm{~km} \mathrm{~W}$ 


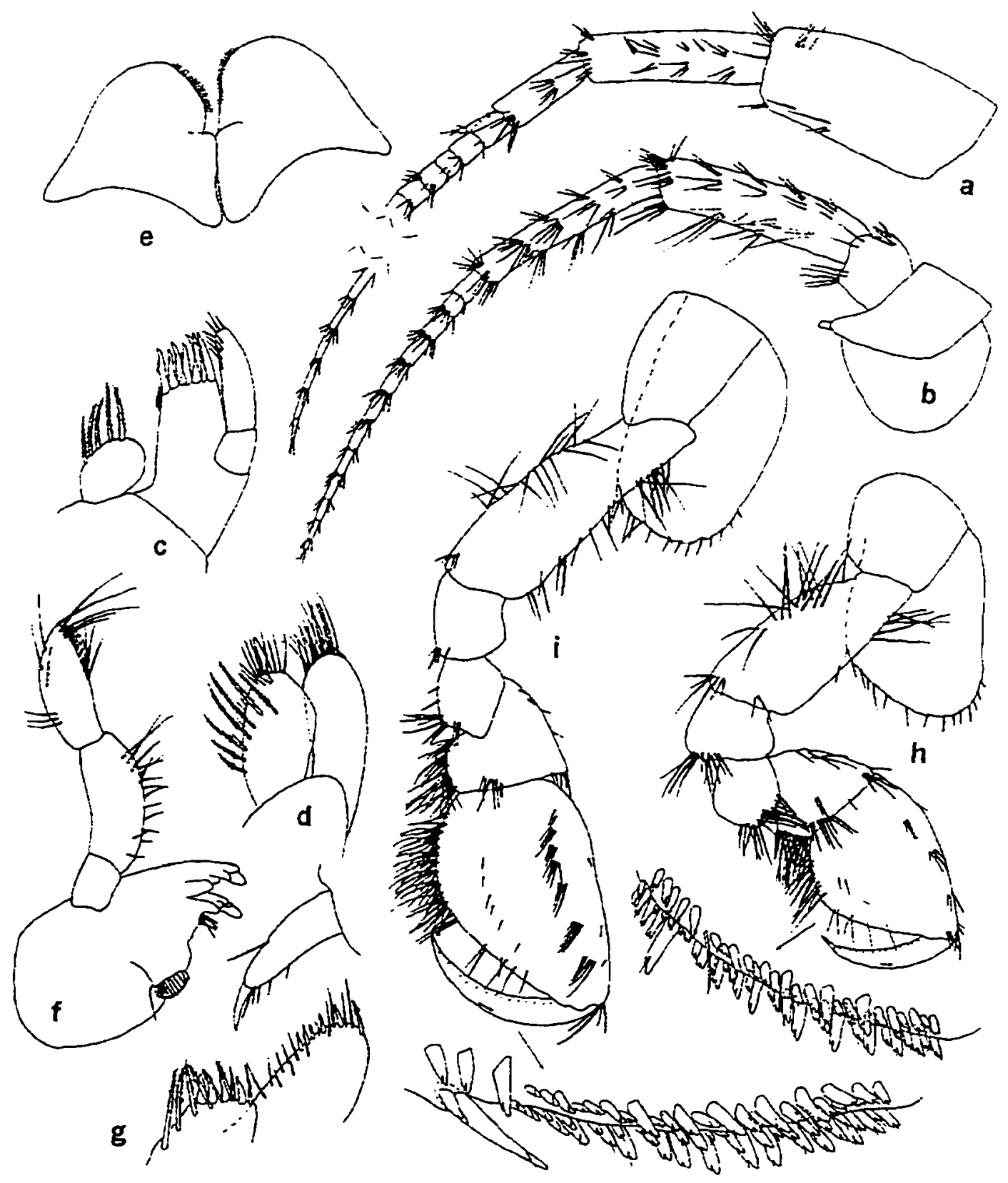

Figure 31 .-Crangomx anomalus Hubricht. Female cotype $(15.5 \mathrm{~mm})$. spring. $1.6 \mathrm{~km} \mathrm{E}$ of Wartentown. Fayette Co.. Kentucky: a, b. antennae I. 2: c. d. maxillae 1. 2: e. lower lip: f. left mandible: g. inner and outer and palp dactyl of maxilliped. Female cotype (16.6 $\mathrm{mm})$, same location: h. i. gnathopods 1.2 (palmar margins enlarged). 


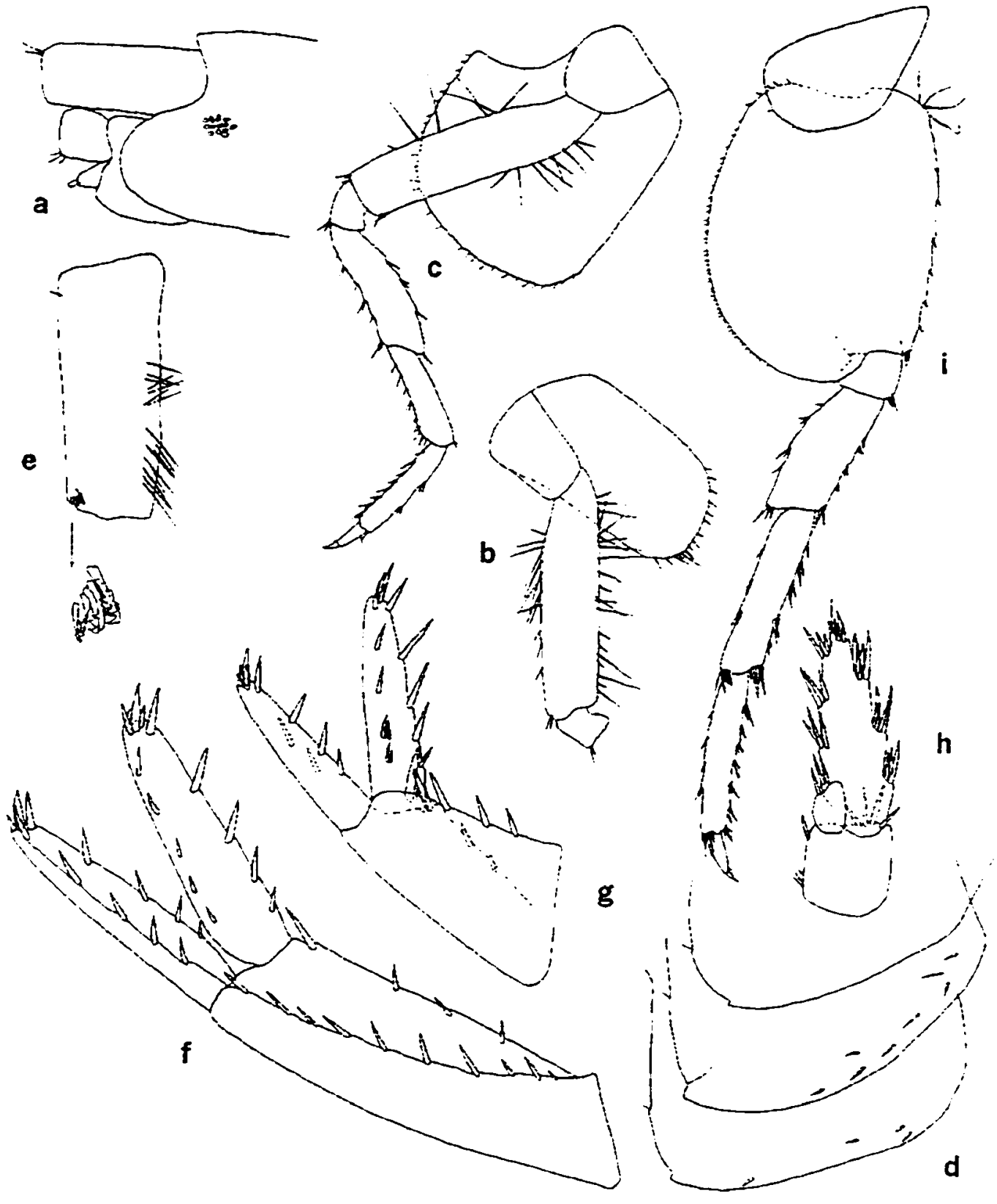

Figure 32.--Crangonyx anomalus Hubricht. Female cotype $(15.5 \mathrm{~mm})$ spring. $1.6 \mathrm{~km} \mathrm{E}$ of Warrentown. Fayette Co., Kentucky: a. head (in part): b. pereopod 3 (in part): c. pereopod 4 : d. pleonal plates: e. peduncle of pleopod $\mathrm{l}$ (retinaculae enlarged); f. g. h. uropods. I. 2. 3. Female cotype (16.6 mm). same location: i. pereopod 7. 


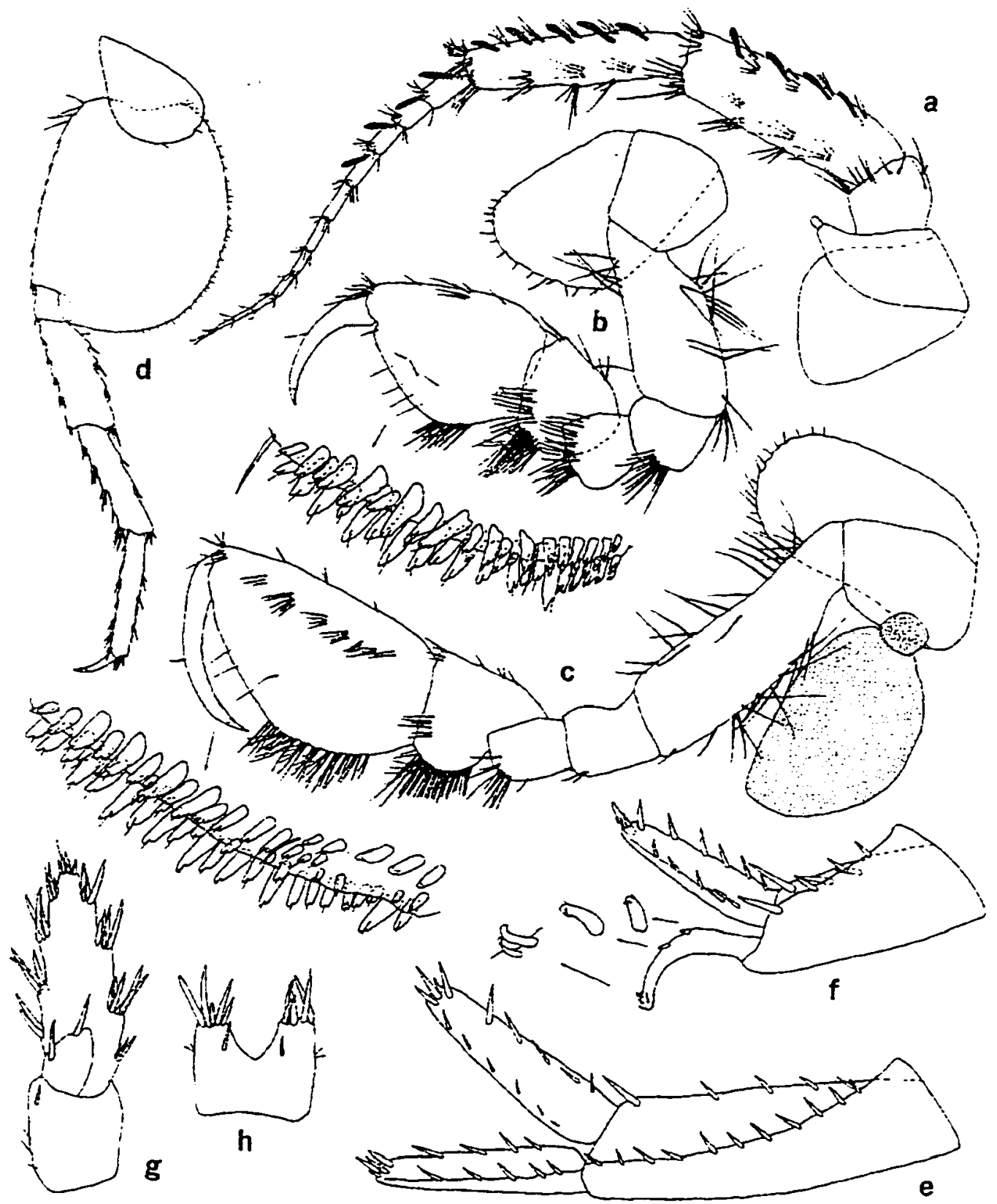

Figure 33.--Crangonyx anomalus Hubricht. Male cotype $(15.4 \mathrm{~mm})$, spring. $1.6 \mathrm{~km}$ E of Warrentown. Fayette Co.. Kentucky: a. antenna 2: b. c. gnathopods 1. 2 (palmar margins enlarged): d. pereopod 7: e. g. uropods 1. 3; f, uropod 2 (some spines enlarged): h. telson. 
of Clark County Court House on Colby Pike. $3 \approx$. R. Fox. I Jan 1971: Fayette Co.: Coldstream Farm. pond Vo. $2.1 \equiv .1: 3$ juvs. collector not known. 30 Jan 1964: Idle Hour spring No. $2.1 \equiv .1 \approx 0$. J. R. Holsinger and R. M. Norton. II Apr 1965: seep-fed swamp off Tates Creek Rd.. Lexington. $15 \Xi .13 \approx^{\circ}$ J. R. Holsinger and R. McAdams. 5 Apr 1965: Maedico Creek, ca. $9.0 \mathrm{~km}$ W of Clark. 1 \%. R. Fox. I Jan 1971: Pompoon creek ca. $11.2 \mathrm{~km}$ W of Clark. $1 \%$. 2 juvs. R. Fox. I Jan 1971 : Waveland Farm spring-fed stream. 1 Q. I $d^{*}$ J. R. Holsinger. 7 Mar 1964 and I \&. II Apr 1965: Bryan Station Rd.. 0.5 km NE of Eastin Rd.. $4.8 \mathrm{~km}$ NE of Lexington. 100 specimens ( $\left({ }^{\circ}\right)$. L. Hubricht. $25 \mathrm{Apr} 1941$ (USNM): spring. 1.6 km E of Warrentown. cotypes. $33 \subsetneq .39 \leftleftarrows$. L. Hubricht. 22 Nov 1940 (USNM): Garrard Co.: spring. 3.2 $\mathrm{km} \mathrm{NW}$ of Lancaster. $3 \Xi 10 \approx$. L. Hubricht, 24 Apr 1941 (USNM): Jefferson Co.: intermittent brook at Poplar Level Rd. and Audubon Rd.. Louisville. I Э. G. A. Cole. 13 Mar 1954 (USNM): Mason Co.: spring. $2.6 \mathrm{~km} \mathrm{~N}$ of Washington. 1 . L. Hubricht. $25 \mathrm{Apr} 1941$ (USNM). OHIO. Brown Co.: spring. 8.0 $\mathrm{km} \mathrm{S}$ of Georgetown. $+\Xi .1 \Xi$ L. Hubricht. $25 \mathrm{Apr} 1941$ (USNM): spring rill. $2.9 \mathrm{~km}$ S of Georgetown. 5 ミ. L. Hubricht. 25 Apr 1941 (USNM): Clermont Co.: Shayler Run (sta.. 4E). 1 Э. 1 :. E. L. Robinson. 9 Mar 1970: Clinton Co.: small spring. 0.7 mi SE of Westboro. $1 \cong .1$ juv. L. Hubricht. 25 Apr 1941 (USNM): Greene Co.: springs. John Bryan State Park. 2 mi W of Clifton. $1 \cong .2: 1$ juv. L. Hubricht. 26 Apr 1941 (USNM): Shelby Co.: "5 mile cave" (=Miami River Cave ?). 3 ₹. A. Pizzini. 27 Nov. 1936 (USNM).

Diagnosis.--A large species easily distinguished from other members in the genus by strongly expanded posterior margins of pereopod bases. more than 30 minute serrations on the posterior margins of bases: and outer ramus of uropod 2 in male. which has 3 - or 4 -notched spines on inside. Largest male. 20.0 $\mathrm{mm}$ : largest female. $19.0 \mathrm{~mm}$.

Female.--Eye round, reduced in size. Antenna 1, 50-55 percent length of body, about 1.7 times longer than antenna 2: primary flagellum with 27-32 segments. Antenna 2: flagellum with up to II segments: peduncular segments 4 and 5 with 4-6 sets of marginal setae radially arranged. Mandibles subequal. spine row with 6-8 spines: segment 2 of palp with about 16-17 long setae: segment 3 with $3-4 A$ setae. $+B$ setae ( 2 sets). $5 \mathrm{C}$ setae. 5-6 E setae and a row of about $15 \mathrm{D}$ setae. Maxilla 1: inner plate with $4-5$ apical plumose setae: palp with 5-7 slender spines and 5 naked setae on apex. Maxilla 2. inner plate with oblique row of 7 plumose setae on inner margin. Maxilliped: inner plate apically with 4-5 bladelike spines. 2 naked setae. and 7-9 plumose long setae extending from inner margin to apex: outer plate with row of naked setae and $4-5$ slender pectinate spines on inner margin and 1 apical plumose seta: inner margin of dactyl with $4-5$ setae. nail long. about $1 / 4$ length of dactyl.

Propod of gnathopod 1 more than 2 times longer than carpus: palm oblique. slightly convex. longer than posterior margin. inside and outside margins each with 2 rows of spine teeth. including $I$ row of about 20 small spine teeth and 1 row of about 24 large spine teeth: defining angle with about 52 -notched or 3notched spine teeth on inner side. I row of 1 large and 4 small 2-notched or 3-notched spine teeth and I row of small $4-5$ spine teeth on outside: 4 sets of superior medial setae in groups of 2-5: posterior margin 
with about 4 sets of setae in groups of 2-6. Dactyl of gnathopod I with row of 10 short setae on inner margin. nail very short. Ventral margin of coxa 1 with 17-18 setae.

Propod of gnathopod 2 more than 2 times longer than carpus: palm strongly convex. oblique. longer than posterior margin, inside and outside margin each with 2 rows notched spine teeth. including $I$ row of 23-25 small spine teeth and 1 row of 23-25 large spine teeth: defining angle with 3 large and 3 small spine teeth on inside. 1 very large. long spine teeth and 9 small spine teeth on outside: 7 sets of superior medial setae. mostly in groups of 5: posterior margin with 10 sets of setae in groups of 3-7. Dactyl of gnathopod 2 with row of very short setae on inner margin: dactyl nail very short. Ventral margin of coxa 2 with 13 setae.

Coxa of pereopod 3 with 16 marginal setae. Coxa of pereopod + with 32 marginal setae: dactyl of pereopod 4 about 35 percent length of propod. Pereopod 5: basis greatly expanded posteriorly. with more than 30 very shallow serrations on posterior margin: 11 sets of spines in groups of $1-3$ on anterior margin: 6 setae on the anteroproximal margin. Pereopod 6: basis greatly expanded posteriorly. distoposterior lobe well developed. more than 30 very shallow serrations along posterior margin: 10 sets of spines in groups of 1-j on anterior margin: 5 long setae on anteroproximal margin. Pereopod 7: coxa with 3 setae along posterior margin and $4-5$ small spines on the posterior margin of basis: basis greatly expanded posteriorly: with more than 30 very shallow serrations along posterior margin: 8 sets of short spines on anterior margin: + long setae on anteroproximal margin: dactyl about 33 percent length of propod.

Pleonal plates: posterior margin of plate I convex. with I short seta. distoposterior comer not produced but weakly acute: posterior margin of plate 2 nearly straight with I seta. distoposterior corner not produced but subacute. ventral margin with up to 12 (subventral) spines: posterior margin of plate 3 nearly straight with I seta. distoposterior corner not produced but subacute. ventral margin with 9-1 | subventral spines. Peduncle of pleopod I with 5-6 retinaculae and many long setae on outer margin.

Uropod 1: inner ramus 60 percent length of peduncle. armed with abolit 14 spines: outer ramus with about 13 spines: peduncle with 10-11 outer and $4-5$ inner spines. Uropod 2: inner ramus armed with about 15 spines: outer ramus armed with about 9 spines: peduncle with 4 outer and $3-4$ inner spines. Lropod 3 : inner ramus with 1-2 spines. outer ramus about 2 times longer than peduncle, inner and outer margin each with $3-4$ sets of spines in groups of $3-5$. Telson broader than long. notched about $1 / 4$ distance to base. each lobe with 5-7 apical spines: spines longer than $1 / 2$ length of telson.

Male.--Differing from female as follows: longer with same body shape or smaller with slender body shape. Antenna 2: calceoli small. present on peduncular segments 4 and 5 and first 4 segments of tlagellum.

Uropod 2: outer ramus with 2 short. 2-. 3-or 4-notched spines on inside. 4-5 curved short spines on apex: inner ramus with about 17 spines: peduncle arcuate. reduced in size, with + outer and + inner spines. Telson slightly broader than long, notched about $3 / 7$ distance to base.

Distribution and ecology.--This species is an inhabitant of springs and spring-fed streams in 
northcentral Kentucky, southern Illinois. southeastern Indiana, and southwestern Ohio (fig. 34). It was also collected from single cave streams in Indiana and Ohio. Ovigerous females were found in collections made in winter and early spring. The southern Illinois population is highly disjunct. inasmuch as it occurs approximately $250 \mathrm{~km}$ from the heart of the range in northern Kentucky.

Remarks.--C. anomalus is unique in the genus Crangonxx by having some males larger than the largest known females. However. this observation is possibly biased by too few samples. In all collections of ${ }^{\circ}$. unomulus examined to date. a majority of females are larger and longer than the males. and only a few males are larger than the largest females. These may also be morphological variation between different populations. resulting in some populations with males that are more robust and larger than females. Since sexually mature females (with larger. well developed brood plates) sometimes have penes in other Crangomx species (e.g. $C$. indianensis), it is also remotely possible that the large males in $C$. anomalus transform from females when brood plates are lost after brooding. Future investigation is needed to answer these questions.

Crangonyx indianensis. new species

Figures $35-38$

Crangonnx gracilis Smith.--Banta. 1907:78.

Material examined (paratypes unless designated otherwise).--INDIANA. Clark Co.: Spring No. 1. Vine Penny Branch Nature Preserve. ca. $4.8 \mathrm{~km} \mathrm{~N}$ of Charlestown. $2 \cong .1$ juv. J. J. Lewis et al.. 29 Apr 1995: Spring No. 7. Nine Penny Branch Nature Preserve. ca. 4.8 km N of Charlestown. 1 ๆ. J. J. Lew is et al.. 29 Apr 1995: spring on Everitt farm. Nine Penny Branch Nature Preserve. ca. $4.8 \mathrm{~km} \mathrm{~N}$ of Charlestown. 1 ?. J. J. Lewis et al., 29 Apr 1995: Greene Co.: Rays Cave. 2 ?. B. Nicoll. Oct 1964 and 3 $\Xi$. Dec 1964 and holotype $\approx .1 \cong .5 \approx .11$ juvs. J. R. Holsinger. R. Nicoll and R. M. Norton. 18 Jun 1965: Lawrence Co.: Donnehues Cave. 1 ๆ. 1 c.. J. R. Holsinger and E. Lavangino. 8 Aug 1964 and $1 \equiv$. S. B. Peck and J. Reddell. 14 Jun 1965: Martin Co.: Gypsy Bill Allen Cave. 1 . $5 \approx$. H. H. Hobbs. III. L. Mullins. H. Kronk. 1 Nov 1992: Rose Cave. 4 O. H. H. Hobbs. III. M. Porter. 2 Aug 1995: Monroe Co.: Buckners Cave. 1 ๑. 1 c. G. A. Coovert. 20 Feb 1972 (Dayton Museum Natural History): Matlocks Cave. $4 \equiv$. J. J. Lewis and H. Huffman. 29 Mar 1995: May's Cave. 4 ₹. N. Hynes. 3 Jan 1963 (USNM): Maýtield Cave, $4 \equiv$. T. C. Barr, 20 Aug 1957: Oliver Spring Cave. I F. H. H. Hobbs, III, no collection date: Patton Cave. 2 ฐ. H. H. Hobbs. III. J. Lapp and C. Frost. 24 Oct 1992: Salamander Cave. 1 ๖. H. H. Hobbs. III. no collection date and $2 \subsetneq .1 \approx$. J. R. Holsinger. D. C. Culver and J. Keith. 19 Jun 1973: Orange Co.: Black Cave. 1 Đ. J. J. Lewis et al.. 6 Feb 1994: Boiling Springs Cave. I §. J. R. Holsinger and R. M. Norton. 15 Jun 1965: Bond Cave. 1 ₹. H. H. Hobbs. III. 24 Apr 1993: Conrad Spring Cave. 1 ‡. H. H. Hobbs. III 


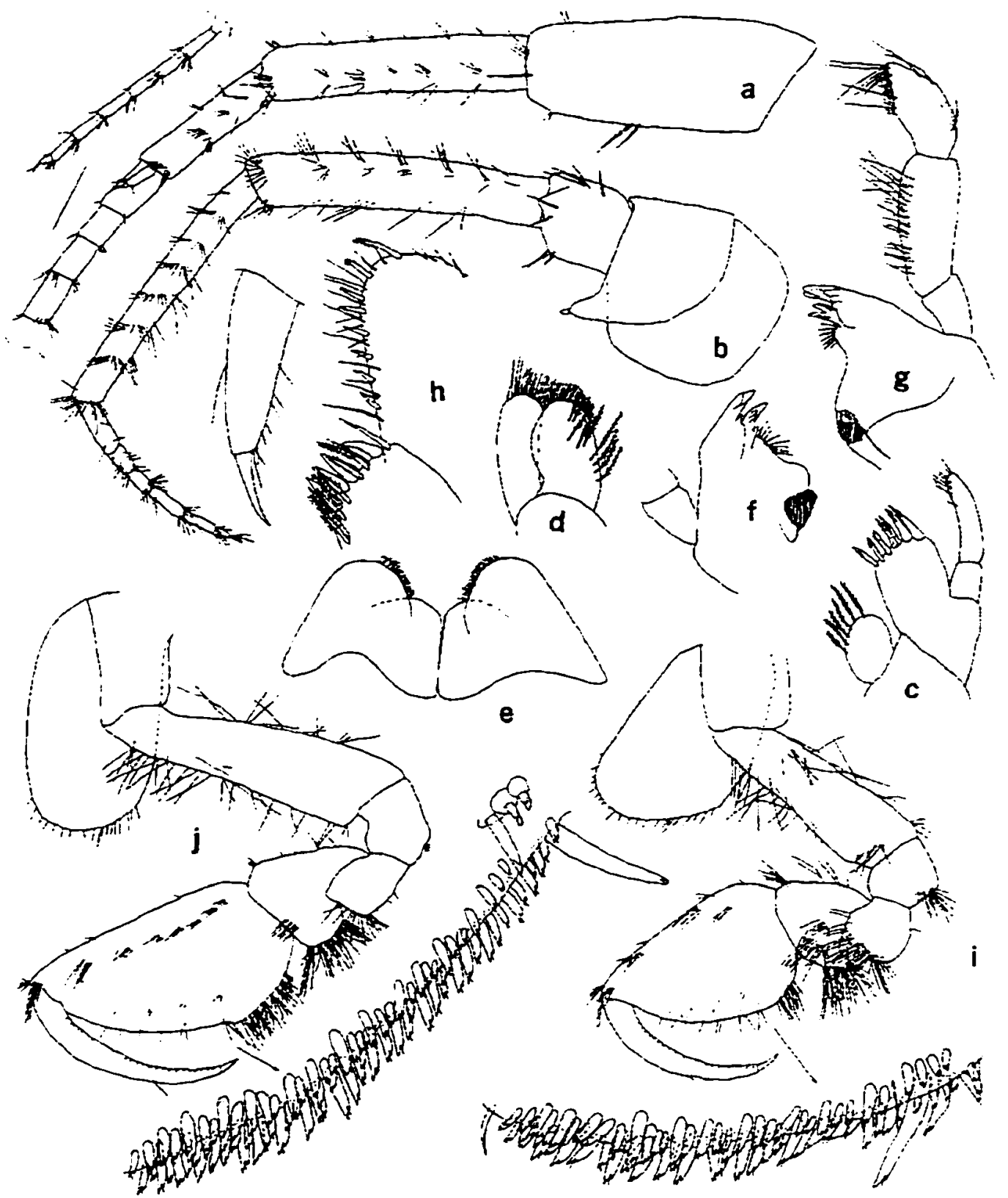

Figure 35.--Crangonvx indianensis, new species. Female paratype $(18.5 \mathrm{~mm})$. Ray's Cave. Green Co.. Indiana: a. b. antenna 1. 2: c. d. maxillae 1. 2: e. lower lip: f. dentate part of left mandible: g. right mandible: h. inner, outer plates and palp dactyl of maxilliped (greatly enlarged): i. j. gnathopods I. 2 (palmar margins enlarged). 


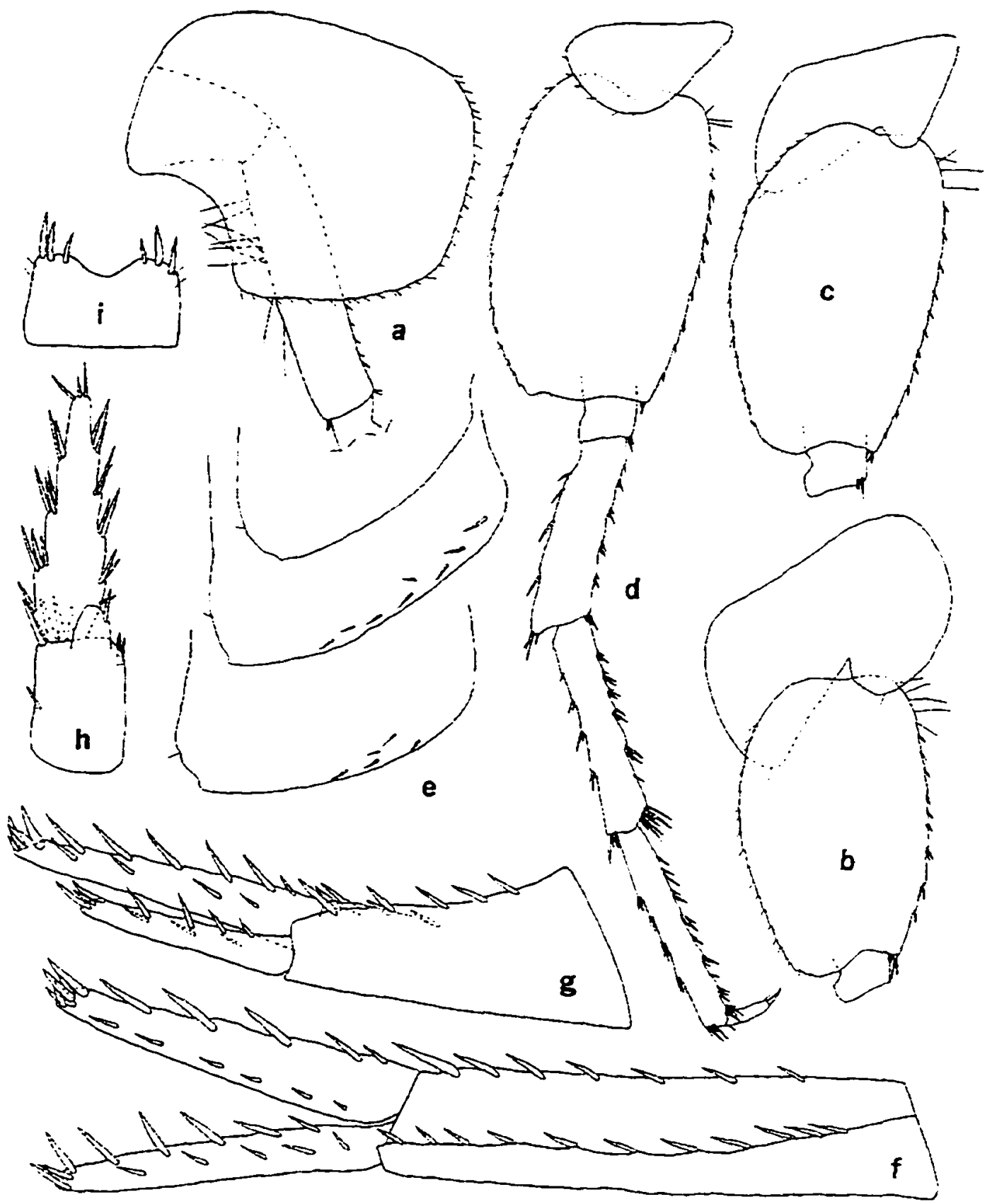

Figure 36.--Crangonyx indianensis, new species. Femate paratype $(18.5 \mathrm{~mm})$. Rays Cave. Green Co.. Indiana: a. b. c. pereopods 4.5,6 (in part): d. pereopod 7: e. pleonal plates: f. g. h. uropods 1. 2. 3: i. telson. 


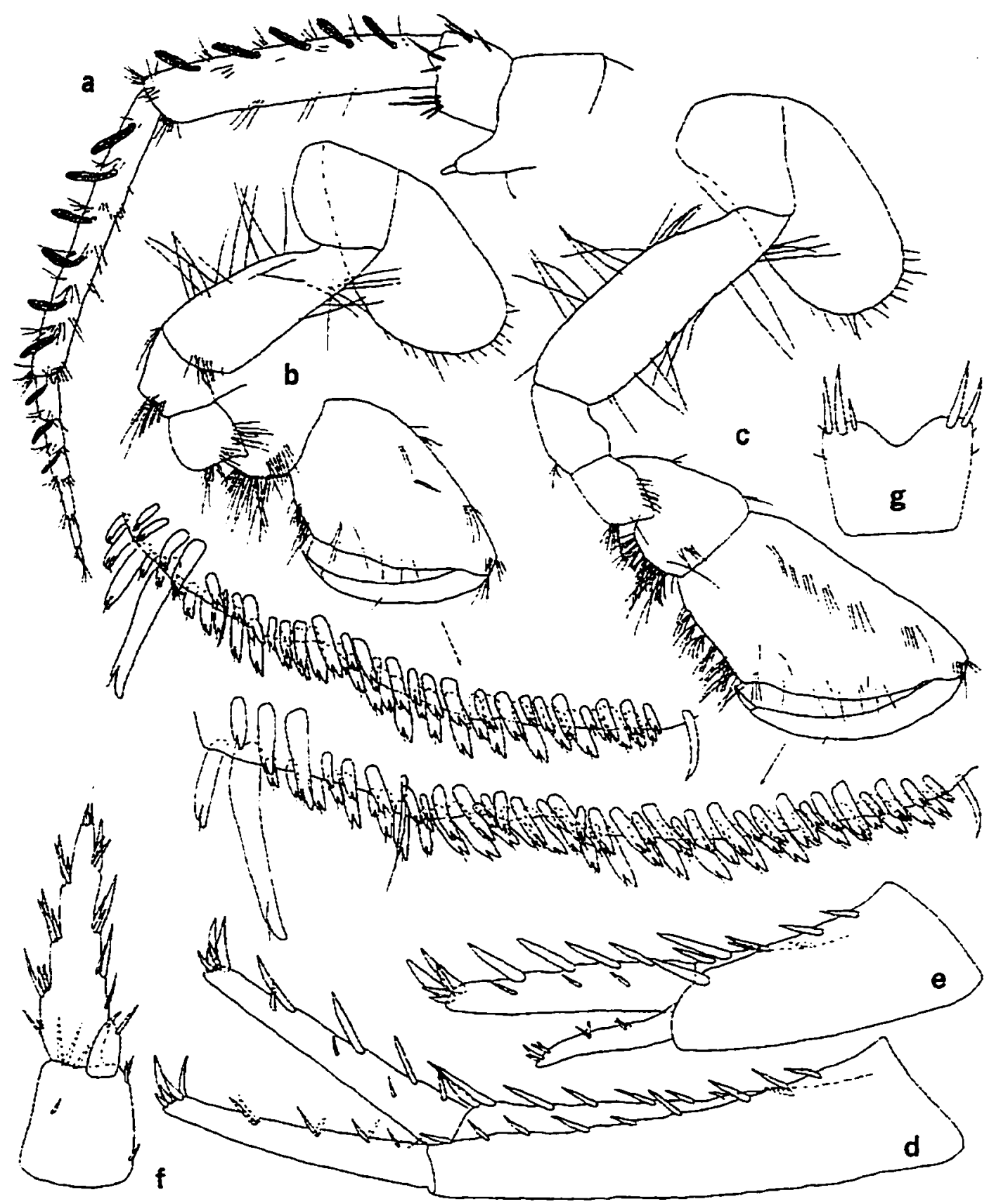

Figure 37.--Crangonyx indianensis, new species. Male paratype (11.5 mm). Rays Cave. Green Co.. Indiana: a. antenna 2: b. c. gnathopods 1, 2 (palmar margins enlarged): d. e. f. uropods 1. 2. 3. Male paratype (12.5 mm). Rays Cave, Green Co., Indiana: g, telson. 


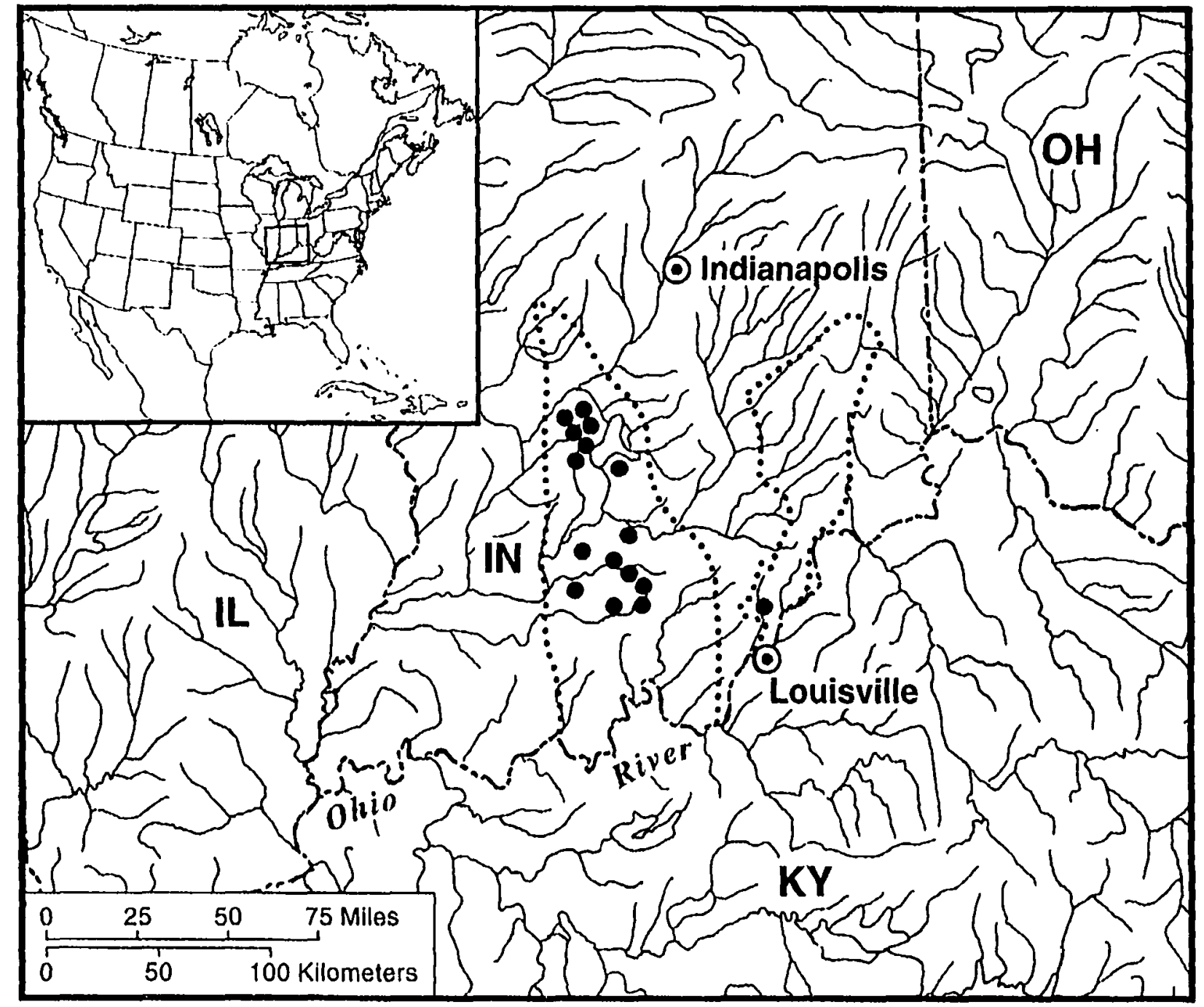

Figure 38.--Distribution of ('rangemyx indiamensix in North America. Solid circles represem 1-2 closely proximate localities. Karst regions of soulhern Indiana are enclosed by donled lines. 
and M. Porter. 2 Aug 1995: Critchfield Cave Spring, 1 ๑. J. J. Lewis et al., 12 Mar 1994: Dillion Cave. 12 э. 1 ๘. H. H. Hobbs. III et al.. 29 Jul 1992; small spring on spring run flowing into true rise of Lost River. $1.1 \mathrm{~km} \mathrm{~S}$ of Orangeville. $1 \% .1$ \%. J. J. Lewis et al.. 12 Mar 1994: Tucker Lake Spring Cave. $1 c^{*}$, H. H. Hobbs. III, and M. Porter. I Aug 1995: Wesley Chapel Cave, 3 \&. I ๔. J. H. Holsinger and R. Norton. I5 Jun 1965 and 6 \%. J. J. Lewis and M. Lewis, 17 Jul 1993: Owen Co.: spring on McCormicks Creek. McCormicks Creek State Park. I ๆ. J. Lewis, 21 Mar 1993.

Diagnosis.--A large species of the richmondensis group. distinguished from other members of this group by unproduced and subacute distoposterior comers of female pleonal plates: posterior margins of pereopods 5-7 with very shallow serrations: maxilla 2 inner plate with oblique row of up to 7-9 piumose setae: and much broader telson of the female. Largest male. $10.0 \mathrm{~mm}$ : largest female. $20.0 \mathrm{~mm}$.

Female.--Eye small and ovate. Antenna 1, about 55 percent length of body. about 2 times longer than antenna 2: primary flagellum with 20 segments. Antenna 2, flagellum with 6 segments. Mandibles subequal. spine row with 7-8 spines: segment 2 of palp with 13-14 short and 7-8 long setae, segment 3 with 4 A setae. 2 B setae. 2-3 C setae. 5-6 E setae and a row of D setae. Maxilla I: inner plate with 5-7 apical plumose setae: palp with 8-9 slender spines on apex. Maxilla 2. inner plate with oblique row of 7-9 plumose setae on inner margin. Maxilliped: inner plate apically with 4 bladelike spines. 2 naked spines. and 5-7 plumose setae on the inner margin and on apex: outer plate with row of naked setae and 5-7 slender pectinate spines on inner margin and I apical plumose seta: dactyl with 6-7 setae on inner margin: dactyl nail short.

Propod of gnathopod 1 about 2 times larger and longer than carpus and much broader: palm about 2 times longer than posterior margin. convex and very oblique. inside and outside each with $2-3$ rows of spine teeth. including 33 spine teeth on inside. 37 on the outside: defining angle with 4 notched but weakly serrate spine teeth on inside. 1 large and 4 small notched spine teeth and 1 row of 4 notched spine teeth on outside: superior medial setae in $3-4$ sets. inserted in groups of $3-4$; setae on posterior margin densely inserted. $4-5$ sets on proximal half. Dactyl with row of short setae on inner margin. nail short. Ventral margin of coxa 1 with 23 setae.

Propod of gnathopod 2 more than 2 times larger and longer than carpus and much broader: inside and outside palmar margin each with 2-3 rows of notched spine teeth. including 45 spine teeth on inside. 49 on outside: defining angle with 3 spine teeth on inside. I very large one on outside: superior medial setae in 7 sets in groups of 3-8: posterior margin with 6-7 sets of setae in groups of 4-10. Dactyl with row of short setae on inner margin: dactyl nail very short. Ventral margin of coxa 2 with 18 setae.

Coxa of pereopod 3 with 18 marginal setae. Coxa of pereopod 4 with 33 marginal setae: dactyl of pereopod 4. 45 percent length of propod. Pereopod 5: basis with 20 shallow serrations along posterior margin: 9 sets of short spines on anterior margin: 4-5 long setae on anteroproximal margin. Pereopod 6: basis with 24 very shallow serrations along posterior margin: 11 short spines on anterior margin: 4 setae on anteroproximal margin. Pereopod 7: coxa with 6-8 setae on posterior margin: basis with 23 very shallow 
serrations along posterior margin: 11 short spines on anterior margin: 3 setae on anteroproximal margin: dactyl about 30 percent length of corresponding propod.

Pleonal plates: posterior margin of plate I convex. with I seta. distoposterior corner subacute but not produced: posterior margin of plate 2 nearly straight with 1 seta, distoposterior corner subacute but not produced, ventra! margin with 8 (subventral) spines: posterior margin of plate 3 slightly convex with 1 seta. distoposterior corner subacute but not produced, ventral margin with 5 (subventral) spines. Peduncle of pleopod 1 with about 7 retinaculae. many setae on outside margin. a few setae on inside margin.

Uropod 1: inner ramus about 75 percent length of peduncle, armed with 16 spines: outer ramus with 14 spines: peduncle with 11 outer and 7 inner spines. Uropod 2: inner ramus much longer than outer ramus. armed with 14 spines: outer ramus with 10 spines: peduncle with 5 outer and 4 inner spines. Uropod 3 : inner ramus with $I$ spine. outer ramus about 2 times longer than peduncle. inner and outer margin each with 4-5 sets of spines in groups of $1-3$. Telson much broader than long (about 1.5 times). notched about $1 / 5-1 / 4$ distance to base. each lobe with 3 apical spines: spines about $1 / 2$ length of telson.

Male.--Differing from female as follows: smaller with more slender. elongate body. Antenna 2: calceoli percent on peduncular segments 4 and 5 . and tirst 3 segments of flagellum.

Propod of gnathopod I with 21 spine teeth on inside. 23 on outside. defining angle with I notched and 4 notched but weakly serrate spine teeth on inside. 10 notched spines on outside ( 1 very large): 3 sets of superior medial setae in groups of 2-3: posterior margin setae densely inserted on proximal half.

Palmar margin of propod of gnathopod 2 strongly oblique. with 28 spine teeth on inside. 28 on outside: defining angle with 6 spine teeth on inside, 10 on outside ( 1 very large): 7 sets of superior medial setae in groups of $2-5$.

Uropod 2: inner ramus with 12 spines: outer ramus reduced in size. curved slightly. with 2 short spines on inner and outer margins. apex with 4 spines: peduncle with 4 outer and 4 inner spines.

Variation.--All females examined had penes and some had calceoli. Therefore it was often difficult to distinguish males from immature females.

Type-locality.--Rays Cave. located in Greene County. Indiana. consists primarily of a single stream passage that is developed in the Beech Creek limestone of Mississippian age (Powell. 1961). According to J. R. Holsinger (pers. comm.). the type series was collected from under rocks in a shallow stream 100-300 meters or more inside the cave. Specimens were light grayish to pale yellowish in color when alive. Darkly pigmented specimens of Gummarus minus were abundant near the entrance and in the stream issuing from the mouth of the cave. There was little or no overlap between these two species in the cave stream.

Distribution and ecology.--Although this species occurs in many caves of southern Indiana. it is noi troglomorphic. Its range. which is restricted to the karsts region of southern Indiana, covers a linear distance of $120 \mathrm{~km}$ (fig. 38 ).

('rangomyx indianensis was collected from cave streams and surface springs. Ovigerous females were found in June and November collections. In caves it was found living with other amphipods. viz.. $C$. 
packardi. Gammurus minus. and the isopods Caecidotea strgia, and Lirceus sp.

Etymology.--The specific name refers to the occurrence of this species in the state of Indiana.

Remarks.--The species that Banta (1907) found in Mayfield Cave and called C. gracilis actually belongs to the new species described above. Although $C$. packardi has also been collected from this cave (see material examined section under C. paCkardi), Banta (1907) stated that the specimens he examined from Mayfield Cave had well-developed and distinctly pigmented eyes and the largest specimens were up to $18 \mathrm{~mm}$ long. These match the characteristics of $C$. indianensis and not $C$. packardi (or C. gracilis). Specimens of $C$. Indianensis were also collected from Mayfield Cave by T. C. Barr. Jr. on 20 Aug 1957 and their identity was verified by me in the present study.

Crangonyx cornutus, new species

Figures $39-42$

Material examined (paratypes unless designated otherwise).--ALABAMA. Jackson Co.: ditch at Stevenson. $4 \subsetneq$. J. E. Cooper and M. R. Cooper. 9 Apr 1966: Madison Co.: craytish burrows in Ka Lea

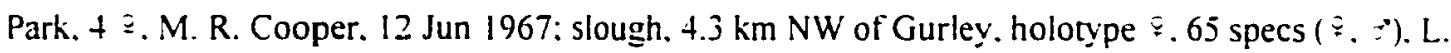
Hubricht. 12 Apr 1941 (USNM). TENNESSEE. Davidson Co.: spring. "Fortland" near Shelby Park. Nashville. $6 \cong .6 \%$. L. Hubricht, 31 May 194I (USNM).

Diagnosis.--A medium-sized species morphologically similar to other species of the richmondensis group but distinguished from them by unique features of uropod 2 of male. which has I-2 large (thick) spines on the middle of outer ramus (c.f.. C. disjunctus): and more setae on maxillae. Largest male, 8.5 $\mathrm{mm}$ : largest female. $14.0 \mathrm{~mm}$.

Female.--Eye small. round. Antenna 1. about 60 percent length of body. about 1.9 times longer than antenna 2: primary flagellum with about 28 segments. Antenna 2. flagellum with 9 segments. Mandibles subequal. spine row with 5-7 spines: segment 2 of palp with 12 setae. segment 3 with 3 A setae. 2 B setae. $3 \mathrm{C}$ setae. $5 \mathrm{E}$ setae and a row of D setae. Maxilla I: inner plate with 5 apical plumose setae: palp with 8 slender spines on apex. Maxilla 2, inner plate with oblique row of 4-5 plumose setae on inner margin. Maxilliped: inner plate apically with 4 bladelike spines. 2 naked spines, and 5-6 plumose setae extending from inner margin to apex: outer plate with row of naked setae and 2-3 slender pectinate spines on inner margin and 1 apical plumose seta: dactyl with 3 setae on inner margin: dactyl nail short. about 14 length of dactyl.

Propod of gnathopod I more than 2 times longer than carpus: palm very oblique. convex. longer than posterior margin. with 13 spine teeth on inside. 16 on the outside: defining angle with 4 notched spine teeth on inside. 2 rows of spine teeth on outside ( 1 row of 5-6 small spines and I row of I slightly large and 3 small notched spine teeth); superior medial setae few in number and mostly doubly inserted: setae on 


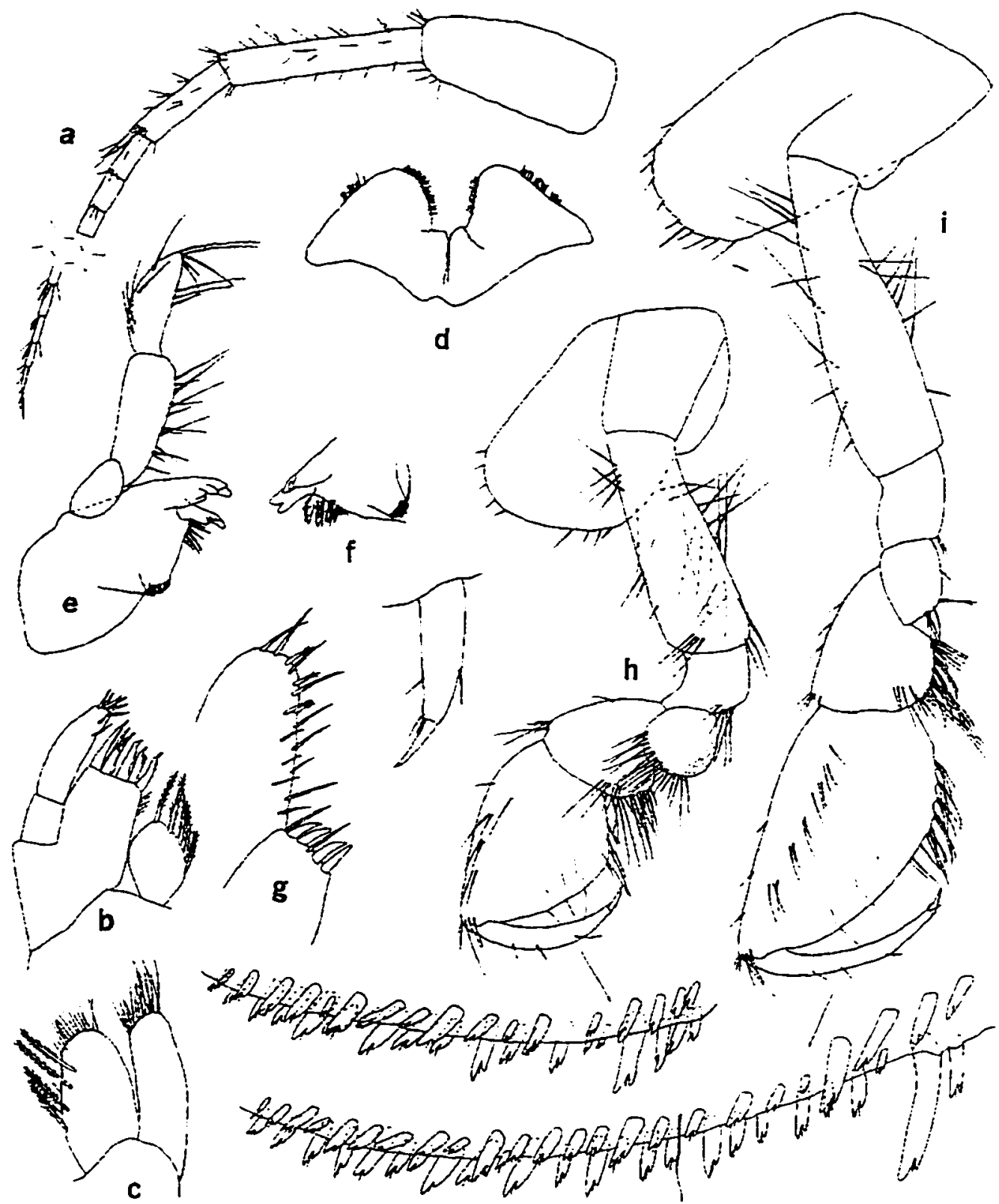

Figure 39.--Crangonyx cornutus, new species. Female paratype (11.5 mm), slough. $4.3 \mathrm{~km} \mathrm{NW}$ of Gurley. Madison Co., Alabama: a. antenna 1: b. c. maxillae 1. 2: d. lower lip: e. left mandible: $f$. dentate part of right mandible: $g$, inner and outer plates and palp dactyl of maxilliped (greatly enlarged): h. i. gnathopods. 1. 2 (palmar margins greatly enlarged). 


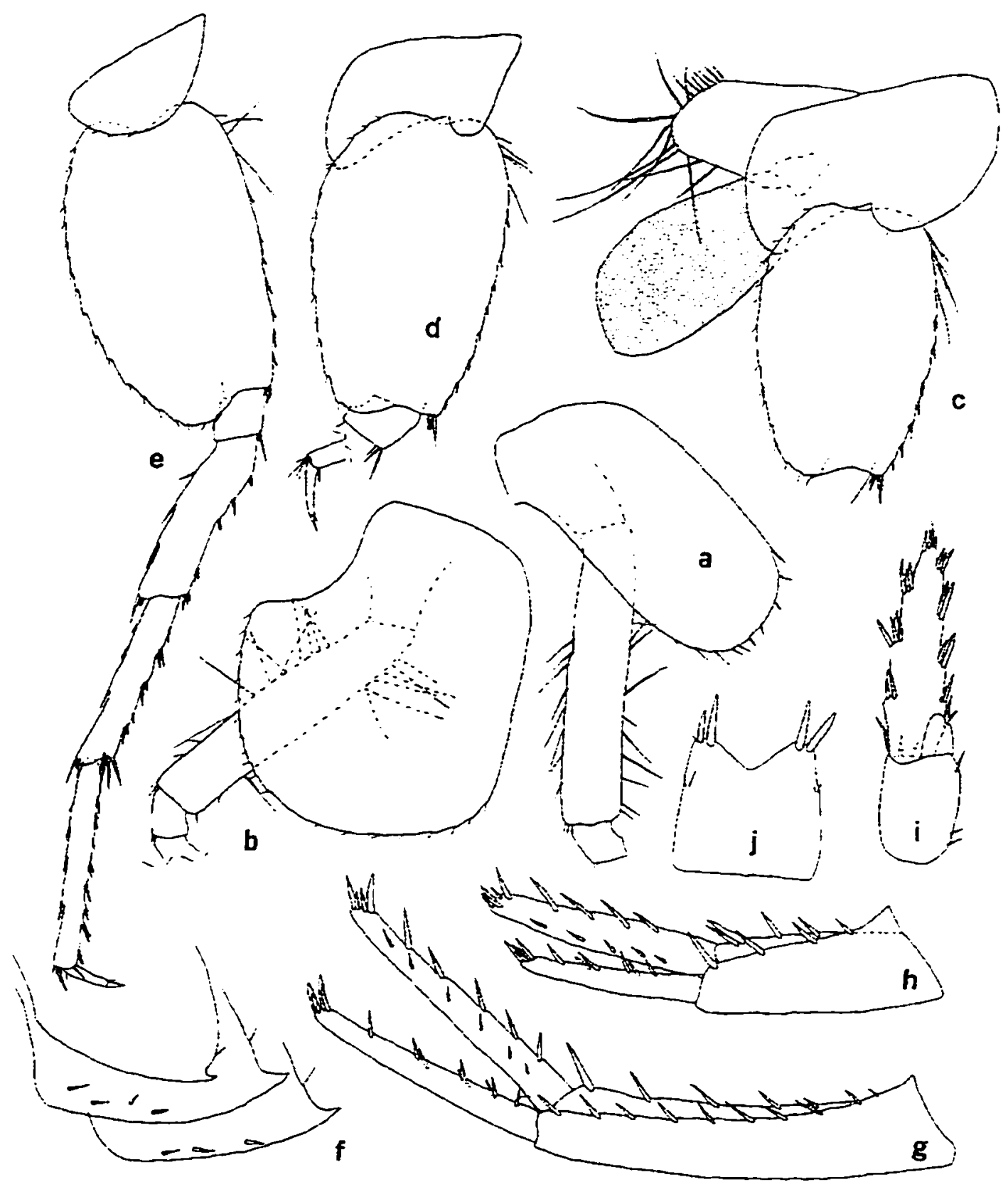

Figure 40.--Crangonyx cornutus. new species. Female paratype $(11.5 \mathrm{~mm})$. slough. $4.3 \mathrm{~km} \mathrm{NW}$ of Gurley. Madison Co.. Alabama: a. b. c. d. pereopods 3. 4. 5.6 (in part): e. pereopod 7: f. pleonal plates: g. h, i, uropods $1,2,3:$ j. telson. 


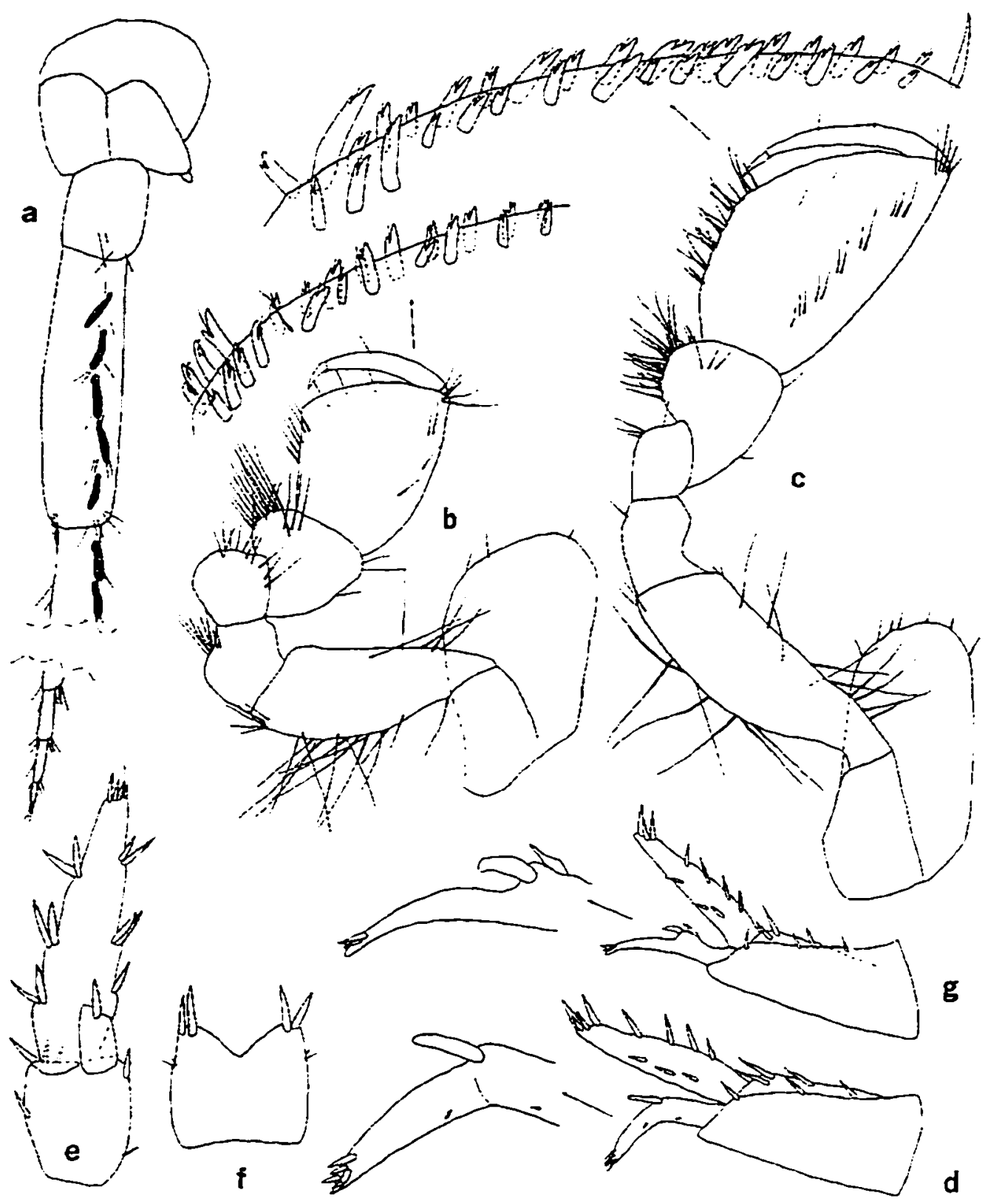

Figure 41 .-Crangonyx cornutus, new species. Male paratype $(7.2 \mathrm{~mm})$, slough. $4.3 \mathrm{~km} \mathrm{NW}$ of Gurley. Madison Co.. Alabama: a. antenna 2 (in part): b. c. gnathopods 1. 2 (palmar margins greatly enlarged): d. uropod 2 (outer ramus enlarged): e. uropod 3: f. telson. Male paratype (7.2 mm). same location: g, uropod 2 (outer ramus enlarged). 


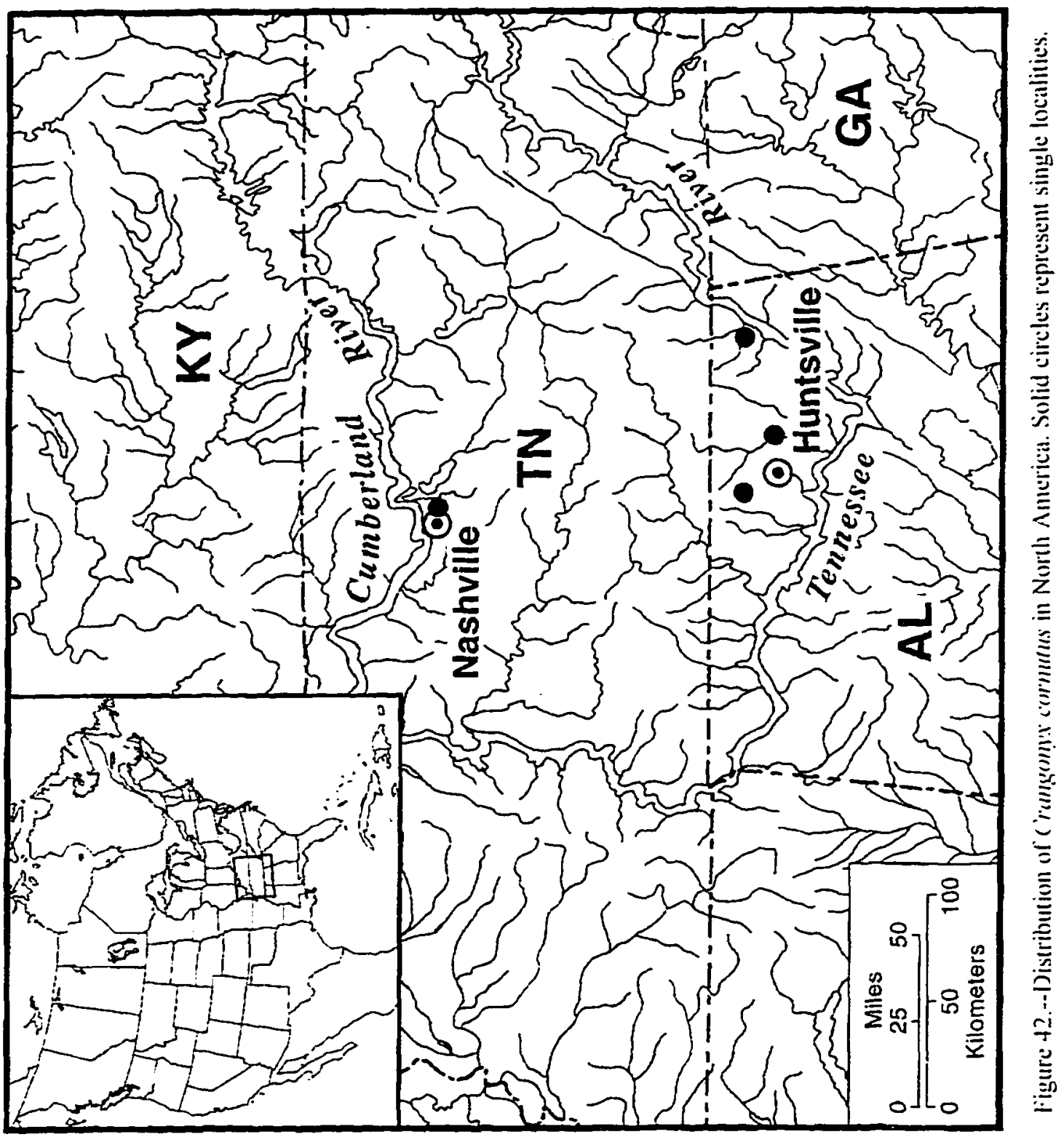


posterior margin in 3 sets. inserted in groups of $1-3$. Dactyl with 3 setae on outer margin, nail short. Ventral margin of coxa I with 9 setae.

Propod of gnathopod 2 more than 2 times longer than carpus: palm very oblique. convex. much longer than posterior margin. with 19 spine teeth on inside. 16 on outside: defining angle with 8 spine teeth on inside. 8 on outside ( 1 very large); superior medial setae in 7 sets inserted in groups of 1-3: posterior margin with 5 sets setae in groups of 3-6. Dactyl with 3-4 setae on outer margin. Ventral margin of coxa 2 with 11 setae.

Coxa of pereopod 3 with 10 marginal setae. Coxa of pereopod 4 slightly longer than broad. with 18 marginal setae. dactyl of pereopod 4 about 35 percent length of propod. Pereopod 5: basis with 14 shallow serrations along posterior margin; 7 short spines on anterior margin: 6 setae on anteroproximal margin. Pereopod 6: basis with 13 shallow serrations along posterior margin: 8 short spines on anterior margin: 5 setae on proximal margin. Pereopod 7: coxa with 3 setae on posterior margin: basis with 17 shallow serrations along posterior margin: 7 short spines on anterior margin: 4 setae on another proximal margin: dactyl about 33 percent length of corresponding propod.

Pleonal plates: posterior margin of plate I slightly convex. with I seta. distoposterior corner acute but not produced: posterior margin of plate 2 nearly straight with I seta. distoposterior comer strongly produced and acute. ventral margin with 4 (subventral) spines: posterior margin of plate 3 nearly straight with I seta. distoposterior corner also strongly produced and acute, ventral margin with 3 (subventral) spines. Peduncle of pleopod 1 with up to 5 retinaculae. many setae on outside margin. few on proximal end of inner margin.

Uropod 1: inner ramus 75 percent length of peduncle. armed with 15 spines: outer ramus with 13 spines: peduncle with 10 outer and 5 inner spines. Uropod 2: inner ramus armed with 15 spines: outer ramus with 10 spines: peduncle with 4 outer and 4 inner spines. Uropod 3 : inner ramus ivith 1 spine. outer ramus about 2 times longer than peduncle. inner and outer margin each with 4 sets of spines in groups of 15. Telson broad. about as broad as long. notched about 1/6 distance to base. each lobe with 2 apical spines: spines less than $1 / 2$ length of telson.

Male.--Differing from female as follows: smaller with more slender. elongate body. Antenna 2: calceoli percent on peduncular segments 4 and 5 . and first 3 segments of flagellum.

Uropod 2: outer ramus strongly curved, with $1-2$ very large spines on inner margin in the middle. 2-3 very small outer spines. apex with 3-5 short spines: peduncle with 3-4 outer and 4 inner spines. Uropod 3 : inner ramus with 1 spine: outer ramus about 2.5 times length of peduncle. inner and outer margins each with 3 sets of spines in groups of $1-2$.

Type-locality.--slough. $4.3 \mathrm{~km} \mathrm{NW}$ of Gurley. Madison County. Alabama.

Distribution and ecology.--The range of this species. which to date is based on 3 collections in northern Alabama and 1 collection in central Tennessee. covers a linear distance about $150 \mathrm{~km}(\mathrm{fig}$. +2 ). Its habitats include a ditch. a slough, springs. and crayfish burrows. It was found together with $C$. consimulis in 
a spring in Tennessee and with C. floridanus in a ditch in Jackson Co.. Alabama. Ovigerous females were collected in April and May.

Etymology.-The epithet cornutus is from Latin meaning "horned," which is in reference to the large. horn-like spine on the outer ramus of male uropod 2 of male.

Crangonvx obliquus (Hubricht and Mackin)

Figures $43-46$

Eucrangenyx obliquus Hubricht and Mackin. 1940:195. fig. + [type-locality: a small creek $W$ of the college chapel. Clarksville. Johnson Co., Arkansas].

Crangonyx obliquzus (Hubricht and Mackin).--Hubricht. 1943:689 [in part].--Bousfield. 1958:89.--

Nicholas. 1960:128 [in part].--Holsinger. 1972:43. figs. 12d. 12i. 17: 1977:252.--McDaniel \& Smith. 1976:57 [in part]: Barnard \& Barnard. 1983:435. mapll [in part].--Fitzpatrick. 1983:145. [Not Kent. 1949:48.--Cole. 1957:36.--Krekeler \& Williams. 1966:394].

Material examined.--ALABAMA. Green Co.: roadside ditch. $1.3 \mathrm{~km} \mathrm{~N}$ of Boligee. $5: 1 \div \mathrm{L}$. Hubricht. 11 Feb 1962 (USNM). ARKANSAS. Faulkner Co.: temporary stream. $1.0 \mathrm{~km}$ S of Wooster. 6 ¿. 5 ‥ L. Hubricht. 3 May 1940 (USNM): Jefferson Co.: slough. $6.9 \mathrm{~km}$ NE of Altheimer. $1 \equiv .1 \because$ L. Hubricht. 7 Apr 1941 (USNM): Johnson Co.: oxbows of small stream behind the college chapel. Clarksville. $4 \subseteq .4 c^{\circ}$. L. Hubricht. 3 May 1940 (USNM); small creek W of the college chapel. Clarksville. holotype $\ddot{x}$. L. Hubricht. $28 \mathrm{Apr} 1936$ (USNM): Monroe Co.: stream $7.2 \mathrm{~km} \mathrm{SW}$ of Clarendon. 1 I. 7 : L. Hubricht. 7 Apr 1941 (USNM); slough. $1.7 \mathrm{~km} \mathrm{~N}$ of Holly Grove. 39.6 . L. Hubricht. 7 Apr 1941 (USNM): Perry Co.: cypress swamp $3.2 \mathrm{~km} \mathrm{~N}$ of Perry. 26 specs (₹. ₹). L. Hubricht. 5 May 1940 (USNM): Phillips Co.: temporary pool $8.8 \mathrm{~km} \mathrm{~N}$ of Barton. 1 ․ 1 ₹. L. Hubricht. 6 Apr 1940 (USNM): Yell Co.: temporary pool $0.6 \mathrm{~km}$ W of Danville. $1 \equiv .3$ juvs. L. Hubricht. 4 May 1940 (USNM). ILLINOIS. Johnson Co.: Heron Pond I.6 km NW of Foreman. 6 ?. L. M. Page and B. M. Burr. 30 Mar 1973. LOUISIANA. Beauregard Par.: $4.8 \mathrm{~km} \mathrm{~N}$ of Newlin (habitat not given). 2 ₹. G. H. Penn. 7 Feb 1941 (USNM): Lafourche Par.: Bayou Boeuf. Kraemer. 7 . 18 Apr 1936 (USNM): Pointe Coupee Par.: roadside pool $3.2 \mathrm{~km}$ E of Lottie, 8 9. 1 c". L. Hubricht. $12 \mathrm{Apr} 1964$ (USNM): Lincoln Par.: slough 1.4 $\mathrm{km}$ S of Dubach. 2 \%. L. Hubricht. 8 Apr 1941 (USNM): Morehouse Par.: ditch on E side of US 165. ca. $7.2 \mathrm{~km} \mathrm{~S}$ of Bastrop. 1 (USNM) and 6 \&. J. E. and M. R. Cooper. 15 Mar 1967: Ouachita Par.: small ditch. 3.2-4.8 km NW of Monroe. 1 ₹. J. E. and M. R. Cooper. 25 Dec 1965. MISSISSIPPI. Forrest Co.: small stream in swamp near Black Creek. $3.2 \mathrm{~km} \mathrm{SW}$ of Brooklyn. 1 @. L. Hubricht. 2 Mar 1963 (USNM): Harrison Co.: Pass Christian (habitat not given), $1 \approx$. J. and W. Rapp. 17 Feb 1946 (USNM): Lauderdale Co.: pool $4.8 \mathrm{~km}$ E of Lost Gap. 1 :. L. Hubricht. 3 Feb 1962 (USNM): roadside pool. US Rt. 80 at 31 St. 


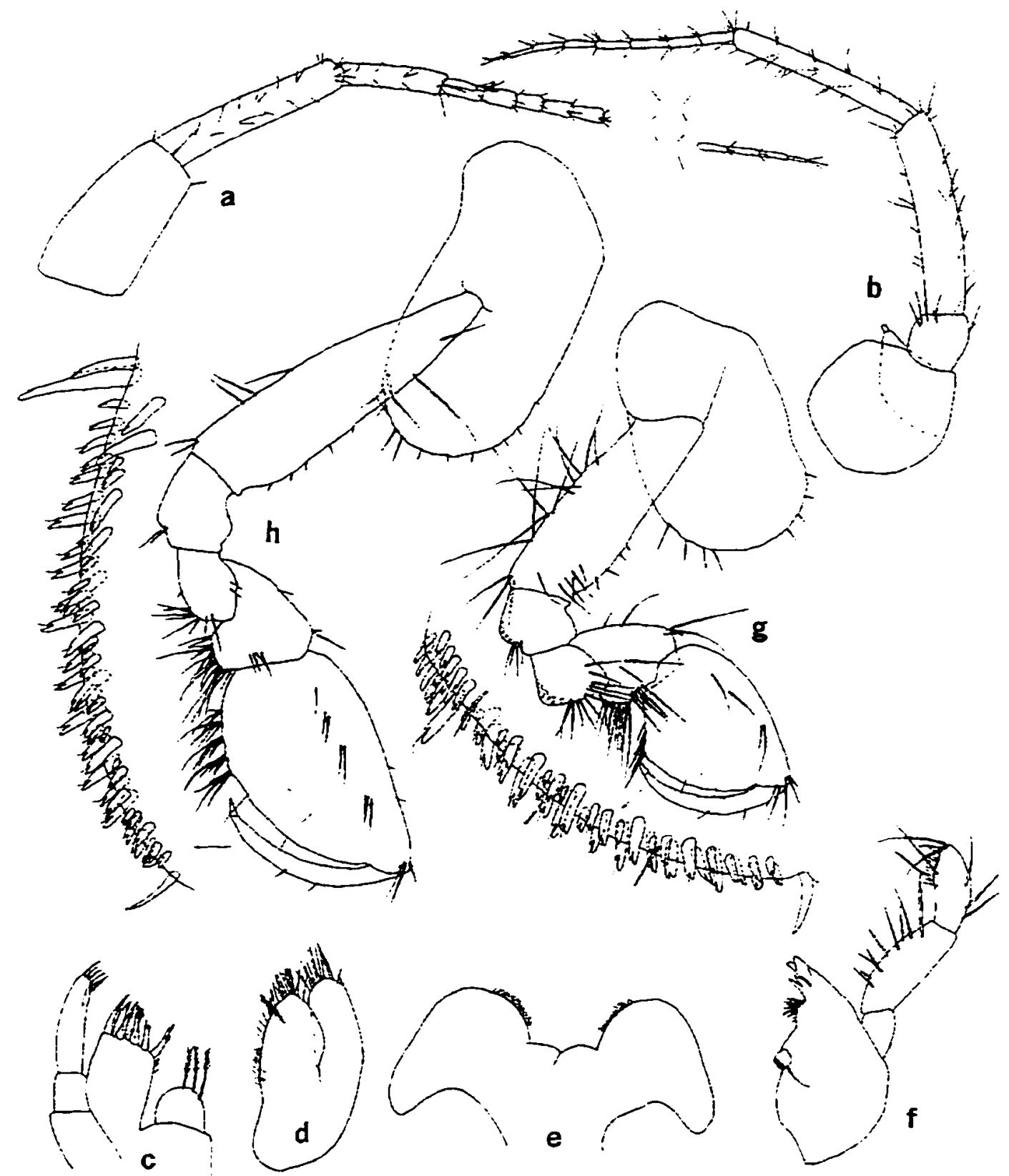

Figure 43.--Crangonyx obliquus (Hubricht and Mackin). Female topotype (12.4 mm). oxbows of small stream behind the college chapel, Clarksville. Johnson Co.. Arkansas: a, b. antennae 1. 2: c. d. maxillae 1. 2: e. lower lip: f. right mandible: g. h. gnathopods !, 2 (palmar margins enlarged). 

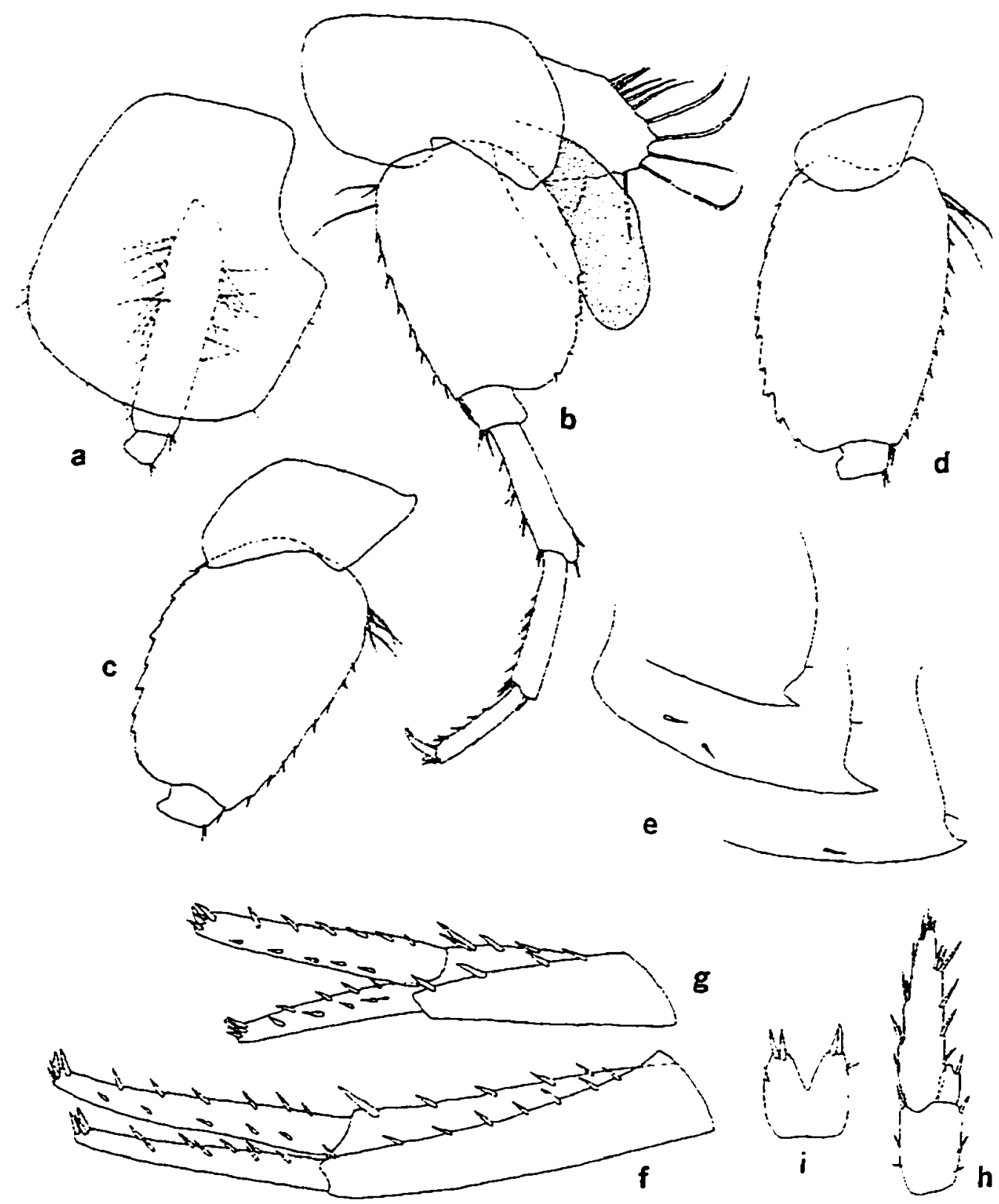

Figure 44.--Crangonyx obliquus (Hubricht and Mackin). Female topotype (12.4 mm). oxbows of small stream behind the college chapel, Clarksville. Johnson Co.. Arkansas: a. d. c. pereopods 4.6.7 (in part): b. pereopod 5: e. pleonal plates: f. g. h. uropods 1. 2. 3: i. telson. 


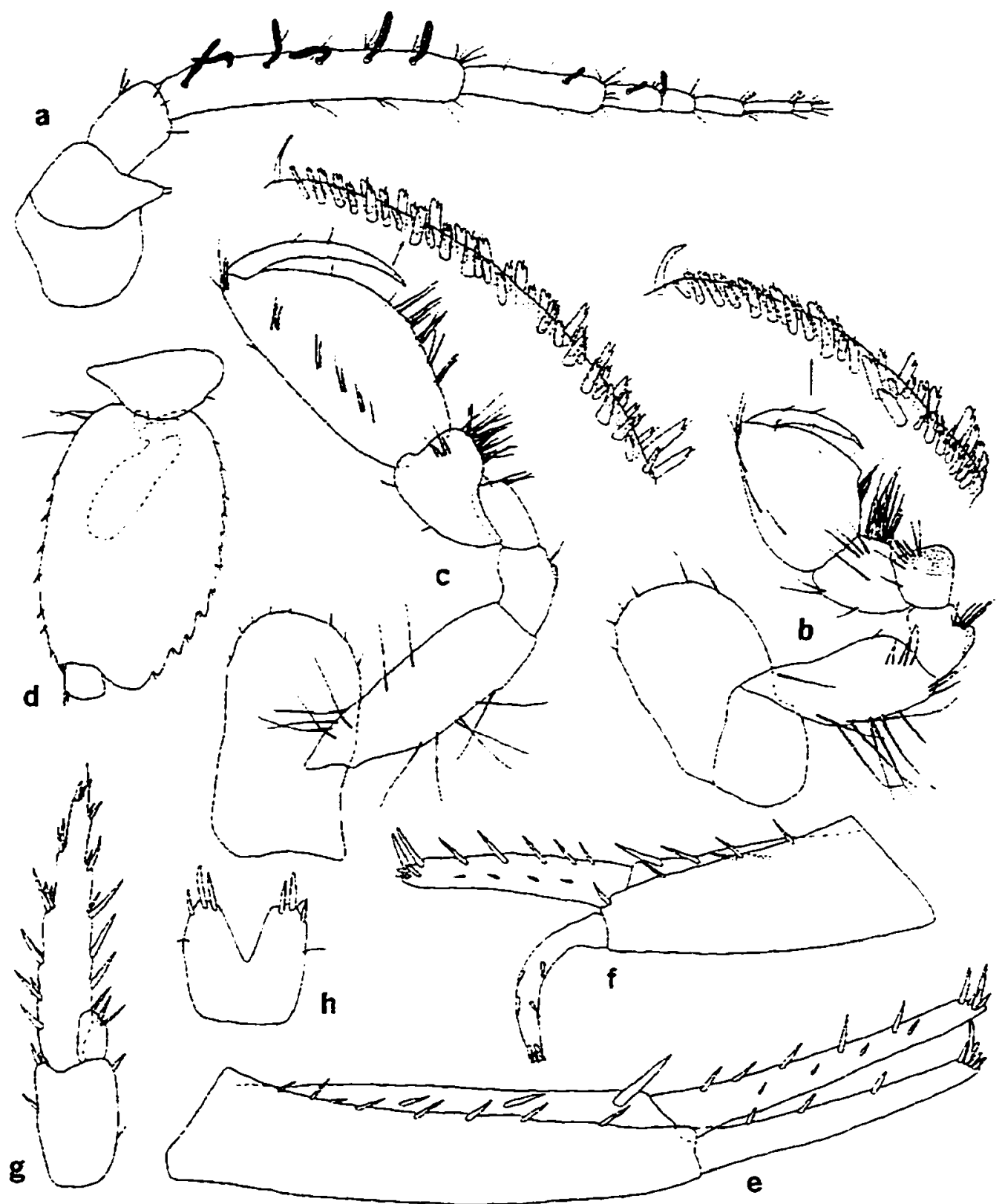

Figure 45.--Crangonyx obliquus (Hubricht and Mackin). Male topotype $(10.1 \mathrm{~mm})$. oxbows of small stream behind the college chapel. Clarksville. Johnson Co.. Arkansas: a. antenna 2: b. c. gnathopods I. 2 (palmar margins enlarged): d. pereopod 7 (in part): e. f. g. uropods I. $2.3:$ h. telson. 
Ave. Meridian. $12 \%, 5 \sigma^{\circ}$. L. Hubricht. 8 Mar 1964 (USNM); Monroe Co.: roadside ditch. 3 q. J. E. And M. R. Cooper. 20 Mar 1968: Quitman Co.: ditch on St. Hwy 6.4.8 km W of Marks. 9 q. 25 juvs. J. E. and M. R. Cooper. 17 mar 1967: Rankin Co.: swamp. Pelahatchee. 1 \%. P. Hartfield. 16 Mar 1987 (USNM): slough. $1.3 \mathrm{~km} \mathrm{~W}$ of Pelahatchee. 5 \%. $1 \sigma^{\circ}$. L. Hubricht. 9 Apr 1941 (USNM). MISSOURI. Butler Co.: slough. $9.8 \mathrm{~km} \mathrm{~N}$ of Neelyville. $5 q .3 \approx$. L. Hubricht. 5 Apr 1941 (USNM), 30 specs ( $q, \approx$. juvs), L. Hubricht. 8 Jun 1940 (USNM); temporary pool, roadside ditch. $4.3 \mathrm{~km} \mathrm{~W}$ of Ash Hill. 15 juvs. L. Hubricht. 21 Apr 1938 (USNM). TENNESSEE. Lake/Obion Co.: Reelfoot Lake. 1 s. V. E. Shelford. 9 Apr 1943 (USNM), I \&. J. and W. Rapp, 12 Apr 1946 (USNM). TEXAS. Liberty Co.: Dayton, $3 \varsubsetneqq$. H. H. Hobbs. Jr. (habitat and collection date not given) (USNM).

Diagnosis.-Morphologically closely similar to $C$. richmondensis but distinguished by having 2-3 setae on outer margins of dactyl of gnathopods in both males and females. Largest male. $10.0 \mathrm{~mm}$ : largest female. $20.0 \mathrm{~mm}$.

Female.--Eye large and ovate. Antenna 1, about 50 percent length of body, about 1.5 times longer than antenna 2: primary flagellum with 28 segments. Antenna 2. flagellum with 7-8 segments. Mandibles subequal. spine row with 5-6 spines; segment 2 of palp with 8-9 long setae. segment 3 with about 2 A setae. $1 \mathrm{~B}$ seta. $2 \mathrm{C}$ setae. $3 \mathrm{E}$ setae and a row of $\mathrm{D}$ setae. Maxilla 1: inner plate with 3 apical plumose setae: palp with 5-6 slender spines on apex. Maxilla 2. inner plate with I plumose seta on inner margin. Maxilliped: inner plate apically with 3-5 bladelike spines, 1 plumose spines, 2 naked spines. and 4-5 plumose setae extending from inner margin to apex: outer plate with row of naked setae and $3-4$ weak pectinate spines on inner margin: dactyl inner margin with 3-4 setae, nail long. about $1: 4$ length of dacty!.

Propod of gnathopod I about 2 times larger and longer than carpus: paim convex. oblique, much longer than posterior margin, inside and outside each with 2 rows of notched spine teeth, about 19 on inside. 23 on the outside: defining angle with 5 notched spine teeth on inside. two rows of spine teeth on outside: inner row with 5 small spines, outer row with I very large and 5 small spines: superior medial setae in 3 sets, singly or doubly inserted; posterior margin with $3-4$ sets of setae singly inserted or in groups of 2-4 on proximal half. Dactyl with row of short setae on inner margin. outer margin with 2-3 setae. nail very short. Ventral margin of coxa I with 7-8 setae.

Propod of gnathopod 2 more than 2 times larger and longer than carpus and much broader: palm convex, very oblique, much longer than posterior margin, inside and outside each with 2 rows of rotched spine teeth, about 22 on inside. about 21 on outside; defining angle with 5 spine teeth on inside. 10 on outside: about 4 sets of superior medial setae in groups of 1-3; posterior margin with 5 sets of setae in groups of 2-4. Dactyl with row of short setae on inner margin. outer margin with $2-3$ setae, nail very small and short. Ventral margin of coxa 2 with 10 seiae.

Coxa of pereopod 3 with 12 marginal setae. Coxa of pereopod 4 with 17 marginal setae. Pereopod 5: basis with 13 shallow serrations along posterior margin: 9 spines on anterior margin: 3 long setae on the anteroproximal margin. Pereopod 6: basis with 14 serrations along posterior margin: 8 short spines on 
anterior margin: 5 long setae on anteroproximal margin. Pereopod 7: coxa with 3 setae on the posterior margin: basis with 13 serrations along posterior margin: 10 short spines on anterior margin: 4 long setae on anteroproximal margin; dactyl about 25 percent the length of propod.

Pleonal plates: posterior margin of plate I convex. with I seta, distoposterior comer produced and acute: posterior margin of plate 2 nearly straight with 1 seta. distoposterior corner distinctly produced and strongly acute. ventral margin with 2-3 (subventral) spines: posterior margin of plate 3 nearly straight with l seta. distoposterior comer produced and acute, ventral margin with 1 (subventral) spines. Peduncle of pleopod 1 with about 4 retinaculae: many setae on outside margin.

Uropod I: inner ramus 75 percent length of peduncle. armed with 16 spines: outer ramus with 14 spines: peduncle with row of 5 outer and 8 inner spines. Uropod 2: inner ramus armed with 16 spines: outer ramus with 13 spines: peduncle with 4 outer and 5 inner spines. Uropod 3 : inner ramus with 1 seta. outer ramus about 2.2 times longer than peduncle, inner and outer margin each with 4-6 sets of spines in groups of 1-3: peduncle with several spines on lateral border and apex. Telson about as broad as long. notched about $1 / 2$ distance to base, each lobe with 2 apical spines up to $1 / 3$ length of telson.

Male.--Differing from female as follows: smaller with more slender, elongate body. Antenna 2: calceoli percent on peduncular segments 4 and 5 , and first 3 segments of flagellum.

Uropod 1: inner ramus about 60 percent of peduncle. lacking inner spines but with 3 long outer spines. apex with 5 spines: outer ramus with 13 spines: peduncle with 8 outer and 4 inner spines. Uropod 2: inner ramus with 13 spines: outer ramus strongly curved laterally, with 2 short inner and 2 short outer spines. apex with 5 spines: peduncle with 4 outer and $3-4$ inner spines.

Distribution and ecology.--The range (about $850 \mathrm{~km}$ across) of this species. which is largely restricted to the Coastal Plain of south-central United States, is situated primarily within the lower Mississippi River drainage basin (fig. 46). Only a few locality records exist outside the Coastal Plain and occur just to the north of it.

This species is found in a variety of habitats. including roadside ditches. small streams. small creeks. sloughs. cypress swamps. ponds, and pools. Sexually mature female. 13.5-20.0 mm. sexually mature male. $8.0-10.0 \mathrm{~mm}$. It has been found in association with the amphipods $C$. pseudogracilis and Sinurella hifurca.

Remarks.-Nicholas (1960) mistakenly called this species a troglobite and Krekeler and Williams (1966) repeated Nicholas' error by listing it as a true cave animal from Indiana. They did not give any collection records for it. however. McDaniel and Smith (1976) also mistakenly listed this species as a troglophile in the cave fauna of Arkansas, another mistake undoubtedly influenced by the original error of Nicholas (1960). 
Crangonyx disjunctus, new species

Figures 47-50

Material examined (paratypes unless designated otherwise).--NORTH CAROLINA. Gates Co.: Hamburg ditch and marsh. Dismal Swamp. 9 9. 6 c". S. Hetrick. 2 Mar 1974: Hartford Co.: Winton. 2 . (collector and habitat not given), 23 Mar 1940 (USNM). VIRGINIA. Chesapeake (Norfolk County): roadside ditch. $1.6 \mathrm{~km}$ S of Wallaceton, 2 \%. J. R. Holsinger and C. H. Holsinger, $18 \mathrm{Apr}$ 1971: sphagnum bog. VA Chain ferry, North Ditch. Dismal Swamp. 4 \%. R. Rose. 15 Nov 1985: Elizabeth City Co.: small stream. $6.4 \mathrm{~km} \mathrm{NW}$ of Hampton. 7 \%, $80^{\circ}$. L. Hubricht. 23 Jan 1944 (USNM); Hampton (Elizabeth City County): shallow poor near forested wetland on NASA Langley Research Center. 1 ๑. R. T. Tuner et al.. 14 Mar 1994: small pool. forested wetland on western side of NASA Langley Research Center. Hampton. $1 \approx z$ R. L. Bedenbaugh. 17 mar 1994: Henrico Co.: Brook Hill N of Richmond. $5 \Xi 20 こ$. A. Pizzini. 30 Apr 1939 (USNM): Highland Co.: spring on Sapling Ridge. 2 \&. C. Pague. May 1990 (not paratype): Isle of Wight Co.: outlet of drain tile on Taylor Farm, $5.6 \mathrm{~km} \mathrm{~N}$ of Chuckatuck. $2 \sqsubseteq$. J. R. Holsinger. $24 \mathrm{Apr}$ 1983: small stream, $1.6 \mathrm{~km} \mathrm{~N}$ of Bartlett. 5 Z. L. Hubricht, 2 Apr 1944 (USNM): roadside ditch. $0.5 \mathrm{~km} \mathrm{~N}$ of Carrollton. 129.40 . L. Hubricht. 30 Jan 1944 (USNM): small stream. $1.6 \mathrm{~km}$ S of Rescue. holotype $\cong$. $74 \operatorname{specs}(\because$.$) ). L. Hubricht, 12$ Mar 1944 (USNM); vernal pond in wetland. ca. $6.4 \mathrm{~km} \mathrm{NW}$ of Windsor. I ¡. H. Jones. 12 Apr 1994: Nansemond Co.: small spring and stream $3.2 \mathrm{~km}$ ESE of Chuckatuck. 1 \&. J. R. Holsinger and D. Culver. 5 Feb 1969: Washington ditch. Dismal Swamp. $3.2 \mathrm{~km}$ ENE of Saunders. 46 specs $(2.3)$. L. Hubricht. 21 Nov 1943 (USNM): pool in Disaml Swamp near Washington Ditch. 2 . K. Garrert and B. H. Poweil. 28 Apr 1971: Dismal Sivamp. just $N$ of Washington Ditch. $5 \Xi$. J. R. Holsinger. 8 May 1971: pool 70 meters from Lynn Ditch. Dismal Swamp. $1 \fallingdotseq .5 己$. K. Garrett. 30 Jan 1972: temporary pool in woods $1.6 \mathrm{~km}$ E of Driver. $39.4 c^{\circ} 1$ juv. L. Hubricht. 26 Mar 1944 (USNM): stream

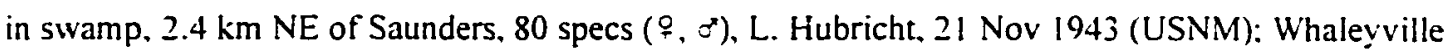
(habitat not given). $5 \bar{q} 1 \mathrm{c}$. R. Bray, 22 Mar 1940 (USNM); small stream in swamp. $2.4 \mathrm{~km}$ W of Baker Store. 20 ๆ. 1 \%. L. Hubricht, 12 Nov 1944 (USNM); New Kent Co.: roadside pond. $3 \mathrm{mi}$ E of Providence Ford. $5 \cong .1$ juv. J. R. Holsinger, 13 Apr 1972: Norfolk Co.: roadside ditch. Bowers Hill. $9 \subsetneq .2 \approx 2 . \mathrm{L}$. Hubricht. 25 Feb 1945 (USNM): small stream. $0.8 \mathrm{~km}$ W of Churchland. $8 \nsubseteq .13 \approx$. L. Hubricht. 30 Jan 1944 (USNM): stream in swamp. $3.2 \mathrm{~km} \mathrm{~S}$ of North Landing, $79 .+3.9$ juvs. L. Hubricht. 9 Apr 1944 (USNM): stream in swamp. $4.8 \mathrm{~km}$ S of North Landing. 11 \&. $3 \approx$. L. Hubricht. 9 Apr 1944 (USNM): temporary pond. $3.2 \mathrm{~km}$ WSW of Bowers Hill, 8 \%. L. Hubricht. 2 I Nov 1943 (USNM): temporary pool. Dismal Swamp. $3.2 \mathrm{~km}$ SSE of Bowers Hill, 80 specs ( $₹, 0^{\circ}$ ), L. Hubricht. 14 Nov 1943 (USNM): Northampton Co.: small stream, $1.6 \mathrm{~km}$ S of Capeville, 15 ₹. L. Hubricht. 16 Apr 1944 (USNM); Princess Anne Co.: temporary pool in swamp $1.6 \mathrm{~km}$ SE of Thalia. L. Hubricht. 45 specimens. 25 Nov 1943 (USNM): Southampton Co.: Charles Plantation (habitat not given), Franklin. $7 \subseteq .1 \approx$. R. Bray. 22 Mar 


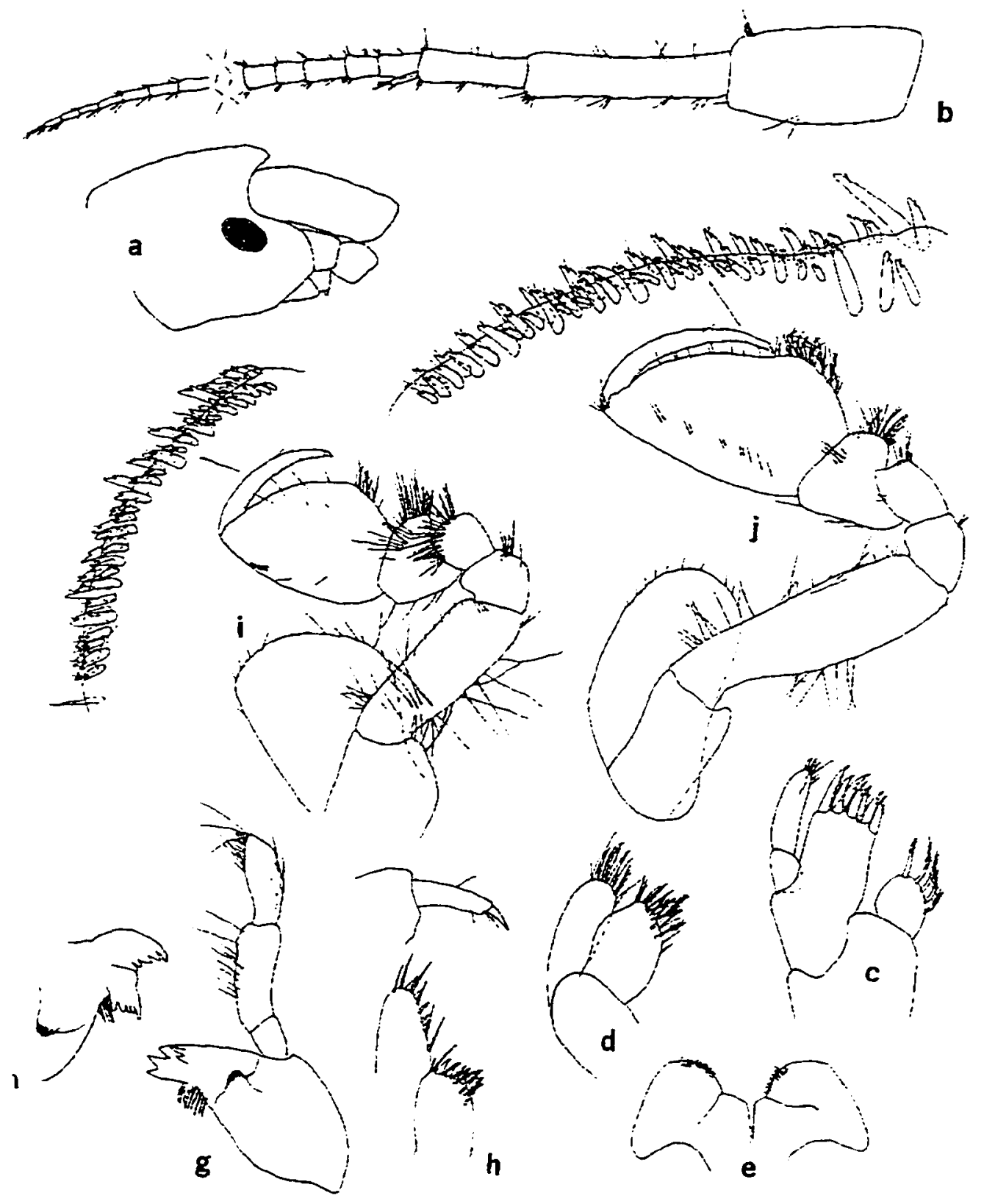

Figure 47.--Crungonvx disjunctus, new species. Female paratype $(13.3 \mathrm{~mm})$, small stream. $1.6 \mathrm{~km} \mathrm{~S}$ of Rescue. Isle of Wight Co., Virginia: a, head (in part); b. antenna I: c. d. maxillae 1. 2: e. lower lip: f. dentate part of left mandible: g. right mandible: $h$, inner and outer plates and palp dactyl of maxilliped (greatly enlarged); i. j. gnathopods 1, 2 (palmar margins enlarged). 


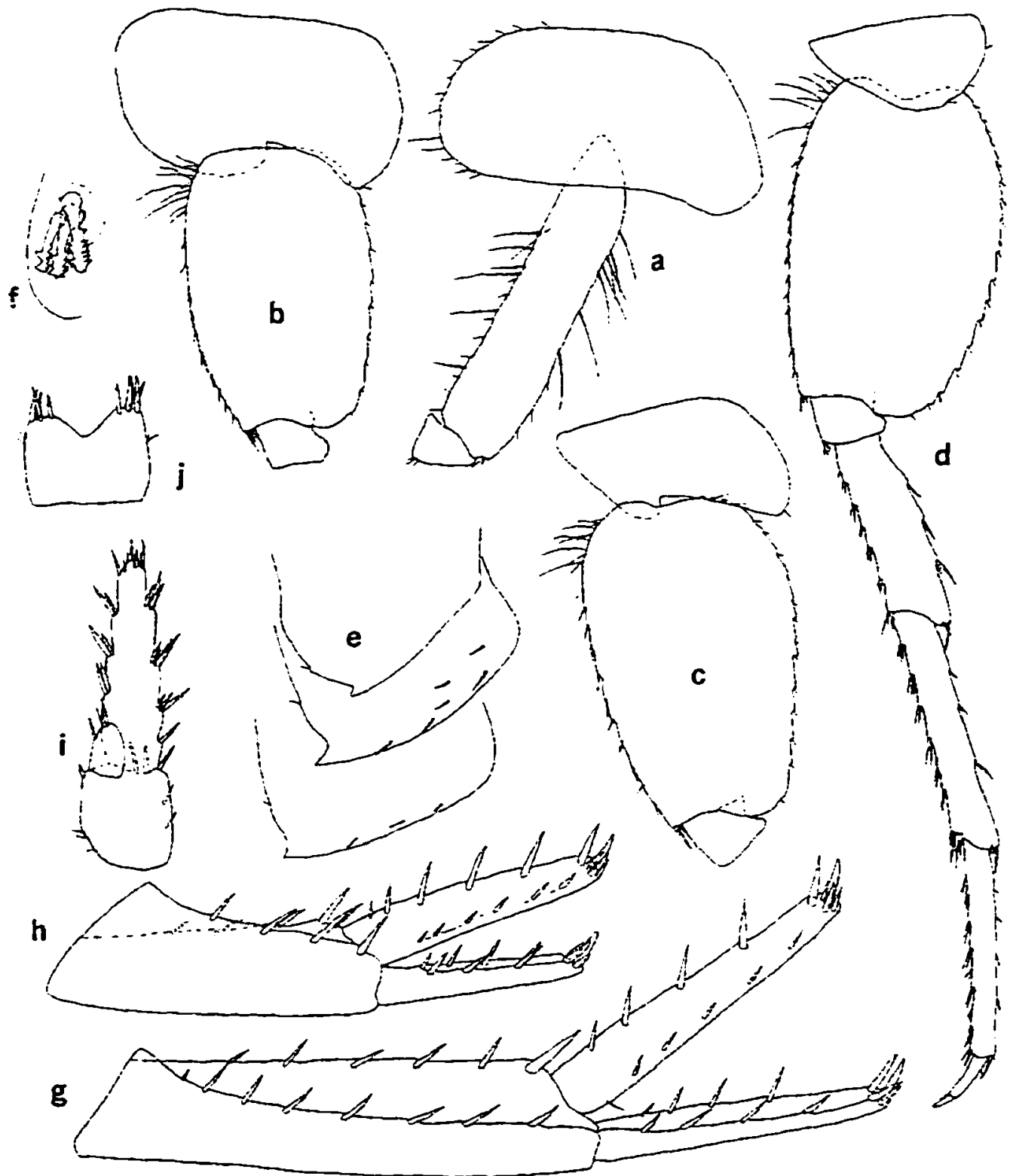

Figure 48.--Crangon!x disjunctus. new species. Female paralyze $(13.3 \mathrm{~mm})$. small stream. $1.6 \mathrm{~km} \mathrm{~S}$ of Rescue. Isle of Wight Co.. Virginia: a. b. c. pereopods 3. 5.6 (in part); d. pereopod 7: e. pleonal plates: f. retinacula of pleopod (enlarged); g, h. i. uropods I. 2. 3; j, telson. 


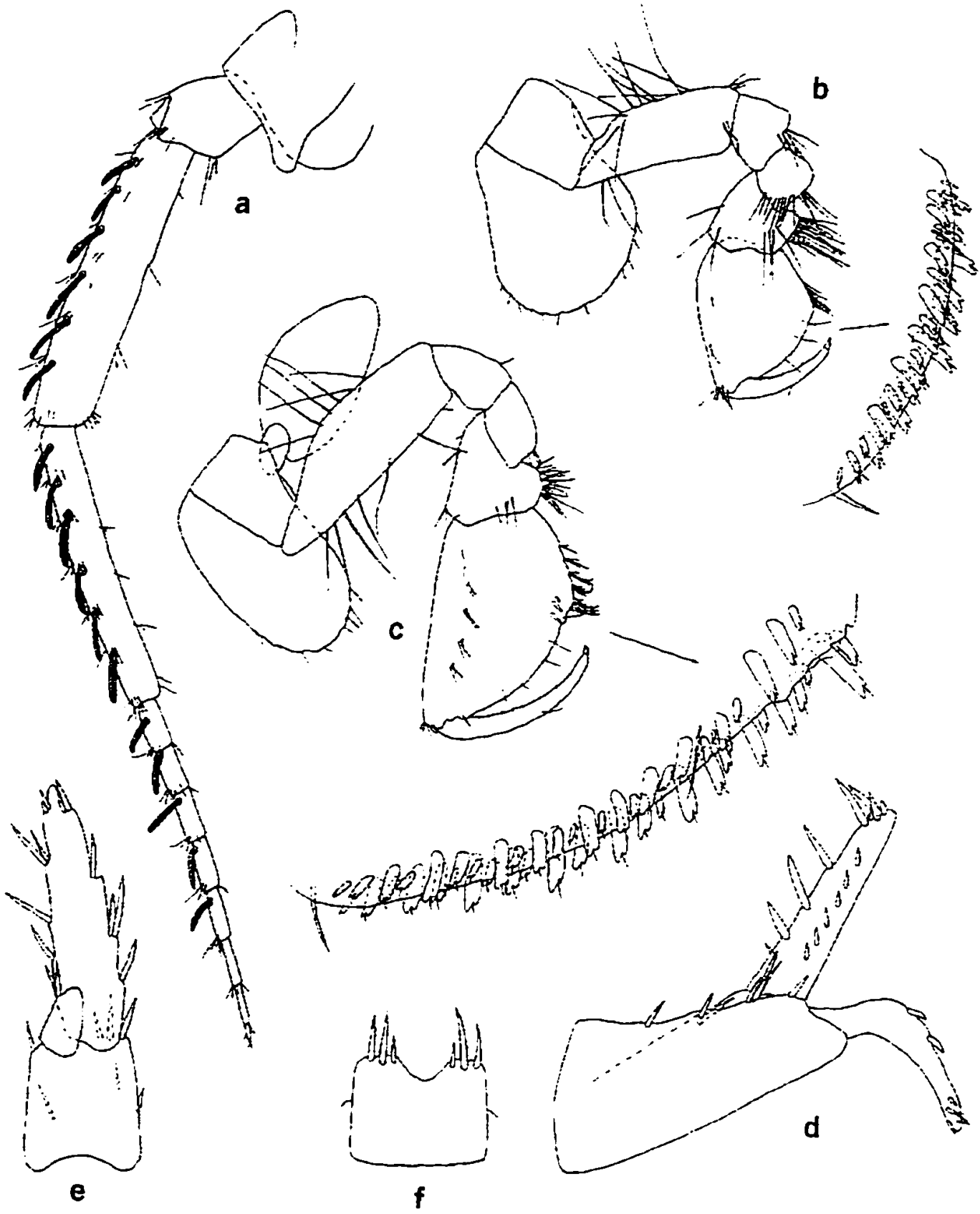

Figure 49.--Crangonyx disjunctus, new species. Male paratype $(9.5 \mathrm{~mm})$, small stream. $1.6 \mathrm{~km} \mathrm{~S}$ of Rescue. Isle of Wight Co.. Virginia: a, antenna 2: b. c. gnathopods 1. 2 (palmar margins enlarged): $d$. e. uropods 2. 3: f, telson. 
1940 (USNM): Virginia Beach (Princess Anne County): Stumpy Lake near golf course. $8.0 \mathrm{~km} \mathrm{~S} \mathrm{of}$ Kempsville. 1 \% 10 juvs. J. R. Holsinger and C. H. Holsinger. 2 Mav 1971: Warwick Co.: swamp. 0.8 km NE of Harpersville. $7 \% .16 \approx$. L. Hubricht. 23 Jan 1944 (USNM): ditch. 2.4 km NE of Morrison. $37 \equiv .22$ $\therefore$ L. Hubricht. $23 \mathrm{Jan}$ I944 (USNM); pool in woods. $4.0 \mathrm{~km} \mathrm{NW}$ of Newport News. $1 \cong .7 \approx$. L. Hubricht. 23 Jan 1944 (USNM); York Co.: small stream $0.5 \mathrm{~km}$ SW of Tabb. 4 . $12 \approx$. L. Hubricht. 23 Jan 1944 (USNM); pond $0.6 \mathrm{mi}$ of Grafton Church. 3 \%. P. H. Stevenson and S. H. Holbrook. 13 Feb 1993: pond on Rt. 17. $1.1 \mathrm{~km} \mathrm{~S}$ of Harris Grove. 14 \&. 11 ठ.J. R. Holsinger et al.. 9 Mar 1994.

Diagnosis.--A large species of the richmondensis group distinguished from other members of the group by inner plate of maxilla I with up to 5 apical plumose setae: inner plate of maxilla 2 with $3-4$ plumose setae on inner margin near apex: uropod 2 of male with only 2-4 thick bladelike spines on outside. Largest male. $11.5 \mathrm{~mm}$ : largest female. $19.5 \mathrm{~mm}$.

Female.--Eye small and round. Antenna 1.55 percent length of body, about 1.8 times longer than antenna 2: primary flagellum with 27 segments. Antenna 2. flagellum with 8 segments. Mandibles subequal. spine row with 6 spines: segment 2 of palp with 10-11 long setae. segment 3 with $3-4$ A setae. 1 $B$ seta. $3 \mathrm{C}$ setae. $34 \mathrm{E}$ setae and a row of D setae. Maxilla 1 : inner plate with 5 apical plumose setae: palp with 5-6 slender spines on apex. Maxilla 2, inner plate with 3-4 plumose setae on inner margin near apex. Maxilliped: inner plate apically with 4 bladelike spines, I naked spine. and 5-6 plumose setae extending from inner margin to apex: outer plate with row of naked spines and $4-5$ slender pectinate spines on inner margin: dactyl with 2-3 setae on inner margin near nail: dactyl nail short. less than 14 length of dactyl.

Propod of gnathopod 1 more than 2 times larger and longer than carpus and much broader: palm very: oblique. convex. longer than posterior margin. inside and outside each with $2-3$ rows of notched spine teeth. 21 on inside. 23 on the outside: defining angle with 7 spine teeth on inside. 1 row of 1 large and 5 small spine teeth and 1 row of 7 small spine teeth on outside: posterior margin with $4-5$ sets of setae inserted in groups of $1-5$ on proximal half. Dactyl with row of short setae on inner margin. nail very short. Ventral margin of coxa I with 12 setae.

Propod of gnathopod 2 more than 2 times larger and longer than carpus: palm more oblique. convex. much longer than posterior margin. inside and outside each with 2 rows of spine teeth. about 20 on inside. 18 on outside: defining angle with 8 spine teeth on inside. 15 on outside ( 1 very large): superior medial setae doubly or singly inserted: posterior margin with 6 sets of setae in groups of 2-6. Dacty/ with row of short setae on inner margin. Ventral margin of coxa 2 with II setae.

Coxa of pereopod 3 with 15 marginal setae. Coxa of pereopod 4 with 24 marginal setae. dactyl of pereopod 4. 33 percent length of corresponding propod. Pereopod 5: basis with 12 shallow serrations along posterior margin: 10 short spines on anterior margin: 9-11 long setae on the anteroproximal margin. Pereopod 6: basis with 15 shallow serrations along posterior margin: 10 short spines on anterior margin: 12 short and long setae on anteroproximal margin. Pereopod 7: coxa with 3 setae on the posterior margin: basis with 18 shallow serrations along posterior margin: 14 short spines on anterior margin: 9 short and 
long setae on anteroproximal margin: dactyl about 30 percent the length of corresponding propod.

Pleonal p!ates: posterior margin of plate I slightly convex. with I seta. distoposterior corner acute but not produced: posterior margin of plate 2 nearly straight with 1 seta, distoposterior corner strongly produced and acute, ventral margin with 6 (subventral) spines: posterior margin of plate 3 nearly straight with 1 seta. distoposterior corner produced and acute, ventral margin with 3 (subventral) spines. Peduncle of pleopod 1 with 3 retinaculae. about 5 setae on distal end of outside margin. about 10 setae on proximal half of inside and outside margins.

Uropod 1: inner ramus 80 percent length of peduncle. armed with 14 spines: outer ramus with 12 spines: peduncle with row of 8 outer and 6 inner spines. Uropod 2: inner ramus armed with 16 spines: outer ramus with 11 spines: peduncle with 4 outer and 5 inner spines. Uropod 3 : inner ramus with $1-2$ spines. outer ramus about 2 times longer than peduncle. inner and outer margin each with $4-5$ set of spines in groups 1-5. Telson broader than long, notched about $1 / 4$ distance to base. each lobe with $3-4$ apical spines: spines less than $1 / 2$ length of telson.

Male.--Differing from female as follows. Smaller with more slender. elongate body. Antenna 1: primary flagellum with 15 segments. Antenna 2: flagellum with 8 segments. calceoli percent on peduncular segments 4 and 5 and first 5 segments of flagellum.

Propod of gnathopod 1 palm with 12 notched and 4 simple spine teeth on inside. 13 notched spine teeth on outside: defining angle with 3 spine teeth on inside and I large and 6 small spine teeth on outside.

Propod of gnathopod 2 palm margin strongly oblique, with 19 spine teeth on inside. 16 on outside: defining angle with 7 spine teeth on inside and 10 on outside.

Pereopod 7: basis with 16 serrations along posterior margin: 5-6 short spines on anterior margin: 2 long setae on anteroproximal margin: dactyl about 33 percent length of corresponding propod.

Uropod I: inner ramus with 12 spines: outer ramus with 11 spines: peduncle with 7 outer and $f$ inner spines. Uropod 2: inner ramus with 15 spines; outer ramus strongly curved laterally. with 2-4 outer bladelike spines. no inner spines, apex with 5 spines: peduncle with 4 outer and 3 inner spines. Uropod 3 : inner ramus with 1 spine: outer ramus about 1.8 times length of peduncle. inner and outer margins each with 3-4 sets of spines in groups of 1-4. Telson broader than long. notched about 1:5 distance to base: each lobe with 3 apical spines: spines about $1 / 2$ length of telson.

Type-locality.--Small stream, about $1.6 \mathrm{~km} \mathrm{~S}$ of Rescue. Isle of Wight County. VA.

Distribution and ecology.--The range of this species is restricted to the Coastal Plain of southeastern Virginia and northeastern Norti Carolina. with exception of a single highly disjunct locality in extreme western Virginia. approximately $200 \mathrm{~km}$ west of the eastern localities (fig. 50 ). To date this species has not been found in the intervening Blue Ridge Mountains or Piedmont.

On the Coastal Plain. $C$. disjunctus was found in ditches, marshes, streams, swamps. pools, and ponds. Sexually mature females are $10-19.5 \mathrm{~mm}$, and sexually mature male are $9.0-11.0 \mathrm{~mm}$. Ovigerous females 
were collected during January, March. April and May. This species has been found together with other amphipods, including $C$. serratus. $C$. polustris and $C$. orientalis. It co-occurs with $C$. polustris and $C$. orientalis in Grafton Ponds (in York Co., Virginia).

Etymology.--The epithet disjunctus is from Latin meaning "disunited." so named because of the distinctly disjunct population in extreme western Virginia.

Crangomx dearolfi Shoemaker

Figures $51-54$

Crangontx dearolfi Shoemaker. 1942:20-24. figs. 7-8 [type-locality: Hobo Cave. Wernersville. Berks Co.. Pennsylvania].--Nicholas. 1960:127.--Holsinger, 1969:25: 1972:34. fig. 13. 1977:252: 1986:97.--

Franz \& Slifer. 1971:26.--Barnard \& Barnard. 1983:434, map 11.--Fitzpatrick. 1983:145.

Material examined.--MARYL.AND. Washington Co.: Cave in field at Charles Mill. 3 juvs. R. Franz. 7 Sep 1968: Natural Well near Downsville. $1 \subsetneq 1$ c. R. Franz. II Jun 1969: Hogmaw Cave (Rohrersville caves). 1 こ. R. Franz. 2! Jan 1967 and 2 9. 9 Dec 1967. PENNSYLVANIA. Berks Co.: Hobo Cave. holotype $\sigma$ (USNM 78266), 4 ․ $1 \sigma^{*}$ K. Dearolf, 28 Jul 1938 (USNM): Cumberland Co.: Carnegie Cave. $1 \Xi$. B. P. Smeltzer. collection date unknown (USNM): Dauphin Co.: Brownstone Cave. $1 \approx *$ K. Dearolf. 3 Sep 1937 (USNM): Franklin Co.: Python Pit Cave. 1 ₹. B. Barton. 25 Jun 1993: York Co.: York Cave (or North York Cave), 1 §. P. J. Spangler. 1948 (USNM): "cave in Pennsylvania" (county not given). $1 \doteq . R$. E. Hotfmaster. 1949 (USNM).

Diagnosis.--A large troglobitic species of the richmondensis group easily distinguished from all other species of Crangonyx by having tri-tid or quadri-fid spine teeth on paims of gnathopod propods: longer antenna 1, and more spinose appendages. Largest male, $15.4 \mathrm{~mm}$ : largest female, $22.0 \mathrm{~mm}$.

Female.--Eye consisting of only few degenerate elements or completely absent. Antenna 1. about 50 percent length of body, about I.4 times longer than antenna 2: primary flagellum up to 37 segments. Antenna 2, flagellum with 15 segments. calceoli may be present on first several segments of flagellum: peduncular segments 3.4 and 5 with many sets of marginal setae (3-10 setae per set). Mandibles subequal. spine row with 8-9 spines: segment 2 of palp with 12-13 long setae. segment 3 with + A setae. 6 B setae. 2 C setae. 5-6 E setae and a row of D setae. Maxilla 1: inner plate with 3-4 apical plumose setae: palp with 57 slender spines and 5-6 setae on apex. Maxilla 2. inner plate with oblique row of 2-3 plumose setae on inner margin. Maxilliped: inner plate apically with 5-6 bladelike spines, 4 naked setae, and 5-6 plumose setae on inner margin: outer plate with row of naked setae and naked slender bladelike spines on inner margin: dactyl inner margin with 5-6 setae, outer margin with 4-5 setae: dactyl nail short. about 1:5 length of dactyl. 


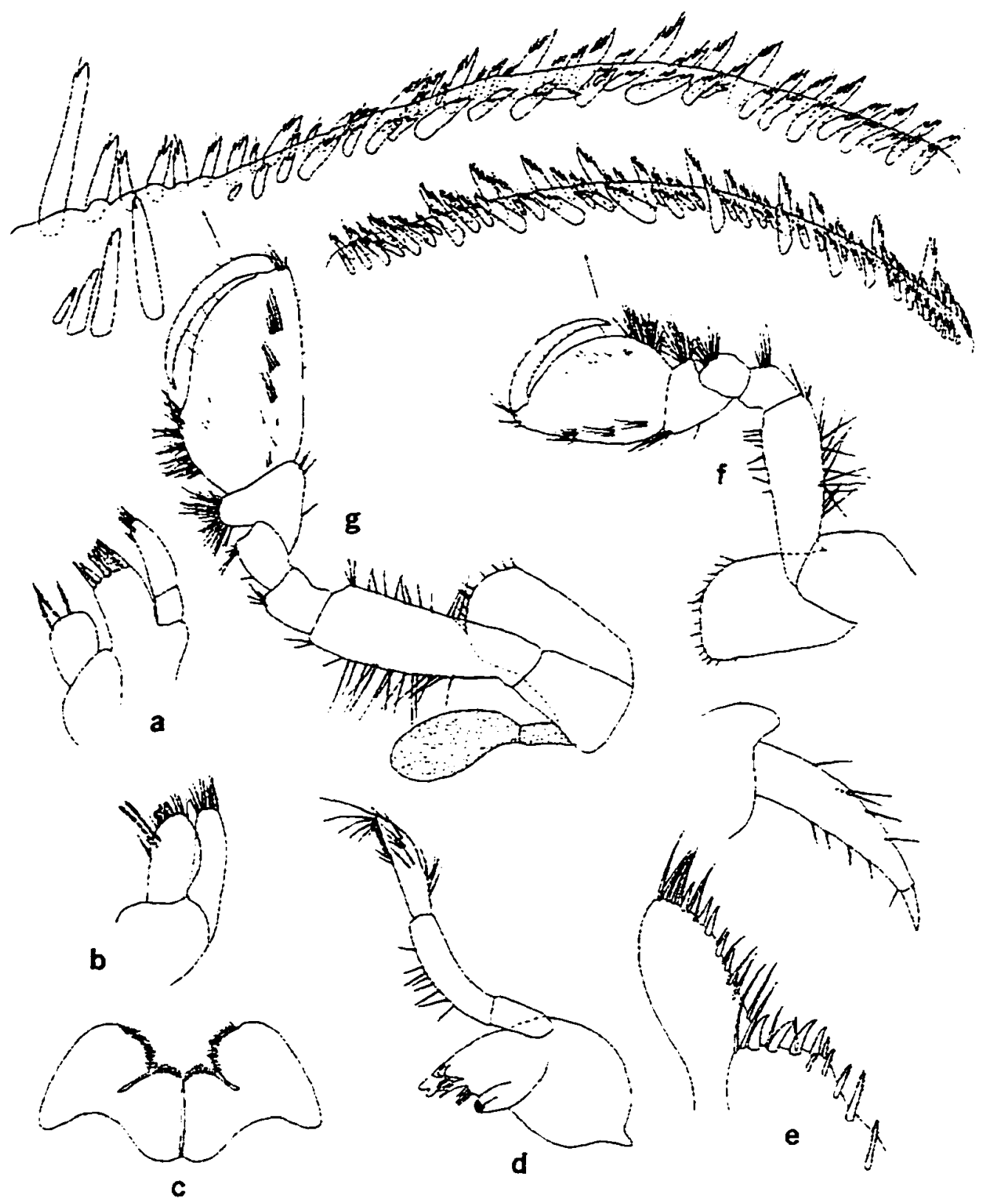

Figure $51 .--$ Crangonyx dearolfi Shoemaker. Female $(19.0 \mathrm{~mm})$, natural well near Downsville. Washington Co., Maryland: a. b. maxillae 1. 2: c. lower lip: d. right mandible: e. inner and outer plates and palp dactyl of maxilliped (greatly enlarged); f. g. gnathopods 1. 2 (palmar margins enlarged). 


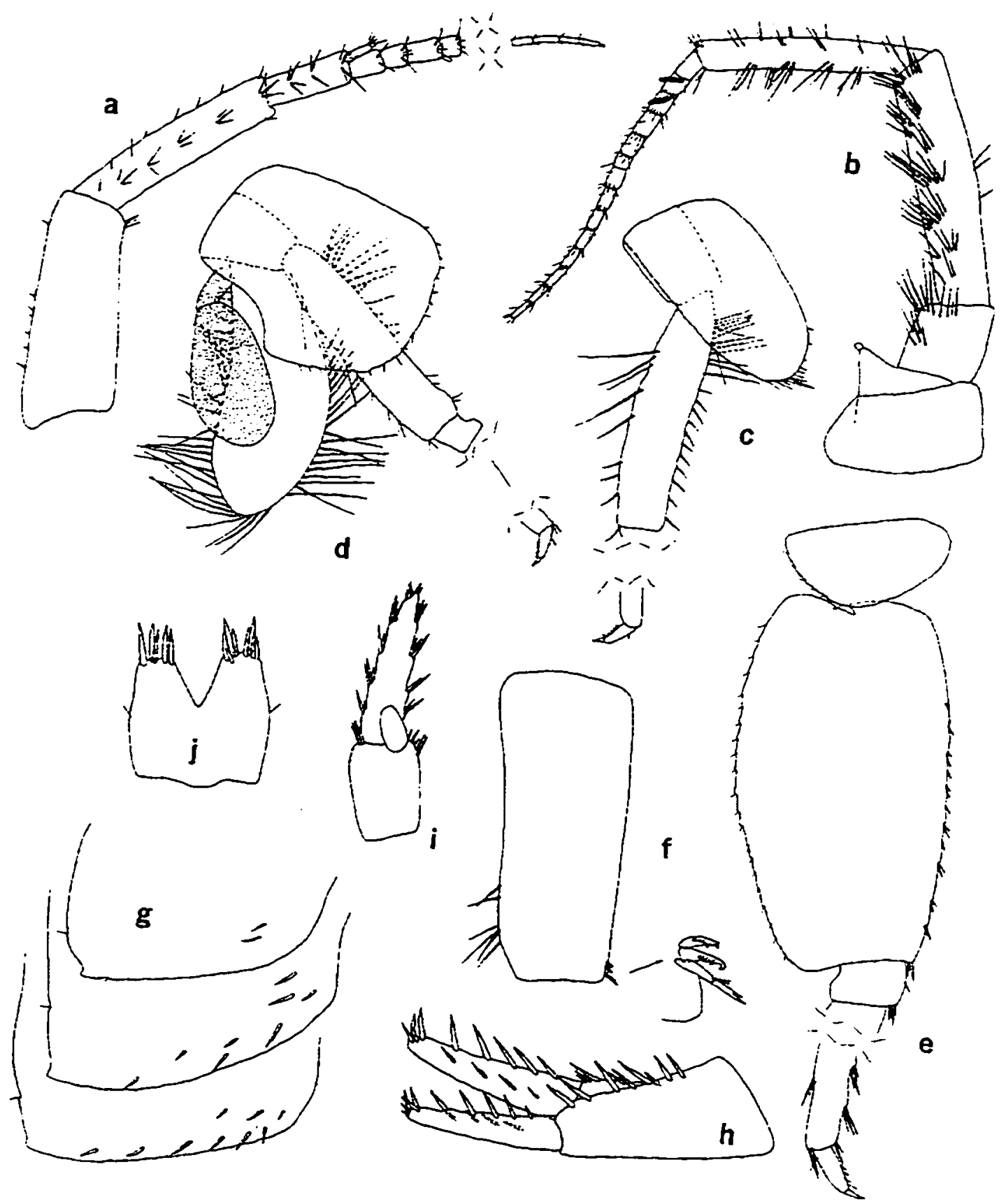

Figure 52.--Crangonvx dearolfi Shoemaker. Female $(19.0 \mathrm{~mm})$. natural well near Downsville. Washington Co.. Maryland: a. b. antennae 1. 2: c. d. e. pereopods 3. 4. 7 (in part): f. peduncle of pleopod I (retinacula enlarged): g, pleonal plates: h. i. uropods 2. 3: j. telson. 


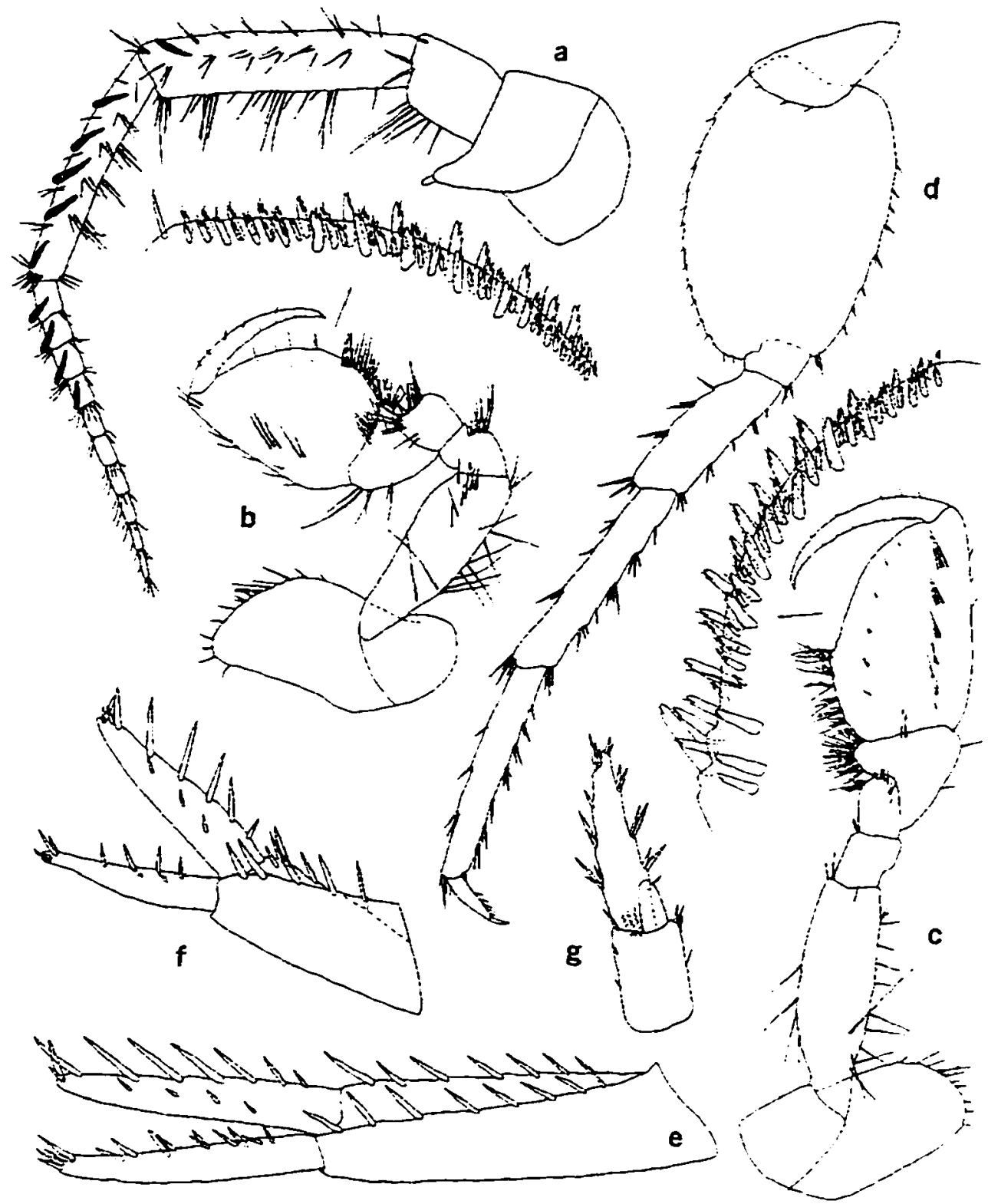

Figure 53.--Crangonyx dearolfi Shoemaker. Male (14.0 mm), natural well near Downsville. Washington Co.. Maryland: a, antenna 2: b, c. gnathopods I. 2 (palmar margins enlarged): d. pereopod 7: e. f, g. uropods 1. 2.3. 


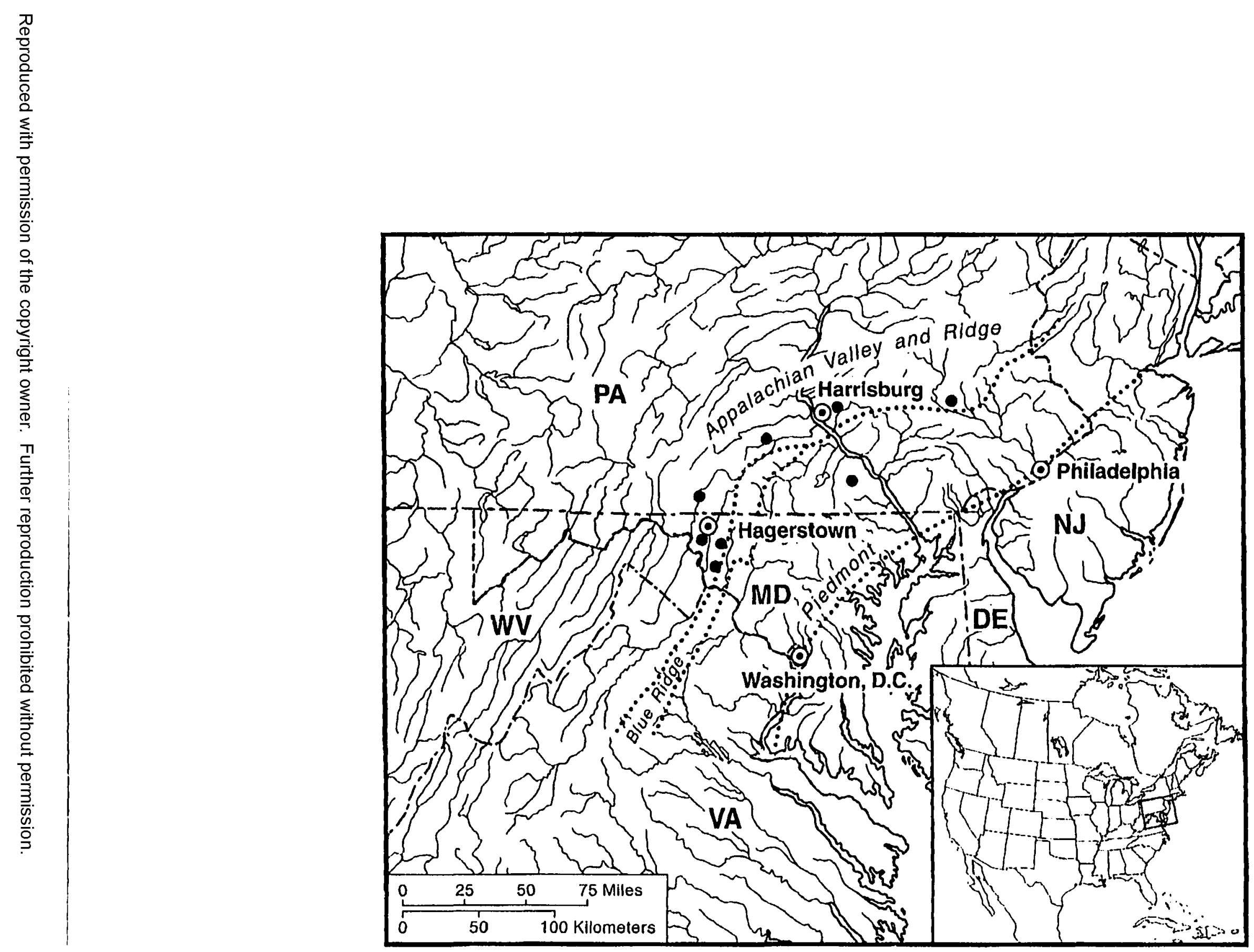

Figure 54.--1)istribution of ( 'rungen!y de'urolfi in North Americia. A solid circle represents 1-3 closely proximate locialities. 
Propod of gnathopod $I$ much broader than. and more than 2 times larger and longer than carpus: palm almost 2 times longer than the length of posterior margin. strongly oblique and convex: inside and outside each with 2 or 3 rows of spine teeth. largest spine teeth in outer row. more than 30 spine teeth on each side. large spine teeth especially near hinge of dactyl tri-fid or quadri-fid: defining angle with 7-9 spine teeth on inside. outside with two rows spine teeth. inner row with 7-10 small. weakly notched-serrate teeth and outer row with I large notched and about 7 serrate spine teeth: superior medial setae in $3-4$ sets in groups of 3-5: inferior lateral setae more than 10 and mostly singly inserted: posterior margin with 5-6 sets of setae in groups of 2-10. Dactyl with a row of very short setae on inner margin: outer margin with up to 8 setae: dactyl nail very short: Ventral margin of coxa I with 16-19 setae.

Propod of gnathopod 2 more than 2 times larger and longer than carpus: palm strongly oblique. convex in the middle and slightly concave at the defining angle. about 2 times longer than posterior margin. inside and outside each with 2 rows of notched spine teeth. more than 30 spine teeth on each side. small spine teeth in inner row. large spine teeth especially near hinge of dactyl tri-fid. quadri-fid. or quinti-tid: defining angle with I large spine tooth on outside. 4 on inside: about 6 sets of superior medial setae in groups of 2-6: posterior margin with 5-6 sets of setae in groups of 5-10. Dactyl with row of very short setae on inner margin. outer margin with up to 9 setae. Ventral margin of coxa 2 with $11-20$ setae.

Coxa of pereopod 3 with 12-13 marginal setae: dactyl with 5 setae on inner margin. Coxa of pereopod 4 with 19-22 marginal setae. dactyl with 5 setae on inner margin. Pereopod 5: basis with 17-21 shallow serrations along posterior margin: 11 sets of spines in groups of $1-3$ on anterior margin: dactyl with 5 setae on inner margin. Pereopod 6: basis with 25 serrations along posterior margin: 11 sets of spines in groups of 1-3 on anterior margin. Pereopod 7: coxa with 6 setae on the posterior margin: basis with 24 very shallow serrations along posterior margin: 12 sets of spines in groups of $1-2$ on anterior margin: dactyl of pereopod 7 about 25 percent the length of corresponding propod. 4 setae on inner margin.

Pleonal plates: posterior margin of plate I slightly convex. with I seta. distoposterior comer very small, subacute but not produced. ventral margin with 1-2 (subventral) spines: posterior margin of plate 2 nearly straight with I or 2 setae. distoposterior corner slightly produced. acute: ventral margin with 8-9 (subventral) spines: posterior margin of plate 3 nearly straight with 1 seta. distoposterior corner rounded. not produced. ventral margin with 7-9 (subventral) spines. Peduncle of pleopod I with 2-3 retinaculae. one of which may be 2-segmented and notched at end of second segment: up to 6 long setae on outside margin distally.

Uropod 1: inner ramus slightly shorter than peduri:le. armed with 14 spines: outer ramus with 14 spines: peduncle with 11 outer and 7 inner spines. Uropod 2: inner and outer ramus each with 14 spines: peduncle with 6-7 outer and 6 inner spines. 3. Uropod 3: inner ramus lacking spines. outer ramus about 1.7 times longer than peduncle. inner and outer margin each with $4-5$ sets of spines in groups 2-4. Telson slightly broader than long, notched about 2/5 distance to base. each lobe with 5-8 apical spines: spine length less than $1 / 2$ length of telson: may with 1-2 dorsal spines. 
Male.--Differing from female as follows. Smaller size at sexual maturity. Antenna 2: flagellum with up to 12 segments, calceoli percent on peduncular segments 4 and 5 , and first 5 segments of flagellum.

Uropod 2: inner ramus with 10 spines; outer ramus weakly deflected. narrowing at apex. with 2 spines on inside. 4 short spines on outside. 4 spines on apex; peduncle with 5 outer and 6 inner spines.

Distribution and ecology.-This rare troglobitic species is recorded from five caves in southeastern Pennsylvania and three in central Maryland (fig. 54). It occurs in pools and small streams. Sexually mature males are 14 to $15.4 \mathrm{~mm}$. sexually mature females are 19.0 to $22.0 \mathrm{~mm}$ and newly hatched young are $3.5 \mathrm{~mm}$. Only a single ovigerous female is recorded from a January collection. It was made from Vatural Well near Downsville in Maryland.

Remarks.--Shoemaker (1942) first described this species from Hobo Cave. Wernersville. Berks County. Pennsylvania. Although he described only a male. the description and figures are quite definitive for the species. Two important points should be mentioned. In Shoemakeri's drawing of the head (fig. 7a). the lateral lobe is not broadly rounded and has a weak inferior antennal sinus. The presence of an inferior antennal sinus in the lateral lobe of the head is atypically for Crangonx. inasmuch as most species in the genus have a broadly rounded lobe without a sinus. Shoemaker also pointed out that the coxal gill of pereopod 7 did not originate from the inner surface of the coxa but from the upper margin of the inside of the basis. Steele and Steele (1991) also reported this condition in other amphipods and believed it to be the normal situation for gammaridean amphipods with the so called "7th pereopod coxal gill". All species of Crangonvx observed in this study have the "7th pereopod coxal gill" attached to the upper margin of the basis and not to the coxa.

Crangomx grandimanus Boustield

Figures $55-59$

Crangomyx grandimanus Bousfield, 1963:6-8, fig. 3 [type-locality: Indian Cave. 7 mi SW of Ocala. Martin Co.. Florida].--Holsinger, 1972:41. figs. 12h. 16: 1977:252,--Barnard \& Barnard. 1983:434. map 11..Fitzpatrick. 1983:145.

Material examined---FLORIDA. Alachua Co.: Dudley Cave. $1 \%$ J. Martin. 16 Oct 1937 (USNM):

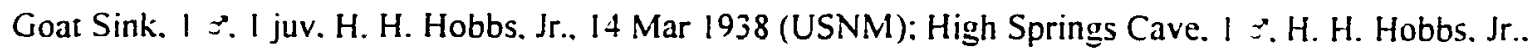
28 Feb 1935 (USNM); Huggins Cave. 3 ․ $1 c^{2}$. L. Hubricht. 17 Apr 1941 (USNM), holotype $\subsetneq$. R. D. Warren. 17 Dec 1961 (CMN): Citrus Co.: Gum Tree Cave (=Gum Cave or Sweetgum Cave). $1:$ collector unknown. 30 Apr 1937 (USNM): Dade Co.: Little Bird Nursery well. collections in USNM made by G.

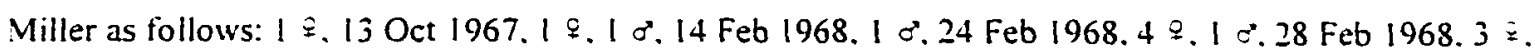
3 c. 12 Mar 1968. 15 specs, 27 Mar 1968. 8 specs. 15 Apr 1968. 1 c. 2 May 1968. 1 \&. 22 May 1968. $1 \equiv$. 


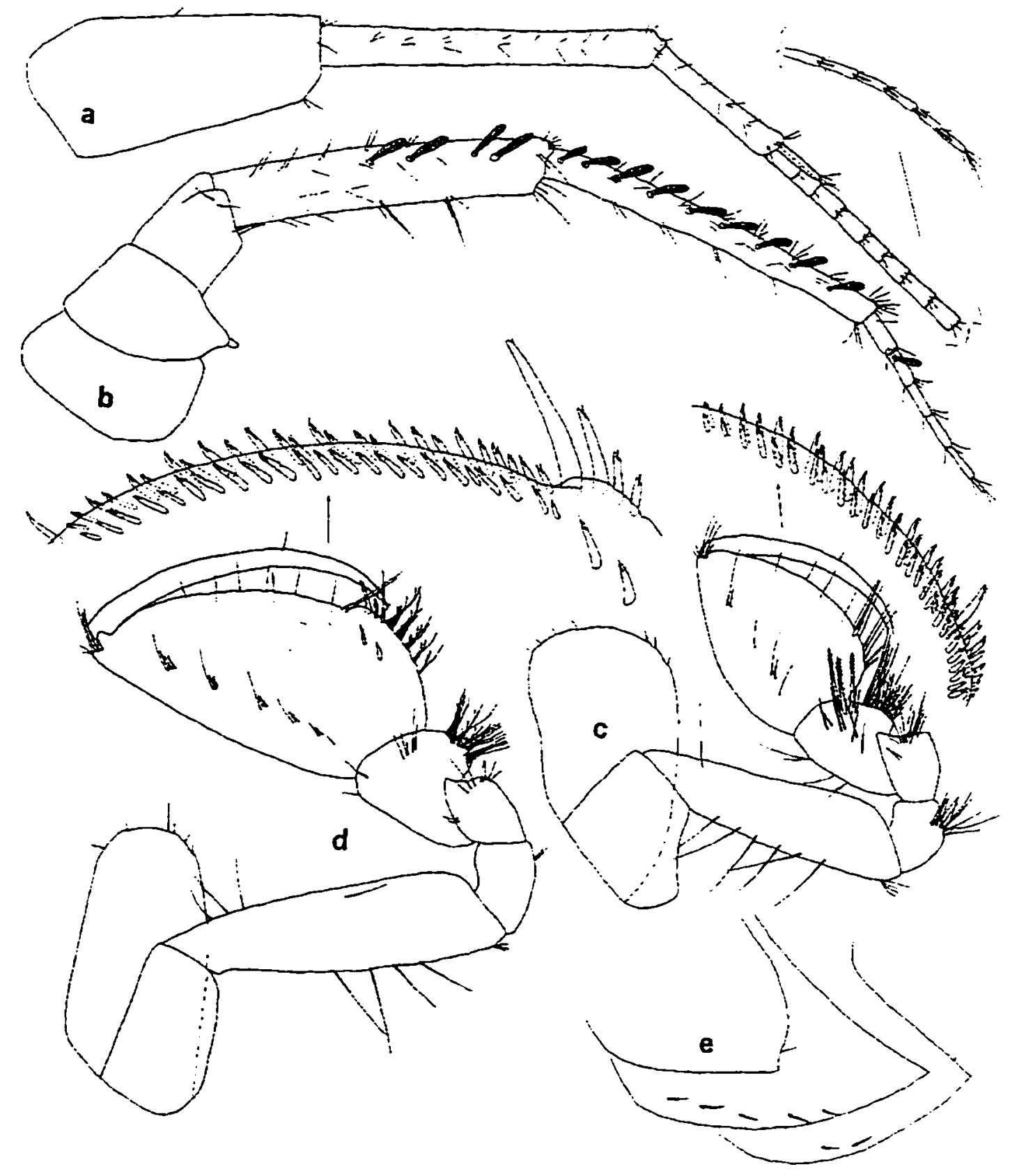

Figure 55.--Crangonlx grandimanus Bousfield. Female (1 $1.7 \mathrm{~mm})$, River Sink. Wakulla Co.. Florida: a. b. antennae I. 2: c. d. gnathopods 1.2 (palmar margins enlarged): e. pleonal plates. 


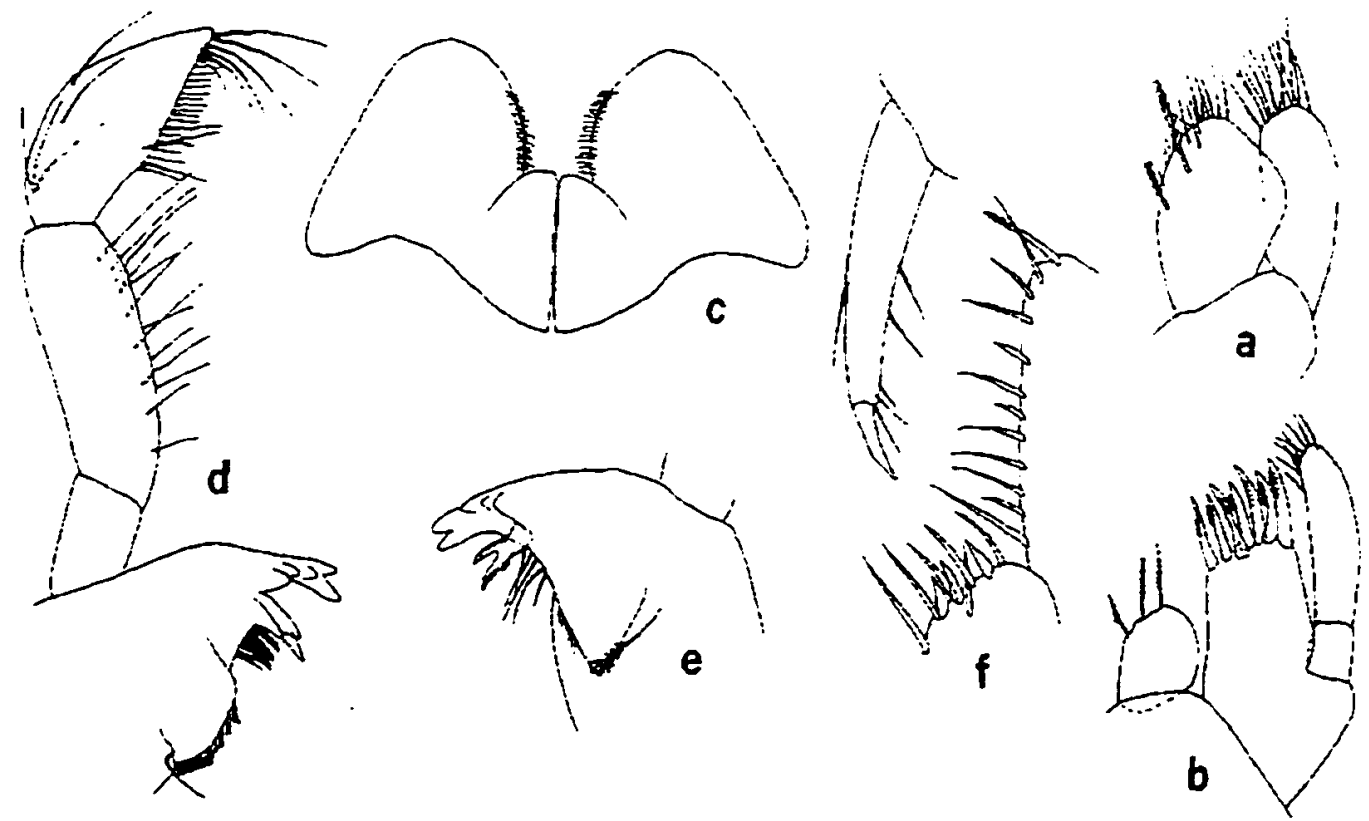

Figure 56.--Crangonyx grandimanus Bousfield. Female ( $11.7 \mathrm{~mm})$. River Sink. Wakulla Co.. Florida: a. b. maxillae I. 2: c. lower lip: d. left mandible: e. dentate part of right mandible: $f$. inner and outer plates and palp dactyl of maxilliped. 


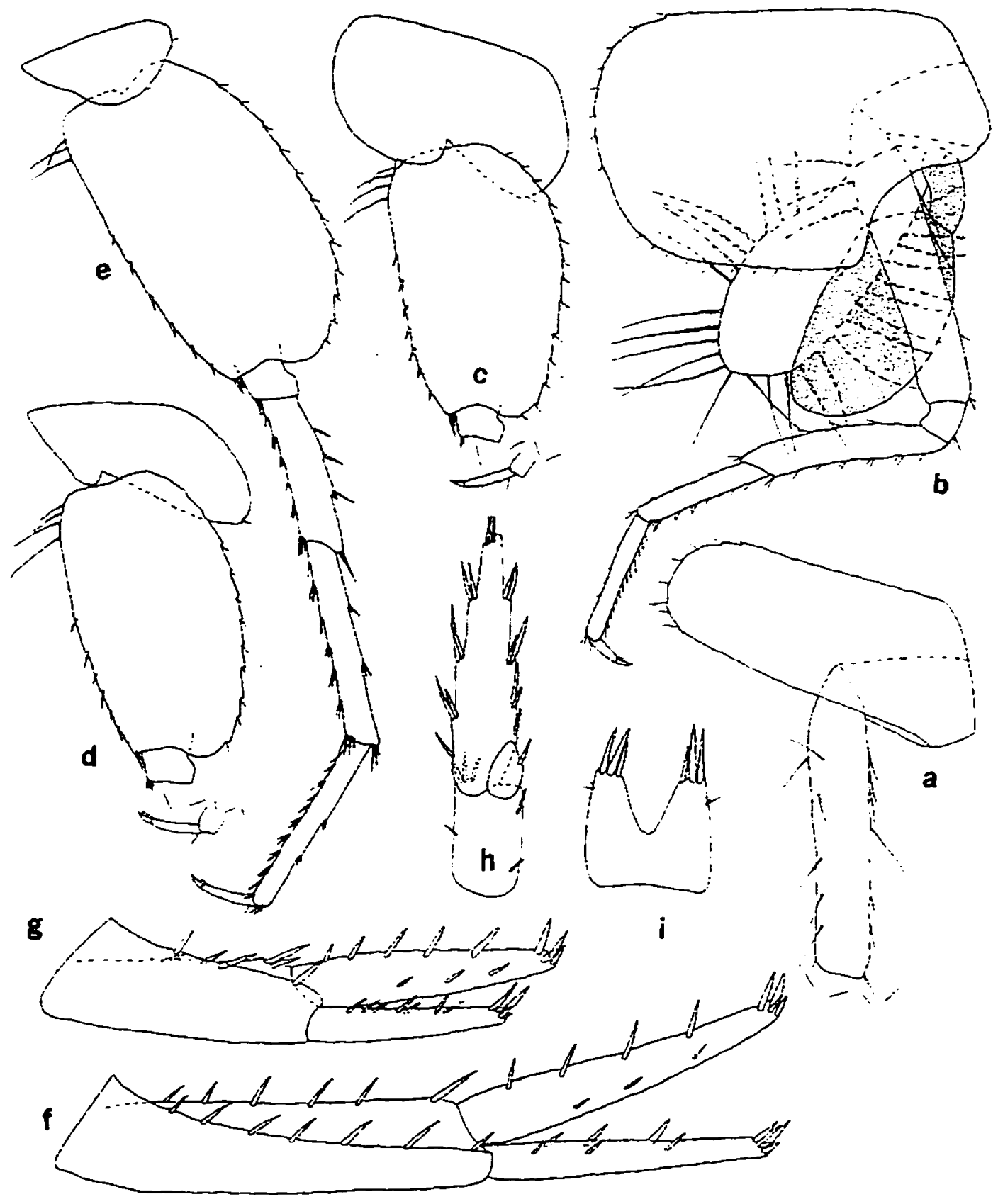

Figure 57.--Crangonyx grandimanus Bousfield. Female (11.7 mm), River Sink. Wakulla Co.. Florida: a. c. d. pereopods 3. 5, 6: b, e, pereopods 4,$7 ; \mathrm{f}, \mathrm{g}, \mathrm{h}$, uropods I, 2, 3: i. telson. 


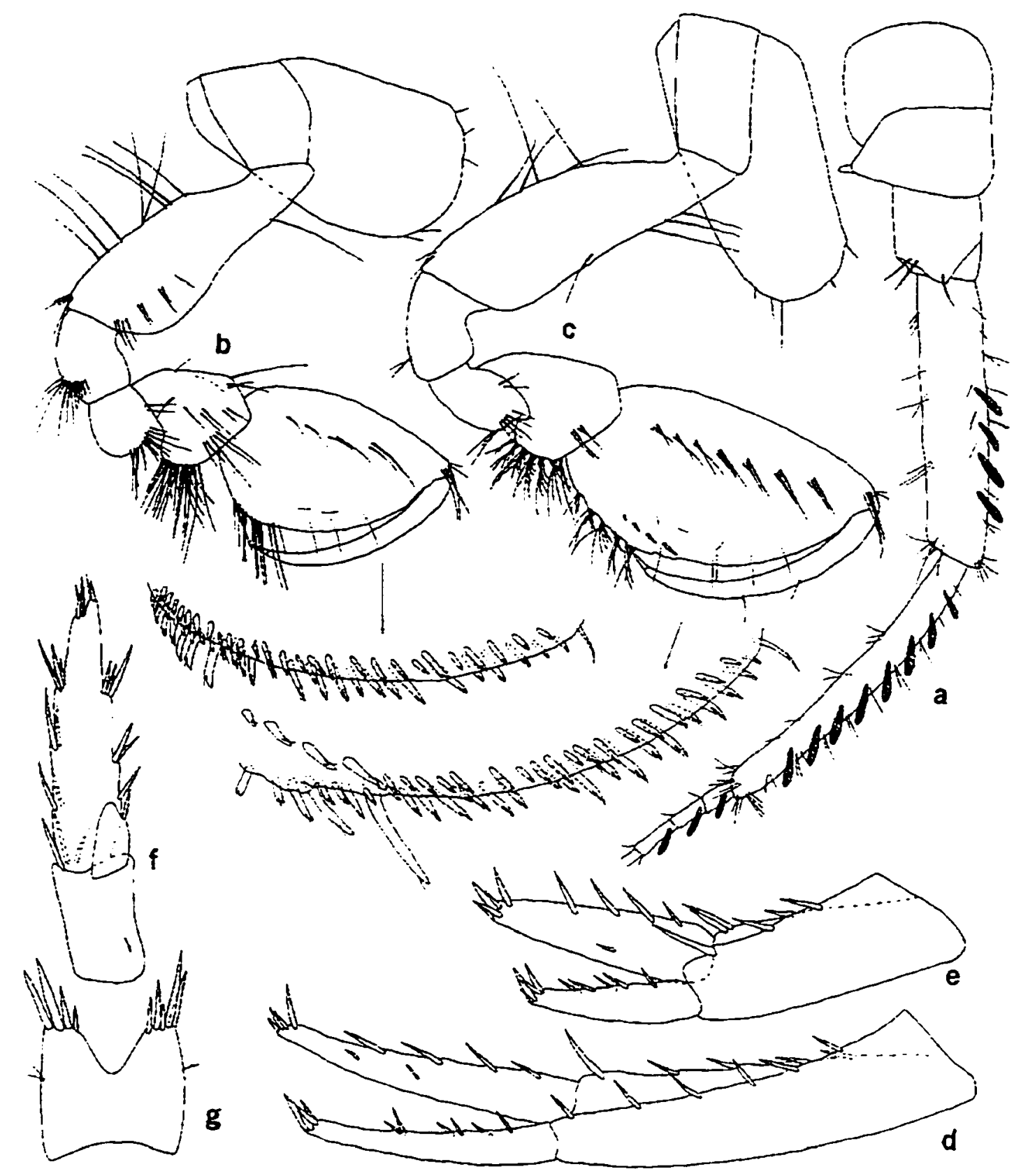

Figure 58.--Crangonyx grandimanus Boustield. Male (10.3 mm), River Sink. Wakulla Co.. Florida: a. antenna 2: b. c. gnathopods 1. 2 (palmar margins enlarged): d. e. f. uropods 1. 2. 3: g. telson. 


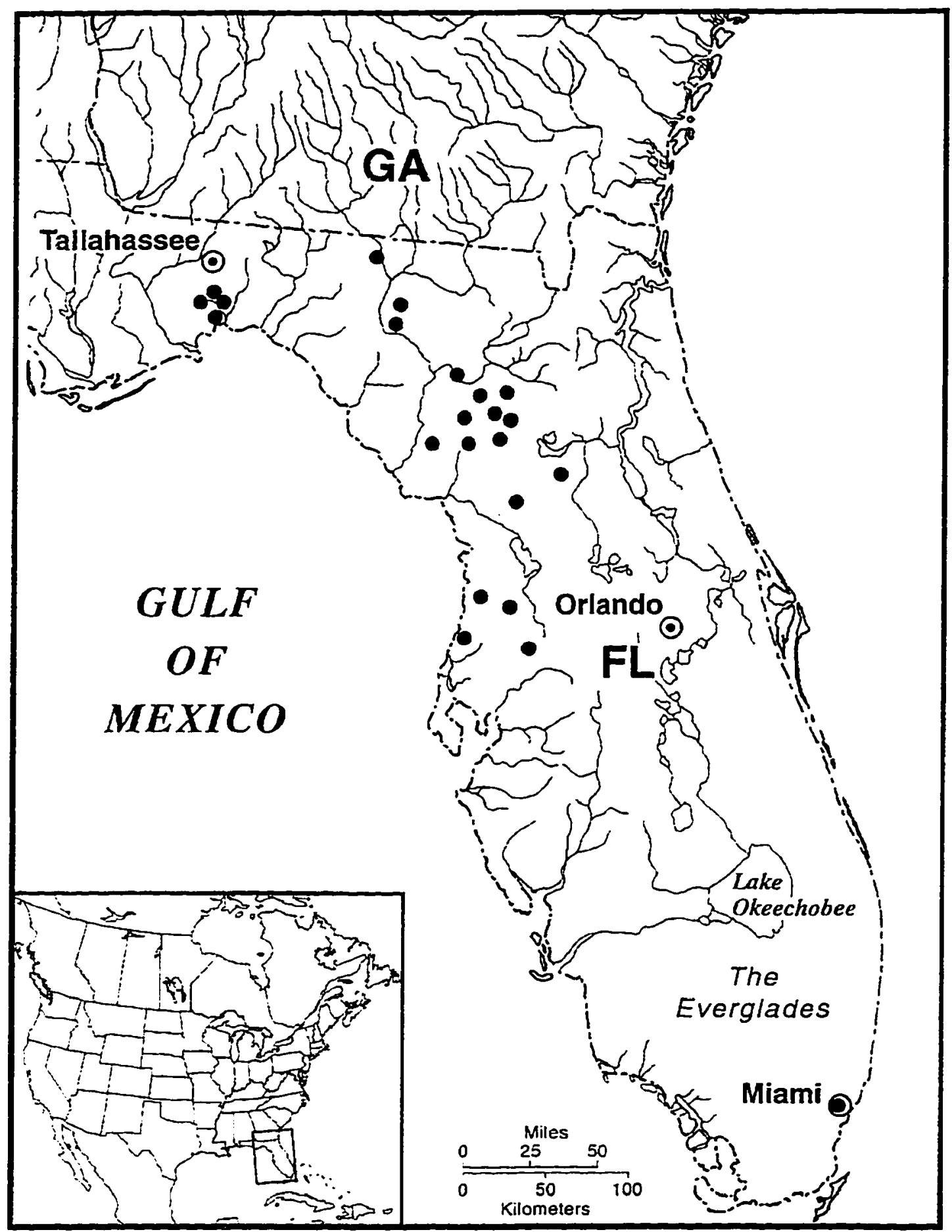

Figure 59.--Distribution of Crangonyx grandimanus in North America. A solid circle represents 1-5 closely proximate localities. 
$1 \approx .29$ Oct 1968. 1 ₹.1 $\% 29$ Nov 1968. $2 \approx .8$ Jan 1969: Bird Road well (probably Little Bird Nursery well). Miami. $1:$ G. Miller. 26 Jan 1968 (USNM): Gilchrist Co.: Devils Eye Spring on Sante Fe River. 1 ‡. A. T. Leitheuser et al. 13 Sep 1979: Devils Eye ( 1 entrance to Ginnie Spring Cave system). 1 ‡. J. Bozanic. 21 Jan 1986: Hernando Co.: Eagles Nest Sink. I פ. A. T. Leitheuser and J. Bentz. 15 Sep 1979: Leon Co.: Little Dismal Sink. 1 \% 3 juvs. P. Heinroth, 5 Mar 1977: Levy Co.: Archer Cave. $1 ‡$. B. Honha. 16 Mar 1976 (FSM); well at Chiefland. 3 o. A. H. Verrill. Apr 1940 (AMNH) and I 1941 (USNM): Madison Co.: Blue Springs. I . J. Bozanic, 30 Aug 1985: Marion Co.: Indian Cave. $11.2 \mathrm{~km}$ SW of Ocala. holotype ?. R. D. Warten. 18 Feb $1961(\mathrm{CMN})$; well $3.2 \mathrm{~km} \mathrm{NE}$ of Authony. I . H. H. Hobbs. Jr.. I Apr 1944 (USNM); Pasco Co.: Nexus Sink. I ̊. A. T. Leitheuser and R. Heinerth. 1 | Jan 1983 (FSM): Suwanne Co.: Cisteen Sink ( 1 of several entrances to Peacock Springs Cave system). $1 \cong$. A. T. Leitheuser and J. Kasserman. 16 Feb 1979: Orange Grove Sink (Peacock Cave system). 1 ¿. J. Bauer and C. Bailey. 2 Jan 1983 (FSM): Peacock Cave System. I §. J. Bozanic. 20 Jan 1985: Wakulla Co.: Emerald Sink. I $\approx$. S. Gerrard and M. Poskey. 31 Oct 1982 (FSM): River sinks. $+\cong .3$ : E. A. Caine. 27 Aug 1972: Shepard Blue Spring. 2 9. W. Fehring. 22 Jul 1983: Wakulla Springs (Wakulla Springs Cave system ). ! ₹. T. Morris. 23 Nov 1987. I Р. I c.29 Nov 1987 and ! c*. Dec 1987.

Diagnosis.--A large trogobitic species of the richmondensis group distinguished from other members of the group by having strongly produced distoposterior comers of pleonal plates 2 and 3 ; posterior margins of basis of pereopods 3-7 with shallow serrations: peduncle 5 longer than peduncle 4 of antenna 2: and peduncle 2 much longer than peduncle 3 of antenna 1 . Largest male $13.0 \mathrm{~mm}$ : largest female $17.0 \mathrm{~mm}$.

Female.--Eyes absent. Antenna I, about 65-70 percent length of body. about 1.8-2.0 times longer than antenna 2: primary flagellum about $30(25-37)$ segments. Antenna 2: peduncle 5 longer than peduncle 4 : flagellum with up to 9 segments; with calceoli in some specimens. Mandibles subequal. spine row with 7 spines: segment 2 of palp with 14 long setae. segment 3 with $3 \mathrm{~A}$ setae. $2 \mathrm{~B}$ setae. $3 \mathrm{C}$ setae. $6 \mathrm{E}$ setae and a row of $20 \mathrm{D}$ setae. Maxilla I: inner plate with 2-3 apical plumose setae: palp with 2 slender spines and 2 rows of setae on apex. Maxilla 2, inner plate with 1 plumose seta on inner margin. Maxilliped: inner plate apically with 4 bladelike spine teeth. 2 naked setae. and 4 plumose setae extending from inner maryin to apex: outer plate with row of naked setae and 2 small slender pectinate spines on inner margin: inner margin of dactyl with 5 setae; dactyl nail short, about $1 / 5$ length of dacty.

Propod of gnathopod I more than 2 times larger and longer than carpus and much broader: palm strongly oblique, convex, almost 2 times longer than posterior margin. with about 20 spine teeth on inside. 24 on the outside: defining angle with 6 spine teeth on inside. outside with two rows spine teeth. inner row with 4 small and outer row with ! large and 8 small spine teeth; superior medial setae few in number and singly or doubly inserted: posterior margin with about 13 setae in a cluster in the defining angle area: dactyl of gnathopod I lacking short setae on inner margin. nail very short. ventral margin of coxa 1 with 7 . 8 setae.

Propod of gnathopod 2 more than 2 times larger and longer than carpus: palm oblique. convex, about 2 
times longer than posterior margin. with 21 spine teeth on inside. 26 on outside: defining angle with 2 spine teeth on inside. 2 simple spine teeth on inner surface: I very large unnotched and 3 small notched spine teeth on outside; 6 sets of medial setae in groups of 3-4; posterior margin with 5 sets of setae in groups of 5-6. Dactyl lacking short setae on inner margin. nail very short. Ventral margin of coxa 2 with 5 setae.

Coxa of pereopod 3 with 8 marginal setae. Coxa of pereopod 4 with 19 marginal setae. Pereopod 5 : basis with 13 shallow serrations along posterior margin: 7 short spines on anterior margin: 4 long setae on the anteroproximal margin. Pereopod 6: basis with II serrations along posterior margin: 6 short spines on anterior margin: 5 long setae on anteroproximal margin. Pereopod 7: coxa with 2 setae on the posterior margin: basis with 12 shallow serrations along posterior margin. deeper at apex: 6 short spines on anterior margin: 3 setae on anteroproximal margin: dactyl about 35 percent length of corresponding propod.

Pleonal plates: posterior margin of plate I strongly convex. with l-2 setae. distoposterior corner very small. not produced: posterior margin of plate 2 with up to 4 setae. strongly oblique. ventral margin with 2 (subventral) spines: posterior margin of plate 3 also oblique. with $1-4$ setae. distoposterior corner subquadrate. ventral margin with $2-4$ (subventral) spines. Peduncle of pleopod 1 with + retinaculae: 3 setae on inside margin proximally, 9 setae on outside margin.

Uropod 1: inner ramus 60 percent length of peduncle, armed with 12 spines: outer ramus with 11 spines: peduncle with row of 7 outer and 6 inner spines. Uropod 2: inner ramus armed with 12 spines: outer ramus with 12 spines: peduncle with 3 outer and 4 inner spines. Uropod 3 : inner ramus with 1 spines. outer ramus about 2.1 times longer than peduncle. inner and outer margin each with + sets of spines in groups of $1-3$. Telson as broad as long, notched from $1 / 4$ to $1 / 2$ distance to base. each lobe with 3-4 apical spines: spines about $1 / 2$ length of telson.

Male.--Differing from female as follows. Smaller in size. Antenna 2: calceoli percent on peduncular segments 4 and 5 and first $j$ segments of flagellum.

Uropod 2: inner ramus with 11 spines: outer ramus smaller and narrower than inner ramus. with 2 inner and $j$ outer spines. apex with 4 spines; peduncle with 4 outer and 5 inner spines.

Distribution and ecology.--This range of this large. distinct troglobitic species, which is restricted to Florida. extends from Madison County in the north to Dade County in the south. covering a linear distance of about $625 \mathrm{~km}$. However, a large disjunction of about $350 \mathrm{~km}$ separates the southern most record in Dade County from those in central Florida west of Orlando (fig. 59).

C. grandimanus is recorded from caves. wells, and karst springs. It is almost always associated with $C$ : hobhsi and sometimes with C. floridanus in nature. Sexually mature males are 8.0-13.0 mm and sexually mature females are $10.0-17.0 \mathrm{~mm}$. Ovigerous females have been collected during February. March. and December and sexually mature females also occur in October samples. 
Crangonvx hubrichti. new species

Figures 60-62

Material examined (paratypes unless designated otherwise).--ILLINOIS. Champaign Co.: drainage ditch. Savoy. 5 ミ. H. J. Van Cleave. 9 May 1942 (USNM): Effingham Co.: ditch. $3.0 \mathrm{~km} \mathrm{WSW} \mathrm{of}$ Altamont. holotype $₫ .14 \subsetneq$. L. Hubricht. $16 \mathrm{Apr} 1942$ (USNM): temporary stream. $1.6 \mathrm{~km} \mathrm{SW}$ of Funkhouser. $1 \fallingdotseq$. L. Hubricht, 16 Apr 1942 (USNM); Madison Co.: spring. $0.64 \mathrm{~km}$ SW of Troy. $5 \cong$. L. Hubricht. 16 Apr 1942 (USNM).

Diagnosis.--A medium-sized species of the richmondensis groups distinguished from other members of the group by inner plate of maxilla 1 which bears 3 apical plumose setae and inner plate of maxilla 2 . which has 2 plumose setae on inner margin: weak serrations along posterior margin of basis of pereopods 5-7: unproduced distoposterior comers of pleonal plates. Largest female. $15.5 \mathrm{~mm}$ : males unknown.

Female.--Eye large and ovate. Antenna 1 , about 50 percent length of body. about 2 times longer than antenna 2: primary flagellum with about 22 segments. Antenna 2. tlagellum with 6-8 segments. Mandibles subequal. spine row with 4-5 spines: segment 2 of palp with 12 setae. segment 3 with 3 A setae. 3 B setae. 3-5 C setae. 5-6 E setae and a row of D setae. Maxilla 1 : inner plate with 3 apical plumose setae: palp with 5-6 slender spines on apex. Maxilla 2, inner plate with 2 plumose setae on inner margin. Maxilliped: inner plate apically with 4 bladelike spines. I naked spine. and 5-6 plumose setae extending from inner margin to apex: outer plate with row of naked setae and 5-6 slender spines (not pectinate) on inner margin and 1 apical plumose seta: inner margin of dactyl with 4 setae: dactyl nail long. about 1.4 length of dactyl.

Propod of gnathopod 1 about 2 times larger and longer than carpus: palm convex. longer than posterior margin. inside and outside margins each with 2 rows of notched spine teeth. 13 on inside. 14 on outside: defining angle with 8 spine teeth on inside, 6 small and I large notched spine teeth and I row of 8 simple spine teeth on outside: superior medial setae in $3-4$ sets inserted in groups of $3: 4$ sets of setae on posterior margin singly inserted in groups of 2-3. Dactyl with row of 4 short setae on inner margin. nail long. Ventral margin of coxa 1 with 14 setae.

Propod of gnathopod 2 about 2 times larger and longer than carpus: palm convex. oblique. slightly longer than posterior margin. inside and outside each with 2 rows of notched spine teeth. 15 on inside. 17 on outside: defining angle with 4 spine teeth on inside. 7 on outside: superior medial setae in 6-7 sets inserted in groups of 2-4: posterior margin with 5-6 sets of setae in groups of 3-6. Dactyl with row of 7 short setae on inner margin. Ventral margin of coxa 2 with 14 setae.

Coxa of pereopod 3 with 12-14 marginal setae. Coxa of pereopod 4 with 27 marginal setae: dactyl of pereopod 4. 33 percent length of corresponding propod. Pereopod 5: basis with 18 shallow serrations along posterior margin: 10 short spines on anterior margin: 6 long setae on the anteroproximal margin. Pereopod 6: basis with 18 shallow serrations along posterior margin: 8 short spines on anterior margin: 6 


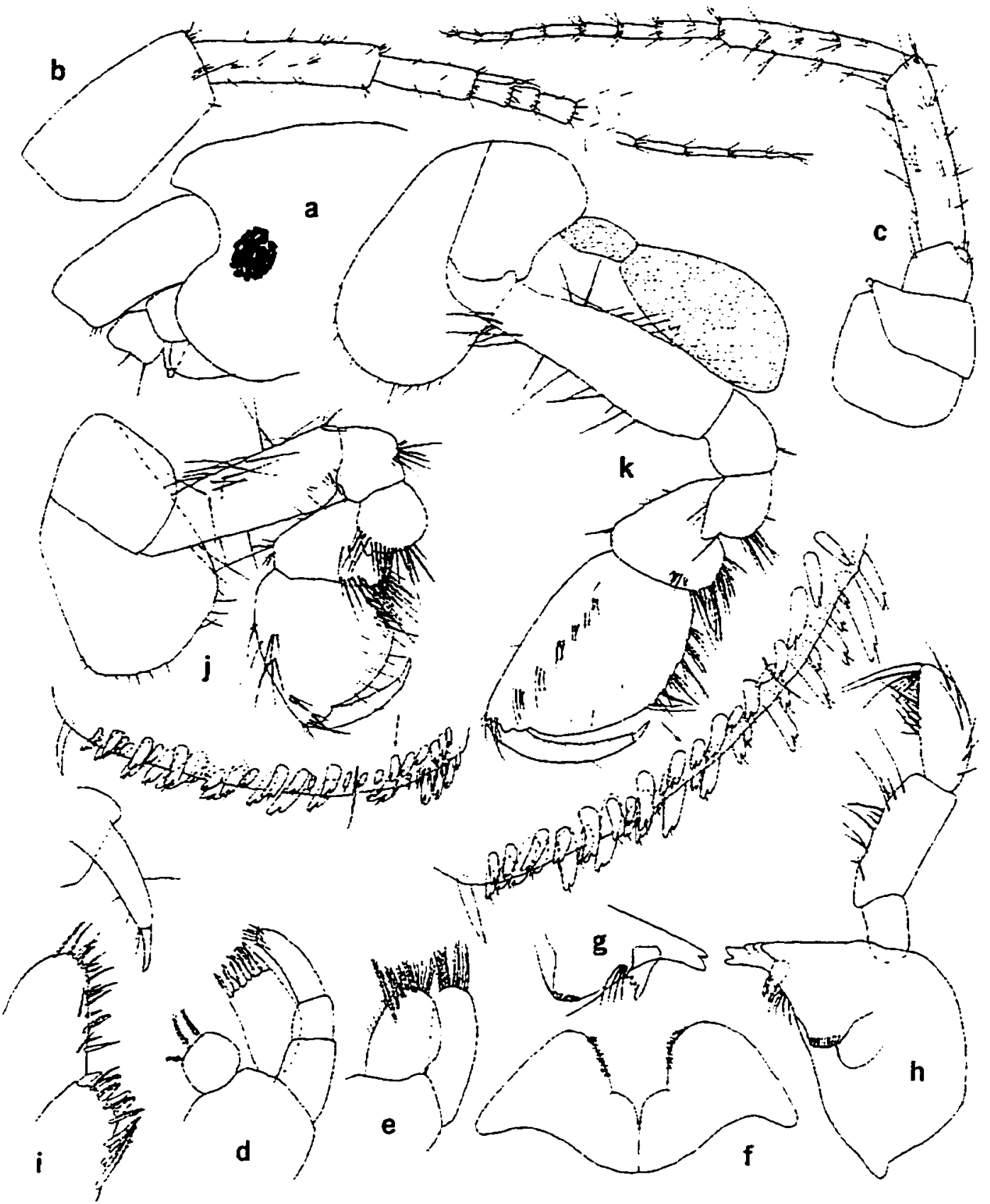

Figure 60.--Crangonyx hubrichti. new species. Female paratype ( $13.2 \mathrm{~mm})$, ditch. $3.0 \mathrm{~km} \mathrm{WSW}$ of Altamont. Effingham Co.. Illinois: a. head (in part): b. c. antennae 1. 2: d. e. maxillae 1. 2: t. lower lip: g. dentate part of left mandible: $h$. right mandible: i. inner and outer plates and palp dactyl of maxilliped (greatly enlarged); j, k. gnathopods 1.2 ( palmar margins enlarged). 


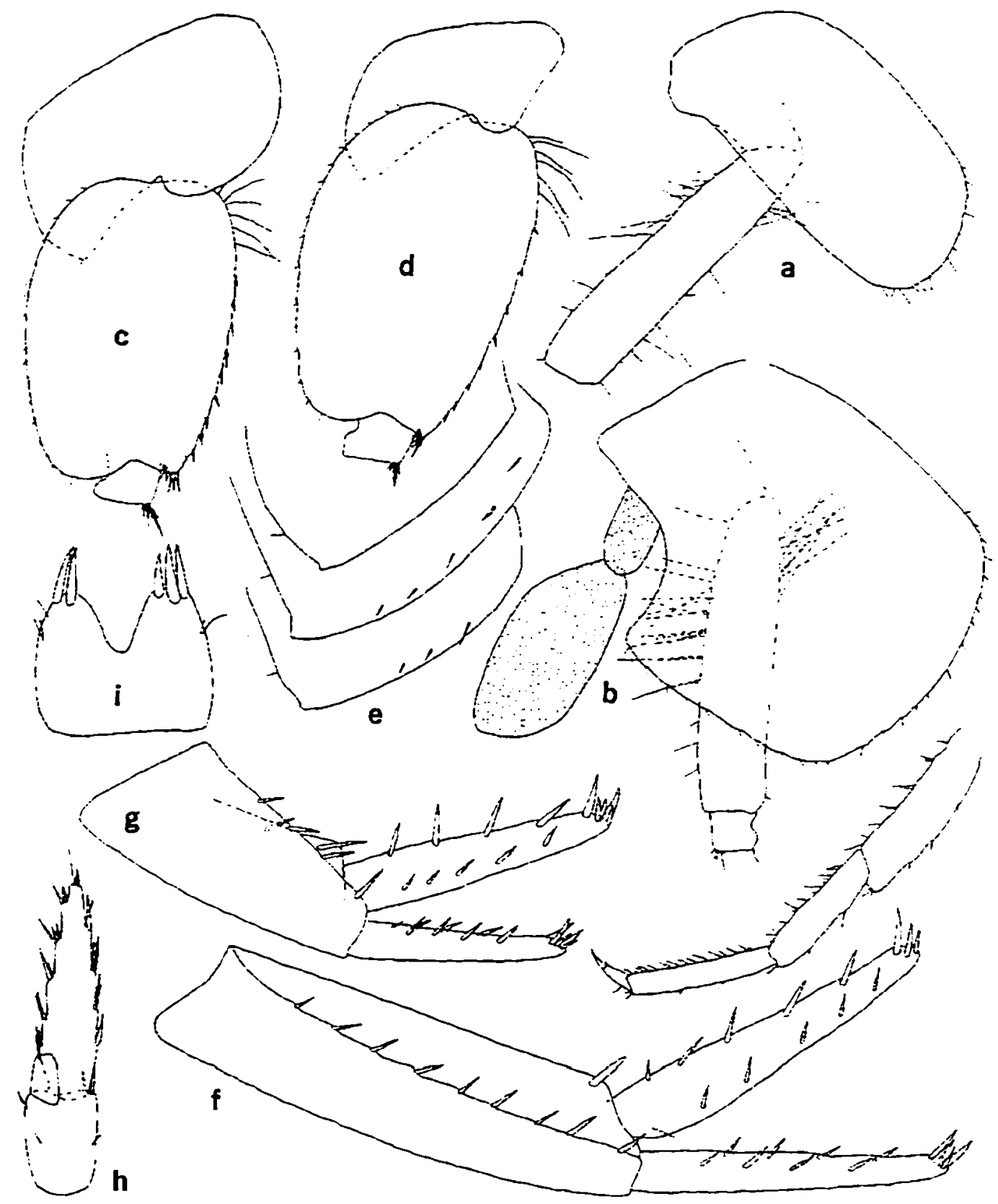

Figure 61.--Crangonvx hubrichti, new species. Female paratype $(13.2 \mathrm{~mm})$. ditch. $3.0 \mathrm{~km} \mathrm{WSW}$ of Altamont. Effingham Co.. Illinois: a. b. c. d. pereopods 3. 4. 5. 6: e. pleonal plates: f. g. h. uropods 1. 2. 3: i. telson. 


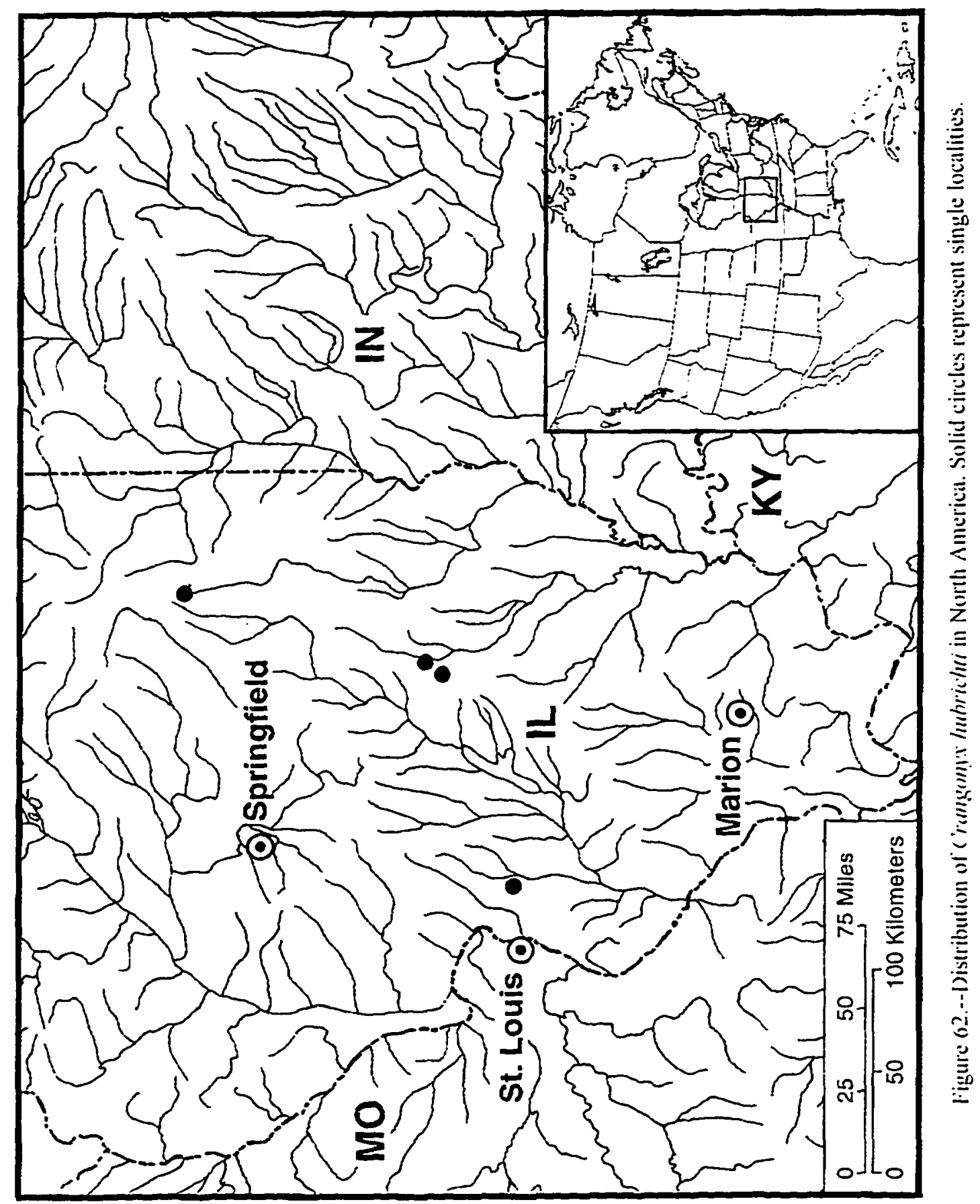


short setae on anteroproximal margin. Pereopod 7: coxa with 5 setae on the posterior margin: basis with 20 very shallow serrations along posterior margin: 7 short spines on anterior margin: 4 long setae on anteroproximal margin: dactyl about 30 percent length of corresponding propod.

Pleonal plates: posterior margin of plate 1 slightly convex. with 1 seta. distoposterior corner acute but not produced: posterior margin of plate 2 nearly straight with 1 seta. distoposterior comer produced and acute. ventral margin with 5 (subventral) spines: posterior margin of plate 3 nearly straight with 1 seta. distoposterior corner subacute but not produced. ventral margin with 3 (subventral) spines. Peduncle of pleopod 1 with 5 retinaculae. about 20 long setae on outside margin. 7-8 short setae on proximal half of inside margin.

Uropod I: inner ramus 70 percent length of peduncle. armed with 13 spines: outer ramus with 12 spines: peduncle with row of 9 outer and 1 inner spines. Uropod 2: inner ramus with 14 spines: outer ramus with 13 spines: peduncle with 3 outer and 2 inner spines. Uropod 3 : inner ramus with 1 spine. outer ramus about 2 times longer than peduncle. inner and outer margin each with $4-5$ set of spines in groups of $1-4$. Telson a little broader than long. notched about 1/2 distance to base, each lobe with 2-3 apical spines: spines slightly less than $1 / 2$ length of telson.

Type-locality.--Ditch, $3.0 \mathrm{~km}$ WSW of Altamont. Effingham Co.. Illinois.

Distribution and ecology.--The range of this species covers approximately $200 \mathrm{~km}$ in south-central Illinois (fig. 62). It is recorded from ditches. a temporary stream. and a spring. Ovigerous temales (13.0$15.5 \mathrm{~mm}$ ) were collected in April. Crangonyx hubrichti was always found together with $C$. minor.

Etymology.--It is a pleasure to name this species in honor of its discoverer. Leslie Hubricht. who has made numerous positive contributions to our knowledge of the freshwater invertebrates of North America.

The forbesi group

Diagnosis.--Closed related to the richmondensis group but distinguished from that group as follows. Adult size range. 14.0-22.0 mm. Maxilla 1: inner plate with about 11 apical plumose. Maxilla 2: inner plate with oblique row of 9-12 plumose setae on inner margin. Propod of gnathopod 1 : defining angle inside with notched-serrate spine teeth. outside with 1 row of 6-7 small notched spine teeth and 1 row of 1 large notched and 7 serrate spine teeth. Propod of gnathopod 2: 7-9 sets of superior medial setae mostly inserted in groups of 4-6: posterior margin with 10 sets of setae in groups of 4-9. Peduncle of pleopod 1 with 8-9 retinaculae. about 12 setae on outer margin and about 7 setae on inner margin proximally.

Remarks.--This group is composed of a single species. C. forbesi. which is widely distributed in eastcentral United States. 
Crangonvx forbesi (Hubricht and Mackin)

Figures $63-67$

Eucrungonyx forbesi Hubricht and Mackin. 1940:196-197, fig. 5 [type-locality: outlet of drain (spring).

Osage Hills Golf Course. Kirkwood, St. Louis Co.. Missouri].

Crangonxx forbesi (Hubricht and Mackin).--Hubricht, 1943:689.--Kenk. 1949:48.--Hubricht. 1950:16.--

Dearolf. 1953:228.--Bousfield. 1958:87-89, fig. 11.--Nicholas. 1960:127.--Black. 1971:7.--

Holsinger.1972:36.--Page. 1974:93.--Pflieger. 1974:36.--Craig. 1977:83.--McDaniel \& Smith.

1976:57.--Crawford. 1977:18.--Holsinger. 1977:252.--Barnard \& Barnard. 1983: +34. map 11.--

Fitzpatrick. 1983:145.--Gardner. 1986:15.--Pennak. 1989:483.

Crangonyx gracilis Smith.--Hay. 1882:241.

Crangonyx shoemakeri (Hubricht \& Mackin).--Hubricht. 1943:690 [in part].

Material examined.-ARKANSAS. Fulton Co.: Mammoth Spring. 1 ๑. L. Hubricht. 9 Apr 1939 (USNM): small spring near Mammoth Spring, 5 specs. L. Hubricht. 18 Jul 1942 (USNM): Lawrence Co.: spring. $5.9 \mathrm{~km}$ S of Imboden. 40 specs. L. Hubricht. 6 Apr 1941 (USNM). ILLINOIS. Gallatin Co.: seep near salt well. $5 \geq .8$ juvs. J. J. Lewis. 27 Apr 1974: Jackson Co.: small intermittent stream. Little Grand Canyon. $8.0 \mathrm{~km}$ W of Etherton. 40 specs ( $($.$) ). L. Hubricht. 20$ Oct 1940 (USNM): spring in Happy

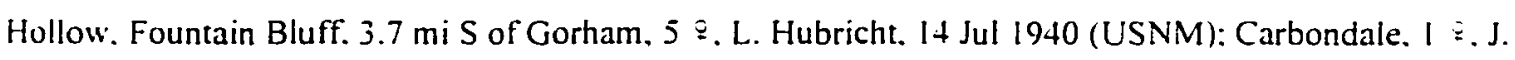
G. Weise. 26 Oct 1951: Cave No.1. I $\approx$. S. B. Peck. 24 Mar 1966: walled spring. Fountain Blutf. $1 \equiv .3 \approx$ 3 juvs. J. J. Lewis. 10 Feb 1974: Jersey Co.: spring near Grafton. 4 ×. S. B. Peck. 26 Nov 1965: Johnson Co.: Firestone Creek Cave. 1 z. J. J. Lewis. 15 Dec 1973: Monroe Co.: Camp Vandeventer Cave. 1 z". S. B. Peck. 27 Nov 1965; Dry Run Cave. 6 ๑4 juvs. S. B. Peck. 26 Jun 1965: Fogelpole Cave. 1 ミ. 2 : S. B. Peck. 25 Jun 1965: spring, $3.2 \mathrm{~km}$ N of Fountain Gap. 3 \&. L. Hubricht. 26 May 1937 (USNM): Fruths Spider Cave. 1 \. S. B. Peck. 25 Jun 1965: Horsethief Cave. 1 2. 1 ₹. S. Peck. 29 Nov 1965: Pautler Cave. 1 : . S. B. Peck. 27 Nov 1965: Terry Spring (Long Slash) Cave. 3 g. 1 : . S. B. Peck. 27 Nov 1965: Wildes Cave. $4 \cong .1 \approx$. L. Hubricht. 31 Jul 1938 (USNM): spring. $3.2 \mathrm{~km}$ E of Waterloo. $5 \equiv$. L. Hubricht. 24 Oct 1937 (USNM): ditch leading into Moredock Lake. $6.2 \mathrm{~km} \mathrm{NE}$ of Valmeyer. 14 . L. Hubricht. 26 May 1937 (USNM): spring. 4.8 km N of Fountain Gap. 20 specs. L. Hubricht. 26 May 1937 (USNM): Illinois Caverns (Morrison Cave), 40 specs. L. Hubricht. 28 Aug 1938 (USNM) and 29 . T. C. Barr. Jr. and T. Wallwork. 20 Oct 1961: Pike Co.: Lost Creek Cave. 10 q. 30 . S. B. Peck. 25 Nov 1965: St. Clair Co.: Stemmlers Cave. 5 ₹. 3 o. 1 juv, L. Hubricht. 16 Jan 1938 (USNM) and $1 ₹ .2$ juvs. J. R. Holsinger and R. M. Norton. 13 Jun 1965: Union Co.: spring. Alto Pass. 5 \&. 1 juv. J. G. Weise. 2 Nov 1951: small spring. just $\mathrm{N}$ of Ecological area. Pine Hills. La Rue. Shawnee National Forest. 1 z. D. Bechler. 12 Mar 1977: spring. $3.2 \mathrm{~km}$ E of Reynoldsville. $9 \subsetneq .2 \%$. L. Hubricht, 5 Nov 1939 (USNM): spring. $15 \mathrm{~m} \mathrm{E}$ of 


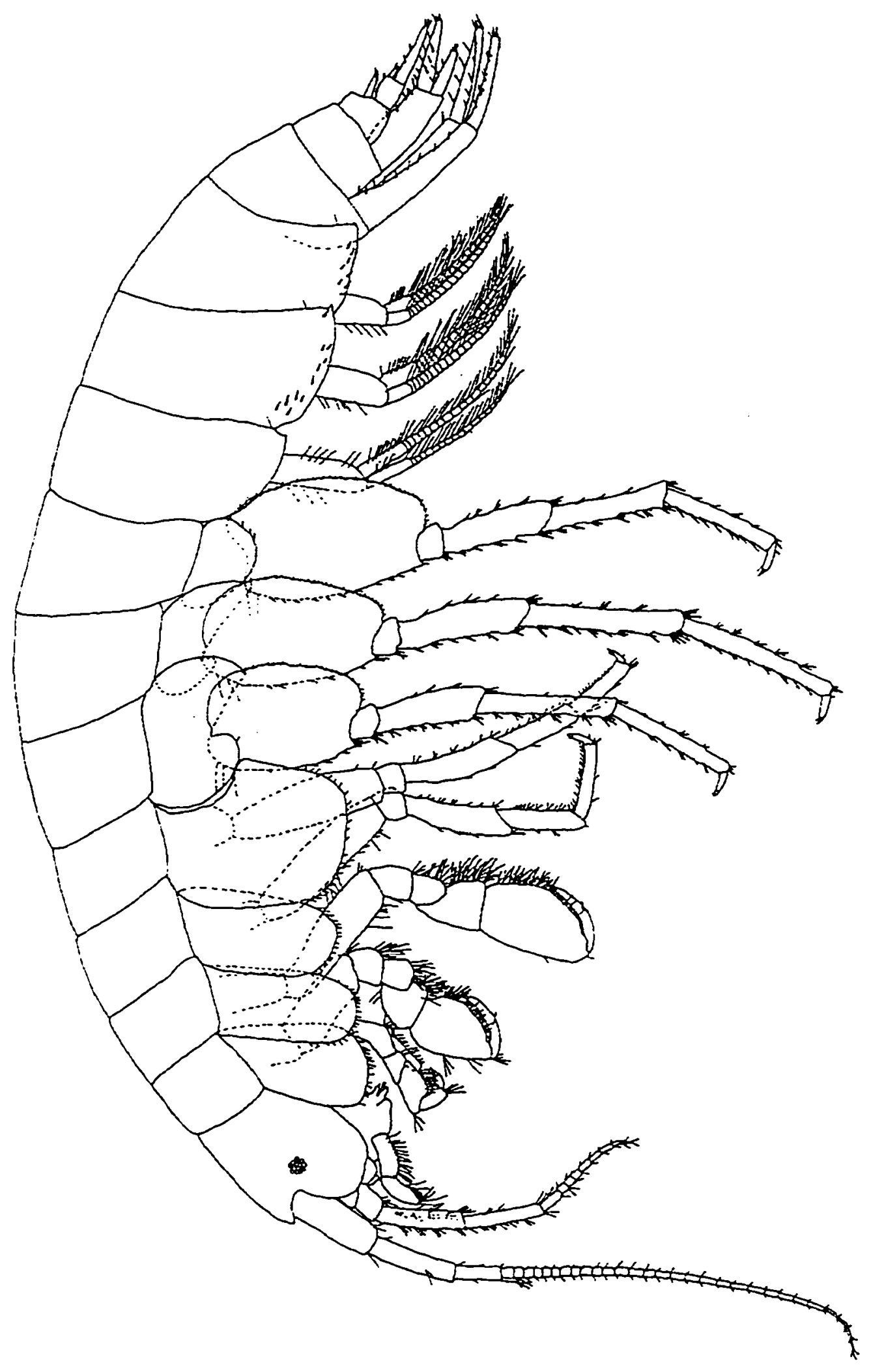




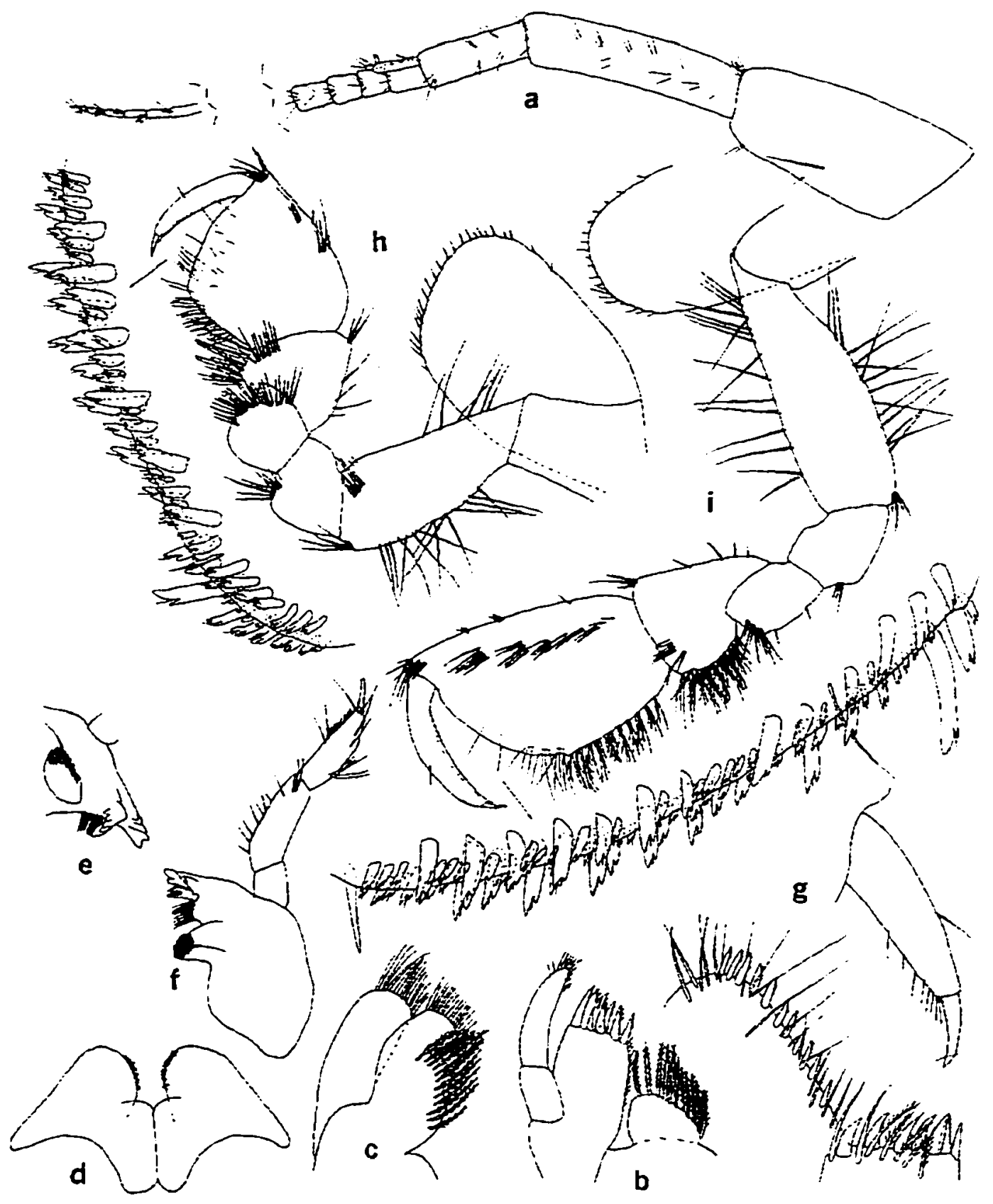

Figure 64.--Crangonyx forbesi (Hubricht and Mackin). Female (17.0 mm), small spring near entrance to Normundy Osteopathic Center. Kirkwood. St. Louis Co.. Missouri: a. antenna 1: b. c. maxillae 1. 2: d. lower lip: e. dentate part of left mandible; $f$. right mandible: $g$. inner and outer plates and palp dactyl of maxilliped: h. i. gnathopods 1.2 (palmar margins enlarged). 


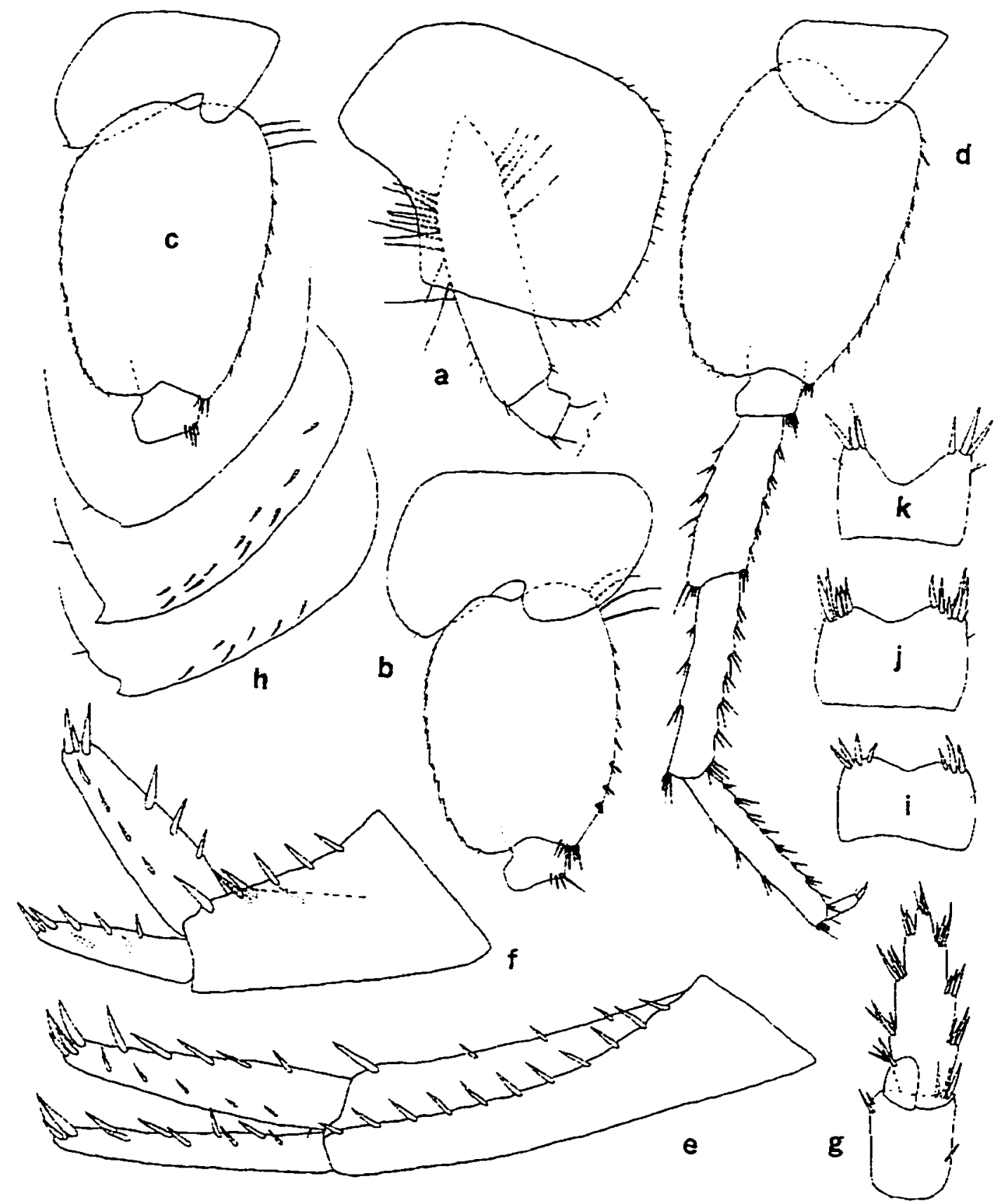

Figure 65.--Crangonyx forbesi (Hubricht and Mackin). Female (17.0 mm). small spring near entrance to Normundy Osteopathic Center. Kirkwood. St. Louis Co.. Missouri: a. b. c. pereopods 4. 5. 6 (in part): d. pereopod 7; e, f. g. uropods 1. 2. 3: h. pleonal plates: i, telson. Female (12.0). McDowell Cave. Miller Co.. Missouri: j. telson. Female (12.5). spring on tributary of Crooked Creek. Guyandotte. Putnam Co.. West Virginia: $k$, telson. 


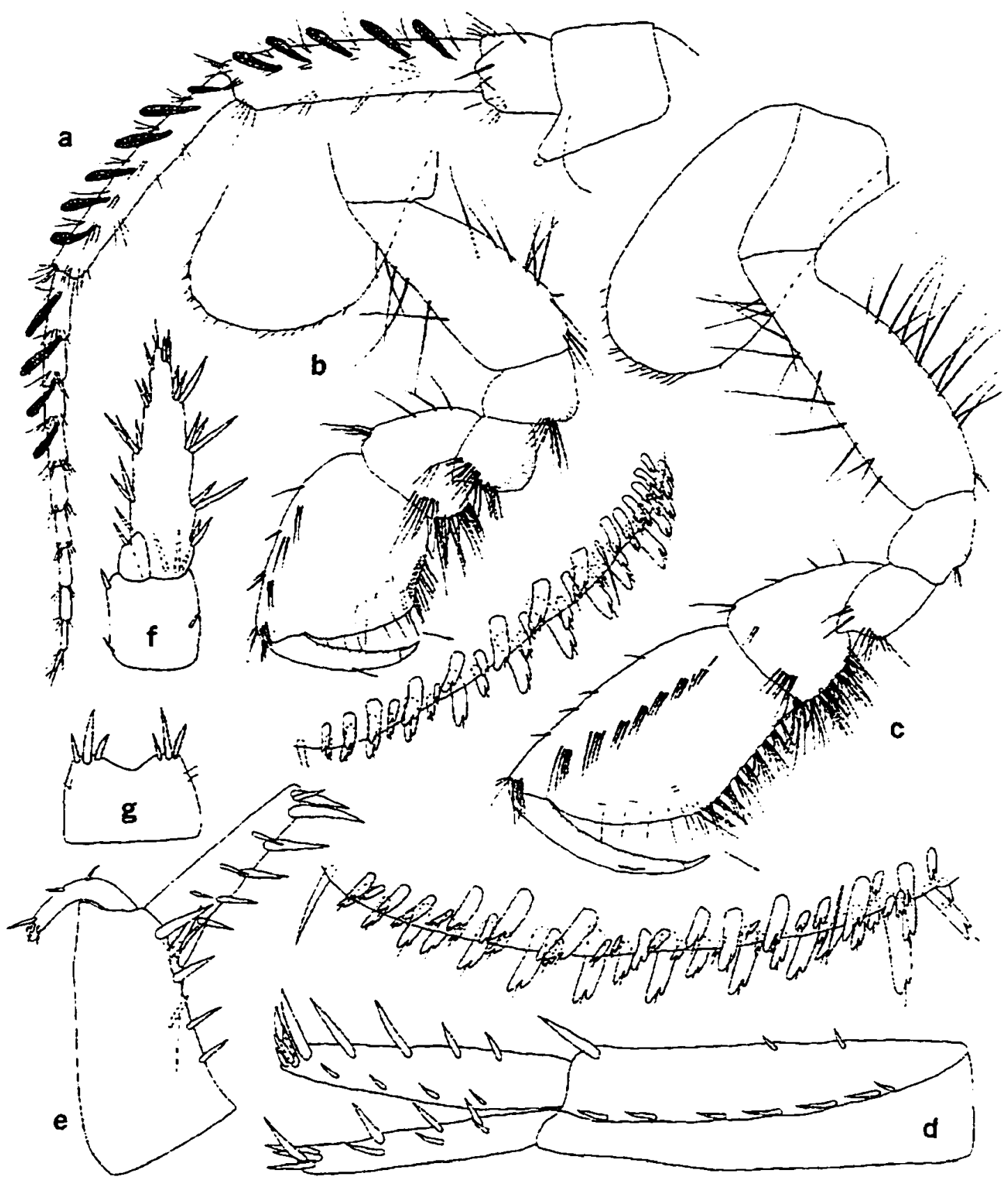

Figure 66.--Crangonyx forbesi (Hubricht and Mackin). Male ( $12.2 \mathrm{~mm}$ ). small spring near entrance to Normundy Osteopathic Center. Kirkwood. St. Louis Co., Missouri: a. antenna 2: b. c. gnathopods 1. 2 (palmar margins enlarged): d, e. f. uropods 1, 2. 3; g. teison. 


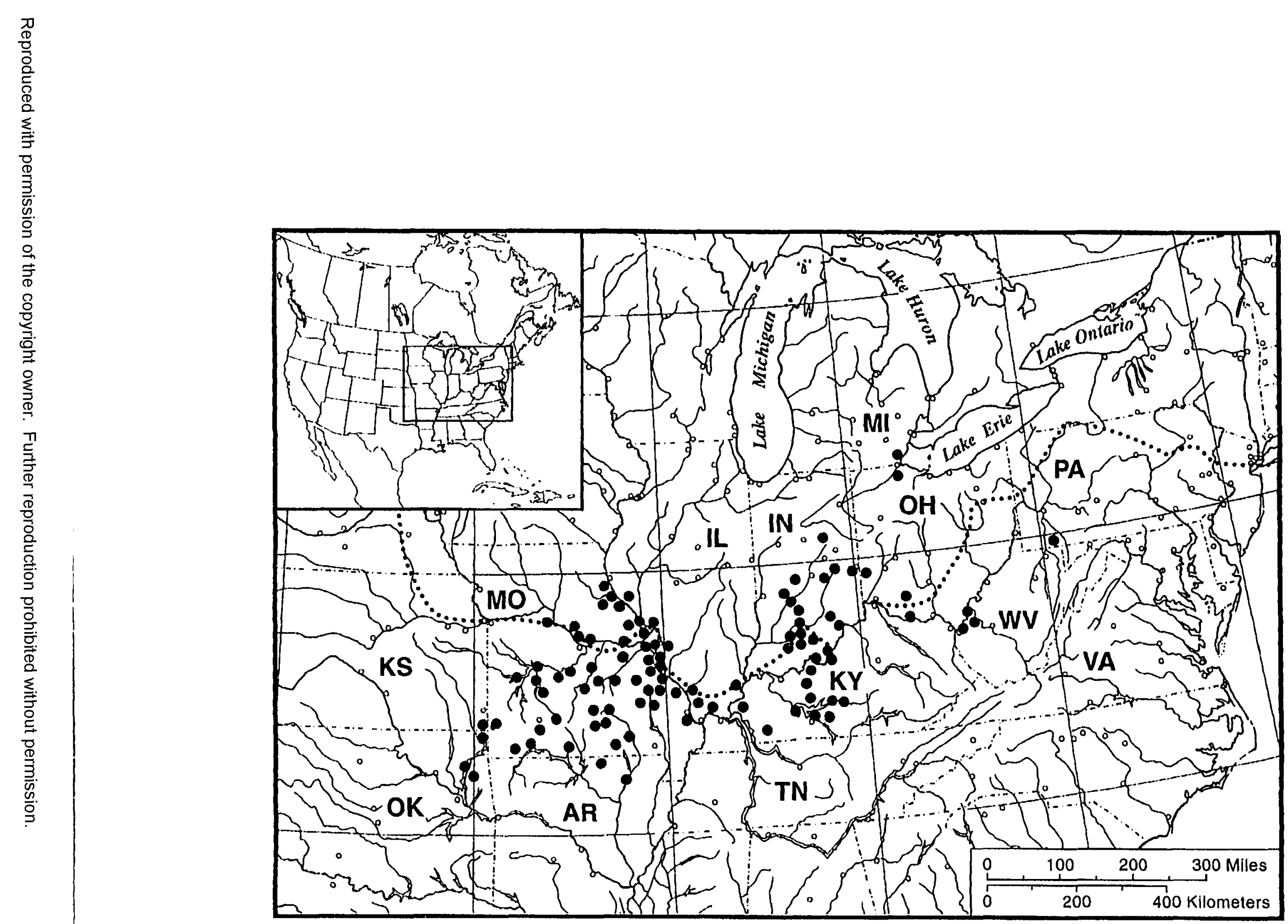

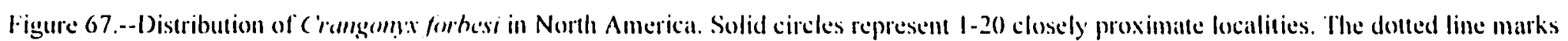
lhe approximate southern extent of glaciation during the Pleistocene. 
Tellor's Farm. 3 c. J. G. Weise. 21 Dec 1951: county not given. $4.8 \mathrm{~km} \mathrm{~N}$ of Hwy 146 on Beach Grove Road. 5 specs. J. G. Weise, 7 Feb 1952. INDIANA. Dubois Co.: Vowell Cave. $1 \Xi .2 \approx$. R. M. Norton. 19 Jul 1967: Floyd Co.: spring and spring-fed stream just $E$ of Edwardsville. 60 specs. J. R. Holsinger and R. M. Norton. 12 Jun 1965: Grant Co.: temporary pools, $8.0 \mathrm{~km} \mathrm{~W}$ of Jonesboro. 35 specs. L. Hubricht. 27 Apr 1941 (USNM): Hancock Co.: ditch. $5.3 \mathrm{~km}$ E of Greenfield. 25 specs. L. Hubricht. 17 Apr 1942 (USNM): Harrison Co.: Wallier Cave. I c*. S. B. Peck. 24 Jan 1964: Hendricks Co.: temporary spring. 6.2 $\mathrm{km} \mathrm{SW}$ of Belleville. 30 specs. L. Hubricht. 16 Apr 1942 (USNM): Henry Co.: outlet of drain. $5.6 \mathrm{~km} \mathrm{~W}$ of Knightstown. 7 ․ 2 . L. Hubricht. 17 Apr 1942 (USNM): small stream. 2.I km NW of Blountsville. 15 specs. L. Hubricht. 27 Apr 194I (USNM): Jefferson Co.: Wilson's Cave. 6 ?. S. B. Peck. 9 Aug 1964: Jennings Co.: Hooker Cave. 1 P. 2 juvs. R. M. Norton. 14 Aug 1967: Lawrence Co.: Gyger Bend I Cave. 2 ¡. H. H. Hobbs. III. collection date not given): Bronson Cave. $29.6 \approx$. 2 juvs. J. R. Holsinger. 19 Jun 1965: Donnehues Cave. 1 s. J. R. Holsinger and E. Lavangino. 8 Aug 1964: Martin Co.: Ellis Spring. 5.6 km E of Loogootee. 50 specs. L. Hubricht. 21 Nov 1941 (USNM): Monroe Co.: Barters Bog. $6 \fallingdotseq .29$. Bleyman. Nov. 1962 (USNM): Jordan River. Bloomington. $3 \mp .7 \approx$. collector and collection date unknown (USNM): small stream $6.2 \mathrm{~km}$ SW Hickory Ridge Fire Towel. Bloomington. $3 \Xi .2$ : D. L. Weaver. Mar 231957 (USNM); Carmichael Cave. $2 \approx$. H. H. Hobbs, Ill. collection date not given: Mays Cave. 1 ₹. H. H. Hobbs, III. collection date not given: Pattons Cave. $3 \& .1 \geq .8$ juvs. W. Tozer. 7 May 1965: Reeves Cave. 5 9. 1 juv. H. H. Hobbs. III, collection date not given: Orange Co.: Elrod Cave. $1 \cong .1$ juv. L. Hubricht. 29 Aug 1942 (USNM): spring, $13.8 \mathrm{~km}$ S of Paoli. $13 \Xi .32$. L. Hubricht. 2 Sep 1939 (USNM): Owen Co.: Porter Cave. $1 \approx^{*}$. R. M. Norton. 1 Aug 1967: Hights Pit Cave. $1 \equiv .3 \approx 21$ juv. W. Tozer. 9 May 1965: Putnam Co.: Triple Springs near Pleasant Gardens. 50 specs. L. Hubricht. 16 Apr 1942 (USNM): Spencer Co.: small tributary of Richland Creek on Rt. 48. 5 . 1 ڤ.. N. Hynes. 5 Jan 1963 (USNM): Washington Co.: Flowstone Cave. $10 \cong$. J. J. Lewis. 15 Sep 1973: Joe Hall Cave. 2 : R. M. Norton. 18 Jul 1967: Wayne Co.: small spring, ca. $12.8 \mathrm{mi}$ W of Economy, 30 specs. L. Hubricht. $27 \mathrm{Apr}$ 1941 (USNM): County and habitat not given: Irvington. 8 ฯ. W. P. Hay. collection date unknown. (USNM). KANSAS. Cherokee Co.: Schermerhorn Park Cave spring. $2.4 \mathrm{~km} \mathrm{~S} \mathrm{of} \mathrm{Galena.} 1 \Xi .+\approx 0 . \mathrm{J}$. Danoff-Burg, 18 Jan 1991: spring-run from cave at Schermerhorn Park. $3.7 \mathrm{~km} \mathrm{~S}$ of Galena. 4 2. 2 ¿.P. Lerchti et al.. $18 \mathrm{Mar}$ 1981. KENTUCKY. Barten Co.: swale. $1.6 \mathrm{~km}$ E of Bonayr. $5 \Xi .7 \Xi$. L. Hubricht. $20 \mathrm{Mar} 1956$ (USNM): Breckinridge Co.: spring, $5.6 \mathrm{~km}$ E of Cloverport. $6 \doteq .1 \approx$. L. Hubricht. 12 May 1957 (USNM): Penitentiary Cave. 1 . R. M. Norton. 19 Oct 1963: Butler Co.: Jeff Tavlor Spring. $1.6 \mathrm{~km}$ SSW of Martin Ferry, 28 specs ( $9 . \approx *$. juvs). L. Hubricht. 2 Jun 1941 (USNM): Christian Co.: Chandler Cave. $1 ~ z$. T. C. Barr. Jr. and W. Andrews, 16 Jul 1965: spring on Pollard Farm. 26.4 km NW of Hopkinsville. 27 specs, 30 May 1941 (USNM): Crittenden Co.: Blowing Spring close to Ellen Clark Cave. 11 ב. T. C. Barr. Jr. and W. Andrews. 7 Jul 1965: Ellen Clark Cave, 10 ?. 1 juv. W. Andrews and T. C. Barr. Jr.. 7 Jul 1965: Edmonson Co.: spring, 2.4 km E of Brownsville. Mammoth Cave National Park. $1 \Xi$. $1: 1$ juv. L. Hubricht. 19 May 1956 (USNM); stream in small cave $1.6 \mathrm{~km} \mathrm{NW}$ of Arthur. 1 . L. 
Hubricht. I Dec 1956 (USNM): Grayson Co.: Condor Cave. 2 §. T. C. Barr. Jr. and R. M. Norton. 6 Aug 1966: Willis Cave. 1 ご. R. M. Norton. 6 Aug 1966: Green Co.: Woodard Cave. 1 ご. J. R. Holsinger. 28 Sep 1963: Hart Co.: Riders Mill Cave. I 9.2 c*. R. M. Norton. 5 Oct 1963: Jefferson Co.: spring at Seneca Park Golf course. Louisville. 2 ๑. 2 s, G. A. Cole. 14 Mar 1954 (USNM): spring $4.8 \mathrm{~km}$ SSE of Okalona. 1 Æ. R. Fox. 12 Feb 1971: spring on Seneca Park golf course. Louisville. 19 ९. G. A. Cole. 14 Mar 1954 (USNM): Meade Co.: Cold Spring Cave, 1 \%, $2 \sigma^{\circ}, 1$ juv, 29 Jun 1965: Doe Run. $5 \approx^{*}$. N. Hynes. 12 Mar 1985 (USNM): Youtsler Cave, 2 9. 1 c. T. C. Barr, Jr. and W. Andrews. 30 Jun 1965. MICHIGAN. Monroe Co.: Outlet of drain, $0.5 \mathrm{mi} \mathrm{N}$ of Ottawa lake, $2 \mathrm{~J}$. L. Hubricht. $18 \mathrm{Apr} 1942$ (USNM). MISSOURI. Barry Co.: Chimney Rock Cave. 4 c.. J. E. Gardner. 14 Mar 1979. 1 . 1 1.1 juv. 22 Jan 1980: Icebox Spring. $11.6 \mathrm{~km}$ S of Cassville. $17 \Xi$. J. E. Gardner. 3 Apr 1981: Mushroom Rock Cave. 4 ‡. $3 \approx$ J. E. Gardner. 12 May 1981: seep spring. $9.6 \mathrm{~km}$ NNE of Cassville. $3=2.1$ juv. 8 Mar 1979: Trailside Cave. 1 §. J. E. Gardner. 6 May 1982: Benton Co.: Elisha Estes Cave. 2 ‡. 3 :. J. Reynolds. 6 Apr 1968 (USNM): Flippen Cave. 6 9. 2 c. J. Reynolds. 30 Mar 1958 (USNM): Boone Co.: Devil's Icebox Cave. 39.3 . J. Reynolds et al.. I Dec 1957 (USNM) and 32.1 s. J. E. Gardner. 29 Jan 1981: Pollys Pot Cave. 7 P. 1 \&. J. E. Gardner. 28 Jan 1981: small overflow pool on Brush Creek. $6.2 \mathrm{~km}$ of Ashland. 20 specs ( $尹$. $:$. juvs). L. Hubricht. 30 Apr 1938 (USNM): spring in Rockbridge State Park. $7 \geq$ J. E. Gardner. $25 \mathrm{Feb}$ 198I: walled-in spring. Rockbridge State Park. $3 \equiv .7$ こ̊. J. E. Gardner. $25 \mathrm{Feb}$ 1981: University of Missouri. Columbia. $1 \subseteq .1 \approx$ W. Chipman, habitat and collection date not given (USNM): Callaway Co.: Spaghetti Cave. 6 9. 16 c. J. E. Gardner. 26 Feb 1981: Camden Co.: Hahatonka Spring. 8 ․ 1 L. Hubricht. 26 Sep 1937 (USNM): Lower Burnt Mill Cave. 3 ¡. C. Hawksley. 7 Jan 1958: Fiery Folks Spring. $12.8 \mathrm{~km} \mathrm{~N}$ of Mocks Creek. 18 \&. 2 z. 4 juvs. J. E. Gardner. 16 Dec 1982: Moles Cave. $10 \cong .2 \approx$ J. E. Gardner, 7 Sep 1979: Carter Co.: a cave. $9.6 \mathrm{~km} \mathrm{~N}$ of Van Buren. $12 \cong .1 \approx$ Creaser. 24 Apr 1930 (USNM): Barkdull Cave. $29.3 \approx$. J. E. Gardner. 23 May 1984: Ebb and Flow Spring. 2.4 km SW of Big Spring State Park. 8 \& 1 0.. Creaser. 10 Aug 1930 (USNM): Lower Camp Yarn Cave. 10 specs. J. E. Gardner. 10 Jul 1982: spring on Curent River, near mouth of Mill Creek. $6.2 \mathrm{~km}$ NW of Van Buren. 30 specs. L. Hubricht. 3 May 1942 (USNM): Christian Co.: Math Branch Cave. 6 ¡. J. E. Gardner. 9 Aug 1981: Smallin Cave (at entrance). 40 specs. L. Hubricht. 21 Oct 1939 (USNM): Crawford Co.: Bear Cave. 3 ๑. J. L. Craig. 23 May 1974 and $4 \subseteq$. J. E. Gardner. 6 Aug 1980: Hidden Spring Cave. 1 $\equiv .1$ ×. J. J. Lewis. et al.. 29 July 1995: Little Crystal Cave. 1 \&. J. E. Gardner. 13 Mar 1982: Mud River Cave. $13 \equiv .10 .1$ juv. J. Craig. 15 Dec 1973: Greens Cave. 3 ?. L. Hubricht. 20 Jul 1940 (USNM): Dallas Co.: spring. $1.0 \mathrm{~km}$ E of Louisburg, 100 specs. L. Hubricht. 16 May 1942 (USNM): spring. $5.9 \mathrm{~km}$ E of Louisburg. 50 specs. 16 May 1942 (USNM); spring. $6.7 \mathrm{~km} \mathrm{E} \mathrm{of} \mathrm{Louisburg.} 30$ specs. L. Hubricht. 16 May 1942 (USNM): Franklin Co.: Camp Cave, 3 ๑. J. E. Gardner. 6 May 1982: Elm Spring. Meramec State Park. 3.2 km E of Sullivan. 7 \&. L. Hubricht, 25 Jul 1937 (USNM); Fisher Cave. Meramec State Park. 2 ₹. L. Hubricht. $25 \mathrm{Jul} 1937$ (USNM) and 4 9. 12 c. J. E. Garner. 11 Mar 1982: Lone Hill Onyx Cave. 11 ¿. 9 Jan 1980: Lost Cave. 1 spec. J. E. Gardner. 13 Mar 1981: spring at Port Royal. $15 \Xi .5 \approx$ L. 
Hubricht. 21 Apr 1937 (USNM): Quarty Cave No.1. 14 P. 4 2. J. E. Gardner. 17 Mar 1981: temporary pond. $3.2 \mathrm{~km}$ S of Gray Summit. 1 Đ. L. Hubricht. $28 \mathrm{Mar} 1937$ (USNM): spring. $3.2 \mathrm{~km}$ S of Gray summit. 500 specs ( $\& . \sigma^{\circ}$ juvs), L. Hubricht. 29 May 1937 (USNM): Mushroom Cave. 1 ९. J. L. Craig. 1 Jun 1974: Onyx Cave. I \&. M. L. Grumbine. 8 Jan 1966: Hickory Co.: Vanderman Cave. $6 \cong .6$ ङ. J. E. Gardner. 15 Dec 1982: small stream 6.2 km NE of Weaubleau. 23 specs. L. Hubricht. 17 May 1942 (USNM): Iron Co.: small spring. $3.2 \mathrm{~km} \mathrm{~N}$ of Belleview. 75 specs. L. Hubricht. 29 Sep 1940 (USNM): Jasper Co.: Dowlers (Days) Cave Spring. Sarcoxie. 3 ९. 1 こ., 6 juvs. L. Hubricht. 22 May 1942 (USNM): Sefferson Co.: Sims Cave. 2 ₹. T. March, 27 Mar 1966: spring. $0.2 \mathrm{~km} \mathrm{~S} \mathrm{of} \mathrm{Ware.} 30$ specs. L. Hubricht. $30 \mathrm{Mar} 1941$ (USNM): small spring. $1.6 \mathrm{~km}$ W of Horine. 200 specs. L. Hubricht. 21 May 1936 (USNM): small spring. $2.4 \mathrm{~km} \mathrm{~N}$ of Antonia. $10 \cong$. L. Hubricht. 6 Jun 1937 (USNM): Rice's Spring. $4.8 \mathrm{~km} \mathrm{NE}$ of Goldman. 7 乏. L. Hubricht. 20 Nov 1938 (USNM); spring on hillside on Selma Creek. $0.8 \mathrm{~km} \mathrm{NNW}$ of Selma. 1 ¿. L. Hubricht. 2 Jun 1937 (USNM): unnamed stream on Jefferson College campus at Hillsboro.

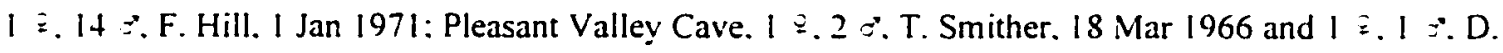
and M. Bechler. 20 Mar 1977: spring at Selma. I co. R. Kenk. 4 Oct 1967: Laclede Co.: spring at mouth of Davis Cave. 18 9. 9 J. L. Hubricht. 4 Jul 1940 (USNM): spring. $3.8 \mathrm{~km}$ W of Hazelgreen. $17 \fallingdotseq .7:$ L. Hubrichr. 23 May 1942 (USNM): smail stream near Bennett Spring. 24 specs. L. Hubricht. 16 May 1942 (USNM): Mary Lawson Cave. 2 @. L. Hubricht. $26 \mathrm{Mar} 1958$ (USNM): Lincoln Co.: Cave Spring. $4.0 \mathrm{~km}$ S of Wintield. $3 \Xi$. L. Hubricht. 22 Mar 1941 (USNM); Sherwood Forest Cave. $7.6 \mathrm{~km} \mathrm{NE}$ of Froy. $1 \cong .1$ $\approx$ ¿. J. E. Gardner. 15 Apr 1982; Madison Co.: spring. $0.8 \mathrm{~km}$ S of Mill Creek. 25 specs. L. Hubricht. 5 Apr 1941 (USNM): Maries Co.: Boulware Cave, 30 specs. L. Hubricht. 17 Apr 1941 (USNM): Marion Co.: spring. $6.2 \mathrm{~km} \mathrm{~N}$ of Palmyra, I P. L. Hubricht. 22 Mar 194 I (USNM): Miller Co.: Barnett Cave. 20 specs. L. Hubricht. 24 Aug 1940 (USNM): De Graffenreid Spring Cave. $3 \nexists .9 \Xi$. J. E. Gardner. 15 Mar 1982: Ferguson Crawl Cave. $1 \cong$. J. E. Gardner. 23 Feb 1984: McDowell Cave. 12 Ð. 1 ₹. J. E. Gardner. 19 May: 1981: small stream in small cave. $0.5 \mathrm{~km} \mathrm{SE}$ of Miller City Home. $4.8 \mathrm{~km}$ E of Tuscumbia. + juvs. L. Hubricht. 24 Aug 1940 (USNM): Oregon Co.: Blowing Spring Cave. 2 ?. 1 =’. M. Sutton. 5 Apr 1992: Brawley Hollow Cave No.1. 1 ₹. M. Sutton, 17 Dec 1994: Greer Springs. $2.1 \mathrm{~km} \mathrm{~N}$ of Greer. 100 specs. L. Hubricht. 23 Oct 1939 (USNM): Greer Spring Cave. 3 ə. 1 . J. E. Gardner. 29 Sep 1981: Lower Cane Bluff Cave. $2 \approx$. M. Sutton. 10 Sep 1994: McDowell Cave. $9 \cong .1 \approx 0$. J. E. Gardner. 21 Jul 1981: spring in Cook Hollow. 9 §. $3 \gtrsim$. T. Aley, 1972: Posy Spring Cave. 2 ₹. M. Sutton. 11 Jan 1992: Onyx Cave. $1 \equiv .1$ $\because 1$ juv. M. Sutton. 3 May 1992: Bat Cave. 3 z. J. E. Gardner. 21 Jul 1979 and $2 \equiv$. M. Sutton. 30 Oct 1993: Adams Cave No.2. 1 ₹. 1 . M. Sutton. 24 Aug 1991: Adams Cave No.1. 3 I. 2 :. M. Sutton. 24 Aug 1991: Barrett Spring Cave. $1 c^{\circ}$. M. Sutton. 10 Mar 1991: Bliss Spring Cave. $1:$ M. Sutton. 17 May 1993: Dead Man Cave. 6 ₹.2 $\approx$. J. E. Gardner. 31 Oct 1979: Turner Spring Cave. $3 \cong .5 \approx$. J. E. Gardner. 8 : May 1980: Willow Tree Cave. 11 Z. J. E. Gardner, 6 Nov 1979: Ozark Co.: Bat Spring Cave. $10 \cong$. J. E. Gardner. 27 Jun 1979 and 34 ९. J. E. Gardner. 12 Jul 1979: Perry Co.: Garbage Hole Cave. 39.2 juvs. J. J. Lewis. 3 Feb 1973: Mystery Cave. 3 9. 21 juvs, J. J. Lewis, 31 Mar 1974; Schindler Cave (= Mertz Cave 
of Mertz-Creice Cave system). 2 \&. 1 \%. L. Hubricht. 20 Sep 1941 (USNM): Tom Moore Cave. 1 . J. J. Lewis, 30 Nov 1973: Phelps Co.: Gourd Creek Cave. 30 specs. L. Hubricht. 8 Oct 1939 (USNM): Pike Co.: "inlet of cave". 30 specs. L. Hubricht. 22 Mar 1941 (USNM): Pulaski Co.: Henshaw Cave. $1 \%$. collector not given, 30 Dec 1985: Maxey (=Inca) Cave. 20 specs. L. Hubricht. 8 Oct 1939 (USNM) and 3 §. $40^{\circ}$. J. R. Holsinger and R. M. Norton and 6 ․ 1 c.. J. E. Gardner. 22 Jun 1979: Piquet Cave. $1 \equiv$. L. Hubricht. 24 Aug 1940 (USNM); Riden Cave Spring, $3.2 \mathrm{~km} \mathrm{~W}$ of Duke, 30 specs. L. Hubricht, 7 Jun 1942 (USNM): Gremp Cave. 4 9. 2 \%, L. Hubricht. 25 Aug 1940 (USNM): Asy Cave. 1 . 1 : L. Hubricht. 27 Jul 1940 (USNM); stream at mouth of Piquet Cave. 30 specs. L. Hubricht. 24 Aug 1940 (USNM): Wind Cave. 2 ค. L. Hubricht. 17 Aug 1940 (USNM): Spring Cave. 7 . 1 \&. L. Hubricht. 17 Aug 1940 (USNM): Miller Spring. $4.8 \mathrm{~km}$ NE of Big Piney. 30 specs. 21 Sep 1940 (USNM): Stockpen Cave. 1 . J. E. Gardner. 28 Nov 1978: Ralls Co.: Fisher Cave. L. Hubricht. 7 ¡. I Jun 1941 and $1 \doteqdot . J$. G. Weise. 21 Apr 1953 and $1 \cong .16$ Oct 1954: cave stream. $3.2 \mathrm{~km}$ SSW of llasso. $10 \vdots .4 z^{*}$ J. G. Weise. 18 Mar 1955: creek. $3.2 \mathrm{~km}$ W of Spalding. 3 \%. J.G. Weise. I Apr 1955: St. Charles Co.: spring at Weldon Springs, 50 specs. L. Hubricht, 2 Feb 1941 (USNM); St. Clair Co.: spring at Collins. 21 specs. L. Hubricht. 17 May 1942 (USNM): St. Francis Co.: small spring, $2.4 \mathrm{~km}$ NE of Doe Run. 100 specs. L. Hubricht. 11 Jan 1941 (USNM): St. Genevieve Co.: spring on south side of Beckett Hills. 40 specs. L. Hubricht. 11 Jan 1941 (USNM): Kolm's (=Kohms) Cave. I . L. Hubricht. 24 Aug 1941 (USNM) and I T. C. Barr. Jr.. 26 Dec 1965. 2 §. J. J. Lewis. Feb 1973: Gegg Cave. 1 §. S. B. Peck. 15 May 1966: Gillam

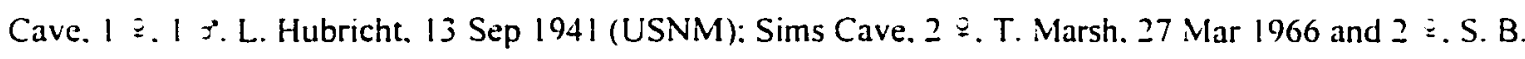
Peck. 15 May 1966: spring at Zell. 60 specs. L. Hubricht. 9 Dec 1939 (USNM): St. Louis Co.: walled spring on Keifer Creek. $1.0 \mathrm{~km} \mathrm{NW}$ of Fern Glen. 30 specs. L. Hubricht. 1 Mar 1936 (USNM): spring. Carondelet Park. 50 specs. L. Hubricht. 25 Nov 1937 (USNM): outlet of drain near hole 17. Osage Hills Country Club. Kirkwood. 14 s. L. Hubricht. 2 Dec 1934 (USNM): Spring on Glencoe Creek. $6.6 \mathrm{~km} \mathrm{NW}$ of Glencoe Station. 1 §. I ¿. L. Hubricht. I Mar 1936 (USNM): Cave of the Falls. Oakville. $1 \sqsubseteq . J$. E. Gardner. 5 Jul 1984: small spring near entrance to Normundy Osteopathic Center. Kirkwood. 28 specs. D. Bechler and N. Aspinall. 20 May 1977: temporary pond. Buder Park. $1.6 \mathrm{~km}$ SE of Valley Park. $8 \equiv .9=$ L. Hubricht. 15 Mar 1936 (USNM); outlet of drain (spring), Osage Hills Country club. Kirkwood. 200 specs. L. Hubricht. 2 Dec 1934 (USNM): pool in dry stream. 2 ङ. 1 Æ. B. M. Turlow. 2 Apr 1972 (USNM): temporary pool, $4.8 \mathrm{~km} \mathrm{NW}$ of Pattonville. 2 juvs. L. Hubricht. collection date not given (USNM): Saline Co.: Arrow Rock Cave No.2. 5 ๆ. 9 s. J. E. Gardner. 22 Apr 1982: Scott Co.: small pool on road. $0.8 \mathrm{~km} \mathrm{~N}$ of Fornfelt. $3 \approx$. W. L. Pflieger and G. M. Donald. 27 Feb 1976: spring seeps on gravel road. $2.8 \mathrm{~km} \mathrm{NW}$ of Illmo. 60 specs. W. L. Pflieger and G. M. Donald. 27 Feb 1976: Shannon Co.: Cave Spring in Cave Hollow. near Ebb and Flow spring, $9.6 \mathrm{~km} \mathrm{~N}$ of Montier. 14 $\cong$. L. Hubricht. 6 Jul 1940 (USNM): Keyhole Cave. 1 juv. J. E. Gardner. 28 Aug 1979: Bay Branch Natural Arch Cave. $1 \geq .1$ z. J. E. Gardner. 21 Mar 1984: Blue Spring. $17.6 \mathrm{~km}$ E of Eminence. 2 ₹. J. E. Gardner. 4 Jul 1980: Gillmore Spring. $9.6 \mathrm{~km} \mathrm{NW}$ of Birch Tree. $3 £ .1 \subset .3$ juvs. J. E. Gardner. 14 Mar 1984: Hollow Cave. $4 \cong .2 \approx . J$. 
E. Gardner. 14 Sep 1983: Little Gem Cave. 1 \. 2 ×. J. E. Gardner. 14 Jan 1983: Nill Cave. $11 \geqq .2 \therefore 2$ juvs. J. E. Gardner. 27 Oct 1982: Powder Mill Cave, 15 ?. 7 juvs. J. E. Gardner. 29 Aug 1979: Round Spring Caverns Spring, $19.2 \mathrm{~km} N$ of Eminence, 25 specs, L. Hubricht. 25 Sep 1938 (USNM): Blair Creek Cave. 2 ฐ. 9 ơ. J. E. Gardner. 29 May 1984: Blue Lower Cave, 9 9. 2 o. I Juv. J. E. Gardner. 2 May 1984: Flying W Cave. 1 \&. J. E. Gardner. I Dec 1982; Pump Lead Spring Cave. 1 juv. J. E. Gardner. 24 May 1984: Welch Spring Cave. $4.8 \mathrm{~km}$ NW of Akers. $30^{\circ}$, J. E. Gardner. 13 Sep 1983: Williams Ford Cave. 6 ミ. 9 juvs. J. E. Gardner. 15 Jan 1983: Wind Cave. $12 \approx$ J. E. Gardner. 30 Dec 1982: Spring near Jack's Fork. N of Teresita. $14 q .1 \approx$. L. Hubricht. I Sep 1940 (USNM): Cave Hollow Cave. $10 \cong .2 \approx 7$ L. Hubricht. 6 Jul 1940 (USNM): Pipestem Spring Cave. 1 ๔.. M. Sutton. 17 Apr 1993: Stone Co.: small stream in cave. $0.8 \mathrm{~km}$ SE of Fairy Cave and near Marvel Cave. 1 . J. G. Mackin. 19 Jul 1937 (USNM): Texas Co.: Bear Cave, 8 a. 3 c'. 1 juv. 15 Nov 1982: spring mouth of Bat Cave. $3 \equiv .1 \Xi$. L. Hubricht. 5 Jul 1940 (USNM): Dam Cave. $19.4 c^{*}$ J. E. Gardner. 13 Mar 1984: Warten Co.: small spring at the Big Rock. $2.6 \mathrm{~km}$ NE of Case. $139.1 \approx$. L. Hubricht. 7 Dec 1940 (USNM): tributary to Dry Fork. $5.6 \mathrm{~km}$ SSE of Warrenton. 169.8 ऽ. J. E. Gardner. 16 Apr 1982: Washington Co.: Hamilton Cave. $3 \cong$. L. Hubricht. $20 \mathrm{Jul} 1940$ (USNM): Great Scott Cave. 11 ₹. J. E. Gardner. 13 Sep 1979: small spring on hillside. Washıngton State Park, 2 \&. $1 \div 2$ juvs, L. Hubricht, 4 Jul 1937 (USNM): Greens Cave. $1: 1$ juv. J. L. Craig. 12 Jul 1974: Wright Co.: Smittle Cave No.2. $9 \subsetneq 3 \approx$. J. R. Holsinger et al.. 19 Aug 1968. OHIO. Adams Co.: Cave Hill Cave. $3 \approx$. T. C. Barr. Jr. and R. M. Norton. 22 Jun 1967: Cedar Fork Cave. $2 \geq *$ J. R. Holsinger. 16 Jul 1966: Turkey Creek Cave. $1 \approx$. T. C. Barr. Jr. and R. M. Norton. 22 Jun 1967: Highland Co.: cave on Baker Fork nr. Ft. Hill St Mem. I ₹. G. A. Coovert. 24 Mar 1972 (Dayton Mus. Nat. Hist): Preble Co.: outlet of drain. $0.3 \mathrm{~km} \mathrm{NW}$ of New Hope. $20 \cong$. L. Hubricht. 17 Apr 1942 (USNM): Wood Co.: outlet of drain. $2.1 \mathrm{~km} \mathrm{SE}$ of Perrysburg. 2 ₹. 2 . L. Hubricht. 18 Apr 1942 (USNM). OKLAHOMA. Cherokee Co.: Lucky Spring about $4.0 \mathrm{~km}$ E of Peggs. 2 ₹. J. J. Hoover and $W$. B. Milstead. 20 May 1981 and 11 \&. 3 Jun 1982: Dressler Cave. 4 ะ. 1 juv. C. Vavghn et al.. 26 Sep 1991: Mayes Co.: spring at roadside park $1.6 \mathrm{~km}$ E of Locust Grove. $17 \supseteq .4 \geq$. L. Hubricht. 24 May 1940 (USNM): spring at Girl Scout Camp. $4.8 \mathrm{~km} \mathrm{~S}$ of Locust Grove. 16 ¿. L. Hubricht. 22 May 1940 (USNM): spring on E side of Locust Grove. $6 \mp .3 \mathrm{c}^{3}$ J. Black. 3 Aug 1970: spring. $5.9 \mathrm{~km} \mathrm{~W}$ of Locust Grove. 50 specs. L. Hubricht. 21 May 1942; Ottawa Co.: Cave Spring ca. $8.8 \mathrm{~km}$ E of Fairland. 31 @. J. J. Hoover and W. B. Milstead. 2 Jun 1981 and $6 \%, 1 \sigma^{\circ}, 10$ juvs. 2 Jun 1982: unnamed cave spring ca. $6.2 \mathrm{~km} \mathrm{E}$ of Wyandotte. $1 \subsetneq$. J. J. Hoover and W. B. Milstead, 2 Jun 1981. PENNSYLVANIA. Fayette Co.: spring on spur of Allegheny Front Mountain. near Dulaney's Cave. ca. $19.2 \mathrm{~km}$ SE of Uniontown. $33 \equiv .1$ :. J. P. E. Morrison. $20 \mathrm{Apr} 1941$ (USNM): $210 \mathrm{~m}$ feet outside entrance of Dulany Cave. $2 \dot{\mp} .2 \prec$. R. E. Hoffmaster (collection date not given) (USNM). WEST VIRGINIA. Mason Co.: Flatfoot Creek. $3 \equiv .1 \approx$. D. Tarter. 28-29 Apr 1977: Cabell Co.: Miller Fork of Fourpole Creek. 2 . D. Tarter and Hamilton. 8 May 1974: Putnam Co.: spring on tributary of Crooked Creek. Guyaudotte. 14 . M. Mills. 7 Jun 1974.

Diagnosis.--A large species distinguished by having comparatively more spinose body than most other 
species of Crangonyx. Also having up to 10 sets of setae on posterior margin of propod of gnathopods. more flagellar segments (up to 36 ) and setae on antennae 1, 8-9 retinaculae on pleopod peduncular segment. and 6-9 setae on inner margin of dactyl of maxilliped: teison much broader than long. notched less than $1 / 3$ distance to base: outer ramus of male uropod 2 curved backward. with inner marginal spines only. Largest male, $18.0 \mathrm{~mm}$; largest female. $22.0 \mathrm{~mm}$.

Female.-- Eye small and ovate. Antenna I. about 50 percent length of body. about 1.8 times longer than antenna 2: primary flagellum with 36 segments. Antenna 2. flagellum with 8-9 segments. peduncular segments 4 and 5 with 6-7 sets of marginal setae. Mandibles subequal. spine row with 8 spines: segment 2 of palp with 18 long setae. segment 3 with 4 A setae. 2 B setae. $4 \mathrm{C}$ setae. $6 \mathrm{E}$ setae and a row of $19 \mathrm{D}$ setae. Maxilla I: inner plate with 11 apical plumose setae: palp with 5 slender spines on apex. Maxilla 2: inner plate with oblique row of $9-12$ plumose setae on inner margin. Maxilliped: inner plate apically with 8 bladelike spine teeth. 2 naked setae. and up to 17 plumose setae extending from inner margin to apex: outer plate with row of naked setae and 11 slender pectinate spines on inner margin: dactyl with 6-9 setae on inner margin: dactyi nail long. about $1 / 3$ length of dactyl.

Propod of gnathopod 1 about two times larger and longer than carpus and much broader: palm slightly longer than posterior margin. almost straight, inside and outside each with 2 rows of spine teeth, inner row with small spines and outer row with larger spines. 25 on inside. 28 on outside: defining angle with 3 notched spine teeth and 4 notched-serrate spine teeth on inside. 1 inner row of 6-7 small notched spines and I large notched and 7 serrate spine teeth on outside: $2-4$ sets of superior medial setae in groups of $2-4$ : posterior margin with 4-6 sets of setae in groups of 2-4. Dactyl with a row of many short setae on inner margin. nail short. Ventral margin of coxa 1 with 24 setae.

Propod of gnathopod 2 about 2 times larger and longer than carpus: palm oblique. convex. subequal in length to posterior margin. inside and outside margin each with 2 rows of spine teeth (larger teeth in outer row. smaller teeth in inner row), about 22-28 spine teeth on inside. 20-30 on outside: defining angle with 4 spine teeth on inside. 7-9 on outside: 7-9 sets of superior medial setae, mostly inserted in groups of 4-6: posterior margin with 10 sets of setae in groups of 4-9. Dactyl with row of many short setae on inner margin. nail short. Ventral margin of coxa 2 with 20 setae.

Coxa of pereopod 3 with 14 marginal setae. Coxa of pereopod 4 with 30 marginal setae. dactyl of pereopod 4. 30 percent length of corresponding propod. Pereopod 5: basis with 16 shallow serrations along posterior margin: 9 sets of 1-2 short spines on anterior margin: 4 long setae on anteroproximal margin. Pereopod 6: basis with 21 serrations along posterior margin: 9 short spines on anterior margin: 3 long setae on anteroproximal margin. Pereopod 7: coxa with 3 setae on the posterior margin: basis with 25 serrations along posterior margin: 13 short spines on anterior margin: dactyl about 31 percent length of corresponding propod.

Pleonal plates: posterior margin of plate 1 slightly convex. with I seta. distoposterior corner not produced: posterior margin of plate 2 nearly straight with I seta. distoposterior corner produced. ventral 
margin with up to 1 I (subventral) spines: posterior margin of plate 3 nearly straight with I seta. distoposterior corner slightly produced, ventral margin with 6 (subventral) spines. Peduncle of pleopod 1 with 8-9 retinacula: with 12 setae on outer margin. 7 setae on proximal half of inside margin.

Uropod I: inner ramus about 60 percent length of peduncle, armed with 15 spines: outer ramus with 13 spines: peduncle with row of 11-12 outer and about 2-4 inner spines. Uropod 2: inner ramus much longer and thicker than outer ramus. armed with 13 spines: outer ramus with 10 spines: peduncle with 5 outer and 3-4 inner spines. Uropod 3 : inner ramus with 1 spine, outer ramus about 1.8 times longer than peduncle. inner and outer margin each with $3-4$ set of spines in groups 2-3. Telson much broader than long. notched $1 / 5$ to little less than $1 / 2$ distance to base. each lobe with $3-7$ apical spines: spines up to 12 length of telson.

Male.--Differing from female as follows. Smaller size at maturity. Antenna 2: calceoli present on peduncular segments 4 and 5 and first 5 segments of flagellum. Uropod 2: inner ramus with 11 spines: outer ramus much smaller than inner ramus. strongly curved backward. with 2 spines on inside. lacking outer spines. apex with 5 spines: peduncle with 4 outer and 3 inner spines.

Variation.--Females were always found to have penes. The facial setae on inner lobe of maxilla 2 and plumose setae on inner lobe of maxilla I may be reduced to 4-6. Eyes are reduced in some cave populations.

Distribution and ecology.--This large. somewhat variable species. is a common inhabitant of cave streams and springs in central and southern Missouri, southern Illinois. northern Arkansas. northeastern Oklahoma. southeastern Kansas. central and southern Indiana. west-central Kentucky and southwestern Ohio. It is also recorded from southeastern Michigan. northern Ohio. western West Virginia and southwestern Pennsylvania (fig. 67). It also occurs in surface streams and ponds.

Crangony forbesi is equally common in springs and caves and does not show a significant loss of pigment or eye structure when it occurs in caves. The size is quite variable: sexually mature males range from 10.0 to $18.0 \mathrm{~mm}$ and sexually mature females from 14.0 to $22.0 \mathrm{~mm}$. Newly hatched young are 2.0 to $3.0 \mathrm{~mm}$ in length. Ovigerous females are known from all four seasons, implying that breeding is continuous throughout the year.

Crangonyx forbesi is often associated with other amphipods--commonly with Gammarus troglophilus and occasionally with Gammarus acherondytes and Bactrurus brachicaudus.

The insolitus group

Diagnosis.--Closely related to the $C$. forbesi (group) but distinguished from that species as follows. Adult size range. $5.6-9.9 \mathrm{~mm}$ : spine teeth on inner margin of dactyl of maxilliped: propod of gnathopods less than 2 times larger and longer than carpus, palms with fewer spine teeth: detining angle of propod of gnathopod I of male on outside with only one row of I large non-serrate and 2-3 serrate spine teeth: 
pleonal plates with ventral spines and setae: short lateral spines on peduncle of uropods 1 and 2 .

Remarks.--The insolitus group is based on a single species from a cave in southern Missouri. It is the only species in the family Crangonyctidae with ventro-lateral spines on the peduncles of uropods I and 2 .

Crangonx insolitus. new species

Figures 68-71

Material examined.--MISSOURI. Shannon Co.: Blue Spring Cave. $17.6 \mathrm{~km}$ E of Eminence. holotype $\Xi .2 \Xi$ (paratype), $1 \circlearrowright$ (paratype), J. E. Gardner. 2 May 1984.

Diagnnsis.--A relatively small species easily distinguished from all other species of Crangonyx by having 2 tiny ventrolateral spines on peduncles of uropods 1 and 2: spine teeth on inner margin of dactyl of maxilliped: and pleonal plates with ventral spines and setae. Largest male. $5.6 \mathrm{~mm}$ : largest female. 9.9 $\mathrm{mm}$.

Female.--Eye large and ovate. Antenna I. about 60 percent length of body. about 2 times longer than antenna 2: primary flagellum with about 32 segments. Antenna 2. flagellum with 10 segments. Mandibles subequal. spine row with 7-9 spines: segment 2 of palp with 16 setae. segment 3 with 3 A setae. 3 B setae. jC setae. $5 \mathrm{E}$ setae and a row of D setae. Maxilla $\mathrm{l}$ : inner plate with 8 apical plumose setae: palp with 9 slender spines on apex. Maxilla 2, inner plate with oblique row of 10 plumose setae on inner margin. Maxilliped: inner plate apically with 5 bladelike spines. 2 naked spines. and 5-6 plumose setae extending from inner margin to apex: outer plate with row of naked setae and 8 slender pectinate spines on inner margin: $3-4$ spine teeth and 4-5 setae on inner margin of dactyl: dactyl nail very long. 1.3 of dactyl.

Propod of gnathopod I less than 2 times larger and longer than carpus: palm almost straight. subequal in length to posterior margin. with 9 spine teeth on inside. 11 on the outside: detining angle with 5 notchedserrate spine teeth on inside. 1 large notched and 4 serrate spine teeth on outside: 3 sets of superior medial setae inserted in groups of 1-3: 6 sets of posterior marginal setae inserted in groups of 1-3. Dactyl with 2 setae on outer margin, nail long. Ventral margin of coxa 1 with 27 short setae.

Propod of gnathopod 2 less than 2 times larger and longer than carpus: palm slightly convex. subequal in length to posterior margin. with 11 spine teeth on inside. 13 on outside: defining angle with 3 spine teeth on inside. 1 large and 1 small tooth on outside: 6 sets superior medial setae inserted in groups of 2-5. rypically with 4 setae per set: posterior margin with 5 sets of setae in groups of $3-6$. Dactyl with row of short setae on inner margin and 2 setae on outer margin. Ventral margin of coxa 2 with 20 setae.

Coxa of pereopod 3 with 22 marginal setae. Coxa of pereopod 4 with 40 marginal setae: dactyl of pereopod 4, 45 percent length of corresponding propod. Pereopod 5: coxa with 3 setae on distoposterior corner: basis with 25 shallow serrations along posterior margin: 12 short spines on anterior margin: 2 short setae on anteroproximal margin. Pereopod 6: coxa with 6 setae on distoposterior comer and 3 setae on 


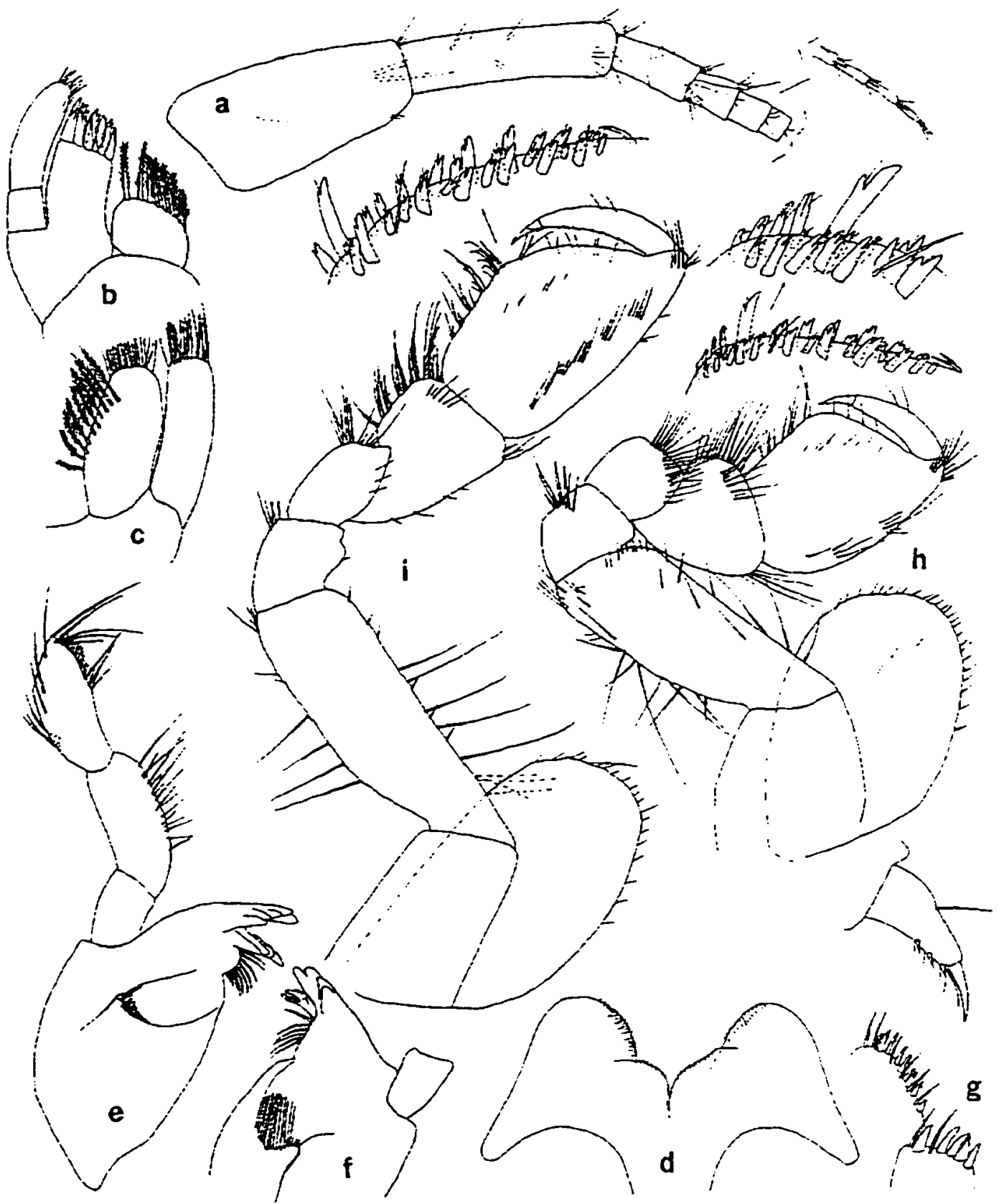

Figure 68.--Crangonyx ınsolitus, new species. Female paratype $(9.4 \mathrm{~mm})$. Blue Spring Cave. $17.6 \mathrm{~km}$ E of Eminence. Shannon Co.. Missouri: a, antenna 1: b. c. maxillae I. 2: d. lower lip: e. left mandible: f. dentate part of left mandible: g. inner and outer plates and palp dactyl of maxilliped (greatly enlarged); h. i, gnathopods 1. 2 (palmar margin enlarged). 


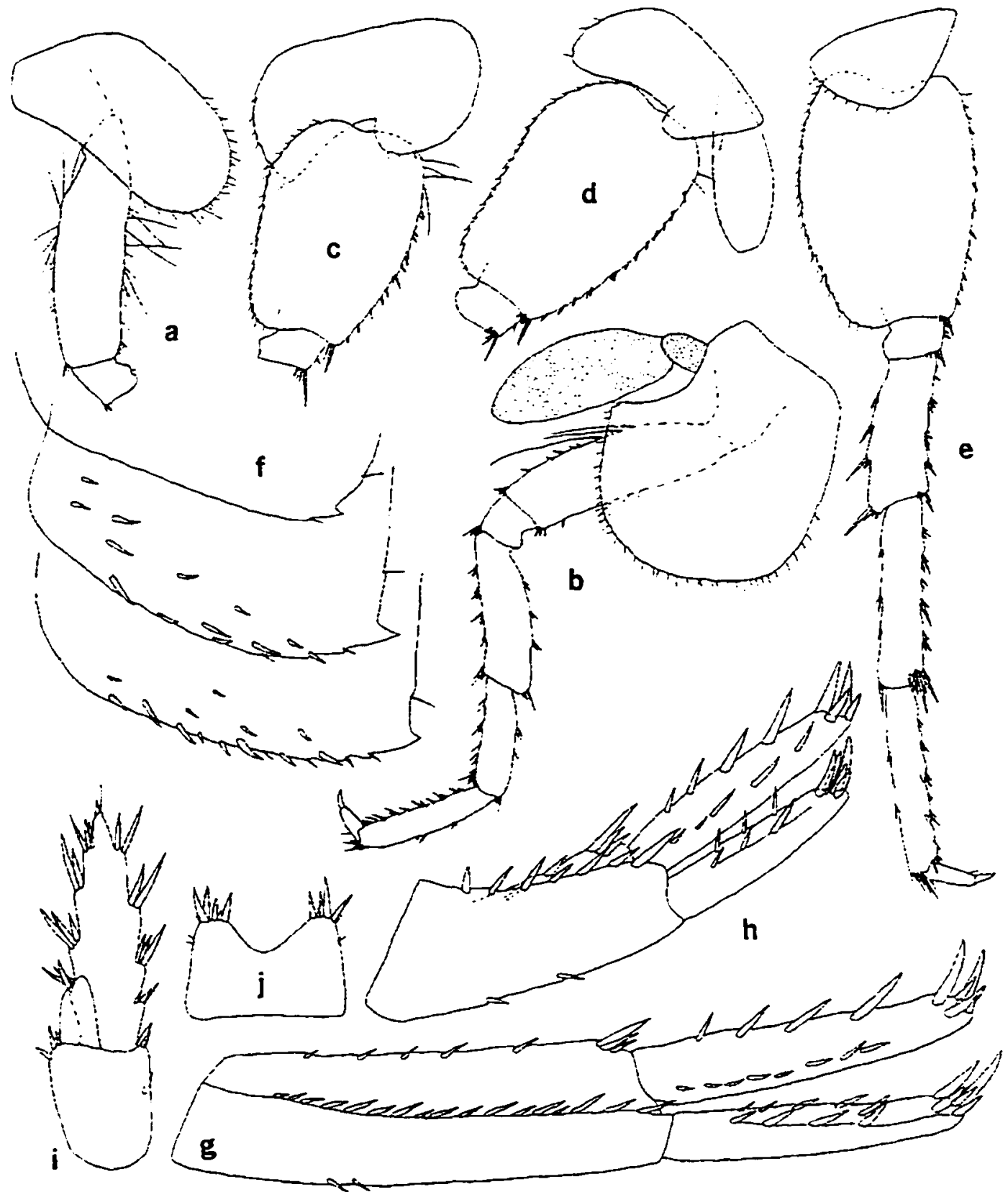

Figure 69.--Crangonyx insolitus. new species. Female paratype $(9.4 \mathrm{~mm})$. Blue Spring Cave. $17.6 \mathrm{~km}$ E of Eminence. Shannon Co.. Missouri: a. c. d. pereopods 3.5.6 (in part): b. e. pereopods 4. 7: $f$. pleonal plates: g. h, i, uropods 1.2. 3: j. telson. 


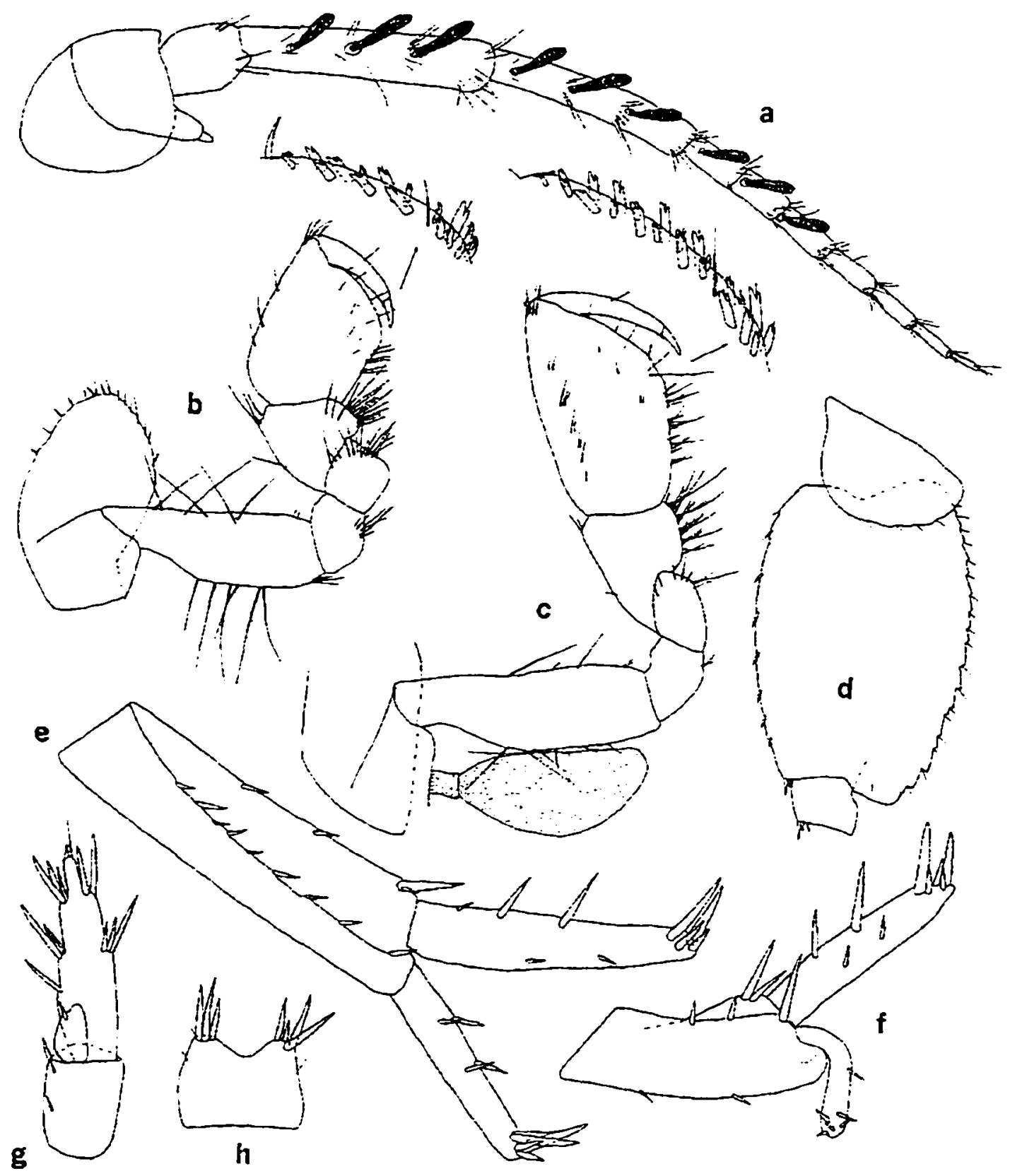

Figure 70.--Crangonyx insolitus, new species. Male paratype $(5.6 \mathrm{~mm})$. Blue Spring Cave. $17.6 \mathrm{~km} \mathrm{E}$ of Eminence. Shannon Co., Missouri: a. antenna 2: b. c. gnathopods 1. 2: d. pereopod 7 (in part): e. f. g. uropods 1. 2. 3: h. telson. 


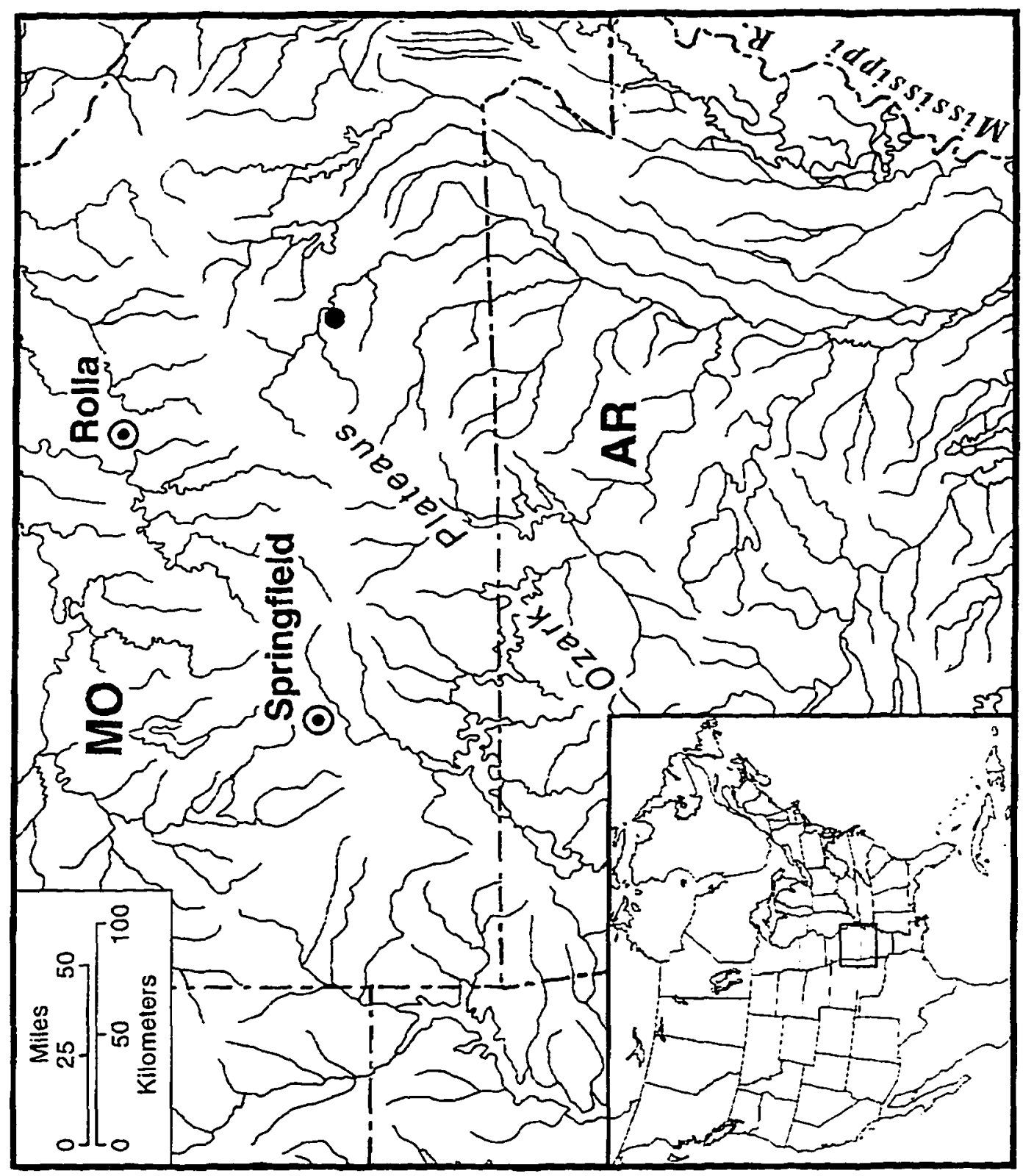


anteroventral margin; basis with 23 shallow serrations along posterior margin: 8 sets of short spines in groups of 1-2 on anterior margin: 4 long setae on anteroproximal of anterior margin. Pereopod 7 : coxa with 7 setae on the posterior margin; basis with 28 shallow serrations along posterior margin: 12 short spines on anterior margin: dactyl 33 percent length of corresponding propod.

Pleonal plates: posterior margin of plate 1 slightly convex, with 1 seta. distoposterior corner acute but not produced. with I seta on ventral margin near corner: posterior margin of plate 2 nearly straight with 1 setae. distoposterior corner produced and acute. ventral margin ivith 6-7 (subventral) spines. 3 ventral spines and 2 ventral setae: posterior margin of plate 3 nearly straight with I seta. distoposterior comer acute. ventral margin with 8 (subventral) spines. 4 ventral spines and 5 ventral setae. Peduncle of pleopod 1 with up to 5 retinaculae. about 10 setae on outside distally.

Uropod 1 : inner ramus 70 percent length of peduncle. armed with 17 spines: outer ramus with 14 spines: peduncle with row of 17 outer and 8 inner spines and 2 tiny lateral spines near ventral margin. Uropod 2: inner ramus armed with 13 spines: outer ramus with 11 spines: peduncle with 7 outer and 5 inner spines and 2 tiny lateral spines near ventral margin. Uropod 3 : inner ramus with 1 spine. outer ramus about 2 times longer than peduncle. inner and outer margin each with 4 sets of spines in groups $2-3$. Telson distinctly broader than long. notched about $1 / 3$ distance to base. each lobe with 5 apical spines: spines less than 1,2 length of telson.

Male.--Differing from female as follows. Smaller with more slender. elongate body. Antenna 2: calceoli present on peduncular segments 4 and 5 . and first 3 segments of flagellum. Propod of gnathopod 1 more than 2 times larger and longer than carpus: palm with 4 spine teeth on inside. 7 on outside: defining angle with 3 notched-serrate spine teeth on inside. I large notched and 2 serrate spine teeth on outside.

Propod of gnathopod 2 more than 2 times larger and longer than carpus: palmar margin strongly oblique. with 8 spine teeth on inside. 6 on outside: defining angle with $j$ spine teeth on inside and 4 notched spine teeth on outside.

Uropod 1: inner and outer ramus each with 9 spines: peduncle with 9 outer and 4 inner spines. lacking tiny ventrolateral spines. Uropod 2: inner ramus with 9 spines: outer ramus curved to complete right angle with peduncle. with I inner spine, outer spines absent. apex with 5 small spines: peduncle with 3 outer spines. 2 inner spines and 2 tiny lateral spines near ventral margin.

Type-locality.--Blue Spring Cave. $17.6 \mathrm{~km}$ E of Eminence. Shannon County. Missouri.

Distribution and ecology.--This rare species is known only from its type-locality (fig. 71 ), where it was collected from a pool in the entrance, in company with 5 specimens of the common epigean amphipod Hialella usteca.

Etymology.--The epithet insolitus is from Latin, meaning "unusual." "uncommon" and "strange". 


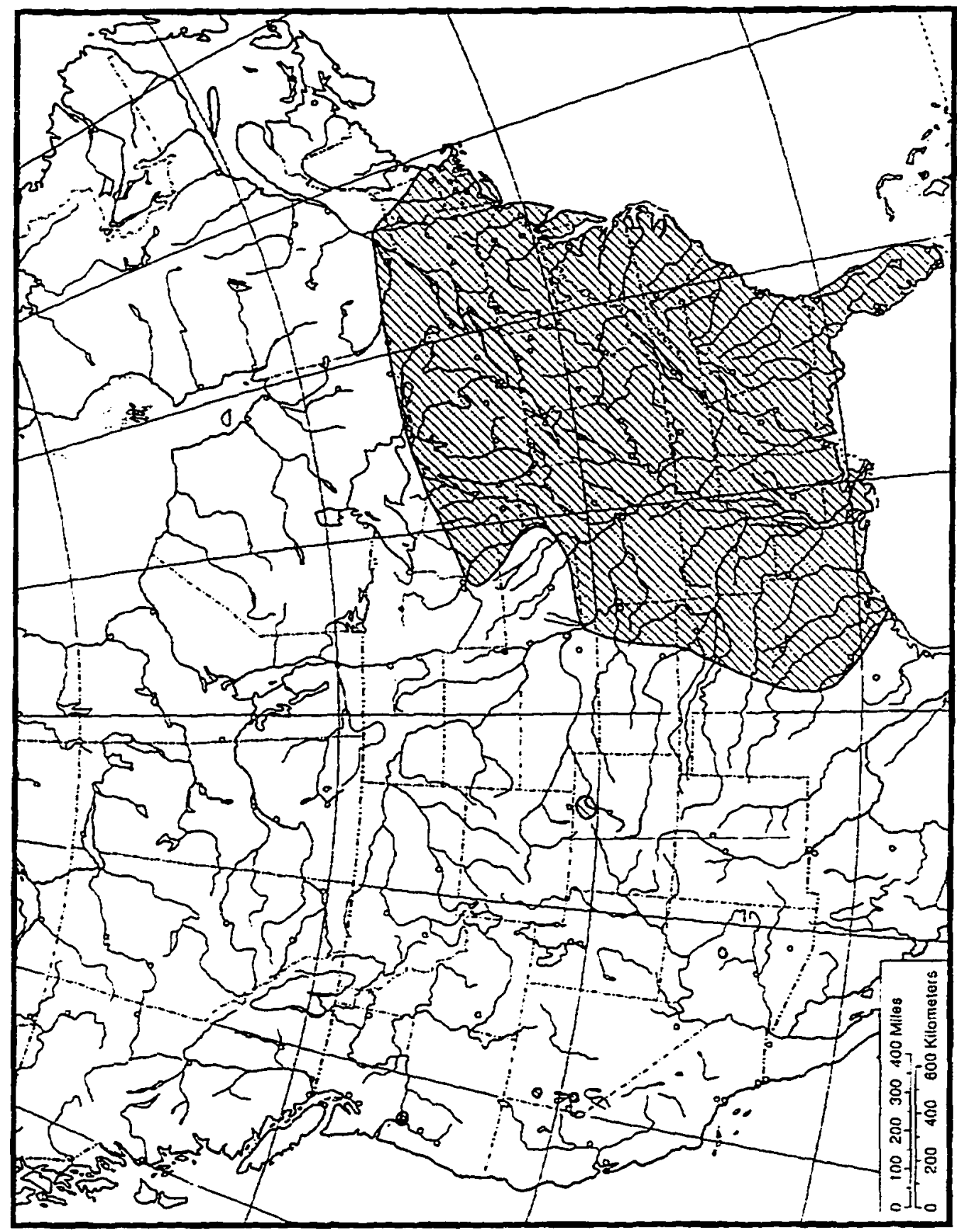

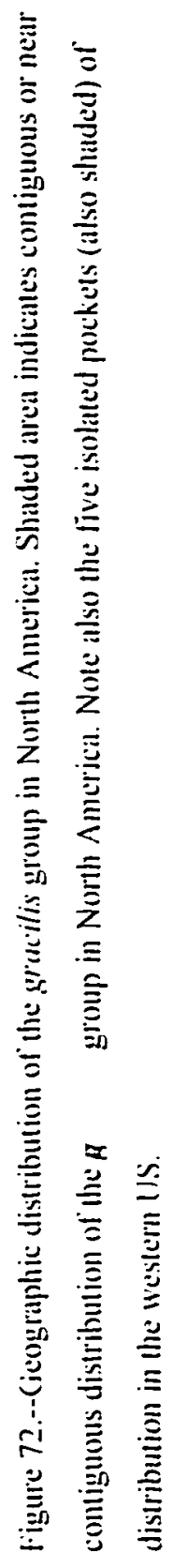




\section{The grucilis group}

Diagnosis.--Adult size. $5.0-11.0 \mathrm{~mm}$. Segment 3 of palp with only $1-2$ A setae. lacking B setae. Maxilla 1: inner plate with 5-6 apical plumose setae. Maxilla 2. inner plate with oblique row of 5-6 plumose setae on inner margin. Gnathopods with distinct sexually dimorphism. Gnathopods of female: propod less than 2 times larger and longer than carpus: palm of propod shorter than posterior margin. armed with simple spine teeth: defining angle with notched-serrate and serrate spine teeth: superior medial setae singly inserted. Gnathopods of male: carpus proportionately smaller and shorter. propod proportionately larger and broader. propod more than 2 times larger and longer than carpus: palm of propod shorter. equal or longer than posterior margin. armed with notched spine teeth: detining angle of gnathopod 1 with notched-serrate and serrate spine teeth. that of gnathopod 2 may or may not have serrate spine teeth. Posterior margins of pereopods 5-7 with fewer serrations, and anterior margin with fewer spines in both female and male in comparison with other species in the genus.

Remarks.--This group is composed of 24 species. and like the richmondensis group. it is widespread (fig. 72). However, species of the richmondensis group are larger. have stouter gnathopods with many notched spine teeth. in contrast to species of the gracilis group. which are distinctly smaller and have weak gnathopods with simple spine teeth. Many species of these two groups share the same habitat. suggesting that they have different ecological niches. For example. the large. well armed gnathopods of the richmondensis group species suggest the possibility of a carnivorous life style. whereas the weak. poorly armed gnathopods of gracilis group species suggest a significantly different feeding habit.

Key to the species of the gracilis group

1. Carpus and propod of female gnathopods very long: carpus of gnathopod 2 longer than propod

Carpus of gnathopods of female shorter than propod: propod and carpus much broader than other segments of the gnathopods.

2. Eyes absent: carpus and propod of male gnathopods very long with simple and few notched spines C. hohbsi

Eles present: carpus much short than propod of male gnathopods propod with unnotched. rod-like spines C. baculispina

3. Outer ramus of male uropod 2 with comb spines. ..+

Outer ramus of male uropod 2 without comb spines

4. Posterior margins of pleonal plates 1-3 with 2-3 setae. but lacking a seta barely above the distoposterior corner

Posterior margins of pleonal plates $\mid-3$ with 1 seta only 
5. Pleonal plates with few subventral spines (less than 8): outer ramus of uropod 3 about 1.5 times longer than peduncle. .. consimilis

Pleonal plates with more than 14 subventral spines: outer ramus of uropod 3 about 1.9 times longer than peduncle. .. longidacinlus

6. Outer ramus of male uropod 2 with ventral spines.. C. pseudogracilis

Outer ramus of male uropod 2 without ventral spines. C. floridanus

7. Posterior margins of pleonal plates $1-3$ with 2 setae. one just above distoposterior corner C. stagnicolous

Posterior margins of pleonal plates $1-3$ with 1 seta each. 8

8. Propod of female gnathopod 2 with I very large spine on distal end of outer margin of defining angle

Propod of female gnathopod 2 with I or more small spines on distal end of outer margin of detining angle. 10

9. Eyes greatly reduced to specks: inner and outer margin of outer ramus of male uropod 2 with evenly distributed spines. C. harri

Eyes large: outer ramus of male uropod 2 with 1 spine on inside and 3 spines on outside

C. montanus

10. Inside of outer ramus of male uropod 2 with several thickspines near distal end. outside with setal-like spines.

Lacking spine cluster near the distal end of male uropod 2. and outside lacking setal-like spines

11. Eyes reduced to specks or lacking: telson of female broader than long: distoposterior corners of pleonal plates 1 and 3 neither produced nor acute.

C. custellantum

Eyes normal: telson of female about as broad as long: distoposterior corners of pleonal plates

$1-3$ strongly acute C. acicularts

12. Inside of outer ramus of male uropod 2 with $1-2$ spines near end. 13 Inside of outer ramus of male uropod 2 with $0-1$ spine in the middle or with more than 3 evenly distributed spines.

13. Eyes present (not reduced); inside of outer ramus of male uropod 2 with 1 spine in the notch near end C. polustris

Eyes greatly reduced or absent: inside of outer ramus of male uropod 2 with $1-2$ spines near end but without notch

C. ohioensis

14. Inside of outer ramus of male uropod 2 with more than 3 evenly distributed spines........................15

Inside of outer ramus of male uropod 2 with 0 -1 spines...................................................... 17

15. Eyes greatly reduced or absent; defining angle of gnathopod propods of both sexes with only notched spine teeth. C. puckardi 
Eyes large: defining angle of gnathopod propods of both sexes with notched and serrated spine teeth 16

16. Telson longer than broad. notched $1 / 2$ distance to base C. $a k a$

Telson broader than long. notched less than $1 / 2$ distance to base. C. rivularis

17. Eyes large: distoposterior corners of pleonal plates $1-3$ produced and acute C. gracilis

Eyes absent or represented by specks: distoposteri or corners of pleonal plates $1-j$ not produced C. calecus Species of the gracilis group unassigned to subgroups

The following 12 species of the grarilis group remain unassigned to subgroups pending further study.

Crangonix gracilis Smith

Figures $73-77$

Crangonvx gracilis Smith. $1871: 452-453$ [type-locality: Lake Superior near St. Ignace Island. Ontario]: 1874:654-656 [in part].--Forbes. 1876:6 [in part].--Huntsman. 1915:152 [in part|.--Schellenberg. 1936:35.--Kenk. 1949:47.--Bousfield, 1958:105-108, figs. 18-19: 1970:113.--Bell. 1971:35.-Holsinger. 1972:36.--Page. 1974:94.--Barton \& Hynes, 1976:207.--Pennak \& Rosine. 1976:329.-Holsinger. 1977:252.--Barnard \& Barnard. 1983:434. map 11.--Fitzpatrick. 1983:145 [in part].-Pennak. 1989:482.--Jass\& Klausmeier. 1995:7. [Not Hay. 1888:241. 1896:206.--Banta.1907:78.-Hỹnes. 1955a:378: 1955b:624.--Cole, 1957:36].

Eucrangonyx gracilis (Smith).--Stebbing, 1899:423: 1906:388.--Weckel. 1907:32 [in part].--Pearse. 1910:73.--Hubricht and Makin.1940:199-200 [in part]. [Not Kunkel. 1918:94.--Saunders.1933:4.-. Shoemaker.1933:15.--Fleming. 1939:305.--Mackin. 1941:29.--Hynes. 1951:152].

Crangonyx gracilis gracilis Smith.--Hubricht. 1943:691 [in part].

Material Examined.--ONTARIO. Holotype $₹$ and small series of $\doteq$ and $\Xi$ topotypes. all from Lake Superior S of St. Ignace Island. S. I. Smith collector. summer 187I (USNM); stream and large pond. Beattis Pt. 5 9. 5 đ. E. L. Bousfield. 5 May 1957 (USNM); near Ottawa. I ₹. F. Johansen. summer 1918 (USNM): pool and stream at Deschines. Ottawa. I $\%$. F. Johansen. 20 Apr 1919 (USNM): pool at Yatineau River. Ottawa, 3 ․ F. Johansen. 13 May 1917 (USNM). ILLINOIS. Champaign Co.: oxbow. Urbana. 30 ב. J. G. Mackin. spring 1931 (USNM): St. Joseph (habitat not given), 3 9. J and H. Papp. 31 Mar 1946 (USNM): Cook Co.: temporary pools in Chicago, 95th Street E of 96th Ave., 50 specs (₹. :). L. Hubricht. 3 May 1941 (USNM): Marsh. $0.6 \mathrm{~km}$ W of Chicago Ridge. 200 specs ( 9 . e). L. Hubricht. 2 May $19+1$ 


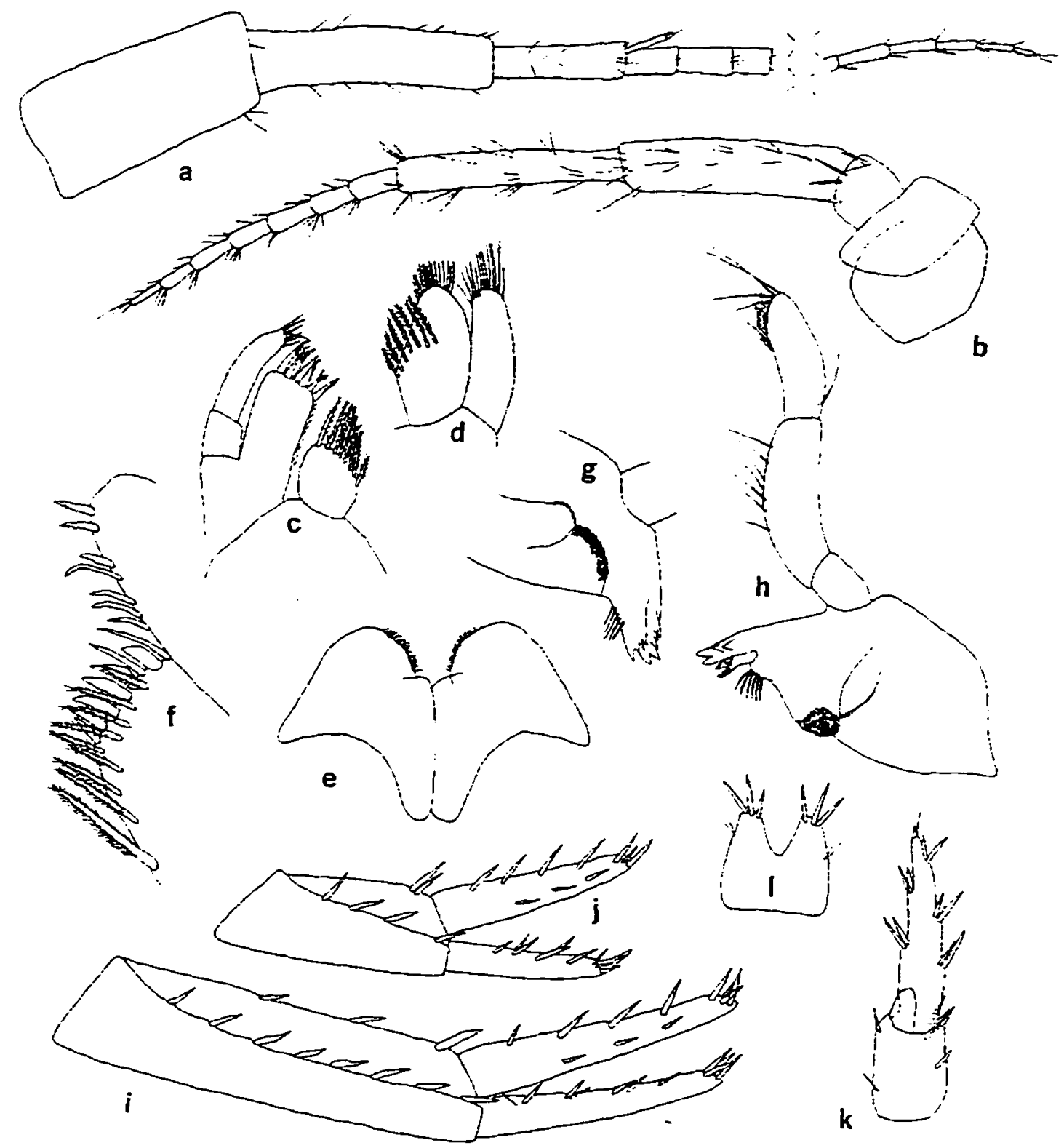

Figure 73.--Crangonvx gracilis Smith. Female $(9.2 \mathrm{~mm})$. pond just $\mathrm{S}$ of Ypsilanti Airport. $8.0 \mathrm{~km} \mathrm{SE}$ of Ann Arbor. Washtenaw Co.. Michigan: a. b. antennae 1. 2: c. d. maxillae I. 2: e. lower lip: f. inner and outer plates of maxilliped (greatly enlarged): g. dentate part of left mandible: h. right mandible: $i$. j. k. uropods 1. 2, 3: I. telson. 


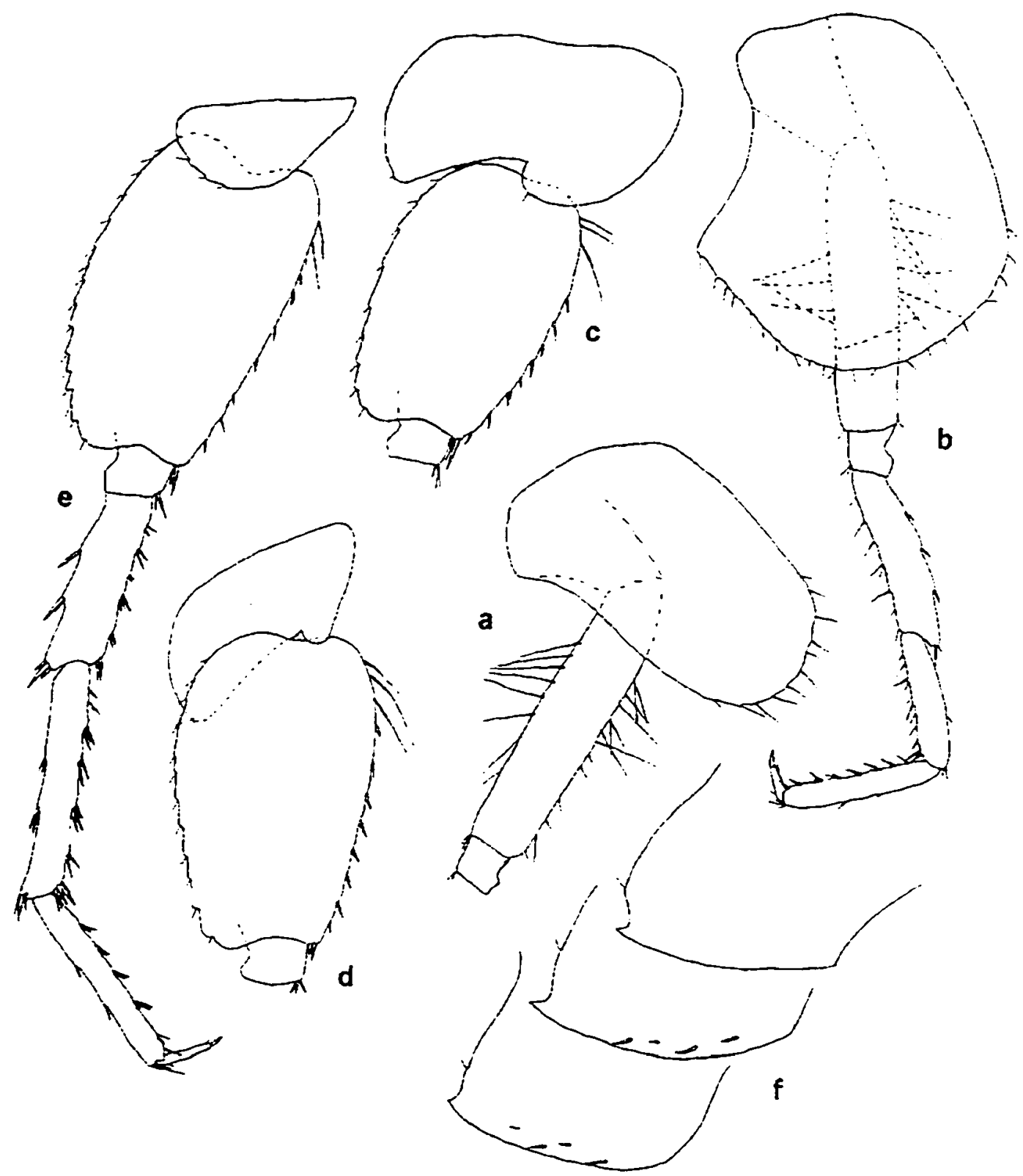

Figure 74.--Crangonyx gracilis Smith. Female $(9.2 \mathrm{~mm})$. pond just $S$ of Ypsilanti Airport. $8.0 \mathrm{~km} \mathrm{SE}$ of Ann Arbor. Washtenaw Co.. Michigan: a. c. d. pereopods 3. 5. 6: b. e. pereopods 4. 7: f. pleonal plates. 


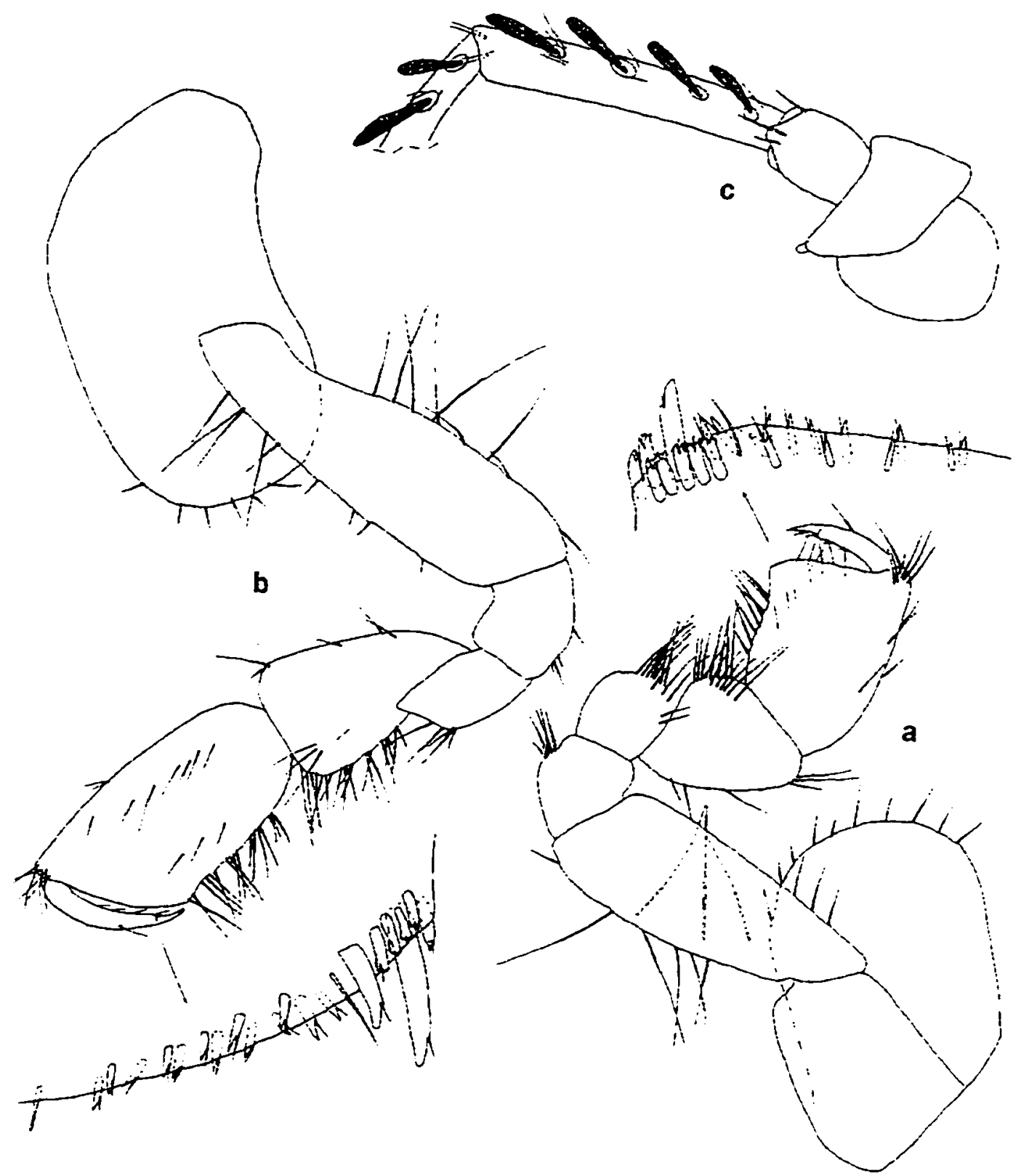

Figure 75.--Crangonyx gracilis Smith. Female $(9.2 \mathrm{~mm})$. pond just $\mathrm{S}$ of Ypsilanti Airport. $8.0 \mathrm{~km} \mathrm{SE}$ of Ann Arbor. Washtenaw Co.. Michigan: a. b. gnathopods 1. 2 (palmar margins enlarged). Male (7.0 $\mathrm{mm}$ ), same location: c, antenna 2 (in part). 


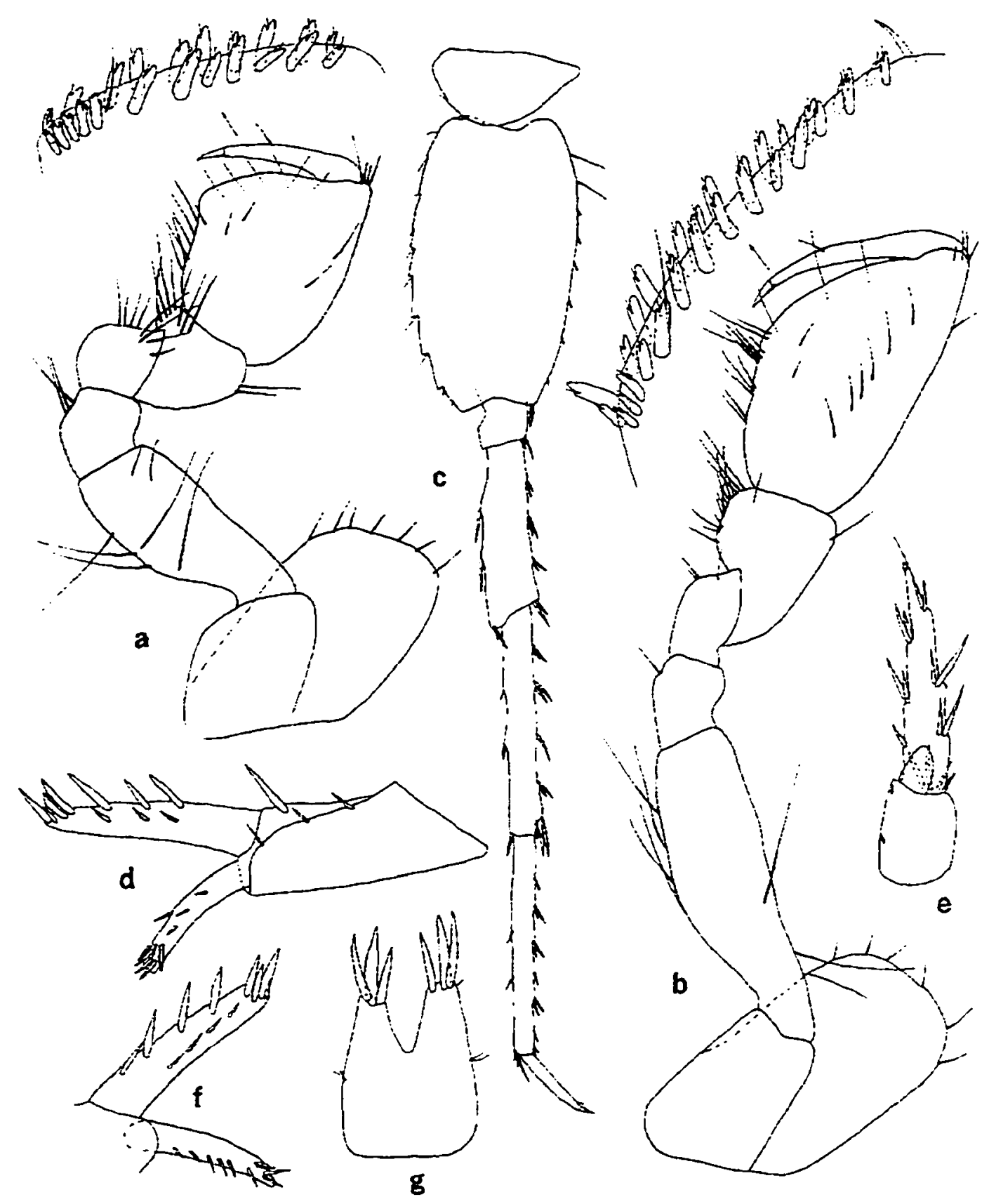

Figure 76.--Crangonyx gracilis Smith. Male $(7.0 \mathrm{~mm})$, pond just S of Ypsilanti Airport. $8.0 \mathrm{~km} \mathrm{SE}$ of Ann Arbor. Washtenaw Co.. Michigan: a. b. gnathopods 1. 2 (palmar margins enlarged): c. pereopod 7: d. e. uropod 2. 3. Male ( $5.7 \mathrm{~mm}$ ), same location: f. uropod 2 (in part): g. telson. 


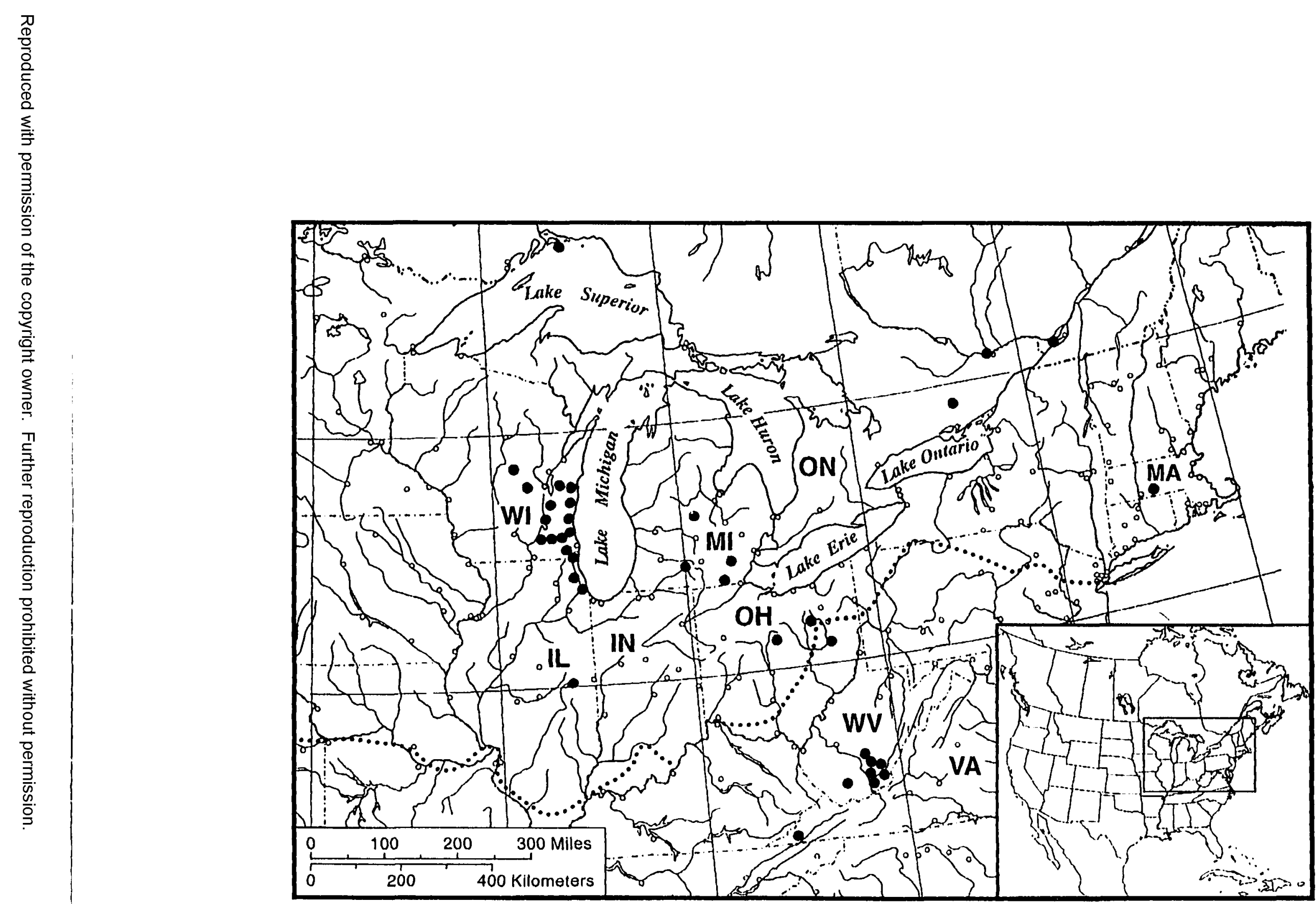

Figure 77.--1) istribution of ('rangennyx gracilis in North America. Solid circles represent I-13 closely proximate localities.

The doned line indicates the approximate southern extent of glaciation during the Pleistocene. 
(USNM): soil water in ant nest. Palatine. 1 spec. A. M. Holmquist. 15 Feb 1925 (USNM): MASSACHUSETTS. Hampton Co.: spring-fed pond. 8 ₹.4 D. G. Smith. 10 Jun 1988. MICHIGAN. Calhoun Co.: Battle Creek. Bailey Park. 3 \&. L. Hubricht. 26 Apr 1947 (USNM): outlet of drain. 10.9 km $\mathrm{N}$ of Marshall. $16 \% .6 \%$ L. Hubricht. 19 Apr 1942 (USNM): temporary pond. $3.2 \mathrm{~km} \mathrm{~N}$ of Tekonsha. 1 spec.. L. Hubricht. I May 1941 (USNM): Gratiot Co.: pond in woods, $4.0 \mathrm{~km} \mathrm{NW}$ of Alma. I g. E. P. Creaser. 20 Apr 1929 (USNM): Lenawee Co.: swale $5.0 \mathrm{~km} \mathrm{~N}$ of Addison. 100 specs ( 29 Apr 1941 (USNM); Washtenaw Co.: seep on Huron River. 2.4 km E of Foster. 50 specs ( $\Xi$. $:$ juvs). L. Hubricht. I May 1941 (USNM): temporary pond. $1.6 \mathrm{~km}$ E of Lima Center. $8 \equiv .3$ z. L. Hubricht. 1 : May 194 I (USNM): pond on Platt Road. $0.8 \mathrm{~km} \mathrm{~N}$ of Bemis Road. 150 specs ( $\subsetneq$. ڤ). L. Hubricht. 30 Apr 1941 (USNM): pond just S of Ypsilanti Airport. $8.0 \mathrm{~km} \mathrm{SE}$ of Ann Arbor. 50 specs ( $\left.\bar{\Xi} . z^{*}\right)$. L. Hubricht. 29 Apr 1941 (USNM) and 70 specs ( $₹$. $z$. juvs). 30 Apr 1941 (USNM): temporary pond west side of US 23 ca. $8.8 \mathrm{~km}$ SE of Ann Arbor, numerous specimens in 11 collections (USNM). R. Kenk. 1940-1941: temporary pond. W side of Milford Road. $16 \mathrm{~km} \mathrm{NE}$ of Ann Arbor. numerous specimens in 9 collections (USNM). R. Kenk. Feb-Jun 1941: OHIO. Holmes Co.: Butler Spring, Holmesville. $12 \cong$. A. Weaver. 4 May 1962: Morrow Co.: pond in thicket. Marengo. $1 \cong$. N. C. Furtos. 6 Apr 1932 (USNM): New Philadelphia. $6 \equiv$. V. Sterki. collection date not given (USNM): Wayne Co.: habitat not given. New Pittio. I spec. MLK. 13 Apr 1962: spring. Wooster. 5 specs. MLK. 20 Apr 1962 and 5 spec. 24 Apr 1962: spring. Shreve. 20 specs. MLK. \& May 1962: Moreland vernal pool. 9 specs. A. Weaver. 19 Apr 1964:3 specs. MLK. 9 Apr 1962. I

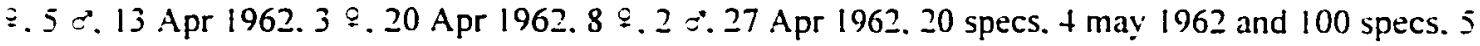
May 1962: Funk (habitat not given), 3 . A. Weaver. 26 Apr 1960. VIRGINIA. Scott Co.: seep in Glady' Fork Wetland. ca. $200 \mathrm{~m} \mathrm{~S}$ of FR 29 I, Jefferson National Forest. 9 ?. 2 :. S. M. Roble. 22 Sept 1993: WEST VIRGINIA. Fayente Co.: seep. $0.5 \mathrm{~km} \mathrm{NW}$ of Hico. $13 \Xi .4$ ₹.4 juvs.. L. Hubrisht. 23 Apr 1949 (USNM): Greenbrier Co.: Benedicts Cave. 4 2. West Virginia Association or Cave Studies, 8 Jan 1966. 2 $\Xi 1$ D. W. Fong and D. C. Culver. 14 Jan 1986: Fast spring. $4 \cong .1$ \&. S. W. Hetrick. 12 May 1972: Jarrets Water Cave. I juv.. D. C. Culver and R. Baroody. Oct 1970: Jewel Cave. $4 \mp .2 \approx$. J. R. Holsinger. 16 Sep 1967: Organ Cave System. I 9. L. G. Conrad. 14 Jan 1960 (USNM). $2: .5 \approx$. D. W. Fong. 31 Jul 1993: Organ Cave resurgence. 6 ?. 2 . D. C. Culver and D. W. Fong. It Jan 1986: Piereys Cave. $5 \vdots .1$ $\because$ J. R. Holsinger. 13 Aug 1966: pond, $3.2 \mathrm{~km} \mathrm{~N}$ of Rainelle. $13 \cong .1 \approx$. J. R. Holsinger. 6 May 1966: Monroe Co.: Cross Road Cave. 3 ․ 1 c. 1 juv., J. R. Holsinger. I Sep 1967: Hunt Cave. $5 \cong$. West Virginia Association for Cave Studies. II Dec 1965. 3 9. I o. D. C. Culver and R. Baroody. Oct 1970: Laurel Creek Cave. $2 \cong .1$ juv., J. R. Holsinger et al.. I Sep 1967: Nicolas Co.: small sluggish silt. Carnitex Ferry Battlefield State Park. I 2. W. A. Shear. 2 Apr 1967: Summers Co.: seep pool on Lick Creek Rd.. $9.6 \mathrm{~km} \mathrm{~W}$ of Athens. $19.3 \mathrm{~g}$. W. Shear. 28 Aug 1966: seepage and stream at base of cliff. Lick Creek. 11 specs.. W. A. Shear, 22 Mar 1968: Wyoming Co.: temporary pool near community swimming pool. Mullins. I $2.1 c^{*}$ J. J. Lewis, 26 Feb 1967. WISCONSIN. Calumet Co.: W branch Plum Creek at County Trunk Highway KK. 2 ९. J. P. Jass and B. Klausmeier. 23 Sep 1994 (MPM): Dodge Co.: small stream. 1.1 
$\mathrm{km}$ S of Oak Grove, 6 \%. 5 c, L. Hubricht. 21 Apr 1942 (USNM): Reeseville pool of stream into Trib. Beaver Dam River, 1 ₹. 6 juvs. J. P. Jass and B. Klausmeier, 22 Jul 1994 (MPM): Fond Du Lac Co.: creek. $4.3 \mathrm{~km} \mathrm{SW}$ of Lamartine, 30 specs $\left({ }^{\circ}, \sigma^{\prime}\right)$, L. Hubricht. 21 Apr 1942 (USNM): swampy water. Auburn township. $2 \cong$. collector unknown. 12 May 197 ! (MPM); Jefferson Co.: Near Palmyra. ponds on Emma Carlin Trail. many juvs. collector unknown. 31 Mar 1980 (MPM): Kenosha Co.: Near New Munster. $2 \cong$. collector unknown. 29 Sep 1989 (MPM): unnamed stream at County Trunk Highway AH. $3.5 \mathrm{~km} \mathrm{E} \mathrm{jct}$ Hwy $83.8 \subseteq .4$ C. J. P. Jass and B. Klausmeier. 6 May 1994 (MPM): marsh N of Junction Co. Highways $F$ \& JI. 2 ₹゙. J. P. Jass and B. Klausmeier. 6 May 1994 (MPM): pool S of Wheatland on Co. Hwy W just N. junct Hwy 50. 5 ค. 9 o. J. P. Jass and B. Klausmeier, 6 May 1994 (MPM); Manitowoc Co.: Mud Creek at County Trunk Highway J. $2.9 \mathrm{~km} \mathrm{~S}$ of Valders, I juv. B. Klausmeier. 9 Sep 1994 (MPM): Milwaukee Co.: City of Oak Creek. Bender Park. I ミ. collector unknown. 2I Apr 1990 (MPM): Menomonee River. just W of 70th St bridge. 2 . B. Klausneier. 16 Sep 1994 (MPM): Ozaukee Co.: $2.4 \mathrm{~km}$ S of Newburg. 25 specs. collector unknown. 5 May 1980 (MPM): Near Saukville. 1 ₹. 1 collector unknown. 12 May 1989 (MPM): Sapa Spruce Bog. W of Saukville, $19.12 \approx$. J. P. Jass and B. Klausmeier. 15 Jul 1994 (MPM): Portage Co.: temporary pool. $16 \mathrm{~km} \mathrm{~N}$ of Stevens Point. 50 specs. L. Hubricht. 22 Apr 1942 (USNM): Racine Co.: Goosel Branch on Olson Rd., $0.8 \mathrm{~km} \mathrm{E} \mathrm{of} \mathrm{Hwy} \mathrm{S} \mathrm{south.} 34$ specs J. P. Jass and B. Klausmeier. 13 May 1994 (MPM): pond at County Trunk Highway G \& Waukesha Co. line. 7 ₹. 1 . J. P. Jass and B. Klausmeier. 13 May 1994 (MPM): Root River at 7 Mile Road just W of Hwy 194. 2 . J. P. Jass and B. Klausmeier, 13 May 1994 (MPM): grassy pool. N side. and 7 Mile Rd.. just W of Root River Canal bridge. $12: 8 \approx$ J. P. Jass and B. Klausmeier. 13 May 1994 (MPM): Wind Lake outlet channel. $20 \cong .6 \approx$ J. P. Jass and B. Klausmeier. 13 May 1994 (MPM): Sheboygan Co.: Sheboygan River at County. Trunk Highway M. less $1.6 \mathrm{~km} \mathrm{~S}$ junct J.4פ. $1 \gtrsim 6$ juv. J. P. Jass and B. Klausmeter. 5 Aug 1994 (MPM): Waukesha Co.: Skettle Moraine State Forest. 1 ?. many juvs. collector unknown. 3 Jun 1979 (MPM): Just $\mathrm{N}$ of Eagle, $4 e^{*}$. collector not given. 9 May 1980 (MPM): Scuppernong Creek. 1 . collector not given. 20 Sep 1979 (MPM): Bark River, near Dousman at Hwy 18 Bridge. 1 \&. J. P. Jass and B. Klausmeier. 3 Jun 1994 (MPM); roadside ditch cattails. County Trunk Highway (Watertown) just W of J. 64 specs. J. P. Jass and B. Klausmeier. I Apr 1994 (MPM): unnamed stream. 2 specs. J. P. Jass and B. Klausmeier. 13 Oct 1994 (MPM): Cattail Pond, 9 ๑. J. P. Jass and B. Klausmeier. 13 Oct 1994 (MPM): marsh N of Co. Hivy NN. less $0.8 \mathrm{~km}$ E of Co. Hwy Z north. 32 specs. J. P. Jass and B. Klausmeier. Z Jun 1994 (USNM): Waushara Co.: cattail pond $N$ side County Trunk Highway XX. 9 specs. J. P. Jass and B. Klausmeier. 13 Oct 1994 (MPM): unnamed stream at County Trunk Highway XX. 2 specs. J. P. Jass and B. Klausmeier. 13 Oct 1994 (MPM): ditch, $4.8 \mathrm{~km}$ S of Aurorahville, 30 specs, L. Hubricht, 22 Apr 1942 (USNM):

Diagnosis.--A relatively small species closely allied morphologically with $C^{\circ}$. pseudogracilis and $C$. floridunus but distinguished from them by lacking comb spines on outer ramus of uropod 2 of male, and having either I spine or lacking spines on the outside margin of the outer ramus. Largest male. $5.5 \mathrm{~mm}$ : largest female. $9.0 \mathrm{~mm}$. 
Female.--Eye large. ovate. Antenna 1, about 50 percent length of body. about 1.8 times longer than antenna 2: primary flagellum with about 20-23 segments. Antenna 2. flagellum with 8-9 segments. Mandibles subequal, spine row with 5 spines: segment 2 of palp with 7 long setae: segment 3 with $1 \mathrm{~A}$ seta. I-2 C setae. 3-4 E setae and a row of I0 D setae. lacking B seta. Maxilla I: inner plate with 4-7 apical plumose setae: palp with 5-6 slender spines on apex. Maxilla 2, inner plate with oblique row of $4-8$ plumose setae on inner margin. Maxilliped: apically with 4 bladelike spine teeth. 2 naked spines. and 8 plumose setae extending from inner margin to apex: outer plate with row of naked setae and $3-4$ slender pectinate spines on inner margin, I apical plumose seta: dactyl with 1-2 setae on inner margin near nail: dactyl nail very long, about $1 / 3$ length of dactyl.

Propod of gnathopod I less than 2 times larger and longer than carpus: palm length shorter than posterior margin. slightly convex. with about 5 unnotched spine teeth on inside. about 8 on the outside: defining angle with 6 notched-serrate spine teeth on inside. I large notched and 3 serrate on outside: superior medial setae few in number and singly inserted: 5 posterior margin setae singly inserted. Dactyi with a row of $3-4$ short setae on inner margin. nail long. Ventral margin of coxa 1 with 7-8 setae.

Propod of gnathopod 2 less than 1.5 times larger than carpus which up to $3 / 4$ length of corresponding propod: palm slightly convex and oblique, much shorter than posterior margin. with about 4 unnotched spine teeth on inside. 10 on outside: defining angle with I notched spine tooth and $3-4$ notched-serrate spine teeth on inside. I large notched and I small notched-serrate spine teeth on outside: superior medial setae up to 7. singly inserted: posterior margin with 4-5 sets of setae in groups of $1-5$. Dactyl with row of short setae on inner margin. Ventral margin of coxa 2 with 7 setae.

Coxa of pereopod 3 with 6-8 marginal setae. Coxa of pereopod 4 with $13-15$ marginal setae. dacty! of pereopod 4. 35 percent length of corresponding propod. Pereopod 5: basis with 9 shallow serrations along posterior margin: 7 sets of $1-2$ short spines on anterior margin: 2 long setae on the anteroproximal of anterior margin. Pereopod 6: basis with 12 serrations along posterior margin: 6 short spines on anterior margin: 4 long setae on anteroproximal of anterior margin. Pereopod 7: coxa with 3 setae on the posterior margin: basis with 15 serrations along posterior margin: deeper near apex: 5-6 short spines on anterior margin: 3 setae on anteroproximal: dactyl about 35 percent length of corresponding propod.

Pleonal plates: posterior margin of plate I slightly convex. with I seta. distoposterior corner produced and acute: posterior margin of plate 2 nearly straight with 1 seta. distoposterior corner strongly produced and acute. ventral margin with 3-4 (subventral) spines; posterior margin of plate 3 nearly straight with 1 seta. distoposterior comer slightly produced and acute, ventral margin with 4 (subventral) spines. Peduncle of pleopod I with 2 retinaculae: lacking setae on outside margin. 1 spine on proximal inner margin.

Uropod I: inner ramus about 70 percent length of peduncle, armed with about 12 spines: outer ramus with II spines: peduncle with row of 7-8 outer and 3 inner spines. Uropod 2: inner ramus slightly shorter than peduncle. inner ramus armed with 12 spines: outer ramus with 9 spines: peduncle with 4 outer spines. 2 inner spines. Uropod 3 : inner ramus lacking spines. outer ramus about 1.8 times longer than peduncle. 
inner and outer margin each with 3 set of spines in groups $1-4$. Telson broader than long. notched about $1 / 3-1 / 2$ distance to base. each lobe with about 3 apical spines.

Male.--Differing from female as follows. Smaller, with more slender. elongate body. Antenna 2: calceoli present on peduncular segments 4 and 5 and first 4 segments of flagellum. Propod of gnathopod I more than 2 times larger and longer than carpus and broader: palm slightly oblique and convex, subequal in length to posterior margin: with 7 spine teeth on inside, 8 on outside. defining angle with $4-5$ notchedserrate spine teeth on inside: 2 notched and $1-3$ serrate spine teeth on outside. and with have another row of 4 small serrate spine teeth in larger specimens.

Propod of gnathopod 2 more than 2 times larger and longer than carpus: palmar margin oblique. slightly convex. subequal in length to posterior margin. with 9-10 spine teeth on inside. 10 on outside: defining angle with 2 notched spine teeth and 3 serrate spine teeth on inside and $4-5$ notched spine teeth on outside. Uropod 2: outer ramus deflected laterally about 45 degrees from midline: with either I spine or lacking spines on inside. bearing $2-4$ short spines on outside. apex with 5 short spines: peduncle with 3 spines on outside. about 4 spines on inside.

Distribution and ecology.--C. gracilis is found primarily in the Great Lakes region. where it has been collected from Wisconsin. Michigan. Illinois. Ohio. and Ontario (fig. 77). The eastem-most record is in central Massachusetts and the southern-most records are in southeastern West Virginia and southeastern Virginia.

C. gracilis inhabits lakes. creeks. ponds, rivers, streams. marshes. ditches. swales. and spring-ted ponds throughout most of its range. but it is found in caves in southeastern West Virginia. Ovigerous females were found in collections made in April to June, and September.

Crangonyx harri. new species

Figures $78-82$

Material examined (paratypes unless designated otherwise).--INDIANA. Clark Co.: spring at Falls of the Ohio River State Park. 1 શ. J. J. Lewis. 24 Jul 1995 and 1 \&. J. J. Lewis. 25 Jul 1995: Crawford Co.:

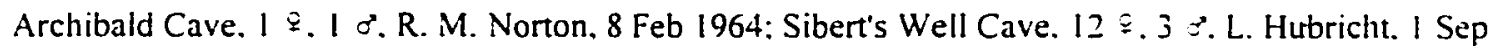
1939 (USNM), 3 ₹. 2 c. J. R. Holsinger and R. M. Norton. 12 Jun 1965 and I $\Xi . J$. J. Lewis and $M$. Lewis. 7 Dec 1992: Wyandotte Cave. 6 . 1 c. 15 juvs. L. Hubricht. I Sep 1939 (USNM): Wymans Cave. $1 \approx$ J. J. Lewis, et al. 25 May 1996: small cave on Texas Creek. $1.6 \mathrm{~km} \mathrm{~N}$ Leavenworth. 2 ‡. J. J. Lewis and M. Lewis, 26 Jun 1993: Harrison Co.: Black Medusa Cave. 1 q. J. J. Lewis and M. Lewis. 27 Jun 1993: Binkley Cave system. 3 \&. J. J. Lewis and M. Lewis, 26 Jun 1993: Horsemans Hidaway Cave. $1 \Xi$. J. J. Lewis. 27 Dec 1974: Jug Hole Cave, 3 ₹. $4 \sigma^{*}$. B. Tozer, 8 Nov 1964: Squire Boone Caverns. $69.40^{\circ}$ J. J. Lewis. 21 Jul 1975: Wallier Cave. I . B. Tozer. 31 Aug 1965: Zallmans Cave. I §. J. J. Lewis. et al.. 


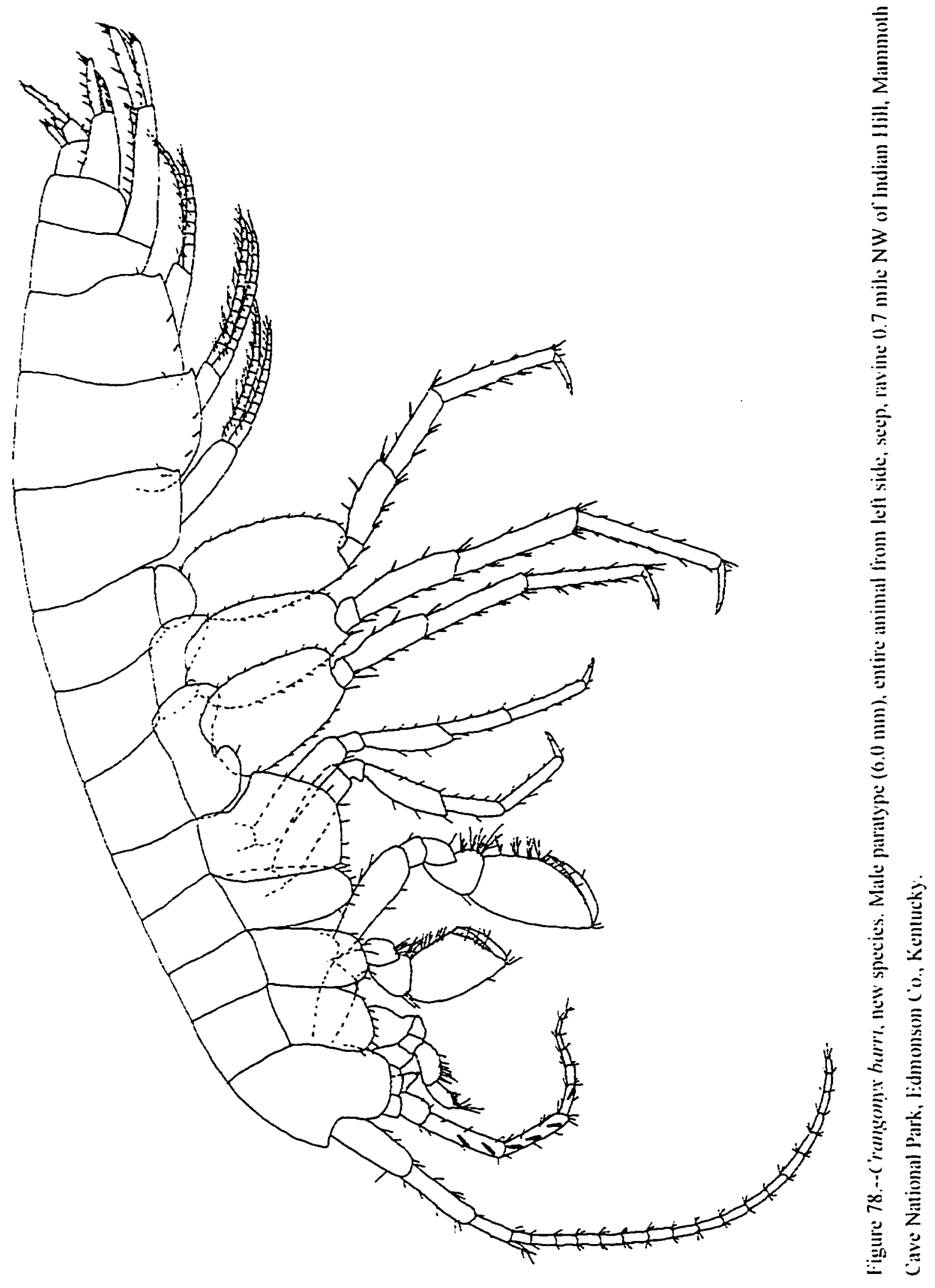




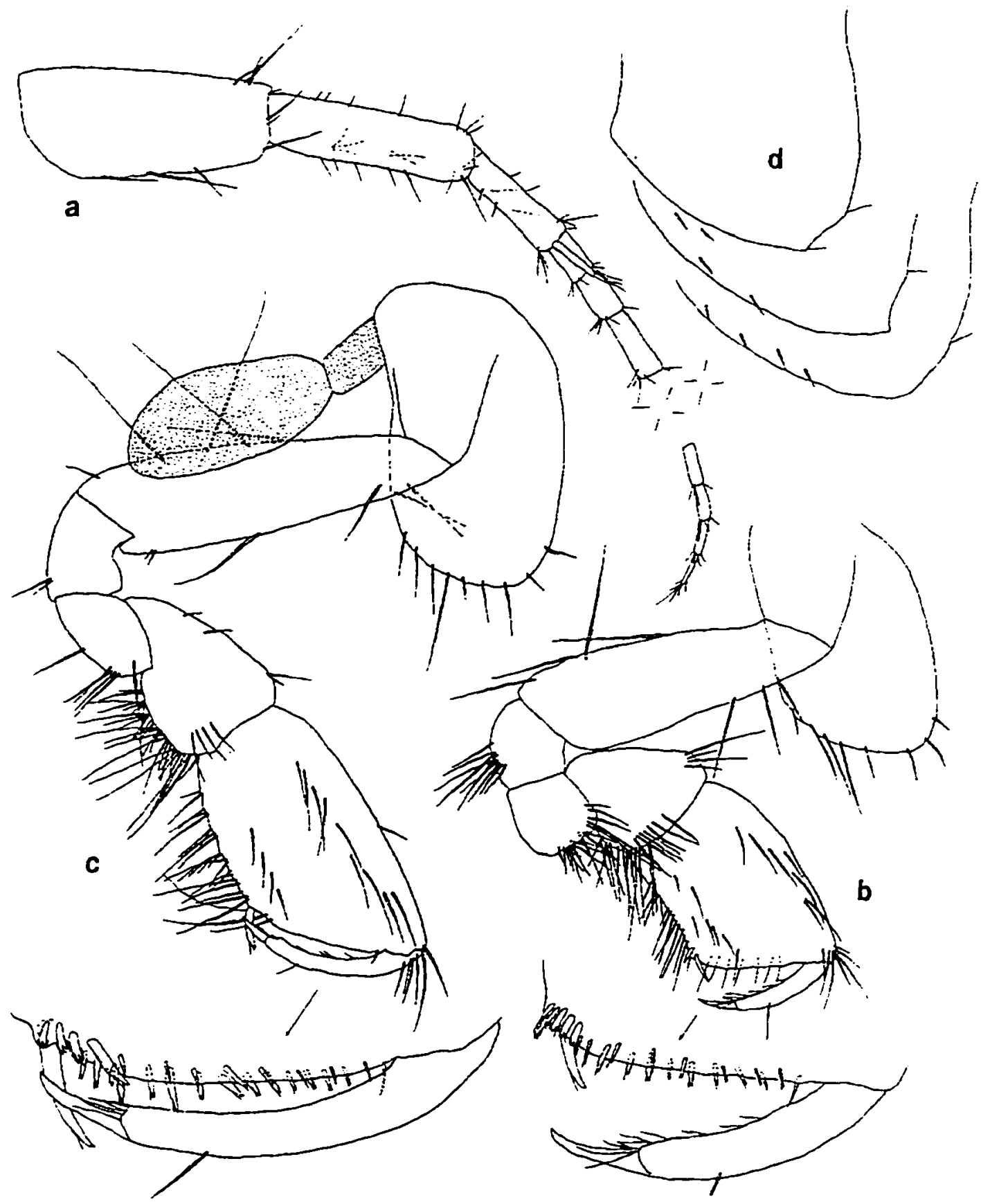

Figure 79.--Crangonix barri. new species. Female paratype $(7.8 \mathrm{~mm})$. Mammoth Cave system (Cathedral Domes). Edmonson Co.. Kentucky: a. antenna 1: b. c. gnathopods 1.2 (palmar margins enlarged): d. pleonal plates. 


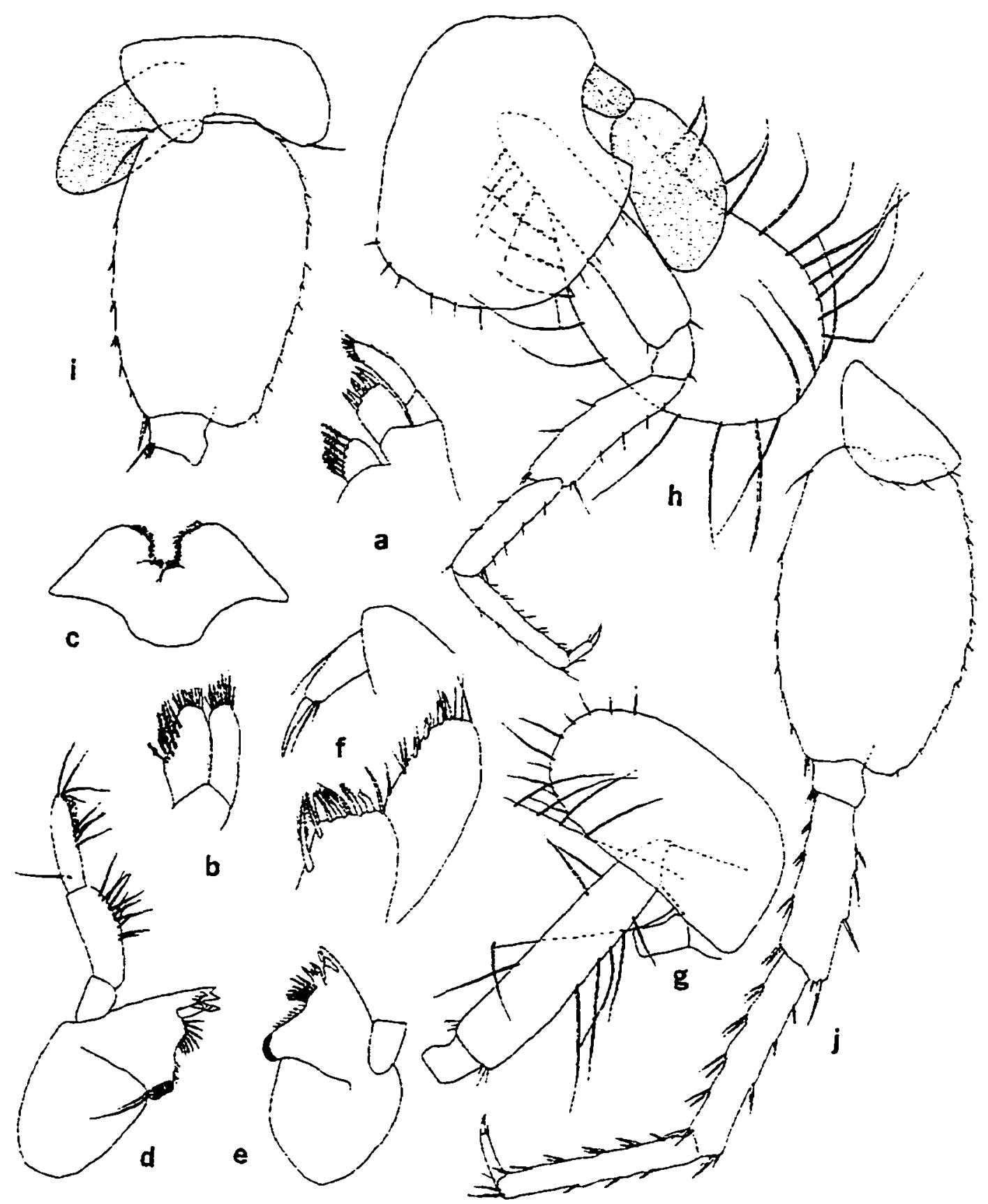

Figure 80.--Crangonyx barri. new species. Female paratype $(7.8 \mathrm{~mm})$. Mammoth Cave system (Cathedral Domes). Edmonson Co.. Kentucky: a. b. maxillae 1. 2: c. lower lip: d. left mandible: e. dentate part of right mandible: $f$. inner and outer plates and palp dactyl of maxilliped: g. i. pereopod 3. 6 (in part): h, j. pereopods 4.7 . 


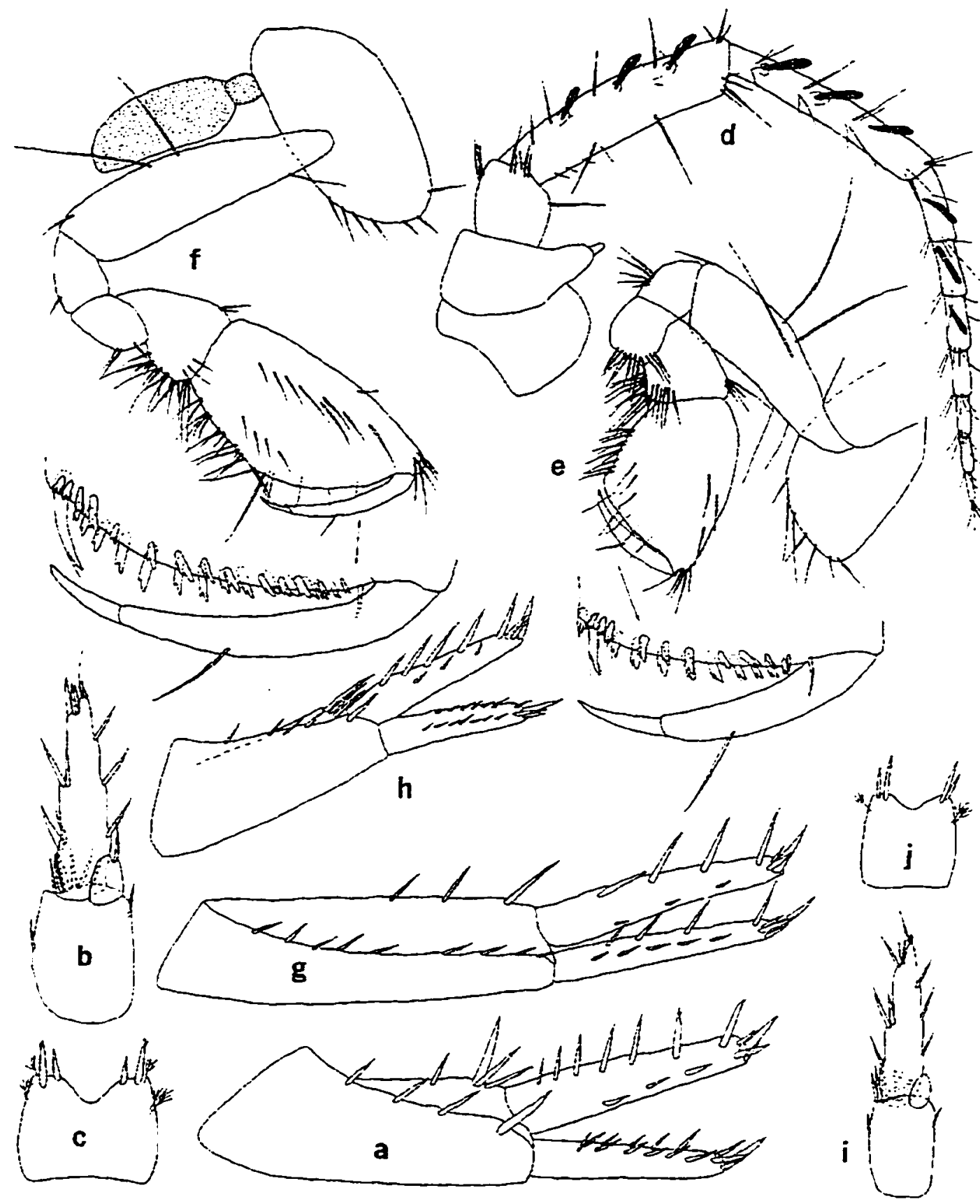

Figure 81 .--Crangonyx harri. new species. Female paratype $(7.8 \mathrm{~mm})$. Mammoth Cave system

(Cathedral Domes), Edmonson Co.. Kentucky: a. b. uropods 2. 3: c, telson. Male paratype (5.8 mm). same location: d. antenna 2: e. f. gnathopods 1.2 (palmar margins enlarged): g. h. i. uropods 1. 2 . 3: j. telson. 
6 July 1996. I Р. 1 × 18 July 1996. Martin Co.: Rose Cave. $2 \cong .1 \approx *$ H. H. Hobbs. III and M. Porter. 2 Aug 1995. KENTUCKY. Adair Co.: Cue Cave. 4 ๆ. 1 ¿. T. C. Barr. Jr.. and S. B. Peck. 29 Jul 1964: Fanny Holiday Cave, 10 ๆ, $2 \approx, 1$ juv T. C. Barr. Jr. and S. B. Peck. 29 Jul 1964: Roger's Cave. $39.4 \geqslant 1$ juv R. M. Norton, 16 Oct 1965: Scout Cave. 2 2. 2 ๔. R. M. Norton, 16 Oct 1965: Walker Cave. $3 \Xi$. S. B. Peck and T. C. Barr. Jr.. 22 Jul 1964: Barren Co.: Bowls Branch Cave. 2 ๑. T. Marsh. 18 Mar 1966: Brown Cave. 1 ฐ. R. M. Norton. 25 Sep 1965; Crowhorn Cave. $1 \subseteq .1$. T. Marsh. 19 Feb 1966: Edmonds Cave. 2 ๑. I $~ \&$. T. Marsh. R. M. Norton and T. C. Barr. Jr.. 19 Feb 1966: L and N Cave. $3 \equiv .1$ $\therefore$ I juv. J. J. Lewis et al., 17 Oct 1982 and 3 Q. I $s$. J. R. Holsinger and A. T. Leitheuser. 6 Jul 1983:

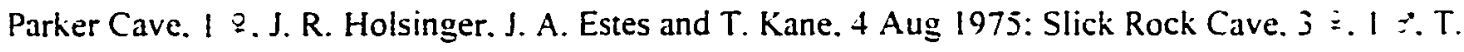
Marsh. 17 Mar 1966: Walnut Hill Cave. 1 ₹. J. R. Holsinger. G. W. Dickson. and J. A. Estes. 4 Aug 1975: Breckinridge Co.: Bandy Cave. 11 ₹. 4 \%. J. R. Holsinger and R. M. Norton. 19 Sep 1964: Boot Hill Cave. $8 \equiv .2 \geq 3$. T. C. Barr. Jr. and W. Andrews. 30 Jun 1965: Harrison Spring Cave. $1 \equiv .3 \geq$. R. M. Norton. 19 Oct 1963: Penitentiary Cave. $1 \equiv .3 \approx$. R. M. Norton. 19 Oct 1963: Thorn Hill Cave. $4 \Xi .6 \approx$. R. Fox et al. 14 Nov 1970: Casey Co.: Cedar Grove Cave, 3 ₹. T. Marsh. 20 Nov 1965: Clark Co.: Jones Cave. $4 \cong$. $1: 1$ juv. J. R. Holsinger and $14 \operatorname{Sep} 1963.99 .11$ ع. 2 juvs J. R. Holsinger and R. M. Norton. 31 Oct. 1964: Clinton Co.: Caney Branch Cave. 2 9. 2 c. A. Bosnak. 3 Oct 1980: Cumberland Co.: Mud Lick Cave. $I$ ₹. L. Hubricht (collection date not given) (USNM) and 9 ?. I $\approx$. S. B. Peck and T. C. Barr. 22 July 1964: Edmonson Co.: Big Spring Cave, $7 £ .1 \approx " .8$ juvs L. Hubricht. 10 Nov 1956 (USNM): Cathedral Cave (=Buzzard cave), 20 9. $4 \sigma^{*}$. L. Giovannoli. 22 Aug 1929 (USNM): 3 ミ. T. C. Barr. Jr.. 7 Jun 1967 and $5 \varsubsetneqq .6 c$.J. R. Holsinger. 30 Aug 1963: Cooper Spring pumphouse. Flint Ridge. Mammoth Cave Nationa! Park. $7 \cong .7 \approx^{*} 2$ juvs A. T. Leitheuser, 20 Feb - 2 Mar. 1982: Crystal Cave. $1 \equiv$. i $\approx$ A. T. Leitheuser et al., 14 Aug 1982: Mammoth Cave system (Annettes Dome). 5 § $+\jmath^{\circ}$. T. C. Barr. Jr.. 1964: Mammoth Cave system (Gauit's Way). 1 ₹. J.Lewis. 10 Dec 1983: Mammoth Cave system (Cathedral

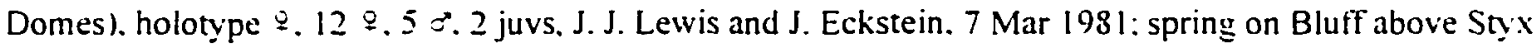
River. 4 ‡. $2 \approx$. L. Hubricht. 13 May 1956 (USNM). 2 9. T. C. Barr. 29 Sep 1973: small cave. $1.6 \mathrm{~km}$ NW of Arthur, I $\approx$. L. Hubricht. I Dec 1956 (USNM); small cave, $1.6 \mathrm{~km} \mathrm{SW}$ of Cade. $6 \cong$. L. Hubricht. 10 Nov 1956 (USNM): small cave at head of Big Hollow, $2 \cong .1 \approx$. 1 juv L. Hubricht. 9 Dec 1956 (USNM): seep. 1.1 km NW of Indian Hill. Mammoth Cave National Park. $5 \cong .1$ ऽ. L. Hubricht. 7 Apr 1957 (USNM): spring. $1.1 \mathrm{~km} \mathrm{NE}$ of Brownsville and $0.8 \mathrm{~km} \mathrm{~S}$ of Indian Hill, $1 \beth_{.}+\epsilon^{*} 1$ juv J. R. Holsinger an A. T. Leitheuser. 31 Jul 1984: Fayette Co.: stream just $S$ of Lexington off Tates Creek Road. I $\equiv$. J. R. Holsinger. 5 Apr 1965: Phelps Cave. I 9.2 , J. R. Holsinger. 15 Jul 1964: Russell Cave. $4 \fallingdotseq .3=1$. R. Holsinger and R. M. Norton. 16 Nov 1963: Grayson Co.: Condor Cave. 6 c. R. M. Norton and T. C. Barr. Jr.. 6 Aug 1966: Green Co.: Brush Creek Cave. 5 ૧. 9 §. J. R. Holsinger. 28 Sep 1963: Newt Cave. $2 \varsubsetneqq .1$ $\therefore 3$ juvs R. M. Norton. 2 Oct 1965: Saltpeter Cave. 4 \&. J. R. Holsinger and T. C. Barr. Jr.. 28 Sep 1963: Scott Cave. 1 G. T. C. Barr. Jr.. 2 Apr 1966: Three Room Cave, $2 \nsubseteq .3 \approx .3$ juvs R. M. Norton. 25 Sep 1965: Whitlock Cave. $4 \cong$. T. Marsh and R. M. Norton, 16 Apr 1966: Wilsons Cave. $5 \cong .2 \leftleftarrows$ J. R. 
Holsinger. 31 Aug 1963: Wisdom Cave. 2 q. I $\sigma^{*}$, R. M. Norton. 2 Oct 1965 and $1 \bar{z}$. I juv S. B. Peck and A. Fiske. 28 Aug - 1 Sep 1967; Woodard Cave. 6 \&. 3 ๑. J. R. Holsinger and T. C. Barr. Jr.. 28 Sep 1963: Hardin Co.: small spring. $2.9 \mathrm{~km}$ W of Summit. 4 \&. L. Hubricht. 23 Nov 1940 (USNM): Hart Co.: Cooch Webb Cave. 2 ?. 4 C. 2 juvs J. J. Lewis an T. Lewis. 27 May 1979: Cub Run Cave. $3 \mp .2$ ๔. L. Hubricht. 29 Dec 1956 (USNM) and 6 \&. T. C. Barr. Jr. and W. Andrews, 28 Nov 1964: Hidden River Cave. 89.2 $\approx .4$ juvs L. Hubricht, 30 Aug 1939 (USNM): Lone Star Paccary Cave. 1 ९. $1 \curvearrowright$. E. A. Lisowski. 22 Jun 1974: Puckett Cave, 5 9. 20 . L. Hubricht. 11 Aug 1957 (USNM): Rider's Mill Cave. $6 \equiv .1 \approx 2$ R. M.

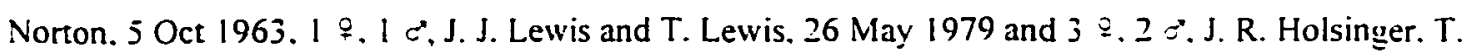
Kane and E. Benedict. 27 Jul 1981: Harrison Co.: Beaver Cave. 4 ?. J. R. Holsinger and T. C. Barr. Jr.. 16 Jul 1966: Jackson Co.: Bowman's Saltpeter Cave. 5 פ. 1 \%. R. M. Norton. 12 Sep 1964: Jefferson Co.: Highball Cave. 1 ․ N. Whitehead, 6 Oct 1964: Oxmoor Cave. $1 \subsetneq .1 \approx *$. A. George. March. 1965 and $1 \cong$. 2 3 . C. Gray. 17 Aug 1965: Jessamine Co.: Meeche Cave. 39 . R. McAdams and W. Andrews. 17 Feb 1964: Larue Co.: Cat Den Cave. 1 モ. R. M. Norton. 5 Oct 1963: Madison Co.: Adams Farm Cave. $3 \vdots .3$ :. 1 juv R. M. Norton. 7 Sep 1964 and $1 \cong$. R. McAdams. 19 Sep 1964: Meade Co.: Cold Spring Cave. 1 $\because$. W. Andrews. 29 Jun 1965: Limekiln Cave. 5 Р. T. C. Barr. Jr. and W. Andrews. 29 Jun 1965: Shackletts Cave. I ९. T. C. Barr. Jr. and R. Kuehne. 2 Dec 1961: Youstler Cave. 2 . T. C. Barr. Jr. and W. Andrews, 30 Jun 1965: small spring in Rock Haven Picnic Area. Otter Creek Park. $10 \doteq$. L. Hubricht. 6 May 1956 (USN.M): Schacklett Cave. 4 ‥ L. Hubricht. 28 Sep 1957 (USNM); Mercer Co.: Old Well Cave. 4 ₹. 2 Æ. J. R. Holsinger, 26 Jun 1965: cave near Brecklyn Bridge. $10 \Xi .5 \approx$. L. Hubricht, 28 Jun 1958 (USNM); Metcalfe Co.: Cave Hill Cave. 3 ₹. R. M. Norton. 2 Oct 1965: Piercy Cave. $19 \cong .3 \approx$. L. Hubricht. 27 Jul 1957 (USNM) and 2 ₹. T. C. Barr. Jr.. 29 Jul 1963: Monroe Co.: Bromtield Cave. $6 \cong .4$ $\approx 1$ juv J. R. Holsinger and A. T. Leitheuser. 6 Jul 1983: Nelson Co.: St. Joseph Cave. $3 \leftleftarrows .2 \approx$. T. C. Barr. Jr. and R. M. Norton. 27 Jun 1967; walled spring behind Bardstown High School. 29.2 :. J. J. Lewis and T. Lewis, 25 May 1979: Ohio Co.: Cave Hill Cave. 5 q. 2 ะ. T. C. Barr. Jr. 30 Oct 1965: Russell Co.: Miller Cave. I $\subsetneq$, S. B. Peck. 31 Jul 1964 and 6 q. 2 c". 14 juvs J. R. Holsinger and A. T. Leitheuser, 5 Jul 1983: Payne Cave. 4 \%. I C. S. B. Peck. 31 Jul 1964: Rowe Cave. $7 \varsubsetneqq$. S. Peck. 31 July 1964: Scott Co.: Slacks Cave, 3 o. J. R. Holsinger and R. M. Norton. I Feb 1964: Warren Co.: Pruett Saltpeter Cave. 3 F. T. C. Barr. Jr.. 30 Nov 1963 and 11 I 5.2 juvs. J. R. Holsinger and J. Fitzpatrick. 25 Jul 1964: Wayne Co.: Clark Cave. 1 \&. J. R. Holsinger. 3 Jul 1964: Woodford Co.: Brittons cave. 2 : 1 $\because 2$ juvs R. M. Norton, 2 Oct 1966: Clifton Cave, J P. J. R. Holsinger and T. C. Barr. Jr.. 24 Jun 1963. 1 z.. J. R. Holsinger. 3 Jul 1965. 7 \&. 1 juv R. M. Norton and T. Marsh. 5 Sep 1965. 1 ‡. T. Barr. Jr.. 2 Aug 1966: Mondays Landing Cave. $1 \% .2 \approx 1$ juv J. Reddell and W. Andrews. + Feb 1967: Nonesuch Cave. 1 juv T. C. Bart. Jr.. 10 Aug 1961.

Diagnosis.--A relatively small stygobiont species, distinguished from other species of the gracilis group by propod of gnathopod 2 of female, which bears a very large spine tooth on outer margin at end of defining angle: unproduced pleonal plates: and spines on outer ramus of uropod 2 of male distributed 
evenly on both inside and outside margins. Largest male. $7.0 \mathrm{~mm}$ : largest female. $8.5 \mathrm{~mm}$.

Female.--Eye greatly reduced. with several facets only. Antenna 1. 55-60 percent length of body. about 2.0 times longer than antenna 2: primary flagellum with 20 segments. Antenna 2. flagellum with 7-9 segments. Mandibles subequal. spine row with 7-8 spines: segment 2 of palp with 12 long setae. segment 3 with I A seta. $4 C$ setae. 4-5 E setae and a row of D setae. lacking B seta. Maxilla 1 : inner plate with 6-8 apical plumose setae: palp with several slender spines on apex. Maxilla 2. inner plate with oblique row of 6-8 plumose setae on inner margin. Maxilliped: inner plate apically with 2 bladelike spines. I naked spine. and 5-6 plumose setae on the inner margin to on apex; outer plate with row of naked setae and 3-4 slender pectinate spines on inner margin: dactyl with 2 setae near nail on inner margin: dactyl nail long. $1 / 3$ length of dactyl.

Propod of gnathopod I less than 2 times larger and longer than carpus: palm almost straight. shorter than posterior margin. with 9 unnotched spine teeth on inside. 7 on the outside: defining angle with 5 notched-serrate spine teeth on inside, I large unnotched and I serrate spine teeth on outside: interior and superior medial setae singly or doubly inserted: posterior marginal setae singly or doubly inserted. Dactyl with a row of short setae on inferior margin. nail long. Ventral margin of coxa I with 7-8 setae.

Propod of gnathopod 2 less than 2 times larger and longer than carpus: palm convex. shorter than posterior margin. with 8 unnotched spine teeth on inside. 11 on outside: defining angle inside with 1 large and $j$ notched-serrate spine teeth. outside with 2 unnotched teeth and I very large unnotched spine teeth at end: superior medial setae singly or doubly inserted: postericr margin with 6-7 sets of setae in groups of 24. Dactyl with row of short setae on inner margin. Ventral margin of coxa 2 with 9 setae.

Coxa of pereopod 3 with 10 marginal setae. Coxa of pereopod 4 with 12 marginal setae. dactyl of pereopod 4. 33 percent length of corresponding propod. Pereopod 5: basis with 14 shallow serrations along posterior margin; 9 short spines on anterior margin: 3 long setae on anteroproximal margin. Pereopod 6: basis with 12 serrations along posterior margin: 6 short spines on anterior margin: 2 setae on anteroproximal margin. Pereopod 7: coxa with 3 setae on the posterior margin: basis with 12-15 serrations along posterior margin: 6 short spines on anterior margin: I long seta on anteroproximal margin: dactyl of pereopod 7. 35 percent length of corresponding propod.

Pleonal plates: posterior margin of plate 1 convex. with 1 (rarely 2) setae. distoposterior corner neither produced nor acute: posterior margin of plate 2 nearly straight with 1 seta. distoposterior corner not produced, subacute, ventral margin with 4-5 (subventral) spines: posterior margin of plate 3 nearly straight with 1 seta. distoposterior corner indistinct rounded. ventral margin with 4 (subventral) spines. Peduncle of pleopod 1 with 2 retinaculae, lacking setae on outer margin.

Uropod I: inner ramus 80 percent length of peduncle, armed with 12 spines: outer ramus with 14 spines: peduncle with 10 outer and 3 inner spines. Uropod 2: inner ramus armed with 14 spines: outer ramus with 15 spines: peduncle with 4 outer and 4 inner spines. Uropod 3 : inner ramus with 1 spine. outer ramus about 2 times longer than peduncle, inner and outer margin each with 2-3 set of spines in groups 1-3. 
Telson broader than long. notched about $1 / 4$ distance to base. each lobe with 2 apical spines: spines about $1: 2$ length of telson.

Male.--Differing from female as follows. Smaller than with more slender. elongate body. Antenna 2: calceoli present on peduncular segments 4 and 5 and first 3 segments of flagellum.

Propod of gnathopod 1 more than 2 times larger and longer than carpus: palm with 6 spine teeth on inside. 8 on outside: defining angle with 4 notched-serrate spine teeth on inside. 1 large notched and 1 small serrate spine teeth on outside. Propod of gnathopod 2 more than 2 times larger and longer than carpus: palm strongly oblique, with 9-10 spine teeth on inside. 12 on outside: defining angle with I notched and 3 notched-serrate spine teeth on inside. 3 notched and I very large notched spine teeth on outside. Uropod 2: inner ramus with 12 spines; outer ramus curved. with 6 or more short spines on both outer margins. apex with 4 spines: peduncle with 4 outer and 4 inner spines.

Type-locality.--Mammoth Cave system (Cathedral Domes). Edmonson County. Kentucky.

Distribution and ecology.--This subterranean species is widely distributed in karst regions of southern Indiana and central Kentucky (fig. 82). Although it is primarily an inhabitant of small cave streams. it also occurs in cave drip pools. In addition. there is one record from a surface stream (presumably a wash-out) and several springs and seeps. Ovigerous females were collected from May to December and brood 10-20 yellow eggs. Sexually mature males. 4.0 to $7.0 \mathrm{~mm}$ : sexually mature females. 5.0 to $8.5 \mathrm{~mm}$.

Crungonix burri has occasionally been found in association with the amphipods $C$. forhest. C. lewrsi. Singobromus exilis. and Syntrella dentata, and the isopods Lirceus sp.. and Caecidotea sp..

Etymology.--It is pleasure to name this species in honor of Dr. Thomas C. Barr. Jr.. Emeritus Professor of Zoology. University of Kentucky, for his assistance with the collection of many of the samples examined in this study and to honor his many important contributions to our knowledge of biospeleology in North America.

Crangonvx montanus, new species

Figures $83-86$

Material examined (paratypes unless designated otherwise).--VIRGINIA. Alleghany Co: small swamp. Fairview-Clifton Forge. 33 specs, no collector given, 17 May 1940 (USNM) and $3 \supseteq .2 \approx 2.20$ May 1940 (USNM): Bath Co.: small spring-fed swamp. Nimrod Hall. $18 \nsubseteq .10 \geq$ T. K. Ellis. 20 May 1940 (USNM): large spring, $8.0 \mathrm{~km} \mathrm{~S}$ of Nimrod Hall. 16 $2.3 \circlearrowright$. 1 juv. T. K. Ellis. 20 May 1940 (USNM): spring at edge of road upper end of Panthers Gap. holotype $\supseteq .40$ specs ( $\left(z^{*}\right)$, no collector given. 18 May 1940 (USNM): Lyle Chapel. $13 \div .26$, no collector and habitat given. 21 May 1940 (USNM): Rockbridge Co.: spring on Rt. 42 in Pathers Gap. 6.4 km W of Goshen. $2 \&$ (not paratypes). R. L. Hoffman. Mar 1948 (USNM): spring-fed pond on Rt. 759 SE of Natural Bridge Station. (not paratypes). C. K. Biernbaum. 10 


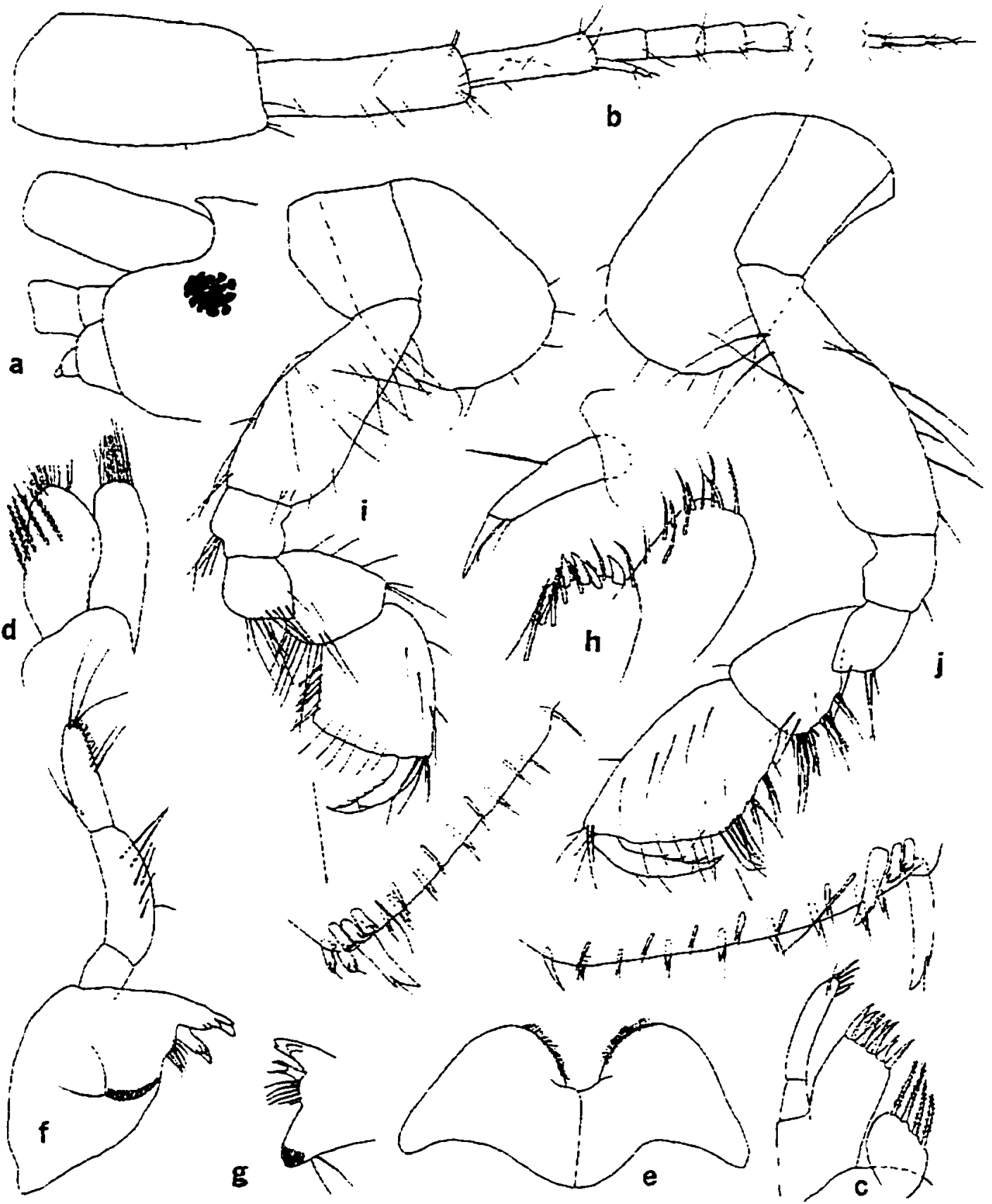

Figure 83.--Crungonyx montanus. new species. Female paratype $(7.0 \mathrm{~mm})$. small spring-fed swamp. Nimrod Hall. Bath Co., Virginia: a. head (in part): b. antenna 1: c, d. maxillae 1. 2: e. lower lip: $f$. left mandible: g. dentate part of right mandible: $h$, inner and outer plates and palp dactyl of maxilliped (greatly enlarged): i. j. gnathopods I. 2 (palmar margins enlarged). 


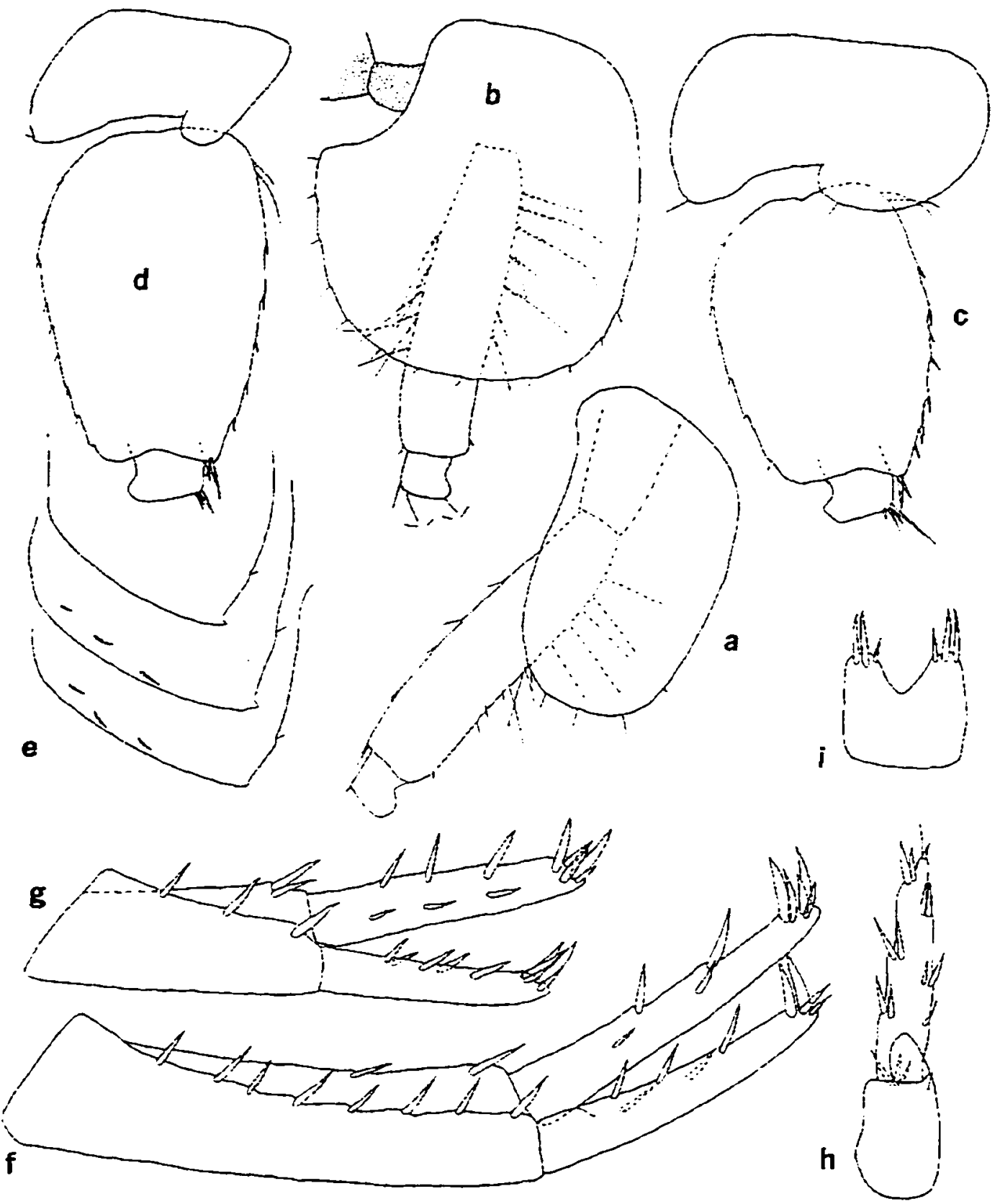

Figure 84.--Crangonyx montanus, new species. Female paratype (7.0 mm). small spring-fed swamp. Nimrod Hall. Bath Co.. Virginia: a. b. c. d. pereopods 3. 4. 5. 6 (in part): e. pleonal plates: f. g. h. uropods I, 2. 3: i, telson. 


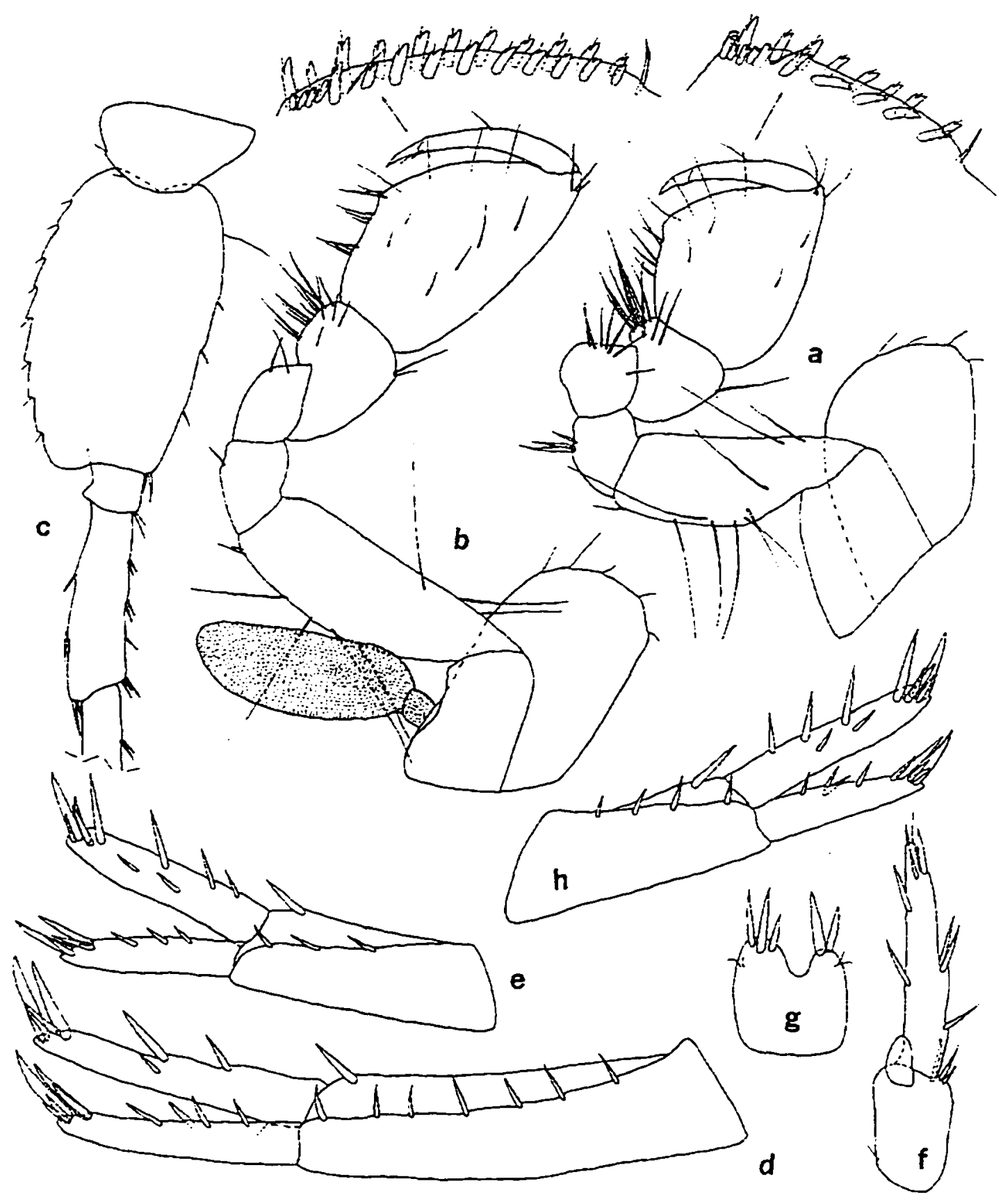

Figure 85 .--Crangonyx montanus. new species. Male paratype $(4.0 \mathrm{~mm})$. small spring-fed swamp.

Nimrod Hall. Bath Co., Virginia: a. b. gnathopods 1.2 (palmar margins enlarged): c. pereopod 7 (in part): d. e. f. uropods 1. 2, 3: g. telson: h. uropod 2. 
July 1989. WEST VIRGINIA. Greenbrier Co.: Bell karst window near Bell entrance to Ludington Cave. 5 $\Sigma$ (not paratypes), D. Fong, 5 Mar 1995.

Diagnosis.--A small species of the gracilis group morphologically very similar to $C$. harri but distinguished from it by having well developed eyes: more distinct corners of produced pleonal plates: and fewer spines on outer ramus of uropod 2 of male. Largest male. $5.0 \mathrm{~mm}$ : largest female. $8.0 \mathrm{~mm}$.

Female.--Eye large. ovate. Antenna I. about 50 percent length of body, about 1.8 times longer than antenna 2: primary flagellum with about 20 segments. Antenna 2. flagellum with 8-9 segments. peduncle 4 with 2-j sets and peduncle 5 with $3-4$ set marginal setae. Mandibles subequal. spine row with 6 spines: segment 2 of palp with 6-7 long setae. segment 3 with I A seta, $2 \mathrm{C}$ setae. $3 \mathrm{E}$ setae and a row of $\mathrm{D}$ setae. lacking B seta. Maxilla 1: inner plate with 4 apical plumose setae: palp with 5-6 slender spines and 3 setae on apex. Maxilla 2. inner plate with oblique row of 5 plumose setae on inner margin. Maxilliped: inner plate apically with 3 bladelike spines. 2 naked spines. and 5-6 plumose setae extending from inner margin to apex: outer plate with row of naked setae and $3-4$ pectinate slender spines on inner margin. 1 apical plumose seta: dactyl with 2-3 setae near nail on inner margin: dactyl nail very long. 1 , 3 length of dactyl.

Propod of gnathopod I less than 2 times larger and longer than carpus: palm almost straight. subequal in length to posterior margin. with about 4 unnotched spine teeth on inside. 6 on outside: defining angle with 1 large notched and 2 small serrate spine teeth on outside. 3 small notched-serrate spine teeth on inside: superior medial setae few in number and singly inserted; posterior margin with 4 sets of setae singly inserted or in groups of 2. Dactyl with a row of 5-6 short setae on inferior margin. nail long. Ventral margin of coxa I with 6 setae.

Propod of gnathopod 2 less than 2 times larger and longer than carpus: palm almost straight. oblique. slightly shorter than posterior margin. with about 6 unnotched spine teeth on inside. 8 on outside: defining angle with 1 large notched and two small notched-serrate spine teeth on inside. I large notched spine tooth on outside end: superior medial setae singly inserted. posterior margin with 5 sets of long setae in groups of 1-6. Dactyl with row of 3-4 short setae on inner margin. Ventral margin of coxa 2 with 6 setae.

Coxa of pereopod 3 with 6 marginal setae. Coxa of pereopod 4 with 12 marginal setae. dactyl about 40 percent length of corresponding propod. Pereopod 5: basis with 7 shallow serrations along posterior margin: 7 short spines on anterior margin: 2-3 long setae on anteroproximal margin. Pereopod 6: basis with 10 shallow serrations along posterior margin: 5 short spines on anterior margin: 2-3 long setae on anteroproximal margin. Pereopod 7: coxa with 2 setae on the posterior margin: basis with 10 shallow serrations along posterior margin: 5 short spines on anterior margin: I seta on anteroproximal margin: dactyl about 35 percent length of corresponding propod.

Pleonal plates: posterior margin of plate I slightly convex, with I seta, distoposterior corner small. acute: posterior margin of plate 2 nearly straight with 1 or 2 setae, distoposterior corner weakly produced and acute. ventral margin with 3 (subventral) spines; posterior margin of plate 3 slightly convex with 1 seta. distoposterior corner not produced, subacute, ventral margin with 3 (subventral) spines. Peduncle of 
pleopod I with 2 retinaculae. lacking setae on outside margin.

Uropod 1: inner ramus about 70 percent length of peduncle. armed with 9 spines: outer ramus with 9 spines: peduncle with 8 outer and 2 inner spines. Uropod 2: inner ramus. inner ramus armed with 11 spines: outer ramus with 9 spines: peduncle with 3 outer spines. 2 inner spines. Uropod 3 : inner ramus with lacking spines, outer ramus about 2 times longer than peduncle. inner and outer margin each with $3-4$ set of spines in groups $1-3$. Telson slightly broader than long, notched about $1 / 3$ distance to base. each lobe with 3 apical spines; spines about $1 / 2$ length of telson.

Male.--Differing from female as follows. Smailer with more slender. elongate body. Antenna 2: calceoli present on peduncular segments 4 and 5 , and first 2 segments of flagellum.

Propod of gnathopod 1 more than 2 times larger and longer than carpus: palm with 5 spine teeth on inside. 7 on outside: defining angle with 4 notched-serrate spine teeth on inside and 1 notched and 2 serrate spine teeth on outside. Propod of gnathopod 2 more than 2 times larger and longer than carpus: palm longer than posterior margin, palm strongly oblique. slightly convex. with 9 spine teeth on inside. 8 on outside: defining angle with I notched and 4 notched-serrate spine teeth on inside. 3 notched spine teeth on outside.

Uropod 2: inner ramus with 10 spines: outer ramus slightly deflected laterally. with $0-1$ inner spines. 3 outer spines, apex with 4-5 spines: peduncle with 4 outer and 1 inner spines.

Type-locality.--Spring at edge of road. upper end of Panthers Gap. Bath County. Virginia.

Distribution and ecology.--This species is recorded from several localities in west-central Virginia and I locality in eastern West Virginia. all in the Appalachian Valley and Ridge province (tig. 86). The range covers a linear distance (east to west) of about $80 \mathrm{~km}$. This species inhabits springs. spring-fed swamps. and spring-fed ponds. Ovigerous females were collected in May.

Etymology.--The epithet montanus is from Latin. "pertaining to mountains." which refers to the occurrence of this species in the mountains of western Virginia and eastern West Virginia.

Crangonvx rivularis Bousfield

Figures $87-90$

Crangonyx rivularis Bousfield. 1958:100-102. fig. 16 [type-locality: Rocky Saugeen River. small tributary $4 \mathrm{mi}$ N. of Durham. Grey Co.. Ontario. Canada].--Judd. 1963:2.--Bousfield. 1970a:I11.--Holsinger. 1972:46. fig. 15: 1977:252.--Barnard \& Barnard. 1983:435. map 11.--Fitzpatrick. 1983:145.

Material Examined.-ONTARIO. Halton Co.: Speyside stream. 1 ¿. N. Hynes. 3 Jun 1975 (USNM): Grey Co.: Rocky Saugeen River, small tributary $6.4 \mathrm{~km}$ N of Durham, holotype $\approx$ allotype $\supseteq .18 \equiv .7 \approx$. paretypes. E. L. Bousfield. 30 May 1957 (CMN): Dufferin Co.: water cress roots. Orangeville. $5 \dot{\Xi}, 4 \nexists^{*}$. E. L. Bousfield. 30 May 1957 (USNM): Spring W of Toronto. 2 \& A. Pizzini. 17 May 1935 (USNM) and 6 


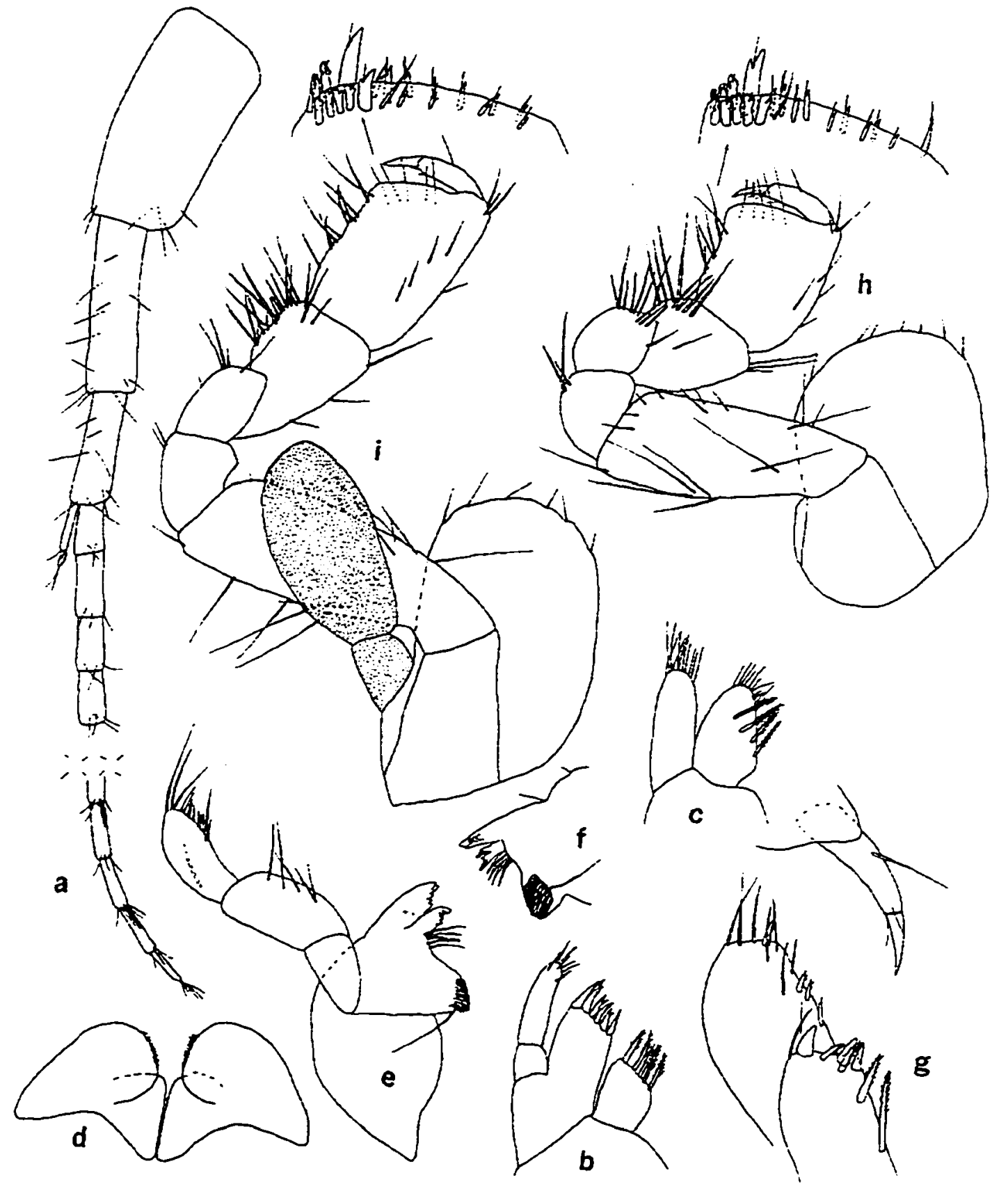

Figure 87.--Crangonvx rivularis Bousfield. Female $(6.0 \mathrm{~mm})$, Orangeville. Ontario. Canada: a. antenna I: b. c. maxiliae I, 2: d. lower lip; e. left mandible: $f$. dentate part of right mandible: $g$. inner and outer plates and palp dactyl of maxilliped (greatly enlarged): h. i. gnathopods 1. 2 (palmar margins enlarged). 


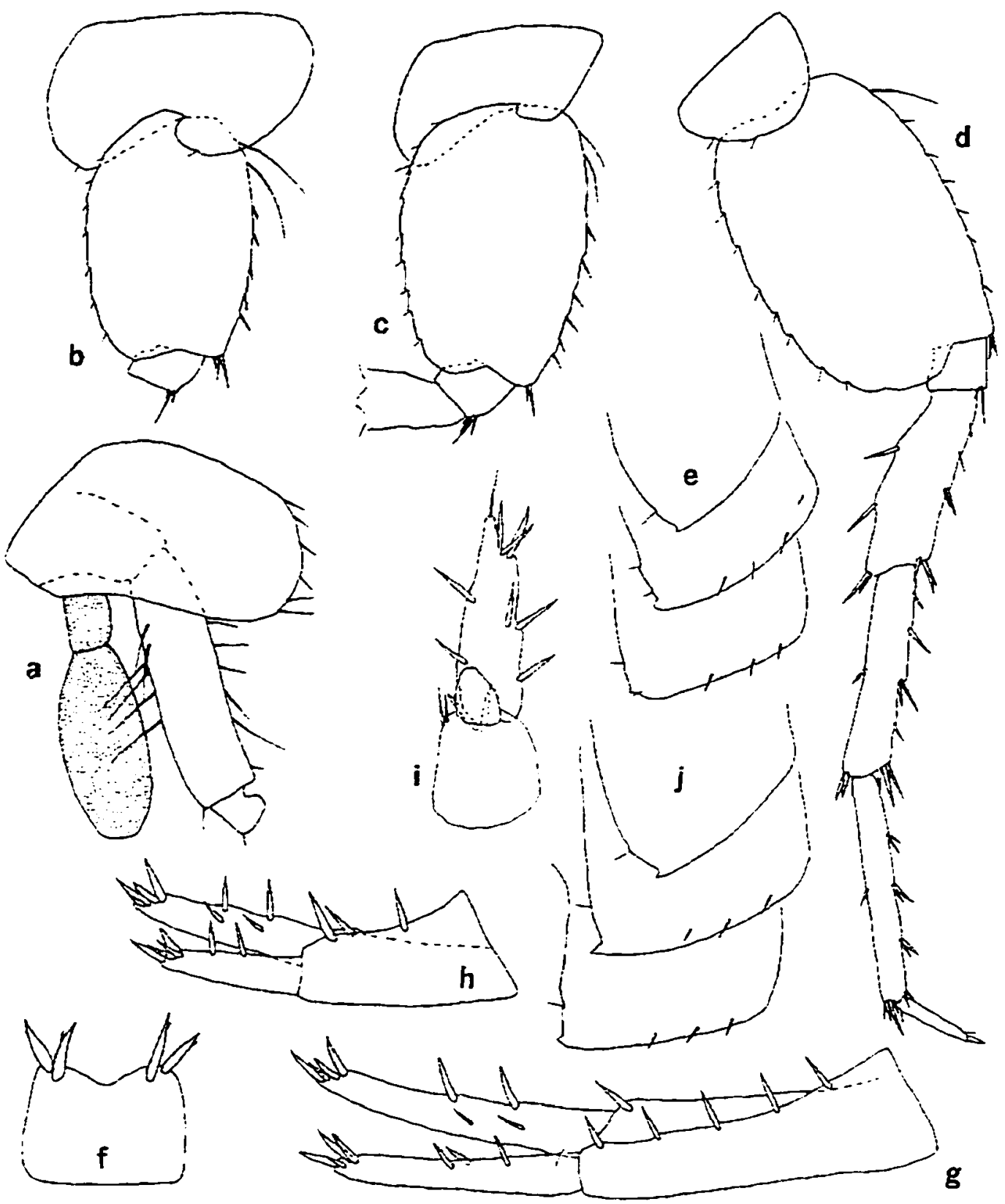

Figure 88.--Crangonvx rivularis Bousfield. Female $(6.0 \mathrm{~mm})$. Orangeville. Ontario. Canada: a. b. c. pereopods 3.5.6 (in part): d. pereopod 7: e. pleonal plates: f. telson: g. h, i. uropods 1. 2. 3. Female $(6.5 \mathrm{~mm})$, same location: $\mathrm{j}$, pleonal plates. 


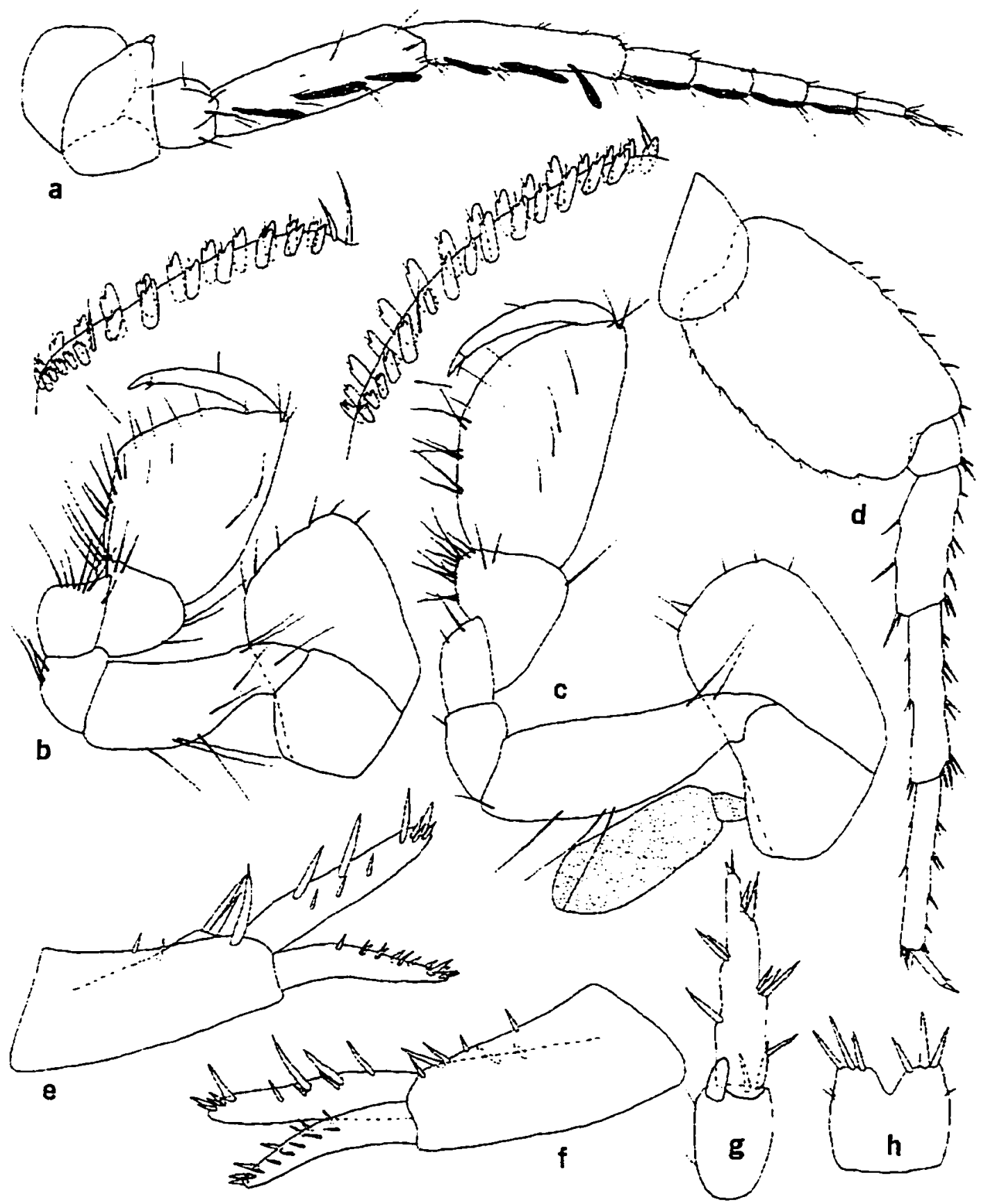

Figure 89.--Crangonvx rivularis Bousfield. Male $(4.1 \mathrm{~mm})$. Orangeville. Ontario. Canada: a. antenna 2: b. c. gnathopods 1. 2. (palmar margins enlarged): d. pereopod 7: e. f. uropods 2: g. uropod 3: h. telson 


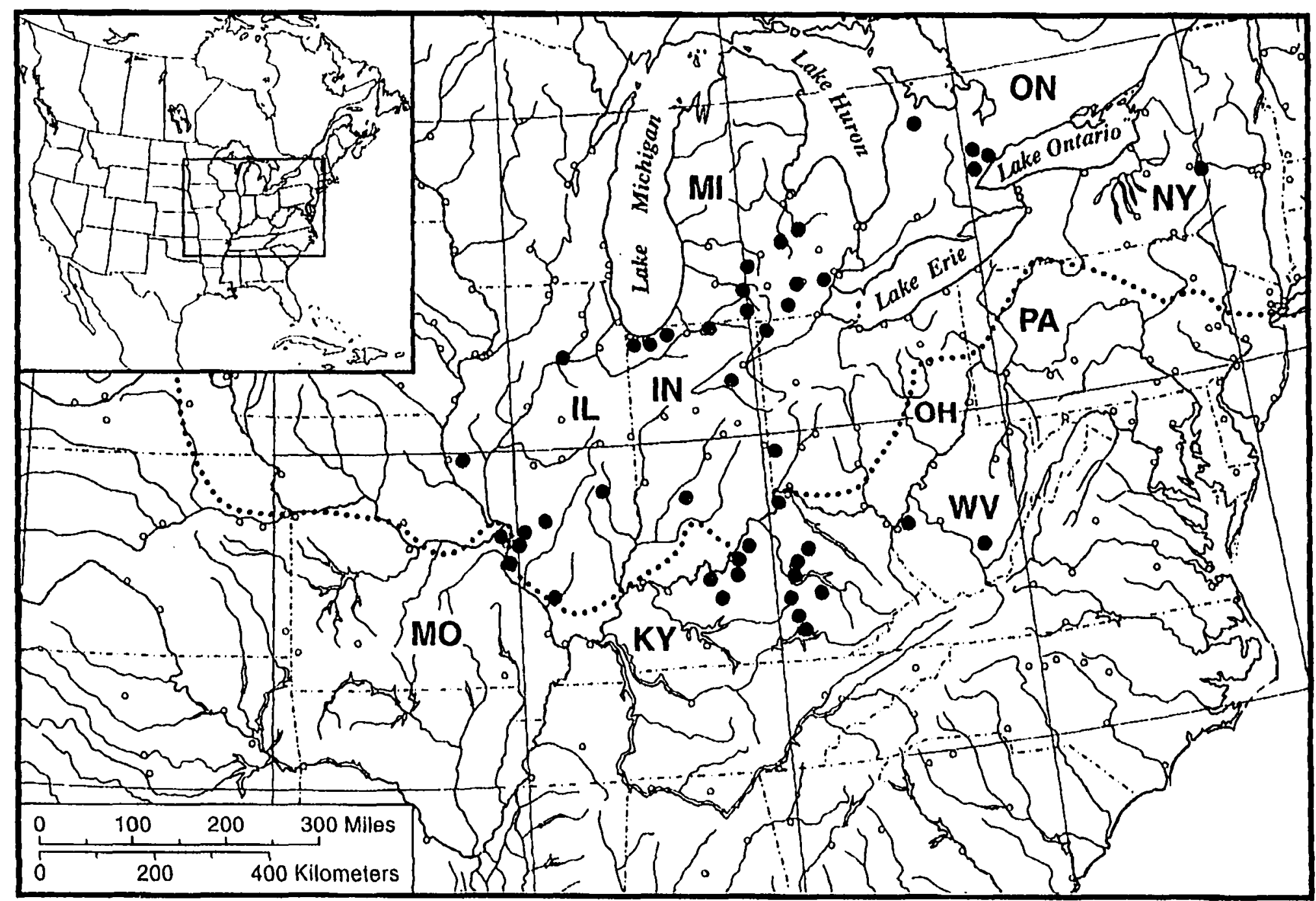

Figure 90.--1)istribution of ('rungenner rivuluris in North America. Solid circles represent 1-8 closely proximate locialities. The donted line indicaltes the approximate southern extent of glateiation during the Pleistocene. 
$\equiv .1 \approx 5 \mathrm{Jul} 1935$ (USNM). ILLINOIS. Adams Co.: spring near house. Siloam Spring State Park. $5 \Xi$. S.

B. Peck. 22 Apr 1966: Bond Co.: temporary pond. 1.0 km E of Baden. $7 \cong .16:$ L. Hubricht. 16 Apr 1942 (USNM): Cumberland Co.: ditch. $5.8 \mathrm{~km}$ ENE of Greenup. 31 \%. 19 ๘. L. Hubricht. 16 Apr 1942 (USNM): Jackson Co.: seeps in bluff on nature trail at cave. Makanda entrance of Giant City State Park. 1 ミ. J. Lewis, 5 May 1974: LaSalle Co.: temporary pond, $0.8 \mathrm{~km}$ NE of Seneca. 50 specs. L. Hubricht. 3 May 1941 (USNM); Madison Co.: temporary pool, near Silver Creek. $5.8 \mathrm{~km} \mathrm{~W}$ of St. Jacob. $2 \approx$. L. Hubricht. 13 Apr 1942 (USNM) and 100 specs. 16 Apr 1942 (USNM): McDaniel Lake. 209 . L. Hubricht. 12 May 1937 (USNM): Monroe Co.: Moredock Lake, 3. km N of Valmeyer. 7 ฐ. L. Hubricht. 26 May 1937 (USNM): temporary pond. roadside ditch. North Dupo. 38 ミ. $12 \approx 0$. L. Hubricht. 24 Apr 1938 (USNM): St. Clair Co.: temporary pool. ditch, $1.6 \mathrm{~km} \mathrm{~S} \mathrm{of} \mathrm{Falling} \mathrm{Spring.} 200$ specs. L. Hubricht. 24 Apr 1938 (USNM): marsh at 5300 State Street. East St. Louis. 20 specs. L. Hubricht. 12 May 1937 (USNM). INDIANA. Elkhart Co.: seep. $22.4 \mathrm{~km}$ E of Nappanee. $199.11 \approx$. L. Hubricht. 19 Apr 1942 (USNM): Huntington Co.: slough. $5.3 \mathrm{~km}$ NE of Huntington. 100 specs. L. Hubricht, 27 Apr 1941 (USNM): LAKE Co.: temporary pond. $1.0 \mathrm{~km}$ W of New Chicago. 30 specs. L. Hubricht. 2 May 1941 (USNM): LaPorte Co.: temporary pond. $7.7 \mathrm{~km}$ NE of La Porte. $10 £ .3 \varepsilon$. L. Hubricht. 2 May 1941 (USNM): Monroe Co.: spring near Jacks Defeat Creek. Bloomington. I $\supseteq .1$ juv. N. Hỵnes. 20 Oct 1962 (LSNM) and $17 \equiv .15$ Dec 1962 (USNM): Porter Co.: outlet of drain. $0.8 \mathrm{~km}$ E of River. 50 specs. L. Hubricht. 20 Apr 1942 (USNM). KENTUCKY. Bourbon Co.: small spring. $4.5 \mathrm{~km} \mathrm{SW}$ of Millersburg. 25 specs. L. Hubriclit. 25 Apr 1941 (USNM): spring. $9.1 \mathrm{~km}$ SW of Paris. 8 9. 7 \%. L. Hubricht. 25 Apr 1941 (USNM): Boyle Co.: Salt River system. Atoka. 2 specs, collector not given, 6 Mar 1971: Bullitt Co.: Creek at T-junction. W of Crutchmere Hollow. $79.4 c^{*}$ N. Hynes. 9 Jan 1985 (USNM): Estill Co.: Clifford Pearson Cave. $2 \cong .1 \approx$. J. H. Carpenter. 20 Jan 1968: Hardin Co.: slough just W of West Point. $8 \equiv .1 \approx$. L. Hubricht. 21 Apr 1956 (USNM): Fayette Co.: temporary pond. near Bluegrass Field. Parkers Mill Road. 50 specs. J. R. Holsinger. 25 Jan 1965. 20 specs. 22 Mar 1965. 118 specs (£. $є$. juvs), 19 Apr 1965 and 100 specs. 5 May 1965: pond on Coldstream Farm. 25 specs ( $\subsetneq . \approx$. juvs), J. R. Holsinger and R. M. Norton. I I Apr 1965: grass roots in stream on Coldstream Farm. 2 c. J. R. Holsinger. 11 Apr 1965: Crasmere Farm No.I spring. Higbee Mill Road. I ९. J. R. Holsinger. 7 Mar 1964: Idle Hour spring No.1. on Rd 25. $1 \cong$. J. R. Holsinger and R. M. Norton. 11 Apr 1965; Idle Hour Spring No.2, near Rd 25. 1 ə. I ‡. J. R. Holsinger and R. M. Norton, 11 Apr 1965: Russell Cave Spring, $11.2 \mathrm{~km}$ NE of Lexington, 4 ₹. L. Hubricht. 22 Nov 1940 (USNM) and I §. J. R. Holsinger, 16 Nov 1963: seep-fed swamp off Tates Creek Road. Lexington. 1 ¿'. J. R. Holsinger. I Mar 1965: spring-fed stream on Waveland Farm near Lexington. $29.2 \approx$. J. R. Holsinger and I $\bar{x} .10 .11$ Apr 1965: Jefferson Co.: Cave Hill Cave. Louisville, I $\Xi .1$ c". J. J. Lewis. 9 Jun 1975: intermittent ditch in George Rogers Clark Park. $5 \approx$. G. A. Cole. 9 Jan 1955: small tributary of Pope Lick. $1 \equiv .9$ Jan 1985 (USNM): pond in Shawnee Park. Louisville. 5 \%. 1 . Ilaria. 29 Mar 1954: shinks branch crosses Old Heady Rd, 2 ?. G. A. Cole, collection date not given: Jessamine Co.: spring. $4.3 \mathrm{~km} \mathrm{~N}$ of Nicholasville. $69.4 \%$. L. Hubricht. 24 Apr 1941 (USNM); spring $9.8 \mathrm{~km} \mathrm{~N}$ of Nicholasville. $1 \cong . \mathrm{L}$. 
Hubricht. 24 Apr 1941 (USNM): Lincoln Co.: small spring. $1.6 \mathrm{~km}$ of Preacherville. $7 \nsubseteq .6 \overbrace{}^{\rtimes}$. L. Hubricht. 24 Apr 1941 (USNM); Meade Co.: spring near Ohio River. Otter Creek State Park. 5 Z. $2 c^{*}$. J. J. Lewis. 24 Mar 1975: Oldham Co.: tributary of Harrods Creek. 21 9. 2 o. R. Fox. 3 Apr 1971. Pulaski Co.: Girdler Cave No.2. 8 \%. J. R. Holsinger and T. C. Barr. Jr.. 10 Oct 1964. MICHIGAN. Branch Co.: ditch. 5.0 km S of Coldwater. 40 specs. L. Hubricht. 19 Apr 1942 (USNM): outlet of drain. $6.9 \mathrm{~km} \mathrm{~S} \mathrm{of} \mathrm{Coldwater.} \mathrm{I} \mathrm{.} \mathrm{L.}$ Hubricht. 19 Apr 1942 (USNM); Calhoun Co.: pools in swamp, $5.6 \mathrm{~km}$ ESE of Battle Creek, 40 specs. L. Hubricht. 13 Apr 1947 (USNM): Eaton Co.: outlet of drain. $1.6 \mathrm{~km}$ NE of Olivet. 20 specs. L. Hubricht. 19 Apr 1942 (USNM): Genesee Co.: small stream. 7.2 km N of Fenton. 2 @. 3 c. L. Hubricht. 19 Apr 1942 (USNM): Lenewee Co.: slough. $0.8 \mathrm{~km} \mathrm{~W}$ of Hudson, 9 q. 13 đ. L. Hubricht. 29 Apr 1941 (USNM): Shiawassee Co.: small stream. $11.2 \mathrm{~km}$ WSW of Perry. 28 ․ L. Hubricht. 19 Apr 1942 (USNM): Washtenaw Co.: swamp. $9.0 \mathrm{~km}$ NE of Saline, $18 \% .2 \approx$. L. Hubricht. 29 Apr 1941 (USNM): Wayne Co.: woodland pools. Southfield Rd and Oakwood Blvd. Dearborn. 148.2 :. L. Hubricht. 7 Apr 1946 (USNM). MISSOURI. St. Louis Co.: temporary pool. $4.8 \mathrm{~km} \mathrm{NW}$ of Pattonville. 150 specs. L. Hubricht. 17 Apr 1938 (USNM): temporary pond. Benbush. 47 specs. L. Hubricht. 8 Mar 1936 (USNM): pond. E of Morschels. $+2.19:$ L. Hubricht (USNM); slough. $8.0 \mathrm{~km}$ W of Florissant. 20 specs. L. Hubricht. 24 May i 941 (USNM): temporary pool. $0.8 \mathrm{~km}$ W of Vigus, 100 specs. L. Hubricht. 17 Apr 1938 (USNM): slough near Mississippi River. $1.6 \mathrm{~km}$ S of Grimsby. 36 specs. L. Hubricht. 25 Apr 1938 (USNM). NEW YORK. Herkimer Co.: marsh near Mohawk River, Ilion. 8 \%. 6 c*. L. Hubricht. 28 Mar 194j (USNM): temporary pool. Frankfort. $21 \% .200$. L. Hubricht. 4 Apr 1943 (USNM); spring near Mohawk River. 15.2 km SE of Little Falls. 50 specs. 18 Apr 1943 (USNM). OHIO. Preble Co.: small stream. $3.2 \mathrm{~km} \mathrm{~W} \mathrm{of}$ Alexandria. $239.4 c^{\circ}$. L. Hubricht. 17 Apr 1942 (USNM): Williams Co.: outlet of drain. $0.3 \mathrm{~km} \mathrm{NE}$ of Wiliams Center. 40 specs ( $\Xi$, $)$. L. Hubricht. 28 Apr 1941 (USNM). WEST VIRGINIA. Cabell Co.: Green Bottom Swamp, $4 \approx$ D. C. Tarter, collection date not given, and 4 ₹.6 60 Mar 1995: Greenbrier Co.: Meadow River Wetlands. 6 §. D. C. Tarter, 19 Apr 1996.

Diagnosis.--Distinguished from other members of the gracilis group by having notched-serrate spine teeth on defining angle of propod of gnathopods of both sexes, shallow serrations on posterior margins of pereopod 5-7. inner and outer margins of outer ramus of male uropod 2 with up to 7 short. well spaced spines. Largest male. $4.5 \mathrm{~mm}$ : largest female $8.5 \mathrm{~mm}$.

Female.--Eye large, ovate. Antenna 1, about 50 percent length of body. about 1.8 times longer than antenna 2: primary flagellum with up to 20 segments. Antenna 2. flagellum with 7-9 segments. Mandibles subequal, spine row with 4-5 spines: segment 2 of palp with 4-5 long setae. segment 3 with 1 A seta. IC seta. 3-4 E setae and a row of D setae. lacking B seta. Maxilla I: inner plate with 4-5 apical plumose setae: palp with 5-6 slender spines on apex. Maxilla 2. inner plate with oblique row of 4-5 plumose setae on inner margin. Maxilliped: inner plate apically with 3 bladelike spines, 1 naked spine, and 3-4 plumose setae extending from inner margin to apex: outer plate with row of naked setae and 2-4 slender pectinate spines on inner margin: dactyl with 2 setae on inner margin near nail: dactyl nail long about 13 length of dactyl. 
Propod of gnathopod I less than two times larger and longer than carpus: palm almost straight. shorter than posterior margin. with 5 unnotched spine teeth on inside. 4 on the outside: defining angle with 4-5 notched-serrate and $I$ serrate spine teeth on inside, 1 large notched and 3 serrate spine teeth on outside: superior medial setae few in number and singly inserted: 4 sets of setae on posterior margin. singly inserted or in groups of 2. Dactyl with a row of short setae on inner margin. nail very long. Ventral margin of coxa 1 with $7-8$ setae.

Propod of gnathopod 2 less than 2 times larger and longer than carpus: palm almost straight. much shorter than posterior margin, with 4 unnotched spine teeth on inside. 7 on outside: defining angle with ! large notched spine tooth and 2-3 serrate spine teeth on outside. I notched and 4-5 notched-serrate spine teeth on inside: superior medial setae singly inserted: posterior margin with 4 sets of setae in groups of $1-5$. Dactyl with row of short setae on inferior margin. nail very long. Ventral margin of coxa 2 with 6 setae.

Coxa of pereopod 3 with 6-8 marginal setae. Coxa of pereopod 4 with 13 marginal setae. dactyl of pereopod 4.40 percent length of corresponding propod. Pereopod 5: basis with 7 very shallow serrations along posterior margin: 5-8 short spines on anterior margin: 2 long setae on the anteroproximal margin. Pereopod 6: basis with 9 shallow serrations along posterior margin: 6 short spines on anterior margin: 2 long setae on anteroproximal margin. Pereopod 7: coxa with 3 setae on the posterior margin: basis with 10 shallow serrations along posterior margin. deeper at apex: 6 short spines on anterior margin: 1.2 long setae on anteroproximal margin: dactyl about 35 percent length of corresponding propod.

Pleonal plates: posterior margin of plate I slightly convex. with I seta. distoposterior corner variable. weakly produced and acute or not: posterior margin of plate 2 nearly straight with I (rarely two) setae. distoposterior comer produced and acute (or somewhat subacute), ventral margin with + (subventral) spines: posterior margin of plate 3 nearly straight with I seta. distoposterior corner produced and acute. ventral margin with 3 (subventral) spines. Peduncle of pleopod 1 with 2 retinaculae. lacking setae on outside margin.

Uropod I: inner ramus 80 percent length of peduncle. armed with 8 spines: outer ramus with 7 spines: peduncle with 5 outer and 1 inner spines. Uropod 2: inner ramus with 8 spines: peduncle with 2 outer and 1 inner spines. Uropod 3: inner ramus lacking spines. outer ramus about 1.7 or more times longer than peduncle. inner and outer margins each with 2-3 set of spines in groups of $1-3$. Telson broader than long. notched about 1/8-1/4 distance to base, each lobe with 2-3 apical spines: spines more than 12 length of telson.

Male.--Differing from female as follows. Smaller, body more slender and elongate. Antenna 2: calceoli present on peduncular segments 4 and 5 . and first 4 segments of flagellum.

Propod of gnathopod I more than 2 times larger and longer than carpus and much broader: palm almost straight. longer than posterior margin, with 7 spine teeth on inside. 8 on outside: defining angle with 5 notched-serrate spine teeth on inside and 2 notched and 2 serrate spine teeth on outside. Propod of gnathopod 2 more than 2 times larger and longer than carpus; palmar margin strongly oblique. convex. 
longer than posterior margin. with 9-12 spine teeth on inside. 9 on outside: defining angle with 2 notched and 3 serrate spine teeth on inside and 4 notched-serrate spine teeth on outside.

Uropod 2: inner ramus with 11 spines; outer ramus curved, with 4-6 short well spaced inner spines. 57 short outer spines. apex with 2-3 spines: peduncle with 4 outer and 3 inner spines.

Distribution and ecology.--This species is widespread in east-central North America. being recorded from southeastern Ontario. northern and southern Indiana. northern and southeastern Illinois. central Kentucky. northern and southeastern Ohio. central and southern Michigan. eastern Missouri. and southerm West Virginia (fig. 90).

Crangonve rivularis inhabits rivers. streams, springs. ditches. ponds. small lakes and rarely caves. Sexually mature males. 3.5 to $4.5 \mathrm{~mm}$ : sexually mature females. 5.5 to $8.5 \mathrm{~mm}$. Ovigerous females were collected in March. April. May, July and November.

\section{Crangonix packardi Smith}

Figures $91-94$

Crangonyx packardi Smith. 1888:35-36. figs. 5-11 [type-locality: wells in Orleans. Indiana].--Hay. 1 896:207.--Fage.1931:361.--Schellenberg, 1936:35.--Holsinger, 1972:44, figs. 11a. IIc. 16 [in part]: 1977:252.--Lewis. 1983:36 [in part].--Barnard \& Barnard. 1983:435. map 12 [in part].--Fitzpatrick. 1983:145 [in part].--Holsinger. 1986:97. fig.7 [in part]. [Not Barr. 1968:161]. Crangonxx gracilis packardi Hubricht. 1943:691 [in part].--Hubricht.1950:16.--Nicholas. 1960:127.--

Ḱrekeler \& Williams. 1966:394.

Eucrungonvx packardi (Smith).--Stebbing. 1899:423: 1906:389.--Weckel. 1907:35.

Material examined.--INDIANA. Clark Co.: Indian Cave. 5 ๆ. 2 juvs. J. J. Lewis. 25 Mar 1975: seep. $4.8 \mathrm{~km} \mathrm{SW}$ of Bethlehem. $79.9 \approx$. L. Hubricht. 21 Apr 1956 (USNM): Crawford Co.: Marengo Cave. 1 巨. R. Rhoades. 30 Dec 1938 (USNM): Old Town Spring Cave. I \&. 1 juv B. Tozer. 21 Nov 1964: Decatur

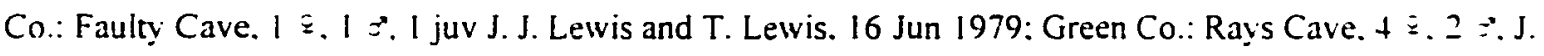
R. Holsinger. R. Nicoll and R. M. Norton. 12 Jun 1965: Harrison Co.: Baker Hollow Cave. 2 ミ. J. J. Lewis. et al.. 25 May 1996: Steerstetter Cave. $2 \varsubsetneqq .1$ *. L. Hubricht. 17 Aug 1957 (USNM): Jefferson Co.: Cricket Cave. 1 spec.. J. J. Lewis and T. Lewis, 6 Nov 1977: Mud Cave. 1 ¡. J. J. Lewis and L. Everitt. 21 Oct 1978: Lawrence Co.: Bronson Cave. 4 7. 2 juvs. J. R. Holsinger and R. M. Norton. 19 Jun 1965 and 1 Đ. J. J. Lewis. 1 Dec 1974: Donaldson Cave. 19.1 ๔. T. C. Barr. Jr. and R.M. Norton. 13 Nov 1965 and 7 E. $2 \approx$ J. R. Holsinger and R. M. Norton. 15 Jun 1965: Donnehues Cave. $139.5 \approx *$ J. R. Holsinger and E. Lavangino. 8 Aug 1964: Shiloh Cave. 10 \%, L. Hubricht. 2 Sep 1939 (USNM). 6 \&. 1 \%. J. R. Holsinger. 8 Aug 1964 and $1 \approx n$. R. M. Norton. 19 Jun 1965: Jennings Co.: Biehle Cave. 29 . J. J. Lewis and M. Lewis. 


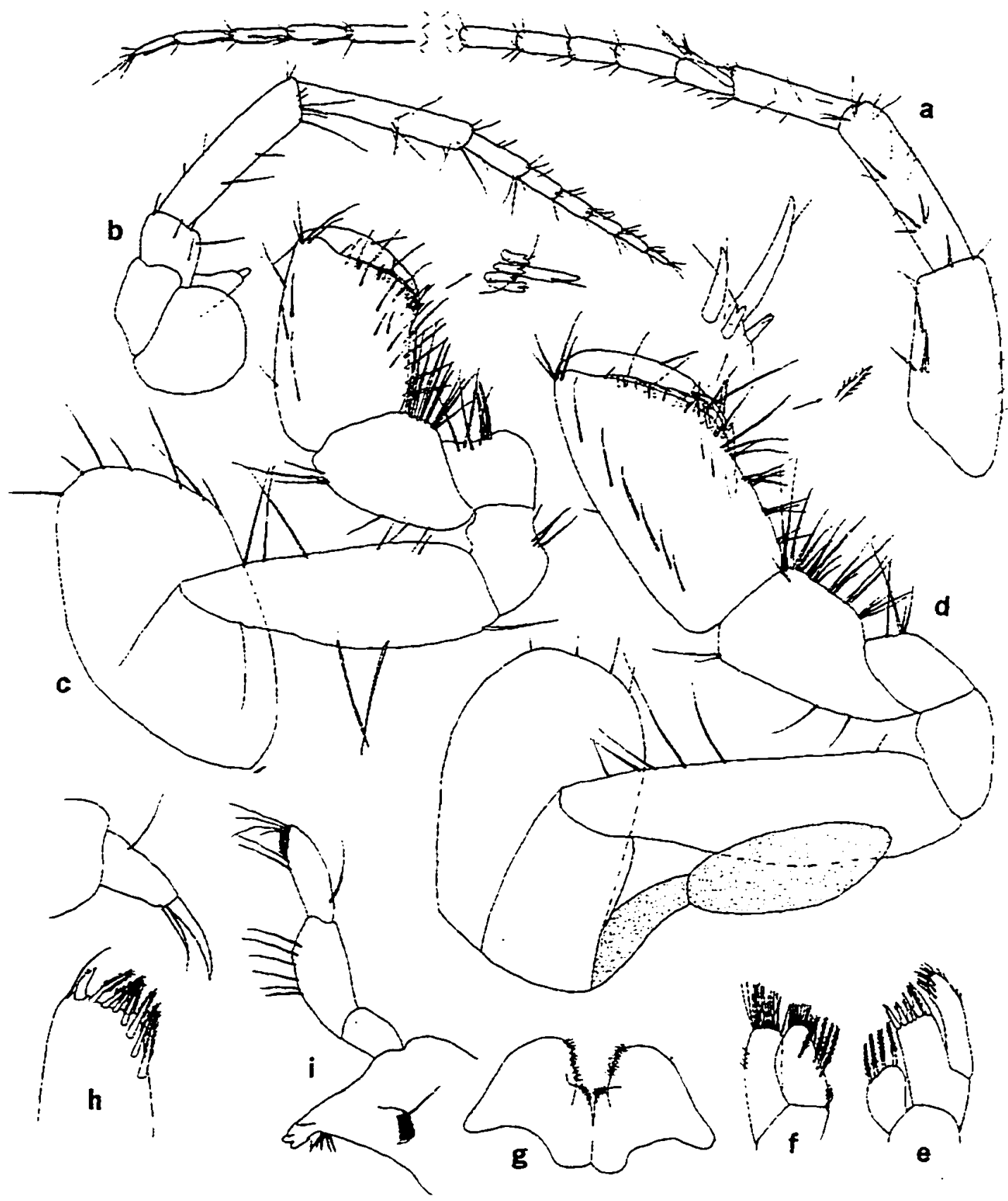

Figure 91 .--Crangonxx packardi Smith. Female $(5.7 \mathrm{~mm})$. Donaldson Cave. Lawrence Co.. Indiana: a. b. antennae I. 2: c. d. gnathopods 1, 2 (spine teeth on defining angle enlarged): e. f. maxillae 1. 2: g. lower lip: $h$. inner plate and palp dactyl of maxilliped; i. right mandible. 


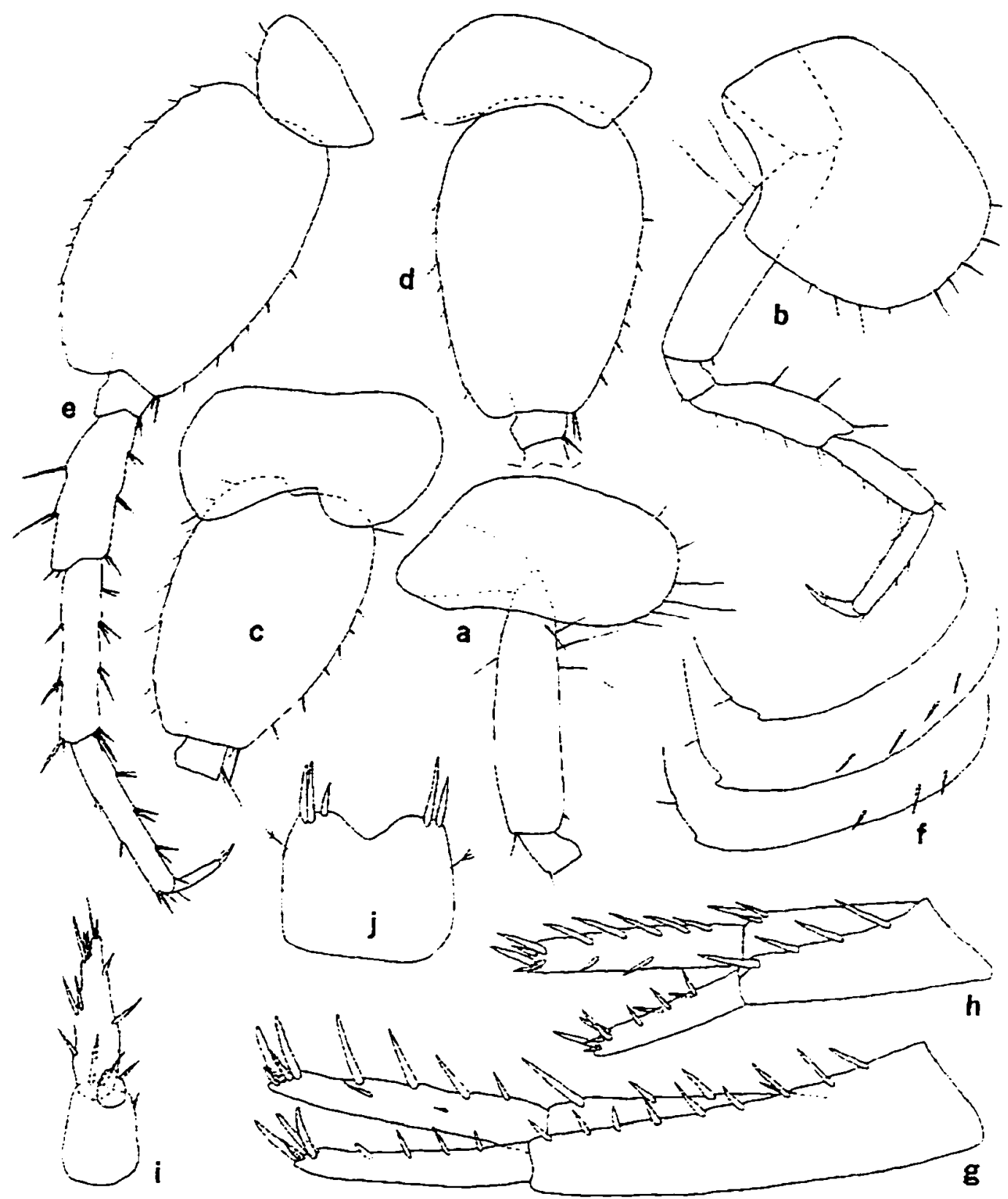

Figure 92.--Crangonxx packardi Smith. Female $(5.7 \mathrm{~mm})$. Donaldson Cave. Lawrence Co.. Indiana: a. c. d. pereopods 3. 5.6 (in part): b. e. pereopods 4. 7: f, pleonal plates: g. h. i. uropods 1. 2. 3: j. telson. 


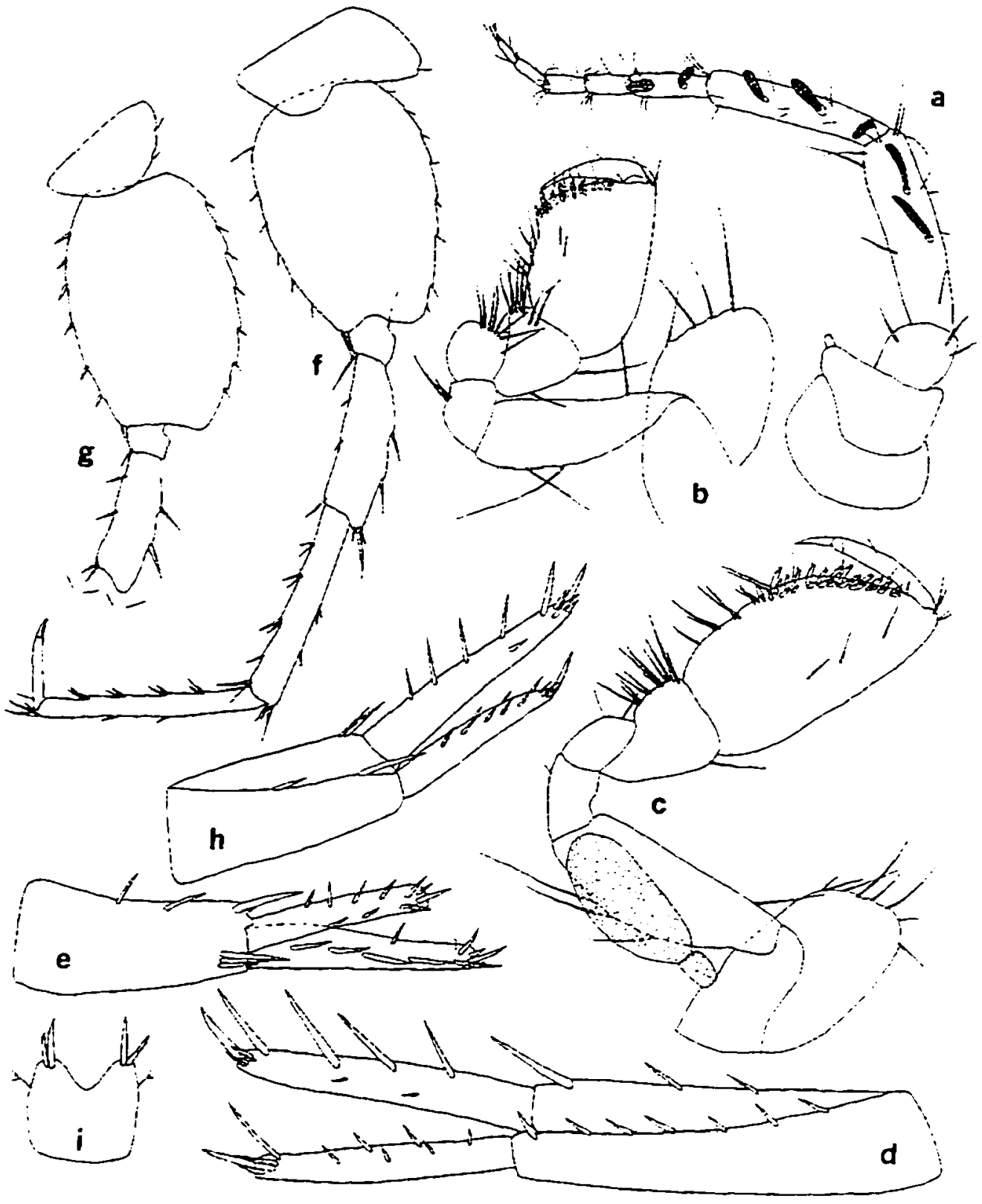

Figure 93.--Crangonvx packurdi Smith. Male (3.4 mm), Donaldson Cave. Lawrence Co.. Indiana: a. antenna 2: b. c. gnathopods 1, 2: d. e. uropods I. 2. Male (3.2 mm). same location: t. pereopod 6: g. pereopod 7 (in part): h. uropod 2: i. telson. 


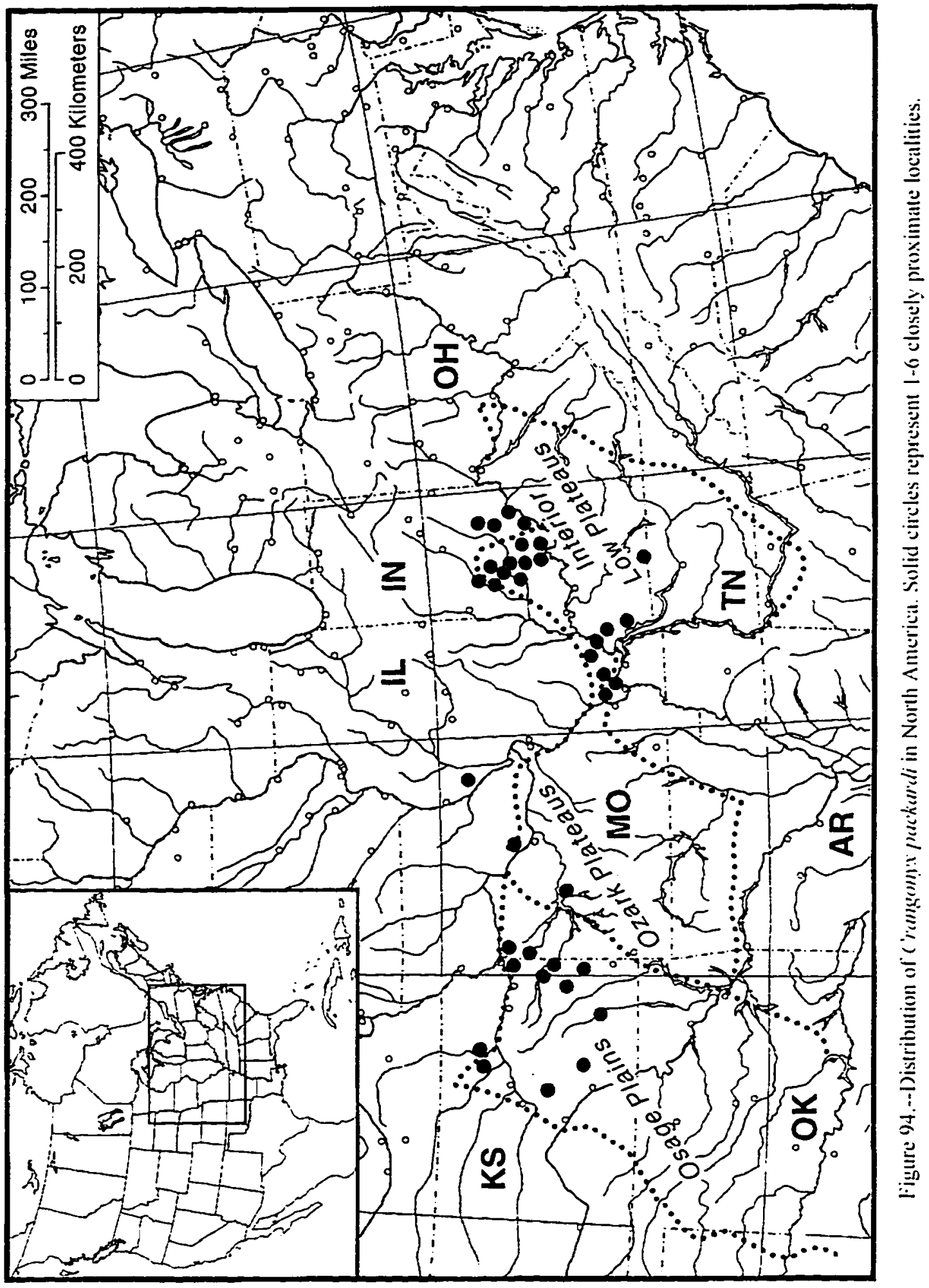


22 May 1994: Crosley Canyon Cave. 1 q. J. J. Lewis. 30 Jul 1994: Horsethief Cave. 1 ‡. J. J. Lewis. 30 May 1994: Martin Co.: Tow Cave. 1 . R. M. Norton. 19 July 1967: Monroe Co.: Matlocks Cave. $4 \vdots .1$ juv. j. j. Lewis and II. Huffman. 29 Mar 1995; May's Cave. I ๆ. 2 c:. N. Hynes. 3 Jan 1963: Mayfield

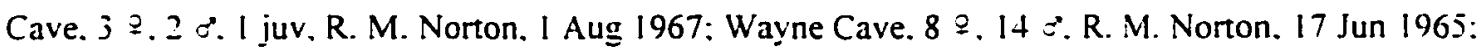
seep under Jordon Hall. Indiana University, $29.1 \approx$ J. R. Holsinger and R. Nicoll. 18 Jun 1965: Salamander Cave. $1 \%$ J. J. Lewis and H. Huffman. 29 Jul 1995: Orange Co.: Campground Cave. $1:$ H. H. Hobbs. III. and M. Porter, I Aug 1995: Hudelson Cave. 39. J. J. Lewis and C. Quillet. 19 Mar 1994: Lost River Cave No. 1. $10 \sqsubseteq .2 \approx 76$ juvs J. J. Lewis and M. Lewis. 31 July 1993: Mount Horeb Cave. 6 . $2 \because$ J. J. Lewis and M. Lewis. 26 Dec 1993: Peacher Cave. $3 \mp .1$ o. J. J. Lewis et al.. 26 Nov 1993. $2 \equiv .1$ $\because$ J. J. Lewis, 12 Feb 1994: William Cleveland Cave. 2 \&. J. J. Lewis et al.. 26 Nov 1993: Owen Co.: Porter Cave. 1 \&. B. Tozer. 16 Jun 1965 and 2 2. 1 . R. M. Norton. 1 Aug 1967: Washington Co.: May Cave. 1 I. 3 . R. M. Norton. 17 Jul 1967. ILLINOIS. Hardin Co.: Cave Spring Cave. 1 ミ. S. B. Peck and J. Peck. 27 Jun 1965: Griffith Cave. $1 \cong .1$ Æ’. S. B. Peck. 19 Sep 1965: Layoff Cave. I Ł. J. J. Lewis. 17 Feb 1974 and $I \Xi 26$ Oct 1974, and I ₹. J. J. Lewis and M. Meister. 21 Jun 1977: spring. north of Illinois from Furnace. 1 \&. J. J. Lewis, 6 Apr 1975: Johnson Co.: Firestone Creek Cave. 2 ₹. 2 z’. J. J. Lewis. 15

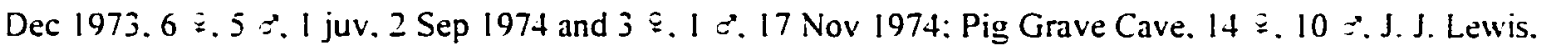
I Nov 1975: Saline Co.: Cave Hill Cave (=Equality Cave). $179.5 \approx .2$ juvs. L. Hubricht. 22 Jun 1940 (USNM). $20 \% .15$ ๘. J. R. Holsinger and R. M. Norton. 14 Jun 1965. 4 ₹. 3 s. S. B. Peck. 23 Oct 1965 and 6 ?. $3 \approx * 2$ juvs. D. A. Hubbard. Jr., 8 Aug 1992: Union Co.: Cricket Cave. 1 ‡. J. R. Holsinger and R. M. Norton. 14 Jun 1965 and 1 juv. J. J. Lewis. 5 Jun 1974: Sensemeyer Cave. 1 \&. S. B. Peck. 15 Jun 1966: Wet Cave. 1 \&. 11 juvs. L. Hubricht. I Jul 1940 (USNM). KANSAS. Anderson Cu.: hand duy Well. $5 \mathrm{mi}$ NE of Colony. I $\&$. J. Danoff-Burg and W. R. Brecheisen. 16 May 199I: Bulter Co.: Stone Cave. $1.5 \mathrm{mi}$ SE of El Dorado. 2 . W. H. Busby and D. Figg. 3 May 1989. 3 §. W. H. Busby and J. J. Young. 7 Sep 1991: Bourbon Co.: Spring, $3.7 \mathrm{~km}$ S of Bronson. $3 \subsetneq 17$ May 1942 (USNM): Seep. $3.5 \mathrm{~km}$ E of Uniontown. $24 \bar{\mp} .1 \cong .2$ Juvs. L. Hubricht. 17 May 1942 (USNM): Franklin Co.: Stock Well. Wheeler Farm. 8.0 km SE of Ottawa. $5 \subsetneq$. L. Hubricht. 31 Aug 1941 (USNM): Johnson Co.: Blockbob Cave. $6.4 \mathrm{~km} \mathrm{SW}$ of Stauley. 5 g. 5 c*.W. H. Busby and J. J. Young. 7 Apr 199I: Miami Co.. Yates Well. $6.4 \mathrm{~km} \mathrm{SW}$ of Osawatomie. 149.60 .3 Juvs. L. Hubricht. 31 Aug 1941 (USNM): Cave Spring Cave. 0.8 km E of Somerset. 3 F. 3 s. W. H. Busby and J. J. Young. I 3 Apr 1991: Poppauatomie Co.: Gluntz Cave. $8.0 \mathrm{~km} \mathrm{SW}$ of Olsburg. 5 ₹. J. Danoff-Burg et al.. 23 Jun 1991: Riley Co.: Shallow well flow, Kouza Prairie. 17 ๆ. 10 c. M. Whiles and K. Hooker. 23 Jul 1986; Wilson Co.: Harper Spring Cave. $3.2 \mathrm{~km} \mathrm{NE}$ of Guilford, I $\varsubsetneqq$.W. H. Busby and J. J. Young. 7 Apr 1991. KENTUCKY. Crittenden Co.: Cannon Cave $1=$ Kinnin Cave). 1 c. T. C. Barr. Jr. and W. Andrews. $8 \mathrm{Jul} 1965$ and 2 Q. J. R. Holsinger et al.. $15 \mathrm{Jun}$ 1978: Caldwell Co.: Watsons Cave. 1 ₹ . J. R. Holsinger et al.. 12 Jun 1978: Simpson Co.: Old Smokey Cave. 1 ₹. J. R. Holsinger. 25 Jul 1981. MISSOURI. Benton Co.: Flipper Cave. 1 . J. Reynolds. 30 Mar 1958 (USNM): Boone Co.: Devils Icebox Cave. $4.8 \mathrm{~km} \mathrm{~S}$ of Columbia. 2 *. J. J. and T. Lewis. 4 Aug 
1979: Cass Co.: Harrisonville, 4 Z. 2 c. A. N. Newman. 24 Feb 1917 (USNM): well. about $6.6 \mathrm{~m}$ deep. Belton. 1 9. 1 \%. J. Adamski, Apr 1983: Jackson Co.: Seep, Independence, 1 ミ. J. L. Craig. 1 Apr 1975.

Diagnosis.--A relatively small stygobiont species of the gracilis group. which is distinguished from other members of the group by lacking notched-serrate spine teeth on defining angle of propod of gnathopods of both sexes: having shallow serrations on posterior margins of pereopods 5-7: and outer ramus of uropod 2 of male, which bears 5-6 short inner and $4-5$ short outer spines. Largest male. $5.5 \mathrm{~mm}$ : largest female. $8.0 \mathrm{~mm}$.

Female.--Eye greatly reduced to few specks or completely absent. Antenna 1. about 50 percent length of body. about 2 times longer than antenna 2: primary flagellum with 16 segments. Antenna 2. flagellum with 6 segments. Mandibles subequal. spine row with 5 spines: segment 2 of palp with 8-9 long setae. segment 3 with $1-2$ A setae. $2-j \mathrm{C}$ setae. $3-4 \mathrm{E}$ setae and a row of $D$ setae. lacking $B$ seta. Maxilla 1: inner plate with 4-8 apical plumose setae: palp with 5-6 slender spines on apex. Maxilla 2. inner plate with oblique row of 5-6 plumose setae on inner margin. Maxilliped: inner plate apically with $j$ bladelike spine teeth. I naked setae, and 4-7 plumose setae extending from inner margin to apex: outer plate with row of naked setae and $2-3$ slender pectinate spines, dactyl with 2 setae on inner margin near nail: dactyl nail very long. about $1: 2$ length of dactyl.

Propod of gnathopod 1 less than 1.5 times larger than carpus, palm straight. shorter than posterior margin. with 4 unnotched spine teeth on inside. 6 on outside: defining angle with 3-4 spine teeth on inside. I large unnotched and I laterally notched spine teeth on outside: superior medial setae few in number and singly inserted: posterior margin with 5 sets of setae singly or doubly inserted. Dactyl with 2-3 short setae on inner margin, nail very long. Ventral margin of coxa 1 with 4 long setae.

Propod of gnathopod 2 slightly longer than carpus: palm almost straight. shorter than posterior margin. with 4 unnotched spine teeth on inside. 6 on outside: defining angle with 2-3 spine teeth on inside. 1 large and I smail unnotched spine teeth on outside: 5 superior medial setae singly inserted: posterior margin with 5 sets of setae in groups of 1-5. Dactyl with row of short setae on inner margin. Ventral margin of coxa 2 with 6 setae.

Coxa of pereopod 3 with 6 marginal setae. Coxa of pereopod 4 with 9-12 marginal setae. dactyl of pereopod 4. 40 percent length of corresponding propod. Pereopod 5: basis with 8 very shallow serrations along posterior margin: 5 short spines on anterior margin. Pereopod 6: basis with 8 very shallow serrations along posterior margin: 5 short spines on anterior margin. Pereopod 7: coxa with 2 setae on the posterior margin: basis with 10 shallow serrations along posterior margin; 6 short spines on anterior margin: dactyl about 35 percent length of corresponding propod.

Pleonal plates: posterior margin of plate I slightly convex. with I seta. distoposterior corner not produced. subacute: posterior margin of plate 2 nearly straight with $\mid$ seta. distoposterior corner acute but not produced. ventral margin with 4 (subventral) spines: posterior margin of plate 3 nearly straight with I seta. distoposterior corner slightly subacute but not produced. ventral margin with 3 (subventral) spines. 
Peduncle of pleopod 1 with 2 retinaculae. lacking setae on outside margin.

Uropod 1: inner ramus 65 percent length of peduncle, armed with 11 spines: outer ramus with about 10 spines: peduncle with 9 outer and 4 inner spines. Uropod 2: inner ramus armed with 12 spines: outer ramus with 8 spines: peduncle with 2-3 outer spines. 3 inner spines. Uropod 3 : inner ramus with I spine. outer ramus about 1.7-2 times longer than peduncle, inner and outer margin each with 2-3 set of spines in groups of $1-3$. Telson about as broad as long, notched about $1 / 6-1 / 3$ distance to base. each lobe with $1-2$ apical spines.

Male.--Differing from female as follows. Smaller with more slender. elongate body. Antenna 2: calceoli present on peduncular segments 4 and 5 . and first 2 segments of flagellum.

Propod of gnathopod I more than 2 times larger and longer than carpus: palm shorter than or equal in length to posterior margin. with 5 spine teeth on inside. 7 on outside: defining angle with + spine teeth on inside and 1 notched and 1 serrate or unnotched spine teeth on outside. Propod of gnathopod 2 more than 2 times larger and longer than carpus: palmar margin strongly oblique. convex. equal or slightly longer than posterior margin. with 10 spine teeth on inside. 12 on outside: defining angle with 2 spine teeth on inside. 2 on outside. Uropod 2: inner ramus with 11 spines: outer ramus slightly deflected. with 5-6 inner and 4-5 outer spines. apex with about 4 spines: peduncle with 2 outer and $2-3$ inner spines.

Distribution and ecology.--This species is recorded from southern Indiana. southern Illinois. southcentral and westerm Kentucky. west-central Missouri. and eastern Kansas (fig. 94). Its range. which is extensive for a stygobiont amphipod. covers a linear distance about $1000 \mathrm{~km}$ and crosses three physiographic provinces. extending from the Interior Low Plateaus in the east to the Osage Plains in the west.

In caves. C. packurdi is found in drip pools. streams. and stream pools. It is also recorded from surface seeps and springs. In Indiana caves. it is sometimes associated with $C_{\text {. Indiunensis or }} C_{\text {. lewisi. In Kansas }}$ caves. it is sometimes associated with the large troglobitic amphipod Sngohromus clantont. Ovigerous females were collected from March to August and in November. Sexually mature females are 4.5 to 8.0 $\mathrm{mm}$ and sexually mature males are $3.0-5.5 \mathrm{~mm}$.

Remarks.--Earlier references to literally all Crangomx from caves in the Interior Low Plateaus region were this species. However. it is obvious from the present study that several species were once called "packardi."

\section{Crangonyx stagnicolous. new species}

Figures $95-98$

Material examined (paratypes unless designated otherwise).--MARYLAND. Calvert Co.: Grays Cypress Swamp. 1 F. C. R. Shoemaker. 17 Apr 1938 (USNM): Dorchester Co.: swamp. $5.3 \mathrm{~km} \mathrm{~W} \mathrm{of}$ 


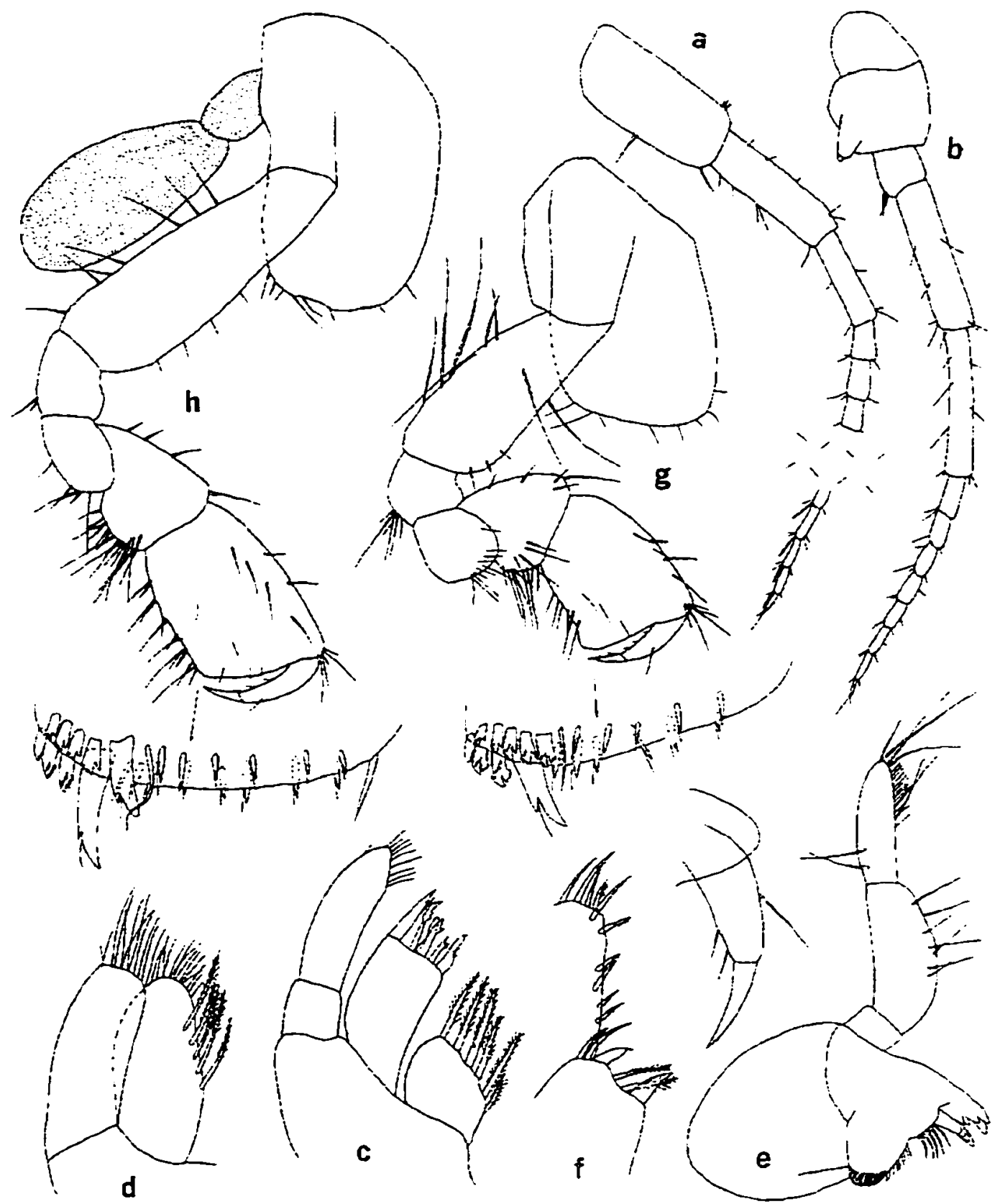

Figure 95.--Crangonyx stagnicolous, new species. Female paratype $(6.4 \mathrm{~mm})$, swamp near Mount Vernon, Fairfax Co.. Virginia: a. b. antennae 1. 2; c. d. maxillae 1. 2: e. left mandible: $f$. inner and outer plates and palp dactyl of maxilliped (greatly enlarged); g, h. gnathopods 1. 2 (palmar margins enlarged). 


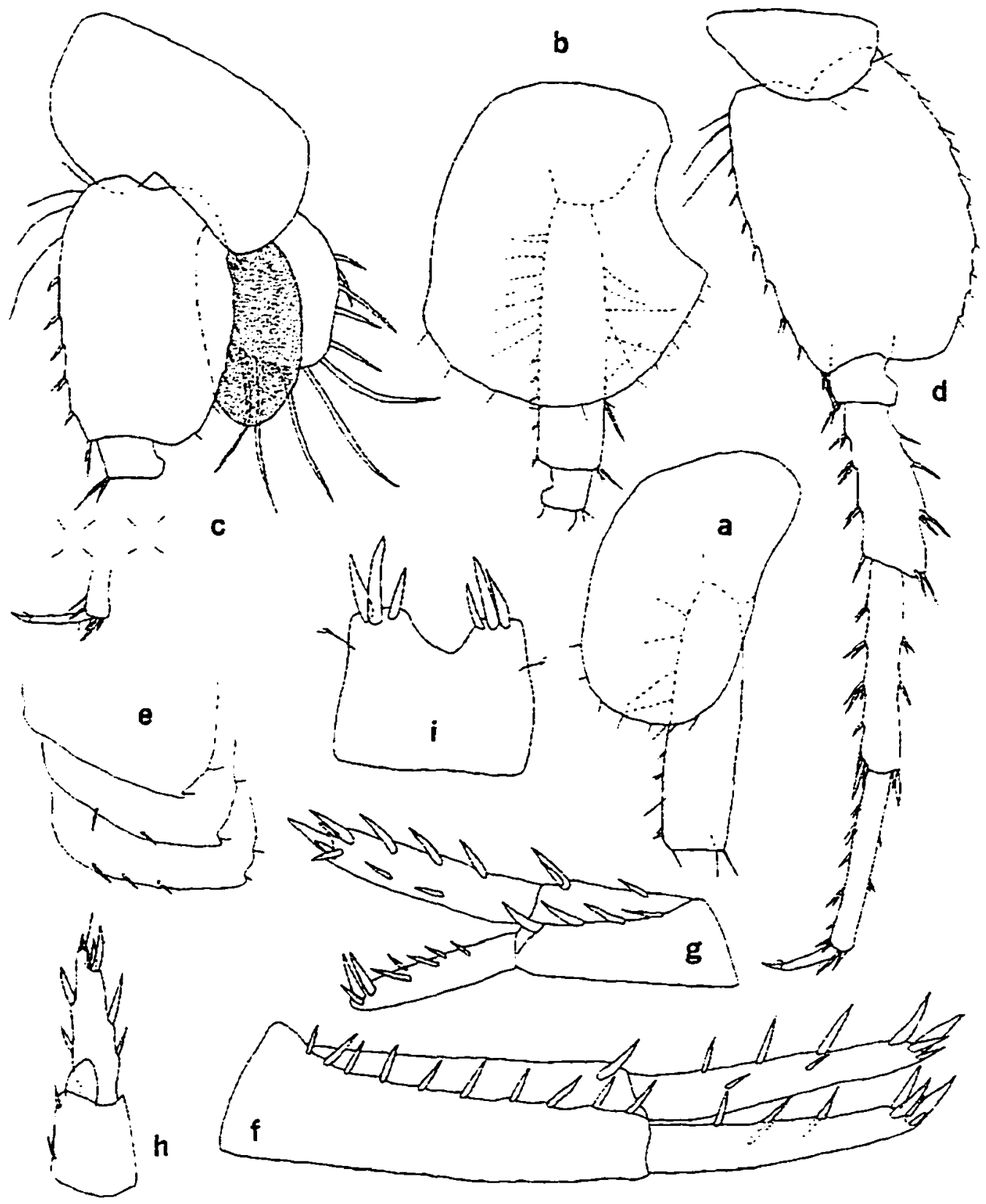

Figure 96.--Crangonyx stagnicolous, new species. Female paratype $(6.4 \mathrm{~mm})$. swamp near Mount Vernon. Fairfax Co.. Virginia: a. b. c. pereopods 3. 4. 5 (in part): d. pereopod 7: e. pleonal plates: f. g. h. urupods 1. 2. 3: i. telson. 


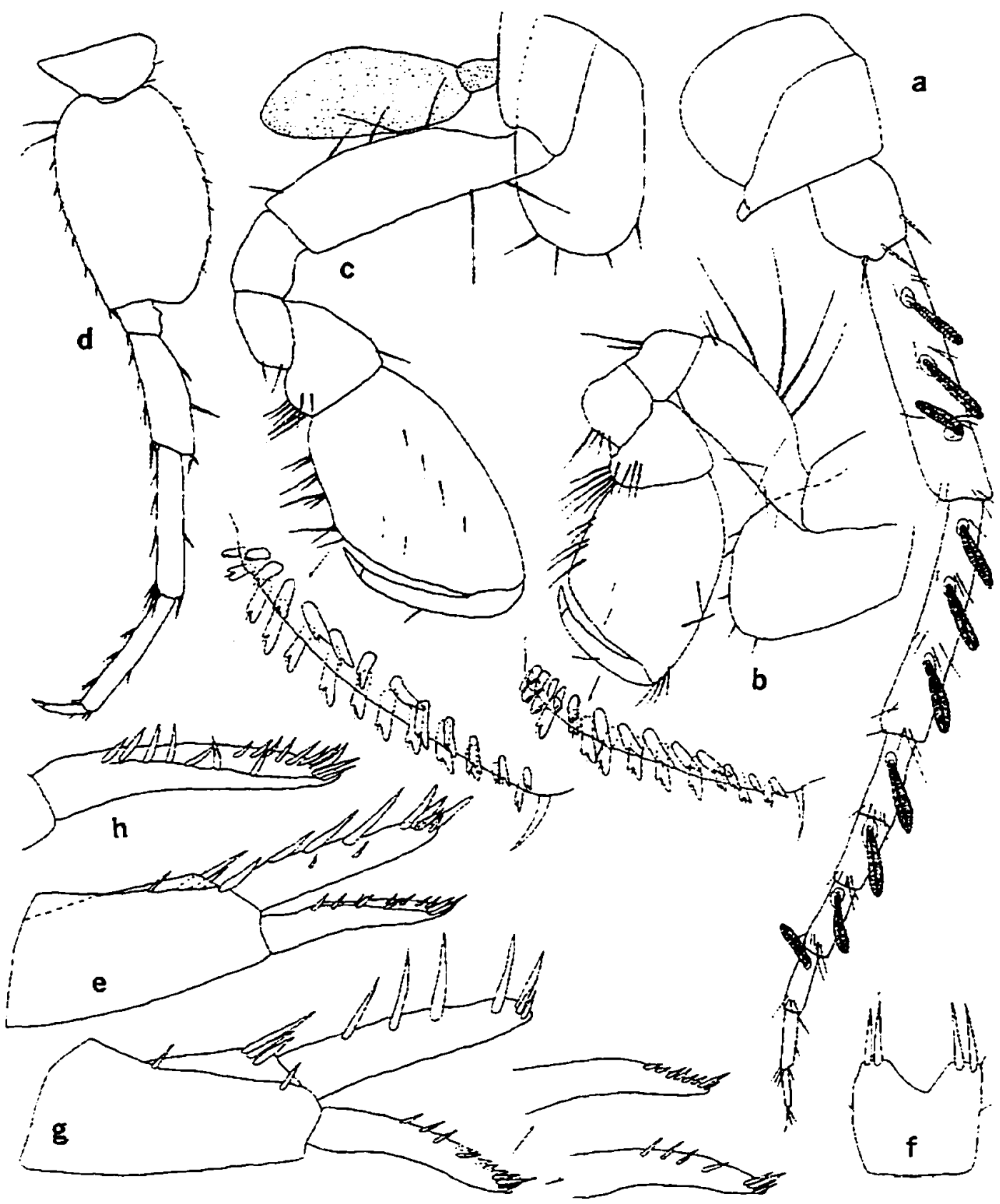

Figure 97.--Crangonyx stagnicolous, new species. Male paratype (3.8 mm), swamp near Mount Vernon. Fairfax Co., Virginia: a, antenna 2: b. c, gnathopods I, 2 (palmar margins enlarged): d. pereopod 7: e. uropod 2: f. telson. Male paratype $(4.1 \mathrm{~mm})$. Swamp near Mount Vernon. Fairfax Co.. Virginia: g. uropod 2 (spines on inner and outer margins of ramus shown separated). Male ( $5.5 \mathrm{~mm}$ ). Ridge. St. Marys Co.. Maryland: h. outer ramus of uropod 2 (enlarged). 


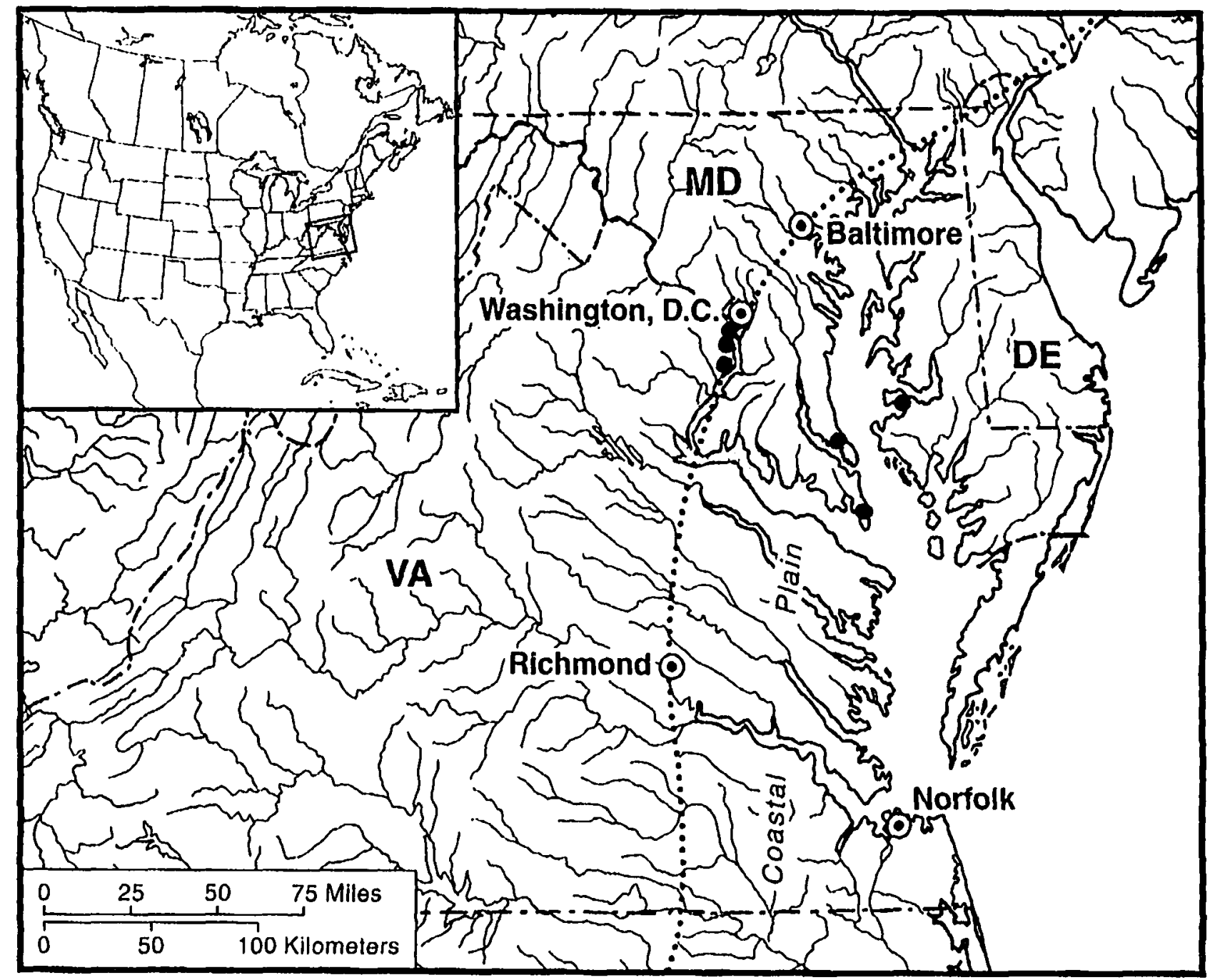

Figure 98.--1)istribution of ( ramgemex stagnicoloms in North Americat. Solid circles represent 1-5 closely proximate locialities. The donted line denotes the "fall line" of "lall zene" between the Coastal Plain and Piedmont. 
Cambridge. 1 . R. W. Jackson. 20 Apr 1943 (USNM): St. Marys Co.: ditch on Ridge. $7 \cong .11$ W. W. Ball. 26 Apr 1930 (USNM). VIRGINIA. Fairfax Co.: marshy ponds. Springfield. holotype $9.4 \supseteq .3 \approx$. J. R. Holsinger. 7 Apr 1973; Mount Vernon. 1 \% M. K. Brady, 10 May, 1924 (USNM): pools near Black Pond. 65 specs ( $q, 0$, juvs), W.D. Webb. 25 Nov 1915 (USNM); small pool between Dyke and Belle Haven. $8 \subseteq, 2 \sigma^{\circ}$. W. H. Ball. 14 Apr 1935 (USNM): small pools near Dyke. 1 ๑. W. H. Ball. 27 Apr 1935 (USNM): swamp near Mount Vernon, 22 ₹. $100^{\circ}$. W. D. Webb. 22 Apr 1917 (USNM): pool in Sweet Gum stand. New Alexandria. 3 ○. W. Ball. 24 Apr 1935 (USNM): small pools near New Alexandria. 2 $\because$ W. H. Ball. 22 Apr 1935 (USNM): seeps ca. 1.2 km NE of Cheney School. Dogue Creek drainage. Ft. Belvoir. 15 specs. C. S. Hobson and D. J. Stevenson. 13 Apr 1995: seep on E bank of Accotink Creek. ca. 0.6-0.7 km WNW of jet. 613 and 617.5 specs.. C. S. Hobson and D. J. Stevenson. 13 Apr 1995.

Diagnosis.-A small species of the gracilis group distinguished from other members of the group by having 2 setae on posterior margins of pleonal plates. I of which is inserted just above distoposterior corner: shorter uropod $j$ with ratio of length of outer ramus to peduncle less than 1.5: male with large well spaced comb-like spines on outer ramus of male uropod 2. Largest male. $5.2 \mathrm{~mm}$ : largest female. $9.0 \mathrm{~mm}$.

Female.--Eye large and ovate. Antenna 1 . about 50 percent length of body. about 1.8 times longer than antenna 2: primary flagellum with about 20 segments. Antenna 2. flagellum with 7-9 segments. Mandibles subequal. spine row with 5 spines: segment 2 of palp with 7 long setae. segment 3 with 1-2 A setae. $2 \mathrm{C}$ setae. $4-5 E$ setae and a row of $D$ setae. lacking $B$ seta. Maxilla 1: inner plate with 6 apical plumose setae: palp with 9 slender spines on apex. Maxilla 2. inner plate with oblique row of 5-6 plumose setae on inner margin. Maxilliped: inner plate apically with 2 bladelike spines. I naked spine. and 5-6 plumose setae extending from inner margin to apex: outer plate with row of naked setae and $j-4$ slender pectinate spines on inner margin: $2-3$ setae on inner margin of dactyl near nail: dactyl nail very long. about 1.3 length of dactyl.

Propod of gnathopod 1 less than 2 times larger and longer than carpus: palm almost straight. shorter than posterior margin. with 5 unnotched spine teeth on inside. 6 on the outside: defining angle with 5 notched-serrate spine teeth on inside. I large notched and 3 serrate spine teeth on outside: superior medial setae few in number and singly inserted: posterior margin with 6 singly inserted setae. Dactyl with row of short setae on inner margin. nail very lorig. Ventral margin of coxa I with 7-8 setae.

Propod of gnathopod 2 lees than 2 times larger and longer than carpus: palm almost straight. shorter than posterior margin. with 6 unnotched spine teeth on inside. 8 on outside: defining angle with 1 large notched spine tooth and 4 notched-serrate spine teeth on outside: superior medial setae singly inserted: posterior margin with 2-4 setae. Dactyl with row of short setae on inner margin. Ventral margin of coxa 2 with 8 setae.

Coxa of pereopod 3 with 8 marginal setae. Coxa of pereopod 4 with 12 marginal setae. dactyl of pereopod 4. 40 percent length of corresponding propod. Pereopod 5: basis with 8 very shallow serrations along posterior margin: 6-8 short spines on anterior margin: 4 long setae on the anteroproximal margin. 
Pereopod 6: basis with 8-9 shallow serrations along posterior margin: 9 short spines on anterior margin: 1 seta on anteroproximal margin. Pereopod 7: : coxa with 3 setae on the posterior margin: basis with 11 shallow serrations along posterior margin: 7 short spines on anterior margin: 3 long setae on anteroproximal margin: dactyl about 40 percent length of corresponding propod.

Pleonal plates: posterior margin of plate I slightly convex. with 2 setae. I at distoposterior corner: distoposterior corner slightly acute but not produced: posterior margin of plate 2 slightly convex with 2 setae. I at the distoposterior comer, distoposterior comer acute but not produced. ventral margin with 2 (subventral) spines; posterior margin of plate 3 slightly convex with 2 setae. I at distoposterior comer. distoposterior comer acute but not produced, ventral margin with 3 (subventral) spines. Peduncle of pleopod 1 with 2 retinaculae. lacking setae on outside margin.

Uropod I: inner ramus 75 percent length of peduncle. armed with 9 spines: out ramus with 10 spines: peduncle with 10 outer and I inner spines. Uropod 2: inner ramus armed with 10 spines: outer ramus with 8 spines: peduncle with 4 outer and 3 inner spines. Uropod 3 : inner ramus lacking spines. outer ramus about 1.5 times longer than peduncle. inner and outer margin each with $2-3$ set of spines in groups of $1-3$. Telson broader than long. notched about 1/4 distance to base. each lobe with 3 apical spines: spines about 12 length of telson.

Male.--Ditfering from female as follows. Smaller than female. with more slender. elongate body. Antenna 2: calceoli percent on peduncular segments 4 and 5 . and first 4 segments of flagellum.

Propod of gnathopod 1 more than 2 times larger and longer than carpus and much broader. Propod of gnathopod 1 with 8 spine teeth on inside, 9 on outside. defining angle with 5 notched-serrate spine teeth on inside. I notched and 3 serrate spine teeth on outside. Propod of gnathopod 2 more than 2 times larger and longer than carpus: palm margin strongly oblique. with 10 spine teeth on inside. 10 on outside: detining angle with $\mid$ notched and $\mid$ serrate spine tooth on inside and 2 similar spine teeth on outside.

Uropod 2: inner ramus lacking spines or with 3 short inner spines. 3-4 long outer spines. 3-5 spines on apex: outer ramus curved. with 4-7 outer spines and comb-like row of spines on outside on distal half. apex with 3-5 spines: peduncle with 4 outer and 2 inner spines. Uropod 3 : inner ramus lacking spines: outer ramus about 2 times length of peduncle.

Type-locality.--Marshy ponds on Accotink Creek. just below Lake Accotink. Springfield. Fairtax County, Virginia.

Distribution and ecology.--The range of this species. which is restricted at present to the western margin of the Coastal Plain in northern Virginia and a small part of the Coastal Plain in southern Maryland. covers a linear distance of about $100 \mathrm{~km}$ (fig. 98).

C. stagnicolous inhibits cypress swamps, marshy ponds. and small pools and is sometimes associated with $C$. poiustris. Ovigerous females were collected in April and August. Sexually mature males. 4.0-5.5 $\mathrm{mm}$ : sexually mature fermales. $6.5-9.0 \mathrm{~mm}$.

Etymology.--The epithet stagnıcolous is from Latin, meaning "living in a swamp." 
Remarks.-C. stagnicolous shares with $C$. bousfieldi the presence of a seta just above the distoposterior comer of the pleonal plate. Although this character is unique to these two species of Crangonx. it is probable a homoplasy.

Crangomy aka, new species

Figures 99-102

Material examined (paratypes unless designated otherwise).--ARKANSAS. Pope Co.: unmarked stream ca. $0.8 \mathrm{~km}$ S of Hector on state rd. 27, holotype ₹. 25 \%. 15 \%. R. Fox. 28 Dec 1970: Saline Co.: seep, $8.0 \mathrm{~km}$ N of Paron. 30 specs ( $\left.{ }^{\circ}, 0^{\circ}\right)$. L. Hubricht, 5 May 1940 (USNM): Van Buren Co.: unmarked stream. $8.0 \mathrm{~km} \mathrm{~W}$ of Rupert. on state rd. 16.8 F. $3 \mathrm{c}$. R. Fox. 28 Dec 1970: unmarked stream. $5.0 \mathrm{~km} \mathrm{~W} \mathrm{of}$ Rupert. 4 q. 4 s. R. Fox. 28 Dec 1970.

Diagnosis.--A small species of the gracilis group closely allied morphologically with $C$. rivuluris but distinguished from that species by the telson, which is notched 1/2 distance to base: spines on inner margin of outer ramus of male uropod 2 distributed evenly; gnathopod 2 of male lacking notched-serrate spine teeth on propod defining angle; setae absent from anteroproximal margin of pereopod 5-7. Largest male. $5.5 \mathrm{~mm}$ : largest female. $7.4 \mathrm{~mm}$.

Female.--Eye large and ovate. Antenna 1, about 50 percent length of body, about 1.8 times longer than antenna 2: primary flagellum with about 24 segments. Antenna 2, flagellum with 8 segments. Mandibles subequal, spine row with 4-5 spines: segment 2 of palp with 7-8 long setae. segment 3 with 1-2 A setae. 1 B seta. 1-2 C setae. $4 \mathrm{E}$ setae and a row of $\mathrm{D}$ setae. Maxilla 1: inner plate with 6 apical plumose setae: palp with 5-7 slender spines on apex. Maxilla 2, inner plate with oblique row of 6 plumose setae on inner margin. Maxilliped: inner plate apically with 3 bladelike spines. 1 naked spine, and 5-6 plumose setae extending from inner margin to apex: outer plate with row of naked setae and 7-8 slender pectinate spines on inner margin; dactyl with $1-2$ setae on inner margin near nail: dactyl nail very long. $1 / 3$ length of dactyl.

Propod of gnathopod 1 less than 2 times larger and longer than carpus: palm almost straight. subequal to posterior margin, with 7 unnotched spine teeth on inside. 6 on the outside: defining angle with 4 notched-serrate spine teeth on inside, 1 large notched and 2 serrate spine teeth on outside: superior medial setae singly inserted; 4 sets of posterior marginal setae inserted in groups of 2-5. Dactyl with row of short setae on inner margin, nail long. Ventral margin of coxa 1 with 5 short setae.

Propod of gnathopod 2 less than 2 time larger and longer than carpus: palm almost straight, shorter than posterior margin, with 8 unnotched spine teeth on inside, 9 on outside; defining angle with I notched and 2 notched-serrate spine teeth on inside, 1 large notched and 2 unnotched spine teeth on outside: superior medial setae singly inserted. posterior margin with 5 sets of setze in groups of $1-5$. Dactyl with row of short setae on inner margin. Ventral margin of coxa 2 with 6 setae. 


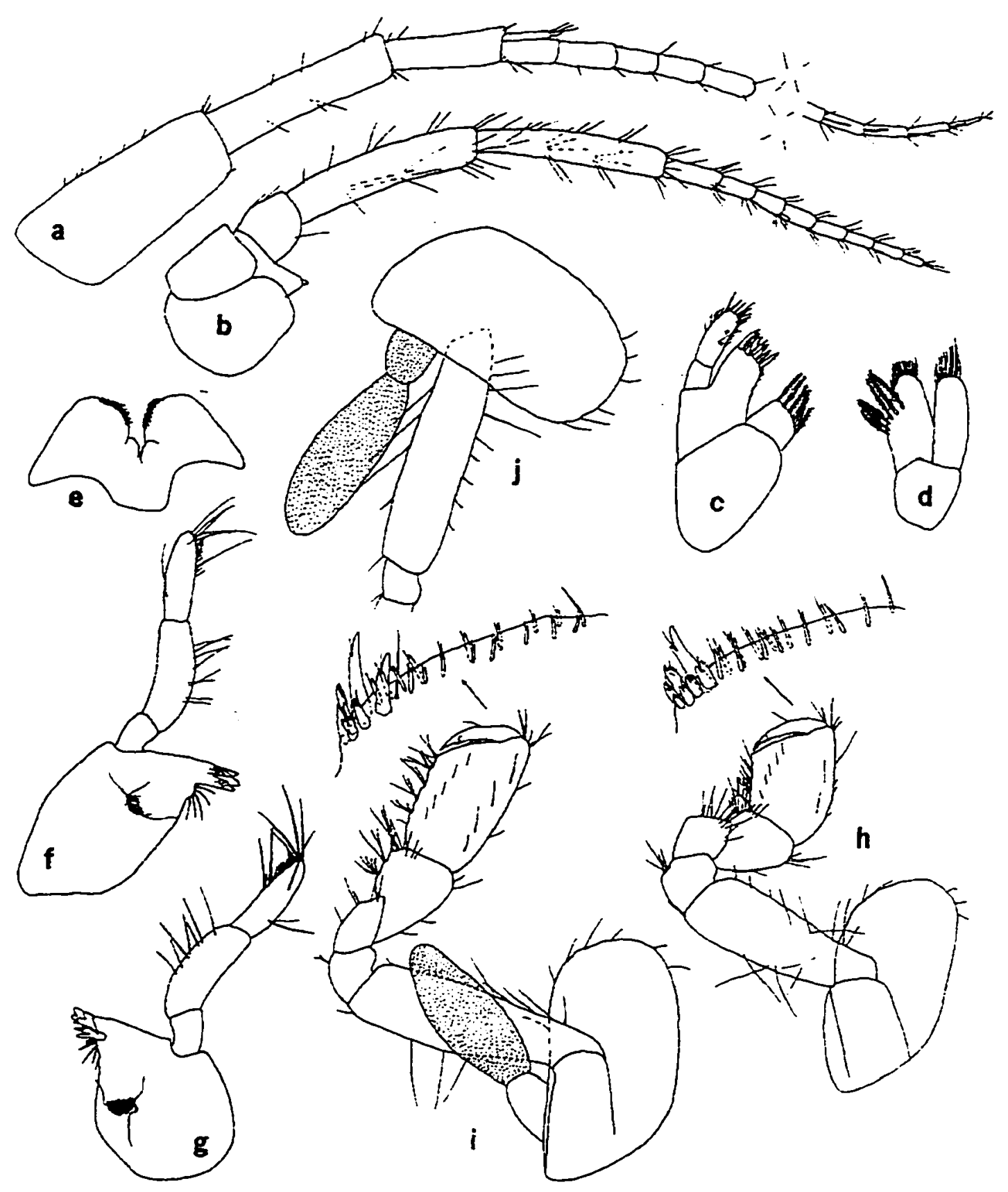

Figure 99.--Crangonvx aka. new species. Female paratype $(6.9 \mathrm{~mm})$. unmarked stream ca. $0.8 \mathrm{~km} \mathrm{~S}$ of Hector on state rd. 27. Pope Co., Arkansas: a. b. antennae 1. 2: c. d. maxillae 1. 2: e. lower lip: f. g. left and right mandible; h, i. gnathopods I, 2 (palmar margins enlarged): j. pereopod 3 (in part). 


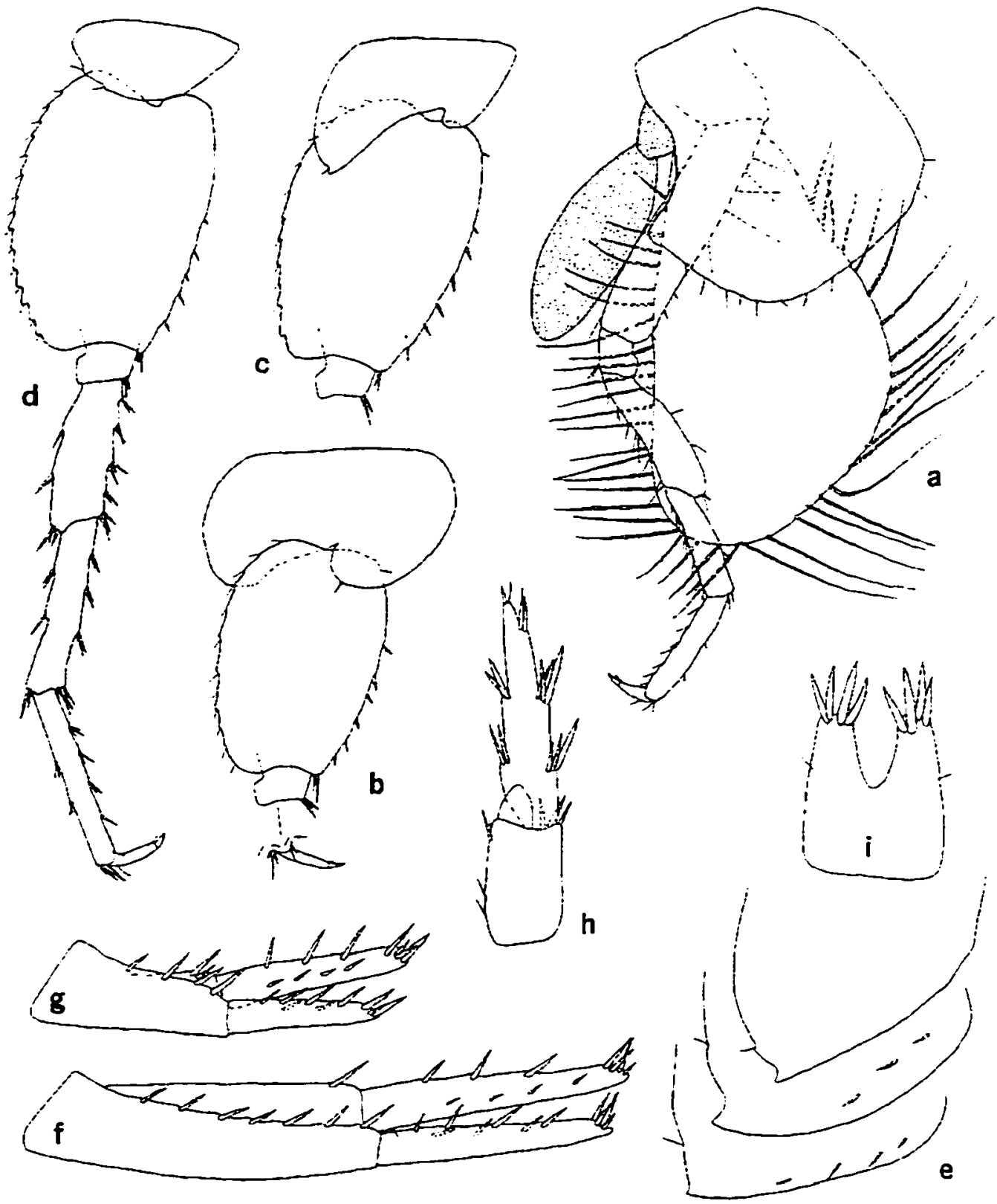

Figure 100.--Crangonyx aka. new species. Female paratype $(6.9 \mathrm{~mm})$. unmarked stream ca. $0.8 \mathrm{~km} \mathrm{~S}$ of Hector on state rd. 27. Pope Co.. Arkansas: a. d. pereopods 4. 7: b. c. pereopods 5.6 (in part): d. pereopod 7; e, pleonal plates; f, g, h. uropods 1, 2, 3: i, telson. 


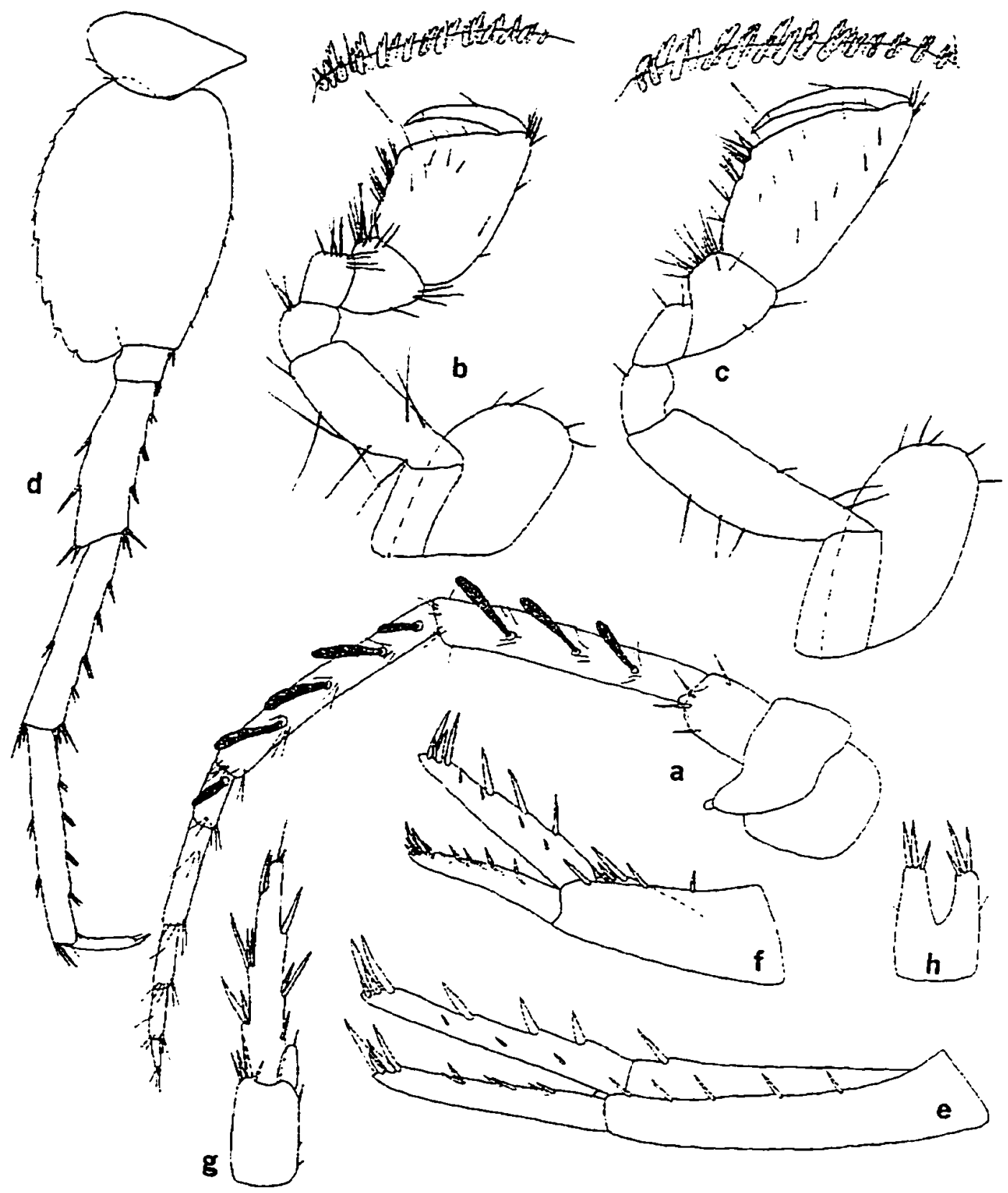

Figure 101 .--Crangonvx aka. new species. Male paratype $(4.8 \mathrm{~mm})$, unmarked stream ca. $0.8 \mathrm{~km} \mathrm{~S}$ of Hector on state rd. 27. Pope Co.. Arkansas: a. antenna 2: b. c. gnathopods I. 2 (palmar margins enlarged): d. pereopod 7: e. f. g. uropods 1. 2. 3: h. telson. 


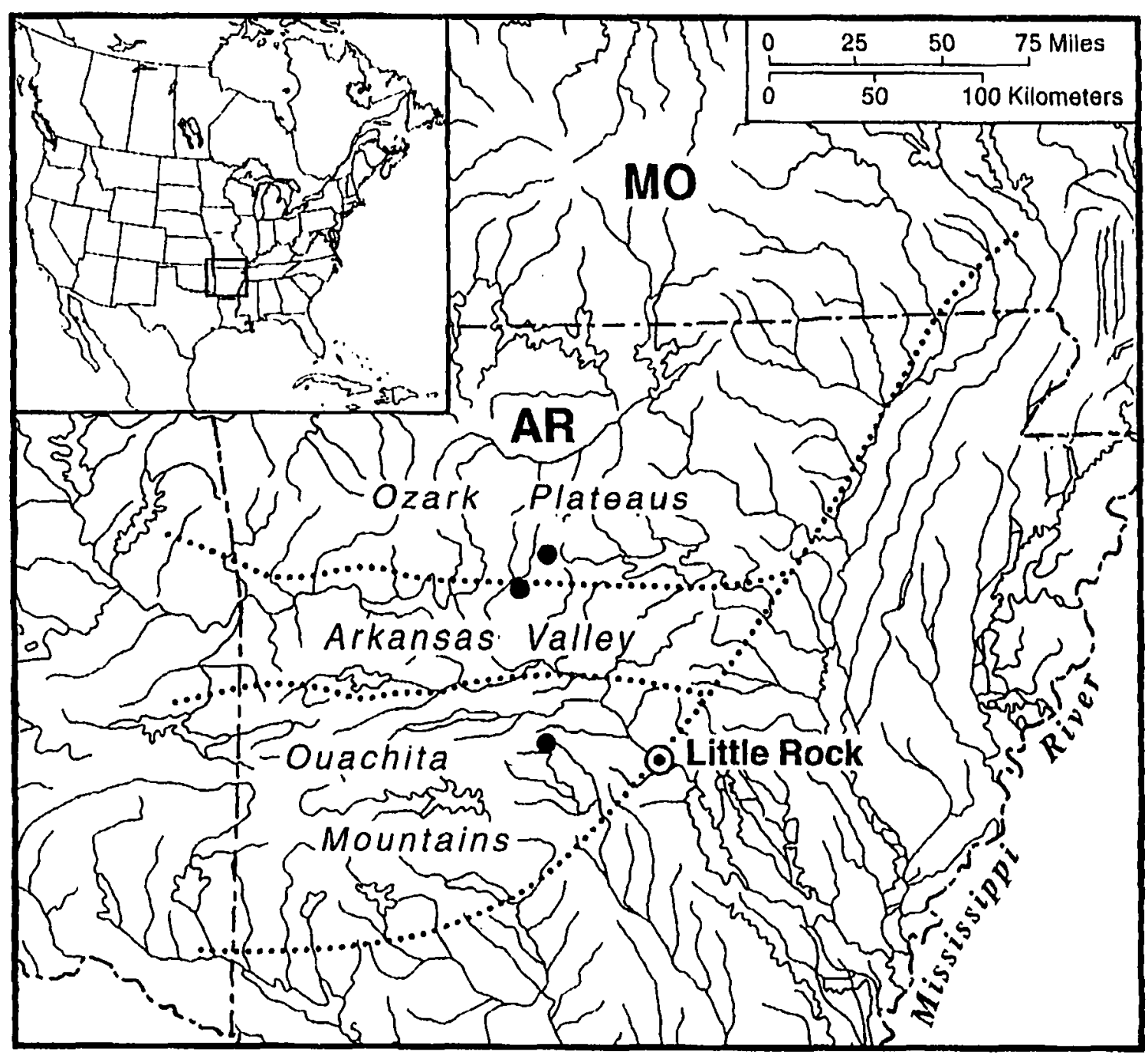

Figure !)2.--1) istribution of ( 'rumgomex aku in North America. Solid circles represent 1-2 closely prosimate localitics. 
Coxa of pereopod 3 with 6 marginal setae. Coxa of pereopod 4 with 11 marginal setae. dactyl of pereopod 4. 35 percent length of corresponding propod. Pereopod 5: basis with 12 very shallow serrations along posterior margin; 7 short spines on anterior margin. Pereopod 6: basis with 13 serrations along posterior margin: 7 short spines on anterior margin. Pereopod 7: coxa with 3 setae on posterior margin: basis with 15 slight serrations along posterior margin; 5 short spines on anterior margin: dactyl about 33 percent length of corresponding propod.

Pleonal plates: posterior margin of plate I convex. with I seta, distoposterior corner produced and acute: posterior margin of plate 2 nearly straight with 1 seta. distoposterior corner strongly produced and acute. ventral margin with 3 (subventral) spines; posterior margin of plate 3 nearly straight with 1 seta. distoposterior corner small and acute but not produced. ventral margin with 4 (subventral) spines. Peduncle of pleopod I with 2 retinaculae, lacking setae on outside margin.

Uropod I: inner ramus 80 percent length of peduncle. armed with II spines: outer ramus with 12 spines: peduncle with 7 outer and 1 inner spines. Uropod 2: inner ramus armed with 10 spines: outer ramus with 8 spines: peduncle with 4 outer spines. and 4 inner spines. Uropod 3 : inner ramus lacking spines. outer ramus about 2 times longer than peduncle. inner and outer margins each with 2-3 set of spines in groups of 2-4. Telson slightly longer than broad, notched $1 / 2$ distance or slightly more to base. each lobe with 4 apical spines.

Male.--Differing from female as follows. Smaller with more slender. elongate body. Antenna 2: calceoli percent on peduncular segments 4 and 5 . and first segment of flagellum.

Propod of gnathopod 1 more than 2 times larger and longer than carpus: palm with 10 spine teeth on inside. 10 on outside: defining angle with 3 notched-serrate spine teeth on inside. 1 large notched and 2 serrate spine teeth on outside. Propod of gnathopod 2 more than 2 times larger and longer than carpus: palm strongly oblique. convex. with 11 spine teeth on inside. 11 on outside: defining angle with 3 spine teeth on inside and 2 on outside.

Uropod 2: inner ramus with 12 spines: outer ramus only slightly modified. with 6 short spines on inner margin along the whole length and 5-6 longer spines on outer margin. apex with 4 spines: peduncle with 3 outer and $3-4$ inner spines. Telson longer than broad. notched about 1/2 distance to base: each lobe with 2 apical spines: spines about $1 / 3$ length of teison.

Type-locality.--Stream, about $0.8 \mathrm{~km} \mathrm{~S}$ of Hector on state route 27. Pope County. Arkansas.

Distribution and ecology.--The range of this species. which at present is only known from 4 collections from central Arkansas, covers a linear distances of about $80 \mathrm{~km}$ from north to south and covers parts of three different physiographic regions (fig. 102).

It was collected from streams and a seep. Ovigerous females are recorded from May and December. Sexually mature females are 6.2 to $7.4 \mathrm{~mm}$ and sexually mature males are 4.2 to $5.5 \mathrm{~mm}$.

Etymology.--The epithet $a k a$ is derived from a contraction of Arkansas. referring to the occurrence of this species in the state of Arkansas. 
Crangonyx caecus, new species

Figures $103-106$

Material examined (paratypes unless designated otherwise).--KENTUCKY. Carter Co.: Bat Cave. Carter Caves State Park. 8 \%. 2 0. 6 Juvs, S. B. Peck. I Sep 1964. 14 \&. 2 c.. R. M. Norton. 23 Jan 1965. and 19. T. C. Barr. Jr., 12 Oct 1966 and 1 \&, 19 Jun 1979: Cascade Cave. 2 2. collector unknown. 24 Jun 1937 (USNM), 19.8 ๙. R. M. Norton and M. D. Hassell. 23 Jan 1965. 2 ๑. T. C. Barr. Jr.. 23 Jan 1978 and holotype ₹. $10 \% .180^{\circ}, 19$ Jun 1979: Laural Cave, 2 ?. collector unknown, 25 Jun 1937 (USNM): Jarvie Rauarks Cave. $2 \cong .3$ juvs. S. B. Peck. 2 Sep 1964: Saltpeter Cave. $1 \cong$. R. M. Norton. 24 Jan 1965

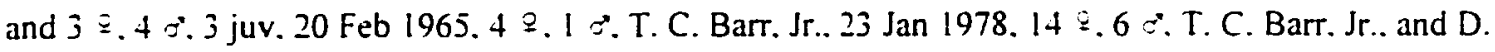
K. Harker. 19 Jan 1979: X Cave. 1 ₹. R. M. Norton. 24 Jan 1965 and 2 ๑. T. C. Barr and D. K. Harker. 29 Jun 1979.

Diagnosis.--A relatively small cavernicolous species closely allied morphologically with C: castellanum but distinguished from that species by lacking calceoli on antenna 2 in mature males. and having fewer spines on inside margin of outer ramus of uropod 2 of male. Largest male, $5.7 \mathrm{~mm}$ : largest female, $7.3 \mathrm{~mm}$.

Female.--Eyes absent. Antenna 1, about 50 percent length of body. about 1.8 times longer than antenna 2: primary flagellum with about 15 segments. Antenna 2. flagellum with 8-9 segments. Mandibles subequal. spine row with 4-5 spines: segment 2 of palp with 10 long setae. segment 3 with 1 A seta. 2-3 C setae. $4 E$ setae and a row of $D$ setae. lacking $B$ seta. Maxilla 1 : inner plate with 5-6 apical plumose setae: palp with 5-6 slender spines on apex. Maxilla 2. inner plate with oblique row of 5-6 plumose setae on inner margin. Maxilliped: inner plate apically with 4 bladelike spines. I naked spine, and 5-6 plumose setae extending from inner margin to apex: outer plate with row of naked setae and 5 weak pectinate spines on inner margin; dactyl with $1-2$ setae on inner margin near nail: dactyl nail very long, about $1 / 2$ length of dactyl.

Propod of gnathopod 1 less than 2 times larger and longer than carpus: palm shoner than posterior margin. almost straight. with 4-6 unnotched spine teeth on inside, 5-7 on the outside: defining angle with 35 notched-serrate spine teeth on inside and 1 large notched or unnotched spine tooth and 1-2 small serrate spine teeth on outside: superior medial setae few in number and singly inserted: 6 sets of setae on posterior margin singly inserted or in groups of 2-4. Dactyl with row of short setae on inner margin. nail very long. Ventral margin of coxa 1 with 9 setae.

Propod of gnathopod 2 less than 2 times larger and longer than carpus: palm almost straight. oblique. shorter than posterior margin. with 6 unnotched spine teeth on inside. 7-9 on outside: defining angle with 1 large notched spine tooth and 1-2 serrate spine tooth on inside, I large unnotched and I serrate or unnotched spine teeth on outside: superior medial setae singly inserted. posterior margin with 6 sets of 


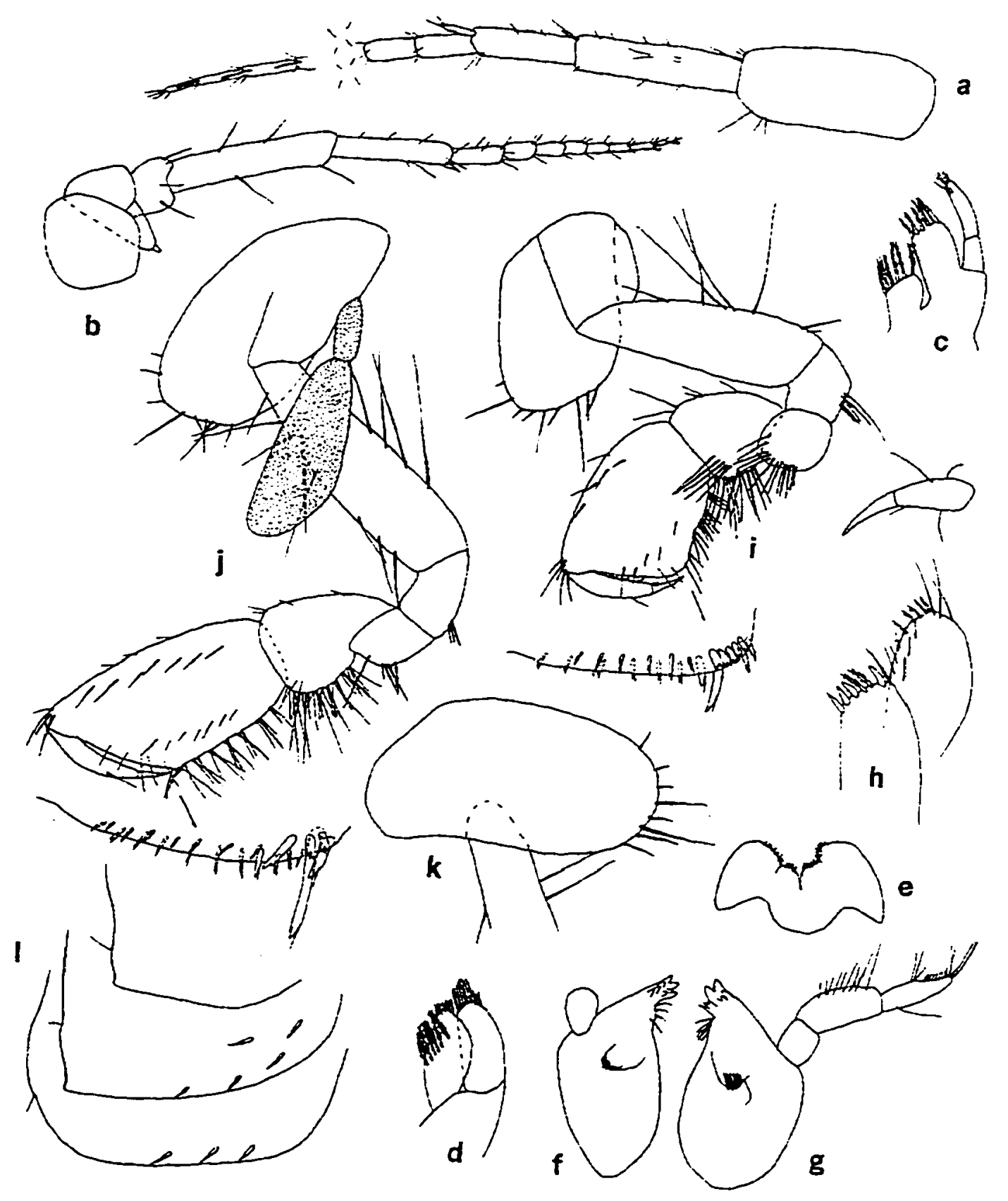

Figure 103.--Crangonyx caecus, new species. Female paratype $(6.5 \mathrm{~mm})$. Cascade Cave. Carter Co.. Kentucky: a. b. antennae 1. 2: c. d, maxillae 1. 2: e. lower lip: f. dentate part of left mandible: g. right mandible: h. inner and outer plates and palp dactyl of maxilliped: i. j. gnathopods 1.2 (palmar margins enlarged): $k$. pereopod 3 (in part): 1 , pleonal plates. 


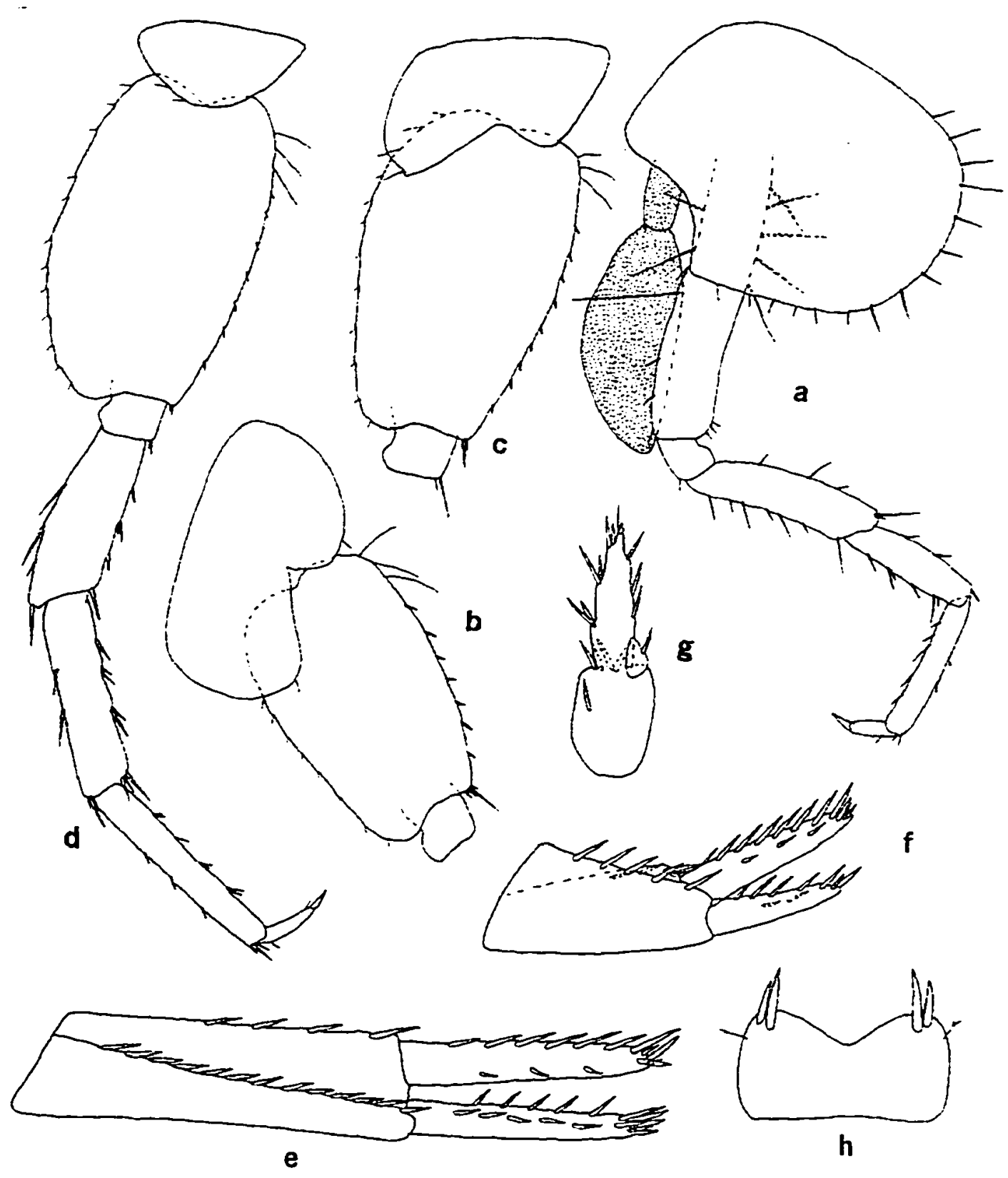

Figure 104.--Crangomx caecus. new species. Female paratype $(6.5 \mathrm{~mm})$. Cascade Cave. Carter Co.. Kentucky: a. d. pereopods 4, 7: b, c. pereopods 5.6 (in part): e. f. g. uropods 1, 2. 3: h, telson. 


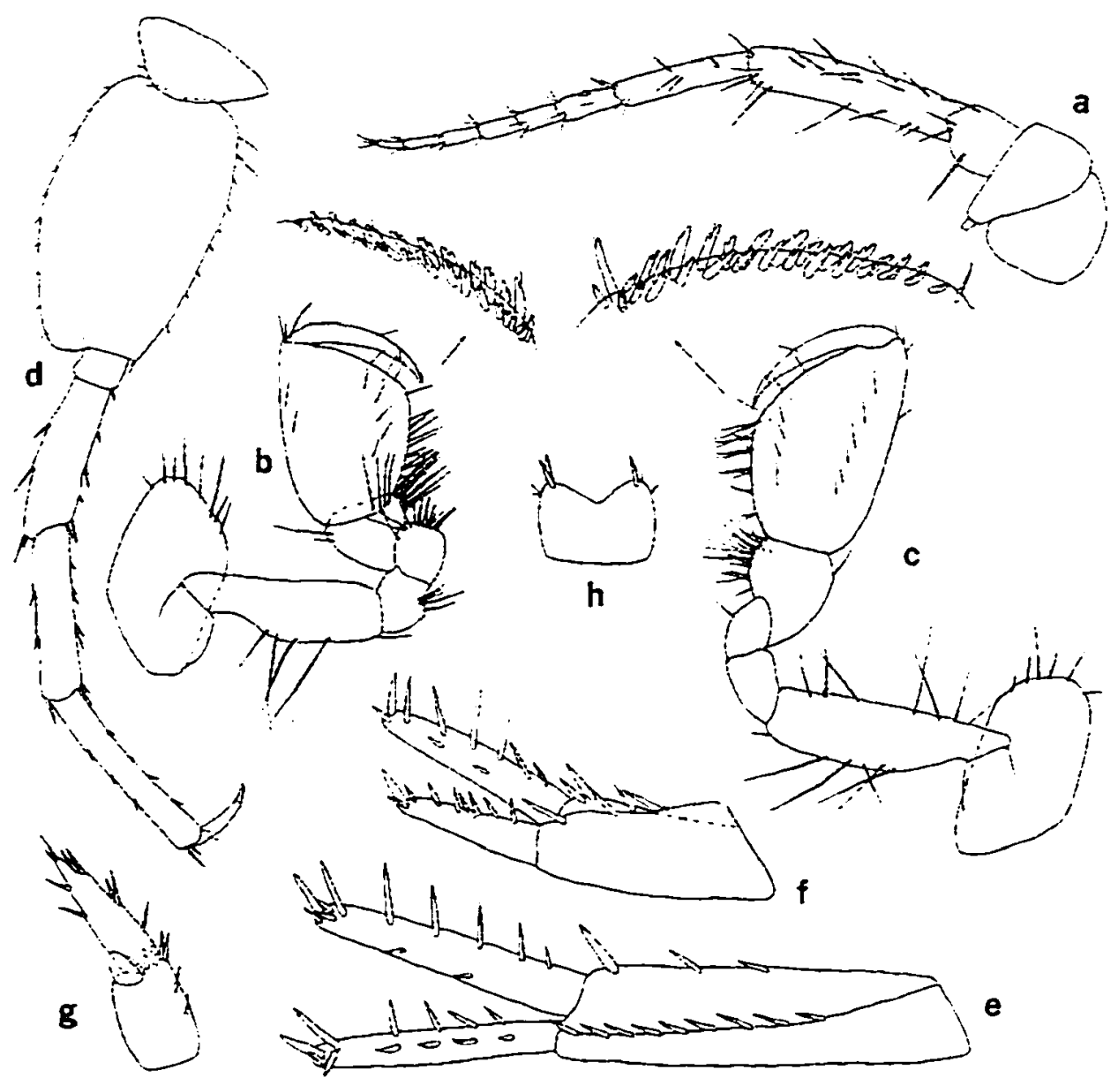

Figure 105.--Crangonvx caecus, new species. Male paratype $(4.7 \mathrm{~mm})$. Cascade Cave. Carter Co.. Kentucky: a. antenna 2: b. c. gnathopods 1, 2 (palmar margins enlarged); d. pereopod 7: e. f. g. uropods $1,2,3: \mathrm{h}$, telson 


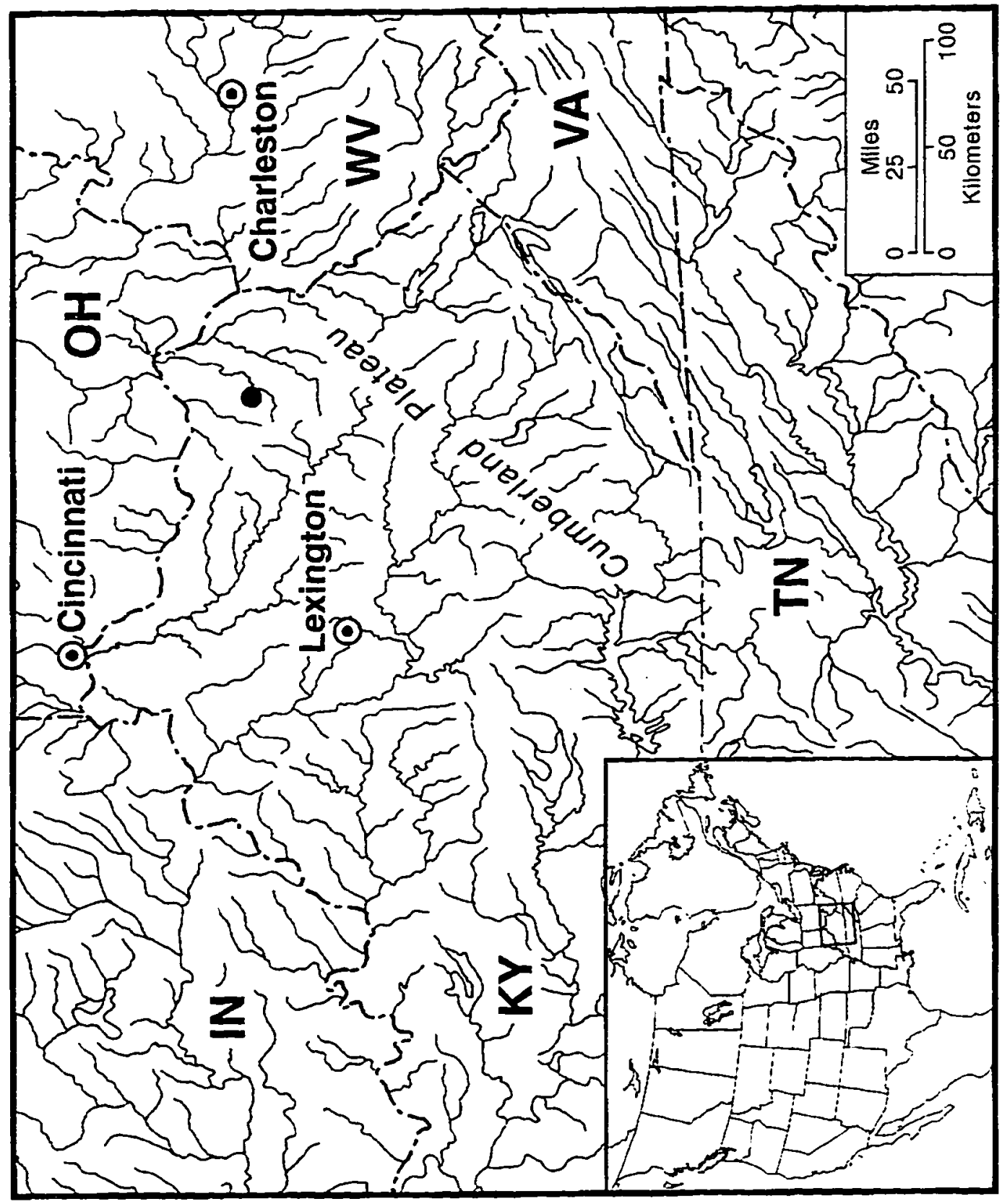

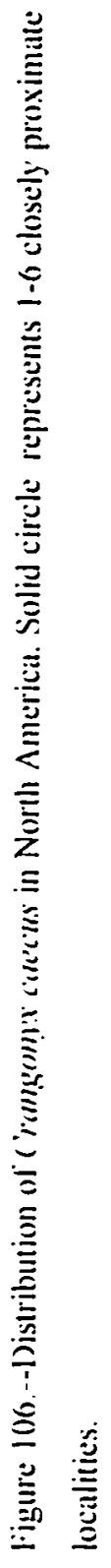


setae in groups of 2-4. Dactyl with row of short setae on inner margin. nail long. Ventral margin of coxa 2 with 9 setae.

Coxa of pereopod 3 with 8 marginal setae. Coxa of pereopod 4 with 15 marginal setae. dactyl of pereopod 4. 45 percent length of corresponding propod. Pereopod 5 : basis with 15 very shallow serrations along posterior margin: 7 short spines on anterior margin: 3 long setae on the anteroproximal margin. Pereopod 6: basis with 12 very shallow serrations along posterior margin: 9 short spines on anterior margin: 3 long setae on anteroproximal margin. Pereopod 7: coxa with 2 setae on posterior margin: basis with 12 shallow serrations along posterior margin: 7 short spines on anterior margin: 3 setae on anteroproximal margin: dactyl about 40 percent the length of corresponding propod.

Pleonal plates: posterior margin of plate I neatly straight. with I seta. distoposterior corner not produced: posterior margin of plate 2 nearly straight with 1 seta. distoposterior comer not produced. subacute, ventral margin with 5 (subventral) spines: posterior margin of plate 3 convex. with 1 seta. distoposterior corner rounded, indistinct, ventral margin with 3 (subventral) spines. Peduncle of pleopod I with 2 retinaculae, lacking setae on outside margin.

Uropod I: inner ramus about 70 percent length of peduncle. armed with 14 spines: outer ramus with 16 spines: peduncle with 13 outer and 4 inner spines. Uropod 2: inner ramus armed with 14 spines: outer ramus with 9 spines: peduncle with 5 outer and 4 inner spines. Uropod 3 : inner ramus with 1 spine. outer ramus about 1.5 times longer than peduncle. inner and outer margins each with $3-4$ sets of spines in groups of $1-2$. Telson much broader than long (about 1.5 times), notched about 1.4 distance to base, each lobe with 2 apical spines: spines more than $1 / 2$ length of telson.

Male.--Differing from female as follows: smaller with more slender. elongate body. Antenna 2: flagellum with 5-6 segment: calceoli absent (at least in specimens examined).

Propod of gnathopod 1 more than 2 times larger and longer than carpus and broader: palm with 10 spine teeth on inside. 10 on outside: defining angle with 3 notched and 2 serrate spine teeth on inside and 1 large unnotched and 1 serrate spine teeth on outside. Propod of gnathopod 2 more than 2 times larger and longer than carpus: palmar margin strongly oblique. convex, with 12 spine teeth on inside. 14 on outside: defining angle with 2 notched-serrate spine teeth on inside and I large unnotched and I small unnotched or serrate spine teeth on outside.

Uropod 2: inner ramus with 10 spines: outer ramus unmodified, with 1 spine on inner margin and 6 spines on outer margin: peduncle with 2-3 outer and 3-4 inner spines. Uropod 3: outer ramus about 1.2 times length of peduncle.

Type-locality.--Cascade Cavern ("North Cave"), Carter Caves State Park. Carter County. Kentucky. The type-locality is one of several caves situated within the boundaries of Carter Caves State Park. In Cascade Cave. $C$. caecus is common in shallow (rimstone) pools with silty bottoms.

Distribution and ecology.--This species is known only from six caves in Carter County in northeastern Kentucky (fig. 106). It inhabits mainly pools, although it is recorded once from a stream and once from a 
drip pool. Ovigerous females were collected in June and September. Sexually females are 5.5 to $7.3 \mathrm{~mm}$ and sexually males are 3.0 to $5.0 \mathrm{~mm}$.

Etymology.--The epithet caecus is from Latin, meaning "blind."

\section{Crangonyx ohioensis. new species}

Figures $107-110$

Material examined (paratypes unless designated othenvise).--INDIANA. Clark Co.: seep. $3 \mathrm{mi} \mathrm{SW}$ of Bethlehem. $59.7 \approx$. L. Hubricht. 21 Apr 1956 (USNM): KENTUCKY. Carter Co.: seep. $8.0 \mathrm{~km}$ E of Olive Hill. holotype ?. 12 ․ 8 \%. L. Hubricht. 22 Apr 1949 (USNM); Lewis Co.: seep. Ohio River Bluff. $0.8 \mathrm{~km}$ SW of St. Paul, $14 \cong .7$ juvs. L. Hubricht. 27 May 1952 (USNM). OHIO. Adams Co.: Cave Hill Cave. 2 . T. Barr and R. Norton. 22 Jun 1967 and 29.1 ¿. H. H. Hobbs. III and M. Porter. 29 Jul 1995. Well in OHIO. $2 ₫ .2 \approx$, no other data (USNM).

Diagnosis.--A relatively small stygobiont species closely allied morphologically with C. castellanum and $C$. caecus but distinguished from them by the telson. which is just slightly broader than long in female: outer ramus of male uropod 2 having only $1-2$ small spines on inner margin near end: and also from the latter by presence of calceoli on antennae 2 of male. Largest male. $5.5 \mathrm{~mm}$. largest female. $7.5 \mathrm{~mm}$.

Female.--Eye greatly reduced to few black specks. Antenna 1, about 50 percent length of body. about 1.8 times longer than antenna 2: primary flagellum with about 20 segments. Antenna 2. flagellum with 8 segments. Mandibles subequal. spine row with 4-6 spines: segment 2 of palp with 5-8 long setae, segment 3 with 1-2 A setae, 2 C setae, 4-5 E setae and a row of D setae. lacking B seta. Maxilla 1: inner plate with 5 apical plumose setae: palp with 7 slender spines on apex. Maxilla 2, inner plate with oblique row of 5 plumose setae on inner margin. Maxilliped: inner plate apically with 2 bladelike spines. 2 naked spines. and 5-6 plumose setae extending from inner margin to apex: outer plate with row of naked setae and 4 slender pectinate spines on inner margin: dactyl with 2 setae near nail on inner margin: dactyl nail very. long. $1 / 2$ length of dactyl.

Propod of gnathopod I less than 2 times larger and longer than carpus: palm slightly convex. shorer than posterior margin. with 6 unnotched spine teeth on inside. 7 on the outside: defining angle with 5 notched-serrate spine teeth on inside. I large unnotched and 3 serrate spine teeth on outside: superior medial setae few in number and singly inserted: 4 sets of setae on posterior margin. singly inserted or in groups of 2-5. Dactyl with row of short setae on inferior margin, nail very long. Ventral margin of coxa 1 with 8 long setae.

Propod of gnathopod 2 less than 2 times larger and longer than carpus: palm convex. shorter than posterior margin. with 7 unnotched spine teeth on inside, 11 on outside: defining angle with 1 large notched and 4 serrate spine teeth on inside. 1 large unnotched and 2 serrated spine teeth on outside: 


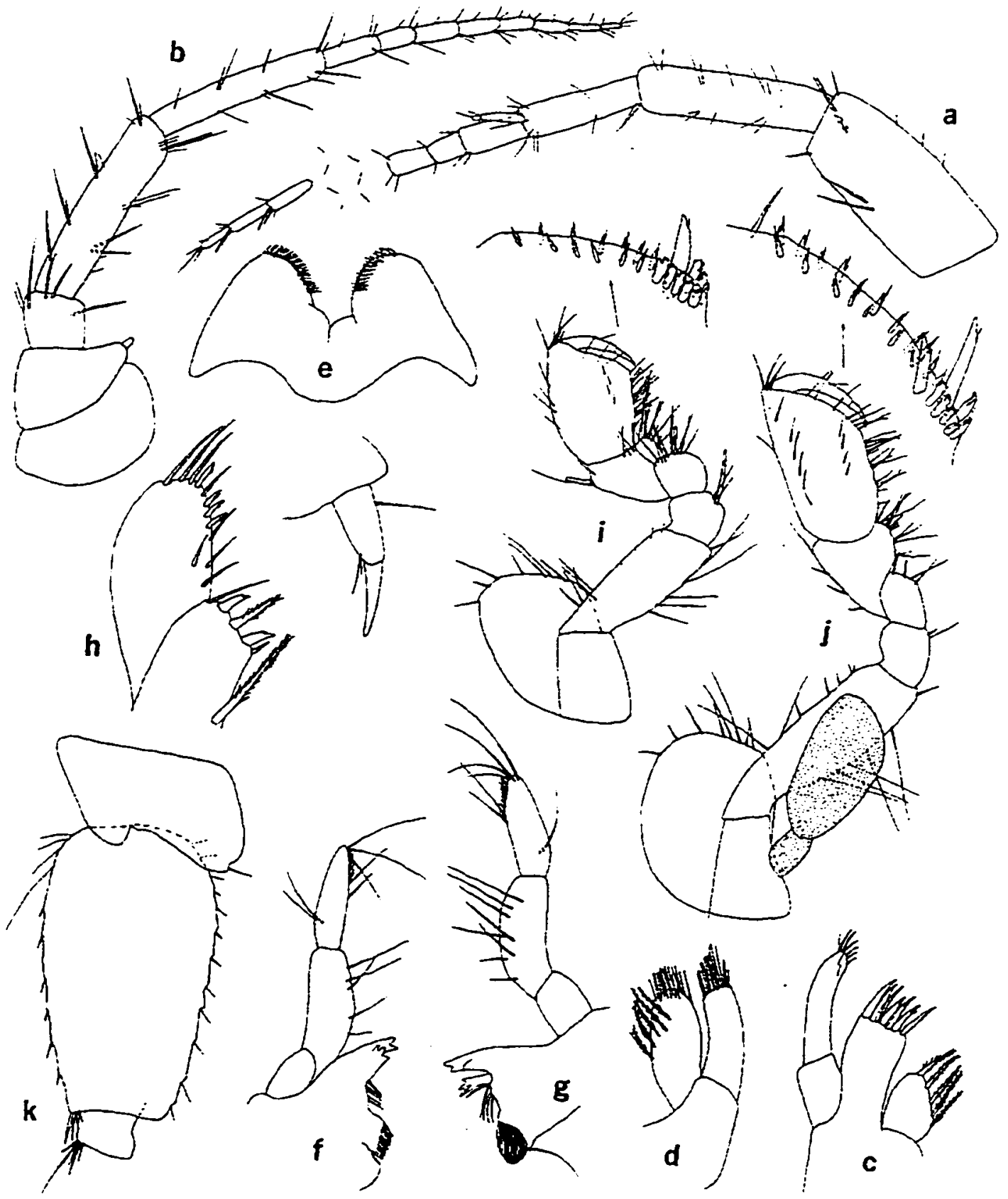

Figure 107.--Crangonyx ohioensis, new species. Female paratype $(6.8 \mathrm{~mm})$, seep. $8.0 \mathrm{~km}$ E of Olive Hill. Carter Co., Kentucky: a, b. antennae I, 2; c, d. maxillae 1. 2: e, lower lip: f. g, left and right mandible: $h$. inner and outer plates and palp dactyl of maxilliped (greatly enlarged): i. j. gnathopods 1 . 2 (palmar margins enlarged): $k$, pereopod 6 (in part). 


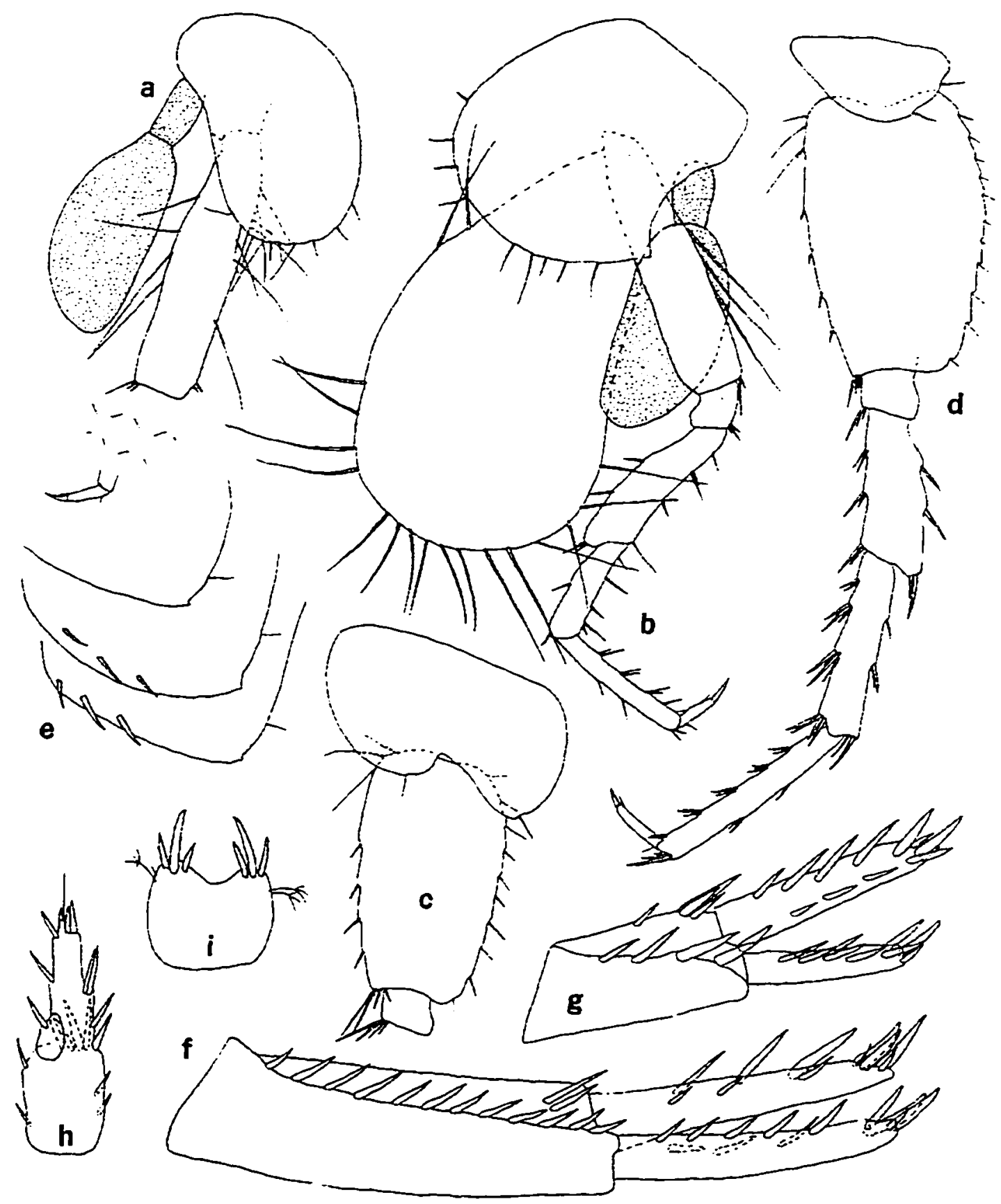

Figure 108.--Crangonyx ohioensis. new species. Female paratype $(6.8 \mathrm{~mm})$. seep. $8.0 \mathrm{~km}$ E of Olive Hill. Carter Co.. Kentucky: a. c. pereopods 3.4 (in part): b. d. pereopods 4. 7: e. pleonal plates: f. g. h. uropods I. 2. 3: i. telson. 


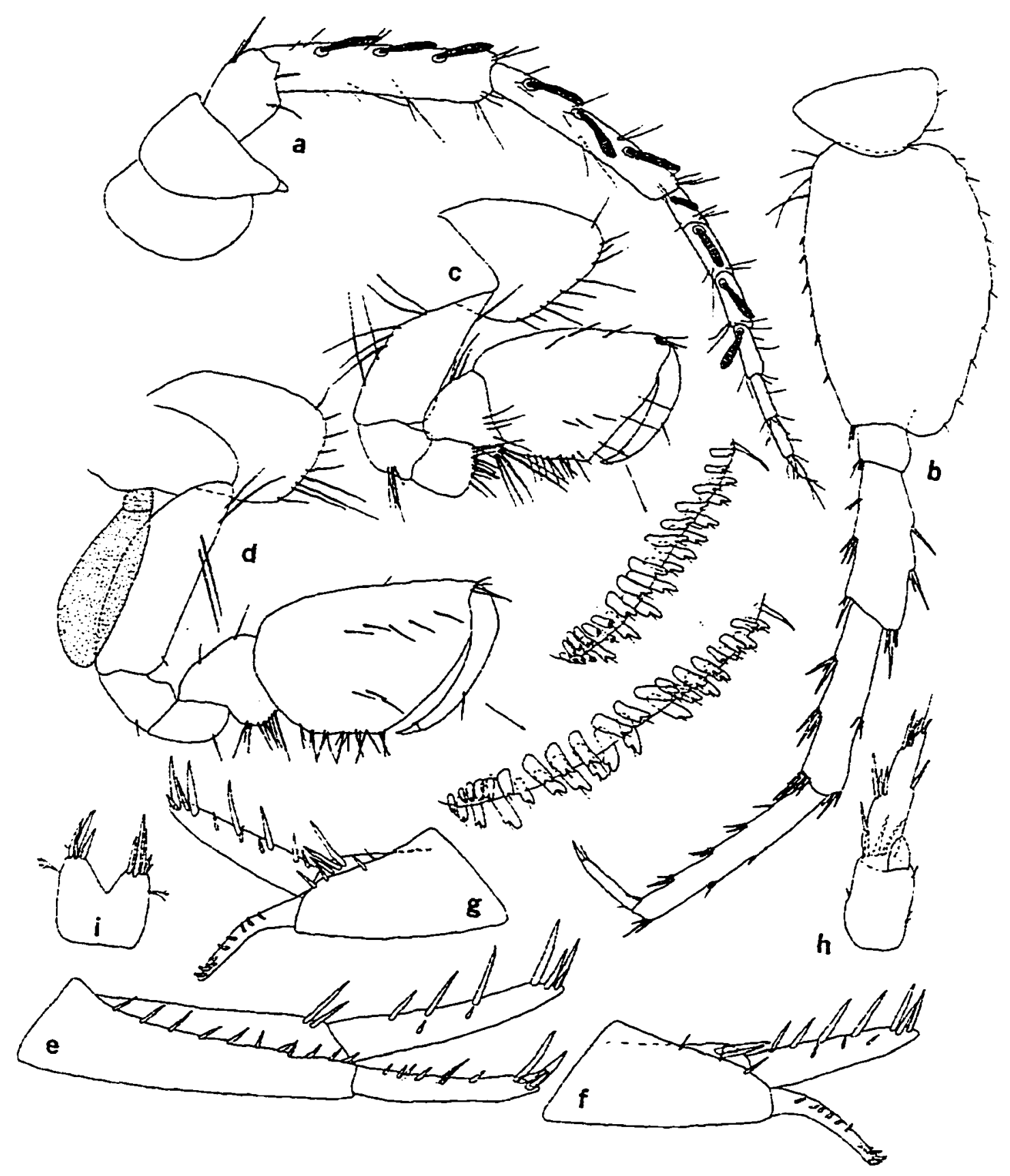

Figure 109.--Crangonyx ohioensis. new species. Male paratype $(5.2 \mathrm{~mm})$. seep. $8.0 \mathrm{~km}$ E of Olive Hill. Carter Co.. Kentucky: a. antenna 2: b. pereopod 7: c. d. gnathopods 1. 2 (palmar margins enlarged): e. uropod 1: f. g, right and left uropod 2: h. uropod 3: i, telson. 


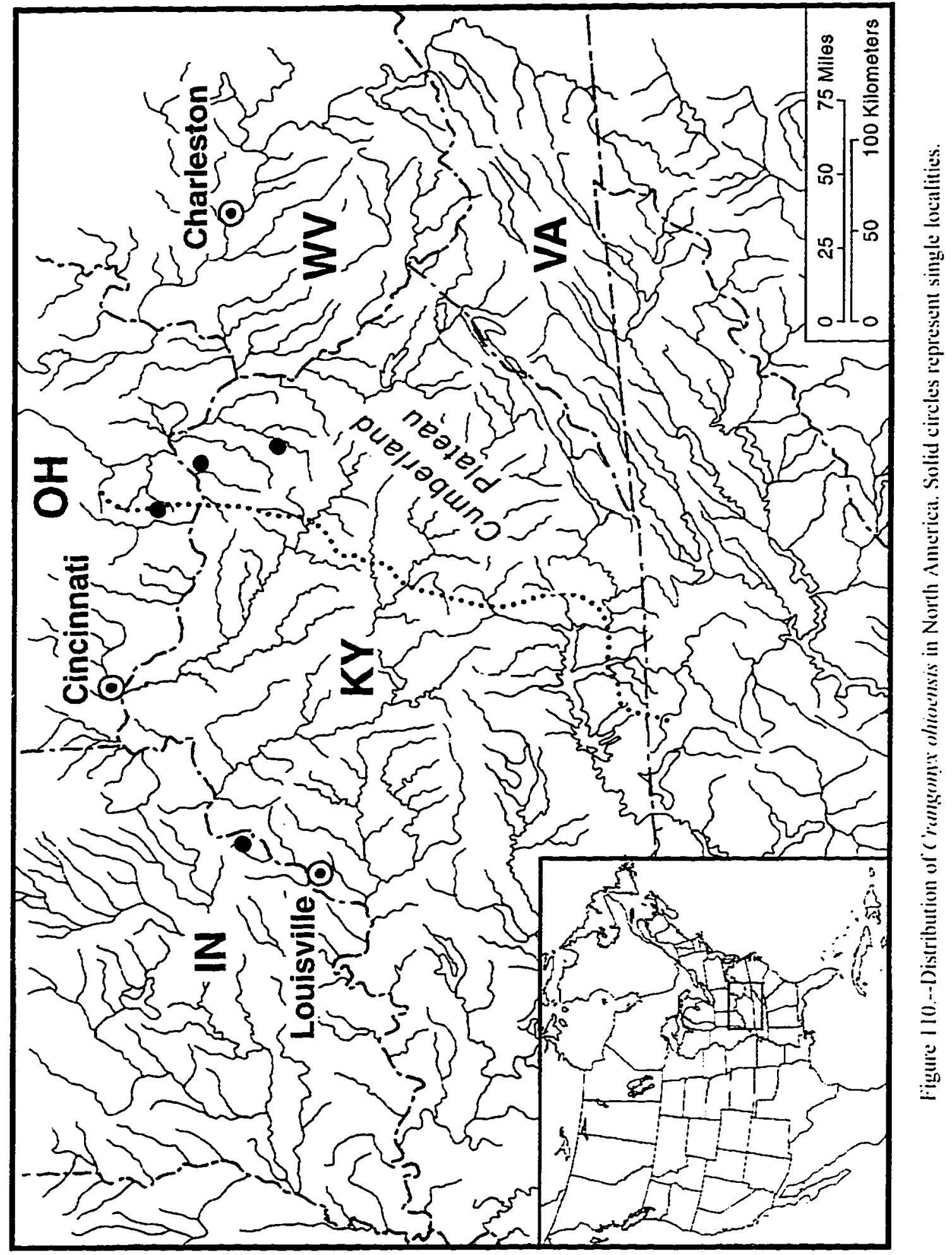


superior medial setae singly inserted: posterior margin with 4 sets of setae in groups of $1-6$. Dactyl with row of short setae on inner margin. Ventral margin of coxa 2 with 8 long setae.

Coxa of pereopod 3 with 10 marginal setae. Coxa of pereopod 4 with 9 marginal setae. dactyl of pereopod 4. 45 percent length of corresponding propod. Pereopod 5: basis with 10 shallow serrations along posterior margin; 4 short spines on anterior margin: 3 long setae on the anteroproximal margin. Pereopod 6: basis with 12 shallow serrations along posterior margin; 6 short spines on anterior margin: 4 setae on anteroproximal margin. Pereopod 7: coxa with 2 setae on the posterior margin: basis with 11 shallow serrations along posterior margin: 4 short spines on anterior margin: 3 long setae on anteroproximal margin: dactyl about 35 percent the length of corresponding propod.

Pleonal plates: posterior margin of plate I slightly convex. with I seta. distoposterior corner small. acute but slightly produced: posterior margin of plate 2 nearly straight with I seta. distoposterior corner acute but not produced. ventral margin with 3 (subventral) spines: posterior margin of plate 3 nearly straight with I seta. distoposterior corner weakly acute. not produced, ventral margin with 3 (subventral) spines. Peduncle of pleopod I with 2 retinaculae. lacking setae on outside margin.

Uropod I: inner ramus 80 percent length of peduncle. armed with II spines: outer ramus with I3 spines: peduncle with 12 outer and 2 inner spines. Uropod 2: inner ramus armed with 12 spines: outer ramus with 10 spines: peduncle with 4 outer spines. and 4 inner spines. Uropod 3: inner ramus with I spine. outer ramus about 1.2 times longer than peduncle, inner and outer margin each with $2-3$ set of spines in groups of 1-3. Telson slightly broader than long, notched about 1/6 distance to base. each lobe with 3 apical spines: spines up to $3 / 4$ length of telson.

Male.--Differing from female as follows. Smaller with more slender. elongate body. Antenna 2: calceoli present on peduncular segments 4 and 5 . and first 4 segments of flagellum.

Propod of gnathopod 1 more than 2 times larger and longer than carpus and much broader: palm straight. with 9 spine teeth on inside. 9 on outside: defining angle with 4 notched-serrate spine teeth on inside. 1 notched-serrate and 1 notched and 3 serrate spine teeth on outside. Propod of gnathopod 2 more than 2 times larger and longer than carpus: palmar margin oblique, slightly concave. with 13 spine teeth on inside. 13 on outside: defining angle with 2 notched and 3 serrate spine teeth on inside and 3 notched spine teeth on outside.

Uropod 2: inner ramus with 10 spines: outer ramus deflected laterally, with 2 inner spines. 5-6 short outer spines. apex with 2-3 spines: peduncle with 3 outer spines. and 2-3 inner spines. Uropod 3 : inner ramus with 1 spine: outer ramus about 1.6 times length of peduncle. Telson broader than long. notched about 1,3 distance to base.

Type-locality.--Seep. $8.0 \mathrm{~km}$ E of Olive. Carter Co. Kentucky.

Distribution and ecology.--This stygobiont species is recorded from a seep in southern Indiana. two seeps in northeastern Kentucky, and one well and one cave in southem Ohio (fig. 110). Although a specific location for the well in Ohio was not given by the collector, it is believed to be in the southern part 
of the state. All specified localities are limited to the Ohio River drainage. From east to west. the range covers a linear distance of about $200 \mathrm{~km}$.

This species is apparently an inhabitant of shallow groundwaters. Ovigerous females were collected in April and July and brood pouches contained up to 30 "yellow" eggs. Sexually mature females. 6.0 to 7.5 mm: sexually mature males. 4.8 to $5.5 \mathrm{~mm}$.

Etymology.--The specific name refers to the occurrence of this species in the Ohio River drainage.

Crangonxx acicularis, new species

Figures $111-114$

Material examined (paratypes unless designated otherwise).--TENNESSEE. Sullivan Co.: spring-fed stream. $8.0 \mathrm{~km}$ E of Bluff City. 20 specs ( $\%$. $)^{\prime}$ ), M. Richmond et al.. 20 Jan 1968: stream. $6.9 \mathrm{~km}$ E of Kingsport. holotype $c^{*}, 49.2 \approx$. R. Larue, 5 Feb 1968; stream just SW of Kingsport, I ¿. R. Larue. 4 Feb 1968: stream just E of Kingsport. $1 \approx$. R. Larue. 5 Feb 1968. VIRGINIA. Montgomery Co.: small spring near Ingles Ferry. $3.2 \mathrm{~km} \mathrm{~S}$ of Radford. $59.5 \mathrm{c}$. L. Hubricht. 17 Dec 1948 (USNM).

Diagnosis.--A small species of the gracilis group very similar to $C$. castlellanum as evidenced by very thin setal-like spines on outside of outer ramus of male uropod 2 and several thick setal-like comb spines near apex on inside. Distinguished from $C$. castlellanum by the produced and acute distoposterior comer of pleonal plate 2 and longer uropod 3 . Largest male, $5.8 \mathrm{~mm}$ : largest female. $7.2 \mathrm{~mm}$.

Female.--Eye large and ovate in shape. Antenna 1. about 55 percent length of body, about 1.8 times longer than antenna 2: primary flagellum with 17 segments. Antenna 2. flagellum with 8-9 segments. Mandibles subequal, spine row with 5 spines: segment 2 of palp with 5-6 long setae. segment 3 with 1 A seta. I C seta. 3-4 E setae and a row of D setae, lacking B seta. Maxilla I: inner plate with 6 apical plumose setae; palp with 6-8 slender spines on apex. Maxilla 2. inner plate with oblique row of 6 plumose setae on inner margin. Maxilliped: inner plate apically with 3 bladelike spines. 2 naked spines. and 5-6 plumose setae extending from inner margin to apex: outer plate with row of naked setae and about 5 slender pectinate spines on inner margin. I apical plumose seta: dactyl with 2 setae on inner margin near nail: dactyl nail very long. more than $1 / 3$ length of dactyl.

Propod of gnathopod 1 less than 2 times larger and longer than carpus: palm slightly convex. not oblique. shorter than posterior margin. with 6 unnotched spine teeth on inside. 6 on the outside: defining angle with 5-6 notched-serrate spine teeth on inside. 1 large notched and 2-3 notched or notched-serrate spine teeth on outside: superior medial setae few in number and singly inserted: $f$ sets of setae on posterior margin setae singly inserted or in groups of 2-4. Dactyl with a row of $4-5$ short setae on inner margin. nail long. Ventral margin of coxa 1 with 8 setae.

Propod of gnathopod 2 less than 2 times larger and longer than carpus: palm almost straight. much 


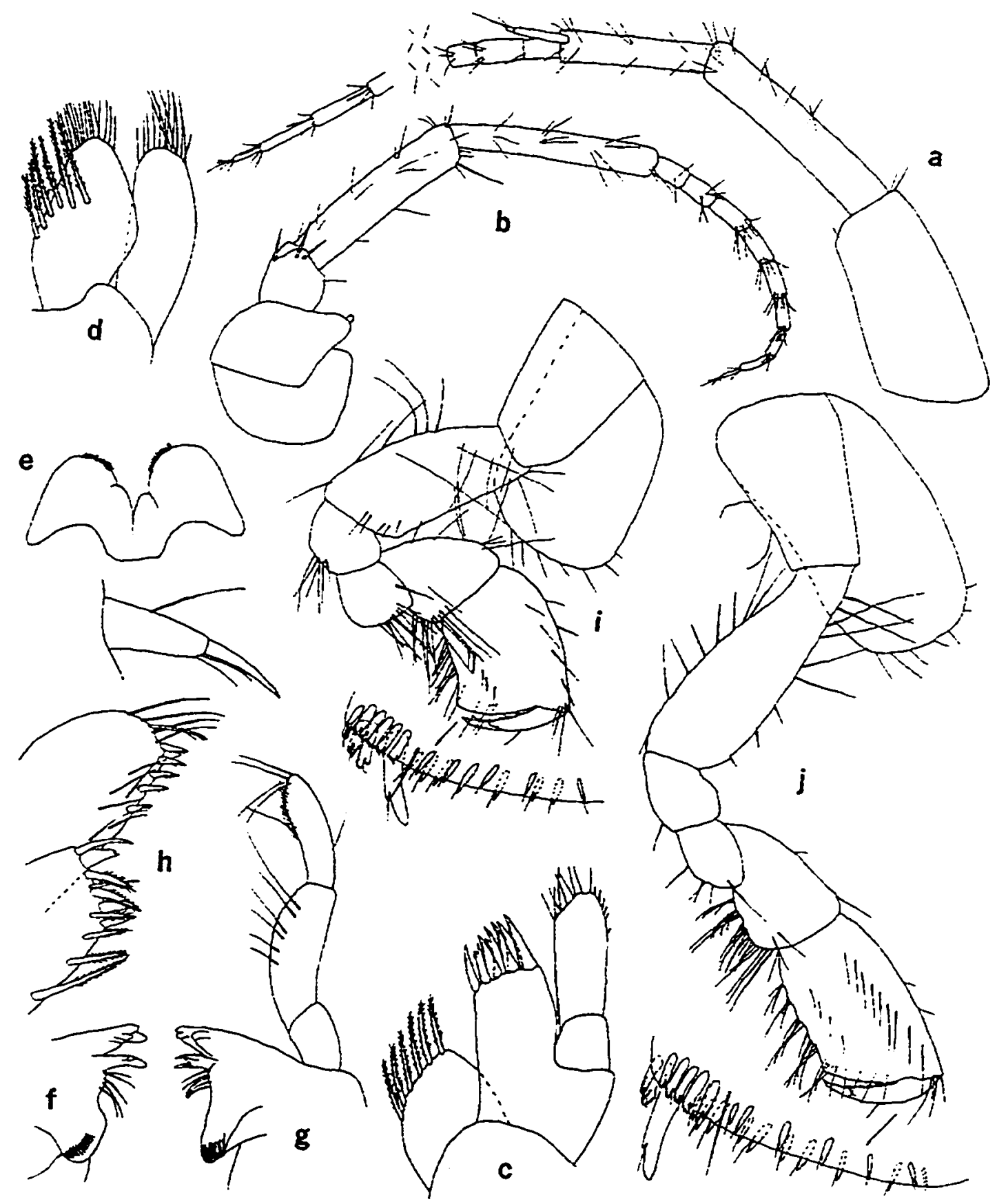

Figure 111 .--Crangomx acicularis, ne:v species. Female paratype $(6.5 \mathrm{~mm})$. stream. $6.9 \mathrm{~km} \mathrm{E}$ of Kingsport. Sullivan Co.. Tennessee: a. b. antennae 1. 2: c. d. maxillae 1. 2: e. lower lip: f. dentate part of left mandible: g. right mandible: h. inner and outer plates and palp dactyl of maxilliped (greatly: enlarged): i. j, gnathopods 1. 2 (palmar margins enlarged). 


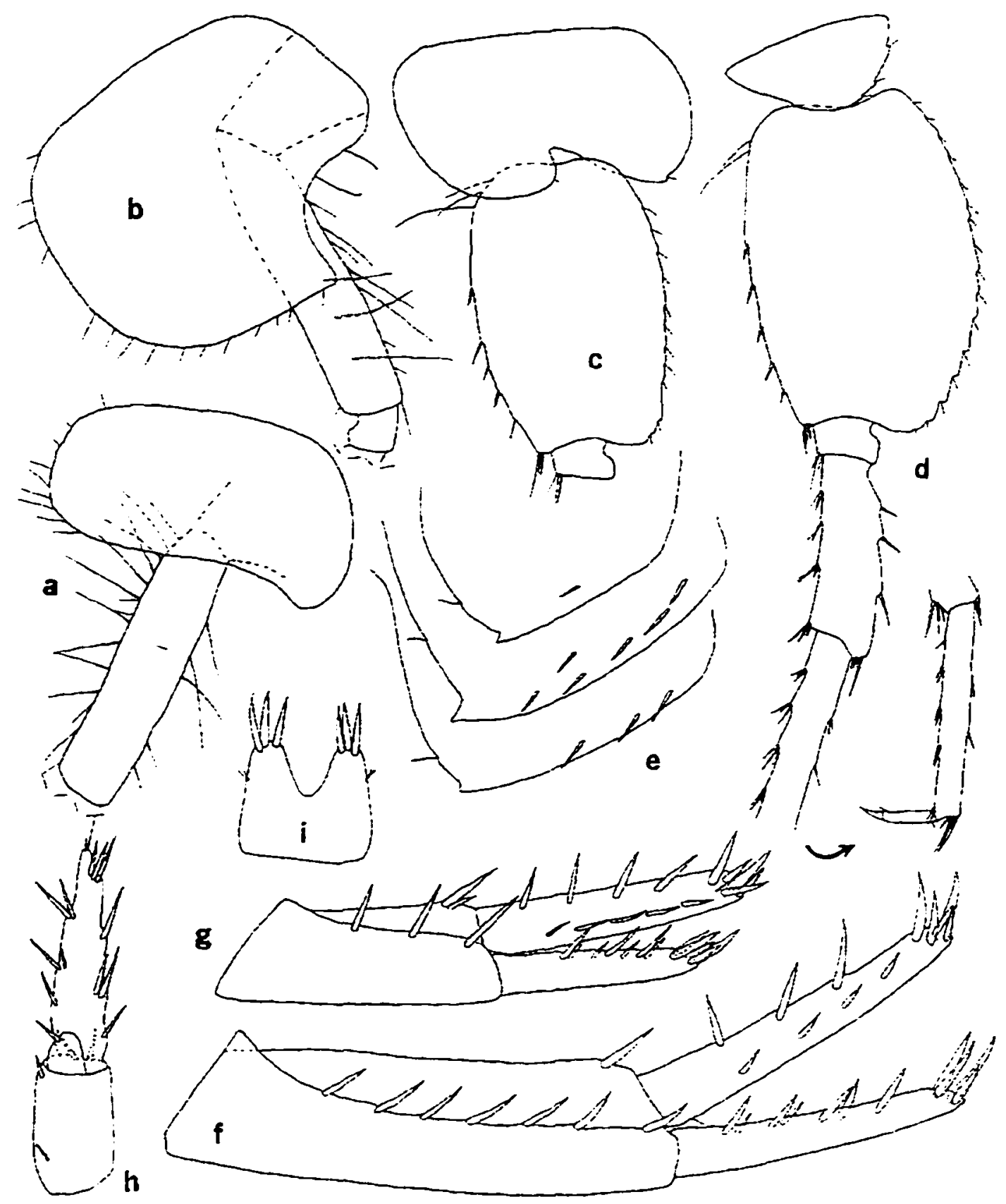

Figure I12.--Crangonvx acicularis, new species. Female paratype $(6.5 \mathrm{~mm})$. stream. $6.9 \mathrm{~km} \mathrm{E}$ of Kingsport. Sullivan Co.. Tennessee: a. b. c, pereopods 3.4. 5 (in part): d. pereopod 7: e. pleonal plates: $f, g$, h. uropods 1, 2, 3: i, telson. 


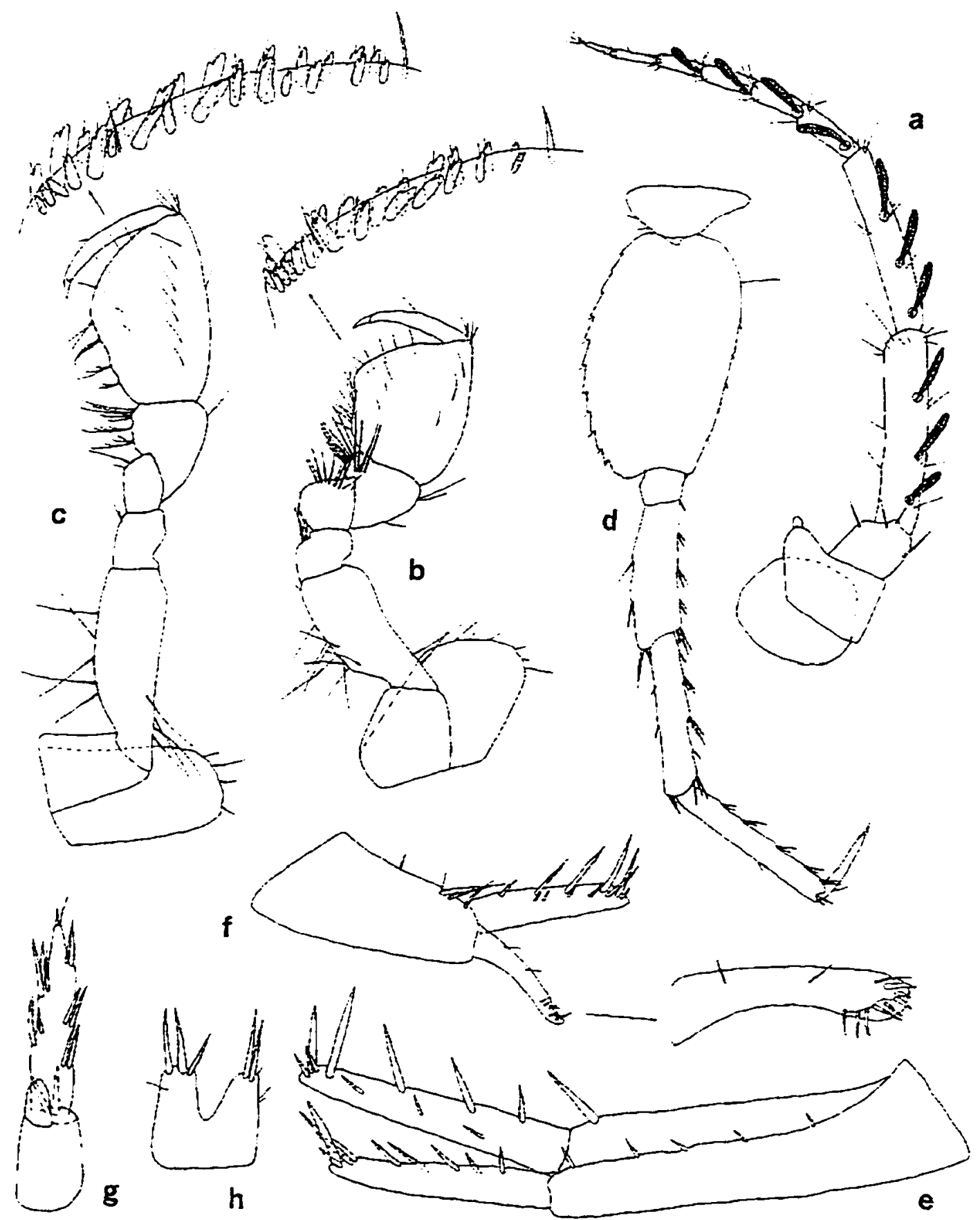

Figure 113 .--Crangonyx acicularis. new species. Male paratype $(4.7 \mathrm{~mm})$. stream. $6.9 \mathrm{~km} \mathrm{E}$ of Kingsport. Sullivan Co.. Tennessee: a. antenna 2: b. c. gnathopods 1, 2 (palmar margins enlarged): d. pereopod 7: e. f. g. uropods 1. 2. 3: h. telson. 


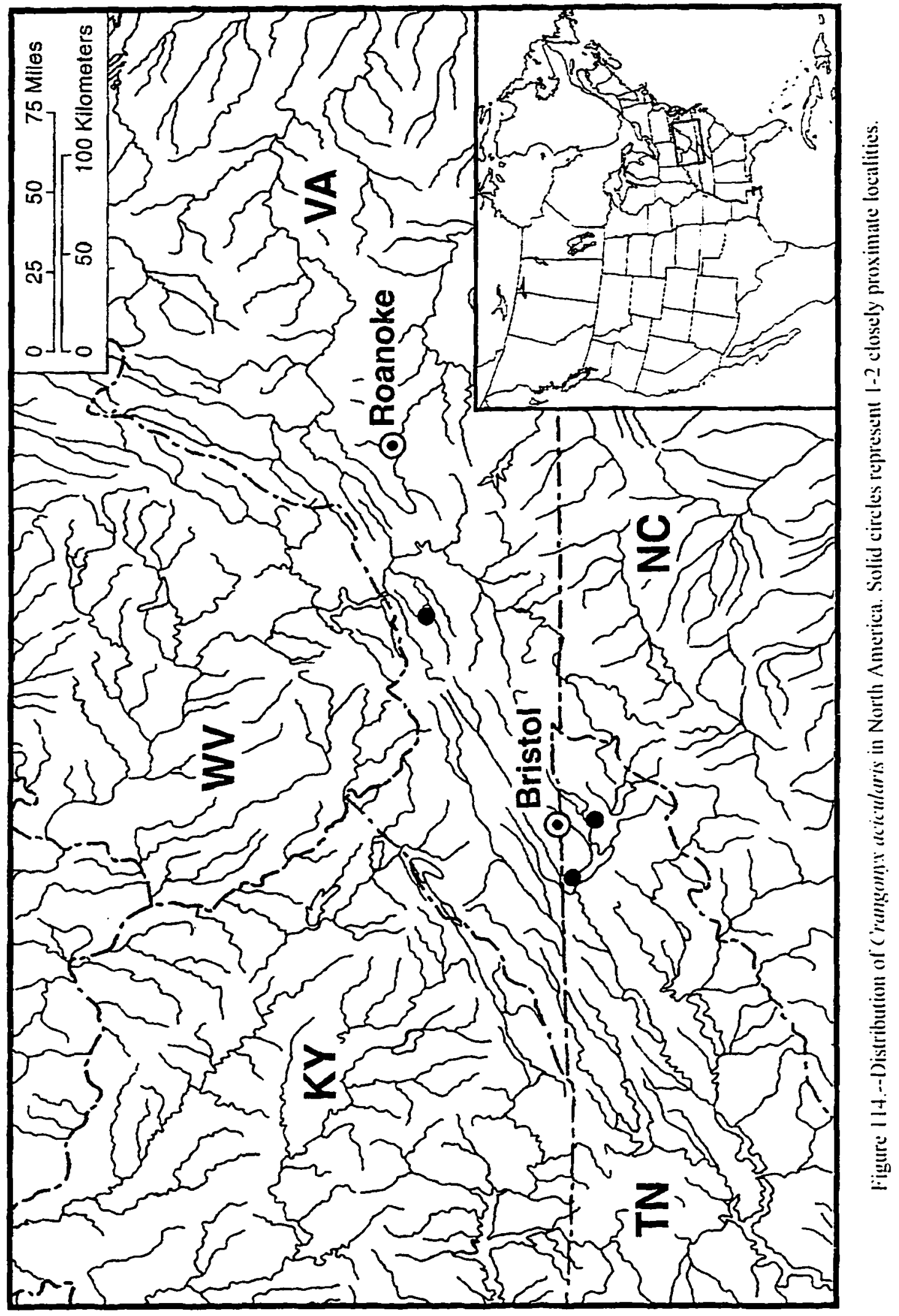


shorter than posterior margin. with 6 unnotched spine teeth on inside. 7 on outside: defining angle with I notched and 4 notched-serrate spine teeth on inside, 3 unnotched and 1 large notched. 2 serrate spine teeth on outside: superior medial setae singly inserted: posterior margin with 5 sets of setae in groups of 3-7. Dactyl with row of 6 short setae on inner margin, nail long. Ventral margin of coxa 2 with 7 setae.

Coxa of pereopod 3 with 9 marginal setae. Coxa of pereopod 4 with 15 marginal setae. dactyl of pereopod 4. 50 percent length of corresponding propod. Pereopod 5: basis with 12 very shallow serrations along posterior margin: 5 short spines on anterior margin: 3 long setae on the anteroproximal margin. Pereopod 6: basis with 13 shallow serrations along posterior margin: 5 short spines on anterior margin: 3 long setae on anteroproximal margin. Pereopod 7: coxa with 3 setae on the posterior margin: basis with 16 shallow serrations along posterior margin: 5 short spines on anterior margin: 2 long setae on anteroproximal margin: dactyl about 35 percent the length of corresponding propod.

Pleonal plates: posterior margin of plate 1 convex. with I seta. distoposterior comer slightly produced and acute. ventral margin may with I (subventral) spine: posterior margin of plate 2 nearly straight with ! seta. distoposterior corner strongly produced and acute. ventral margin with 6 (subventral) spines: posterior margin of plate 3 straightly convex with $\mid$ seta. distoposterior corner subacute. ventral margin with 3 (subventral) spines. Peduncle of pleopod 1 with 2 retinaculae, lacking setae on outside margin.

Uropod 1 : inner ramus 75 percent length of peduncle. armed with 12 spines: outer ramus with 11 spines: peduncle with row of 7 outer and 1 inner spines. Uropod 2: inner ramus armed with 5 inner and 4 outer spines. apex with 5 spines: peduncle with 3 outer and 2 inner spines. Uropod 3 : inner ramus with 1 spine. outer ramus about 1.8 times longer than peduncle. inner and outer margin each with $3-4$ set of spines in groups of $1-3$. Telson slightly broader than long. notched about 2,5 distance to base. each lobe with 3 apical spines: spines about $1 / 2$ length of telson.

Male.--Differing from female as follows. Smaller with more slender. elongate body. Antenna 2: calceoli present on peduncular segments 4 and 5 . and first $3-4$ segments of flagellum.

Propod of gnathopod 1 more than 2 times larger and longer than carpus and much broader: palm slightly convex and slightly longer than posterior margin. with 7 spine teeth on inside. 8 on outside. detining angle with I notched and 4 weakly notched-serrate spine teeth on inside and I notched and 2 serrate spine teeth on outside. Propod of gnathopod 2 more than 2 times larger and longer than carpus: palmar margin oblique, convex. subequal in length to posterior margin. with 9 spine teeth on inside. 11 on outside: defining angle with 4 notched and 1-2 notched-serrate spine teeth on inside. 3 notched spine teeth on outside.

Uropod 2: inner ramus with II spines: outer ramus deflected sightly posteriorly. with 3 inner spines. 3 thin outer spines. apex with 5 spines: peduncle with 2-4 thin outer spines. and 2 large inner spines.

Type-locality.--Surface stream. $6.9 \mathrm{~km}$ E of Kingsport. Sullivan County. Tennessee.

Distribution and ecology.--The range of this species. which is known only from five collections from Sullivan County. Tennessee and 1 coilection from Montgomery County. Virginia. is limited to a relatively 
small part of the Appalachian Valley and Ridge (fig. 114). It covers a linear distance of about $200 \mathrm{~km}$.

The species inhabits small springs and spring-fed streams. Ovigerous females were collected in January. Sexually mature males are 3.5 to $5.8 \mathrm{~mm}$ and sexually mature females are 6.3 to $7.2 \mathrm{~mm}$.

Etymology.--The epithet acicularis is from Latin. meaning "needle" or "pin." in reference to the needle-like spines on uropod 2 of male.

Crangonvx polustris. new species

Figures $115-118$

Material Examined (paratypes unless designated otherwise).--MARYLAND. Anne Arundel Co.: tributary to Paruxent R., Patuxent Refuge. 1 s, collector not given. 8 Feb 1941 (USNM); Charles Co.: rill. $4.3 \mathrm{~km}$ S of La Plata. 5 5. 1 ₹. L. Hubricht, 28 Feb 1959 (USNM): Calvert Co.: pond near beach of North Beach and Chesapeake Beach, 4 9. R. Greenfield. 22 Mar 1932 (USNM): Dorchester Co.: swamp. $5.3 \mathrm{~km}$ W of Cambridge. 4 ?. R. W. Jackson. 20 Apr 1943 (USNM): Prince Georges Co.: seeps in woods just off Suitland Rd.. $0.7 \mathrm{mi}$. SE of Suitland. $23 \bar{z}, 3 \sigma^{\circ}, 1$ juv. J. R. Holsinger. 4 Feb 1973: St. Marys Co.: woodland stream. Mechanicsville. 1 2. W. H. Ball. 11 May 1937 (USNM): spaghnum bog in woods. Ridge. 11 ฐ. 31 ×. W. H. Ball. 26 Apr 1930 (USNM). NEW JERSEY. Burlington Co.: temporary pools. $0.8 \mathrm{~km} \mathrm{SW}$ of Riverside. 100 specs $\left(\Xi . c^{*}\right)$. L. Hubricht. 12 Mar 1938 (USNM): temporary pools. $N$ of Riverton. $2 \equiv .1 \approx$ L. Hubricht. 12 Mar 1938 (USNM). NORTH CAROLINA. Gates Co.: Hamburg ditch and marsh. Dismal Swamp. 3 ○. S. Hetrick. 2 Mar 1974: marsh near Hamburg ditch. Dismal Swamp. 2 . J. Matta. 22 Jan 1974. VIRGINIA. Caroline Co.: seeps, $100 \mathrm{~m} \mathrm{NW}$ of Martins Corner. Ft. A. P. Hill. S. M. Roble. 18 Apr 1994: seeps. $200 \mathrm{~m}$ W of Martins Corner. S. M. Roble. 25 Apr 1994: Elizabeth City Co.: pools. $6.4 \mathrm{~km} \mathrm{~N}$ of Newport News. 3 9. L. Hubricht. 23 Jan 1944 (USNM): Fairfax Co.: pool under pines. Dyke. 3 I.W. H. Ball. 31 Dec 1934 (USNM): small pool between Dyke and Belle Haven. 8 ऽ. 8 ₹.W. H. Ball. 14 Aug 1935 (USNM): small pool near Dyke. 1 . W. H. Ball. 27 Apr 1935 (USNM): pond near Mount Vernon. 2 ․ C. R. Shoemaker. I Mar 1927 (USNM): small pools near New Alexandria. $29.1 \geq$ W. H. Ball. 22 Apr 1935 (USNM): pools in sweet gum stand. New Alexandria. $2 \cong .1 \approx .2+$ Apr 1935 (USNM): marshy ponds on Accotink Creek just below Lake Accotink. Springtield. $11 \supseteq .11 \geq 2 \mathrm{~J}$. R. Holsinger 7 Apr 1973: Hampton (Elizabeth City County): small pool. palustrine forested wetland on

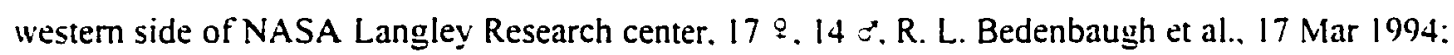
Henrico Co.: Brook Hill N of Richmond. 3 q. A. Pizzini, 30 Apr 1939 (USNM): Isle of Wight Co.: rill in

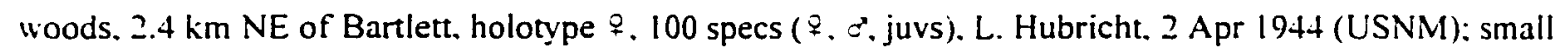
stream. $1.6 \mathrm{~km} \mathrm{~N}$ of Bartlett. 5 \&. $2 \sigma^{\circ}$. L. Hubricht, 2 Apr 1944 (USNM): roadside ditch. $0.5 \mathrm{~km} \mathrm{~N}$ of Carrollton. 1 7. L. Hubricht. 30 Jan 1944 (USNM): seeps. 3.2 km SE of Bartlett. 30 9. 4 : L. Hubricht. 30 Jan 1944 (USNM): small stream. $1.6 \mathrm{~km}$ S of Rescue. $9 £ .2 \approx$. L. Hubricht. 12 Mar 1944 


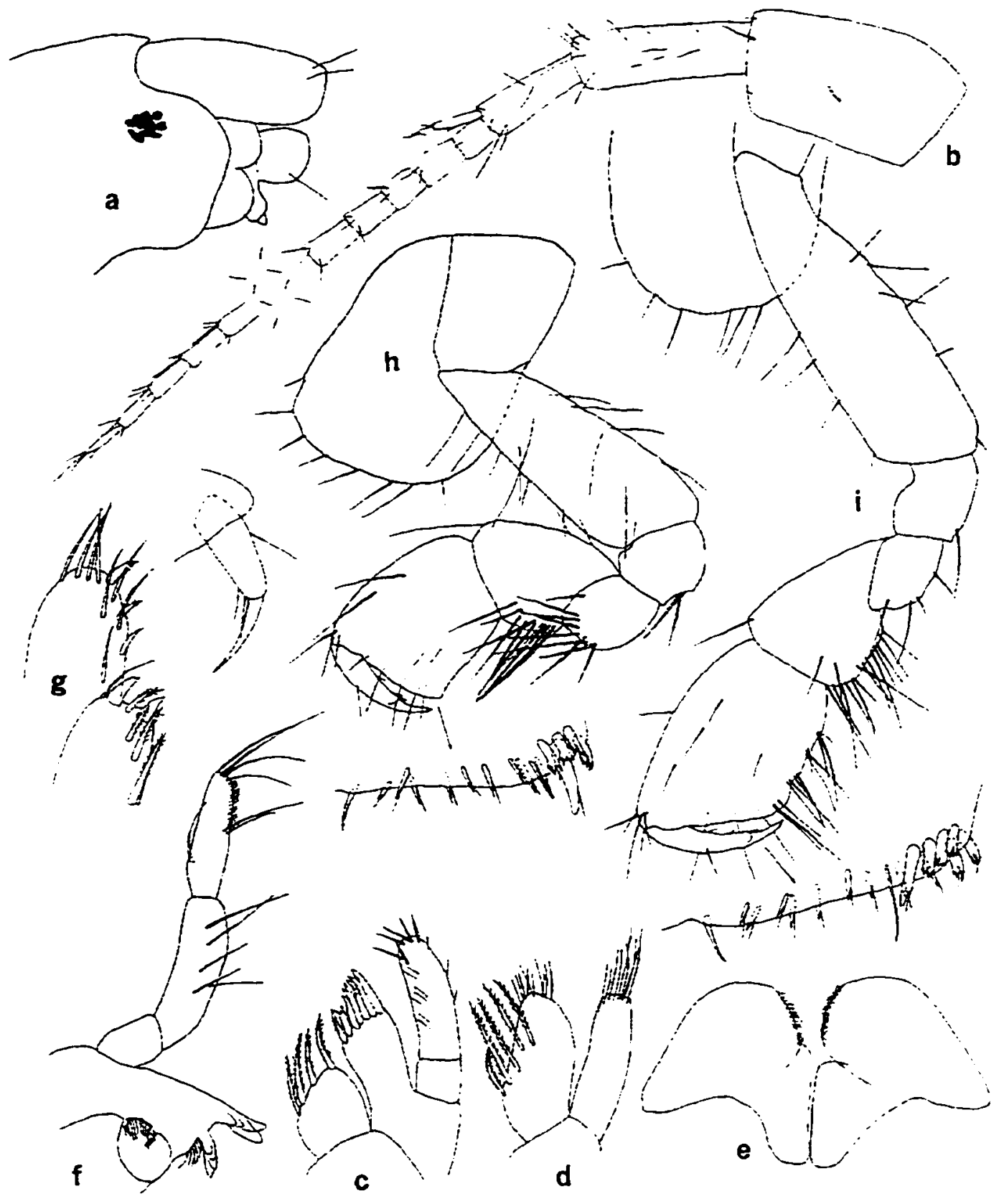

Figure 115.--Crangonyx polustris, new species. Female paratype $(5.5 \mathrm{~mm})$, rill in woods. $2.4 \mathrm{~km} \mathrm{NE}$ of Bartlett. Isle of Wight Co., Virginia: a, head (in part); b, antenna 1: c. d. maxillae 1. 2: e. lower lip: f. left mandible: g. inner and outer plates and palp dactyl of maxilliped (greatly enlarged): h. i.gnathopods 1. 2 (palmar margins enlarged). 


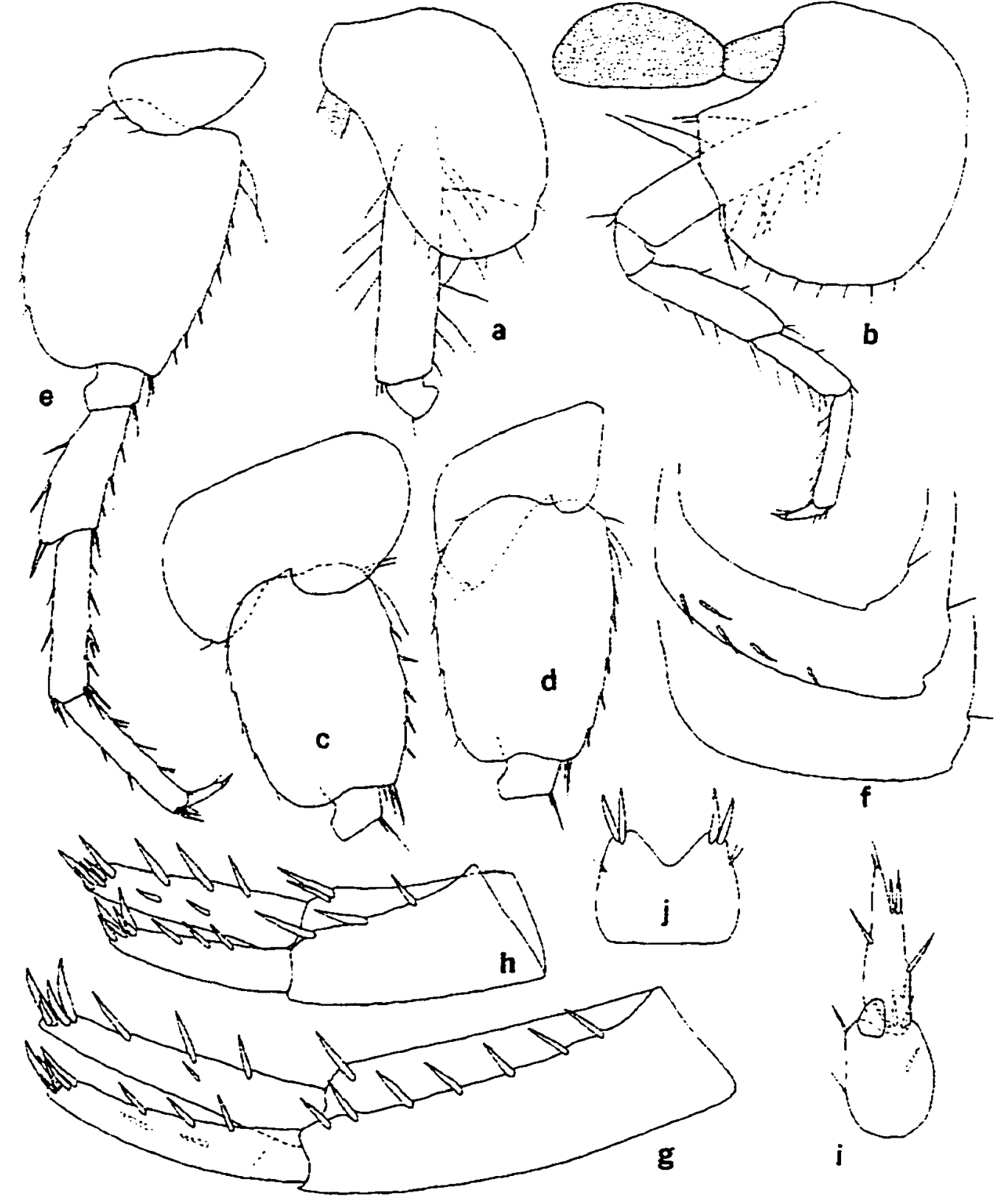

Figure 116.--Crangontx polustris. new species. Female paratype $(5.5 \mathrm{~mm})$, rill in woods. $2.4 \mathrm{~km} \mathrm{NE}$ of Bartlett. Isle of Wight Co.. Virginia: a. c. d. pereopods 3.5.6 (in part): b. e. pereopods 4. 7: f. pleonal plates; g. h. i. uropods 1. 2. 3: j. telson. 


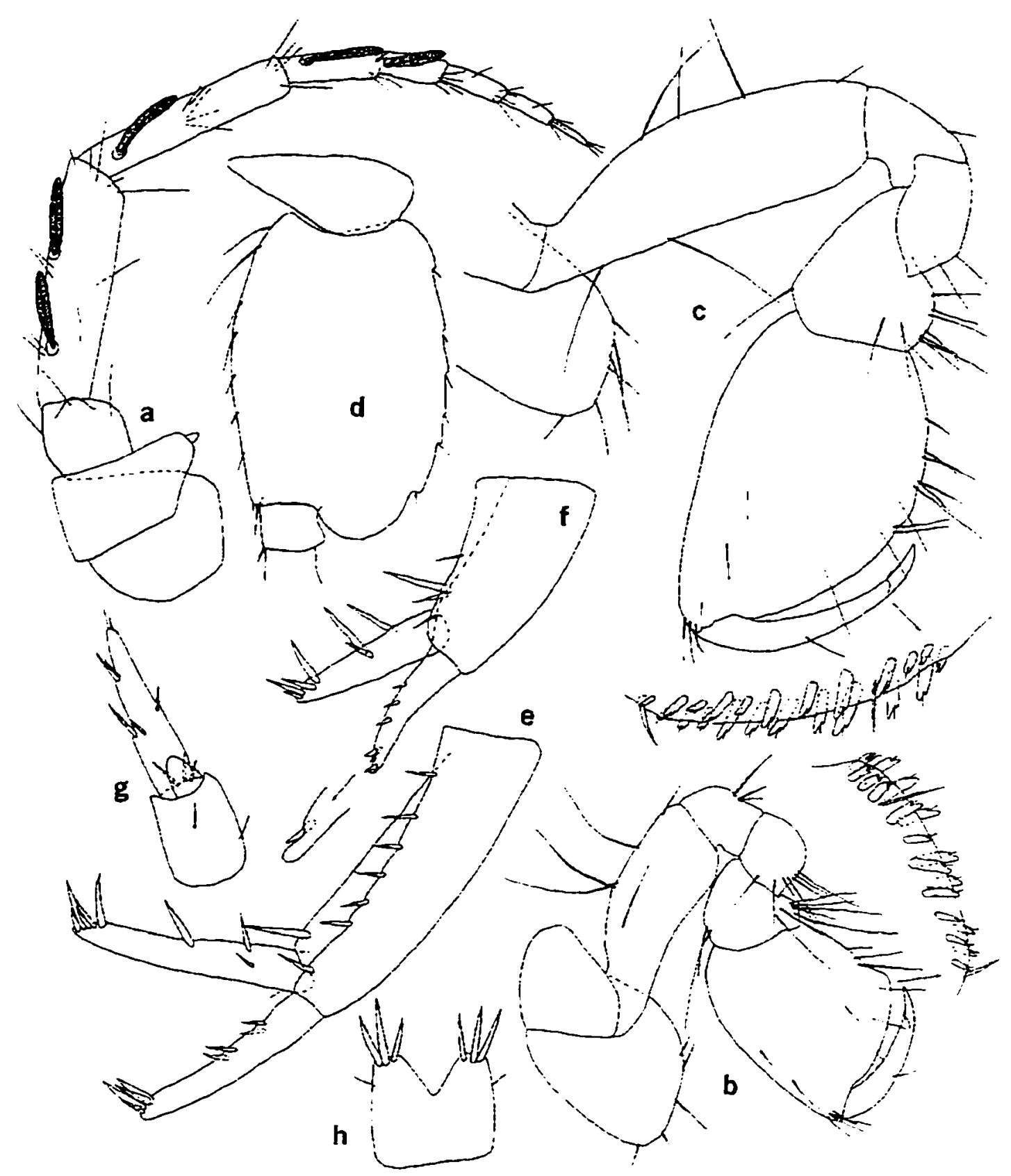

Figure 117.--Crangonyx polustris. new species. Male paratype $(3.2 \mathrm{~mm})$, rill in woods. $2.4 \mathrm{~km} \mathrm{NE}$ of Bartlett. Isle of Wight Co., Virginia: a. antenna 2: b. c. gnathopods 1. 2 (palmar margins enlarged): $d$. pereopod 7 (in part): e, f. g, uropods 1. 2. 3: h. telson. 


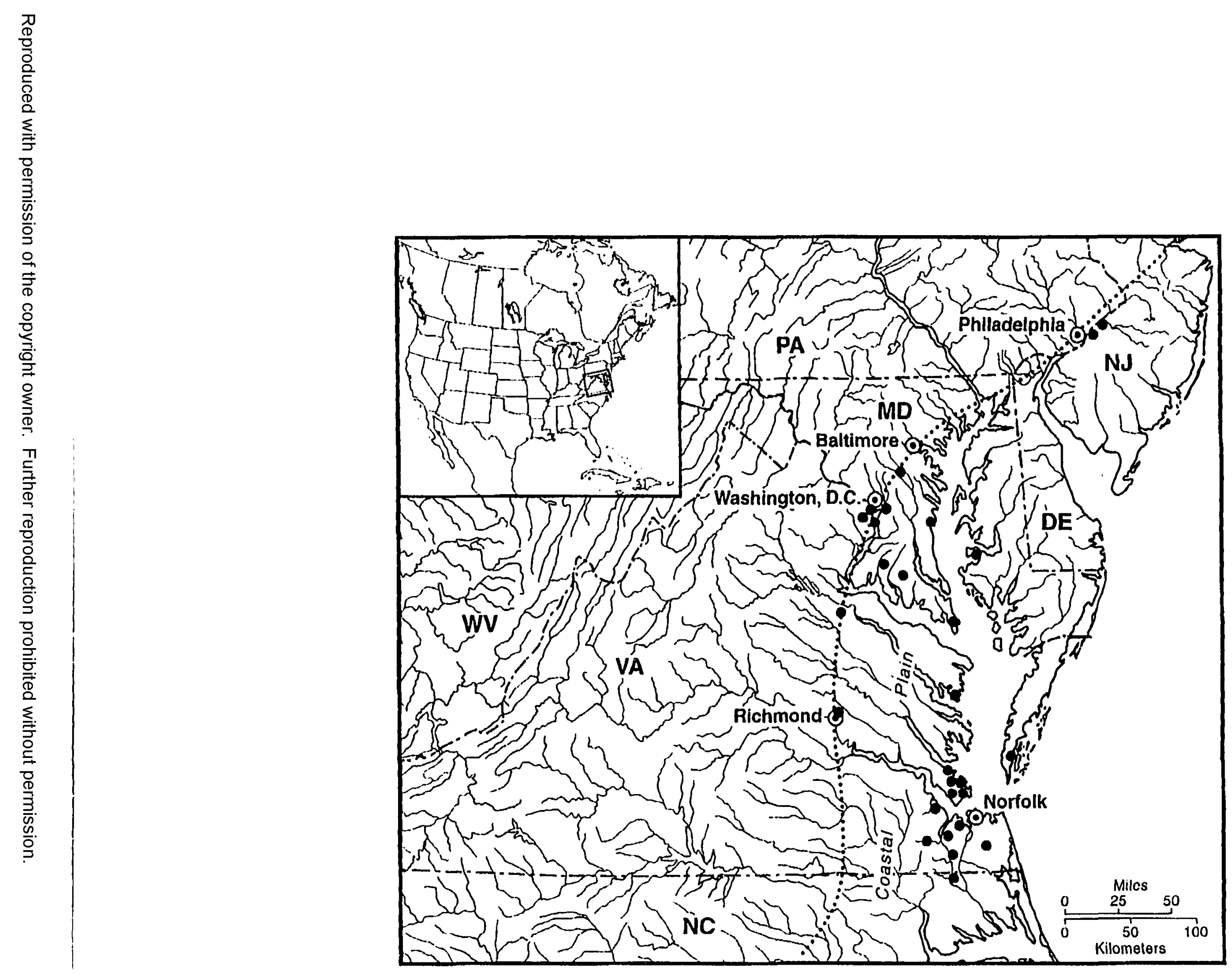

Figure 118.--Distribution of ( 'rangennx po/nstris in North America. Solid circles represent 1-6 closely proximate localities. The doned line denotes the "fall line" or "fall zone" between the Coastal Plain and piedmont. 
(USNM): Lancaster Co.: shallow well and roadside ditch near old trash dump in woods. $4.8 \mathrm{~km} E$ of White Stone. 17 \%. $2 \approx$. S. Hetrick. 10 Mar 1982: seep pool in woods. $4.8 \mathrm{~km}$ E of White Stone. $7 £ .5 \approx$ J. R. Holsinger and R. Godfrey, 17 Apr 1983: shallow well. seep and swamp. $4.8 \mathrm{~km}$ E of White Stone. $26 \equiv .2$ ;. 6 juvs. J. R. Holsinger et al.. 9 March 1994: Nansemond Co.: pool in Dismal Sivamp near Washington Ditch. 4 . $1 \approx$ L. Hubricht. 30 Apr 1944 (USNM); seep-fed pool in woods. ca. $4.8 \mathrm{~km} \mathrm{NW}$ of center of Suffolk, $3 \Xi .1 \bumpeq$ J. R. Holsinger, 25 Mar 1984: pools in Dismal Swamp. K. Garrett and B. H. Powell. 20

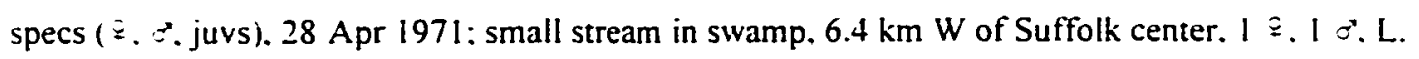
Hubricht. 25 Feb 1945 (USNM): temporary pool in woods. $1.6 \mathrm{~km}$ E of Driver. $3 \subsetneq .1 \approx$. L. Hubricht. 26 Mar 1944 (USNM); small stream. $4.8 \mathrm{~km}$ S of Bay View, 25 ․ 2 \%. L. Hubricht. 16 Apr 1944 (USNM): Norfolk Co.: small spring, $0.8 \mathrm{~km}$ SE of Churchland. $99.2 \%$. L. Hubricht. 11 Mar 1945 (USNM): stream in swamp. $4.8 \mathrm{~km}$ S of North Landing. 4 O. 1 : L. Hubricht. 9 Apr 1944 (USNM): Warwick Co.: ditch 2.4 $\mathrm{km}$ NE of Morrison. 1 . L. Hubricht. 23 Jan 1944 (USNM): pools in woods. $4.0 \mathrm{~km} \mathrm{NW}$ of Newport News. $2 \cong$. L. Hubricht. 23 Jan 1944 (USNM): York Co.: small seeping spring pond in woods. $4.5 \mathrm{~km} \mathrm{SW}$ of Yorktown. $7 \cong .2 \%$ J. G. Mackin. 17 Feb 1946 (USNM); ditch, $1.6 \mathrm{~km} \mathrm{SE} \mathrm{of} \mathrm{Tabb.} 3 \fallingdotseq$. L. Hubricht. 23 Jan 1944 (USNM): small marsh-pond in woods, $4.0 \mathrm{~km} \mathrm{~W} \mathrm{of} \mathrm{Yorktown.} 7$. 1 ₹*. J. G. Mackin. (USNM): pond. $1.0 \mathrm{~km} \mathrm{~S}$ of Grafton Church. 1 \%. P. H. Stevenson and S. H. Holbrook. 13 Feb 1993: Lakeside forest subdivision, ca. $0.8 \mathrm{~km} \mathrm{NE}$ of junction of rds 620 and $17.2 \equiv .7 \approx$. C. S. Hobson. $27 \mathrm{Apr}$ 1994: pond on Ri. 17. $1.1 \mathrm{~km}$ S of Harris Grove. 23 9. 17 \%. J. R. Holsinger et al.. 9 March 1994:

Diagnosis.--A relatively small species of the gracilis groups distinguished from other members of the group by having a single spine in a small notch on the inner side (near the end) of outer ramus of male uropod 2: small, rounded distoposterior corners of pleonal plates: and very short uropod 3 with few spines on peduncle. Largest male. $5.5 \mathrm{~mm}$ : largest female. $9.0 \mathrm{~mm}$.

Female.--Eye small and round. Antenna I, about 46 percent length of body. about 1.7 times longer than antenna 2: primary flagellum with about 15 segments. Antenna 2. flagellum with 6-7 segments. Mandibles subequal. spine row with 4-6 spines: segment 2 of palp with 5 long setae. segment 3 with 1 A seta. $2 \mathrm{C}$ setae. $4 \mathrm{E}$ setae and a row of $\mathrm{D}$ setae, lacking $\mathrm{B}$ seta. Maxilla 1 : inner plate with 4 apical plumose setae: palp with 5-6 slender spines on apex. Maxilla 2, inner plate with oblique row of 5 plumose setae on inner margin. Maxilliped: inner plate apically with 2 bladelike spines. I naked spine. and + plumose setae extending from inner margin to apex: outer plate with row of naked setae and $3-4$ pectinate slender spines on inner margin: dactyl with 2 setae near nail on inner margin: dactyl nail very long. about 12 length of dactyl.

Propod of gnathopod 1 slightly larger and longer than carpus: palm slightly convex. subequal to length of posterior margin, with 4 unnotched spine teeth on inside. 5 on the outside: defining angle with 3 notched-serrate spine teeth on inside. I large unnotched and I serrate spine tooth on outside: superior medial setae few in number and singly inserted: 4 sets of setae on posterior margin singly inserted or in groups of 2. Dactyl with 2-3 short setae on inferior margin, nail long about $1 / 3$ length of dactyl. Ventral 
margin of coxa 1 with 8 setae.

Propod of gnathopod 2 less 2 times larger and longer than carpus; palm slightly shorter than posicrior margin. with 3 unnotched spine teeth on inside, 4 on outside; defining angle with $\mid$ large unnotched spine teeth and three notched-serrate spine teeth on inside, 2 unnotched and 2 serrate spine teeth on outside: superior medial setae singly inserted. posterior margin with 4 sets of setae in groups of 3-4. Dacty/ with 2-3 short setae on inferior margin. Ventral margin of coxa 2 with 6 setae.

Coxa of pereopod 3 with 8 marginal setae. Coxa of pereopod 4 with 12 marginal setae. dactyl of pereopod 4. 40 percent length of corresponding propod length. Pereopod 5: basis with 8 shallow serrations along posterior margin: 5 short spines on anterior margin: 3 long setae on the anteroproximal margin. Pereopod 6: basis with shallow 8 serrations along posterior margin: 5 short spines on anterior margin: 3 long setae on anteroproximal margin. Pereopod 7: coxa with 2-3 setae on the posterior margin: basis with 9 shallow serrations along posterior margin: 7 short spines on anterior margin: 3 long setae on anteroproximal margin: dactyl about 35 percent length of corresponding propod.

Pleonal plates: posterior margin of plate 1 slightly convex. with 1 seta. distoposterior corner small. rounded. not produced: posterior margin of plate 2 slightly convex, with 1 seta. distoposterior comer rounded. not produced. ventral margin with 5 (subventral) spines: posterior margin of plate 3 slightly convex. with I seta. distoposterior comer small. rounded. not produced. ventral margin with 3 (subventral) spines. Peduncle of pleopod 1 with 2 retinaculae. lacking setae on outside margin.

Uropod I: inner ramus about 65 percent length of peduncle. armed with 9 spines: outer ramus with 10 spines: peduncle with row of 7 outer and 1 inner spines. Uropod 2: inner ramus armed with 2 inner and 3 outer spines. apex with 5 spines: outer ramus lacking inner spines. 3 outer spines, apex with 5 spines: peduncle with 3 outer spines. and 2 inner spines. Uropod 3 : inner ramus lacking spines. outer ramus about 1.5 times longer than peduncle. inner and outer margin each with $1-2$ set of spines in groups $1-2$. Telson broader than long. notched about $1 / 3$ distance to base. each lobe bearing 2 apical spines: spines about 12 length of telson.

Male.--Differing from female as follows. Smaller with more slender. elongate body. Antenna 2: calceoli present on peduncular segments 4 and 5 , and first 2 segments of flagellum.

Propod of gnathopod I morc than 2 times larger and longer than carpus: palm with 7 spine teeth on inside. 5 on outside: defining angle with 3 notched-serrate spine teeth on inside and 2 notched and $\mid$ serrate spine teeth on outside. Propod of gnathopod 2 more than 2 times larger and longer than carpus: palm margin oblique. with 7 spine teeth on inside. 8 on outside: defining angle with I notched and 2 weak serrate spine teeth on inside, 3 notched spine teeth on outside.

Uropod 2: inner ramus with 7 spines; outer ramus weakly deflected laterally, with 1 bladelike inner spine on notch near apex. 4 small, bladelike spines on outer margin; peduncle with 2 slender outer spines and 2 large inner spines.

Type-locality.--A rill (small brook or rivulet) in woods, $2.4 \mathrm{~km}$ NE of Bartlett. Isle of Wight County. 
Virginia.

Distribution and ecology.-The range of this species extends from west-central New Jersey southwestward through southern Maryland and eastern Virginia as far south as extreme northeastern North Carolina (fig. 118). With the exception of a few localities just west of the "fall line" immediately south of Washington. DC. C. polustris is restricted to the Coastal Plain.

This species inhabits swamps. springs, ponds. woodland pools. streams. roadside ditches. and seeps. Ovigerous females were collected in February, March. April and August. Sexually mature males are 3.5 to $5.5 \mathrm{~mm}$ and sexually mature females are 4.0 to $9.0 \mathrm{~mm}$.

Etymology.--The epithet polustris is from Latin. meaning "marshy" or "boggy." in reference to one of the primary habitats of this species.

Crangonyx castellanum. new species

Figures 119-122

Material examined (paratypes unless designated otherwise).--KENTUCKY. Rockcastle Co.: Crooked Creek Cave. 3 9. T. C. Barr. Jr.. 27 May 1964: Crooked Creek Cave No.2. 1 ミ. T. Seibert. Dec 1972: Goochland Cave. 1 I. T. Seibert. Sept 1972: Greenhill School Cave. 6 9. $2 \approx *$ R. M. Norton. 5 Sep 1964: Millers Cave No.1. 3 g. T. Seibert. Sep 1972: Mullins Spring Cave. 38.1 . T. Seibert. Nov 1972: Pine Hill Cave. $2 \Xi$. J. Reddell. 4 Mar 1967 and I $\approx$. G. A. Coovert. I Feb 1975: Sinks of Roundstone Cave.

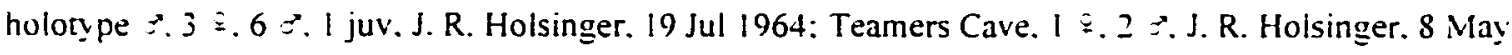
1965: Pulaski Co.: Piney Grove Cave. 2 ₹. L. Hubricht. 16 Dec 1956 (USNM) and 2 ₹. T. C. Barr. Jr.. 16 Dec 1956.

Diagnosis.--A relatively small cavernicolous species of the gracilis group. closely similar to $C$. cuecils but differing from that species by lacking spines on inner margin of outer ramus of male uropod 2 and presence of 2 thin (setal-like) spines on outer margin. Largest male. $5.5 \mathrm{~mm}$ : largest female. $8.2 \mathrm{~mm}$.

Female.--Eyes either absent or greatly reduced. when present limited to several black specks. Antenna 1. about 60 percent length of body, about 2 times longer than antenna 2: primary flagellum with up to 20 segments. Antenna 2. flagellum with up to 8 segments. Mandibles subequal. spine row with 5-6 spines: segment 2 of palp with 7 long setae. segment 3 with 1 A seta. $2 C$ setae. 4-5 E setae and a row of $D$ setae. lacking B seta. Maxilla 1: inner plate with 7 apical plumose setae: palp with 5-7 slender spines on apex. Maxilla 2. inner plate with oblique row of 6-7 plumose setae on inner margin. Maxilliped: inner plate apically with 3 bladelike spines. 2 naked spines, and 5-6 plumose setae extending from inner margin to apex: outer plate with naked long setae and $3-4$ small weak more or less pectinate spines on inner margin. 1 apical plumose seta: dactyl with 2 setae in inner margin near the nail: dactyl nail very long. 13 length of dactyl. 


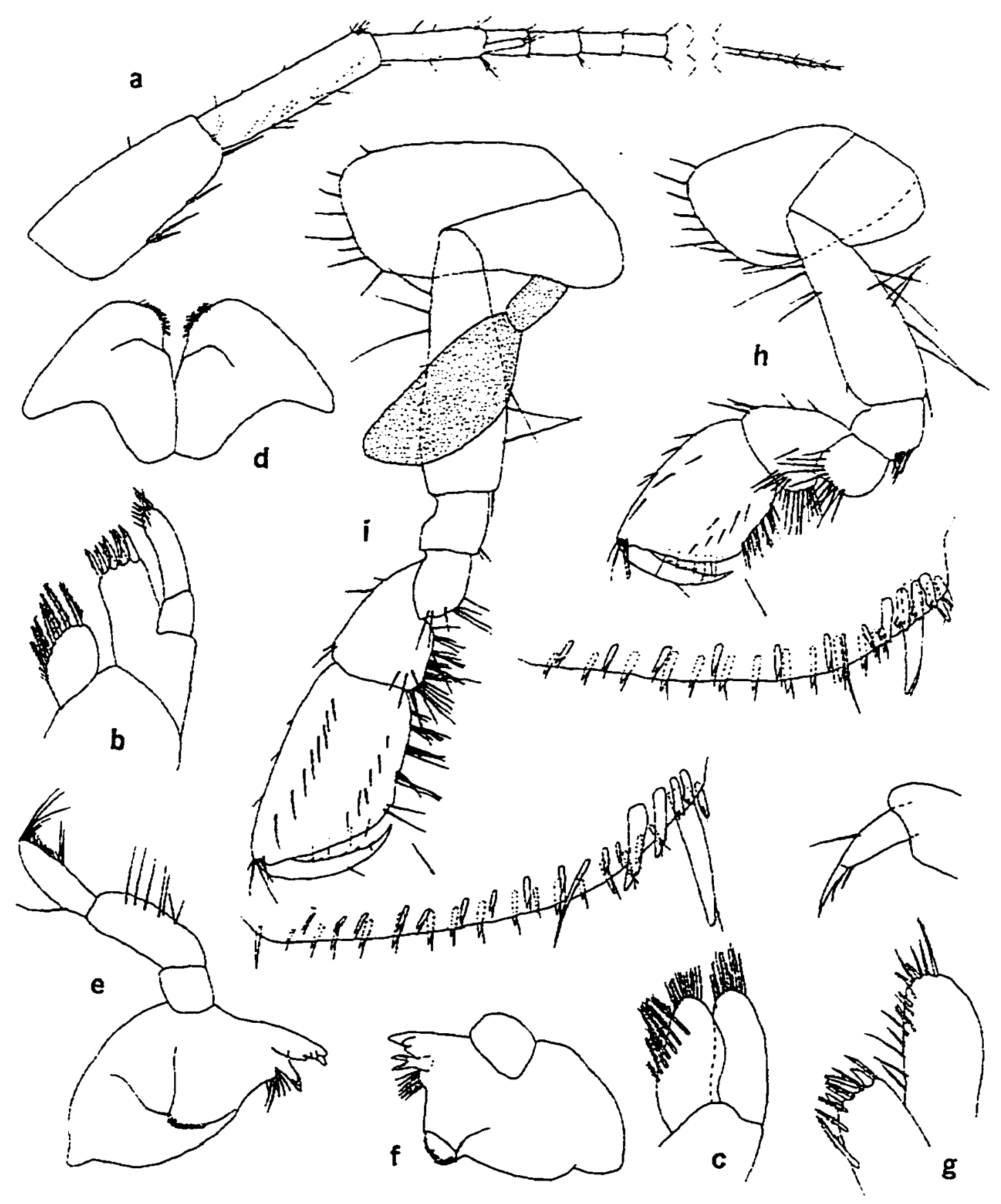

Figure 119.--Crangonyx castellanum, new species. Female paratype $(7.0 \mathrm{~mm})$. Sinks of Roundstone Cave. Rockcastle Co.. Kentucky: a, antenna I. Female paratype $(8.2 \mathrm{~mm})$, same location: b. c. maxillae 1. 2: d. lower lip: e. left mandible: $f$. dentate part of right mandible: g. inner and outer plates and palp dactyl of maxilliped: h. i, gnathopods 1,2 (palmar margins enlarged). 

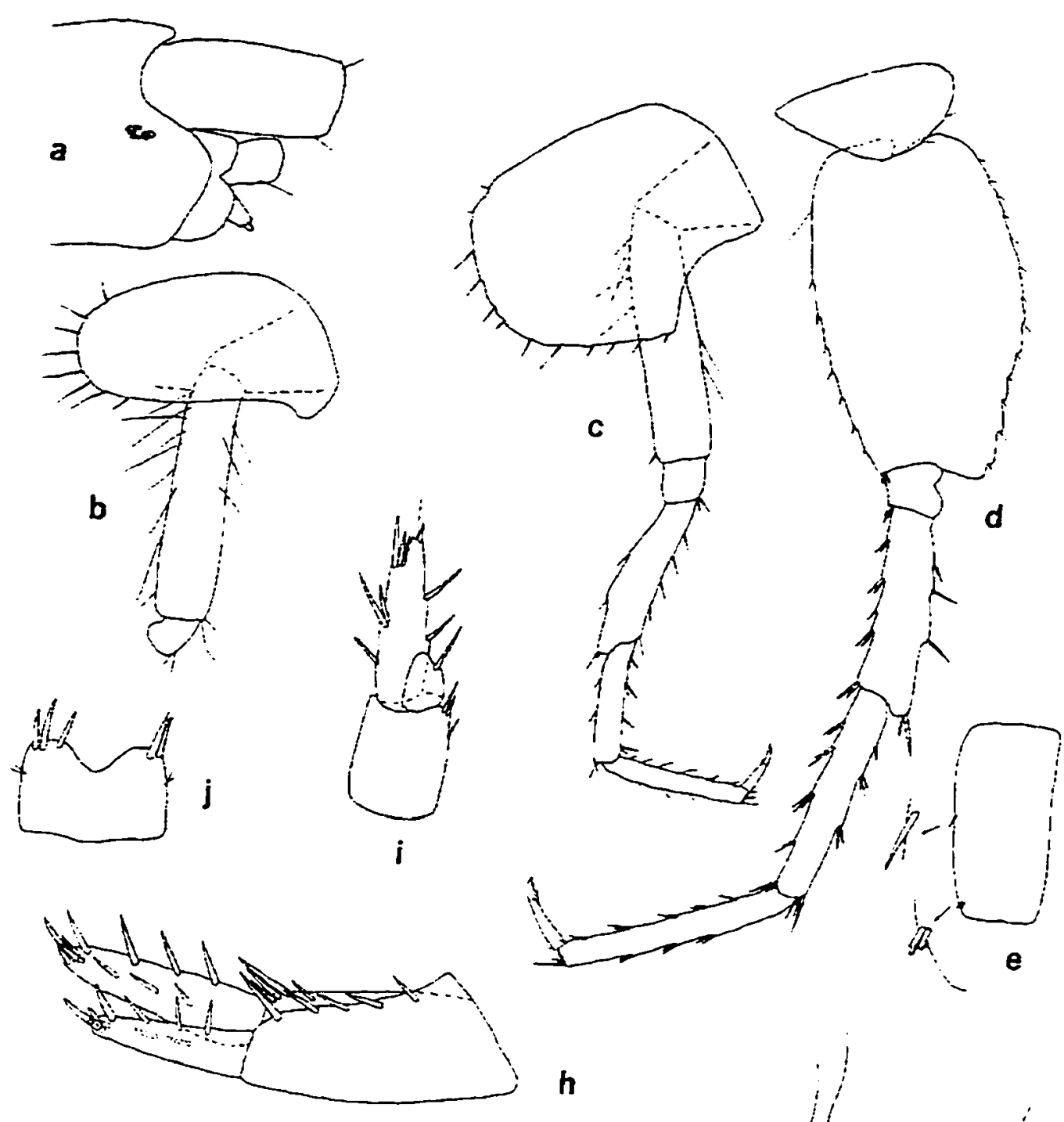

h
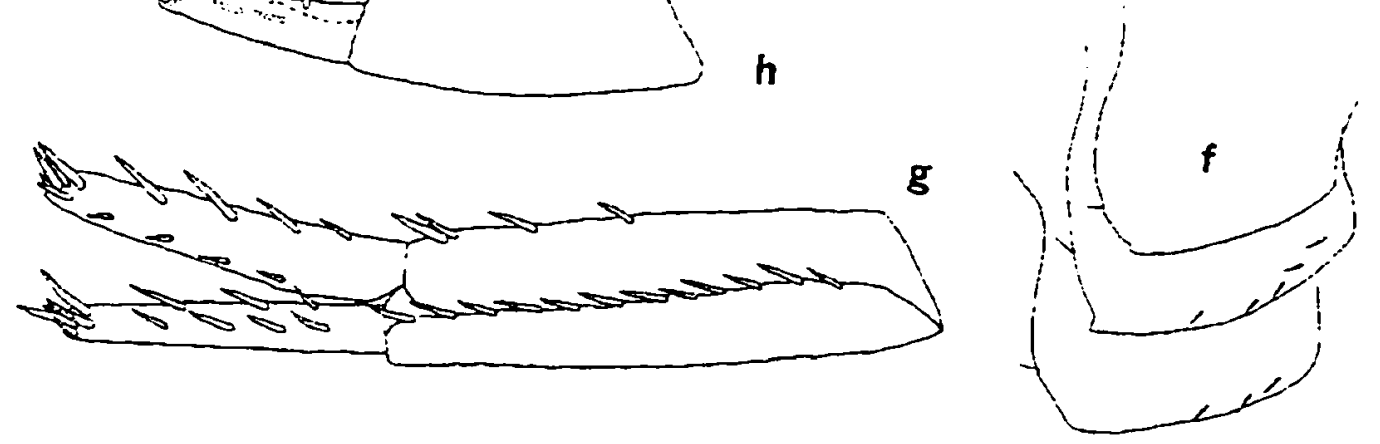

Figure 120.--Crangonyx custellanum. new species. Female paratype $(7.0 \mathrm{~mm})$. Sinks of Roundstone Cave. Rockcastle Co., Kentucky: b. pereopod 3 (in part): c. d. pereopods 4. 7. Female paratype (8.2 $\mathrm{mm}$ ). same location: a. head (in part): e. peduncle of pleopod I (retinacula and other spine enlarged): f. pleonal plates: g, h. i. uropods $1,2,3: \mathrm{j}$, telson. 


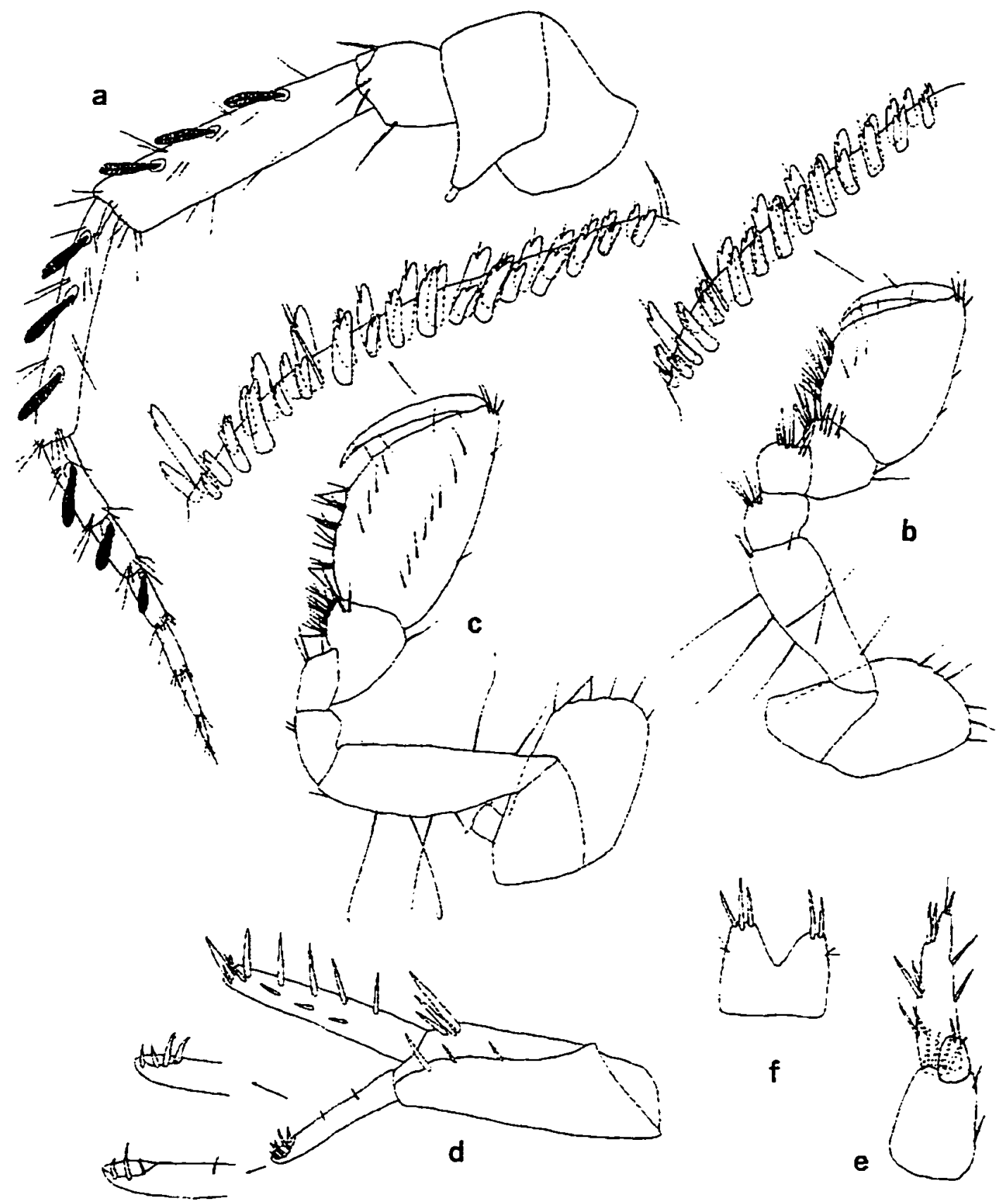

Figure 121.--Crangonyx castellanum. new species. Male paratype $(5.0 \mathrm{~mm})$, sinks of Roundstone Cave. Rockcastle Co.. Kentucky: a. antenna 2: b. c. gnathopods 1. 2 (palmar margins enlarged): d. uropod 2 (end of ouier ramus enlarged): e. uropod 3: f. telson. 


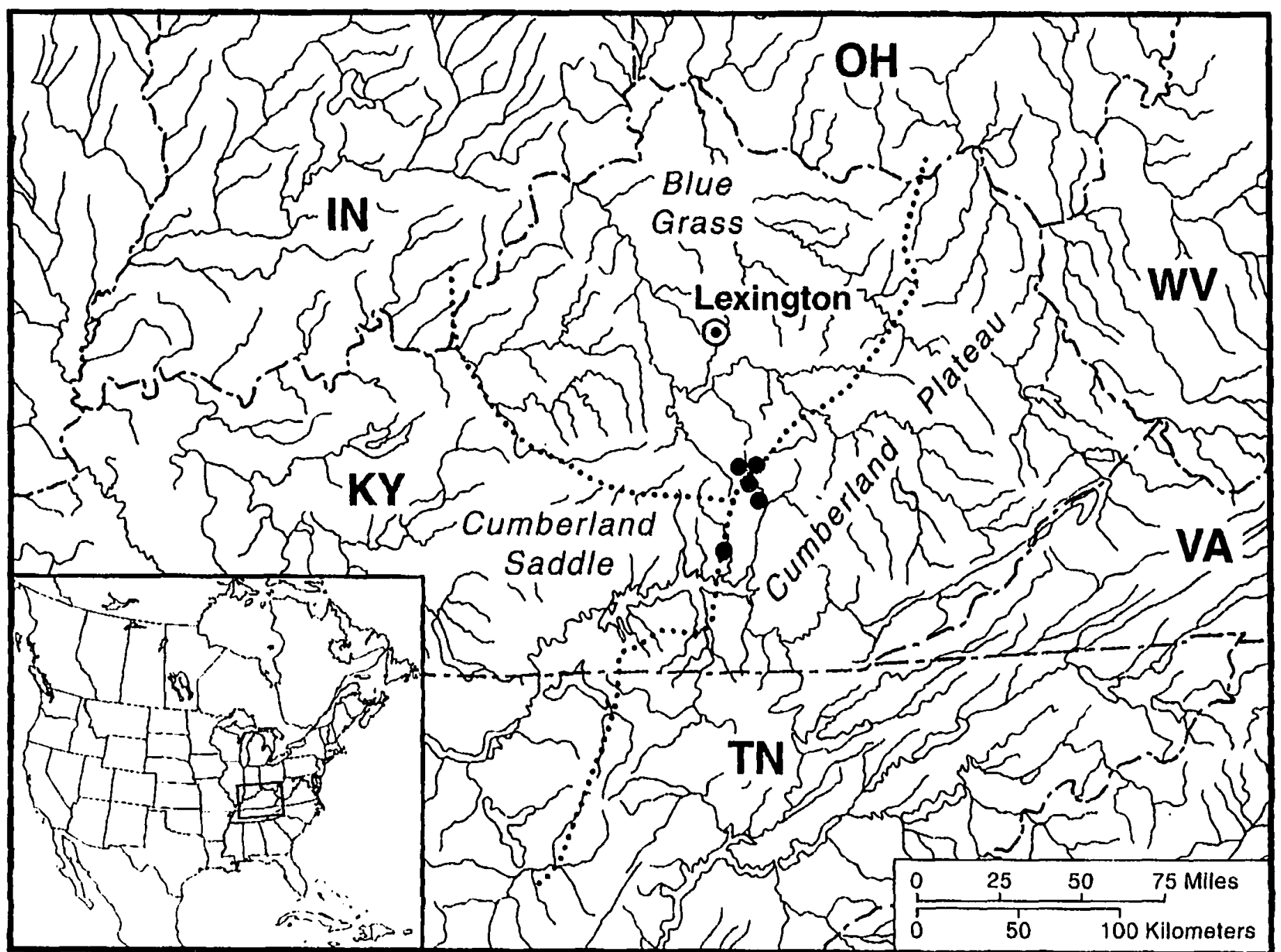

Figure 122.--1) Dstribution of ( 'rangemx castr/lamm in Nonth America. Solid circles represent 1-3 closely proximate localities. 
Propod of gnathopod I less 2 times larger and longer than carpus: palm slightly convex, subequal in length posterior margin, with 6-7 unnotched spine teeth on inside. up to 13 on the outside: defining angle with 4 notched-serrate spine teeth on inside. I large unnotched and 2 serrate spine teeth on outside: inferior and superior medial setae few in number and singly inserted: 4-5 sets of setae on posterior margin inserted in groups of 3-5. Dactyl with a row of 5 short setae inferior margin, nail long. Ventral margin of coxa 1 with 10 setae.

Propod of gnathopod 2 lees 2 times larger and longer than carpus: palm slightly convex and oblique. slightly shorter than posterior margin. with up to 11 unnotched spine teeth on inside. 15 on outside: detining angle with 1 or 2 large notched spine teeth and 2 small notched-serrate spine teeth on inside. 1 or 2 very large and strong unnotched spine teeth and I small serrate spine tooth on outside: up to 8 superior medial setae singly inserted: posterior margin with 5 sets of setae inserted in groups of 2-6. Dactyl with row of 6 short setae on inferior margin. Ventral margin of coxa 2 with 8-9 long setae.

Coxa of pereopod 3 with 8 marginal long setae. Coxa of pereopod 4 with 12 marginal setae. dactyl of pereopod 4.45 percent length of corresponding propod. Pereopod 5: basis with 12 very shallow serrations along posterior margin: 7 short spines on anterior margin: 3-4 long setae on the anteroproximal margin. Pereopod 6: basis with 12 shallow serrations along posterior margin: 6 short spines on anterior margin: 3 setae on anteroproximal margin. Pereopod 7: coxa with 2 setae on the posterior margin: basis with 12-13 shallow serrations along posterior margin: 7 short spines on anterior margin: 1 seta on anteroproximal margin: dactyl about 35 percent length of corresponding propod.

Pleonal plates: posterior margin of plate I convex. with I seta. distoposterior corner not produced. subacute: posterior margin of plate 2 weakly convex. with I setae. distoposterior corner iveakly produced. subacute. ventral margin with 4-5 (subventral) spines: posterior margin of plate 3 convex with I seta. distoposterior corner neither produced nor acute, ventral margin with 3 (subventral) spines. Pleopod I: peduncular segment with 2 retinaculae. I small spine sometimes at middle of inner margin. setae lacking on outer margin.

Uropod 1: inner ramus 60 percent length of peduncle. armed with 13 spines: outer ramus with 13 spines: peduncle with row of 12 outer and $3-4$ inner spines. Uropod 2: inner ramus with 11 spines: outer ramus with 9 spines; peduncle with 5 outer and 4 inner spines. Uropod 3 : inner ramus with 1 long spines. outer ramus about 1.5 times longer than peduncle. inner and outer margin each with 2-3 set of spines in groups of 1-3. Telson much broader than long, notched about li/4 distance to base. each lobe with 2-3 apical spines: spines about $1 / 2$ length of telson.

Male.--Differing from female as follows. Smaller with more slender, elongate body. Antenna 2: calccoii percent on peduncular segments 4 and 5 and first 3 segments of flagellum.

Propod of gnathopod 1 more than 2 times larger and longer than carpus: palm with 9 spine teeth on inside. 11 on outside: defining angle with 3 notched and I notched-serrate spine teeth on inside. 4 notched and 1 serrate spine teeth on outside. Propod of gnathopod 2 more than 2 times larger and longer than 
carpus: palm margin convex and strongly oblique, with 10-12 spine teeth on inside. 12 on outside: defining angle with 5-6 spine teeth on inside and 6 on outside.

Uropod 2: outer ramus deflected laterally, without inner spines but with 2 thin. setal-like outer spines. apex with row of 4 inner and row of 3 outer spines: peduncle with 3 outer spines and 3 inner spines. Telson broader than long, notched about $1 / 3-1 / 2$ distance to base.

Distribution and ecology.--This species is known only from caves on the western margin of the Cumberland Plateau in Rockcastle and Pulaski counties. Kentucky (fig. 122). where it inhabits drip pools and small streams. Of the 37 females collected to date. only one specimen $(6.0 \mathrm{~mm})$ was ovigerous.

Etymology.--The epithet castellanum is from Latin meaning "keepers of a castle." so named in reference to the occurrence of this species in Rockcastle County.

The hobbsi subgroup

Diagnosis.--Adult size range. 7.0-11.0 mm. Eyes absent. Calceoli absent from antenna 2 of male. Maxilla 1 inner plate with up to 16 apical plumose setae. Maxilla 2 inner plate with oblique row of up to 11 plumose setae on inner margin. Outer plate of maxilliped just slightly higher than inner plate. Carpus and propod of gnathopods in both sexes very thin and long. Carpus longer than propod of female gnathopod 2: palmar margin of propod of male with simple spine teeth. very few notched spine teeth: detining angle with notched-serrate spine teeth on inside and serrate spine teeth on outside. Outer ramus of female uropod 3 longer and thicker than in other species of the genus.

Comments.--The hobbsi subgroup is composed of a single species.

Crangonvx hobbst Shoemaker

Figures 123-128

Crangomux hobbsi Shoemaker. 1941:9-! I. figs. 3. 4 [type-locality: Huggin's Cave. Alachua Co.. Florida|.-Hubricht. 1943:690.--Nicholas. 1960:127.--Warren. 1961:60.--Bousfield. 1963:8.--Holsinger. 1972:+1: 1977:252: 1986:97. fig 7.--Barnard \& Barnard. 1983:434. map 11.--Fitzpatrick. 1983:145.

Material examined.--FLORIDA. Alachua Co.: cave in old river bed. High springs. $3 \approx$. H. H. Hobbs. Jr.. 20 Mar 1935 (USNM); Crumbley Sink, 1 fragment. P. Smith and W. Jasper, 9 May 1983 (FSM): Devil's Hole, Gainsville. 1 \&. 9 Jan 1939 (USNM). 1 \&. J. Martin. 27 Nov 1937 (USNM) and $11 \geq .4 z *$. 28 Nov 1937 (USNM): Dudley Cave, I §. J. Martin, 16 Oct 1937 (USNM) and 1 ₹. H. H. Hobbs. Jr. et al.. 31 Oct 1937 (USNM); Goat Sink, 4 \%. H. H. Hobbs. Jr.. 14 Mar 1938 (USNM): High Springs Cave. I ミ. Dickinson and H. H. Hobbs. Jr. 23 Sep 1940 (USNM); Huggins Cave. 14 . $1 \approx$. J. Martin. 23 Jan 1938 


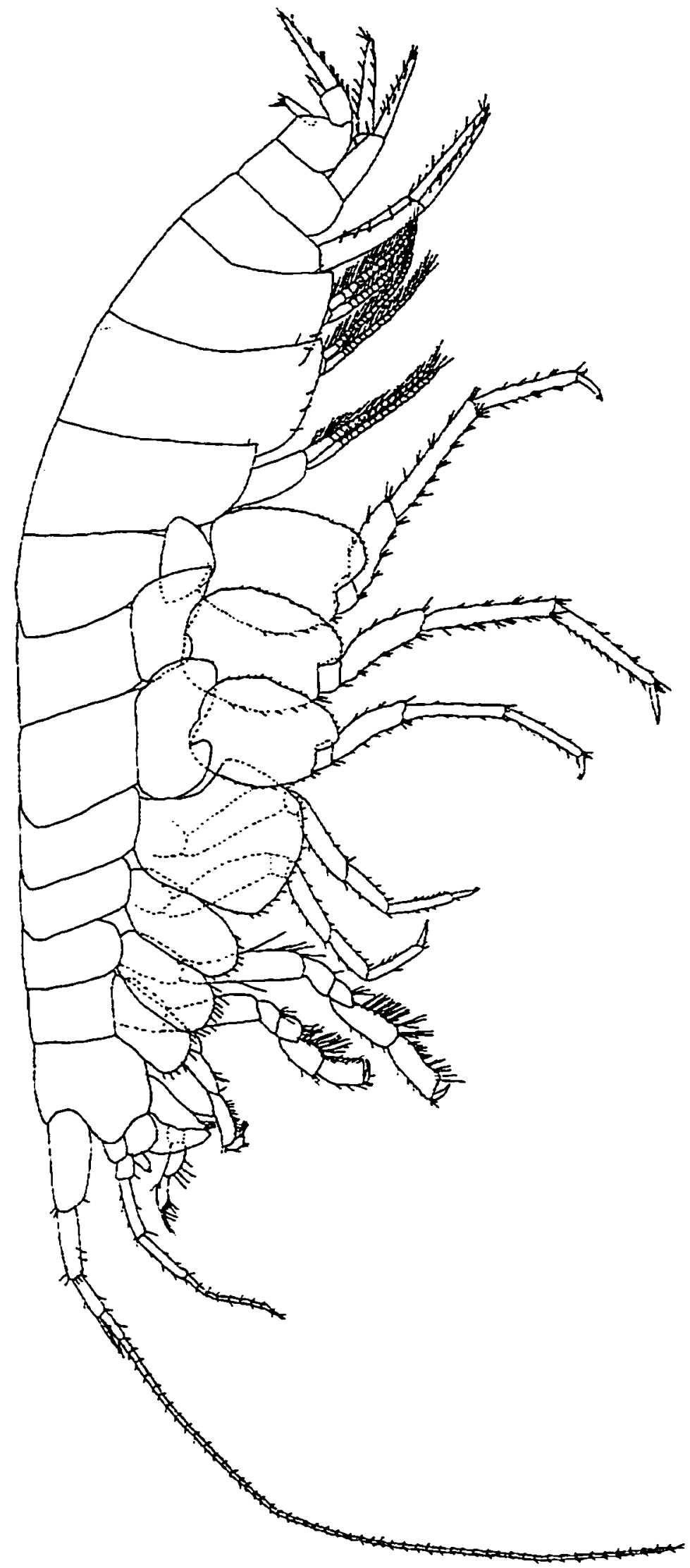

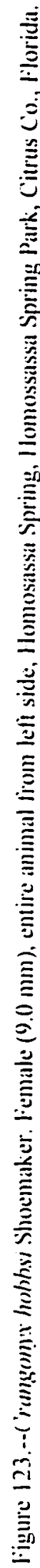




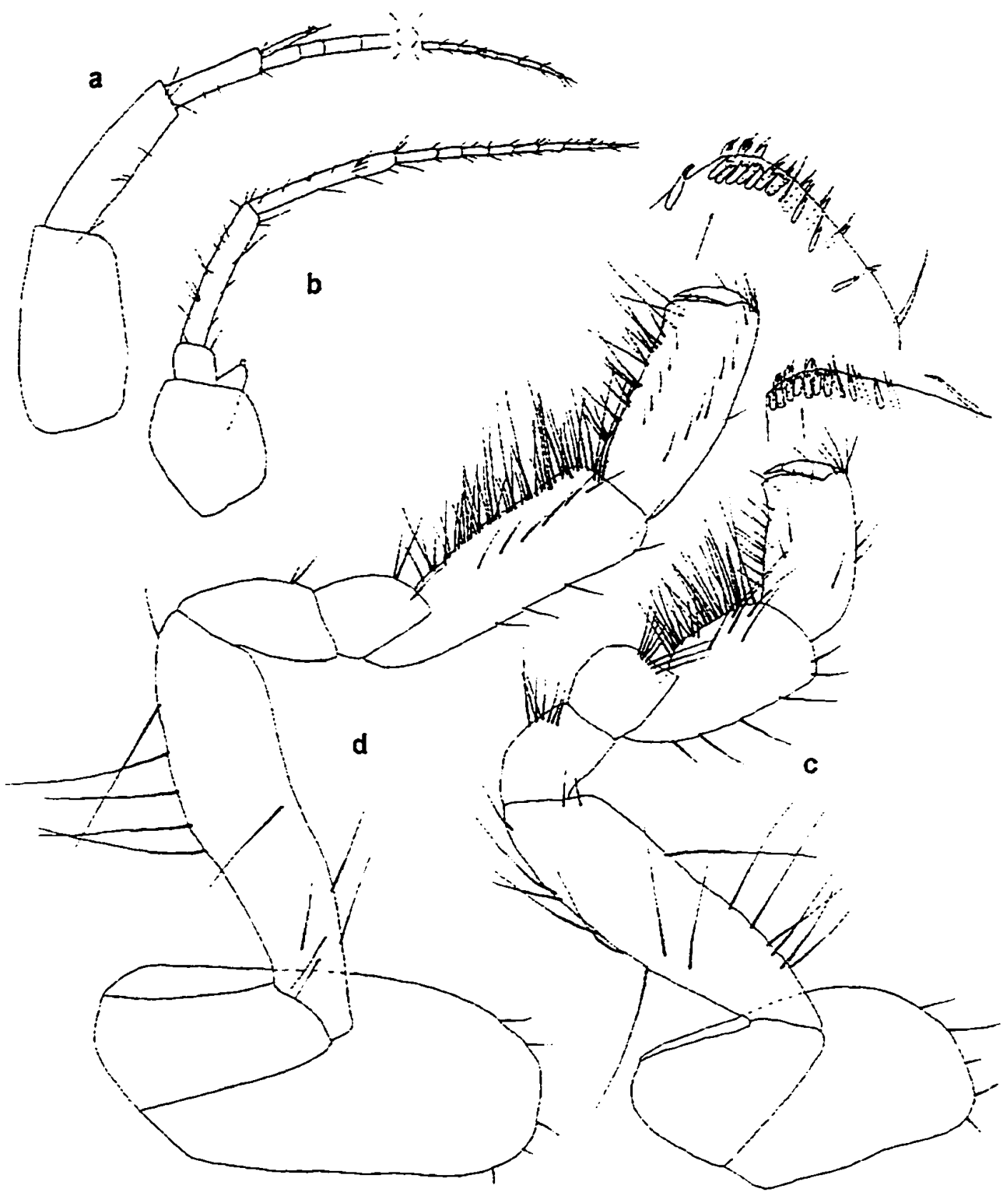

Figure 124.--Crangonvx hobbsi Shoemaker. Female $(9.3 \mathrm{~mm})$. Huggins Cave. Alachua Co.. Florida: a. b. antennae 1. 2: c. d. gnathopods I. 2 (palmar margins enlarged). 


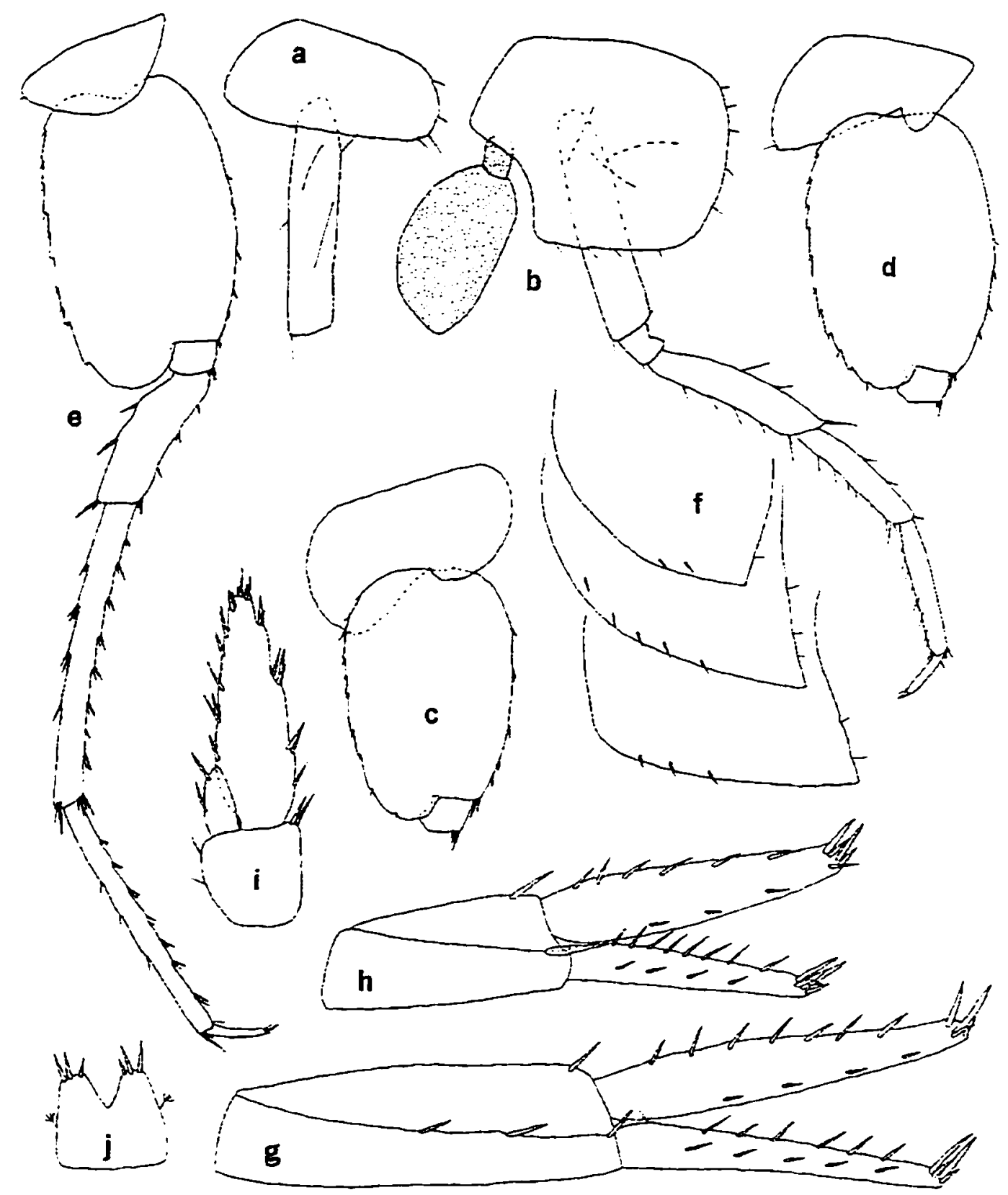

Figure 125.--Crangonyx hobbsi Shoemaker. Female $(7.3 \mathrm{~mm})$. Huggins Cave. Alachua Co.. Florida: a. c. d. pereopods 3. 5.6 (in part): b. e, pereopods 4, 7. Female $(9.3 \mathrm{~mm}$ ). Huggins Cave. Alachua Co.. Florida: f. pleonal plates; g, h. i. uropods 1. 2, 3: j. telson. 

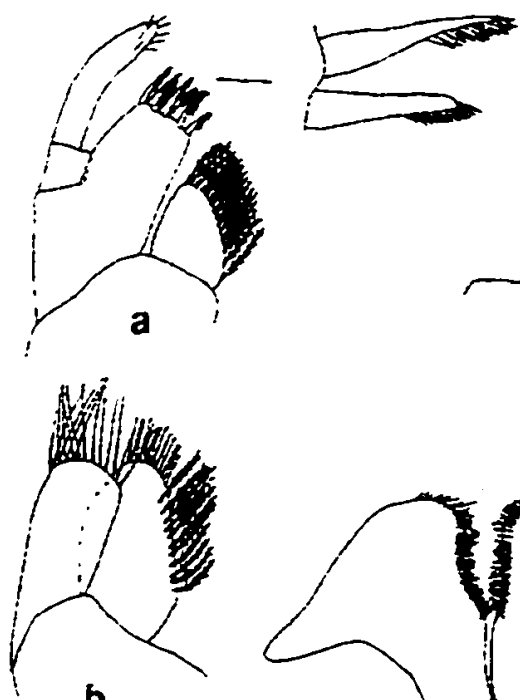

b
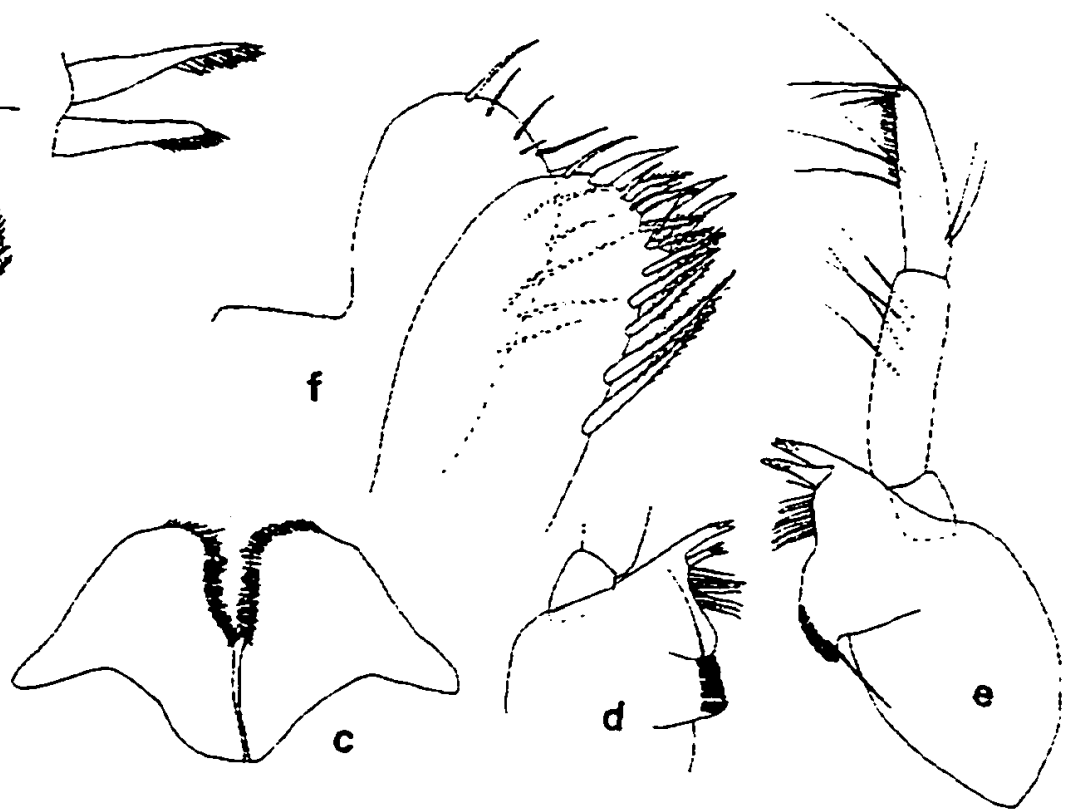

Figure 126.--Crangonıx hobbsi Shoemaker. Female $(9.3 \mathrm{~mm})$. Huggins Cave. Alachua Co.. Florida: a. b. maxillae 1. 2: c. lower lip: d. dentate part of left mandible: e. right mandible: f. inner and outer plates of maxilliped (greatly enlarged). 


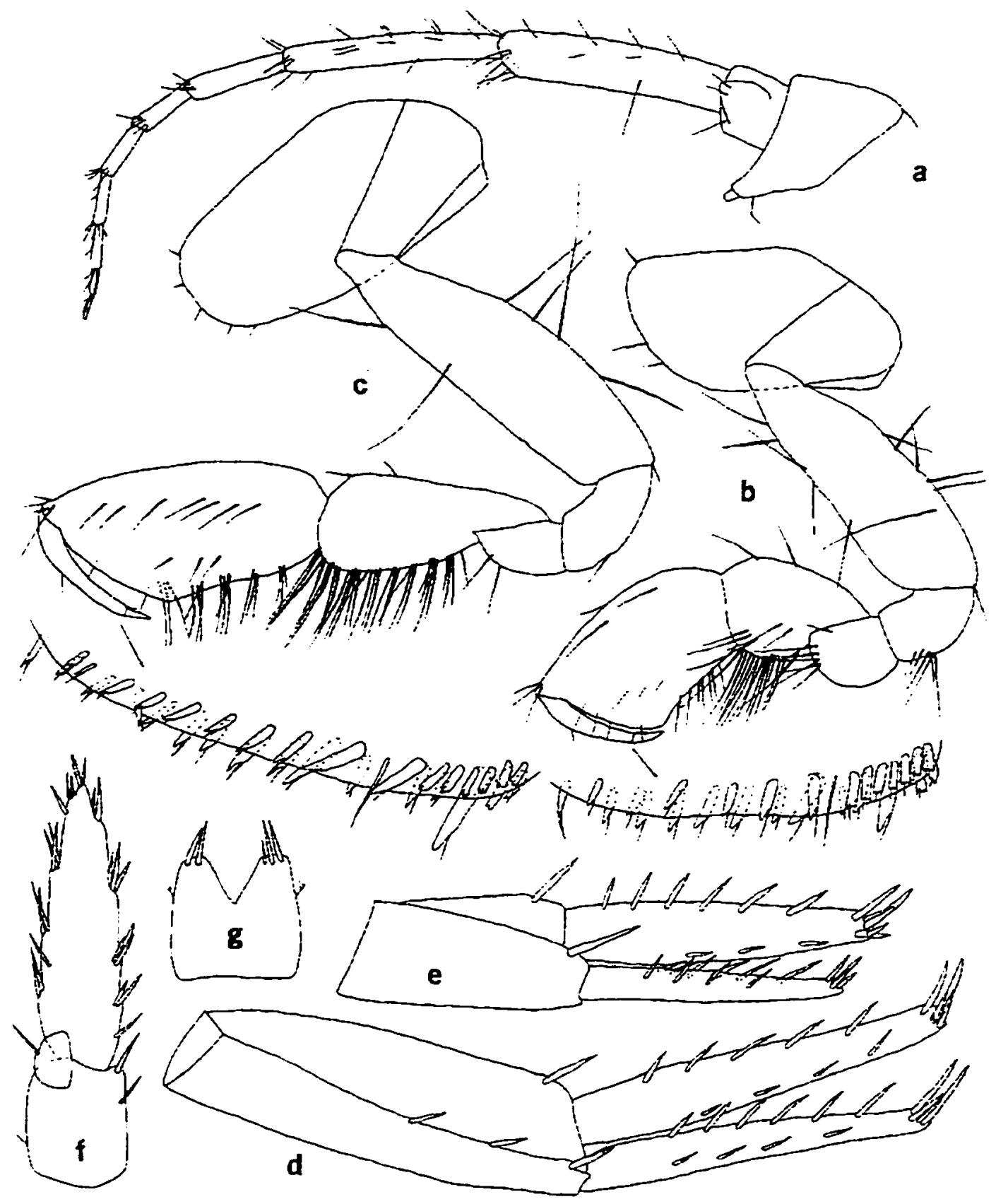

Figure 127.--Crangonyx hobbsi Shoemaker. Male $(6.2 \mathrm{~mm})$. Huggins Cave. Alachua Co.. Florida: a. antenna 2: b. c. gnathopods 1.2 (palmar margins enlarged): d, e. f. uropods 1. 2. 3: g. telson. 


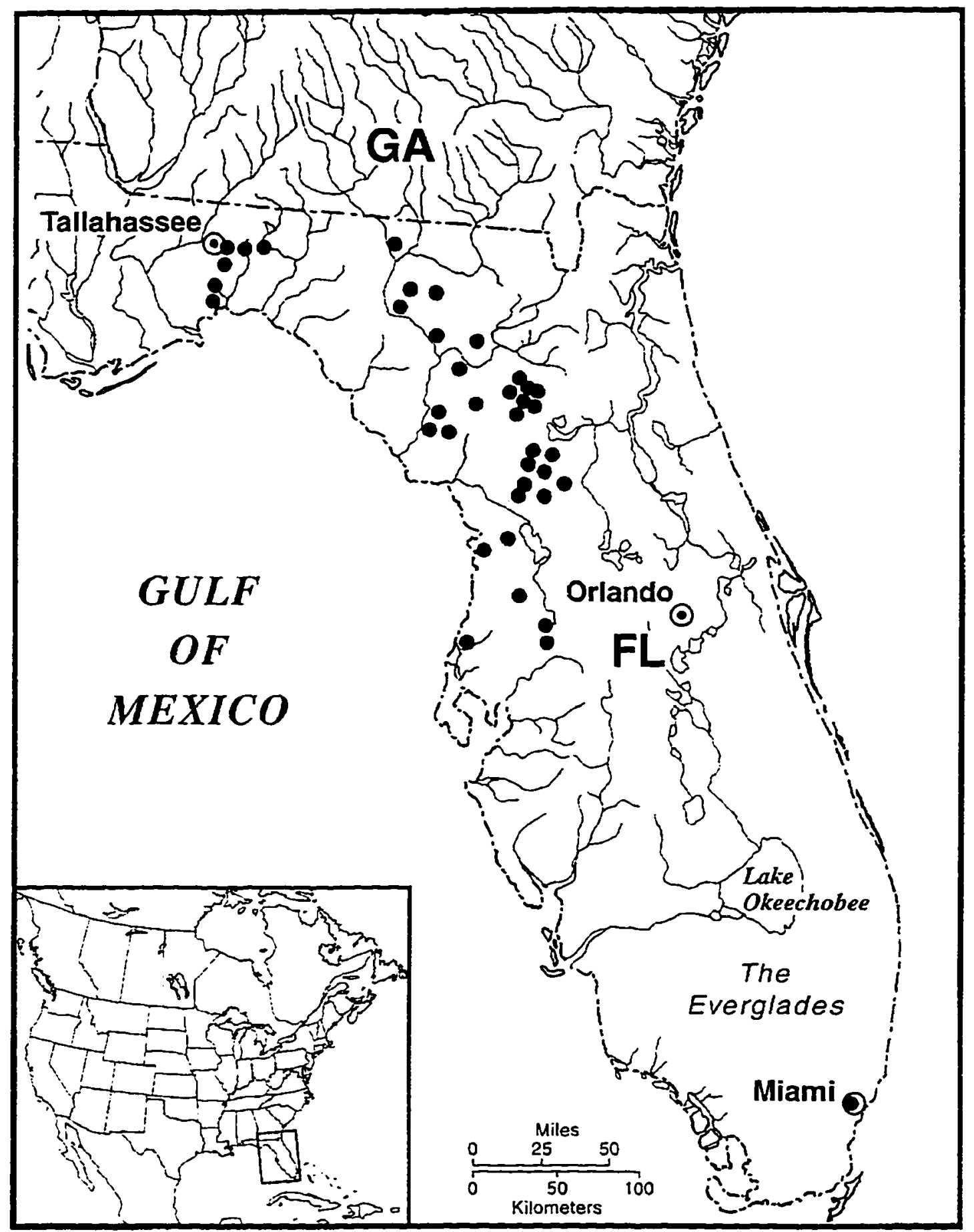

Figure 128.--Distribution of Crangomx hobbsi in North America. A solid circle represents 1-5 closely proximate localities. 
(USNM) and $17 \% .1 \% 12$ Mar 1938 (USNM). 2 \%. $1 \approx$. M. L. Chable. 8 Apr 1939 (USNM), holotype $\approx$ (USNM No. 79362). 12 2. 1 ¿.. H. H. Hobbs, Jr., 12 Jul 1940 (USNM). 7 ?. Dickinson et al.. 22 Sep 1940 (USNM). $30 £ .7 \propto$. L. Hubricht. 17 Apr 1941 (USNM) and 3 \%. R. D. Warren. 13 Jan 1962: Squirrel Chimney, 4 . I E. T. Morris. 1991: well on Carr Farm. W of Micanopy. 3 fragments. A. Carr. 26 May 1975 (USNM): Citrus Co.: Homosassa Springs, Homosassa Spring State Park. $11 \supseteq .2 \approx 11$ Juv. T. Morris. 15 Aug 1991: Gum Tree Cave (=Gum Cave or Sweetgum Cave). 2 ๑. T. H. Hubbell. 8 Mar 1936 (USNM). $2 \equiv .1 \div$ Sherman and H. H. Hobbs. Jr.. 12 Dec 1936 (USNM) and $149.3 \cong .18$ Dec 1936 (USNM). $\equiv$. J. Martin. 18 Dec 1936 (USNM). 19 \&. J. Martin and H. H. Hobbs. Jr.. 26 Feb 1937 (USNM). $12 \cong$. J. Martin. 27 Feb 1937 (USNM), 5 ₹. 1 ¿.2 Apr 1937 (USNM). 1 \&. I fragment. R. Franz and S. Reid. 13 Feb 1982 (FSM): Columbia Co.: Busseys Sink. 1 ९. R. Franz. 17 Jun 1980 (FSM) and 1 ९. 1 :. R. Franz. 8 Jul 1980 (FSM): Rose Creek swallet I (=Duckweed I), 1 ९. T. Morris. 22 Nov 1991: Dade Co.: Little Bird Nursery Well, collections in USNM made by G. Millier as follows--1 $\cong .24$ Jan $1968.14 \supseteq 10=14$

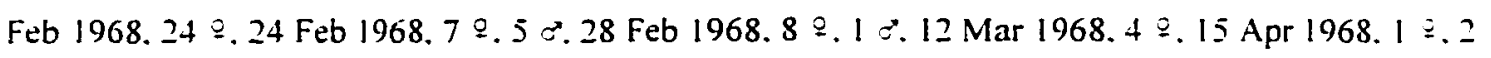

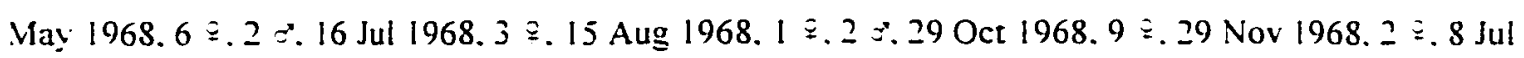
1969. 2 . $2 \approx 13$ Feb 1969: well near Miami (probably same as Little Bird Nursery well). $2 \doteqdot$. collector unknown. Sprıng. 1964: Gilchrist Co.: Devils Eye spring (one entrance to Ginnie Spring Cave Sy stem). 1 ミ. A. T. Leitheuser et al. 13 Sep 1979: 4 Juvs. J. Bozanic. 29 Aug 1985 and 8 Juvs. 21 Jan 1986:

Hernando Co.: Eagles Nest Sink Cave system. 5 ๑. A. T. Leitheuser et al.. 2 Sep 1979: Leon Co.: Bird Sink Swallet. 6 ฐ. T. Morris. 6 Nov 1991 and 9 פ. T. Morris. 17 Jul 1993: Sullivans Tunnel ("Hole in the Ground"). 1 ९. S. Gerrard. 9 Dec 1982 (FSM), 1 . T. Morris. 10 Jun 1990: Levy Co.: Friedmans Sink. Manatee Springs State Park, I c. A. T. Leitheuser, 19 Aug 1979: Manatee springs. $1 \cong$. S. Bauer and P. Smith. 5 Mar 1983 (FSM): Well at Chiefland. I $\cong$. V. H. Verrill. Apr 1940 (USNM) and $1 \cong .1942$ (USNM): Madison Co.: Blue Springs. 2 F. J. Bozanic. 30 Aug 1985: Marion Co.: Chert Cave. $7 \neq 1: 1$ juv. R. Franz and A. T. Leitheuser. 21 Feb 1981 (FSM): Indian Cave. 2‡. R. M. Norton et al.. 16 Mar 1967: Orange Lake Cave. 5 ९. J. R. Holsinger et al.. 5 Mar 1979: Sunday Sink. I פ. R. Franz. 9 Dec 1975: Pasco Co.: Nexus Sink. 8 9. 1 \%. A. T. Leitheuser et al.. II Jan 1983 (FSM); 125-foot well. Lacoochee. 1 ૬. R. A. Pope, 4 Oct 1937 (USNM); Suwanne Co.: Challenge Sink, I \&. A. T. Leitheuser et al.. 10 Mar 1978: Cisteen Sink. 1 \% I juv, A. T. Leitheuser, 11 Feb 1979; Orange Grove Sink. 1 . A. T. Leitheuser et

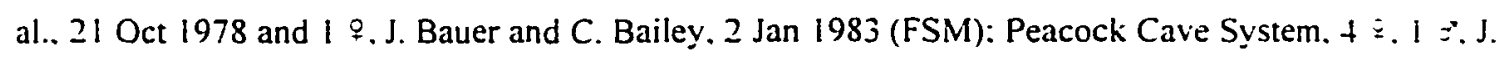

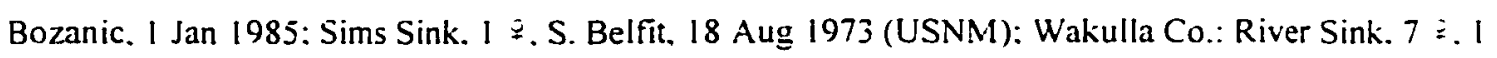
juv. E. A. Caine. 27 Aug 1972: Shepard Blue Spring, St. Marks Wildlife Refuge. $1 \cong$. W. Fehring. 22 Jul 1983: Sally Ward Spring (Wakulla Springs Cave system). 3 2. T. Morris et al.. 23 Nov 1987 and $4 \cong .1$ juv. 9 Dec 1987; McBrides Slough (Wakulla Springs Cave system), 5 ૬. T. Morris et al.. 29 Nov 1987.

Diagnosis.--A medium sized subterranean species distinguished from all other species of the genus by thin. elongate carpus and propod of gnathopods in both sexes (carpus longer than propod of gnathopod 2 in female): setose mouthparts, especially inner plate of maxilla 1 with up to 16 apical plumose setae. and 
inner plate of maxilla 2 with oblique row of up to 11 plumose setae on inner margin: much longer and thicker outer ramus of uropod 3 of female: and absence of calceoli on antenna 2 of male. Largest male. 9.0 $\mathrm{mm}$ : largest fermale. $11.0 \mathrm{~mm}$

Female--Eyes absent. Antenna I. about 80 percent length of body. about 2.2-3.0 times longer than antenna 2: primary flagellum with up to 27 segments. Antenna 2. flagellum with 8-9 segments. Mandibles subequal, spine row with 9-1 I spines: segment 2 of palp with 4 long setae. segment 3 with $1-2$ A setae. 1 B seta (very low location near A setae), $2 \mathrm{C}$ setae, $5 \mathrm{E}$ setae and a row of $\mathrm{D}$ setae. Lower lip: lacking inner lobes. lateral process of outer lobe subacute. Maxilla 1 : inner plate with 16 apical plumose setae: palp with 5-6 slender spines on apex. Maxilla 2. inner plate with oblique row of 11 plumose setae on inner margin. Maxilliped: inner plate apically with 3 bladelike spines. 3 naked spines. and 9 plumose setae extending from inner margin to apex: outer plate with row of naked setae on the inner margin: inner plate slightly higher than outer plate: dactyl with 2 setae on inner margin near nail: dactyl nail long. 13 length of dactyl.

Gnathopod I: propod and carpus very long: carpus slightly longer than propod. posterior margin with very long setae: propod palm with 2 unnotched spine teeth on inside. 4 on the outside: defining angle with 3-7 spine teeth on inside. I large unnotched and 2 serrate spine teeth on outside: inferior and superior medial setae few in number and singly inserted: posterior margin with 4 sets of setae singly or doubly inserted: dactyl nail very long about $1 / 3$ length of dactyl. Ventral margin of coxa 1 with 5 setae.

Gnathopod 2: carpus of gnathopod 2 much longer than propod, posterior margin with very long setae: propod palm with 3 unnotched spine teeth on inside. 4 on outside: defining angle with 3 unnotched and 2 notched-serrate spine teeth on outside. 7 notched-serrate spine teeth on inside: inferior and superior medial setae singly inserted. posterior margin with 6-7 sets of setae in groups of 2-5. Dactyl with several short setae on inner margin. Ventral margin of coxa 2 with 5 setae.

Coxa of pereopod 3 with 3 marginal setae. Coxa of pereopod 4 with 9 marginal setae. dactyl 50 percent length of corresponding propod. Pereopod 5: basis with 10 shallow serrations along posterior margin: 5 short spines on anterior margin: I long seta on anteroproximal margin. Pereopod 6: basis with 12 shallow serrations along posterior margin: 4 short spines on anterior margin: 1 long seta on anteroproximal margin. Pereopod 7: coxa with I seta on the posterior margin: basis with 10 shallow serrations along posterior margin: 5 short spines on anterior margin: dactyl about 25 percent length of corresponding propod.

Pleonal plates: posterior margin of plate I almost straight. with I seta. distoposterior corner not produced and weakly acute. ventral margin with 2 (subventral) spines: posterior margin of plate 2 weakly oblique with 2 or 3 setae, distoposterior corner produced and subqudrate acute. ventral margin with 5 (subventral) spines: posterior margin of plate 3 nearly straight or slightly oblique with I or 2 setae. distoposterior corner produced and subquadrate, ventral margin with 3 (subventral) spines. Peduncle of pleopod 1 with 2 retinaculae: lacking setae on outside margin.

Uropod 1: inner ramus subequal to peduncle length, armed with 16 spines: outer ramus with 15 spines: 
peduncle with 3 outer and 1 inner spines. Uropod 2: inner ramus armed with 14 spines: outer ramus with 17 spines: peduncle with 1 outer and 1 inner spines. Uropod 3: inner ramus with 1-2 spines. outer ramus about 2.5 times longer than peduncle, inner and outer margin each with 4-6 set of spines in groups of $1-3$. Telson broad equal to long, notched about li3 distance to base. each lobe with 3 apical spines: spines less than 12 length of telson.

Male.--Differing from female as follows. Smaller with more slender, elongate body. Antenna 2: flagellum with about 6 segments. peduncle and flagellum lacking calceoli.

Propod palm of gnathopod I with 5 notched or unnotched spine teeth on inside. 8 notched or unnotched spine teeth on outside: defining angle with 7 serrate spine teeth on inside and 3 notched or unnotched and 2 serrate spine teeth on outside. Carpus of gnathopod I very long but shorter than propod. Propod of gnathopod 2 palm margin strongly oblique. with 9 notched or unnotched spine teeth on inside. 11 unnotched spine teeth on outside; defining angle with 6 serrate and I spine teeth on inside. 3 unnotched or notched on outside. Carpus of gnathopod 2 very long but shorter and narrower than propod.

Uropod 2: outer ramus. neither deflected nor curved, outer ramus with 6 inner and 4 outer spines. apex with + spines: peduncle with $I$ outer and $I$ inner spines.

Distribution and ecology.--This species is wildly distributed in the northern and central limestone karst regions of Florida and is also recorded from a well in Dade County in the extreme southern part of the state (fig. 128). It is frequently associated with the stygobiont amphipod Crangonx grandimanus. Breeding apparently takes place the year round but ovigerous females are never abundant at any given time (Holsinger. 1972).

The baculispina subgroup

Diagnosis.--Adult size range. $6.0-7.5 \mathrm{~mm}$. Gnathopods of female: carpus and propod very thin and long: carpus equal or longer than propod, posterior margin armed with long setae: palm of propod with simple spine teeth; defining angle of propod of gnathopod I with serrate spine teeth. that of gnathopod 2 with "normally" notched spine teeth. Gnathopods of male: propod more than 2 time larger and much broader: palm of propod armed with unnotched rodlike spine teeth: defining angle of propod with "normally" notched spine teeth inside.

Comments.--The baculispina subgroup is composed of a single species.

Crangonyx baculispina. new species

Figures 129-132

Material examined (paratypes unless designated otherwise).--VIRGINIA. Lancaster Co.: shallow well 


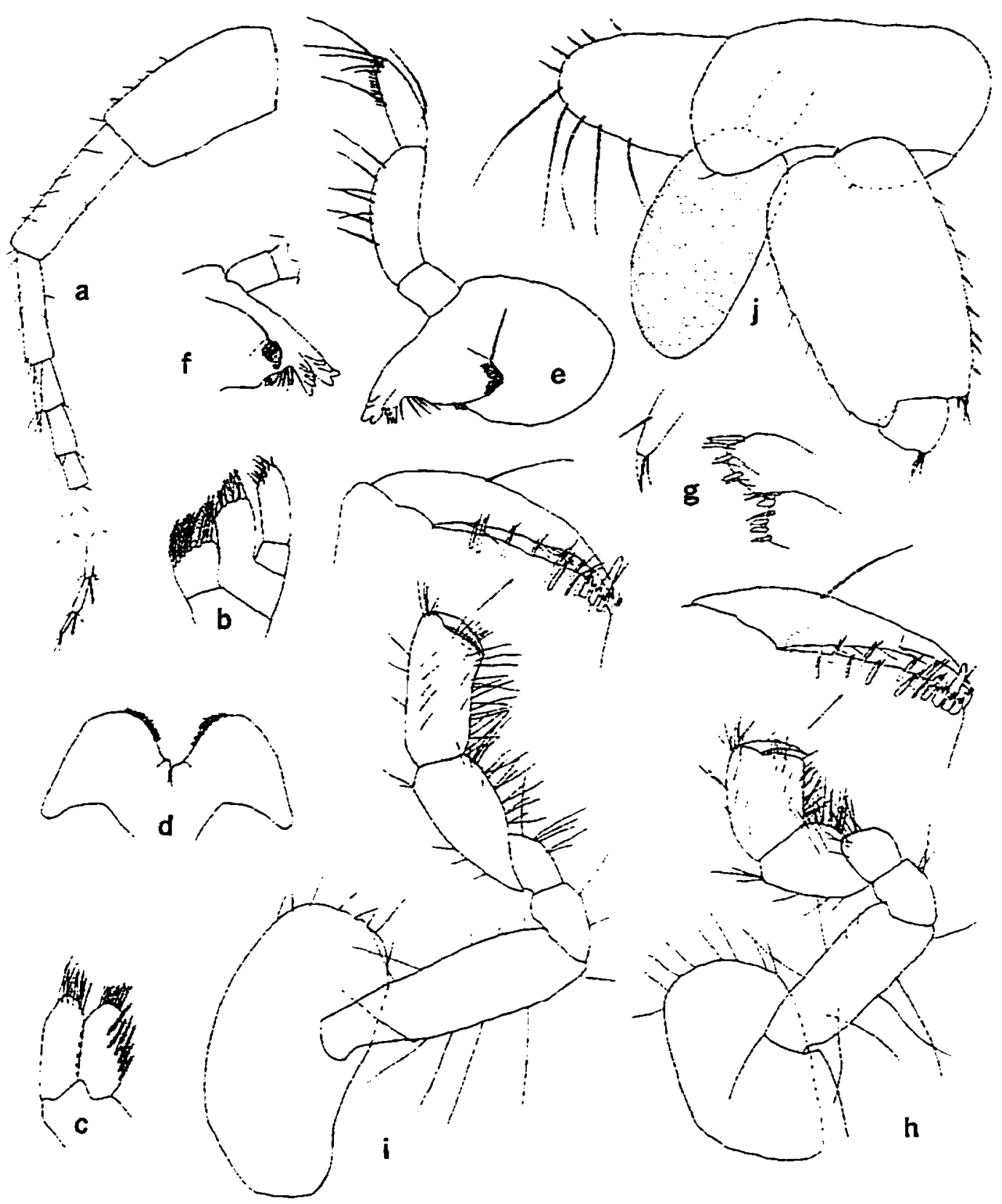

Figure 129.--Crangonyx baculispina, new species. Female paratype $(6.0 \mathrm{~mm})$, seep pool in woods near old dump just off Rt. $645.4 .8 \mathrm{~km}$ E of White Stone. Lancaster Co.. Virginia: a. antenna I: b. c. maxillae 1. 2: d. lower lip: $e$, right mandible: $f$. dentate part of left mandible: $g$. inner and outer ramus and paip dactyl of maxilliped (greatly enlarged): h. i. gnathopods 1.2 (palmar margins enlarged): j. pereopod 5 (in part). 


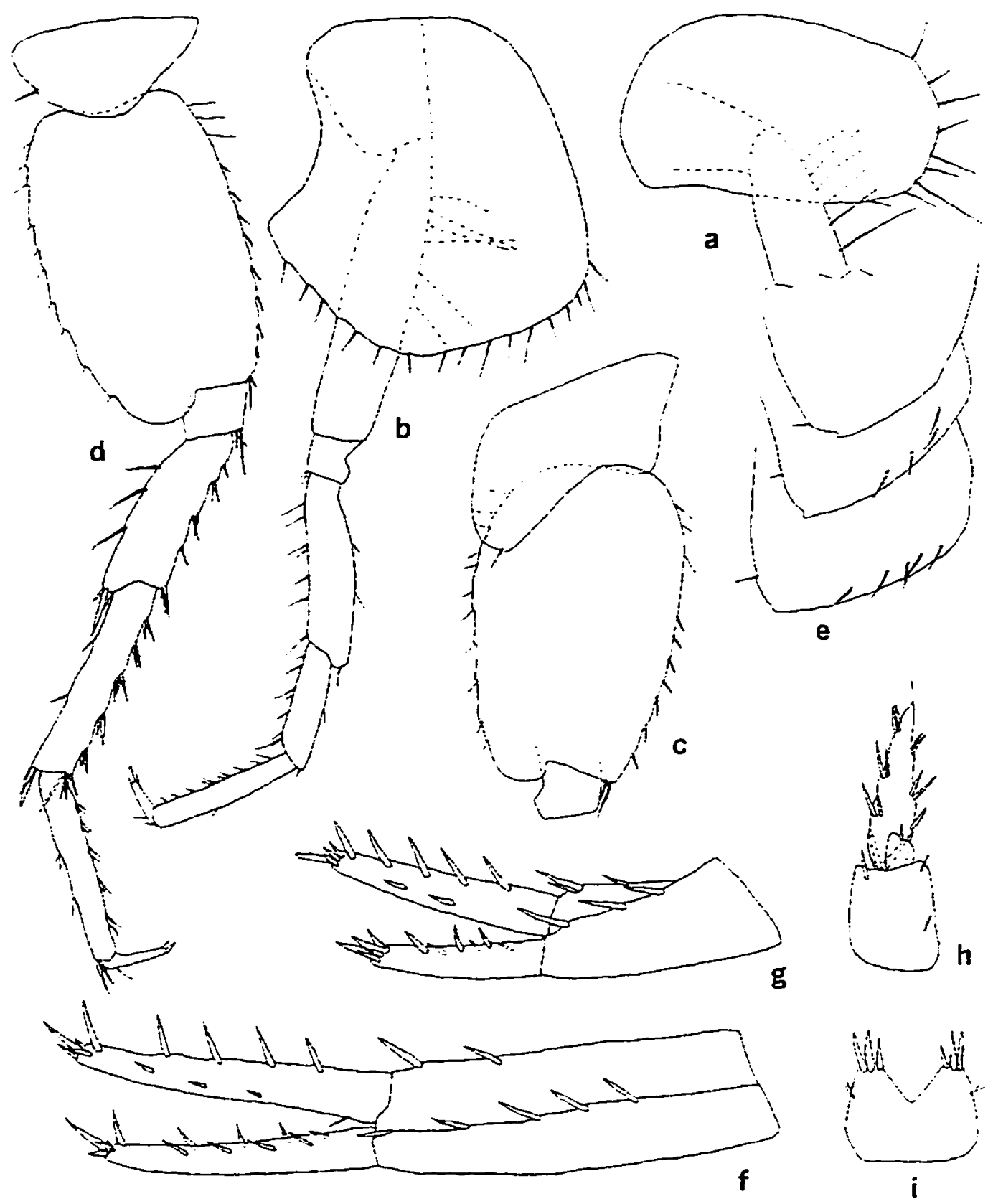

Figure 130.--Crangonyx buculispina, new species. Female paratype $(6.0 \mathrm{~mm})$, seep pool in woods near old dump just off Rt. $645.4 .8 \mathrm{~km}$ E of White Stone, Lancaster Co.. Virginia: a. c. pereopods 3.6 (in part): b. d. pereopods 4. 7: e. pleonal plates: f. g. h. uropods 1. 2. 3: i. telson. 


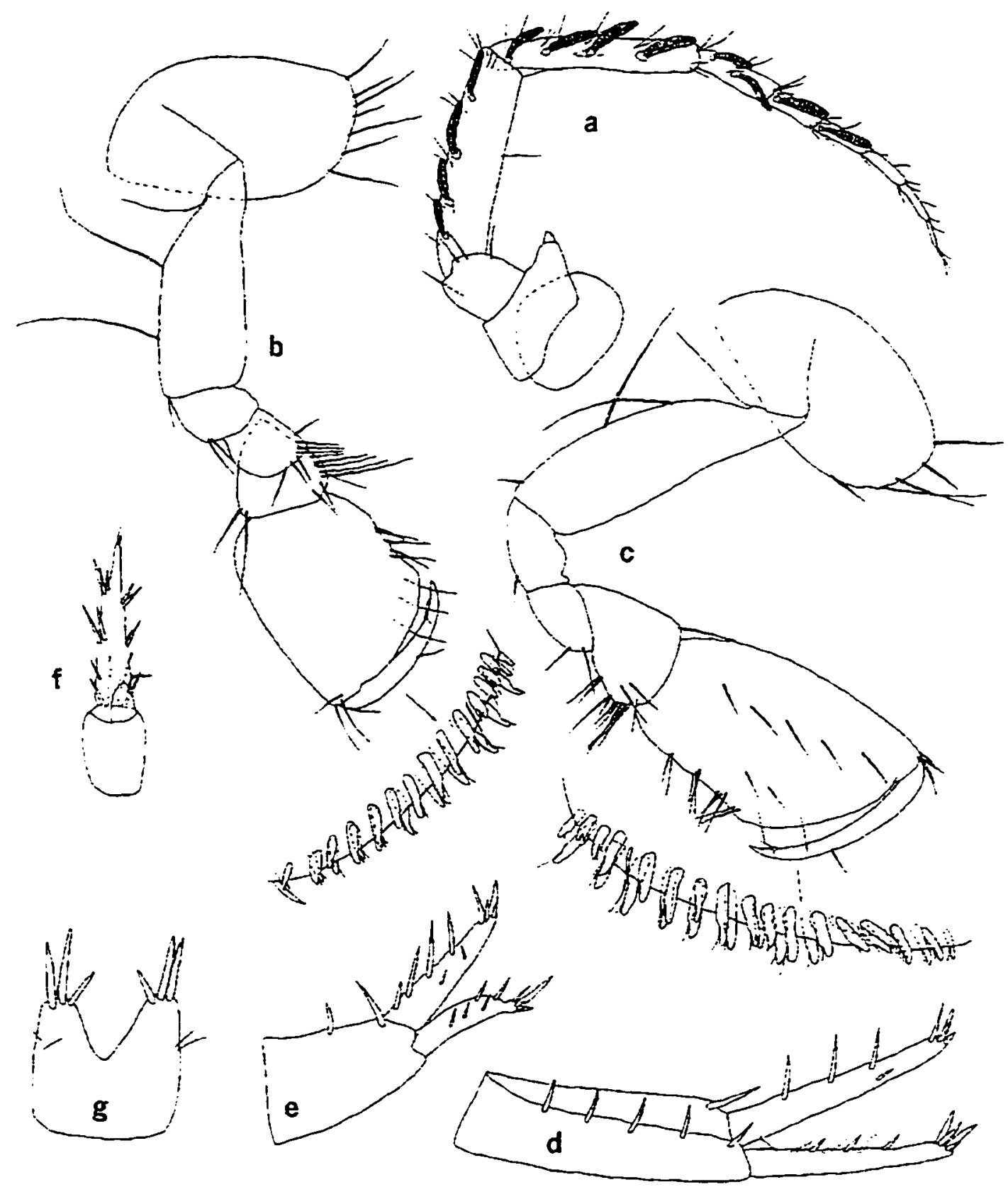

Figure 131.--Crangonvx baculispina. new species. Male paratype $(4.0 \mathrm{~mm})$. seep pool in woods near old dump just off Rt. $645.4 .8 \mathrm{~km}$ E of White Stone. Lancaster Co., Virginia: a. antenna 2: b. c. gnathopods 1. 2 (palmar margins enlarged); d. e. f. uropods 1. 2. 3: g, telson. 


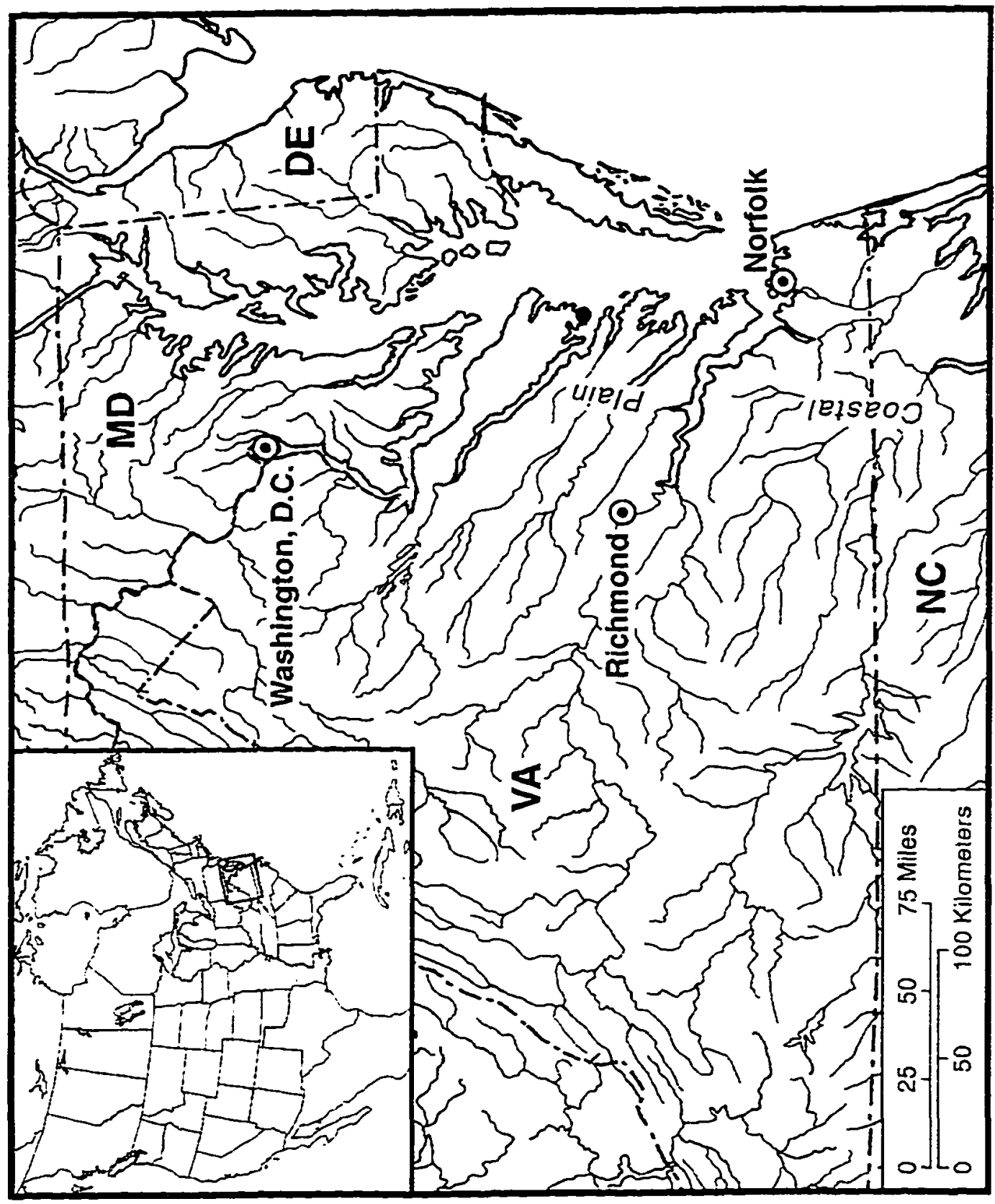

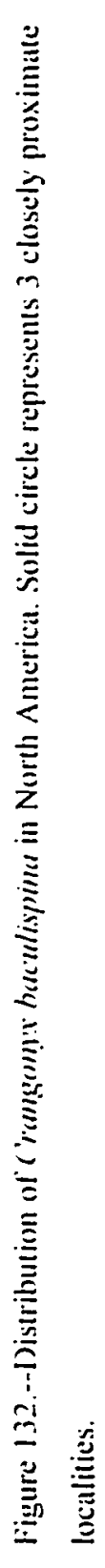


and/or roadside ditch near old dump just off Rt. $645.4 .8 \mathrm{~km}$ E of White Stone. $4 \Xi .2 \approx$. S. Hetrick. 10 Mar 1982: seep pool in woods near old dump just off Rt. $645.4 .8 \mathrm{~km}$ E of White Stone. holotype $\approx .3 \equiv .1$ $\because$ J. R. Holsinger and R. Godfrey, 17 Apr 1983: swamp in woods near old dump just off Rt. $645.4 .8 \mathrm{~km} \mathrm{E}$ of White Stone. 1 \%, J. H. Holsinger et al., 9 Mar 1994. Largest male, $4.0 \mathrm{~mm}$ : largest female. $7.5 \mathrm{~mm}$.

Diagnosis.--A small species morphologically similar to other members of gracilis group but distinguished from them by carpus of gnathopods which is longer than propod in females (ct. $C$. hobhsi): and with unnotched rodlike spines on propods of male gnathopods (unique among all (rangonix species). Largest female. $7.5 \mathrm{~mm}$ : largest male. $4.0 \mathrm{~mm}$.

Female.--Eye large and ovate. Antenna 1, about 50 percent length of body. about 1.8 times longer than antenna 2: primary flagellum with about 16 segments. Antenna 2. flagellum with 8-9 segments. Mandibles subequal. spine row with 5 spines: segment 2 of palp with 7 long setae. segment 3 with 1-2 A setae. I C seta. $5 \mathrm{E}$ setae and a row of D setae. lacking B seta. Maxilla 1: inner plate with 6 apical plumose setae: palp with 7 slender spines on apex. Maxilla 2, inner plate with oblique row of 6 plumose setae on inner margin. Maxilliped: inner plate apically with 3 bladelike spines. 3 naked spines. and 5-6 plumose setae extending from inner margin to apex: outer plate with row of naked setae and about 2 slender pectinate spines on inner margin: dactyl with 2 setae on inner margin near nail; dactyl nail very long. 1 length of dactyl.

Propod of gnathopod $l$ equal in length to carpus: posterior margin relatively long. with long setae: palm almost straight. shorter than posterior margin. with 4 unnotched spine teeth on inside. 4 on outside: defining angle with four serrate spine teeth on inside. I notched. I large and I serrate spine teeth on outside: inferior singly inserted; superior medial setae few in number and singly inserted: 4 sets of posterior margin setae singly inserted or in groups of $1-4$. Dactyl with row of short setae on inner margin. nail very long. Ventral margin of coxa I with 8 long setae.

Propod of gnathopod 2 equal in length to carpus: posterior margin elongate. with long setae: palm almost straight. much shorter than posterior margin. with 2-3 unnotched spine teeth on inside. 5-6 on outside: defining angle with 3 spine teeth on inside. I large unnotched and I serrate spine teeth on outside: inferior medial setae and superior medial setae singly inserted. posterior margin with 5 sets of setae in groups of 1-3. Dactyl with row of short setae on inferior margin. Ventral margin of coxa 2 with 8 setae.

Coxa of pereopod 3 with 9 marginal setae. Coxa of pereopod 4 with 16 marginal setae. dactyl of pereopod 4.35 percent length of corresponding propod. Pereopod 5: basis with 8 shallow serrations along posterior margin: 8 short spines on anterior margin: 1 long seta on anteroproximal margin. Pereopod 6: basis with 11 shallow serrations along posterior margin. 7 short spines on anterior margin: 3 setae on anteroproximal margin. Pereopod 7: coxa with 1 seta on the posterior margin: basis with 10 shallow serrations along posterior margin. 8 short spines on anterior margin: 3 setae on anteroproximal margin: dactyl about 33 percent length of corresponding propod.

Pleonal plates: posterior margin of plate 1 slightly convex. with 1 seta. distoposterior corner acute but not produced: posterior margin of plate 2 nearly straight with 1 or 2 setae, distoposterior corner slightly 
produced and acute, ventral margin with 4 (subventral) spines: posterior margin of plate 3 nearly straight with I seta. distoposterior corner small and subacute but not produced. ventral margin with 5 (subventral) spines. Peduncle of pleopod I with 2 retinaculae, lacking setae on outside margin.

Uropod I: inner ramus 80 percent length of peduncle. armed with 12 spines: outer ramus with 13 spines: peduncle with 5 outer and 2 inner spines. Uropod 2: inner ramus armed with 11 spines: outer ramus with $1 /$ spines: peduncle with 3 outer and 3 inner spines. Uropod 3 : inner ramus with 1 spine. outer ramus about 1.5 times longer than peduncle, inner and outer margins each with $3-4$ set of spines in groups of $1-3$. Telson broader than long, notched about 2/5 distance to base, each lobe with 3 apical spines: spines less than $1: 2$ length of telson.

Male.--Differing from female as follows. Smaller with more slender. elongate body. Antenna 2: calceoli present on peduncular segments 4 and 5 . and first 3 flagellar segments. Propod of gnathopod 1 more than 2 times larger and longer than carpus and much broader: palmar margin with 10 unnotched. curved rodlike spine teeth on inside. 8 on outside: defining angle with 4 normal spine teeth on inside and 1 unnotched. curved rodlike and 2 small laterally notched spine teeth on outside. Propod of gnathopod 2 more than 2 times larger and longer than carpus; palm margin strongly oblique, with I 2 unnotched curved rodlike spine teeth on inside, 12 on outside; defining angle with 1 unnotched curved rodlike and 2 notched teeth on inside and 2 unnotched curved rodlike and 1 laterally notched spine teeth on outside.

Uropod 2: outer ramus curved laterally but not strongly so. shorter and narrower than inner ramus. inside with 3 spines, outside with 2 spines: outer ramus with about 9 spines: peduncle with 2 outer spines and 1 inner spine. Uropod 3: outer ramus about 2 times length of peduncle. Telson longer than broad. notched about $1 / 2$ distance to base: each lobe with 3 apical spines: spines about 12 length of telson.

Type-locality.-- Seep pool in woodland near or old trash dump just off Rt. $645.4 .8 \mathrm{~km} E$ of White Stone. Lancaster County, Virginia.

Distribution and ecology.--This rare species is known only from its type-locality and immediate vicinity, where it was collected from a seep pool. shallow hard-dug well and swamp (fig. I32). Ovigerous female were collected during March and April. Crangonyx baculispina was found living in association with the epigean amphipod C. polustris and the stygobiont amphipod Stygobromus indentatus. and an isopod Cuecidorea sp.

Etymology.--The epithet haculispina refers to the rodlike spines on the margins of the propods of the male gnathopods.

The floridanus subgroup

Diagnosis.--Adult size range. 5.0-10.0 mm. Distoposterior corner of pleonal plates 1-3 strongly produced, acute. Outer ramus of uropod 2 of male with comb spines.

Comments.-- Four species are assigned to this subgroup: $C$. floridanus, $C$. pseudogracilis. $C$. 
consimilis. and C. longidactylus.

Crangomx floridanus Bousfield

Figures $133-136$

Crangonyx floridanus Bousfield. 1963:2-6. figs. 1-2 [type-locality: shallows at edge of cypress swamp. Highlands Hammock State Park. Highlands Co.. Florida].--Holsinger. 1972:34. figs. IIf. 15 : 1977:252.--Karaman. 1978:66-71, figs. 1-3.--Barnard \& Barnard. 1983:434. map 11 .--Fitzpatrick. 1983:145.--Pennak, 1989:482. Crungon!x gracilis Smith.--Hubricht. 1943:691 [in part].--Penak \& Rosine. 1976:329. Eucrangonyx gracilis (Smith).--Shoemaker. 1933:15. Crangomx psendogracilis Bousfield--Bousfield. 1961:5.

Material Examined.--ALABAMA. Bibb Co.: slough of Haysop Creek 1 mi SW of Brent. $1 \equiv .1 \therefore$ L. Hubricht. 10 Apr 1941 (USNM): Fayette Co.: slough. $1.3 \mathrm{~km}$ E of Fayette. 30 specs ( Apr 1941 (USNM): Greene Co.: swamp stream $1.6 \mathrm{~km}$ W of New West Greene. $13 \geq .5 z$. L. Hubricht. i 1 Feb 1962 (USNM): Henry Co.: East Choctawhatochee River. 2 mi W of Capps. $2 \geq .2 \approx *$. L. Hubricht. 25

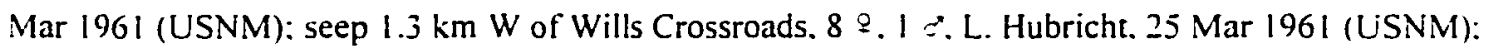
Houston Co.: swamp. $21.6 \mathrm{~km} \mathrm{SW}$ of Dothan. 51 specs ( $\left.¥ . \Xi^{3}\right)$ L. Hubricht. 15 Apr. 1941 (USNM): Jackson Co.: Russell Cave. I spec.. W. B. Jls. 9 Aug 1960. 7 g. 3 c. S. B. and J. Peck.. 3l Jul 1965 and 2 $=1$. H. Hobbs. III. et al. 28 Aug 1992: slough. $2.9 \mathrm{~km} \mathrm{E}$ of Limrock. 40 specs ( $(\approx)$ ). L. Hubricht. 12 Apr 1941 (USNM): slough. $2.6 \mathrm{~km}$ NW of Paint Rock. 50 specs ( $\left.\subseteq . z^{2}\right)$. L. Hubricht. 12 Apr 1941 (USNM): ditch in Stevenson. 2 9. J. E. and M. R. Cooper. 9 Apr 1966: swamp $8.6 \mathrm{~km}$ SE of Section. to specs, L.Hubricht, 13 Apr 1941 (USNM); Lauderdale Co.: Buffler Spring. Florence. 20 specs ( Koch. $13 \mathrm{Apr} 1977,10$ specs ( $\left.8 . \sigma^{\circ}\right), 7$ Jan 1978; Lawrence Co.: springs. $8.0 \mathrm{~km}$ E of Wheeler. 50 specs. 11 Apr 1941 (USNM): swamp $3.7 \mathrm{~km}$ SE of Wheeler. $15 \& .6 \mathrm{c}$. L. Hubricht, II Apr 194I (USNM): spring branch. Wheeler. 50 specs, L. Hubricht, 11 Apr 1941 (USNM): spring-fed slough. $2.7 \mathrm{~km} \mathrm{E} \mathrm{of}$ Courtland. L. Hubricht. II Apr 1941 (USNM); Moulton. I ₹. W. E. Snow. 9 Mar 1952 (USNM). Madison Co.: Spring in Maysville, 4 \&. S. B. Peck (collection date not given); slough $4.3 \mathrm{~km}$ NW of Gurley. 100 specs. 12 Apr 1941 (USNM): Big Spring, Huntsville. 1 \&. L. Hubricht. 22 Jul 1939 (USNM): Perty Co.: slough $8.5 \mathrm{~km}$ E of Uniontown. $7 \bar{\Xi} .4$. L. Hbricht. 9 Apr 1941 (USNM): Sumter Co.: swale $7.8 \mathrm{~km} \mathrm{NW}$ of Coatops. 20 specs. 9 Apr 1941 (USNM): Talladega Co.: Kymulga Cave. 50 specs. L. Hubricht. 18 Jul 1939 (USNM): Tuscaloosa Co.: Black Warrier River below Bankhead dam. 1 juv. L. Hubricht. 26 May 1967 (USNM). COLORADO. Boulder Co.: St. Vrain River at Hygiene, 1 c. J. V. Ward, Jan 1976 and 2 ミ. Apr 1976: Larimer Co.: Paudra River at Lions Park. 1 o. J. V. Ward. 22 Sep 1977: Weld Co.: pipe 


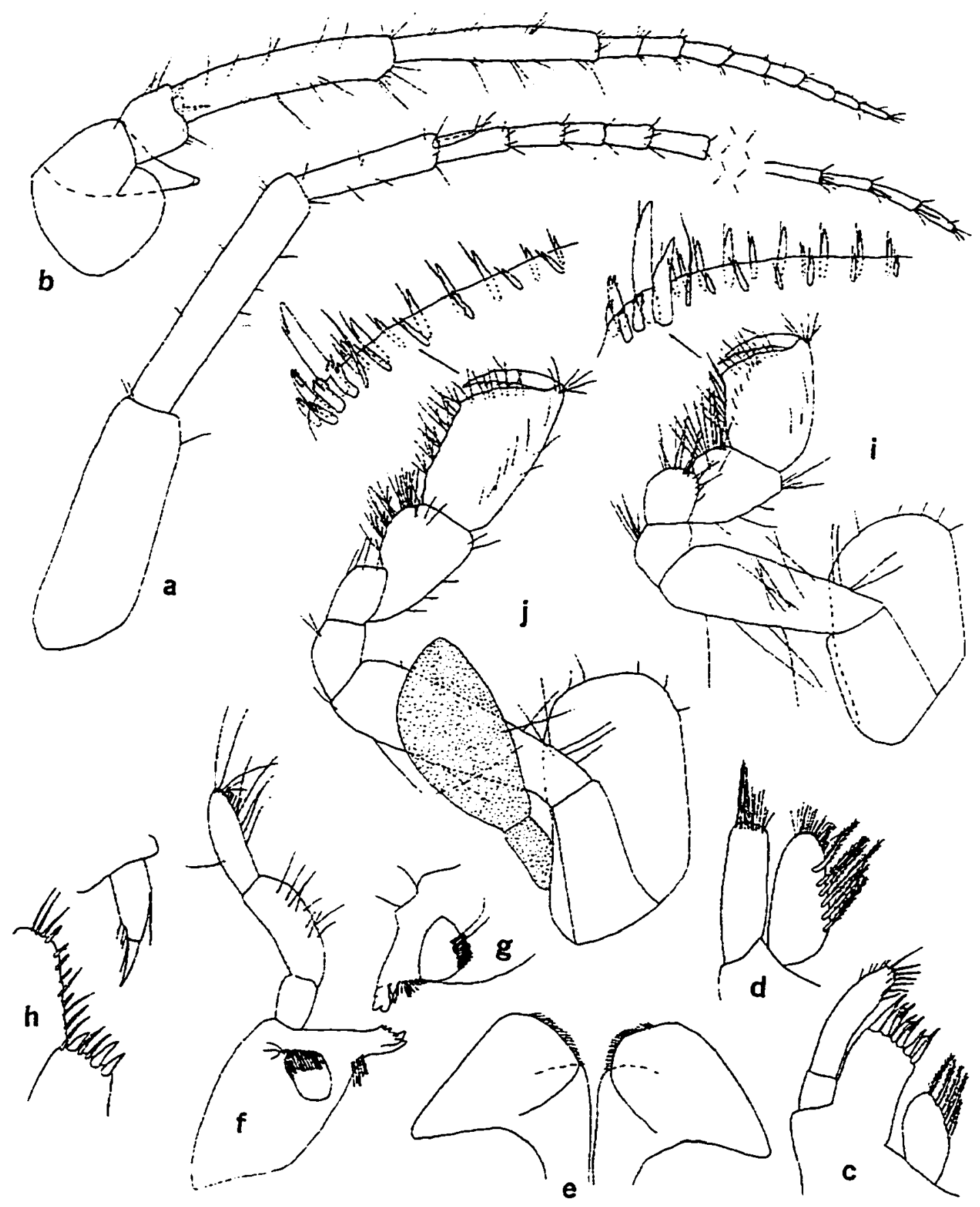

Figure 133.--Crangonyx floridunus Bousfield. Female $(8.2 \mathrm{~mm})$. Stream between Elloree and St. Matthews. Orangeburg Co., South Carolina: a. b. antennae 1. 2: c. d. maxillae 1. 2: e. lower lip: f. left mandible: g. dentate part of right mandible: h. inner. outer plates and palp dactyl of maxilliped (greatly enlarged); i, j, gnathopods 1.2 (palmar margins enlarged). 


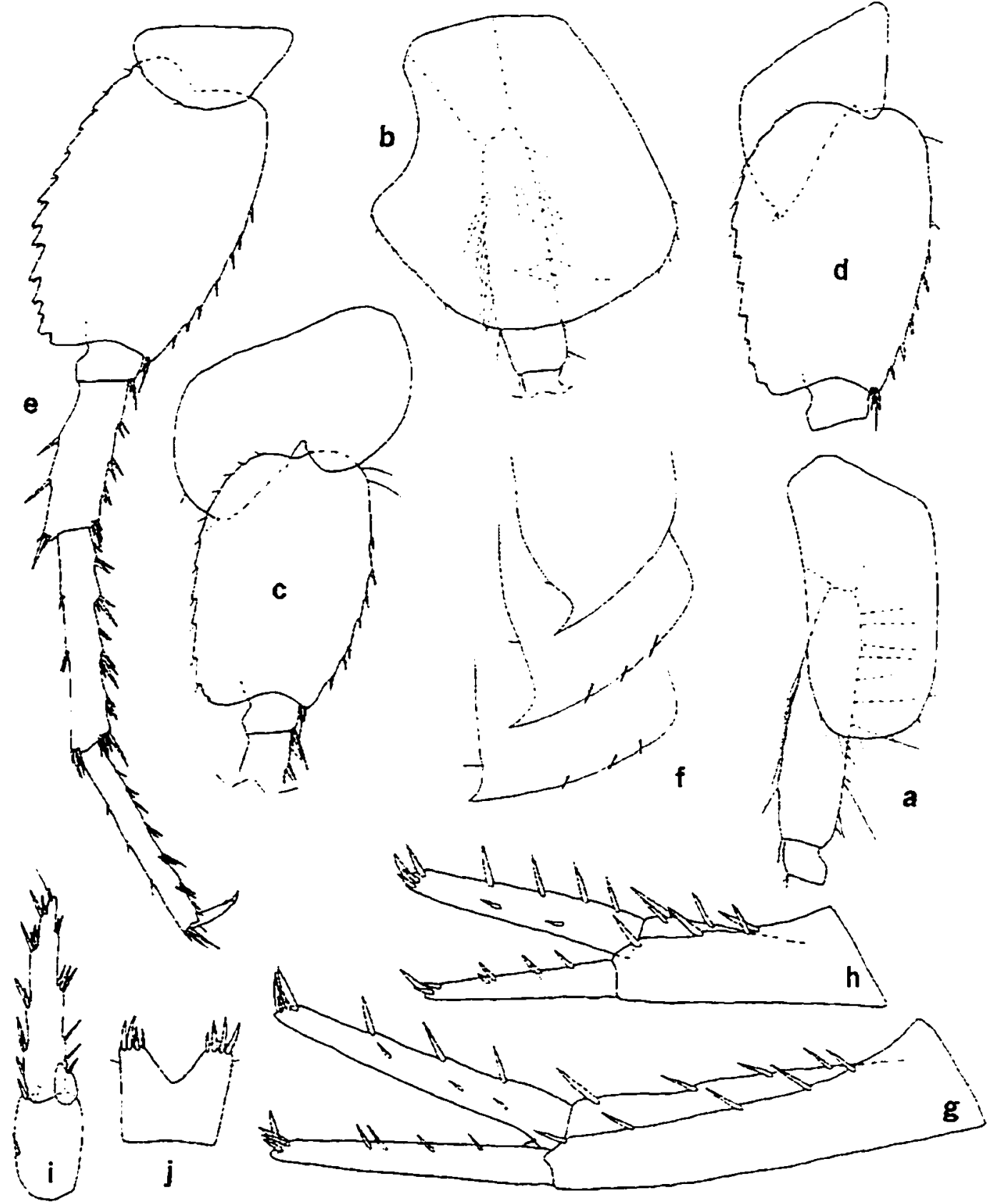

Figure 134.--Crangonyx floridanus Bousfield. Female $(8.2 \mathrm{~mm})$. Stream between Elloree and St.

Matthews, Orangeburg Co., South Carolina: a. b. c. d. pereopods 3.4. 5. 6 (in part): e. pereopod 7: f. pleonal plates: g. h. i. uropods 1. 2. 3: j. telson. 


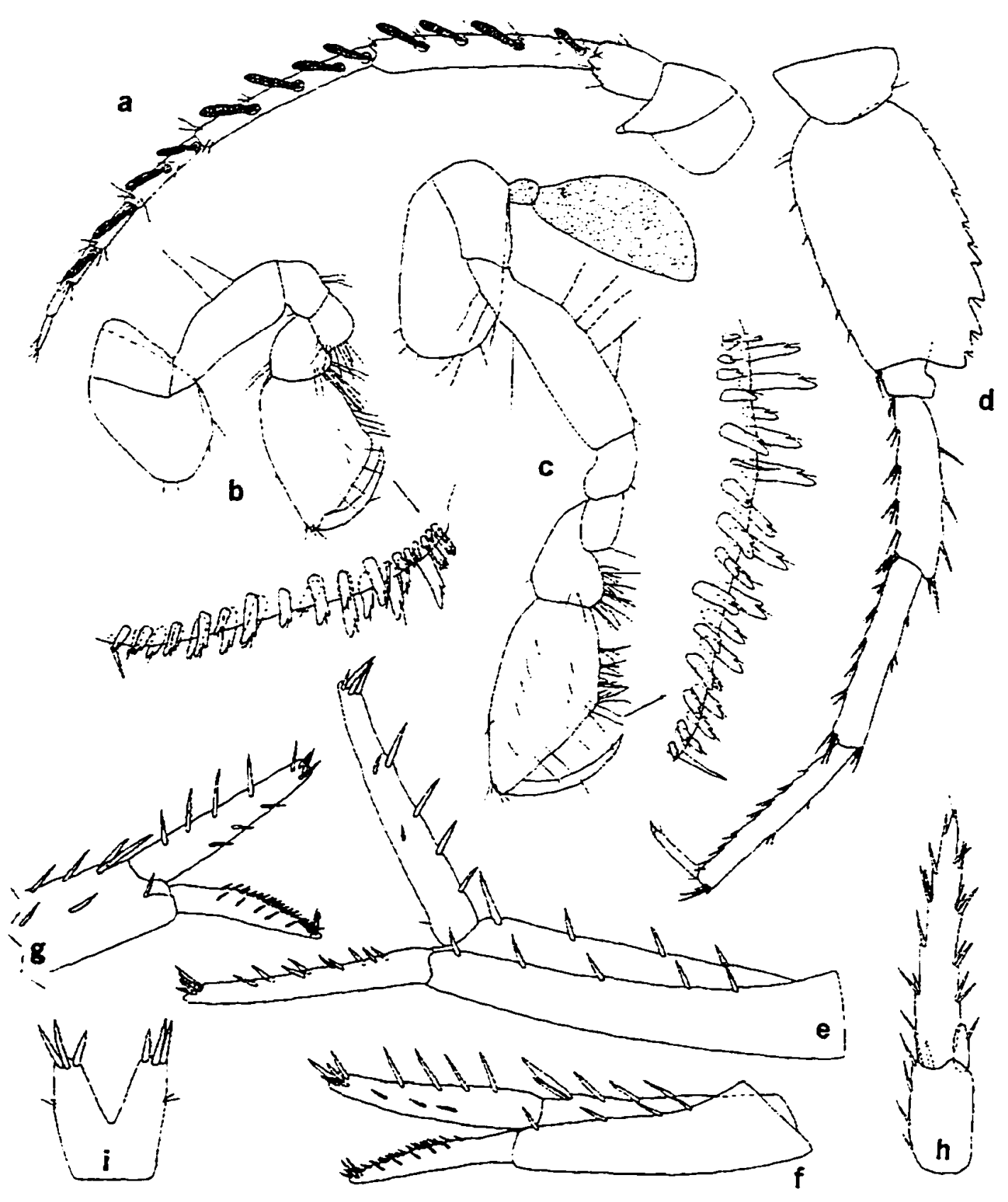

Figure 135.--Crangonyx floridanus Bousfield. Male $(6.0 \mathrm{~mm})$. Stream between Elloree and St. Matthews, Orangeburg Co.. South Carolina:a. antenna 2: b. c. gnathopods 1. 2 (palmar margins enlarged): d. pereopod 7: e. f. h. uropods 1. 2. 3: g. uropod 2 from other side (in part): i. telson. 
outlet N side Milton Reservoir. $9 \Xi .7$ \%. R. W. Pennak. 9 May 1953 (USNM). FLORIDA. Alachua Co.: cypress Swamp at Gainesville. 5 ₹. R. E. Woodruff. 6 Apr 1959 (USNM): Hatchet Creek. $6 \cong .1 \approx$ G. and L. Van Hyming. 8 Feb 1938: Gainesville (habitat not given), I Q. A. G. Mutchber. 29 Sep 1914 (USNM) and 2 ๑. 1 ๔. J. Martin, I May 1937 (USNM); Newman's Lake near Gainesville. $11 \cong .1 \approx$. F. N. Young. 8 Jan 1930 (USNM): N of Highland Springs. 6 \&. 6 c, H. H. Hobbs. Jr., 12 May 1937 (USNM): pond C. 3 ミ. 1 ž. J. Martin. 5 Mar 1934 and 4 9. 4 Apr 1933 (USNM): Santa Fe Lake. 1 \&. J. Martin. 2 Apr 1935 (USNM) and $5 £ .8$ Apr 1937 (USNM): sink. 28 q. 2 c. J. Martin. 14 Mar 1937 (USNM): sivamp 7.2 km E of Gainesville. 100 specs (․ Heights, 70 specs ( $\cong$. $)$. L. Hubricht, 18 Apr 1941 (USNM): Wauberg Lake Station. $7 \cong .7 \Xi$. R. P. Trogdon (collection date not given) (USNM) and 2 \%. J. Martin, 23 Feb 1933 (USNM): II .. J. Martin. 24 Mar 1927 (USNM): Baker Co.: Bayhead Pond, 1 . J. Martin. 12 May 1937 (USNM): stwamp stream. $5 \equiv$. J. Martin. 12 May 1937 (USNM): Brevard Co.: St. Johns R. at Lake Winder. 2 ․ 1 \&.. R. Fox. 9 Feb 1970: St. Johns R.. SW of Lake Hel'n'blazes. 4 . R. Fox. 9 Feb 1970: St. Johns R. at canal on E shore of Lake Poinsett. $1 \fallingdotseq$. R. Fox. 9 Feb 1970: St. Johns R 12 way between Lake Winder and Poinsett. $3 \geqq 1 \div$. Fox. 9 Feb. 1970: Clay Co.: Kingsley Lake. 2 ‥ H. H. Hobbs. Jr.. 30 Apr 1935 (USNM): Columbia Co.: cypress swamp. 5 શ. 1 ×. H. H. Hobbs. Jr., 12 May 1937 (USNM): Mill Pond Spring. Ichatuckenn Springs State Park. 1 s. A. Woodnuff (collection date not given). (Woodruff Collection): Mission Spring. Ichatucknee Springs State Park. 5 §. A. Woodruff (collection date not given). (Woosruff collection): NW of Lake City. $2 \mp$. H. H. Hobbs. Jr.. 4 Feb. 1938 (USNM): Dade Co.: Little Bird Nursery well. Miami. I :. G. Miller. 29 Oct 1968 (USNM) and 2 specs, 29 Nov 1968: Franklin Co.: Crayfish burrow on St. Rt. 65. $4.8 \mathrm{~km}$ E of East Bay. 2 . R. E. Ashton. Jr.. $10 \mathrm{Jul} 1978$ (NCMNH): Hamilton Co.: $6.7 \mathrm{~km}$ N of White Springs. $1 \cong .3 \approx$. H. H. Hobbs. Jr.. 4 Feb 1938 (USNM): $8.0 \mathrm{~km}$ N of White Springs. $29.2:$. H. H. Hobbs. Jr.. 4 Feb 1938 (USNM): Highlands Co.: shallows at edge of cypress swamp. under organic debris.

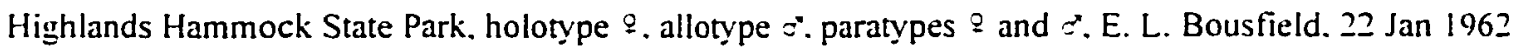
(CMN); Jackson Co.: China Cave, 2 9. P. Moler and B. W. Mansell, 4 Feb 1986: Cottondale (habitat not given). 5 ․ 2 ๔. J. Martin. 17 Apr 1937 (USNM): Gerards Cave. paratypes $\supseteq$ and $\approx$. A. E. Smalley. 20 Oct 1960 (NMC). 1 ?. J. E. and M. L. Cooper, 16 Apr 1965. 3 ๆ. 1 §. H. S. Harris. Jr.. 20 Apr 1967. 39. $2 \approx$. I juv. D. S. Lee. 28 Jul 1968 and 2 fragments. S. B. Peck. 5-9 Sep 1968: Haideotriton Cave. $2 \leftleftarrows .3:$ I juv. D. Franz, 24 Jun 1970: Geromes Cave, 59,1 juv. (collector not given). 15 Oct 1969 and $3 \risingdotseq .1 \%$ D. B. Means et al.. 31 Jul 1969 (USNM): Judge Cave. 7 Z. I juv. S. B. Peck. 9 Apr 1969: Millers Cave. 4 Ð.S. B. Peck, 6 Apr 1969: Tupelo Swamp. $11.1 \mathrm{~km}$ SE of Marianna. 100 specs ( 9.0 . juvs). L. Hubricht. 15 Apr 1941 (USNM): Lake Co.: small stream near Umatilla. 3 ₹. 2 c. J. Martin. 19 Mar 1938 (USNM): Leon Co.: small stream in Silver Lake Recreational Area. Apalachicola National Forest. 1 . R. E. Woodruff. I Feb 1960 (USNM); swamp, $11.7 \mathrm{~km} \mathrm{E}$ of Tallahassee, 30 specs (\%. c"). L. Hubricht. 16 Apr 1941 (USNM): Levy Co.: Waccasassa R. at St. Rt. 24. 2 9. 2 c. R. Fox. 9 Feb 1970: Manatee Springs State Park, 3 ₹. A. Woodruff (collection date not given). (Woodruff Collection): Madison C0.: $12.8 \mathrm{~km} \mathrm{E}$ 
of Lamont. 3 ₹. H. H. Hobbs. Jr.. 5 Feb 1938 (USNM); Okaloosa Co.: Crestview (habitat not given). $4 \equiv$. 1 f. H. H. Hobbs, Jr.. 11 Dec 1937 (USNM); Seminole Co.: In cray fish burrows. $3.5 \mathrm{~km}$ NE of Sanford. 20 juvs, L. Hubricht. 19 Apr 1941 (USNM); Suwannee Co.: Azure Cave. 1 juv. J. Yager. 2 Jan 1987: Wakulla Co.: stream. $32 \mathrm{~km}$ S of Tallahassee. 2 9. 5 juvs. J. G. Mackin. 6 Jul 1937 (USNM). 36 collections. mostly by J. Martin. 1937-38 from north-central Florida (probably from Alachua County).

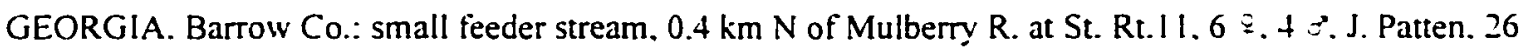
Feb 1978: Catoosa Co.: Catoosa Springs. $8.0 \mathrm{~km}$ E of Ringgold. $29.3 \approx .3$ juvs. J. R. Holsinger. 10 Jun 1967: Chatham Co.: swamp. $4.8 \mathrm{~km}$ NE of Port Wentworth. 36 specs (₹. :"). L. Hubricht. 21 Apr 1941 (USNM): Clarke Co.: Sandy Creek $3.2 \mathrm{~km} \mathrm{~N}$ of Athens. 7 ?. 6 ๙. G. W. Dickson. 19 Feb 1977: Sandy Creek $6.3 \mathrm{~km} \mathrm{~N}$ of Athens. 6 ๑. G. W. Dickson. 16 Feb. 1978: Cobb Co.: Owens Spring near Austell. 11 ¡. 5 : L. Hubricht. 3 Aug 1939 (USNM): Decatur Co.: locality not given. J. Martin. collection date not given (USNM): De Kalb Co.: spring on Walter Candler Estate, Emory University, I E. W. D. Bwrbanck. 19 Apr 1960 (USNM): Lincoln Co.: small spring. base of Graves Mtn. $8.0 \mathrm{~km} \mathrm{SW}$ of Lincolnton. $7 \cong .1 \cong$. L. Hubricht. 15 May 1960: Meriwether Co.: small spring of Warm Springs, $15 \unrhd .7 \sigma^{*}$. J. H. Chandler. Jr.. 28 Jan 1980 (USNM): Polk Co.: spring 3.2 km E of Aragon. 4 ․ 1 ₹. L. Hubricht. 8 May 1951 (USNM): spring below mouth of Deatons Cave. $4.0 \mathrm{~km} \mathrm{SW}$ of Taylorville. L. Hubricht. 8 May 1951 (USNM): Wayne Co.: swamp. $1.8 \mathrm{~km} \mathrm{~N}$ of Jesup. $129.5 \approx$ L. Hubricht. $20 \mathrm{Apr} 1941$ (USNM). KANSAS. Pottanatomie Co.: Pressure Springs. base of Tuttle Creek. Reservoir dam. 7 こ. $16 \approx$. 1 juv. J. Danoff-Burg et al.. 23 Jun 1991 : seepage below Tuttle Creek Reservoir Dam. 11 ?. $1:$ M. Moffett. 27 May 1981. KENTUCKY. Christian Co.: Reeves Cave (=Thomas Cave). $1 \cong$. R. La Val. + Jul 1964 and $2 \varsubsetneqq .4 \cong$. J. R. Holsinger and T. C. Barr. Jr., 12 Aug 1965. MARYLAND. Anne Arundel Co.: Patapsco River at Hammonds Ferry Road. 3 Z. I $s$. L. Hubricht. 28 Mar 1959 (USNM): Frederick Co.: Hunting Creek Lake at Cummingham Falls Park on Rt $77 \mathrm{~W}$ of Thurmont. $2 \Xi .5 \%$. C. K. Biernbaum. 17 Jui 1992: Montgomery Co.: Chilton Woods Spring, $1.7 \mathrm{~km} \mathrm{NW}$ of intersection of Whites Ferry Rd and Martimsburg Rd.. 1 . $1 \div$. D. Feller. 6 Mar 1995: Maryland Line Spring. S side of Beach Dr. at Maryland/DC line. 8 specs. D. Feller, 10 Mar 1995. MASSACHUSETTS. Hampton Co.: Holyoke Power Canals. Connecticut River. 32 9. 3 ๑. D. G. Smith. 5 Jul 1984. MISSISSIPPI. Clarke Co.: pool near Long Creek. W of Middleton. $21 \mp .11 \leqslant$. L. Hubricht, 3 Feb 1962 (USNM): Forrest Co.: small stream in swamp. nr. Black Creek. $3.2 \mathrm{~km} \mathrm{SW}$ of Brooklyn. $3 £ .1$ \%. L. Hubricht. 2 Mar 1963: Lafayette Co.: spring near Oxford. G. Gaston. 25 specs ( $\left.9 . c^{\prime \prime}\right) .11$ May 1991. Lauderdale Co.: pools in swamp. $1.6 \mathrm{~km} \mathrm{NW}$ of Kewanee. 30 specs. L. Hubricht. 4 Feb 1962 (USNM); roadside pool, US 80 at 31 st Ave. 100 specs. L. Hubricht, 8 Mar 1964 (USNM): swamp pool. Bonita. 100 specimens. L. Hubricht, 9 Mar 1963 (USNM): Rankin Co.: slough $1.2 \mathrm{~km}$ W of pelatchee. I $₫$. I juv. L. Hubricht, 9 Apr 194I: Smith Co.: seep near realleaf $4.8 \mathrm{~km} \mathrm{~W}$ of Sylvarana: Winston Co.: pools $4.0 \mathrm{~km}$ S of Noxipater. 2 9. 17 Mar 1963 (USNM): Wayne Co.: roadside ditch $2.4 \mathrm{~km}$ SW of Waynesboro. $48 \operatorname{specs}(9.0)$. L. Hubricht. 16 Feb 1963 (USNM). MISSOURI. Butler Co.: slough. $9.8 \mathrm{~km} \mathrm{~N}$ of Neelyville. 100 specs (?. $\left.\beth^{*}\right)$. L. Hubricht. 5 Apr 194 I 
(USNM): Camden Co.: Camp Resing Sun Cave. $8.8 \mathrm{~km}$ NE of Lenn: Madison Co.: spring. $0.8 \mathrm{~km} \mathrm{~S}$ of Mill Creek. 1 juv. L. Hubricht. 5 Apr 1941 (USNM): Scott Co.: small pool on road, $0.8 \mathrm{~km} \mathrm{~N}$ of Forufelt. 2 g. 1 C.W. W. L. Pfleiger and G. McDonald. 27 Feb 1976: St. Louis Co.: slough near Mississippi River. 1.6 $\mathrm{km} \mathrm{S}$ of Grimsby, 170 specs ( $\% . \sigma^{\circ}$ (USNM) and I $2.1 \sigma^{\circ}$ (NMC), L. Hubricht. 25 Apr 1938: temporary pond. $3.2 \mathrm{~km} \mathrm{~N}$ of Fenton. ca. 130 specs.. L. Hubricht, 12 Jan 1936; temporary pond. near River Kirkwood. $1 \subseteq .3 \approx$ L. Hubricht. 10 Apr 1938: Taney Co.: Lake Taneycomo at Rockaway Beach. 25 specs (ミ. . juvs). W. Pfleiger. 22 Oct 1970. NORTH CAROLINA. Carteret Co.: pond ca. $16 \mathrm{~km} \mathrm{~W}$ of Moorhead City. $5 \Xi .1 \geqslant$ N. Hynes. 26 Apr 1980 (USNM): cypress pond. $11 \mathrm{~km}$ from Moorhead City. 6 ¿. 1 N. Hynes. 21 Apr 1982 (USNM); Chatham Co.: small stream at US $15.0 .8 \mathrm{~km} \mathrm{~N}$ of Bynum. 50 specs. 6 Mar 1972: rill and pool near Rocky River. $11.7 \mathrm{~km} \mathrm{~S}$ of Pittsboro. $33 \approx .25 \mathrm{\&}$. L. Hubricht. 8 Apr 1950 (USNM): Granville Co.: shallow well at end of ST Rt 1947. 1 \& juvs. W. F. Adames. 5 Sep 1991: shallow well on farm at end of Rt 1778, 19.2 juvs. W. F. Adams. 18 Jun 1991: Macon Co.: Club Lake. on Rt 106. just outside Highlands. 20 ९. $60^{\circ}$. C. K. Biernbaun. 14 Jul 199I: Moore Co.: old lake bottom between Southern Pines and Carthage. 20 (collector not given). 4 Feb 1940 (USNM): swamp. NE of Southern Pines. 3 \. $2 \approx$ (collector not given). 19 Mar 1940 (USNM): small spring in pine woods. Southern Pines. 6 ○. 1 T. K. Ellis. 7 Mar 1940 (USNM): swamp on Little River. $4.8 \mathrm{~km} \mathrm{~N}$ of Southern

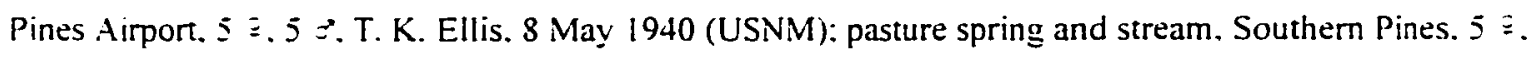
2 juvs (collector not given). 8 Mar 1940 (USNM): Little Crane Creek. Cameron, Rt I. 2 ₹. J. F. Hanson. 13 Dec 1956 (USNM): Orange Co.: marsh, Chapel Hill. $2 \approx 0$ J, Allwein. 19 Jan 1964: Pamlico Cu.: creek. $2.1 \mathrm{~km} \mathrm{~W}$ of Grantsboro. $16 \mp$. L. Hubricht. 23 May 1959 (USNM). Wake Co.: ditch beside St Rt 55. between St Rt 54 and Us 64. 30 ๆ. J. Green. 28 Nov 1972: Little River (Neuse) at Rd 2224. $7.7 \mathrm{~km} \mathrm{E} \mathrm{of}$ Rolesville. 4 . A. L. Braswell. I Dec 1977: intermittent stream near Shearon Harris Lake. 25 specs. J.E. Cooper and A. L. Braswell. 19 Apr 1995: small pond. Reedy Creek Rd. 10 ₹. $10 \approx$. W. M. Brooks. 8 Feb

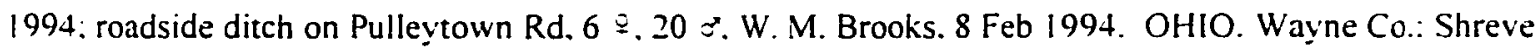
Creek. $2 \Xi .4 \approx$. A. Weaver. 10 Feb 1964. OREGON. Clackamas Co.: small pond on Oswego Lake Co. Club. 3 z. 4 . J. Bond. 2 Mar 1958 (CMN) and $6 x^{2} .+$ juvs. 8 Jan 1961 (CMN): boggy inlet of Lake Oswego. 3 q. J. Bond, 18 Feb 1958 (CMN): Lane Co.: Corner Lake, $3 \fallingdotseq .12$ juvs. F. Ziesenheim, summer.

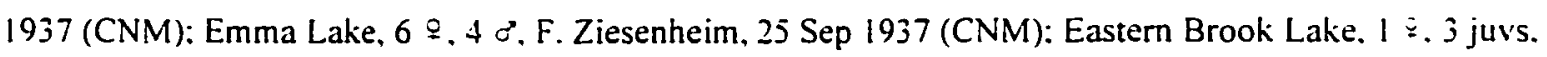
F. Ziesenheim. 27 Sep 1937 (CNM).PENNSYLVANIA. Chester Co.: spring. $1.6 \mathrm{~km}$ E of West Grove. 50 specs $\left(\% . \mho^{*}\right)$. L. Hubricht, 17 Aug 1939 (USNM): Dauphin Co.: intake structure at Three Mile Island. Susquehanna River. 1 ミ. J. L. Evans. 6 Jul 1982: spring-fed pond. Middletown Quad. 4 ?. $4 \approx$. B. Barton. 23 Feb 1995: pond SE of farm off Sandhill Rd.. Middletown Quad. I \&. B. Barton. 16 Feb 1995: Lehigh Co.. Halfrich Spring Cave. $5 \stackrel{2}{2} 1 \approx$. D. A. Hubbard. Jr.. 28 Jun 1991. SOUTH CAROLINA. Bamberg Co.: Brier Branch at US 78. 4 9. P. H. Carlson. 6 Nov 1983: Barnwell Co.: Meyers Branch hyporheic. Savannah River site. 4 juvs. S. Stibbe. 7 Aug 1993: Beaufort Co.: swamp near Frogmore. Saint Helena Island. $11 £ .1 \Im^{*}$ (collector not given), 12 Apr 1940 (USNM): Berkeley Co.: slough $4.8 \mathrm{~km} \mathrm{NW}$ of 
Cordesville. 18 specs ( $\left.₹ . \sigma^{\circ}\right)$, L. Hubricht. 21 Apr 1941 (USNM): small permanent swamp. Cordesville. 6 Đ. T. K. Ellis. 25 Mar 1940 (USNM): Richmond Plantation. Gough. numerous specimens (USNM) from Alligator Pond. Cat Swamp, dammed-up area. Double Swamp. Upper and Lower reserves. Stable Pond. "pond." T. K. Ellis (the majority) and L. Hubricht. 1939-1941: swamp $9.6 \mathrm{~km} \mathrm{~N}$ of Pinopolis. 3 Z. 1 : (collector not given). 4 Apr 1940 (USNM); small temporary pond, Gough. 100 specs ( $\cong$. juvs). (collector not given), $25 \mathrm{Mar} 1940$ (USNM); near Gough (habitat not given), $23 £ .2 \approx$. T. K. Ellis. 1 Jan 1940 (USNM) and 9 \&. $5 \approx^{*}, 28$ Dec 1939 (USNM): Charleston Co.: Caw Caw Swamp (new road). I ₹.T. K. Ellis. 3 Apr 1941 (USNM); Caw Caw Swamp (western end of Charleston Co.). 30 specs ( 2.20 ). T. K. Ellis. 3 Apr 1941 (USNM): swamp on Prospect Hill Road. Edisto Island. $9 \Xi .7 \Xi *$ T. K. Ellis. 3 Apr 1941 (USNM): Dorchester Co.: Edisto River. $20.8 \mathrm{~km}$ W of Summerville. I juv. J. R. Lung. Jr.. 31 Mar 1935 (USNM): swamp. N of Bownan. $9 \Xi .32 .2$ juvs. T. K. Ellis. 28 Mar 1943 (USNM): Greenville Co.: Horse Creek. $7 \cong .1$ : R. M. Shealey. 30 Dec 1966: Jasper Co.: Marsh. $6.7 \mathrm{~km}$ SW of Limehouse. $13 \equiv$. L. Hubricht. 21 Apr 194 I (USNM): slough. $3.2 \mathrm{~km}$ SW of Ridgeland. $5 \cong .1$ I L. Hubricht. 21 Apr 19+1 (USNM): Orangeburg Co.: ditch $7.8 \mathrm{~km} \mathrm{SE}$ of Bowman. 30 specs ( $\&$. $\approx$ ). L. Hubricht. 22 Apr 1941 (USNM): Edisto River Swamp. just W of Branchvillee. $39.2 \approx *$ T. K. Ellis. 11 Feb 1942 (USNM): Edisto River at Hawthorne Field. 4 \&. I $\approx$. T. K. Ellis. 26 Feb 1942 (USNM): Near Betheehem Church (habitat not given). Rt 36 W of Rowesville. 1 . 15 ङ. T. K. Ellis. 12 Feb 1942 (USNM): Santee National Wildlife Refuge. 8 Р. C. K. Biernbaum. 15 Jan $198 j$ (USNM): stream between Elloree and St. Marthews. $14: .5 z^{\circ}$ (collector not given). 4 Apr 1940 (USNM): Richland Co.: pond in Congaree River Swamp. $8 \cong .1=$ (collector not given). 4 Apr 1940 (USNM); small swamp $4.8 \mathrm{~km} \mathrm{~N}$ of Wateree. $18 \cong .6 \geq$ (collector not given). 4 Apr 1940 (USNM): county not given: Harts Bluff (below) Edisto River. 1 ×゙. 6 Mar 1939 (collector not given) (USNM). TENNESSEE. De Kalb Co.: spring at Cripps Mill. 1 . $4 \approx$. J. R. Holsinger. 20 March 1964: Henry Co.: unmarked stream. ca $17 \mathrm{~km} \mathrm{~N}$ of Henry Co. Court House on US Rt 641. 26 specs. R. Fox et al.. 30 Dec 1970: Loudon Co.: spring $11.2 \mathrm{~km} \mathrm{SE}$ of Loudon. 100 specs (ミ. $\Xi$ ). L. Hubricht. 22 May 1963 (USNM): Monroe Co.: spring at Sweetwater. 40 specs (§. ঐ). L. Hubricht. 7 Aug 1939 (USNM): Stewart Co.: Pryor Creek Spring just S of Rd 364.2 2.6 $\approx .1$ juv.. D. L. Gillis. 25 Nov 1988. VIRGINIA. Fairfax Co.: Black pond, 1 juv. C. R. Shoemaker. 19 Sep 1915 (USiNM): Montgomery Co.: ditch beside the Duck Pond on campus of Virginia Tech. Blacksburg. $10 \Xi .5 \approx$.C. K. Biernbaun. 13 Jul 1989: Pittsyivania Co.: swamp on US $56 \mathrm{~N}$ of Danville. 50 specs. L. Hubricht. 16 Jan 1949 (USNM) and 50 specs. 5 Mar 1950 (USNM): seeps N side of White Oak Mtn. $3.2 \mathrm{~km} \mathrm{~N}$ of Spring Garden. 30 specs. L. Hubricht. 5 Feb 1949 (USNM): Rockbridge Co.: pool in old "boat lock" at Ben Salem Wayside on Rt 60. just outside Buena Vista. 30 specs, C. K. Biernbaun. 8 Jul 1989. WEST VIRGINIA. Mason Co.: Flatfoot Creek. 4 2. 13 o. D. Tarter, 28-29 Apr 1977. WASHINGTON. D. C.: seep. south end of National Zoological Park. 14 9. 1 juv. J. R. Holsinger et al., 7 Apr 1978; small spring, south end of National Zoological Park. $23 \subsetneq .4$ c*. 8 juvs. G. Karaman et al.. 7 Apr 1978.

Diagnosis.--A species of the gracilis group closely similar morphologically to C. pseudogracilis but 
distinguished from it and other members of the group by having comb spines on outer ramus of male uropod 2 but lacking ventral spines there: distoposterior corners of pleonal plates $1-3$ well developed: distoposterior margins of pereopods 5-7 with deep serrations. Largest male. $8.0 \mathrm{~mm}$ : largest female. 12.0 $\mathrm{mm}$.

Female.--Eye large and ovate. Antenna 1 , about 55 percent length of body. about $1.5-1.8$ times longer than antenna 2: primary flagellum with up to 19 segments. Antenna 2. flagellum with 7-8 segments. Mandibles subequal. spine row with 5 spines: segment 2 of palp with 6-9 long setae. segment 3 with $1 \mathrm{~A}$ seta. 2-5 C setae. 4-5 E setae, and a row of $9 \mathrm{D}$ setae. lacking B seta. Maxilla 1 : inner plate with 5-6 apical plumose setae: palp with 5-6 slender spines on apex. Maxilla 2. inner plate with oblique row of 6 plumose setae on inner margin. Maxilliped: inner plate apically with 3 bladelike spines. 1-2 naked setae. and 6 plumose setae extending from inner margin to apex: outer plate with about 5 slender pectinate spines and row of naked setae on inner margin. I apical plumose setae: dactyl with 2 setae (rarely + setae) on inner margın: dactyl nail very long. about $1 / 3$ length of dactyl.

Propod of gnathopod I slightly longer than carpus: palm shorter than posterior margin. slightly convex. with about 6 unnotched spine teeth on inside, about 10 on the outside: defining angle with 4 notched-serrate spine teeth on inside. 1 large notched and 2 serrate spine teeth on outside: about 4 superior medial setae. singly inserted: posterior margin with 5 sets of setae in groups of $1-4$. Dactyl with $3-4$ short setae on inner margin: nail very long. Ventral margin of coxa 1 with 8-9 setae.

Carpus of gnathopod 2 very long, $2 / 3$ the length or subequal in length to propod. with up to 5 sets of setae on posterior margin. Propod of gnathopod 2 palm much shorter than posterior margin. slightly convex. oblique. with about 6 unnotched spine teeth on inside. about 10 on outside: defining angle with 1 large notched spine tooth and 3 notched-serrate spine teeth on inside. 1 large notched and 1 small serrate spine teeth on outside: 5-7 superior medial setae. singly inserted: posterior margin with 4 sets of setae in groups of 1-4. Dactyl with 4-5 short setae on inner margin. Ventral margin of coxa 2 with 8 setae.

Coxa of pereopod 3 with 7 marginal setae. Coxa of pereopod 4 with 16 marginal setae. dactyl of pereopod 4. 45 percent length of corresponding propod. Pereopod 5: basis with 9 serrations along posterior margin: 7-8 short spines on anterior margin: 2 setae on the anteroproximal margin. Pereopod 6: basis with 13 serrations along posterior margin: 6 short spines on anterior margin: 2 setae on anteroproximal margin. Pereopod 7: coxa with 2-3 setae on the posterior margin: basis with 13 slightly deep serrations along posterior margin: 4 short spines and 2 short setae on anterior margin: dactyl about 33 percent the length of corresponding propod.

Pleonal plates: posterior margin of plate 1 slightly convex, with I seta. distoposterior corner strongly produced and acute: posterior margin of plate 2 nearly straight with 1 seta. distoposterior corners strongly produced and acute, ventral margin with 2-3 (subventral) spines: posterior margin of plate 3 nearly straight with 1 seta. distoposterior corner strongly produced and acute. ventral margin with 2-3 (subventral) spines. Peduncle of pleopod 1 with 2 retinaculae. lacking setae on outside margin. 
Uropod I: inner ramus 70 percent length of peduncle, armed with 12 spines: outer ramus with 12 spines: peduncle with 5-8 outer and 4 inner spines. Uropod 2: inner and outer ramus each armed with 12 spines: peduncle with 4 outer and 5 inner spines. Uropod 3 : inner ramus with I spine, outer ramus about 1.8-2.0 times longer than peduncle. inner and outer margin each with $j-4$ set of spines in groups $1-2$. Telson about as broad as long. notched about 1/4-1/2 distance to base. each lobe with 2-3 apical spines: spines less than $1 / 2$ length of telson.

Male.--Differing from female as follows. smaller with more slender. elongate body. Antenna 2: calceoli percent on peduncular segments 4 and 5 , and first 2 segments of flagellum.

Propod of gnathopod I more than 2 times larger and longer than carpus: palm slightly shorter than posterior margin. slightly oblique. almost straight. 10 spine teeth on inside. 10 on outside: defining angle with 4-5 notched-serrate and 1-2 serrate spine teeth on inside. I small and 1 large notched spines and 2 serrate spine teeth on outside. Propod of gnathopod 2 more than 2 times larger and longer than carpus: palm margin strongly oblique. convex. slightly shorter than posterior margin. with 13 spine teeth on inside. 13 on outside: detining angle with 4 notched and 2 serrate spine teeth on inside: $2-3$ large notched and 1 small serrate spine teeth on outside.

Uropod 2: inner ramus with $\|$ spines: outer ramus deflected laterally with inner row of comb spines on distal half to two-thirds. outer margin with 5 spines. apex with $j$ spines: peduncle with $j$ outer and $3-4$ inner spines.

Variation.--The row of comb spines on the outer ramus of uropod 2 of mature males extends from the apex and covers $1: 3$ to $2 / 3$ of the length of the ramus. The distoposterior comers of pleonal plates $1-2$ var from just slightly produced and acute in inland populations to strongly produced and acute in coastal plain populations.

Distribution and ecology.--This species is widely distributed in the eastern and east-central United States. occurring as far west as central Kansas (fig. 136).

c. floridanus inhabits swamps. ponds, streams, and occasionally cave pools. Specimens from caves in Florida have comparatively smaller (degenerate) eyes. Sexually mature males. 3.0 to $8.0 \mathrm{~mm}$ : sexually mature females $5.0-10.0 \mathrm{~mm}$. Ovigerous females have been collected during the months of February through October. thus indicating that breeding possibly occurs the year around (Holsinger. 1972).

Remarks.--The specimens examined from Colorado and Oregon are morphologically similar to the eastem populations. but their natural occurrence there is questionable and it is likely that they are introduced populations. Specimens of this species were also found in Japan $(2 \approx * 2 \cong$. pond. Furotone. Chiba Prefecture. Japan. 30 Jan 1992 . H. Kusano) and sent to Dr. Holsinger by Dr. Hiroshi Morino. Ibaraki University, Japan. They were examined by me in this study. The Japanese population is undoubtedly an introduction (See discussions in Chapter V). 
Crangonyx pseudogracilis Boustield

Figures $|37-14|$

Crangonnx pseudogracilis Bousfield. 1958:102-105. fig. 17 [type-locality: Napanee River. at Napanee. Lennox \& Addington Co., Ontario, Canada].-Holsinger. 1972:46, figs. $1 \mathrm{lh} .20$ [in part].--Bousfield. 1973:68 [in part].--Holmes. 1975:225.--Barton \& Hynes. 1976:208.--Thomas, 1976:90.--Holsinger. 1977:252.--Pinkster. Dieleman \& Platvoet. 1980:36.--Pinkster \& Platvoet. 198j:157.--Barnard \& Barnard. 1983:435. map 12 [in part].--Fitzpatrick, 1983:145 [in part].--Platvoet \& Pinkster. 1985:126.--Hautus \& Pinkster. 1987:58.--Platvoet. Scheepmaker \& Pinkster. 1989:198.--Pennak. 1989:482.--Pinkster. Scheepmaker. Platvoet \& Broodbakker. 1992:197.--Gledhill. Scutcliffe \& Williams. 1993:102.--Gledhill. Scutcliffe \& Williams. 1993:102.--Jass \& Klausmeier. 1995:8. [Not Boustield. 1961:5.--Mills. 1964:4.--Bell. 1971:35.--Smith. 1977:256: 1981:11: 1982:316: 1988:46.-Van Maren. 1978:48].

Crungonyx gracilis Smith.--Hynes. 1955a:378: 1955b:624.

Eucrangonyx gracilis (Smith).--Hynes. 1951:152].

Material examined.--ONTARIO. French River. 19.3 juvs. N. Hynes. 29 Jul 1975 (USNM): Napanee River at Napanee. Lennox \& Addington Co.. 3 \& paratypes (NMC 2107). 9 Jun 1950: Rideau River. below Hog's Back. Ottawa. 1 ₹. E. L. Bousfield. 12 May 1956 (NMC): QUEBEC. Ottawa River (Hull Park). 2 . F. Johansen. 6 Jul 1919 (US.NM): Lake St. Louis. Perrot. $2 \cong .1$. J. S. Bleakeny. 28 Aug 1955 (USNM) and lle Perrot. quarty pond. $1 \mp .1$ ב.28 Aug 1955 (NMC). ALABAMA. Greene Co.: roadside ditch. 1.3

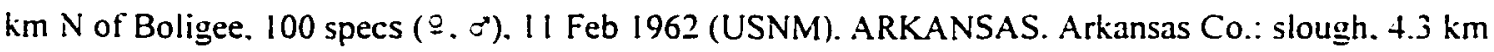
ENE of Humphreys, 7 2. 6 \%. L. Hubricht. 7 Apr 1941 (USNM): Ashley Co.: Chemin-a Haut creek just $N$ of Hamburg. $5 \supseteq$. J. E. and M. R. Cooper. $16 \mathrm{Mar}$ 1967: roadside ditch. $8.0 \mathrm{~km} \mathrm{SW}$ of Hamburg on US 82. $1 戸 .1$ J. E. and M. R. Cooper. 16 Mar 1967: ditch on Ark Hwy 133. $1.3 \mathrm{~km} \mathrm{~S}$ of Crossett Experimental Forest. 2 . J. E. and M. R. Cooper. 16 Mar 1967: Calhoun Co.: small stream. $5.9 \mathrm{~km} \mathrm{~S}$ of Thornton. 14 $6 \approx$. L. Hubricht. 8 Apr 1941 (USNM); Conway Co.: temporary pool. $3.7 \mathrm{~km}$ SE of Menifee. 3 May 1940 (USNM): Cross Co.: temporary pool. $3.7 \mathrm{~km} \mathrm{~N}$ of Cherry Valley. 29.10 ङ. L. Hubricht. 6 Apr 1941 (USNM): Dallas Co.: slough. $3.2 \mathrm{~km} \mathrm{NE}$ of Fordyce. 1 ₹. L. Hubricht. 7 Apr 1941 (USNM): Faulkner Co.: temporary stream. $1.0 \mathrm{~km} \mathrm{~S}$ of Wooster, 30 specs $\left(\dot{F}, \Xi^{\prime}\right)$, L. Hubricht, 3 May 1940 (USNM): Garland Co.: Bear Spring at Bear, 15 q. H. W. Robison. 15 Sep 1979: Grant Co.: unnamed stream on dirt Road. $20 \mp .3$ $\therefore$ R. Fox. 27 Dec 1970: Jefferson Co.: slough, $6.9 \mathrm{~km} \mathrm{NE}$ of Altheimer. $20 ₹ .5 \approx$. L. Hubricht. $7 \mathrm{Apr}$ 1941 (USNM): Arkansas River. backwater at Tarr Camp. ca. $11.2 \mathrm{~km}$ E of Redfield. $2 \varsubsetneqq .2 \approx$. R. Fox. 21 Dec 1970: stream. $1.6 \mathrm{~km}$ W of Jefferson on Jefferson-Sheridan Rd. $10 \cong .5:$ R. Fox. 21 Dec 1970: small creek. $0.8 \mathrm{~km}$ S of Locust Cottage. 40 specs $\left(\$, \sigma^{\circ}\right)$. L. Hubricht. 11 Apr 1936 (USNM): Johnson Co.: 


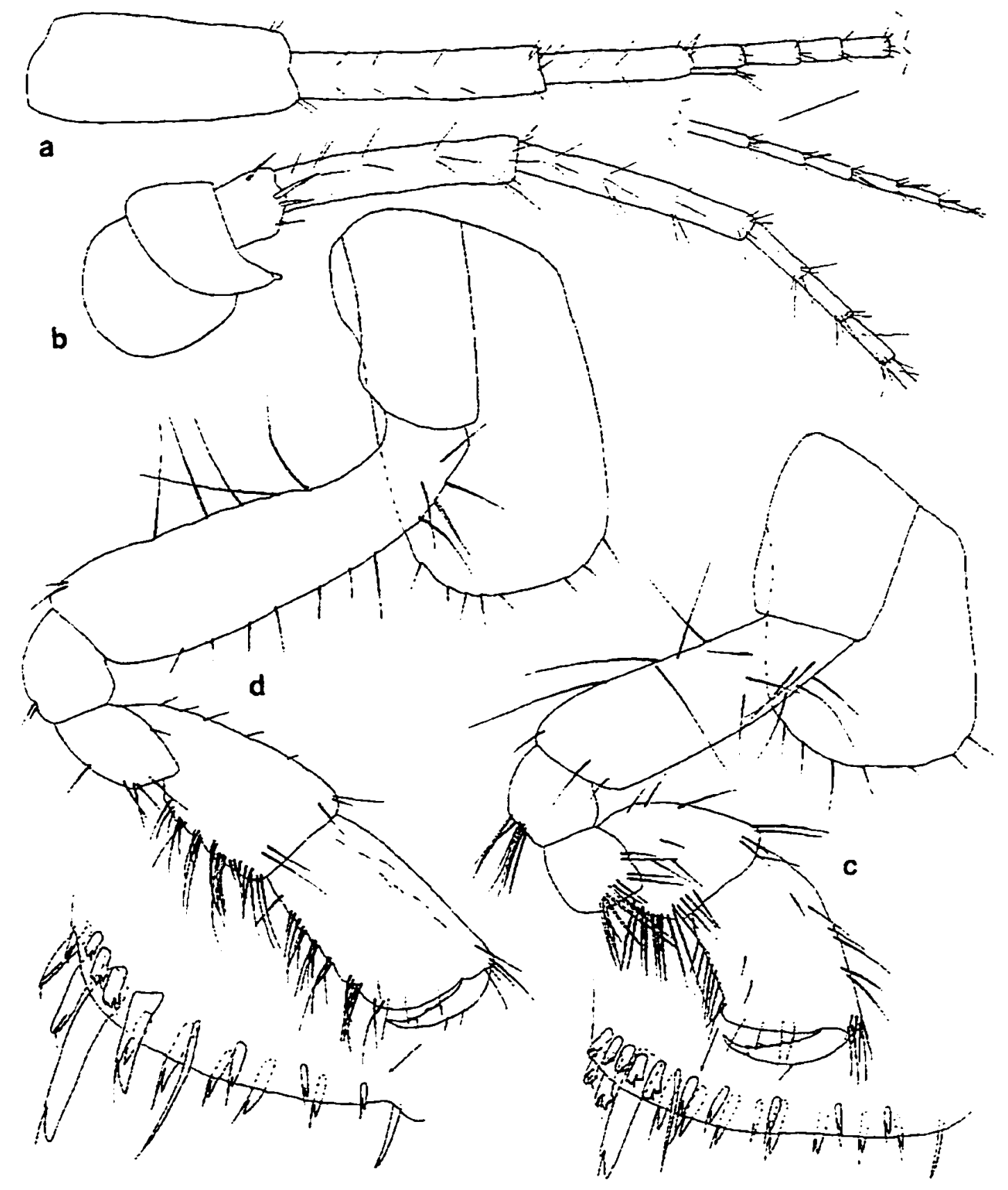

Figure 137.--Crangonyx pseudogracilis Bousfield. Female $(7.9 \mathrm{~mm})$. Lake St. Louis. Perrot. Quebec. Canada: a. b. antennae 1. 2: c. d. gnathopods 1. 2 (palmar margins enlarged). 


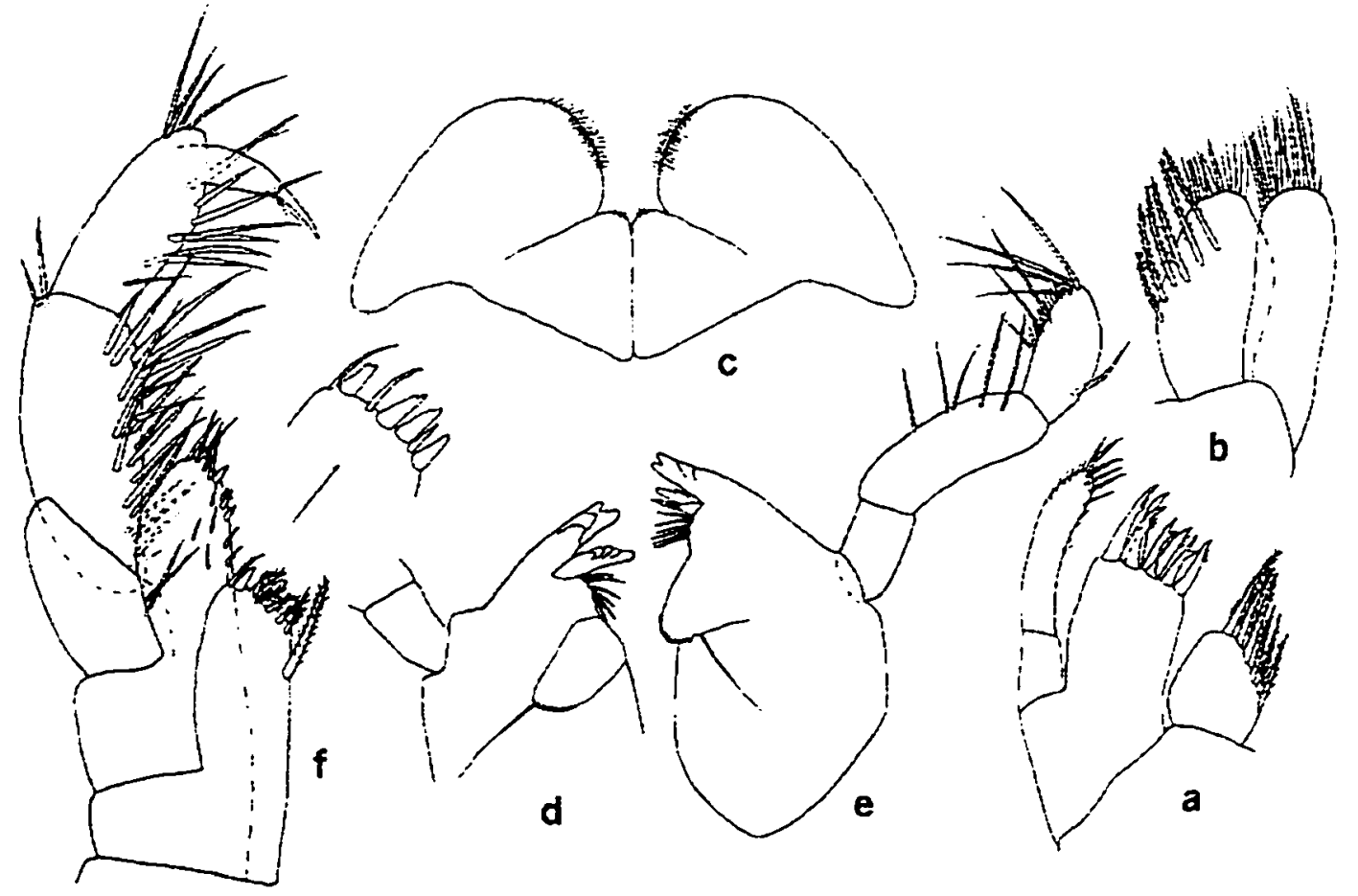

Figure 138.--Crangonvx pseudogracilis Bousfield. Female $(7.9 \mathrm{~mm})$. Lake St. Louis. Perrot. Quebec. Canada: a. b. maxillae 1, 2: c. lower lip: d. dentate part of left mandible: $e$, right mandible: $f$. left half of maxilliped (greatly enlarged). 


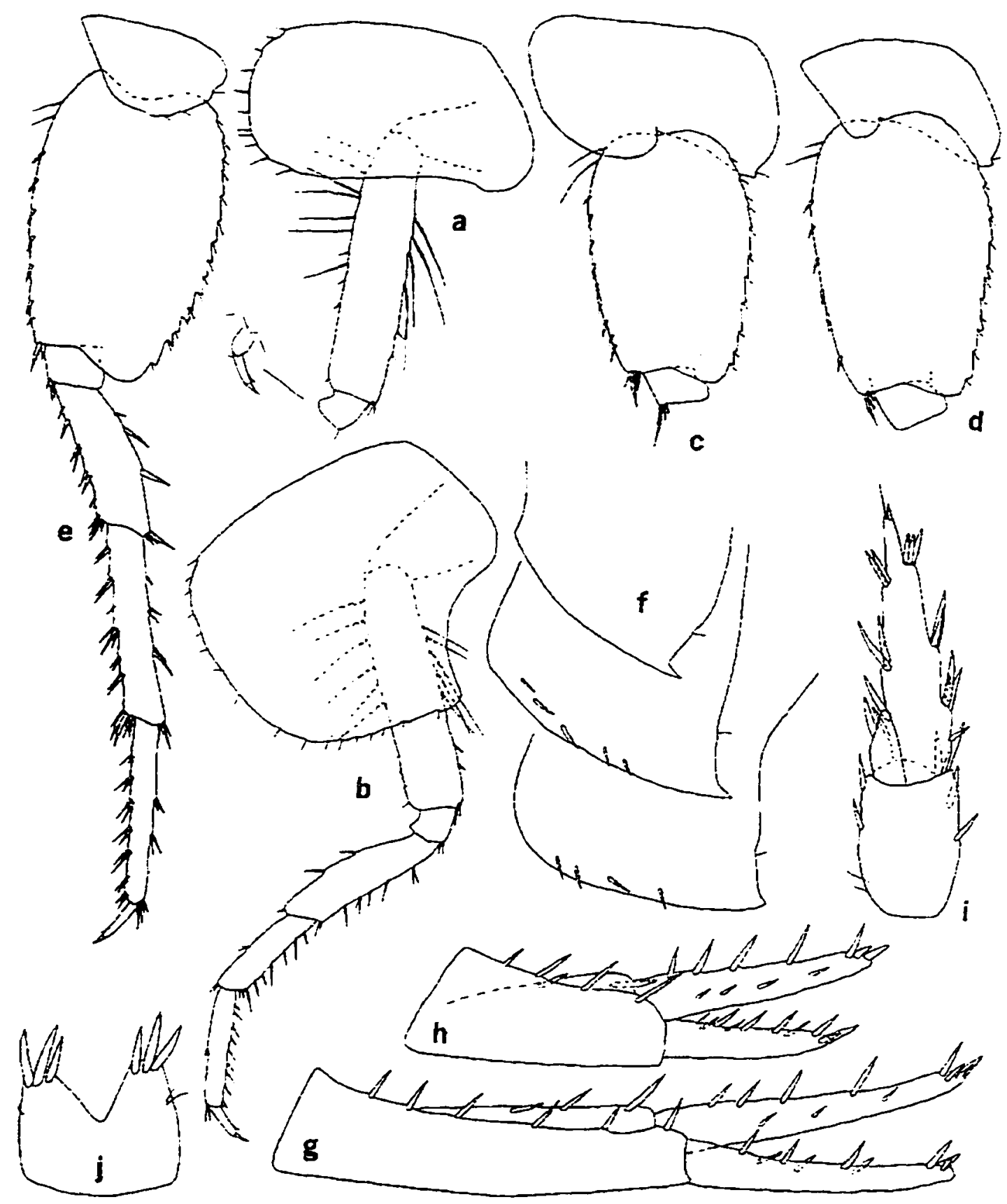

Figure 139.--Crangomx pseudogracilis Bousfield. Female (7.9 mm). Lake St. Louis. Perrot. Quebec. Canada: a. c. d. pereopods 3. 5.6 (in part): b. e, pereopods 4. 7: f. pleonal plates: g. h. i. uropods 1. 2. 3: j. telson. 


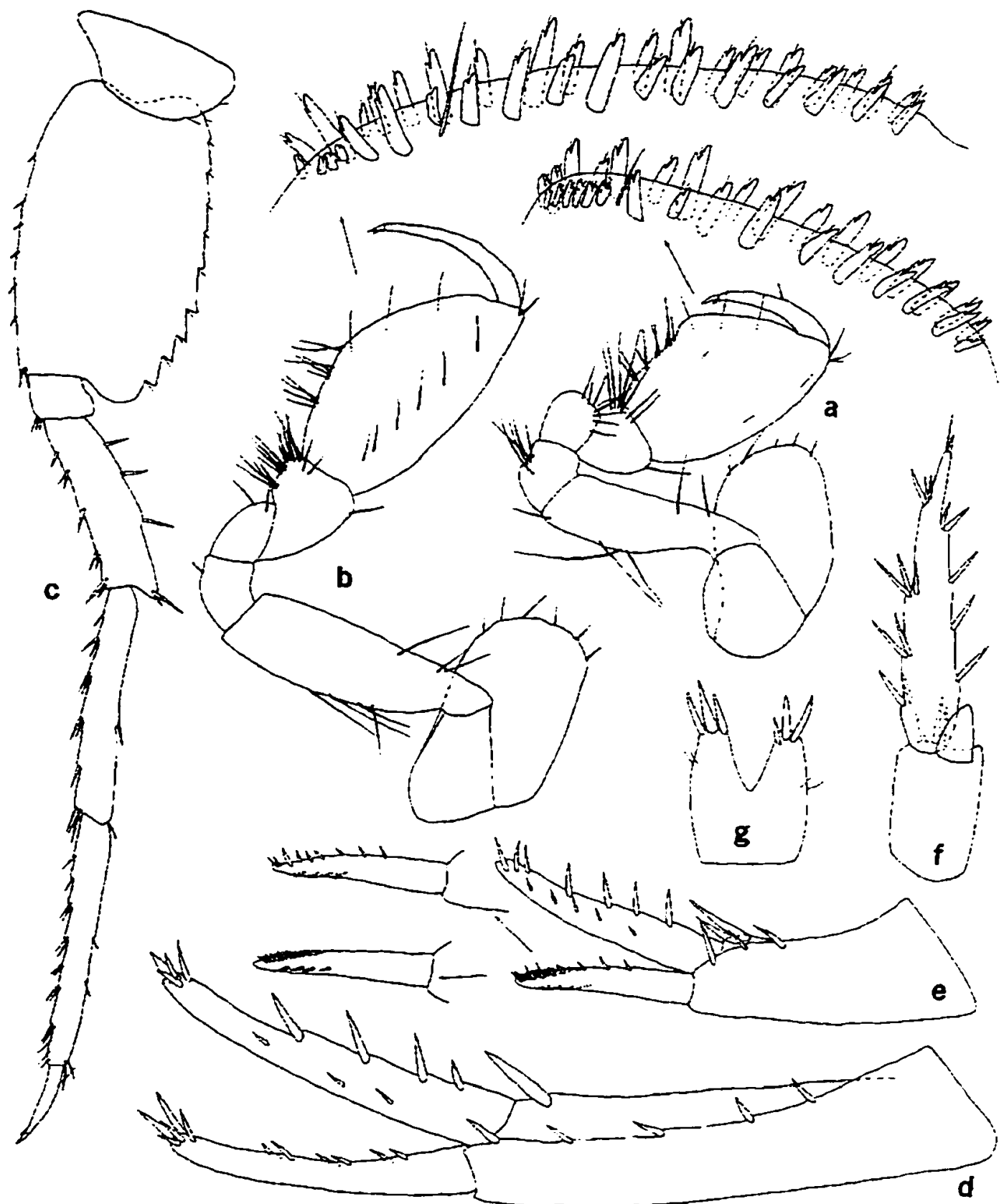

Figure 140.--Crangonyx pseudogracilis Bousfield. Male $(5.0 \mathrm{~mm})$. Lake St. Louis. Perrot. Quebec. Canada: a. b. gnathopods 1.2 (palmar margins enlarged); c. pereopod 7: d. e. f. uropods 1. 2. 3: g. telson. 


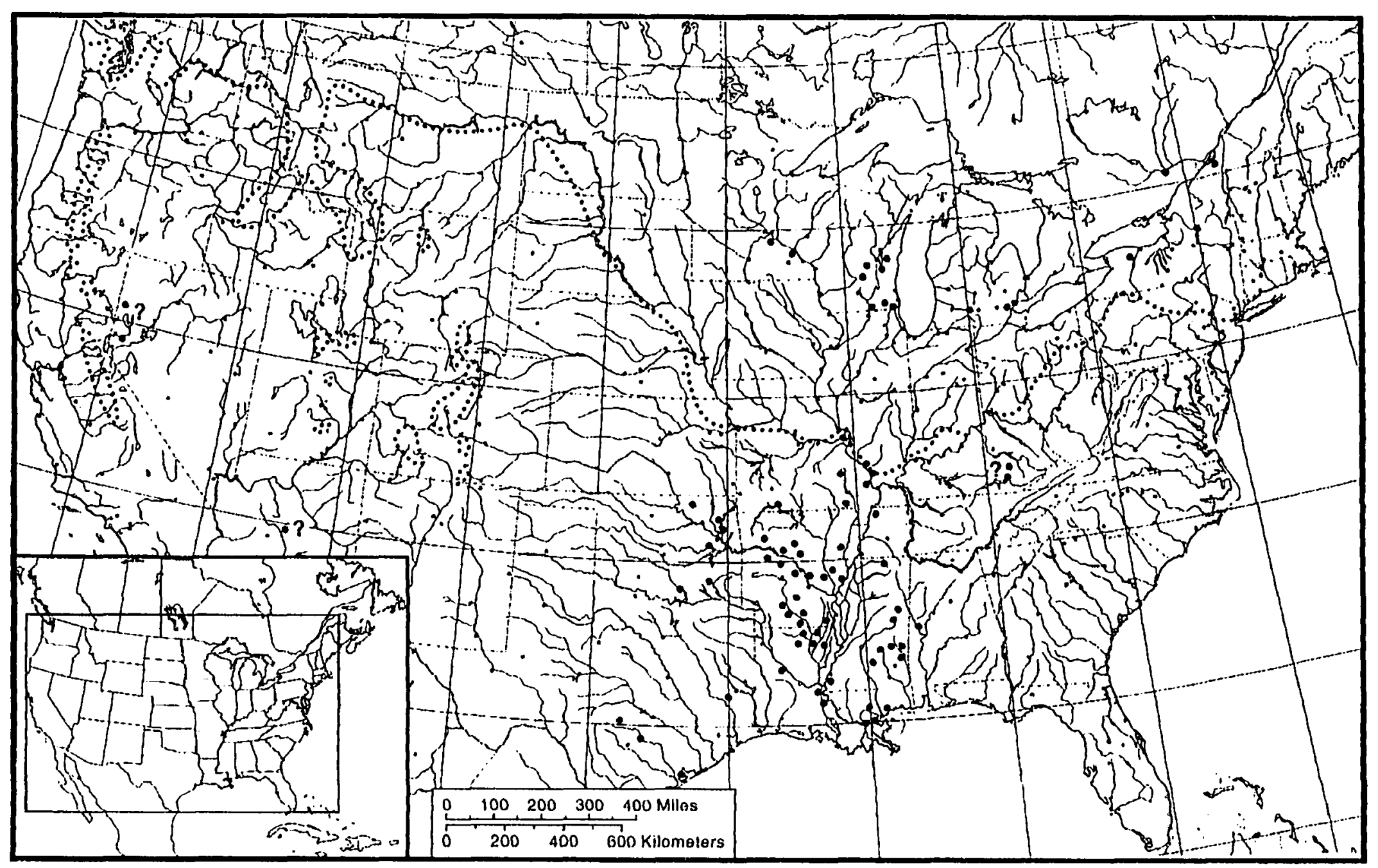

Figure 141.--Distribution of ('rangompx psendegracilis in North America. Solid circles represent 1-5 closely proximate localities. The dotted lines indicale approximate southern extent of glaciation during the Plesstocene and also enclose prockets of alpine glaciation in the west. (ouestion marks

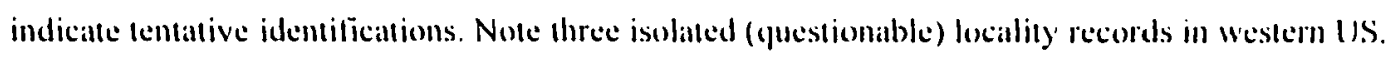


small creek. W of the College Chapel. Clarksville. 50 specs ( 9. \&). L. Hubricht. 28 Apr 1936 (USNM): Lawrence Co.: roadside ditch, $1.0 \mathrm{~km}$ W of Hoxie. 100 specs (\%, $\left.\Xi^{\circ}\right)$. L. Hubricht. 6 Apr 1941 (USNM): Monroe Co.: slough, $1.8 \mathrm{~km} \mathrm{~N}$ of Holly Grove, 70 specs (₹. $\left.0^{\circ}\right)$, L. Hubricht. 7 Apr 1941 (USNM): Newton Co.: large spring near Willcockson, 1 ?. L. Hubricht. 8 Apr 1939 (USNM): Nevada Co.: intermittent stream ca. $100 \mathrm{~m} \mathrm{~N}$ of intersection of St Rds 24 and 36B. 2 ₹. $+\mathrm{cm}^{*}$. R. Fox and Bartlett. 26 Dec 1970: Ouachita Co.: White Oak Creek at St Rd 24 bridge ca. $6.4 \mathrm{~km} \mathrm{~W}$ of Chidester. 6 ?. 10 . R. Fox and Bartlett. 26 Dec 1970: Bragg Lake on St Rd 24 between Bragg City and Chidester. 17 Р. 4 . R. Fox et al. 26 Dec 1970: Perry Co.: cypress swamp, $3.2 \mathrm{~km} \mathrm{~N}$ of Perry. 6 ‡. L. Hubricht, 5 May 1940 (USNM): Phillips Co.: temporary pool. $8.8 \mathrm{~km} \mathrm{~N}$ of Barton. $50 \operatorname{specs}\left(q, \sigma^{\circ}\right)$, L. Hubricht. 6 Apr 194I (USNM): slough. $0.8 \mathrm{~km}$ SE of Turner. 30 specs ( $\left.\% . c^{3}\right)$, L. Hubricht. 7 Apr 1941 (USNM): Pulaski Co.: Clear Creek at St Rd 365 bridge. $2.4 \mathrm{~km} \mathrm{~N}$ of intersection with US-65. 20 specs. R. Fox. 27 Dec 1970: stream crossing St Rd 365 ca. $4.8 \mathrm{~km} \mathrm{~N}$ of intersection with US-65. 50 specs (ङ. ع゙). R. Fox. 27 Dec 1970: Union Co.: temporary pool. $2.9 \mathrm{~km} \mathrm{~N}$ of Eldorado. 30 specs. L. Hubricht. 8 Apr 1941 (USNM): Yell Co.: temporary pool. $0.6 \mathrm{~km}$ W of Danville. $1 \mp .5 z$. L. Hubricht, 4 May 1940 (USNM). ARIZONA.: Yavapai Co.: Page Springs Fish Hatchery. Cornville. $15 \% .2 \approx$. C. Raisanen. 2 Jan 1989. ILLINOIS. Jackson Co.: Turkey Bayou. $2 \varsubsetneqq .3 \approx$ J. J. Lewis. 3 Mar 1974: Union Co.: La Rue-Pine Hills Ecological Area. I I. R. J. Packauskas. 21 Mar 1985 (USNM). KENTUCKY. Laurel Co.: slough. $1.8 \mathrm{~km} \mathrm{~N}$ of Lily. 100 specs.. L. Hubricht. 24 Apr 1941: Little Laurel River, 11 ₹. 12 ๔. R. Fox. II Dec 1970: Jackson Co.: John Rogers Cave, $5 \sqsubseteq .1$ J. J. R. Holsinger. 19 Jul 1964. 39.2 ๙. J. N. Carpenter. 20 Jan 1968: Wild Cave. I §. J. R. Holsinger. 15 Sep 1963. LOUISIANA. A voyelles Par.: (habitat not given). 6 juvs. J. P. Newman. 29 Jul 1975. 2 juvs. 5 Aug. 1975. 3 juvs. 11 Sep 1975 and $1 \subsetneq .1 z^{*} .13$ Oct 1975: Pointe Coupee PAR: roadside pool. $3.2 \mathrm{~km}$ E of Lottie. 50 specs ( 5 . $)$. L. Hubricht. 12 Apr 1964 (USNM): Lincoln Par.: temporary pool. $1.6 \mathrm{~km}$ E of Choudrant. $16 \subsetneq .6 \mathrm{c}$. L. Hubricht. 8 Apr 1941 (USNM): slough. I.t S of Dubach. $27 \cong$. L. Hubricht. 8 Apr 1941 (USNM): Morehouse Par.: Chemin-a-Haut St. Pk.. $129.1 \approx ゙$ J. E. and M. R. Cooper, 15 Mar 1967: ditch on US Hwy 165. ca. $6.2 \mathrm{~km} \mathrm{SW} \mathrm{Bastrap.} 3$ 9. J. E. and M. R. Cooper. 15 Mar

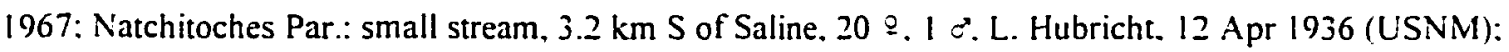
Orleans Par.: roadside ditch on side of Haynes Blvd. $4.5 \mathrm{~km} \mathrm{~S}$ of juct US 90.1 ?. L. Fleming. $29 \mathrm{Nov}$ 1970: Ouachita Par.: slough, $4.8 \mathrm{~km} \mathrm{~S}$ of Swartz. 150 specs ( $\left(0^{\circ}\right)$. L. Hubricht. 8 Apr 1941 (USNM): ditch on St Rd 553 near Lamkin. 6 ₹. 2 ₹. J. E. and M. R. Cooper. 13 Mar 1967: small ditch. $3.7 \mathrm{~km} \mathrm{NW}$ of Monroe. $4 \Xi .3 \%$. J. E. and M. R. Cooper. 25 Dec 1965: borrow pit behind levee of Ouachita R.. 3.2-4.8

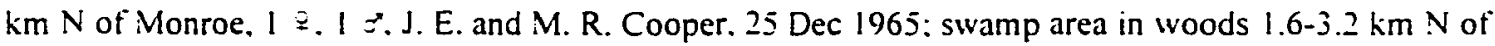
Monroe. 1 ₹. J. E. and M. R. Cooper. 25 Dec 1965: Richland Par.: slough. $1.6 \mathrm{~km} \mathrm{NW}$ of Crew Lake. 19 ¡. 4 *. L. Hubricht. 8 Apr 1941 (USNM): St. Tammany Par.: temporary pond. 35 9. 4 . W. Moore. 18 Feb 1967 (USNM): Union Par.: Lake D'Arbonne, 3 9. J. E. and M. R. Cooper. 14 Mar 1968: Orleans Par.: New Orleans.. $6 \neq .1 \approx$. N. E. Hubert. (collection date not given) (USNM). MICHIGAN. Calhoun Co.: swamp near Kalamazoo River. Urbandale, Battle Creek. $19 \cong .11$ ₹. L. Hubricht, 7 Jun 1947 (USNM): 
Wayne Co.: ditch. $0.8 \mathrm{~km}$ E of Melvindale. 11 \&. $1 \varnothing^{2}$ L. Hubricht. 24 Mar 1946 (USNM). MINNESOTA. Dakota Co.: lower Vermillion River. $19.3 \approx$ J. Muck and R. Newman. 8 Aug 1990: middle Vermillion River. $2 \cong .1 \approx$ J. Muck and R. Newman. 8 Aug 1990; upper Vermillion River. 9 .. I $\approx$. J. Muck and R. Newman. 8 Aug 1990: Wabasha Co.: upper Mazeppa Creek. I ₹. J. Muck and R. Newman. 14 Sep 1990. MISSISSIPPI. Adams Co.: US 6 I Bridge over Homochitto River. swamp-fed stream. 1 \&. 5 \&. L. E. and A. E. Fleming, I Jan 1971: Benton Co.: spring, $1.6 \mathrm{~km} \mathrm{~W}$ of Canaan. $3 \mp .1$ \%. Lubricht. 27 Feb 1961 (USNM): Clarke Co.: swamp. $1.9 \mathrm{~km}$ S of Pachuta. $38 ? .5 c^{\circ}$. L. Hubricht. 16 Mar 1963 (USNM): Hancock Co.: Bayou Bacon. $13.3 \mathrm{mi} \mathrm{N}$ of Kiln. $39.1 \approx .1$ juv. L. Hubricht. 2 Mar 1963 (USNM): Orphan Creek. $9.6 \mathrm{~km}$ N of Kiln. $29.2 \approx$. L. Hubricht. 3 Mar 1963 (USNM): Lauderdale Co.: pool. $+.8 \mathrm{~km} \mathrm{E} \mathrm{of}$ Lost Gap. $11 \cong .2 \unlhd$. L. Hubricht. 3 Feb 1962 (USNM): Newton Co.: slough. $2.7 \mathrm{~km}$ W of Lawrence. $15 \vdots$. 1 . L. Hubricht. 9 Apr 1941 (USNM): Oktibbeha Co.: temporary ponds near Starkville. $7 \leftleftarrows$. S. Walls. Mar 1982 (USNM): Rankin Co.: slough. $1.3 \mathrm{~km} \mathrm{~W} \mathrm{of} \mathrm{Pelahatchee.} 30$ specs. L. Hubricht. 9 Apr 1941 (USNM): Scott Co.: stream. $6.2 \mathrm{~km}$ E of Forest. 2 . L. Hubricht. 9 Apr 1941 (USNM): Winston Co.: pools $4.0 \mathrm{~km}$ S of Noxipater. $2 \subsetneq .17 \mathrm{Mar}$ 1963. NEVADA. Truckee River. Washoe Co. and Storey Co.. 8 ミ. T. C. Frantz. Nov 1955 (USNM): Washoe Co.: Truckee River. Sierra Nevada Mtn. Range. 15 ミ. T. C. Frantz. Nov 1955 (USNM). NEW JERSEY. Burlington Co.: temporary pools, N of Riverton. $1:$. L. Hubricht. 12 Mar 1938 (USNM). NEW YORK. Herkimer Co.: stream in marsh between Frankfort and Ilion. 5 ₹. 1 ¿. L. Hubricht. 4 Apr 1943 (USNM): swamp $1.6 \mathrm{~km}$ ESE of Little Falls. 20 specs. L. Hubricht. 18 Apr 1943 (USNM): swamp. Ilion. 100 specs. L. Hubricht. 20 Jun 1943 (USNM): Monroe Co.: sluggish stream near Allyn Creek. Pittsford. 25 ․ 16 \%. S. F. Salano. Jun 1949 (USNM).

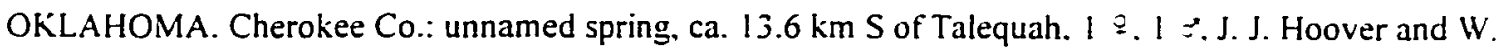
B. Milstead. 22 May 1981 and 2 specs. 4 Jun 1982; Johnston Co.: Cummings Spring. ca. $0.8 \mathrm{~km} \mathrm{~N}$ of Connerville. 11 ₹. 2 ॐ. J. J. Hoover and W. B. Milstead, 14 Jun 1982: Mayes Co.: unnamed Spring. ca. 0.3

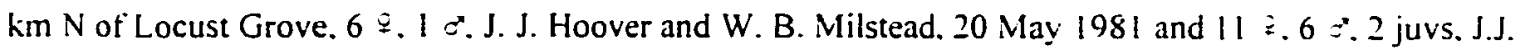
Hoover and W. B. Milstead. 3 Jun 1982: Mcintosh Co.: slough of Canadian River S of Eufaula. $3 z^{\circ}$. L. Hubricht. 10 May 1940 (USNM): Pushmataha Co.: Lily Lake. $3.2 \mathrm{~km} \mathrm{~N}$ of Antlers. 9 ミ. J. G. Mackin.? May 1935 (USNM): Osage Co.: Doddy Miller Spring. ca. $12.0 \mathrm{~km}$ NE of Barnsdall. 3 こ. J. J. Hoover and W. B. Milstead. I Jun 1981 and 2 . I Jun 1982. TENNESSEE. Lake or Obion Co.: Edge sawgrass. Reelfoot Lake, 1 \&. V. C. Sheffoed. 9 Apr 1943 (USNM) and 1 . J. and W. Rapp. 12 Apr 1946 (USNM). TEXAS. Calhoun Co.: roadside ditch. $4.8 \mathrm{~km} \mathrm{SW}$ of Green Lake. 7 . L. Hubricht. 30 Apr 1955 (USNM): Gonzales Co.: ca. $1.6 \mathrm{~km}$ S of Ottine. 3 \%. S. J. Harden and C. F. Lindbloom. 26 Oct - 5 Nov 1986: Kendall Co.: small spring stream, near Sabinas Creek. 2 ₹. I juv. S. J. Harden. 20 Jan 1990: near Boerne (habitat not given). 1 g. S. J. Harden. 14 Oct 1989: San Jacinto Co.: pool beside Big Creek. 2 :. S. J. Harden and C. F. Lindbloom. 28 Nov 1987. WISCONSIN. Calumet Co.: Crystal Creek at county trunk highway KK. 6 ‡. 4 ๘. J. P. Jass and B. Klausmeier. 23 Sep 1994 (MPM): Columbia Co.: Fox River at county trunk highway CM bridge. I $९$. J. P. Jass. 20 Sep 1994 (MPM): Ditch, N side county trunk highway 
O $1.6 \mathrm{~km} \mathrm{~W}$ of Wolfram Rd., $5 \cong .6 c^{2} .2$ Juvs. J. P. Jass. 20 Sep 1994 (MPM): Fond Du Lar Co.: Long Lake. South Shore at Hwy 67. 2 §. J. Jass. 25 Oct 1994 (MPM): Green Lake Co.: Marsh. E side Hwy 49. just S. of Berlin. 2 Z. 2 c, J. P. Jass and B. Klausmeier, 19 Aug 1994 (MPM); Kenosha Co.: Fox River. pk at County Trunk Highway F. near Silverl, 7 \&. I ¿. J. P. Jass and B. Klausmeier. 6 May 1994 (MPM): Rock Co.: near Fulton Rock River trib. at Gibbes Lake Road. I C. J. P. Jass \& B. Klausmeier. 10 Jan 1994 (MPM): Walworth Co.: trib. of Mukwonago River at county trunk highway. 3 Z. $1 \approx$. J. P. Jass and $B$. Klausmeier. 7 Oct 1994 (MPM): Pickerel Lake / L. Beulah juction at Booth Lake Rd (Town Line). 6 ₹ 3 ?. J. P. Jass and B. Klausmeier. 7 Oct 1994 (MPM): Lake Beulah at Springers Bridge Rd.. 5 specimens. J. P. Jass and B. Klausmeier. 7 Oct 1994 (MPM): Green Lake boatlanding. W shore. $3 \equiv .1=\div .5$ Juvs. J. P. Jass and B. Klausmeier. 7 Oct 1994 (MPM).

Diagnosis.-A member of the gracilis group morphologically closely similar to C: floridanus. Distinguished from other members of the group by large eyes: strongly produced distoposterior corners of pieonal plates 1-2: comb spines on outer ramus of uropod 2 of male. Further distinguished by having special ventral spines on inner margin of outer ramus of male uropod 2 (unique among species of (rangonux). Largest male. $6.5 \mathrm{~mm}$ : largest female. $10.5 \mathrm{~mm}$.

Female.--Eye large and ovate. Antenna 1.55-57 percent length of body. about 1.8 times longer than antenna 2: primary flagellum with about 20 segments. Antenna 2. flagellum with 6-8 segments. Mandibles subequal. spine row with 5-6 spines: segment 2 of palp with 6 long setae, segment 3 with 1 A seta. $2-3 \mathrm{C}$ setae. $4 \mathrm{E}$ setae and a row of $D$ setae. lacking $B$ seta. Maxilla 1 : inner plate with 6 apical plumose setae: palp with 5-6 slender spines on apex. Maxilla 2. inner plate with oblique row of 6-7 plumose setae on inner margin. Maxilliped: inner plate apically with 4 bladelike spines. 1 plumose spine. 2 naked spines. and 5-6 plumose setae extending from inner margin to apex: outer plate with row of naked setae and 4-6 slender pectinate spines on inner margin: dactyl with 2 setae on inner margin near nail: dactyl nail very long. about $1 / 3$ length of dactyl.

Propod of gnathopod I slightly larger and longer than carpus: palm almost straight with 5 unnotched spine teeth on inside. 8 on the outside: defining angle with 5 notched-serrate spine teeth on inside. I large notched and 2 small serrate spine teeth on outside: inferior and superior medial setae singly inserted: setae on posterior margin singly or doubly inserted. Dactyl with a row of short setae on inner margin. nail long. Ventral margin of coxa I with 7 setae.

Propod of gnathopod 2: slightly larger than carpus: palm almost straight. much shorter than posterior margin. with about 3 unnotched spine teeth on inside. 5 on outside; defining angle with 1 very large notched spine tooth and 2 serrate spine teeth on outside. I large and 3 small notched-serrate spine teeth on inside: superior medial setae singly inserted: posterior margin with 5-6 sets of setae in groups of 1-6.

Dactyl with row of short setae on inner margin. Ventral margin of coxa 2 with about 8 setae.

Coxa of pereopod 3 with about 11 marginal setae. Coxa of pereopod 4 with about 17 marginal setae. dactyl of pereopod 4.45 percent length of corresponding propod. Pereopod 5: basis with about 11 shallow: 
serrations along posterior margin: 9 short spines on anterior margin: 2 long setae on the anteroproximal margin. Pereopod 6: basis with shallow 12-14 serrations along posterior margin: 6 sets of short spines on anterior margin: 2 short setae on anteroproximal margin. Pereopod 7: coxa with 3 setae on the posterior margin: basis with 12-15 serrations along posterior margin: 7 sets short spines on anterior margin: 2 long setae on anteroproximal margin: dactyl about 30 percent length of corresponding propod.

Pleonal plates: posterior margin of plate I slightly convex. with 1 seta. distoposterior corner produced and acute: posterior margin of plate 2 nearly straight with I seta. distoposterior corner strongly produced and acute. ventral margin with 5 (subventral) spines: posterior margin of plate 3 nearly straight with I seta. distoposterior corner produced. ventral margin with 4 (subventral) spines. Peduncle of pleopod I with 2 retinaculae. lacking setae on outsıde margin.

Uropod 1: inner ramus 65 percent length of peduncle. armed with 11 spines: outer ramus with 9 spines: peduncle with 6 outer and 3 inner spines. Uropod 2: inner ramus armed with 11 spines: peduncle with 4 outer spines. 2 inner spines. Uropod 3 : inner ramus with 1 spines. outer ramus about 2 times longer than peduncle. inner and outer margin each with $3-4$ set of spines in groups $1-3$. Telson slightly broader than long or as broad as long, notched about 2/5-1/2 distance to base. each lobe with about 3 apical spines: spines up to $1 / 2$ length of telson.

Male.--Differing from female as follows. Smaller with more slender. elongate body. Antenna 2: thagellum with 6 segments. calceoli percent on peduncular segments 4 and 5 . and first 4 segments of tlagellum.

Propod of gnathopod 1 more than 2 times larger and longer than carpus and much broader: palm with 9 spine teeth on inside. 11 on outside: defining angle with 6 notched-serrate spine teeth on inside and 3 notched and 2 serrate on outside.

Propod of gnathopod 2 more than 2 times larger and longer than carpus: palm margin strongly oblique. with 11 spine teeth on inside, I I-12 on outside; defining angle with 2-4 notched and 2 weak. notchedserrate teeth on inside and 3-6 notched and/or I unnotched spine teeth on outside.

Uropod 2: inner ramus with 12 spines: outer ramus with row of comb spines on distal 25 of inner margin. outer margin with 7 spines (not in comb row). inner side also with $1-5$ ventral spines on distal half. apex with 2-3 spines: peduncle with 3 outer spines. and 2 inner spines.

Distribution and ecology.--This species is widely distributed in east-central United States and southern Canada (fig. 141). It occurs largely west of the Appalachians. extending approximately from the Great Lakes region south and southwest to Louisiana and Texas. The specimens in populations from eastern Kentucky possess the diagnostic ventral spines on the inner margin of the outer male uropod 2 but occur farther east than most other populations. Although the specimens from Arizona and Nevada are morphologically very similar to the eastern populations, their exact taxonomic species status was not determined in the present study, and their identity remains somewhat questionable.

C. pseudogracilis inhabits rivers. streams, sloughs. lakes, ponds, swamps. ditchs. and almost all kinds 
of surface freshwater habitats as well as two caves in Kentucky. Ovigerous females are collected throughout the year. Sexually mature males are 4.5 to $6.5 \mathrm{~mm}$ and sexually mature female are 7.5 to 10.5 $\mathrm{mm}$.

Remarks.--Crangonyx pseudogracilis was introduced into Great Britain. where it identified as $C$ : gracilis (Hynes 1951. 1955a. b). Subsequently, it was found to have invaded and then spread widely in the Netherlands (Pinkster et al.. 1980. 1983: Platvoet et al.. 1985. 1989). I confirmed the identity of this species in the Netherlands by examination of specimens on loan from the Zoological Museum of the University of Amsterdam from the following localities: stream in Friesland $(48.1)$ and canal in Groningen Province ( 4 ₹.4

Crangonvx consimilis. new species

Figures $142-145$

Material examined (paratypes unless designated otherwise).--ALABAMA. Limesone Co.: Gaston Cave. $33 \Xi .3 \approx$ W. B. Jones et al.. 5 May 1958: Madison Co.: slough. $4.3 \mathrm{~km}$ NW of Gurley. 3 @. L. Hubricht. 12 Apr 1941 (USNM). MISSISSIPPI. Oktibbeha Co.: pond. $5.3 \mathrm{~km}$ from Harved Biology BIdg.. near Starkville. 1 . M. J. Harris, 17 Feb 1968. TENNESSEE. Bedford Co.: spring. $4.0 \mathrm{~km} \mathrm{~W}$ of Shelbyville, 200 specs ( $₹$. $z$. juvs), L. Hubricht. 1 Jun 1941 (USNM); Cheatham Co.: spring near Good Spring Church. 4.2 km SE of Pleasant. 6 \&, L. Hubricht. 31 May 1941 (USNM): Cannon Co.: Davenport Cave spring, $4.0 \mathrm{~km}$ S of Woodburg. 1 ?. J. R. Holsinger and R. B. Godfrey, 9 Jul 1983: Davidson Co.:

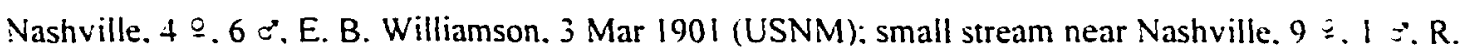
Fleming. 2 Apr 1936 (USNM): Spring. "Fortland". near Shelby Park. Nashville. holotype ₹. ca. $400 \mathrm{spec}$. (F. $\approx$. juvs). L. Hubricht. 31 May 1941 (USNM): Spring on Dr. Fort's Farm near Shelby: Park. $1 \geq$. L. Hubricht. 31 May 1941 (USNM): Shelby Park. Nashville. $10 \cong .7$ c. R. Fleming. 7 Mar 1936 (USNM): Otters Creek. Nashville. 18 ₹. R. Fleming. 26 Oct 1935 (USNM): Radnor Lake. $1 \cong .1 \approx$. M. Wright. 5 May 1945 (USNM): Richland Creek, Nashville. $5 \cong, 20^{\circ}$. R. Fleming. 29 Feb 1936: w ter cress in spring. Nashville. 89.9 \%. R. Fleming. 7 Mar 1936: Dickson Co.: Piney River. $8.6 \mathrm{~km}$ SW of Dickson. 3 §. 1 : L. Hubricht. II Sep 1955 (USNM); spring, $2.1 \mathrm{~km}$ E of Charlotte. 30 specs ( $\left.₹ . \Xi^{*}\right)$. L. Hubricht. 11 Sep 1955 (USNM): Maury Co.: springs. $3.7 \mathrm{~km} \mathrm{SW}$ of Spring Hill. 200 specs ( $\left.\Xi . \beth^{*}\right)$. L. Hubricht. 31 Mar 1941 (USNM): Rutherford Co.: Broyles Cave. $4.0 \mathrm{~km} \mathrm{~N}$ of Beach Grove. 2 9.21 Aug 1967: Oaklands Spring. Murfreesboro, 80 specs ( 9.8 . juvs). C. R. McGhee and L. E. Fleming. 19 Dec 1969; stream in Rainbow

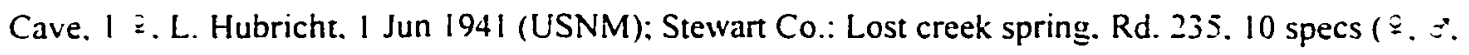
juvs). D. L. Gillis. 14 Jui 1989: Williamson Co.: small spring. $1.9 \mathrm{~km}$ NE of Franklin. 400 specs $(\varepsilon$. juvs). L. Hubricht, 31 May 1941 (USNM): Wilson Co.: spring. $3.5 \mathrm{~km} \mathrm{~N}$ of Bairds Mill, $100 \operatorname{specs}(q .$. juvs). L. Hubricht. I Jun 1941 (USNM). 


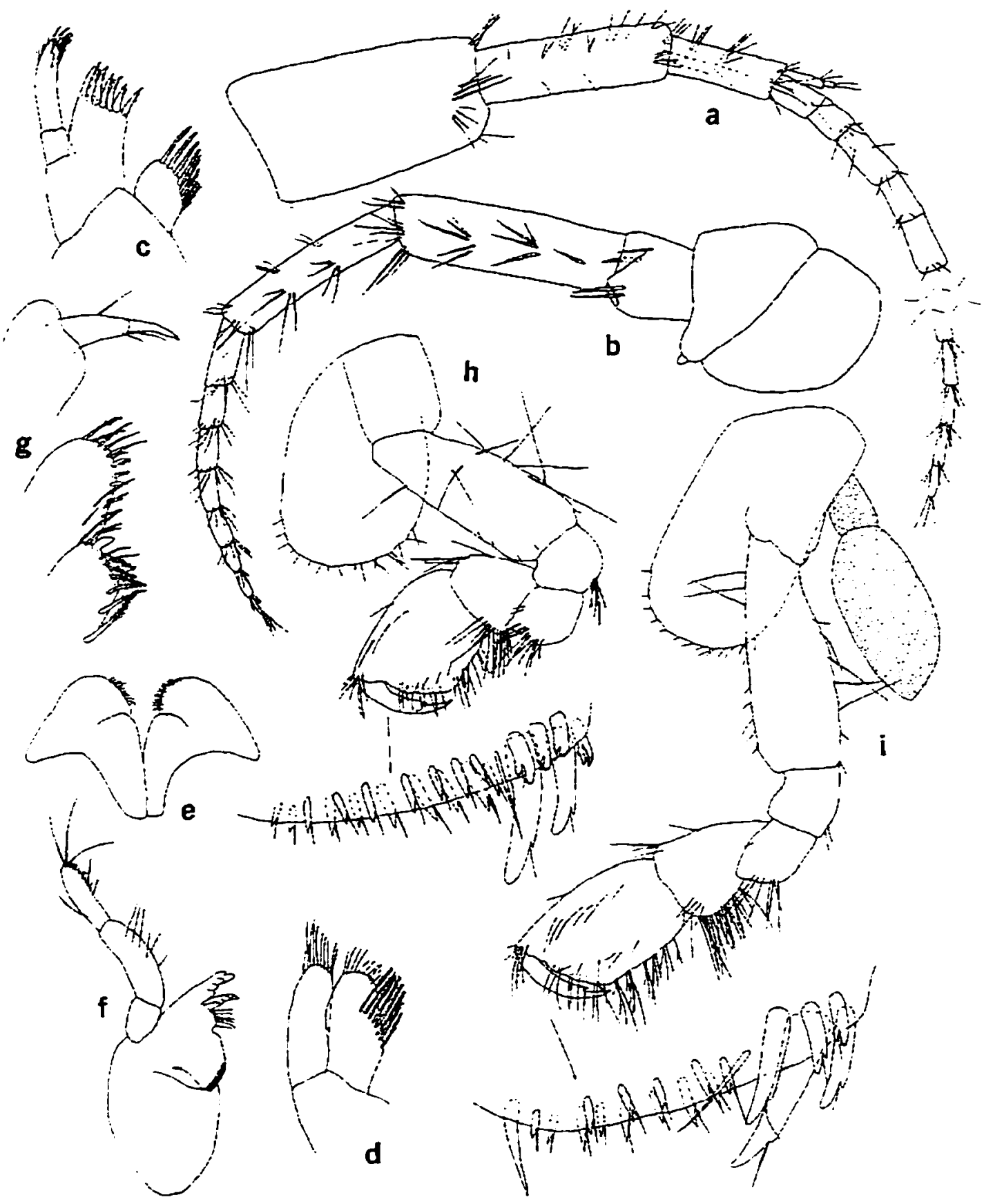

Figure 142.--Crangonyx consimilis, new species. Female paratype $(8.5 \mathrm{~mm})$. spring. near Shelby Park. Nashville, Davidson Co.. Tennessee: a. b, antennae 1, 2: c, d, maxillae 1. 2: e. lower lip: $f$. left mandible: g. inner and outer plates and palp dactyl of maxilliped: h. i. gnathopods I. 2 (palmar margins enlarged). 


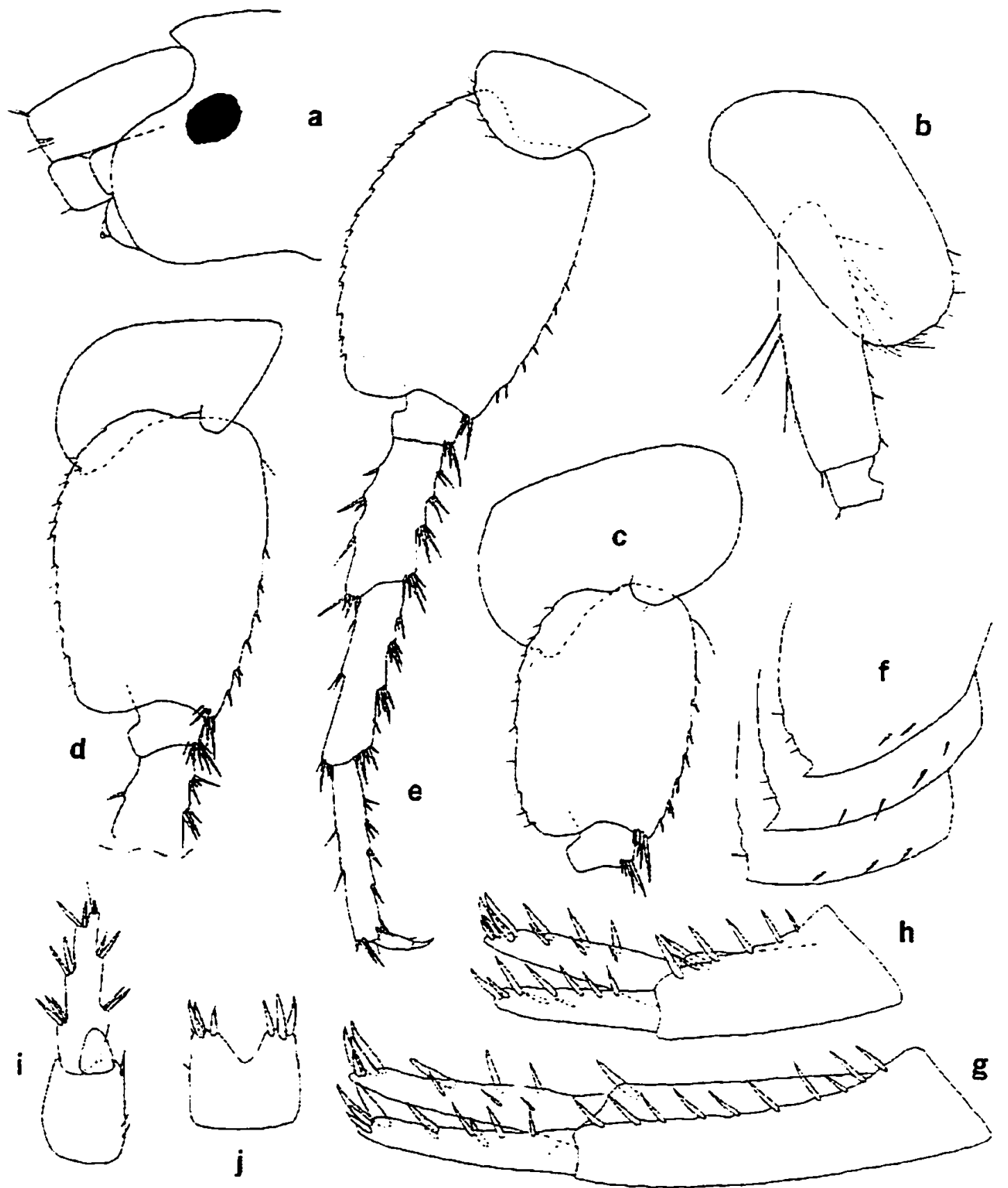

Figure 143.--Crangomxx consimilis, new species. Female paratype $(8.5 \mathrm{~mm})$. spring. near Shelby Park. Nashville. Davidson Co.. Tennessee: a, head (in part); b. c. d. pereopods 3. 5.6 (in part): e. pereopod 7: f. pleonal plates: g. h. i. uropods 1. 2. j: j, telson. 


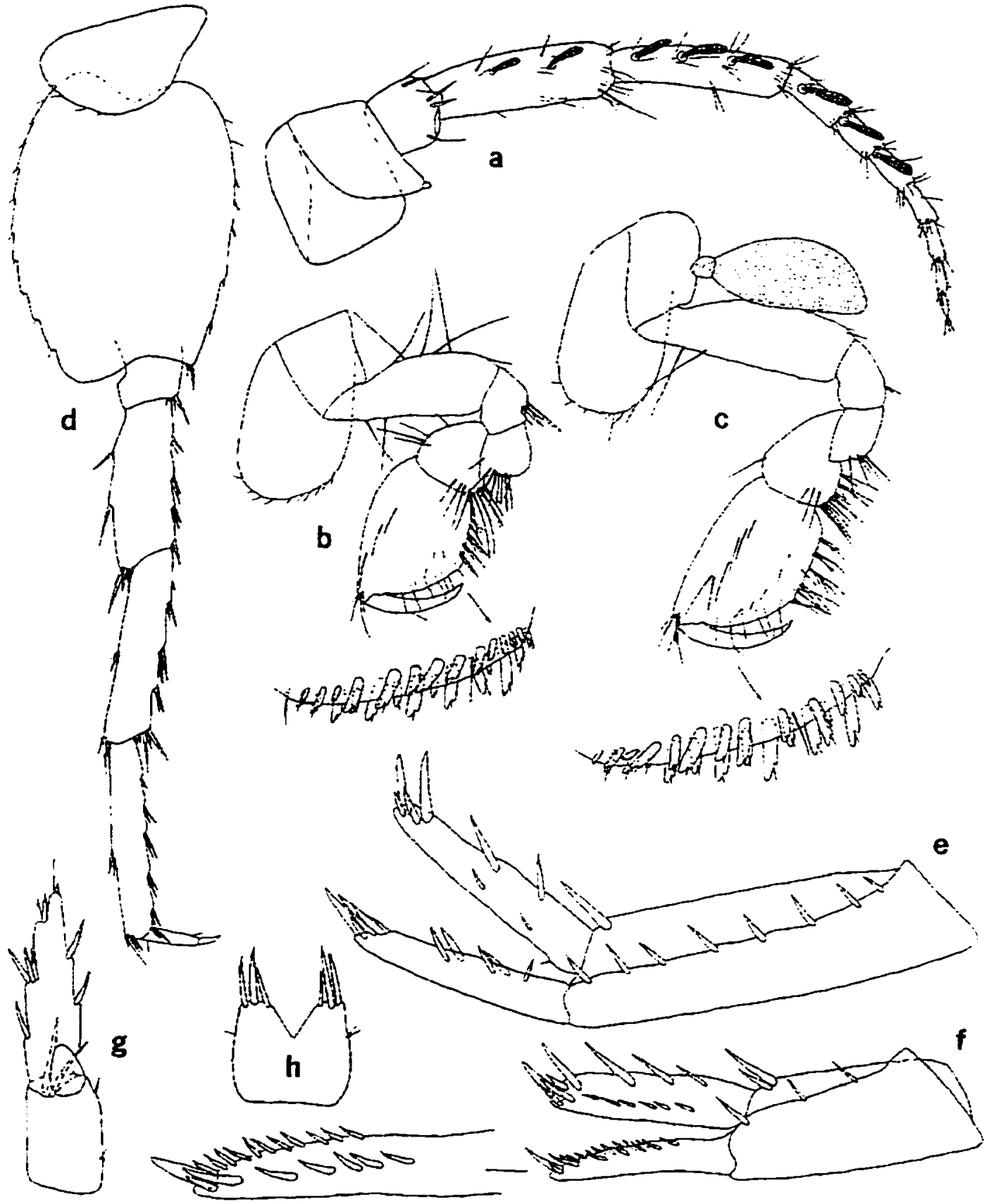

Figure 144.--Crangomxx consimilis, new species. Male paratype $(6.4 \mathrm{~mm})$, spring. near Shelby Park. Nashville. Davidson Co., Tennessee: a. antenna 2: b. c. gnathopods I. 2 (paimar margins enlarged): $d$. pereopod 7: e. g. uropod I. 3: f, uropod 2 (distal end of outer ramus enlarged): h. telson. 


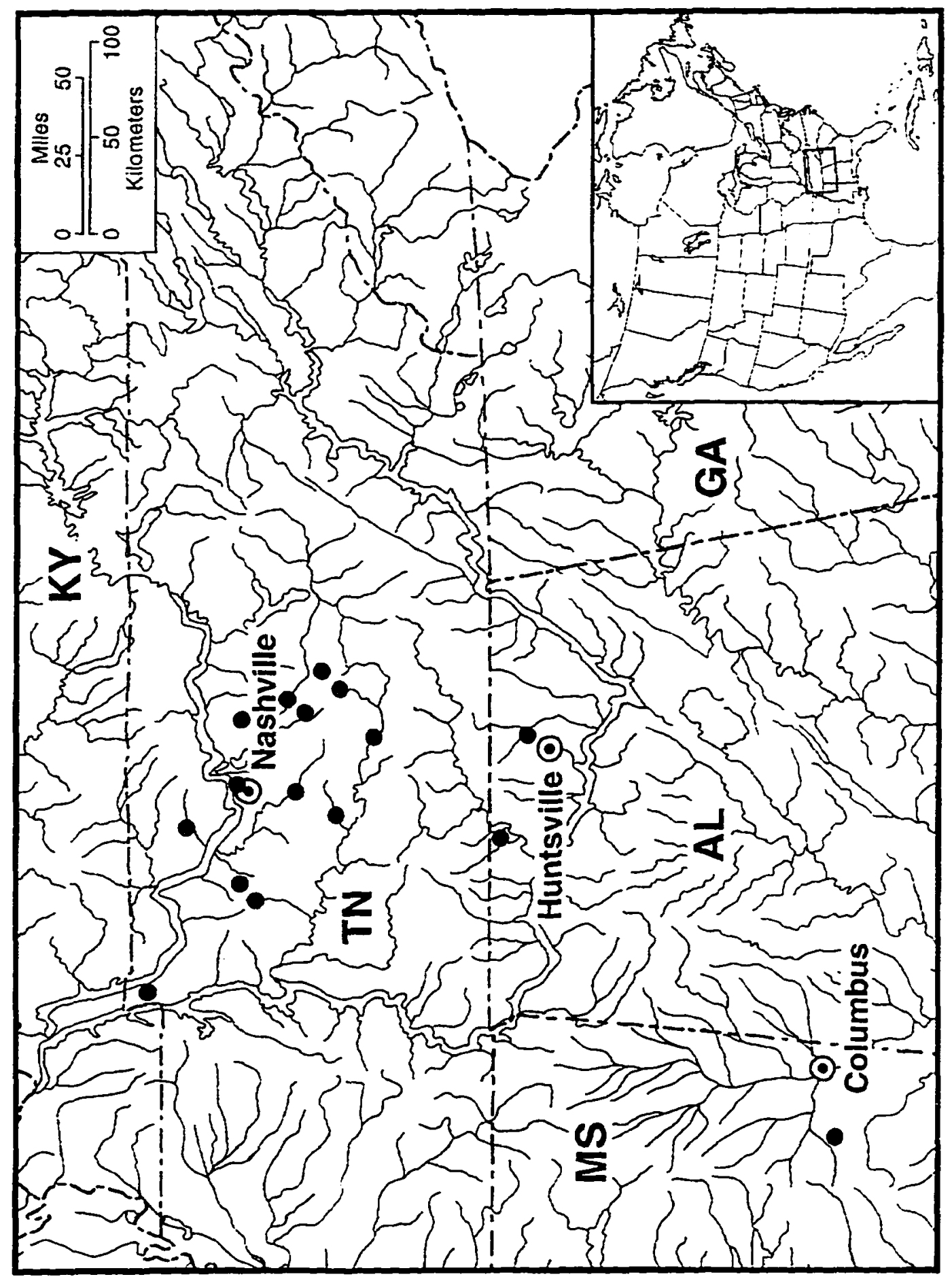

总 
Diagnosis.--A small species of the gracilis group very similar to $C$. longidacty/us but distinguished from it by having fewer subventral spines on pleonal plates and shorter uropod 3 . Largest male. $6.7 \mathrm{~mm}$ : largest female. $10.0 \mathrm{~mm}$.

Female.--Eye large and ovate. Antenna 1, about 50 percent length of body. about 1.8 times longer than antenna 2: primary flagellum with up to 20 segments. Antenna 2. flagellum with up to 8 segments. Mandibles subequal. spine row with 5-6 spines: segment 2 of palp with 6 long setae. segment 3 with I A seta. 2 C setae. 3 E setae and a row of D setae, lacking B seta. Maxilla 1: inner plate with 8 apical plumose setae: palp with 7 slender spines on apex. Maxilla 2, inner plate with oblique row of 8 plumose setae on inner margin. Maxilliped: inner plate apically with 3 bladelike spines. 3 naked spines. and 5-6 plumose setae extending from inner margin to apex: outer plate with row of naked setae and slender pectinate spines on inner margin and 2 apical plumose setae: dactyl with 2 setae on inner margin: dactyl nail very long. 1 ; length of dactyl.

Propod of gnathopod 1 less than 2 times larger and longer than carpus: palm almost straight. slightly shorter than posterior margin. with 7 unnotched spine teeth on inside. 8 on the outside: defining angle with 3 notched-serrate spine teeth on inside. 2 large notched and I small serrate spine teeth on outside: superior medial setae few in number. singly inserted: 4 sets of setae on posterior margin. singly inserted or in groups of 2: dactyl with long nail. Ventral margin of coxa 1 with 18 setae.

Propod of gnathopod 2 less than 2 times larger and longer than carpus: palm slightly convex. shorter than posterior margin. with 6 unnotched spine teeth on inside. 7 on outside: defining angle with 2 notched and 1 notched-serrate spine teeth on inside. 2 large notched spine teeth on outside: superior medial setae singly inserted. posterior margin with 6 sets of setae in groups of 2-6. Ventral margin of coxa 2 with 14 setae.

Coxa of pereopod 3 with 12-14 marginal setae. Coxa of pereopod 4 with 18 marginal setae. dactyl 4 about 35 percent length of corresponding propod. Pereopod 5: basis with 11 shallow serrations along posterior margin: 7 sets of short spines on anterior margin: 1 long seta on anteroproximal margin. Pereopod 6: basis with 12 serrations along posterior margin: 6 short spines on anterior margin: 1 seta on anteroproximal margin. Pereopod 7: coxa with $3-4$ setae on the posterior margin: basis with 17 slight serrations along posterior margin: 8 short spines on anterior margin: dactyl about 33 percent length of corresponding propod. Pleonal plates: posterior margin of plate 1 slightly convex. with 3 setae. distoposterior corner produced and acute, ventral margin with 1-2 (subventral) spines: posterior margin of plate 2 slightly convex with 2 or 3 setae, distoposterior corner strongly produced and acute. ventral margin with 4 (subventral) spines: posterior margin of plate 3 nearly straight with $1-3$ setae. distoposterior corner not produced but weakly acute, ventral margin with 3 (subventral) spines. Peduncle of pleopod 1 with 2 retinaculae: l:rking setae on outside margin.

Uropod 1: inner ramus 70 percent length of peduncle. armed with 10 spines: outer ramus with 10 spines: peduncle with 10 outer spines. and I inner spines. Uropod 2: inner ramus armed with 11 spines: 
peduncle with 5 outer spine, 2 inner spines. Uropod 3 : inner ramus lacking spines, outer ramus about 1.5 times longer than peduncle. inner and outer margin each with 2-3 set of spines in groups of 2-3. Telson slightly broader than long. notched about $1 / 3$ distance to base. each lobe with 3 apical spines: spines about 12 length of telson.

Male.--Differing from female as follows. Smaller with more slender. elongate body. Antenna 2: calceoli percent on peduncular segments 4 and 5 and first 3 segments of flagellum.

Propod of gnathopod 1 more than 2 times larger and longer than carpus: palm with 8 spine teeth on inside. 9 on outside: defining angle with I notched spine tooth and 4 notched-serrate spine teeth on inside. 2 notched and $1-2$ serrate spine teeth on outside. Propod of gnathopod 2 more than 2 times larger and longer than carpus: palmar margin strongly oblique, with 8 spine teeth on inside. 9 on outside: defining angle with 3 notched and 1 serrate spine teeth on inside. 5 notched spine teeth on outside.

Uropod 2: inner ramus with 14 spines of unequal length: outer ramus with row of comb spines on distal 1 2-2 3 of inner margin. face of outer margin with 5 short spines: apex with $3-4$ spines: peduncle with 3 outer spines. and 2 inner spines.

Type-locality.--Spring near Shelby Park in eastern Nashville. Davidson County. Tennessee.

Distribution and ecology.--This species is widely distributed in central Tennessee and also occurs in northem Alabama and east-central Mississippi (fig. 145). Ovigerous females were collected from March to September and in December. suggesting that breeding is continuous throughout the year. Sexually mature males are 3.5 to $6.7 \mathrm{~mm}$ and sexually mature females are 5.0 to $10.0 \mathrm{~mm}$.

Etymology.--The epithet consımilis is from Latin. meaning "like in all respects." in reference to the similarity of this species to other members of the floridanus subgroup.

Crangonyx longidacty/us. new species

Figures $146-149$

Material examined (paratypes unless designated otherwise).--KENTUCKY. Barren Co.. swale. $1.6 \mathrm{~km}$ E of Bonayr, holotype $₹ .150 \operatorname{specs}(7, \approx)$. L. Hubricht. 20 Mar 1956 (USNM): Simpson Co.: Double Entrance Spring Cave resurgence (Sinking Creek Cave system). $4 \sqsubseteq .2 \approx *$ A. T. Leitheuser et al.. 21 Nov 1982: Warren Co.: Barten River Reservoir, 6 juvs, R. Prinz. Jul 1970. NORTH CAROLINA. Macon Co.: Nantahala Reservoir. 100 specs ( $\%$. $\%$, juvs) (not paratypes), W. R. Bonner. Spring 1979.

Diagnosis.--Closely similar to $C$. consimilis but distinguished from it by defining angle of propod of gnathopod 2 with very large spine tooth at end: more subventral species on pleonal plates: longer uropod 3: and small comb spines on outer ramus of uropod 2 of male. Largest male $5.5 \mathrm{~mm}$ : largest female $9.0 \mathrm{~mm}$.

Female.--Eye large and ovate. Antenna 1.53-55 percent length of body. about 1.8-2.0 times longer than antenna 2: primary flagellum with 20 segments. Antenna 2. flagellum with 8 segments. Mandibles 


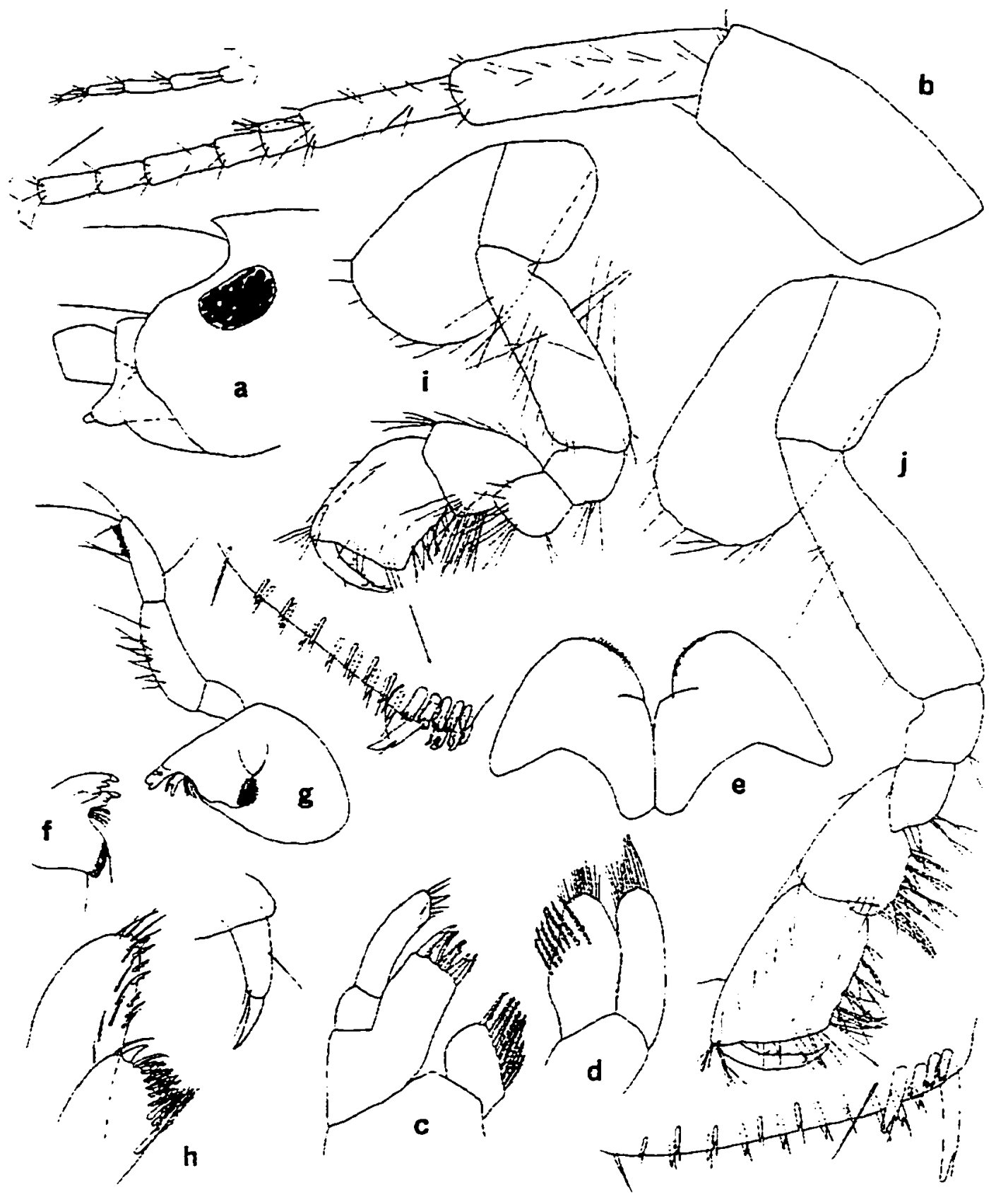

Figure 146.--Crangonyx longidactylus, new species. Female paratype $(9.0 \mathrm{~mm})$. swale. $1.6 \mathrm{~km} \mathrm{E}$ of Bonayr. Barten Co.. Kentucky: a. head (in part): b. antenna 1: c. d. maxillae 1. 2: e. lower lip: f. dentate part of left mandible: g, right mandible: $h$. inner, outer plates and palp dactyl of maxilliped (greatly enlarged): i.j. gnathopods I. 2 (palmar margins enlarged). 


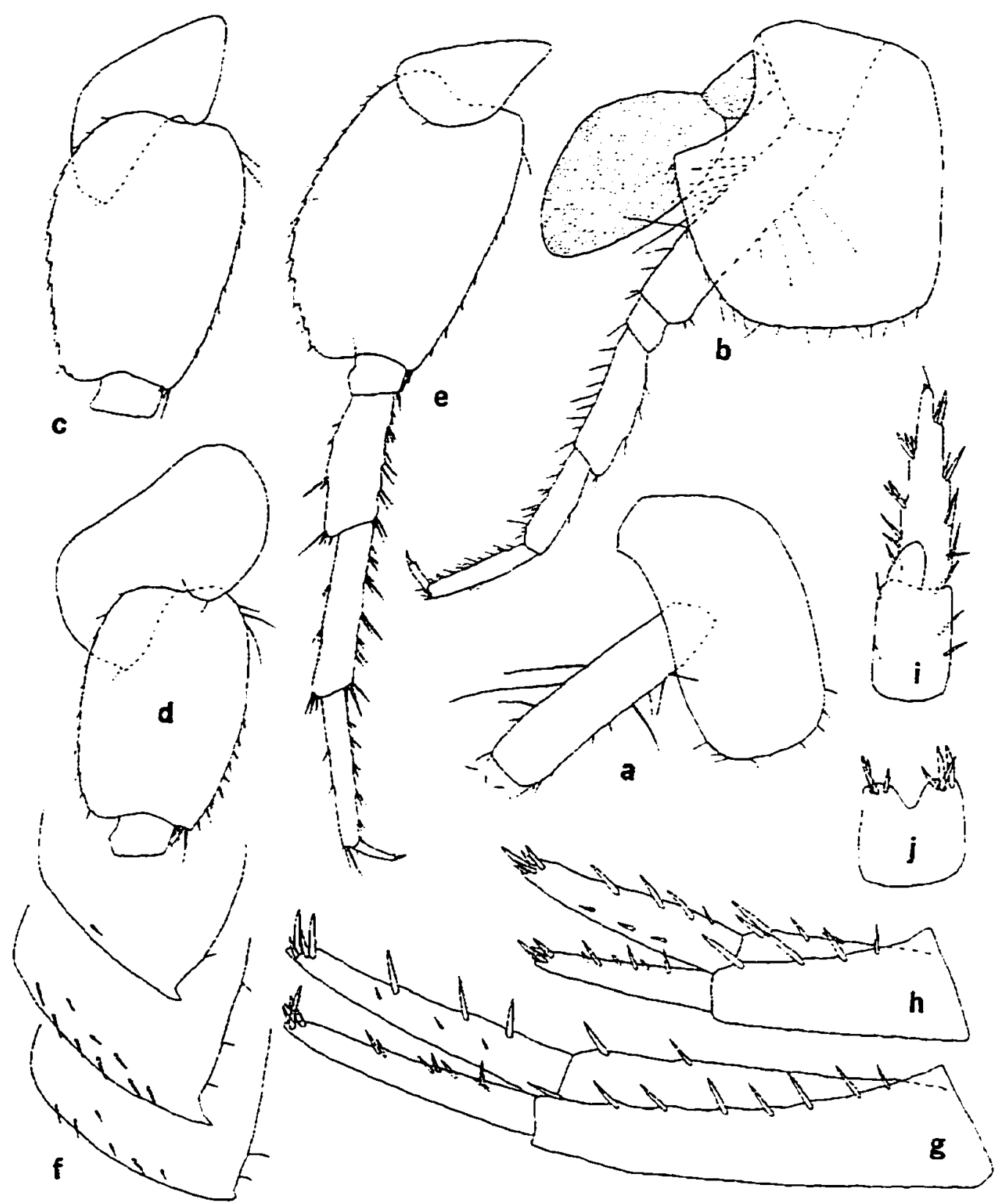

Figure 147.--Crangonyx longidactylus, new species. Fernale paratype $(9.0 \mathrm{~mm})$. swale. $1.6 \mathrm{~km} \mathrm{E}$ of Bonayr. Barren Co., Kentucky: a, c. d. pereopods 3.5.6 (in part): b. e. pereopods 4. 7: f. pleonal plates: g. h. i. uropods 1.2 .3 ; j, telson. 


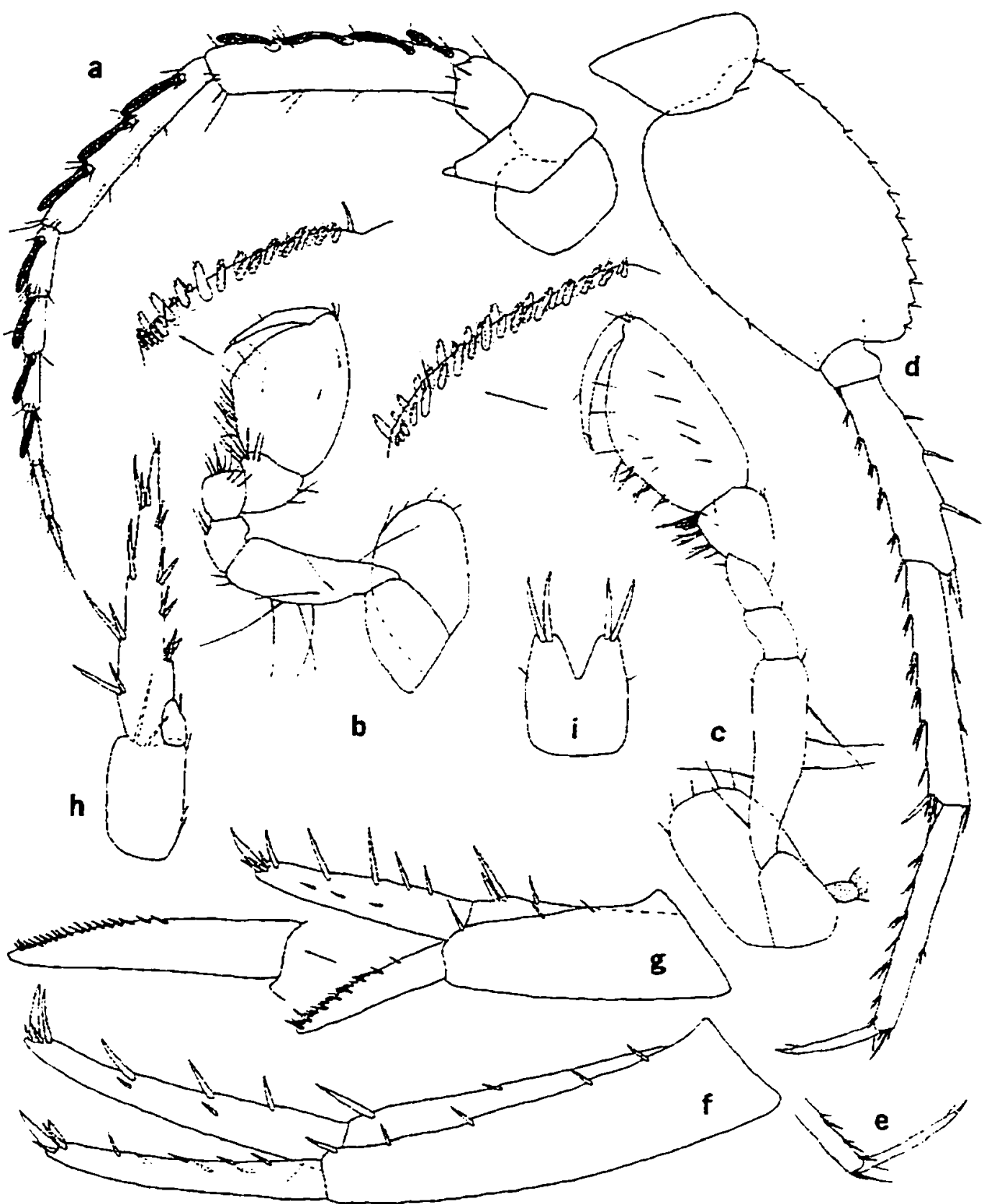

Figure 148.--Crangonyx longidactylus, new species. Male paratype $(5.0 \mathrm{~mm})$. swale. $1.6 \mathrm{~km} \mathrm{E}$ of Bonayr. Barren Co.. Kentucky: a. antenna 2: b. c. gnathopods I. 2 (palmar margins enlarged): d. pereopod 7; e: dactyl of pereopod $5: f, g, h$, uropods $1,2.3$ (comb spine on outer ramus of uropod 2 enlarged): i. telson. 


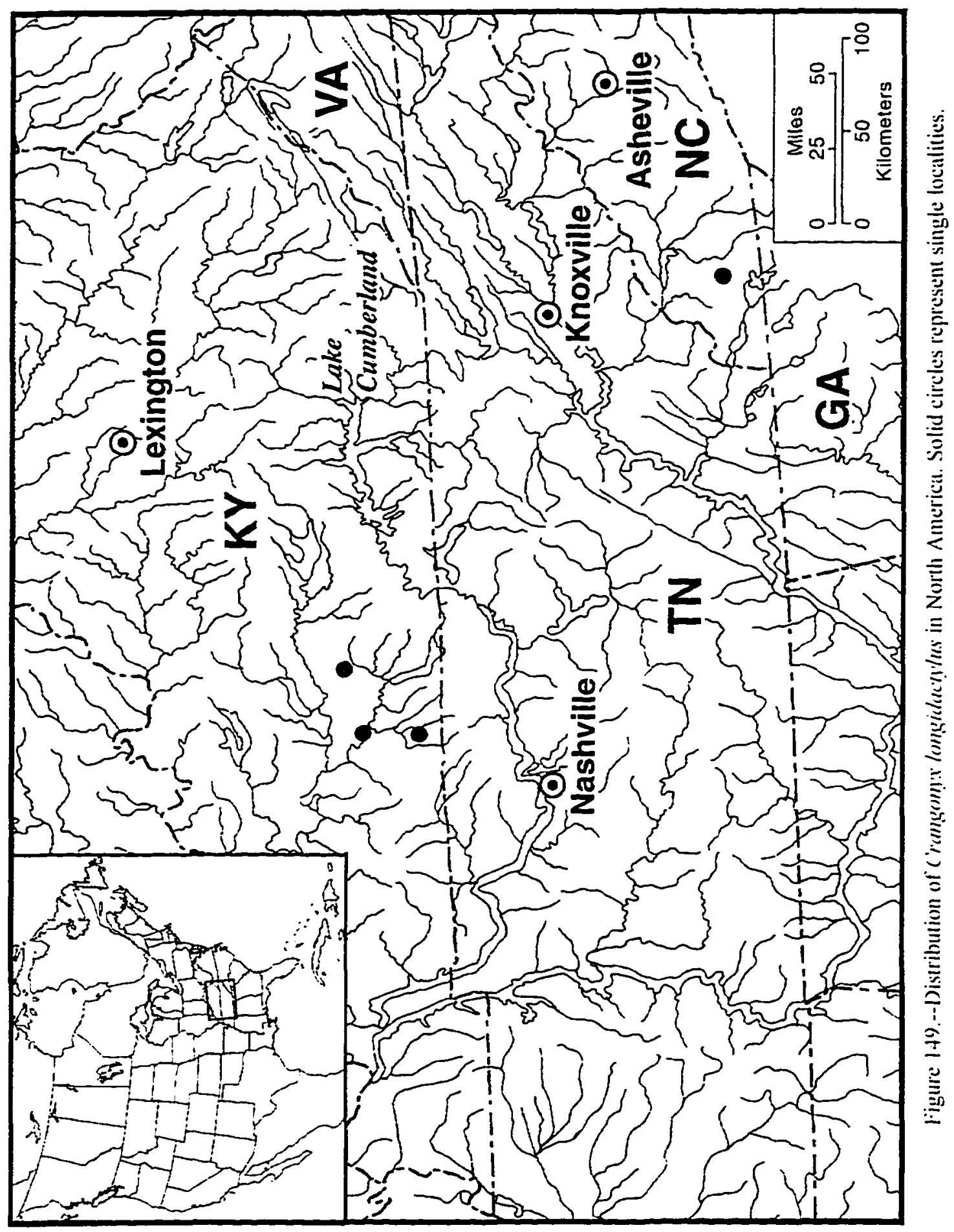


subequal. spine row with 4-5 spines: segment 2 of palp with II setae. segment 3 with 1 A seta. 2 C setae. $3-4$ E setae and a row of D setae. lacking B seta. Maxilla 1: inner plate with 8 apical plumose setae: palp with 6-7 slender spines on apex. Maxilla 2. inner plate with oblique row of 7 plumose setae on inner margin. Maxilliped: inner plate apicaliy with 4 bladelike spines. I naked spine. and 5-6 plumose setae extending from inner margin to apex: outer plate with row of naked setae and 5-6 pectinate spines on inner margin: dactyl with 2 setae on inner margin near nail: dactyl nail long. 1/3 length of dactyl.

Propod of gnathopod 1: propod less than 1.5 times larger and less than 2 times longer than carpus: palm almost straight. slightly shorter than posterior length. with 6 unnotched spine teeth on inside. 8 on the outside: defining angle with 5 small notched-serrate spine teeth on inside. 1 large notched and 3 small serrate small spine teeth on outside: superior medial setae few in number. singly or doubly inserted: about 7 sets of setae on posterior margin. singly inserted or in groups of 2-3. Dactyl with row of 4-5 short setae on inner margin. nail about $1 / 3$ length of dactyl. Ventral margin of coxa I with 9 setae.

Propod of gnathopod 2 less than 1.5 time larger and less than 2 times longer than carpus: palm almost straight or slightly oblique. shorter than posterior margin length. with 5 unnotched spine teeth on inside. 6 on outside: defining angle with I notched and 3 notched-serrate spine teeth on inside: 3 unnotched and 1 large notched spine teeth on outside: superior medial setae singly inserted: posterior margin with 6 sets of setae in groups of 2-8. Ventral margin of coxa 2 with 9 setae.

Coxa of pereopod 3 with 9 marginal setae. Coxa of pereopod 4 with 20 marginal setae. dactyl of pereopod 4. 45 percent length of corresponding propod length. Pereopod 5: basis with $1+$ shallow serrations along posterior margin: 8 short spines on anterior margin: 2 long setae on anteroproximal margin. Pereopod 6: basis with 11 serrations along posterior margin: 9 short spines on anterior margin: 3 long setae on anteroproximal margin. Pereopod 7: coxa with 3 setae on the posterior margin: basis with 19 shallow serrations along posterior margin; 6 short spines on anterior margin: $1-2$ long seta proximally on anterior margin: dactyl long and thin. about 35 percent length of corresponding propod.

Pleonal plates: posterior margin of plate I slightly convex, with I seta. distoposterior corner produced and acute, ventral margin with 1 (subventral) spine: posterior margin of plate 2 nearly straight with 2 or 3 setae, distoposterior corner strongly produced and acute, ventral margin with up to 9 (subventral) spines in 2 rows: posterior margin of plate 3 nearly straight with 2 setae, distoposterior corner weakly produced and subacute. ventral margin with 6 (subventral) spines. Peduncle of pleopod 1 with 2 retinaculae. lacking setae on outside margin.

Uropod 1: inner ramus 70 percent length of peduncle. armed with 10 spines: outer ramus with 11 spines: peduncle with 8 outer and 2 inner spines. Uropod 2: inner ramus armed with 12 spines: outer ramus with 10 spines: peduncle with 4 outer and 4 inner spines. Uropod 3 : inner ramus with 1 spine. outer ramus about 1.9 times longer than peduncle, inner and outer margin each with $3-4$ sets of spines in groups $1-3$. Telson as broad as long. notched about $1 / 4$ distance to base. each lobe with 3-4 apical spines.

Male.--Differing from female as follows. Smaller with more siender, elongate body. Antenna 2: 
calceoli present on peduncular segments 4 and 5 , and first 4 segments of flagellum.

Propod of gnathopod 1 more than 2 times larger and longer than carpus: palm slightly convex and oblique. with II spine teeth on inside. II on outside: defining angle with 4 serrate teeth on inside. I notched and 2 serrate on outside. Propod of gnathopod 2 more than 2 times larger and longer than carpus: palm margin strongly oblique. slightly convex, with 12 spine teeth on inside. 14 on outside: defining angle with 4 notched and 1 notched-serrate spine teeth on inside and 4 spine teeth on outside.

Uropod 2: inner ramus with 12 spines: outer ramus with inner row of comb spines on distal 1:2-2:3. outer margin with 7 spines. apex with 3 spines: peduncle with 3 outer and 3 inner spines.

Type-locality.--A swale. $1.6 \mathrm{~mm}$ E of Bonayr. Barren County. Kentucky.

Distribution and ecology.--The range of this species. which is highly disjunct. is based on 3 localities in south-central Kentucky and I in southwestern North Carolina (fig. 149). It covers a linear distance of about $320 \mathrm{~km}$.

c. Longidactrilus inhabits a swale. a cave spring and two reservoirs. One ovigerous female was found in a March collection. In its type-locality, this species was associated with C. richmondensis. The specimens from the cave spring were lightly pigmented when alive.

Etymology.--The epithet longidactrlus combines the "long" and "dactyl." in reference to the elongate dactyls of pereopods 5-7.

\section{Species Unassigned to Groups}

The eight species treated below have not been assigned to species groups pending further study. Three of them -- Crangonix serratus. C. specus and C. antennatus - have probable affinities with each other. The other five species - $C$. orientalis, $C$. cooperi, $C$. lewisi. C. fontinalis and C. housfieldi -- have affinities with each other and may collectively constitute a species group.

\section{Crangonvx serratus (Embody)}

Figures $150-153$

Eucrangonyx serratus Embody, 1911:299-305. figs. 1-12 [type-locality: spring-fed railroad pond. about 1.5 mi N of Ashland. Hanover Co.. Virginia].--Hubricht \& Mackin. 1940: 194.

Crangonyx serratus (Embody).--Schellenberg, 1936:35.--Hubricht, 1943:689.--Bousfield.1958:72.tig.20.-Holsinger, 1972:46, figs. 12e, 12j. 14, 1977:252.--Van Maren, 1978:48.--Barnard \& Barnard. 1983:435. map 11.--Fitzpatrick. 1983:145.--Biernbaum. 1989:13. 


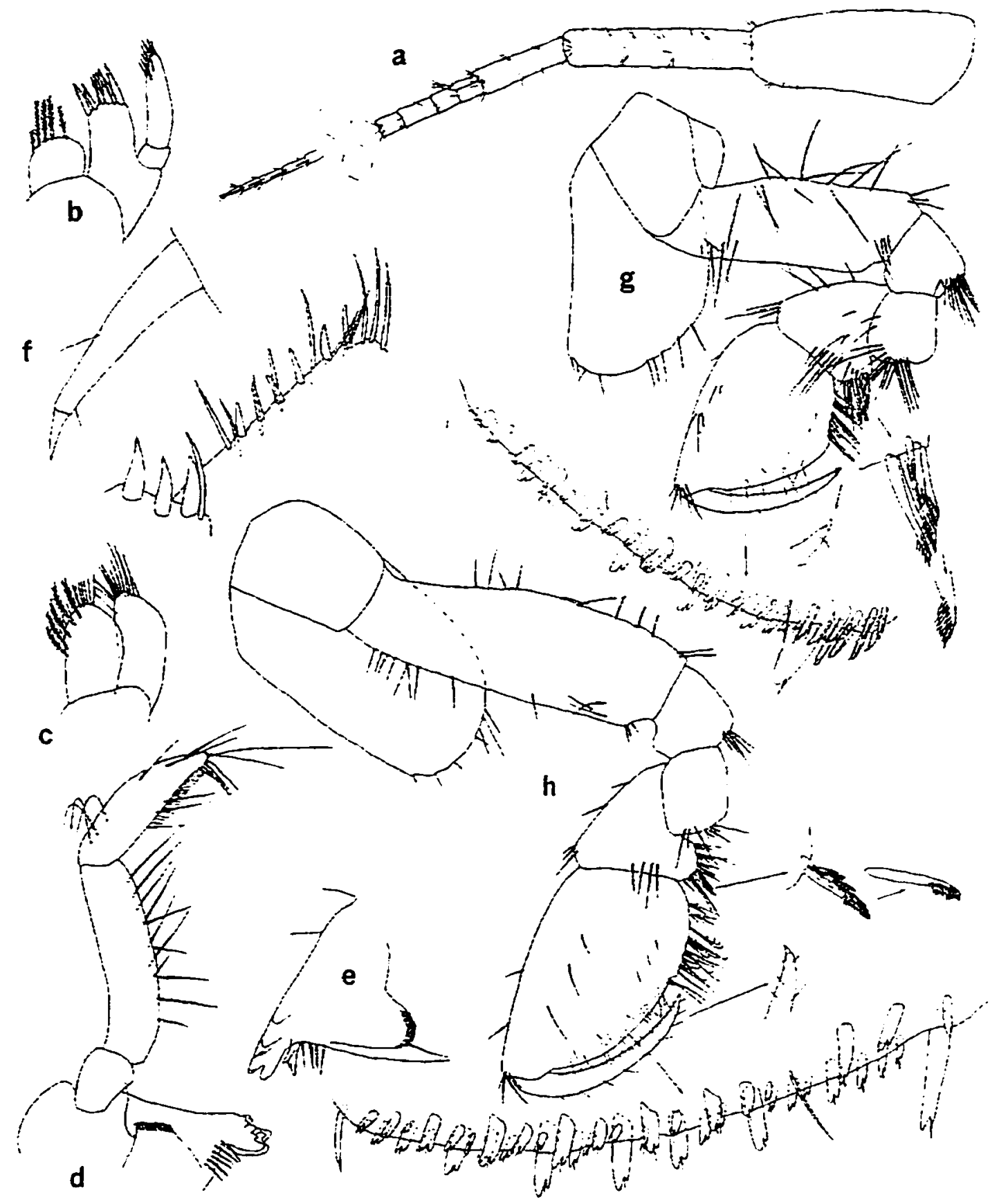

Figure 150.--Crangonx serratus (Embody). Female $(14.5 \mathrm{~mm})$. small stream $0.5 \mathrm{~km} \mathrm{SW}$ of Tabb.

York Co.. Virginia: a. antenna l: b. c. maxillae 1. 2; d. left mandible: e. dentate part of right mandible: $f$. inner and outer plates and palp dactyl of maxilliped (greatly enlarged): g. h. gnathopods 1. 2 (palmar margins enlarged). 


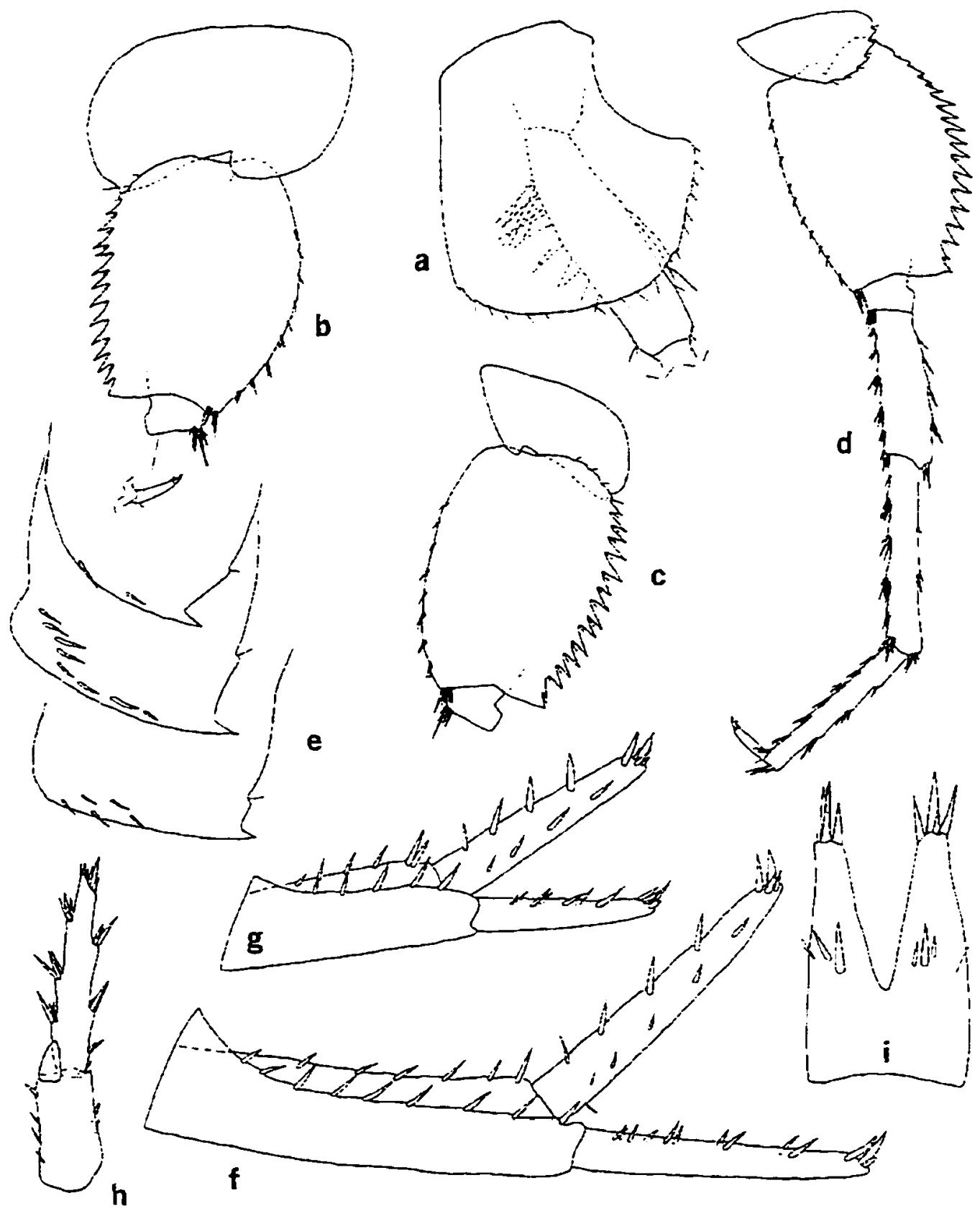

Figure 151.--Crangonvx serratus (Embody). Female (14.5 mm). small stream $0.5 \mathrm{~km} \mathrm{SW}$ of Tabb. York Co.. Virginia: a, b. c. pereopods 4. 5,6 (in part); d. pereopod 7: e. pleonal plates: f. g. h. uropods $1.2 .3:$ i, telson. 


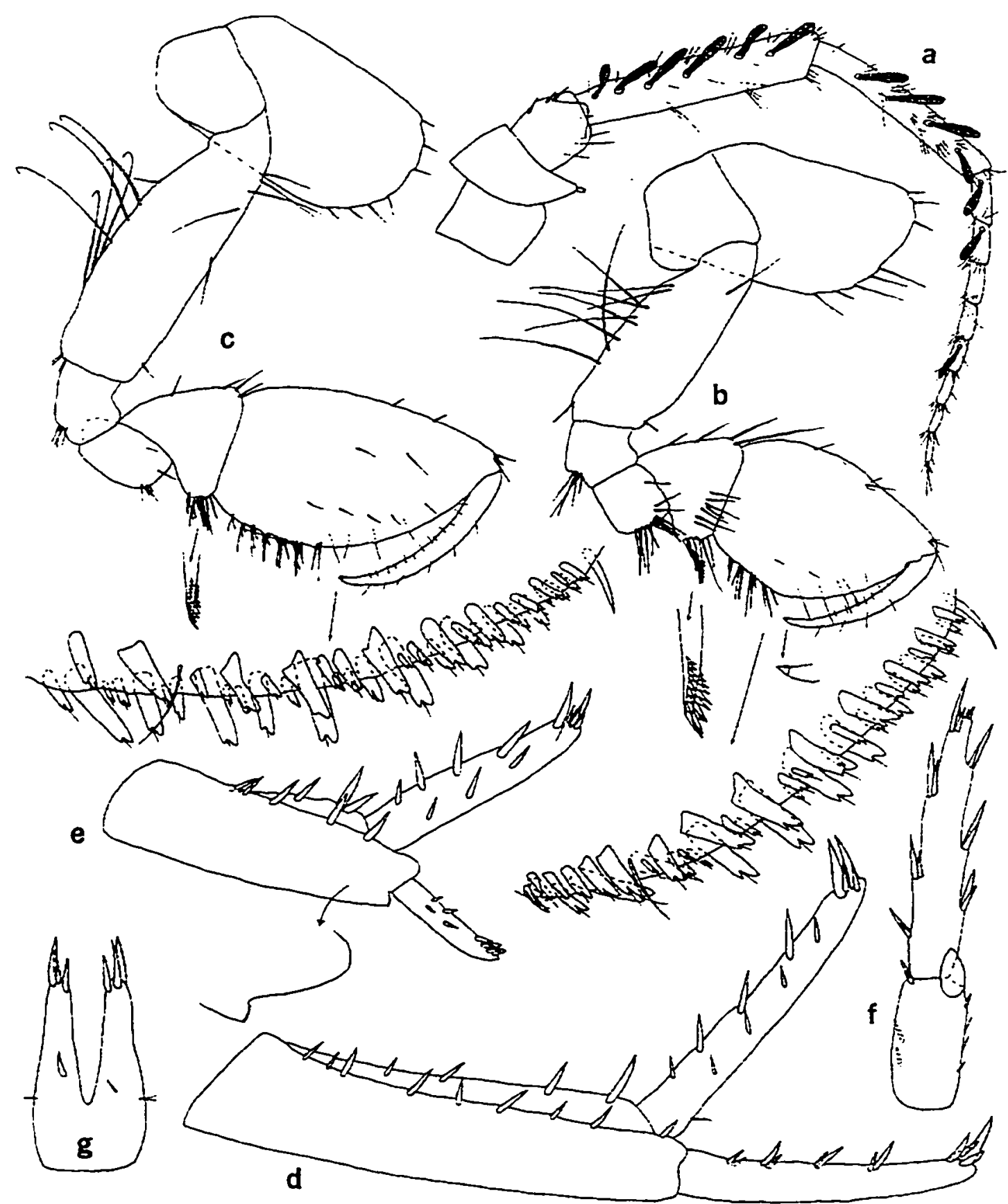

Figure 152.--Crangonyx serratus (Embody). Male $(9.9 \mathrm{~mm})$, small stream $0.5 \mathrm{~km} \mathrm{SW}$ or Tabb. York Co.. Virginia: a. antenna 2: b. c. gnathopods 1.2 (palmar margins enlarged): d. e. f. uropods 1. 2. 3: g. telson. 


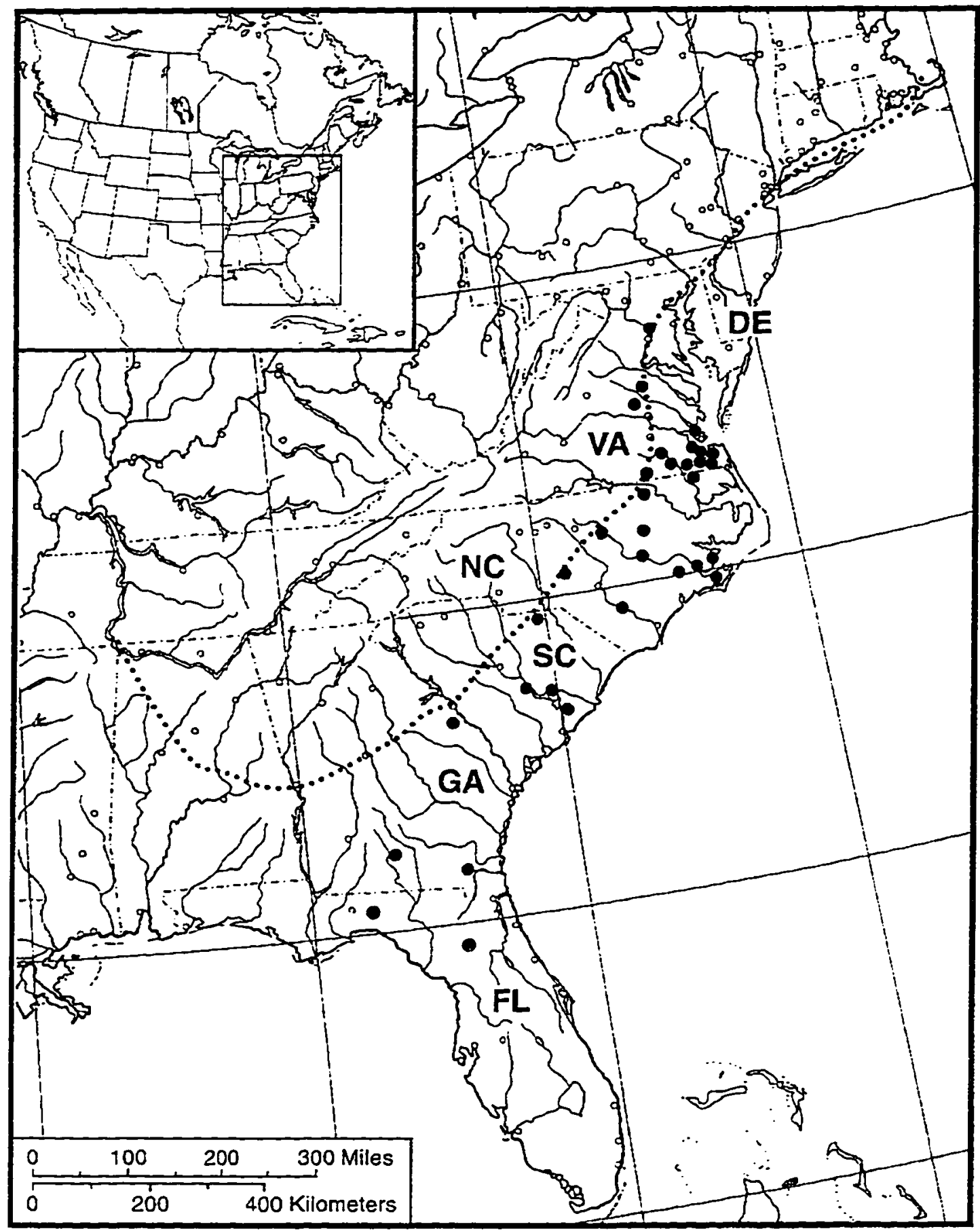

Figure 153.--Distribution of Crangonvx serratus in North America. Solid circles represent 1-5 closely proximate localities. The dotted line denotes the "fall line" or "fall zone" between the Coastal Plain and Piedmont. 
Material examined.-FLORIDA. Alachua Co.: $1 \cong$. Martin. 24 Mar 1927 (USNM): $6 \nsubseteq .5:$ K. Haggart. 15 Feb 1928 (USNM): county not given: 3 ?. J. Martin. habitat and collection date not given (USNM). GEORGIA. Burke Co.: Waynesboro (habitat not given). 1 ?. H. H. Hobbs. Jr.. 30 Dec 1937 (USNM): Cook Co.: swamp at Adel. $1 \%, 10$ juvs. C. K. Shoemaker. (collection date not given) (USNM): Chariton Co.: Okefenokee-Cypress swamp. 18.2 juvs. collector not given. 16 Jul 1942 (USNM). NORTH CAROLINA. Bladen Co.: White Lake, I juv, R. E. Coker. 17 Oct 1937 (USNM), 7 juvs. D. S. Frey. 28 Jul 1947 (USNM): Carteret Co:: pool ca. $16 \mathrm{~km}$ inland from Morehead City. $6 \cong$. N. Hynes. 23 Apr 1978 (USNM): pond ca. $16 \mathrm{~km} \mathrm{~W}$ of Morehead City. 30 specs ( $₫$, $\left.\Xi^{2}\right)$. N. Hynes. 26 Apr 1980 (USNM): cypress pond. Morehead City, 159.30 c. N. Hynes. 21 Apr 1982 (USNM): Broad creek. $8 \subsetneq .1 \subseteq$. R. E. Ashton. Jr. and E. van Riper. 16 Mar 1978: Newport River at St. Rt. 1124. 21 juvs. R. Fox. 9 Aug 1972: Croatan National Forest at Cedar Park Camp between Oak River and St. Rt. 58. $3 \cong$. R. E. Ashton. Jr. and P. S. Ashton. 27 Mar 1978; stream in Croatan National Forest. 8 juvs, R. Fox. 17 May 1972: Craven Co.: Batchelders Creek. $3.8 \mathrm{~km}$ E of Tuscarora, 1 ๆ. L. Hubricht, 10 May 1959 (USNM); Gates Co.: marsh near Hamburg ditch. Dismal Swamp, $\perp \nexists .3 \approx$ J. Matta, 22 Feb 1974: Hamburg ditch and marsh. Dismal Swamp. 5 ₹.1 $\approx$. S. Hetrick. 2 Mar 1974: Halifax Co.: highwater tributary to Little Fishing Creek. ca. 5.1 km ENE of Hollister, 4 ₹. J. E. Cooper and R. E. Ashton. Jr.. 19 Jan 1979 (NCMNH): small tributary to Little Fishing Creek. Medoc Mt. St. Park. $2 £ .1 \approx$. J. E. Cooper and A. L. Braswell. 19 Jan 1979 (NCMNH): Jones Co.: Black Swamp Creek at County Rd 1105. 1 ¿. R. E. Ashton. Jr. and E. van Riper. 16 Mar 1978: Moore Co.: old lake bottom between Southern Pines and Carthage, $35 \Xi .17 z^{*}$. T. K. Ellis. 4 Feb 1940 (USNM): small spring in pine woods. Boyd Estate at Southern Pines. $1 \approx$. collector not given. 7 Mar 1940 (USNM): swamp NE of Southern Pines. $1 \cong$, collector not given. 19 Mar 1940 (USNM): pasture spring and stream. Boyd estate. Southem Pines, $15 \varsubsetneqq .16 \approx$. collector not given. 8 Mar 1940 (USNM): Pamlico Co.: creek $2.1 \mathrm{~km} \mathrm{~W}$ of Grantsboro, $15 \Xi .2 \approx 10$ juvs. L. Hubricht. 23 May I959 (USNM): Wake Co.: Buffalo Creek at bypass 64.4.0 km NW Wendell. 7 ૧. $2 \approx$. A. L. Braswell. 25 Feb 1978: Little River at County Rd 2224, $7.7 \mathrm{~km}$ E of Rolesville. 19 , A. L. Braswell and R. E. Ashton. 17 Nov 1977: Wayne Co.: Buck swamp at St Rt $1120.4 .3 \mathrm{~km}$ W of Dudley. I P. P. S. Freed. 14 Jan 1979 (NCMNH): Wilson Co.: Whitcoak swamp at US Rt. 264. $4.8 \mathrm{~km} \mathrm{NW}$ Saratoga. 3 ₹. P. S. Freed. 4 Feb 1979 (NCMNH). SOUTH CAROLINA. Berkeley Co.: Gough (habitat not given), $25 \Xi .8:$ T. K. Ellis. 23 Dec 1939 (USNM), 9 ₹. 10 \%. T. K. Ellis. I Jun 1940 (USNM): pond at Gough. 4 ₹. T. K. Ellis. II Dec 1939 (USNM): Upper Reserve. Richmond Plantation, 19 9. 8 c. 1 juv. T. K. Ellis. 14 Jan 1940 (USNM): "dammed-up area" on Richmond Plantation. I P. T. K. Ellis. 22 Mar 1939 (USNM): Richmond Plantation Lake, $7 \fallingdotseq$.T. K. Ellis, II Feb 1939 (USNM); Chesterfield Co.: Patrick (habitat not given). $1 \fallingdotseq . J . F$. Hanson. 14 Dec 1956 (USNM); Orangeburg Co.: marshy stream between Paeler and Elloree, $5: 4: T$. K. Ellis. 4 Apr 1940 (USNM): Santee National Wildlife Refuge. 2 . C. K. Biernbaum. 23 Jan 1982 (USNM). VIRGINIA. Caroline Co.: seepage between Swanscorne and Polecat Creek. $2 \equiv . K$. A. Buhimann. I Oct 1991: seep on Catlett Creek. $6 \mathrm{~km} \mathrm{~N}$ of Bowling Green. Ft. A. P. Hill. I juv. P. H. 
Stevenson. 10 Sep 1992: Turkey Track Creek. 7 km NW of Bowling Green. Ft. A. P. Hill. 1 Æ. P. H. Stevenson. 10 Sep 1992: tributary to Permansend Creek. 1 ₹. 1 \&. P. H. Stevenson. 19 Apr 1993: seepage $0.8 \mathrm{~km}$ ESE of Broaddus. 3 ₹. 2 o. P. H. Stevenson. 12 Apr 1993; Chesapeake (Norfolk County): Jericho Ditch near Lake Drummond. 4 9. 5 0", D. H. Hawland. 28 Mar 1972: Washington Ditch at merger with Lake Drummond in Dismal Swamp. 36 specs $\left(q, \sigma^{7}\right.$. juvs), J. R. Holsinger. 5 Oct 1986: Greensville Co.: steep. narrow. swamp stream, $3.7 \mathrm{~km} \mathrm{~S}$ of Sussex Co. line on Rt. 301.1 P. 2 . J. E. and M. L. Cooper. 3 Jan 1965: Hanover Co.: railroad pond $3.2 \mathrm{~km} \mathrm{~N}$ of Ashland. 2 9. L. Hubricht. I Apr 1950 (USNM):

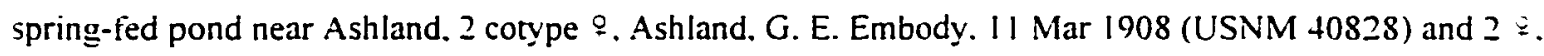
$16 \mathrm{Mar}$ 1909. (collector not given) (USNM): Isle of Wight Co.: cypress swamp. $3.2 \mathrm{~km} \mathrm{NE} \mathrm{Zuni.} 4$ ミ. R. L. Hoffman. 6 Apr 1947 (USNM): Courthouse pond. 2 juvs. R. Bray. 7 Jun 1938 (USNM): seeps $3.2 \mathrm{~km}$ SE of Bartlett. 1 C*. L. Hubricht. 2 A.pr 1944: Nansemond Co.: Jericho Ditch $2.1 \mathrm{~km} \mathrm{SW}$ of Magnolia. 7 . 1 juv. L. Hubricht. 30 Apr 1944 (USNM): Lake Drummond in Dismal swamp. $1 z^{\circ}$. C. R. Shoemaker. 29 May 1922 (USNM): Parkers Pond. Suffolk. 2 ९. R. Bray. 24 Mar 1940 (USNM): stream in swamp. 2.4 $\mathrm{km}$ NE of Saunders. 149.7 \%. L. Hubricht, 21 Nov 1943 (USNM): small stream in swamp $6.4 \mathrm{~km} \mathrm{~W}$ of Suffolk. $33 \Xi .18 \approx$. L. Hubricht. 12 Nov 1944 (USNM): Waleyville. 1 \%. R. Bray. 22 Mar 1940 (USNM): Vorfolk Co.: roadside ditch. Bowers Hill. $9 \approx .15$ C. L. Hubricht. $25 \mathrm{Feb} 1945$ (USNM): roadside ditch. $4.0 \mathrm{~km}$ WSW of Bowers Hill. $139.9 \approx$. L. Hubricht. 21 Nov 1943 (USNM): ditch in Dismal Swamp. 3.2 $\mathrm{km} \mathrm{S}$ of Bowers Hill. $229.5 \approx$. L. Hubricht. 14 Nov 1943 (USNM): stream in swamp. $3.2 \mathrm{~km} \mathrm{~S}$ of North Landing. $5 \bigcirc .20$. L. Hubricht. 9 Apr 1944 (USNM): stream in swamp. $4.8 \mathrm{~km} \mathrm{~S}$ of North Landing. 1 . L. Hubricht. 9 Apr 1944 (USNM): Northwest River. I . L. Hubricht. 25 Mar 1945 (USNM): Southampton Co.: Charles Plantation. Franklin, $59.50^{\circ}$. R. Bray. 22 Mar 1940 (USNM): Franklin. 2 乏. R. Bray. 22 Mar 1940 (USNM): Suffolk (Nansemond County). seep in marsh area in ravine leading to Murphys Pond ca. $4.8 \mathrm{~km}$ NW of Suffolk. I §. J. R. Holsinger. 1 Apr 1984: pond at Viginia Tech agriculture center. Holland. 1 juv. C. Frew. Jun 1992: Sussex Co.: Blackwater River $6.4 \mathrm{~km} \mathrm{~N}$ of Wakefield. $3 \&$. L. Hubricht. 14 Oct 1945 (USNM): Southwest swamp on Rt. 301 just S of Stony Creek. 3 $\Xi$ J. E. and M. E. Cooper, 3 Jan 1965: Warwick Co.: swamp. $0.8 \mathrm{~km} \mathrm{NE}$ of Harpersville. $2 \& .17 \approx 2$ L. Hubricht. 23 Jan 1944 (USNM): York Co.: small stream $0.5 \mathrm{~km}$ SW of Tabb. 59.9 \&. L. Hubricht. 23 Jan 1944 (USNM). WASHINGTON. D. C.: Shaw Lily Pond. Kenilworth, 2 ?. H. S. Barber. 8 Apr 1926 (USNM). 2 juvs. A. Pizzini. 25 Jun 1938 (USNM) and $10^{\circ} .12$ Mar 1941 (USNM). 3 z. $2 \approx$. W. H. Ball. 20 Jan 1934 (USNM) and 1 P, 1 \&. H. S. Barber and C. R. Shoemaker, 10 Apr 1926 (USNM).

Diagnosis.--A relatively large species easily distinguished from all other species of the genus by having very deep serrations along posterior margins of bases of pereopod 5-7: very long and deeply notched telson with mid-dorsal spines: peduncle of uropod 2 of male with unique apical process (not seen in any other species of Crangonvx): and posterior margins of carpus of gnathopods 1 and 2 with strongly developed rastellate setae (not seen in any other Crangonyx but similar to those of Sngobromus). Largest male. $11.0 \mathrm{~mm}$ : largest female. $16.0 \mathrm{~mm}$. 
Female.--Eye small and round. Antenna I, about 50 percent length of body. about 2 times longer than antenna 2: primary flagellum with up to 28 segments. Antenna 2. flagellum with up to 11 segments. Mandibles subequal. spine row with 4-6 spines: segment 2 of palp with $11-12$ long setae. segment 3 with 3 A setae. 2-3 B setae. $3 \mathrm{C}$ setae. 5-6 E setae and a row of D setae. Maxilla l: inner plate with 5 apical plumose setae: palp with 7 slender spines on apex. Maxilla 2. inner plate with oblique row of 5 plumose setae on inner margin. Maxilliped: inner plate apically with 3 bladelike spines, I naked spine. and 5-6 plumose setae extending from inner margin to apex; outer plate with row of naked setae and about 6 slender bladelike spines on inner margin: dactyl inner margin with 1-2 setae near nail: dactyl nail very short.

Propod of gnathopod 1 about 2 times longer than carpus: propod palm little convex. much longer than posterior margin. with 16 spine teeth on inside. 18 on the outside: defining angle with 6 serrate spine teeth on inner side. 2 small and 1 large notched and 4 serrate spine teeth on outside: superior medial setae few in number and inserted in groups of 2-3: posterior margin with about + sets of setae inserted in groups of $1-5$. Dactyl with a row of 11 short setae on inner margin. outer margin with up to 8 setae: dactyl nail very short. Posterior margin of carpus of gnathopod I short. with rastellate setae. Ventral margin of coxa I with 9 setae.

Propod of gnathopod 2 about 2 times longer than carpus; palm convex. oblique. much longer than posterior margin. with 17 spine teeth on inside. 18 on outside: defining angle with 6 spine teeth on outside (the last one very large). 3 small spine teeth on inside: inferior medial setae singly or doubly inserted: superior medial setae singly inserted: posterior margin with 5-6 sets of setae in groups of 3-5. Dactyl with row of up to 16 short setae along inferior margin. outer margin with up to 8 setae: dactyl nail very short. Posterior margin of carpus of gnathopod 2 very short. with rastellate setae. Ventral margin of coxa 2 with 7 setae.

Coxa of pereopod 3 with 12 marginal setae. Coxa of pereopod 4 with 18 marginal setae. dactyl of pereopod 4 about 40 percent length of corresponding propod. Pereopod 5: basis with 13 very deep serrations on posterior margin: 9 sets of short spines in groups of $1-2$ on anterior margin. Pereopod 6: basis with 15 very deep serrations along posterior margin: 8 sets of short spines on anterior margin. Pereopod 7 : coxa with about 6 setae along the posterior margin in deep serrations: basis with 16 very deep serrations along posterior margin: 10 sets of short spines on anterior margin: dactyl about 33 percent length of corresponding propod.

Pleonal plates: posterior margin of plate I convex, with I seta. distoposterior corner strongly produced and acute. ventral margin with 2 (subventral) spines; posterior margin of plate 2 nearly straight with $1-2$ setae. distoposterior comer strongly produced and acute, ventral margin with 8 (subventral) spines: posterior margin of plate 3 uneven. with I seta, distoposterior corner strongly produced, and acute. ventral margin 5 (subventral) spines. Peduncle of pleopod 1 with 2-3 retinaculae, lacking setae on outer margin.

Uropod 1: inner ramus 65 percent length of peduncle. armed with about 13 spines: outer ramus with 
about 13 spines: peduncle with 9 outer and 6 inner spines. Uropod 2: inner ramus armed with about 13 spines: outer ramus with about 12 spines; peduncle with row of 5 outer and 6 inner spines. Lropod 3 : inner ramus lacking spines. outer ramus about 2 times longer than peduncle, inner and outer margin each with 3 4 set of spines in groups of 2-3. Telson much longer than broad. notched about $2 / 3$ distance to base. each lobe with $3-4$ apical spines and $1-3$ mid-dorsal spines.

Male.--Differing from female as follows. Smaller with more slender. elongate body. Antenna 2: calceoli present on peduncular segments 4 and 5 , and first 5 segments of flagellum.

Propod of gnathopod I palm with 16 spine teeth on inside. 16 notched spine teeth on outside: defining angle with $3-4$ notched and 1 serrate spine teeth on inside and 3 notched and 2 serrate spine teeth on outside. Propod of gnathopod 2: defining angle with 2 spine teeth on inside and 5 notched spine teeth on outside.

Uropod 2: inner ramus with II spines: outer ramus much smaller than inner ramus, deflected laterally. with about 9 spines. + or 5 closely spaced near apex: peduncle with 4 outer spines. 4 inner spines. and bearing bluntly rounded apical process.

Distribution and ecology.--The range of this species extends from Washington. DC south along the Cuastal Plain to northern Florida (fig. 153). Crangonyx serratus is an inhabitant of small bodies of water. including ponds. streams. and drainage ditches. Ovigerous female are recorded from November to June (Holsinger. 1972)

Crangonnx antennatus Packard

Figures 154-157

Crangomix antennatus Packard. 1881:880, fig. 2 [type-locality: Nickajack Cave. Marion Co.. Tennessee]:

1888:36. fig. 5.--Shoemaker. 1942:16-20, figs. 5. 6.--Hubricht. 1943:690.--Nicholas.1960:127.--

Holsinger.1963:29: 1969:25: 1972:34: 1977:252: 1986:97.--Holsinger \& Peck. 1971:29.--Barnard \&

Barnard. 1983:434.map 11.--Fitzpatrick. 1983:145.

Eii:rangonyx antennatus Stebbing. 1899:423: 1906:388.

Viphargus antennatus Hay. 1902:430. fig. 6.--Weckel. 1907:36. fig. 6.--Schellenberg. 1936:33

Material examined.--ALABAMA. Blount Co.: Catfish Cave. 4 . J. R. Holsinger et al.. 15 jun 1967: Calhoun Co.: Erby Cave. 10 ₹. 1 juv. S. B. Peck. 3 Sep 1968: Green Valley Cave. $1 \approx^{*}$. G. McCluskey and L. Guy. 23 Jun 1968: De Kalb Co.: Goat House Cave. 2 ๆ. T. Iles. late 1968: Manitou Cave. $24 \bar{Y} .8=$. L.

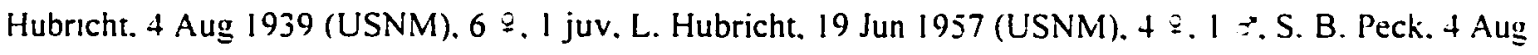
1965 and $6 \mp .25$ Aug 1965: Sequoyah Cave. 1 ₹. S. B. Peck. 9 Aug 1965 and $13 \nexists .2 \approx .1$ juv. 25 Aug 1965: Steward Spring Cave. $3 \varsubsetneqq$. R. Graham et al.. 22 Jun 1968: Jackson Co.: Blowing Cave. $+₹$. 1 juv. L. 


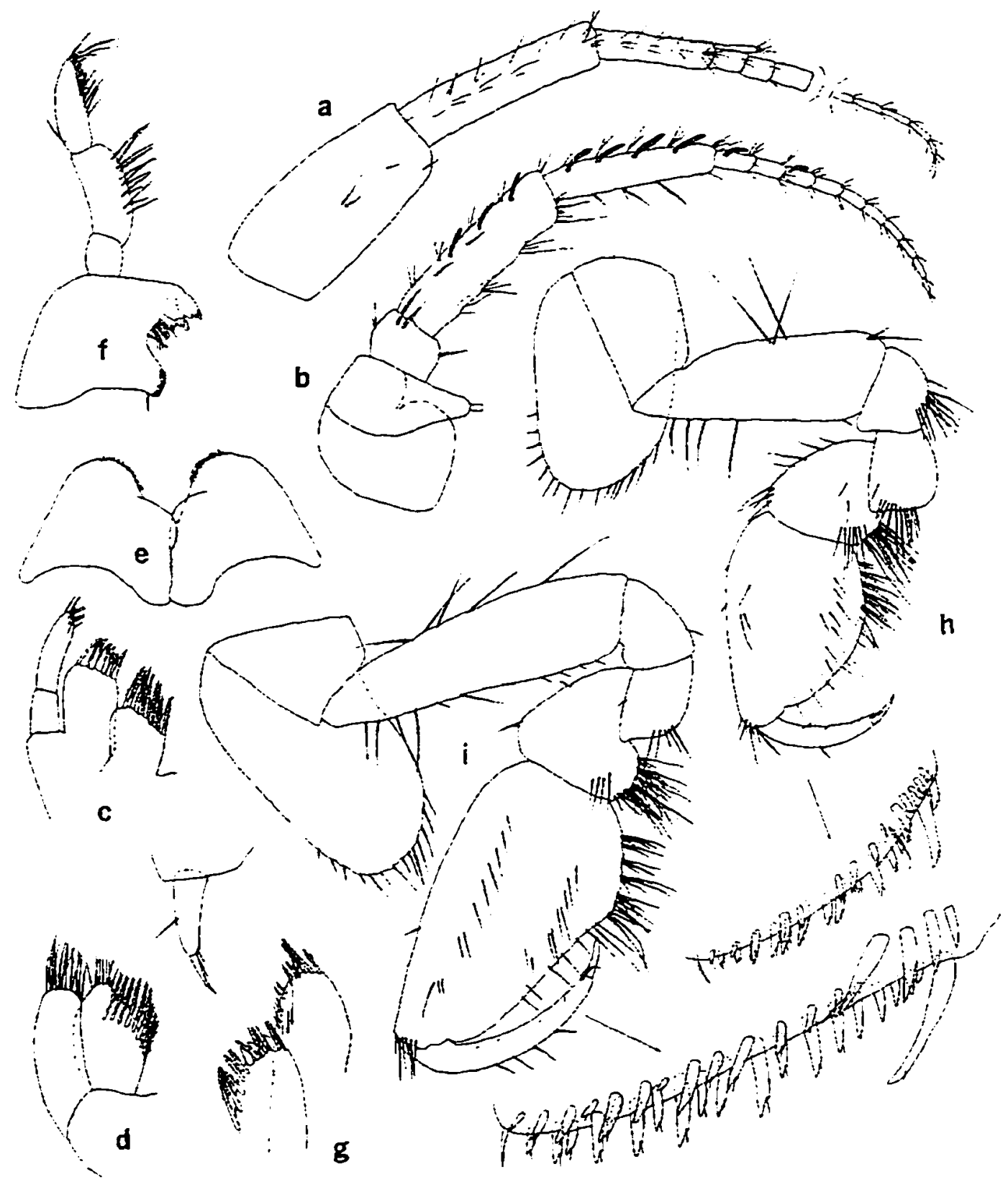

Figure 154.--Crangonnx antennatus Packard. Female $(9.8 \mathrm{~mm})$. Subers Cave. Hancock Co..

Tennessee: a. b. antennae 1. 2: c. d. maxillae 1. 2: e. lower lip: f. left mandible: g. inner and outer plates and palp dactyl (greatly enlarged): h. i. gnathopods I. 2 (palmar margins enlarged). 


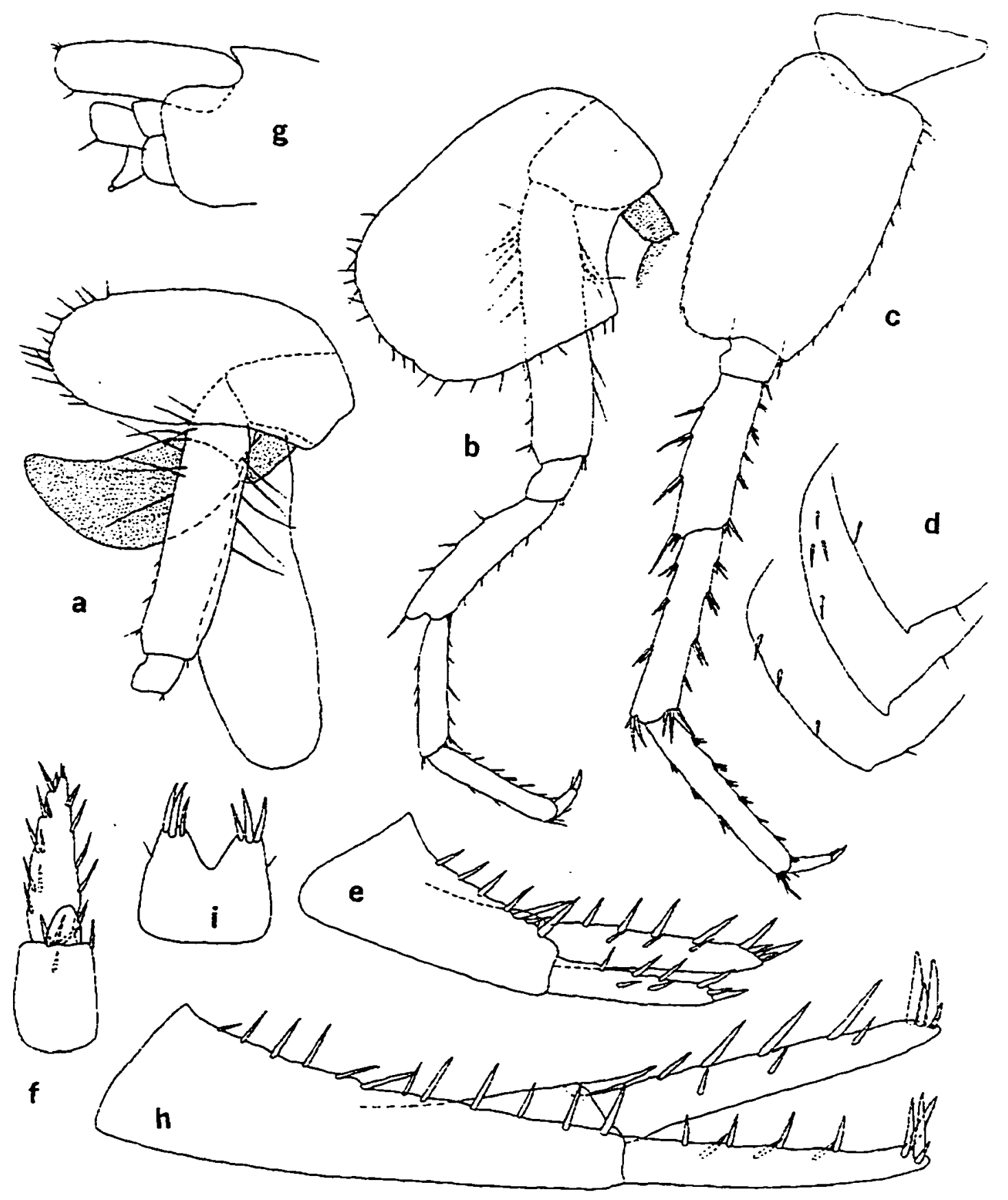

Figure 155.--Crangonyx antennatus Packard. Female $(9.8 \mathrm{~mm})$. Subers Cave. Hancock Co..

Tennessee: a. pereopod 3 (in part): b. c. pereopods 4, 7: d. pleonal plates: e. f. uropods 2. 3. Female $(8.0 \mathrm{~mm})$. Secret Cave. Lee Co.. Virginia: g. head (in part): h. uropod $1: \mathrm{i}$ telson. 


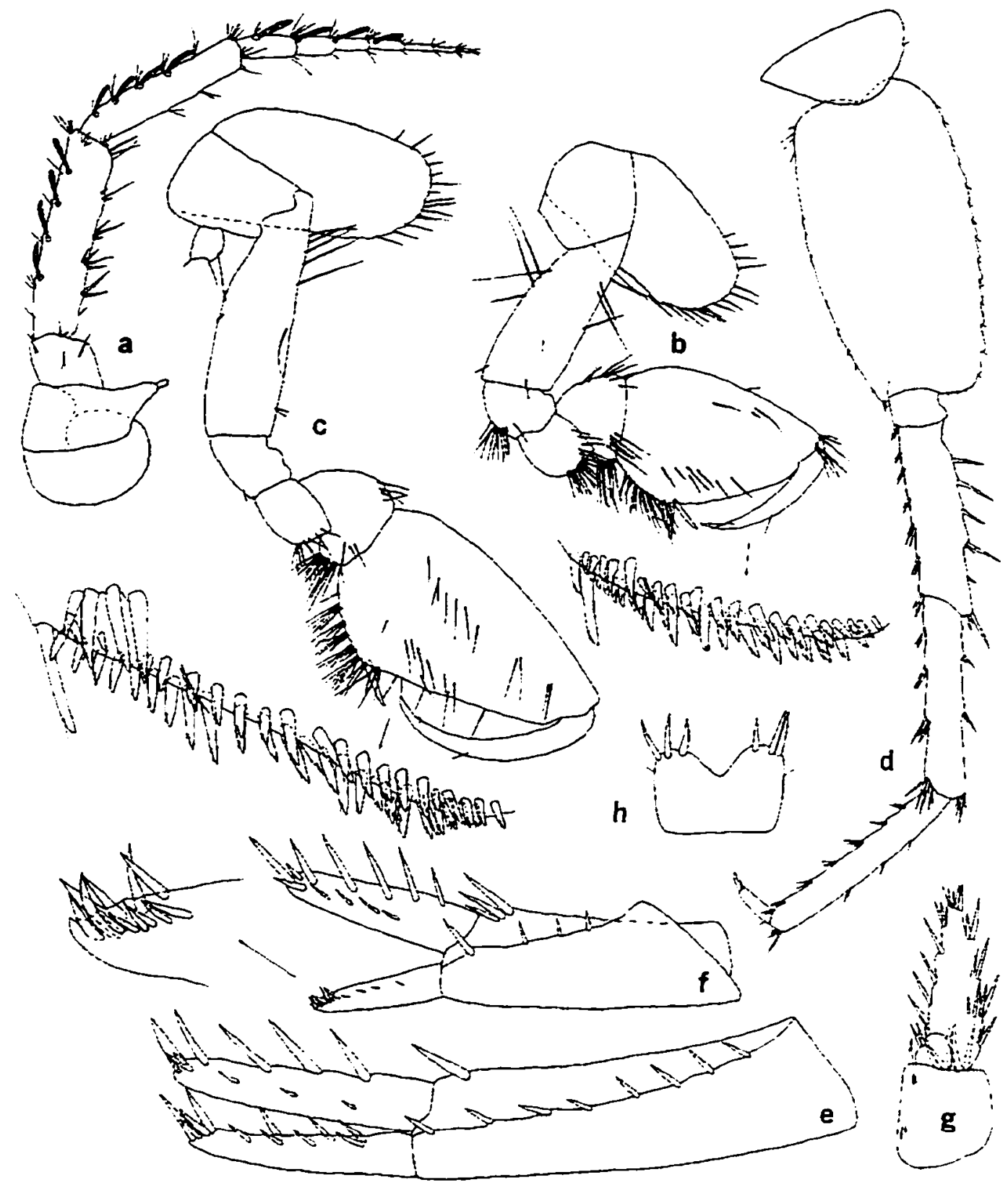

Figure 156.--Crangonyx antennatus Packard. Male $(10.0 \mathrm{~mm})$. Subers Cave. Hancock Co..

Tennessee: a, antenna 2: b. c. gnathopods 1. 2 (palmar margins enlarged): d. pereopod 7: e. g. uropods I. 3: f. uropod 2 (apex of outer ramus enlarged); h. telson. 
Hubricht. 2 I Jul 1939 (USNM): Dry Cave. I 2. R. Graham and J. Wilson. May 1970: Iron Hoop Cave, 3 こ. A. Grubbs et al.. 8 Oct 1988: Jess Elliott Cave, 2 9. S. B. Peck, I Aug 1965 and I ₹. J. E. and M. R. Cooper, 23 Sep 1967; McFarlands Cave. $2 \%$, M. R. and J. E. Cooper. 14 Apr 1965 and $4 \cong .3 \approx$. S. B. Peck. 20 Jul 1965: Russell Cave, I \&. H. H. Hobbs. III et al., 26 Aug 1992 and 2 ?. 28 Aug 1992 and I. S. B. Peck. collection date not given: Jefferson Co.: Alabama Crystal Cave. 4 spes.. S. B. Peck. 18 Mar 1966: Mc Cluvey Cave. 1 \&, S. B. Peck. 23 Jul 1965: Marshall Co.: Beech Spring Cave. 3 s. S. B. Peck. 9 Sep 1965 and 3 \%. J. Wilson. Jul 1969: Eudy Cave. $2 \%$. J. R. Holsinger et al.. 19 Mar 1964: Kirkland Cave. 3 \&. R. Graham. 9 Dec 1967: Guffeys Cave. 1 \%. K. A. Buhlmann. 30 Oct 1993 and 7 ?. 1 ๑. K. A. Buhlmann. 5 Nov 1994: Old Blowing Cave, 32.1 c. S. B. Peck and A. Fiske. 27 Jun 1967: War Eagle

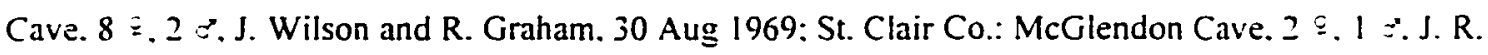
Holsinger et al.. 15 Jun 1967. GEORGIA. Chatooga Co.: Blowing Spring Cave. 3 丹. J. R. Holsinger et al.. 11 Jun 1967: Dade Co.: Byers Cave. $3 \& .3<$. J. R. Holsinger et al.. 13 Jun 1967: Chamblish Cave. $8 \cong$. H. H. Hobbs. III et al., I Sep 1992: Howards Waterfall Cave. I ̊. J. R. Holsinger et al.. 17 Mar 1966: Sittons Cave. 13 9. 6 c. S. B. Peck. 2 Jul 1965 and 4 spec.. K. A. Buhlmann. 17 Sep 1995: Floyd Co.: Cave Springs Cave. $5 \geq .1$ 1 . L. Hubricht, 25 Jul 1939 (USNM) and $2 \mp .1 \approx .2$ juvs. J. R. Holsinger et al.. 13 Jun 1967: Walker Co.: Anderson Spring Cave. 1 o'. I juv. K. A. Buhlmann. 30 Jul 1995 and 3 spec.. 6 Aug 1995: Fricks Cave. 17 spec.. K. A. Buhlmann, 16 Sep 1995: Harrisburg Cave. 1 ฐ. S. B. Peck and A. Fiske. 2 Jul 1967: Horseshoe Cave. 1 Z. I o. J. R. Holsinger et al.. 10 Jun 1967: Mt. Cove Farm Cave. 1 三. $2 \therefore$ S. B. Peck and A. Fiske. 20 Jun 1967: Pettijohn Cave. $1 \subsetneq .1 \approx 1$ juv. J. R. Holsinger et al.. 10 Jun 1967 and 2 q. 1 \%. K. A. Buhlmann. 15 July 1995. TENNESSEE. Anderson Co.: Burress Spring. $7 \cong .4:$ 1 juv. R. M. Norton et al.. 14 Jul 1964; Hill Cave. I o. T. C. Barr. Jr., 28 Sep 1979: Wrights Cave. $1 \geq$. J. A. Payne, 5 Jun 1965: Bledsoe Co.: Aaron Tolletts Cave. 8 \%. l c. J. R. Holsinger. 6 Sep 1964 and $1 \fallingdotseq .1$ $\therefore$ J. R. Holsinger and J. Hixon. 13 Nov 1967; Campbell Co.: Meredith Cave. $2 \equiv .1$. J. R. Holsinger and D. C. Culver. 21 Aug 1972: Claiborne Co.: Blue Moon Cave. 39.1 juv.. S. Smith and C. Holler. 19 Oct 1996: Buis Saltpetre Cave. $4 \cong .1 \Xi * 1$ juv. J. R. Holsinger and D. C. Culver. 19 Aug 1972: Chadwells Cave. $3 \fallingdotseq$. D. Finley. 19 Nov 1963. $19.4 \approx *$ J. R. Holsinger et al.. I Mar 1964 and $6 \supsetneq .6 \approx^{*}$ J. R. Holsinger and C. Rippy. 17 Jul 1965: English Cave. 5 ९. G. P. Engelhardt. 27 Jul 1924 (USNM) and $5 \varsubsetneqq$. $7 \approx$. J. R. Holsinger and C. Rippy. 17 Jul 1965: Hauser Spring Cave. 4 . 1 ¿. D. Finley. Mar 1965: John Lard Cave. 1 o. J. R. Holsinger and D. C. Culver. 20 Aug 1972: Kings Saltpetre Cave. $3 \nexists .3: 2$ juvs. J. R. Holsinger. 24 Nov 1973: Roses Spring at Station Creek Cave. 1 . $1 \approx$ D. C. Culver. II Aug 1973: Station Creek Cave. I juv. J. R. Holsinger, II Aug 1973: spring in Claiborne County (no other data). $17 \equiv$. $1 \propto$. R. Fox and L. Fleming, 12-13 Dec 1970; Cumberland Co.: Run-To-The-Mill Cave. $1 \approx 2.1$ Juv. A. Grubbs. Dec 1985: Decatur Co.: Baugus Cave. 1 \%. D. L. Bechler. 16 Jul 1973: Salt River Cave. 1 I. R. A. Brandon. 27 Nov 1964: Grainger Co.: Horseshoe Cave. $3 \cong .2 \approx$ J. R. Holsinger and D. C. Culver. 23 Aug 1972: Hamilton Co.: Lookout Mountain Cave. 1 juv. J. R. Holsinger et al., 12 Jul 1983: 40-foot well at Chattanooga. $3 \subsetneq .3$ c’. I juvs. R. S. Walker. Jan 1944 (USNM): Mystery Falls Pit. $2 \varsubsetneqq$. H. H. Hobbs. III et 
al. 14 Dec 1992: Hancock Co.: Cantwell Valley Cave. 2 o. J. R. Holsinger and C. W. Maus. 28 Oct 1966: Fairmont School Cave, 2 9. J. R. Holsinger et al., $15 \mathrm{Mar} 1964$ and $3 \subsetneq .12 .1$ juv. J. R. Holsinger and D. C. Culver. 23 Aug 1972: Subers Cave, 1 \&. 3 E*. 1 juv, J. R. Holsinger and D. C. Culver. 25 Aug 1972: Hawkins Co.: Pearson Cave. 9 \&. 1 c J. J. R. Holsinger. 15 Apr 1967 and 4 ₹. J. R. Holsinger et al.. 17 Jul 1979: Knox Co.: Meads Quarry Cave. I 9 . D. Simmons. II Aug 1974: spring run beside Rt. 44. ca. 1.6 $\mathrm{Km}$ S of Knox-Anderson county line. 129.6 \&. R. Fox et al. 4 Jun 1971: Marion Co.: Nickajack Cave. 2 ఏ 1 juv. W. P. Hay, 6 Sep 1901 (USNM), 1 ₹. L. Hubricht. 7 Aug 1939 (USNM). 2 juvs. R. M. Norton. 1

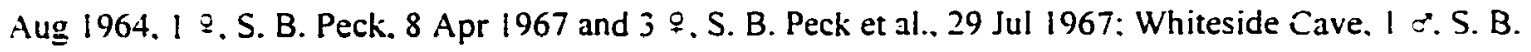
Peck. 29 Aug 1968: Meigs Co.: Sensabaugh Cave. 2 9. R. M. Norton, 4 Jan 1967: Mcmninn Co.: small Cave. $3 \approx$. R. M. Norton. 4 Jan 1967: Rhea Co.: Dayton Quarty Cave. 1 \&. R. M. Norton. 3 Jul 1967 : Grassy Creek Cave. 1 ९. R. M. Norton. 3 Jul 1967: Roane Co.: Berry Cave. 5 §. 2 juvs. R. M. Norton. 15 Jul 1964: Cave Creek Cave. 1 I. R. M. Norton. 15 Jul 1964: Sullivan Co.: Morrills Cave. $1 \cong$. L. Hubricht.

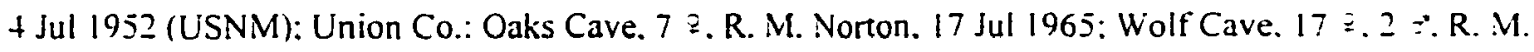
Norton. 6 Jan 1965: Wrights Cave. 1 . collector unknown. 31 Dec 1963: Washington Co.: Clarks Cave. 3 $§ .1$ Juv. J. R. Holsinger, 8 Jun 1968: Wayne Co.: Sheep Cave. $59.2 \approx$. T. C. Barr. Jr. and R. M. Norton. 17 Jun 1967. VIRGINIA. Lee Co.: Baileys Cave. 19.3 . J. R. Holsinger and R. Baroody. 29 Nov 1969 and $3: 2$ juvs. J. R. Holsinger and D. C. Culver, 30 Jul 1974: Bowling Cave. $1 \fallingdotseq$. J. R. Holsinger et al.. 28 Jul 1967; Barney Cave, 9 specs. D. A. Hubbard. Jr. 22 Mar 1994: Burial Cave. $1 \cong .1 \Xi 1$ juv. D. A. Hubbard. Jr.. 7 Apr 1993: Cavin Cave. 5 specs.. D. A. Hubbard et al.. 29 Nov 1996: Cave Springs Cave. 2 juvs. J. J. Lewis. 22 May 1977: Cedar Hill Cave. 4 2. 2 C. J. R. Holsinger and D. Powers. 5 Aug. 1969: Chances Cave. $7 £ 2$ juvs. J. R. Holsinger and D. Powers. 6 Aug 1969: Combs Cave No. 1. $10 \cong .2$ juvs. J. R. Holsinger and D. Powers. 8 Aug 1969: Cope Cave. $8 \cong .6 \approx$. J. R. Holsinger. 23 Aug 1969: Crouse Cave. I . J. R. Holsinger, 16 Aug 1960 (USNM) and I 9.4 C. J. Holsinger et al.. I Mar 1964: Cudjos Cave. $15 \Sigma, 11 \approx * 1$ juv, L. Hubricht, 28 Aug 1939 (USNM) and $1 \approx n$. J. R. Holsinger. 28 Nov 1968: Cumberland Gap Saltpetre Cave, 1 ミ. J. R. Holsinger et al.. 15 Jul 1979: Flanary Bridge spring. 8 km SW of Jonesville. 1 juv, J. R. Holsinger et al.. 24 Nov 1994: Frazier Cave. 6 ९. 1 Æ. J. R. Holsinger and D. C.

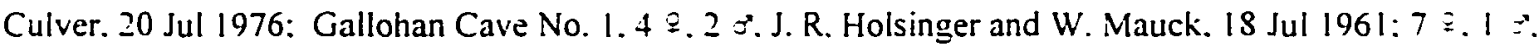
! P. Holsinger. 26 Jun 1963. 2 9. J. R. Holsinger and D. Finley. 10 May 1964 and $3 \varsubsetneqq .18$ Mar 1966: spring near (outside) entrance of Gallohan Cave No. I. $1 ₹ .1 \approx$. J. R. Holsinger. 18 Mar 1966: spring fed concrete basin above entrance to Gallohan Cave No. 1. 4 ๑. J. R. Holsinger et al. 26 Nov 1994: Gallohan Cave No. 2. 3 ․ J. R. Holsinger and W. Mauck. 18 Jul 1961. 39.1 c.. 1 juv. J. R. Holsinger and D.

Finley. 16 Aug 1965 and I 9, G. Dickson et al.. 28 Nov 1974: Garretts Cave. 3 Q. J. R. Holsinger and R. Schultetus. 24 Jul 1966: Gibson Cave. 3 \&. J. R. Holsinger and D. C. Culver. 21 Jul 1976 and $2 ₹ .1=: 1$ juv. D. A. Hubbard. Jr., 2 Nov 1993: Gibson-Frazier Cave. 2 P. $1 \approx^{\circ}$. J. R. Holsinger and D. Finley, 16 Aug 1965: Glen Olingers Cave. 1 ค. J. R. Holsinger. 5 Aug 1969: Golf Course Cave No. 1. 3 \&. 1 . D. C. and N. Culver. 18 Dec. 1973: Golf Course Cave No. 2. 1 ₹. 1 З. J. R. Holsinger and D. Powers. 6 Aug 1969: 
Gregorys Cave. 7 ₹. 3 ఠ. J. R. Holsinger et al.. 23 Jul 1966: Hamblin School Cave. $2 \leftleftarrows .2 \approx *$ K. A. Buhlmann and R. Reynolds. 6 Oct 1992: Indian Burial Cave. 4 ?. 2 . I juv. D. A. Hubbard. Jr.. I Jan

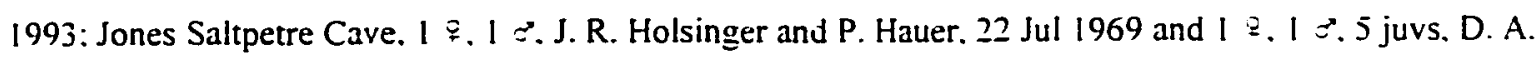
Hubbard. Jr., 25 Nov 1994; Knapper Cave, 5 ๑, J. R. Holsinger, 14 Apr 1968: Lesters Cave. $4 \geq .1: 4$ juvs. J. R. Holsinger et al. 27 Jul 1975: Litton Cave No. 1. $169.5 \odot .17$ Aug 1965 and 4 ๑. $1 \approx$ J. R. Holsinger. 20 Jan 1968: Longs Cave. $29.1 \%$. D. A. Hubbard. Jr., 29 Sep 1993: Lucy Beatty Cave. $10 \cong$. 3 juvs. J. R. Holsinger and D. Martin. Aug 1962 and $3 \cong .2$ ๔. J. R. Holsinger. 9 Mar 1968: McClure Cave. $4 \mp .6 \approx$ J. R. Holsinger et al.. 27 Nov 1971: McCurry Indian cave. 10 specs. D. A. Hubbard. Jr.. 6 Apr 1993: Minors Saltpetre Cave. I ․ J. R. Holsinger and P. Hauer. 29 Nov 1968: Molly Wagle Cave. specimens collected by J. R. Holsinger and asseciates as follows $-209.7=0$. 14 juvs. 18 Aug 1965. $10 \cong$.

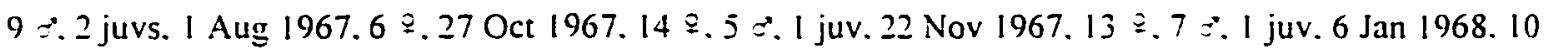

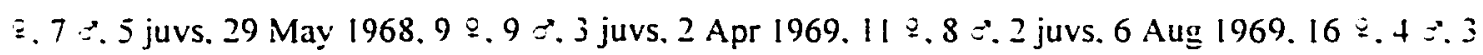

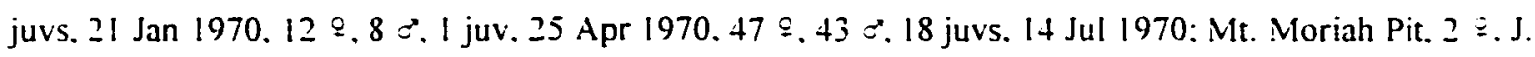
R. Holsinger. 18 Aug 1965: Olinger Cave. 1 \%. J. R. Holsinger and W. Mauck. 17 Jul 196I and 4 ₹. J. R. Holsinger and D. C. Culver. 24 Aug 1972: Reasors Cave. 3 specs. D. A. Hubbard. Jr.. I 8 Jul 1994: Seal Cave. 6 : $2 \approx$ J. R. Holsinger and D. Finley. 10 May 1964: Secret Cave. $7 \equiv .3 \approx$. D. A. Hubbard. 22 Nov 1990: Sims Spring. I juv, J. R. Holsinger et al.. 15-17 May 1990. 3 specs.. C. Hobson et al. I Dec 1996: Sims Spring complex. 6 specs.. C. Hobson et al.. I Dec 1996: shallow spring in "Cedars". 450 meters ENE of Sims Spring, 1 \%. J. R. Holsinger and K. Buhlmann, 24 Nov 1995: Slemp Cave. 29.20 Aug 1969: Smiths Milk Cave. $1 \Xi .2 \approx$ J. R. Holsinger and D. Martin. 17 Aug 1962 and $2 \geqq .1 \because$ J. R. Holsinger. 27 Oct 1967: Spangler Cave. $13 \nsubseteq .5 \propto$ J. R. Holsinger. 17 Aug 1965 and $12 \sqsubseteq .2 ‡$ J. R. Holsinger et al., 26 Nov 1965: Spout Spring, 2 ల. J. R. Holsinger. I Mar 1964: stream in bottom of sink. $2.4 \mathrm{~km}$ NE of Sims Spring. 2 juvs. T. Rowinski and G. Cepko. 2 juvs. 17 May 1990: Sweet Potato Cave. 7

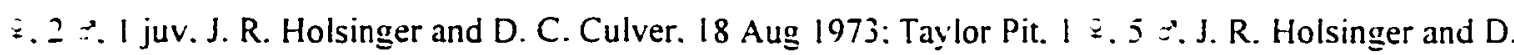
C. Culver. 19 Jul 1976: Unthanks Cave. $4 \cong .2$ \&. J. R. Holsinger and D. Martin. 16 Aug 1962: Watsons Cave No. $1.1 \cong .1$ juv. J. R. Holsinger et al.. 23 Nov, 1977: Young-Fugate Cave. $22 \sqsupseteq .3 \approx 13$ juvs, J. R. Holsinger. 15 Aug 1965: Thompson Cave. 11 P. 4 ङ. I juv. J. R. Holsinger and D. Finley. 19 Aug 1965: Thompson Cedar Cave. 11 q. J. R. Holsinger et al., 30 Jul 1967: Scott Co.: Daniel Boones Spring Cave. 6 2. D. A. Hubbard. Jr.. 25 May 1995: McDavids Cave. I P. J. R. Holsinger and S. Pinkerton. 30 Apr 1967: Speers Ferry Cave. 2 ₹. 2 ঊ. J. R. Holsinger et al. and 14 Jul 1970. 1 \&. J. R. Holsinger and D. C. Culver. 28. 1971 : Spurlock Cave. 1 \%. 2 c. J. R. Holsinger and D. Finley. 11 Jun 1966 and 1 juv. J. R. Holsinger. 11 Mar 1967: Washington Co.: De Busk Natural Caverns No. 3. 3 ₹. D. A. Hubbard. Jr.. 9 Mar 1992:

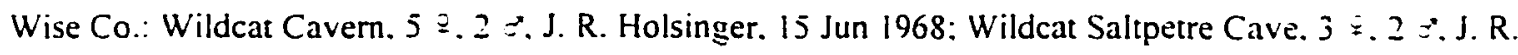
Holsinger et al., 27 Nov 1975.

Diagnosis.--A medium-sized cavernicolous species easily distinguished from other species in the genus by having weakly notched spine teeth on female gnathopods: strongly oblique palms of gnathopod 
propods (especially in mature female); end of defining angle of propod of gnathopod 2 of both sexes with 1 very long. large unnotched spine tooth on the outside; pleonal plate 1 with $1-j$ subventral spines: and outer ramus of male uropod 2 with 4-5 large comb-like spines on inner side. Largest male, $8.0 \mathrm{~mm}$ : largest female. $13.5 \mathrm{~mm}$.

Female.--Eye greatly reduced to few black specks or absent. Antenna 1. about 60 percent length of body, about 1.7 times longer than antenna 2: primary flagellum with up to 29 segments. Antenna 2: flagellum with 8-10 segments. with small calceoli in some larger specimens. Mandibles subequal. spine row with 9 spines: segment 2 of palp with 10-13 long setae, segment 3 with 1 A seta. $5 \mathrm{C}$ setae. $4-5 \mathrm{E}$ setae. and a row of $16 \mathrm{D}$ setae. lacking B seta. Maxilla 1 : inner plate with 7-8 apical plumose setae: palp with 6-10 slender spines on apex. Maxilla 2. inner plate with oblique row of 8 plumose setae on inner margin. Maxilliped: inner plate apically with 3 bladelike spines, 3 naked setae. and about 8 plumose setae extending from inner margin to apex: outer plate with row of naked setae and about $3-4$ slender pectinate spines on inner margin and $2-3$ plumose long setae on apex: dactyl with 2 setae on inner margin near nail: dactyl nail long. about $1 / 3$ of length dactyl.

Propod of gnathopod 1 more than 2 times longer than carpus: palm convex and strongly oblique. much longer than posterior margin. with 11 weakly notched long spine teeth on inside. 14 on the outside: defining angle with 1 notched and 3-4 notched-serrate spine teeth on inside. 1 large notched and I small simple spine teeth on outside: superior medial setae few in number and singly or doubly inserted: $10(-)$ inferior lateral setae, singly inserted: posterior margin with 6 sets of setae in groups of 2-4. Dactyl of gnathopod 1 with a row of short setae on inner margin. outer margin with $1-3$ setae. nail short. Ventral margin of coxa ! with $14-16$ setae.

Propod of gnathopod 2 more than 2 times longer than carpus: palmar margin almost straight. strongly oblique. more than 2 times longer than posterior margin in mature specimens. with 13 weakly notched. long spine teeth on inside. 14 on outside: derinning angle with $3-4$ large notched spine teeth on inner side. 4-5 small notched spine teeth and I very large, long unnotched spine tooth on outside: 8 sets of superior medial setae in groups of $1-3$ but mostly with 1 seta each: 6 sets of inferior medial setae in groups of 1-2: posterior margin with 6 sets of setae in groups of 2-6. Dactyl with about 4 short setae along inner margin, up to 4 setae on outer margin. Ventral margin of coxa I with II setae.

Coxa of pereopod 3 with 14 marginal setae. Coxa of pereopod 4 with 24 marginal setae. Pereopod 5: basis with about 22 shallow serrations on posterior margin: 12 short spines on anterior margin: 3 long setae on the anteroproximal margin. Pereopod 6: basis with 24 shallow serrations along posterior margin: 9 short spines on anterior margin: 3 long setae on anteroproximal margin. Pereopod 7: coxa with I seta along the posterior margin: basis with 21 shallow serrations along posterior margin. deeper near apex: 10 short spines on anterior margin: 3 short setae on anteroproximal margin: dactyl about 33 percent length of corresponding propod.

Pleonal plates: posterior margin of plate I slightly convex. with 1 seta. distoposterior corner not 
produced but acute. ventral margin with 1-j (subventral) spines: posterior margin of plate 2 nearly straight with 1 seta. distoposterior comer produced and acute, ventral margin with 4-5 (subventral) spines: posterior margin of plate 3 nearly straight with I seta. distoposterior comer small. rounded. not produced. ventral margin with 3 (subventral) spines. Peduncle of pleopod 1 with 2 retinaculae, lacking setae on outer margin.

Uropod 1: inner ramus armed with about 13 spines: outer ramus with about 14 spines: peduncle with 9 outer and 1 inner spines. Uropod 2: inner ramus armed with 12 spines: outer ramus with about 10 spines: peduncle with 5 outer and 2 inner spines. Uropod 3: inner ramus with I spine. outer ramus about 1.9 times longer than peduncle. inner and outer margin each with $3-4$ sets of spines in groups of $1-3$. Telson broader than long. notched about $1 / 3$ distance to base. each lobe with 3 apical spines: spines about 1,2 length of telson.

Male.--Differing from female as follows. Smaller with more slender. elongate body. Antenna 2: calceoli present on peduncular segments 4 and 5 , and first 1 or 2 segments of flagellum.

Uropod 2: inner ramus much wider than outer ramus. with about 13 spines: outer ramus deflected laterally. much smaller than outer ramus, distal end with 4-5 large comb-like spines on inside and 4-5 short comb-like spines on outside. outside with 4-5 well spaced short spines also: peduncle with 4 outer and 2-3 inner spines. Telson much broader than long.

Variation.--The eye structure is somewhat variable. Eyes are greatly reduced to a few block specks or are completely absent. The presence or absence of eyes may be variable within or between two closely proximate populations. In juveniles and submature females the palmar margin of the gnathopod propods is not oblique and long. and the propod itself is not 2 times larger than the carpus. When specimens become mature, the palm becomes oblique and longer. and the propod becomes larger. The number of subventral spines on pleonal plate I varies from I to 3 , but I spine is always present. The number of large comb-like spine on the end of the outer ramus uropod 2 varies. but the spines always appear comb-like.

Distribution and ecology.-- This relatively common troglobitic species ranges from the upper Tennessee River drainage basin in Lee, Scott, and Wise counties. Virginia south-southwestward to northeastern Georgia (into the upper Alabama River basin) and west along the Tennessee River valley to northwestern Alabama and extreme southcentral Tennessee; it is also found in the Sequatchie River Valley in Tennessee (fig. 157).

Further details on ecology and natural history are found in Dickson (1977). Dickson and Holsinger (1981) and Dickson (1982).

\section{Crangonyx spectus, new species}

Figures $158-16]$

Material examined (paratypes unless designated otherwise).--KENTUCKY. Pulaski Co.: Girdler Cave 


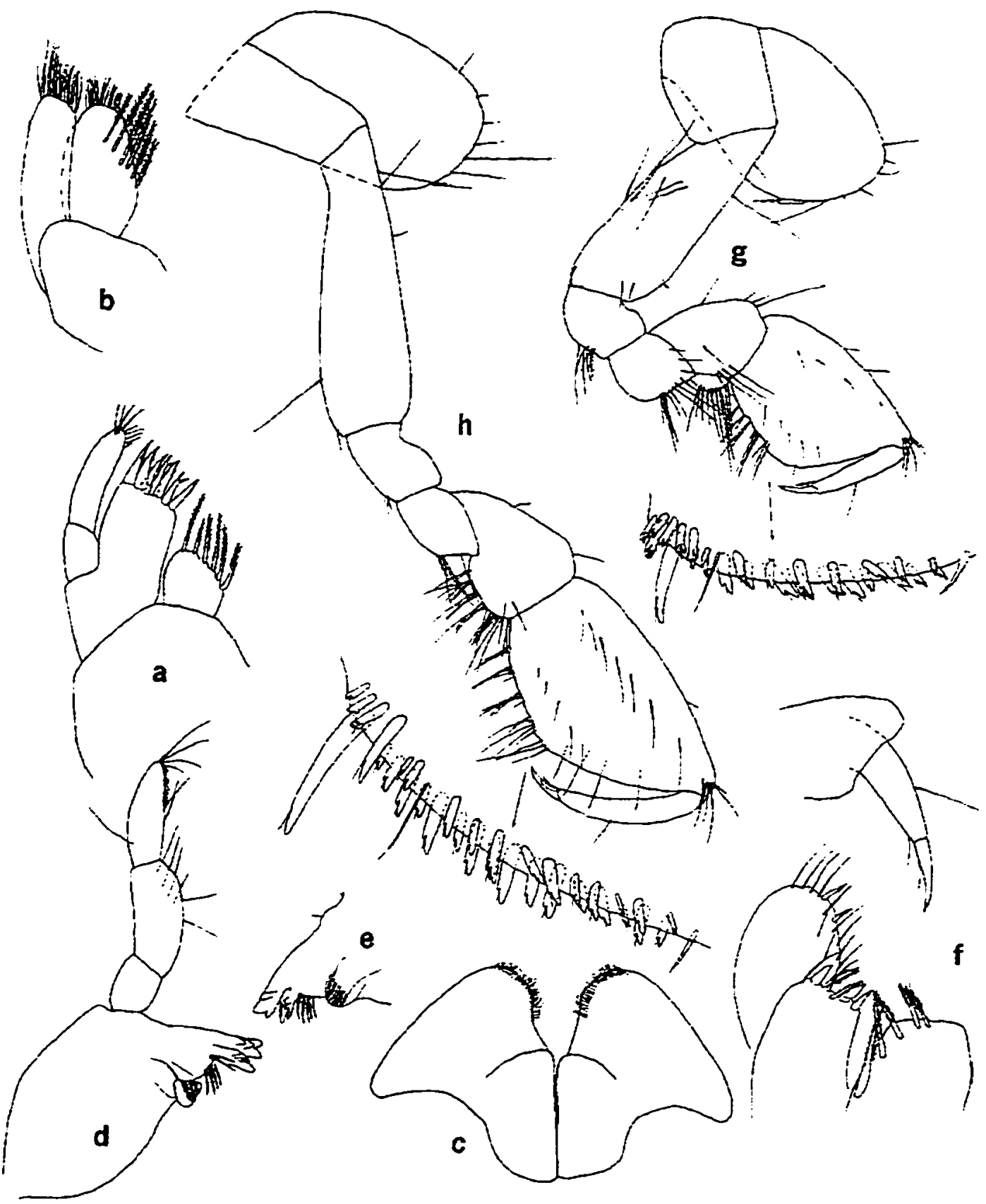

Figure 158.--Crangonyx specus, new species. Female paratype $(7.0 \mathrm{~mm})$. Girdler Cave No.2. Pulaski Co.. Kentucky: a. b, maxillae 1, 2: c. lower lip; $d$. left mandible; e. dentate part of right mandible; $f$. inner and outer plates and palp dactyl of maxilliped (greatly enlarged): g, h. gnathopods 1. 2 (paimar margins enlarged). 


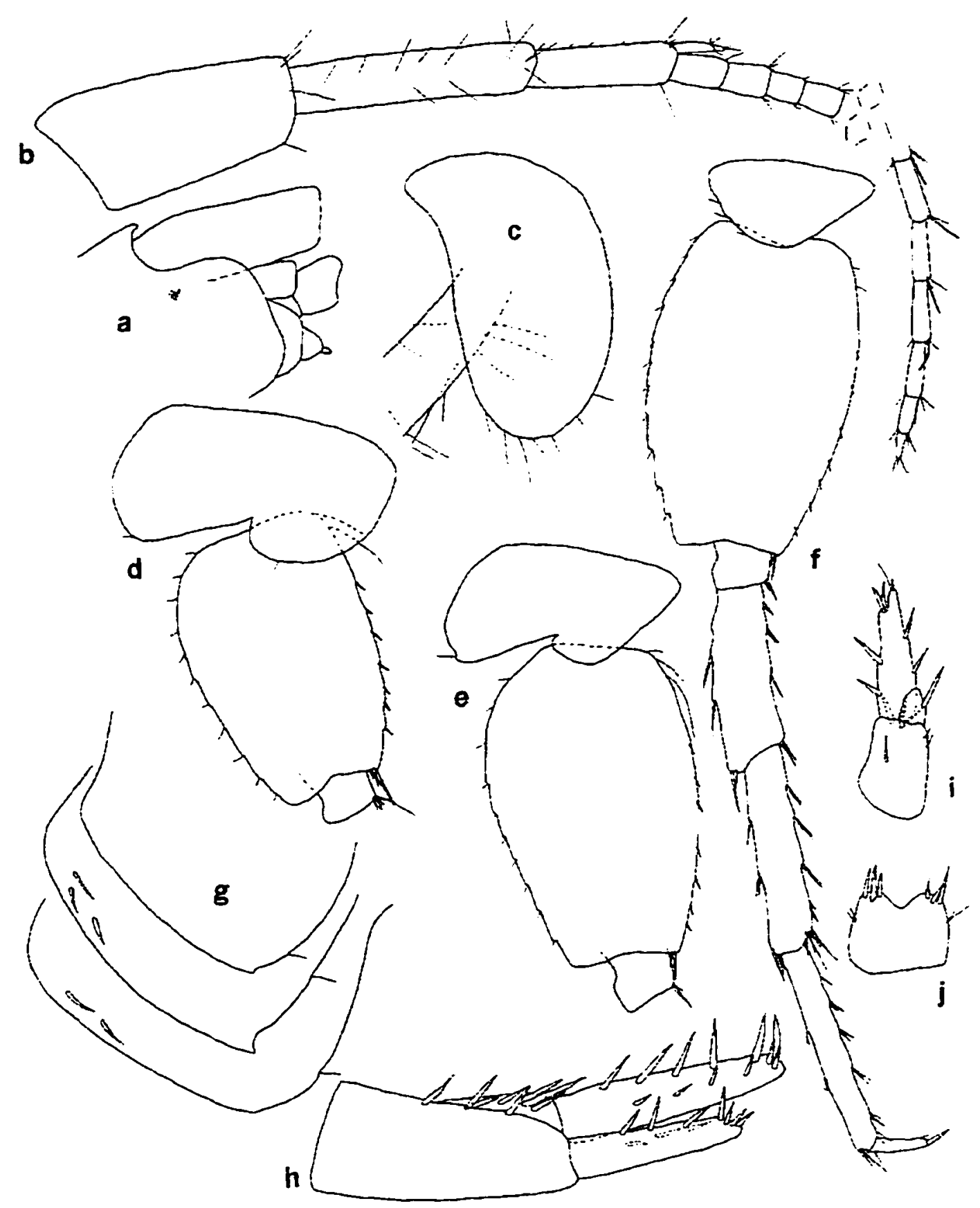

Figure 159.--Crangonyx specus. new species. Female paratype (7.0 mm), Girdler Cave No.2. Pulaski Co., Kentucky: a. head (in part); b, antenna 1; c, d, e, pereopods 3.5.6 (in part); f, pereopod 7: g. pleonal plates: $h$, i, uropods $2,3: j$, telson. 


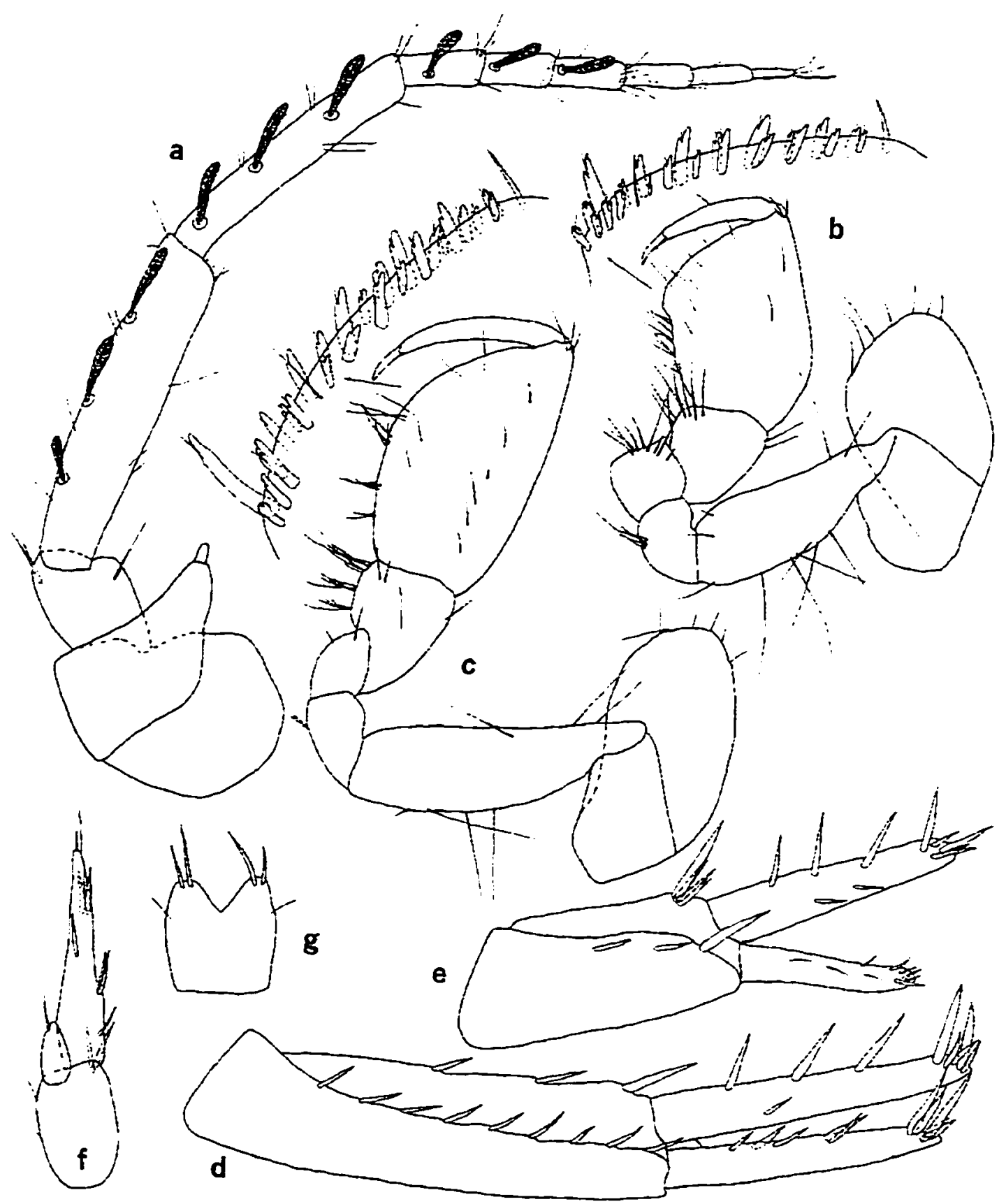

Figure 160.--Crangonyx specus, new species. Male paratype $(4.5 \mathrm{~mm})$. Girdler Cave No.2, Pulaski Co.. Kentucky: a, antenna 2; b, c. gnathopods 1, 2 (palmar margins enlarged); d. e. f. uropods 1. 2. 3: g. telson. 


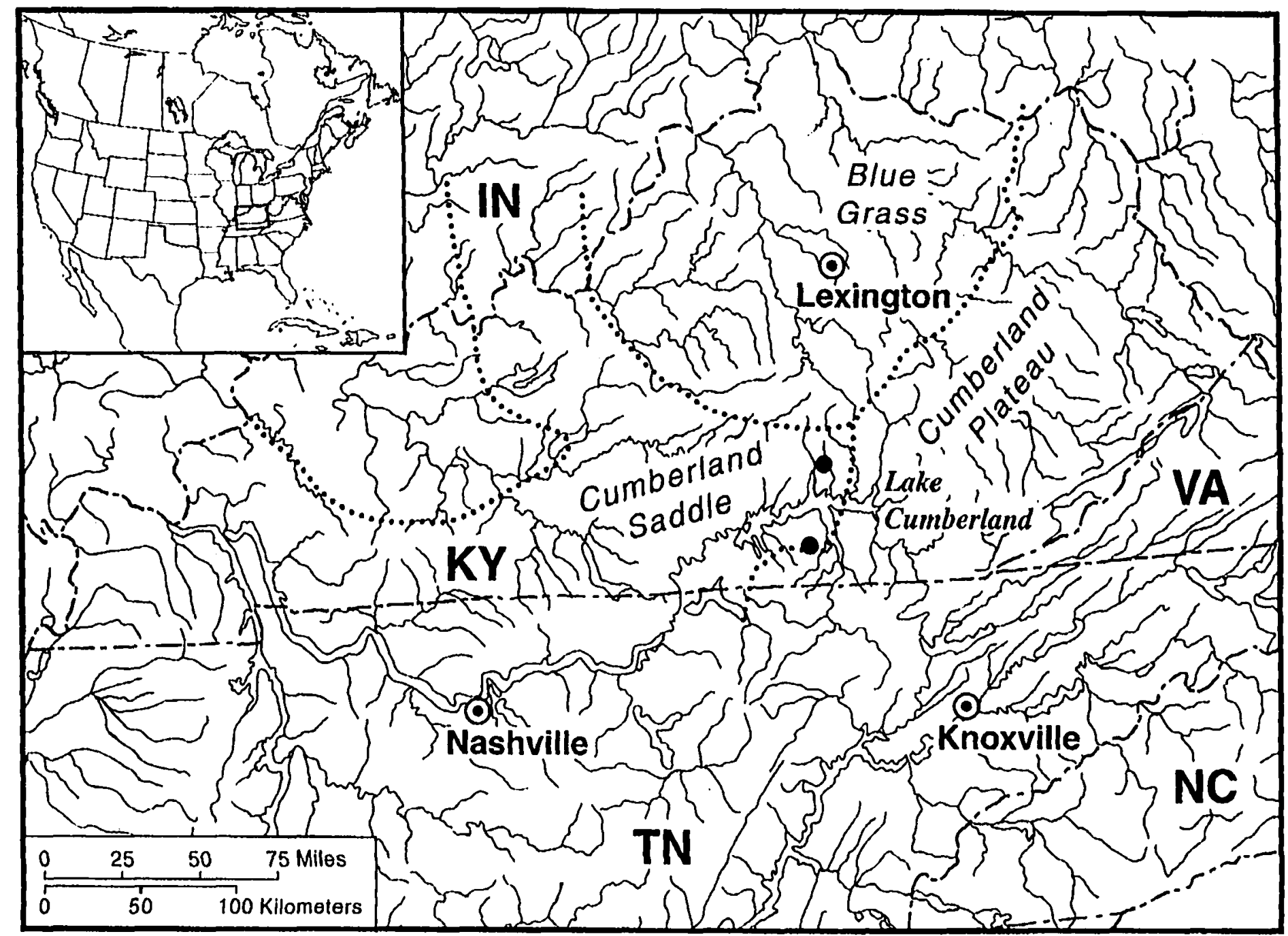

Figure 161.--1)istribution of ( $r$ rmgennx specus in North America. Solid circles represent 1-2 closely proximate localities. 


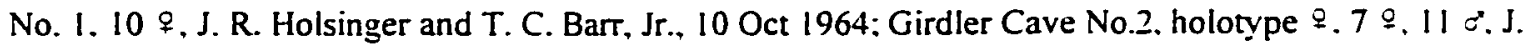

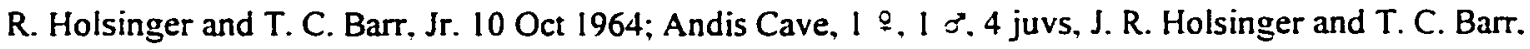
Jr.. 10 Oct 1964: Wayne Co.: Koger Cave, 2 \&, J. R. Holsinger and A. T. Leitheuser, 5 July 1983.

Diagnosis.--A relatively small troglobitic species very similar to $C$. antennatus but distinguished from it by outer ramus of male uropod 2. which has only 1-2 short inner spines and 3 short setal-like outer spines: defining angle of gnathopods 1 and 2 of both sexes with very distinct notched-serrate spines: single seta on dactyl of outer margin of gnathopods: lacking subventral spines on pleonal plate 1: and proportionately shorter uropod 3 in female. Largest male, $4.5 \mathrm{~mm}$; largest female $7.5 \mathrm{~mm}$.

Female.--Eye absent or reduced to few tiny specks. Antenna 1. about 50 percent length of body. about 2 times longer than antenna 2; primary flagellum with about 20 segments. Antenna 2. flagellum with 6-8 segments. Mandibles subequal. spine row with 5-6 spines: segment 2 of palp with 6 long setae. segment 3 with 1 A seta. $2 \mathrm{C}$ setae. $4 \mathrm{E}$ setae and a row of D setae. lacking B seta. Maxilla 1: inner plate with 6 apicai plumose setae: palp with 5-7 slender spines on apex. Maxilla 2. inner plate with oblique row of 6 plumose setae on inner margin. Maxilliped: inner plate apically with 3 bladelike spines. 3 naked spines. and 5-6 plumose setae extending from inner margin to apex: outer plate with row of naked setae and about $2-3$ pectinate slender spines on inner margin: dactyl with 2 setae near nail: dactyl nail very long, about $1 / 3$ length of dactyl.

Propod of gnathopod I about two times longer than carpus: palm slightly convex. slightly longer than posterior margin. with 6 notched spine teeth and 1 simple spine tooth on inside: 9 notched spine teeth and 3 simple spine teeth on outside: defining angle with I simple spine tooth and 4 notched-serrate spine teeth on inside. 1 large notched and 2 serrate spine teeth on outside: superior medial setae singly inserted: posterior margin with 5 sets of setae in groups of 2-5. Dactyl with 2-3 short setae on inner margin. nail about lit length of dactyl. Ventral margin of coxa 1 with 7-8 setae. some very long.

Propod of gnathopod 2 much wider and more than 2 times longer than carpus: palm convex. oblique. longer than posterior margin, with 9 spine teeth on inside, 13 on outside: defining angle with 2 notched and I very large simple teeth and 3 serrate spine teeth on inside. outside with 3 notched and I very large simple spine teeth: superior medial setae singly inserted; posterior margin with 5 sets of setae in groups of 3-6. Dactyl lacking setae on inner margin. Ventral margin of coxa 2 with 7 setae.

Coxa of pereopod 3 with 7 marginal setae. 2 very long. Coxa of pereopod 4 with 11 marginal setae. Pereopod 5: basis with 11 very shallow serrations along posterior margin: 7 short spines on anterior margin: 2 long setae on the anteroproximal margin. Pereopod 6: basis with 10 shallow serrations along posterior margin: 7 short spines on anterior margin: 3 long setae on anteroproximal margin. Pereopod 7: coxa with 3 setae on the posterior margin; basis with 11 shallow serrations along posterior margin: 7 short spines on anterior margin; 2 short setae on anteroproximal margin; dactyl about 35 percent length of corresponding propod.

Pleonal plates: posterior margin of plate I convex, with I seta. distoposterior comer not produced. 
subacute; posterior margin of plate 2 nearly straight with $1-2$ setae, distoposterior corner weakly produced and acute. ventral margin with 3 (subventral) spines; posterior margin of plate 3 convex with I seta. distoposterior comer recessed and indistinct, ventral margin with 2 (subventral) spines. Peduncle of pleopod I with 2 retinaculae, lacking setae on outside margin.

Uropod I: inner ramus 65 percent length of peduncle, armed with 12 spines: outer ramus with 11 spines: peduncle with 10 outer and 4 inner spines. Uropod 2: inner ramus armed with 12 spines: outer ramus with 10 spines: peduncle with 3 outer and 5 inner spines. Uropod 3: inner ramus with 1 large spine. outer ramus about 1.5 times longer than peduncle, inner and outer margin each with 2-3 set of spines in groups of $1-2$. Telson broader than long, notched about 1/6 distance to base, each lobe with 3 apical spines.

Male.--Differing from female as follows. Smaller with more slender, elongate body. Antenna 2: calceoli present on peduncular segments 4 and 5 , and first 3 segments of flagellum.

Propod of gnathopod 1 palm convex, oblique, with 7 spine teeth on inside. 8 on outside. defining angle with 3 notched-serrate spine teeth on inside, 1 large notched and 2 serrate spine teeth on outside. Propod of gnathopod 2 palm oblique, with 7 spine teeth on inside. 10 on outside: defining angle with 3 notched and 1 notched-serrate spine teeth on inside. 3 notched and I very large simple spine teeth on outside.

Uropod 2: inner ramus with 10 spines: outer ramus weakly deflected laterally, with 1-2 short inner spines. and 3 short setal-like outer spines, apex with 5 spines: peduncle with 4 outer and $3-4$ inner spines. Uropod 3 : inner ramus with 1 spine: outer ramus about 2 times length of peduncle. inner and outer margins each with 3 sets of spines in groups of $1-3$. Telson slightly longer than broad. notched about $1 / 4$ distance to base: each lobe with 2 apical spines: spines about $1 / 2$ length of the telson.

Type-locality.--Girdler Cave No. 2, located in Pulaski Co., Kentucky, is a stream passage cave developed in Mississippian-aged limstone. The type specimens were collected from mud-bottom stream pools, where they were relatively abundant (J. R. Holsinger, pers. comm.).

Distribution and ecology.--As presently known, the range of this species is limited to the extreme western margin of the Cumberland Plateau or the eastern margin of the Cumberland Saddle and occupies a small part of the Cumberland River drainage basin (fig. 161). The range. which covers a linear distance of approximately $50 \mathrm{~km}$ from north to south, extends from Pulaski County southward to Wayne County.

All collections made to date have been from stream pools except one from a drip/seep pool. In stream pools it was associated with a stygobiont isopod Caecidotea sp.. while in drip pools it was taken with the stygobiont amphipod Stygobromus exilis and isopod Caecidotea sp. Ovigerous females have not been collected to date: however. females (size range, $6.5-7.5 \mathrm{~mm}$ ) with setose brood plates have been collected in October.

Etymology.--The epithet specus is from Latin, meaning "cave" or "den." 
Crangonxx fontinalis, new species

Figures $162-165$

Material examined (paratypes unless designated otherwise).--VIRGINIA. Bland Co.: spring on Walker Creek just NW of Hamilton Cave, $22 \% .25 \approx$. J. R. Holsinger. 10 May 1965: spring, ca. $300 \mathrm{~m} \mathrm{~N}$ of Repass Saltpeter Cave, holotype $. .35 \%, 16$ c. J. R. Holsinger. 10 May 1965 and 50 ๆ. $12 \approx ゙$. J. R. Holsinger. 28 Jan 1968.

Diagnosis.--A medium-sized species similar to $C$. orientalis but distinguished from it by having about 5 evenly distributed spines on inner margin of outer ramus of male uropod 2. lacking serrate spine teeth on propod of male gnathopod 2. setal-like spines on anterior margin of bases of pereopods of male. Largest male. $6.0 \mathrm{~mm}$ : largest female. $8.0 \mathrm{~mm}$.

Female.--Eye large and ovate. Antenna 1. about 50 percent length of body. about 2 times longer than antenna 2: primary flagellum with about 22 segments. Antenna 2. flagellum with 8 segments. Mandibles subequal. spine row with 5-6 spines: segment 2 of palp with 7-9 long setae. segment 3 with I A seta. $2 \mathrm{C}$ setae. 4-5 E setae and a row of D setae, lacking B seta. Maxilla 1 : inner plate with 7-8 apical plumose setae: palp with 5-6 slender spines on apex. Maxilla 2, inner plate with oblique row of 8 plumose setae on inner margin. Maxilliped: inner plate apically with 4 bladelike spines. 3 naked spines, and 5-6 plumose setae extending from inner margin to apex; outer plate with row of naked setae and 4-5 slender pectinate spines on inner margin: dactyl with 2-3 setae near nail on inner margin: dactyl nail longer than $1: 3$ length of dactyl.

Propod of gnathopod 1 less than 2 times longer than carpus: palm almost straight. slightly shorter than posterior margin. with 8 spine teeth on inside, 9 on the outside: defining angle with 3 notched-serrate spine teeth on inside. I large notched and I small notched-serrate spine teeth on outside: superior medial setae singly inserted; posterior margin with $4-5$ sets of setae singly inserted or in groups of 2-3. Dacty! with a row of 5 short setae on inner margin, nail long. Ventral margin of coxa 1 with 8 setae.

Propod of gnathopod 2: palm slightly convex, oblique, shorter than posterior margin, with 7 spine tceth on inside. 9 on outside: defining angle with 1 notched and 2 notched-serrate spine teeth on inside. 2 notched and 1 large simple teeth on outside: superior medial setae singly inserted: posterior margin with 5 sets of setae in groups of 2-6. Dactyl with row of 5 short setae on inner margin. Ventral margin of coxa 2 with 8 setae.

Coxa of pereopod 3 with 8 marginal setae. Coxa of pereopod 4 with 16 marginal setae, dactyl of pereopod 4 about 45 percent length of corresponding propod. Pereopod 5: basis with deep 10 serrations along posterior margin; 4 short spines on anterior margin; 3 long setae on the anteroproximal margin. Pereopod 6: basis with 11 deep serrations along posterior margin; 6 short spines on anterior margin: 3 short setae on anteroproximal margin. Pereopod 7: coxa with 3 setae on the posterior margin: basis with 14 deep 


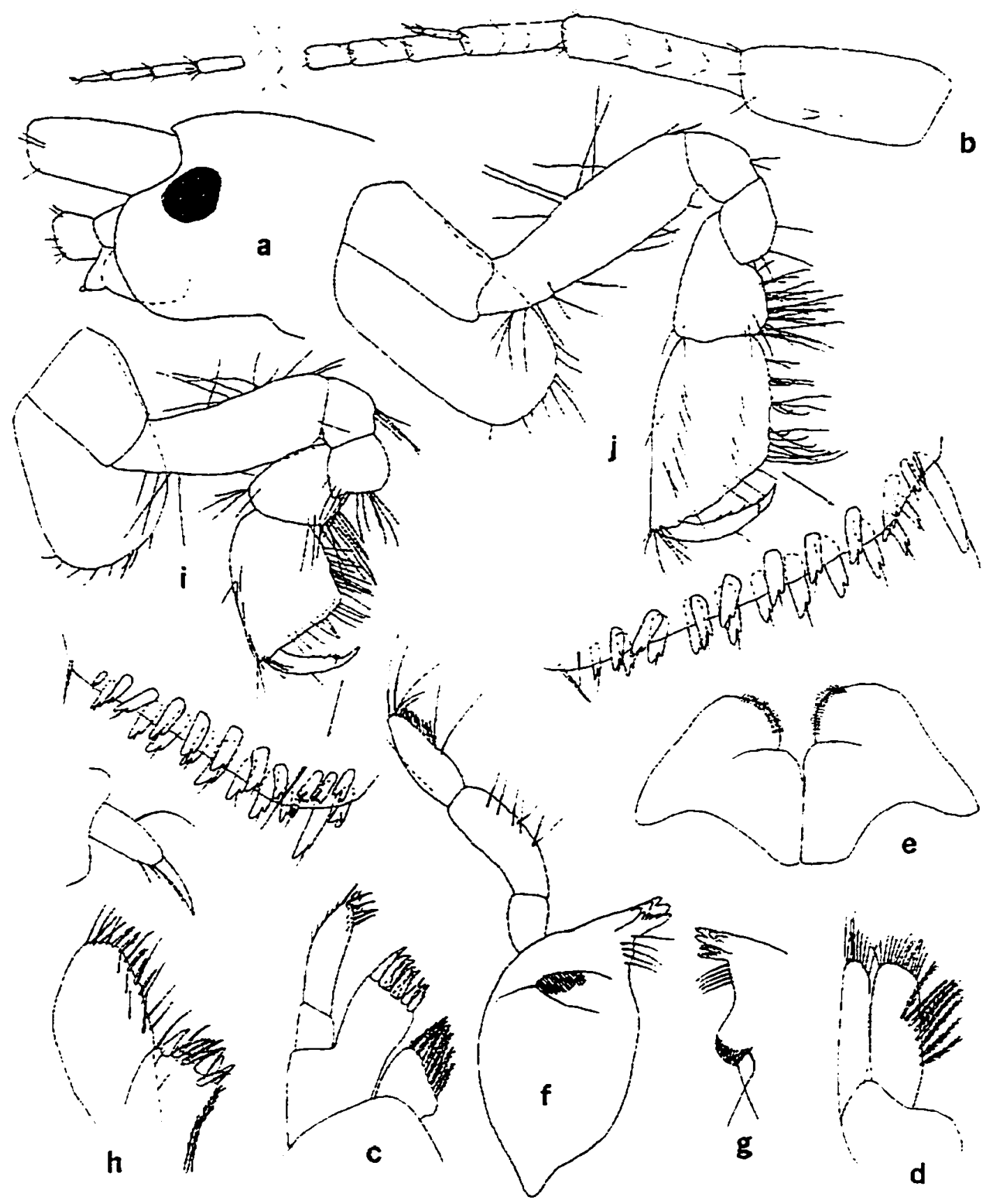

Figure 162.--Crangomx fontinalis, new species. Female paratype $(7.8 \mathrm{~mm})$, spring $\mathrm{N}$ of Repass

Saltpetre Cave. Bland Co.. Virginia: a, head (in part): b. antenna 1: c. d. maxillae 1. 2: e. lower lip: f. left mandible: $g$. dentate part of right mandible: $h$. inner and outer plates and palp dactyl of maxilliped: i. j, gnathopods 1.2 (palmar margins enlarged). 


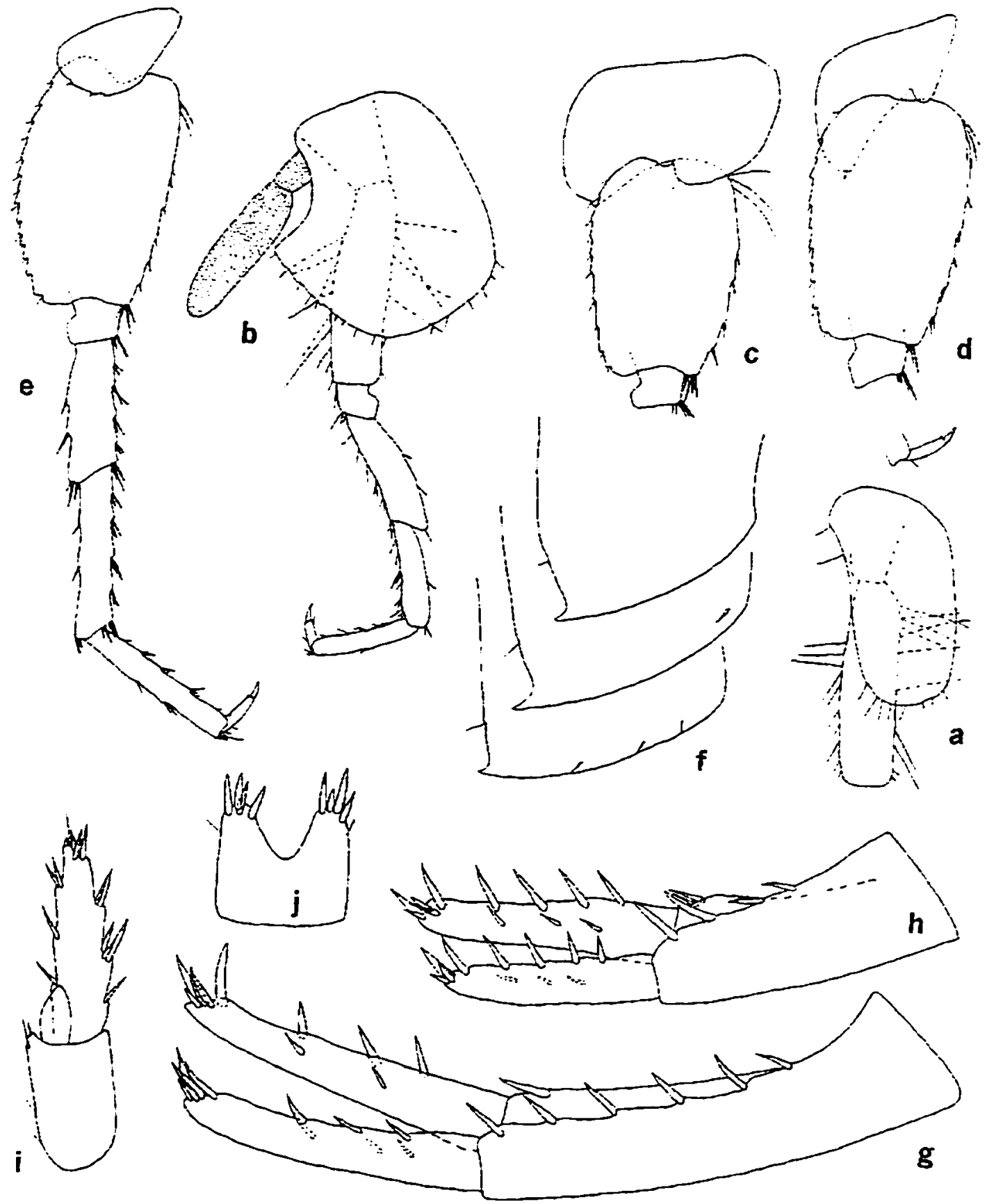

Figure 163.--Crangonvx fontinalis, new species. Female paratype $(7.8 \mathrm{~mm})$, spring $\mathrm{N}$ of Repass Saltpetre Cave, Bland Co.. Virgiiila: a, c. d. pereopods 3. 5.6 (in part): b. e. pereopods 4. 7; f. pleonal plates: g. h, i. uropods 1. 2. 3: j, telson. 


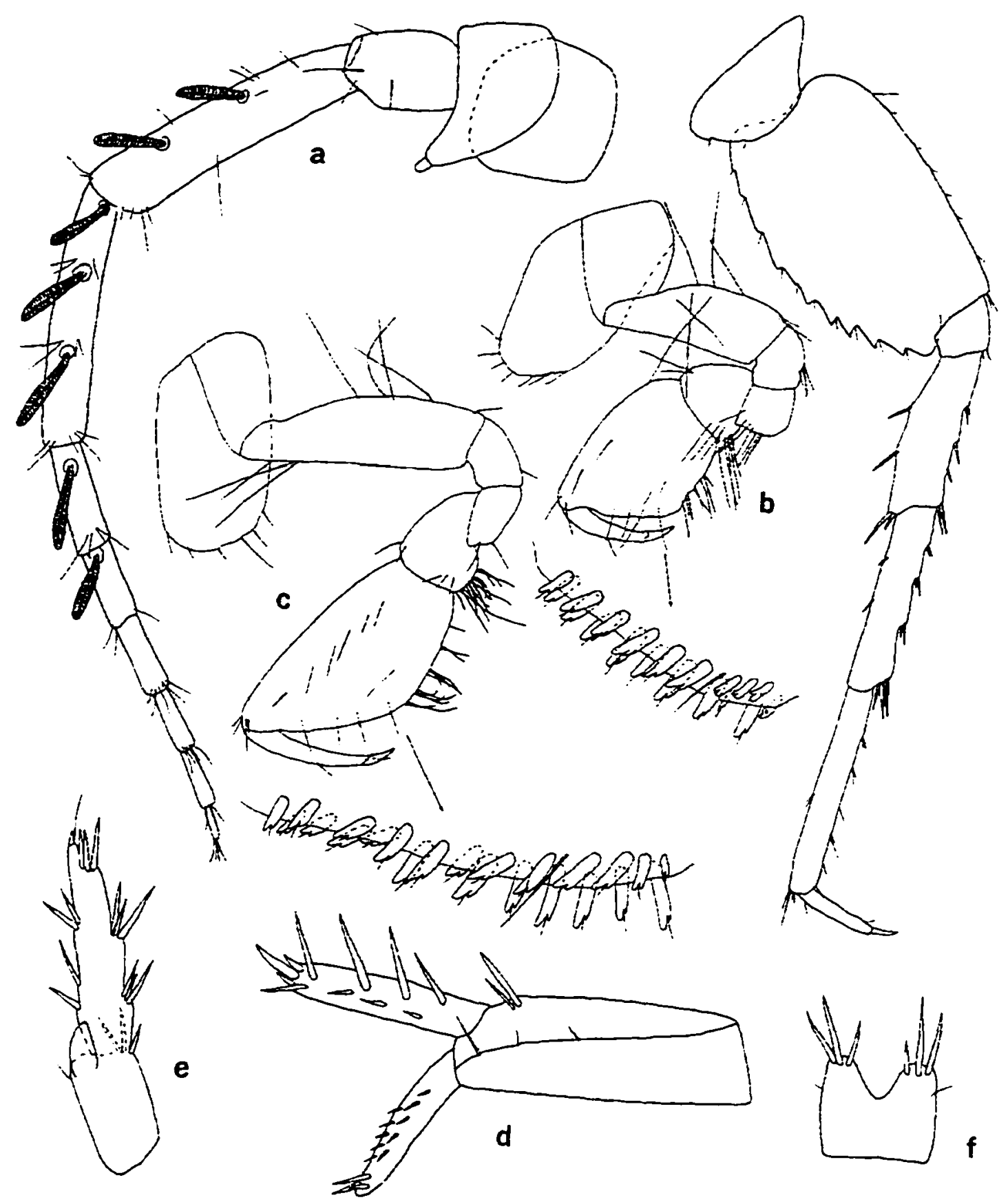

Figure 164.--Crangonyx fontinalis, new species. Male paratype $(5.0 \mathrm{~mm})$, spring $\mathrm{N}$ of Repass

Saltpetre Cave, Bland Co., Virginia: a, antenna 2; b. c. gnathopods I. 2 (palmar margins enlarged); d. e. uropods 2. 3: f. telson: g, pereopods 7. 


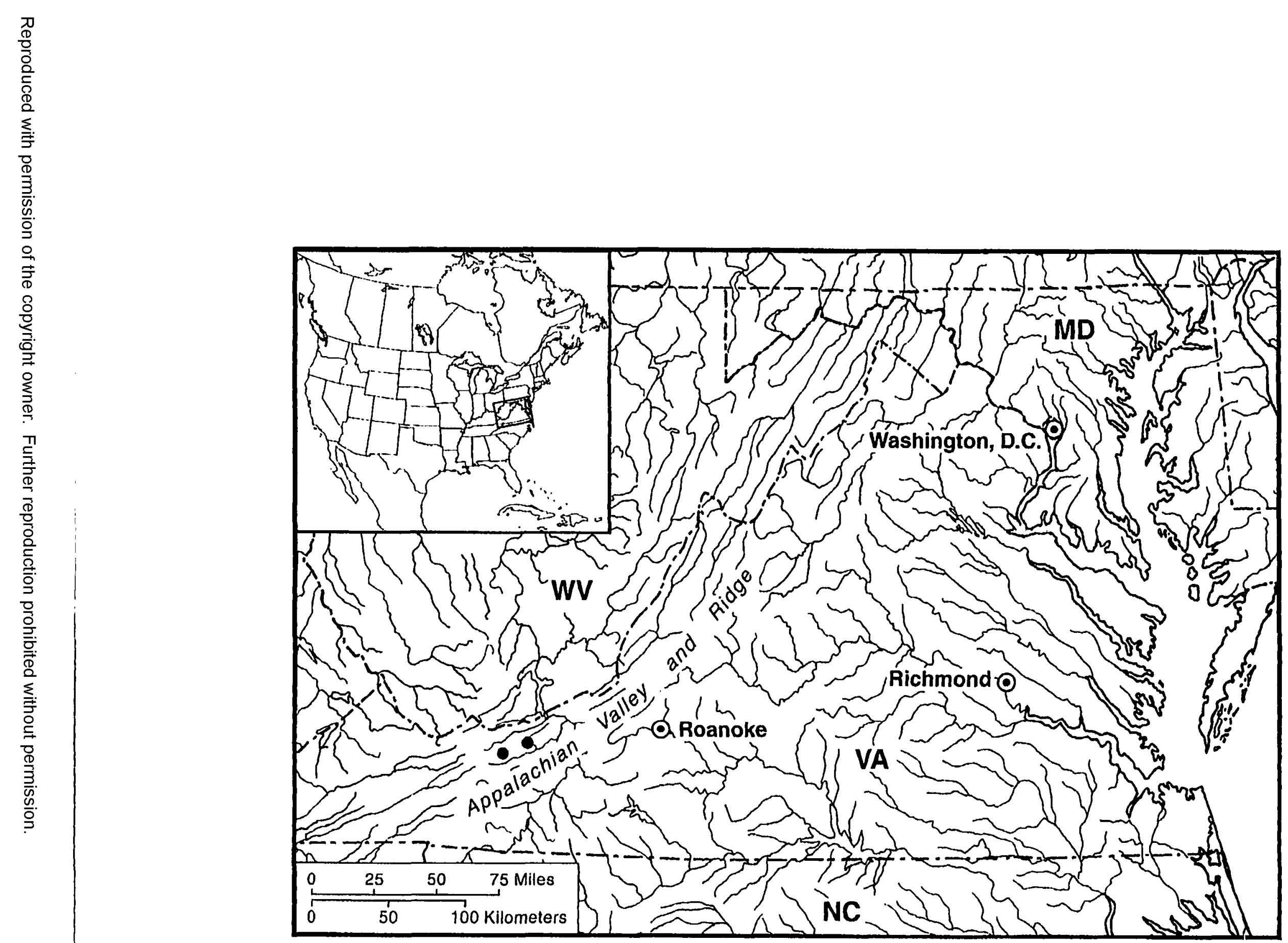

Pigure 165.--1)istribution of ('remgemyx fommind is in North America, Solid circles represent single localities. 
serrations along posterior margin; 6 short spines on anterior margin: 2 long setae on anteroproximal margin: dactyl about 35 percent length of corresponding propod.

Pleonal plates: posterior margin of plate I slightly convex, with 1 seta. distoposterior corner produced and acute: posterior margin of plate 2 nearly straight with I seta. distoposterior corner strongly produced and acute, ventral margin with I (subventral) spine: posterior margin of plate 3 nearly straight with I seta. distoposterior corner produced and acute, ventral margin with 3 (subventral) spines. Peduncle of pleopod 1 with 2 retinaculae. lacking setae on outside margin.

Uropod 1 : inner ramus 70 percent length of peduncle. armed with 10 spines: outer ramus with 11 spines: peduncle with row of 5 outer and $I$ inner spines. Uropod 2: inner ramus armed with 12 spines: outer ramus with 11 spines: peduncle with 4 outer spines. and 2 inner spines. Uropod 3 : inner ramus lacking spines. outer ramus about 1.5 times longer than peduncle. inner and outer margin each with $3-4$ sets of spines in groups $1-3$. Telson slightly broader than long, notched about 1/2 distance to base, each lobe with 4 apical spines: spines less than 1/2 length of telson.

Male.--Differing from female as follows. Smaller with more slender. elongate body. Antenna 2: calceoli present on peduncular segments 4 and 5 . and first 2 segments of flagellum.

Propod of gnathopods 1 and 2 more than 2 times larger and longer than carpus. proportionately much broader. Palm of propod of gnathopod I with 6 spine teeth on inside, 6 on outside: defining angle with 3 notched-serrate spine teeth on inside and 3 notched and 1 serrate spine teeth on outside. Propod of gnathopod 2 palm margin oblique. with 7 spine teeth on inside. 8 on outside: defining angle with 4 spine teeth on inside and 4 on outside.

Pereopod 6-7: basis with about 10 prominent serrations along posterior margin. 6-7 short setal-like spines on anterior margin.

Uropod 2: outer ramus strongly deflected laterally, with about 5 short inner and 5 short outer spines on upper margin. apex with 3 spines: peduncle with 3 thin outer spines. 2 inner spines. Uropod 3 : inner ramus with I spine: outer ramus about 2 times length of peduncle.

Type-locality.--spring, approximately $300 \mathrm{~m} \mathrm{~N}$ of Repass Saltpetre Cave. Bland Co.. VA.

Distribution and ecology.--The presently known range of this species is limited to Bland county. VA. and covers a linear distance of only about $20 \mathrm{~km}$ (fig. 165). This species is known only from two karst springs situated close to several caves. However, the populations appear to be epigean and superficially resemble Gammanus minus when alive in their natural setting. Specimens were abundant on roots and under leaves just beyond the spring outlets (J. R. Holsinger. pers. comm.).

Etymology.--The epithet fontinalis is from the Latin fontinalis, meaning "of a spring" and refers to the only known habitat of this new species. 


\section{Crangomyx cooperi. new species}

Figures 166-169

Material examined (paratypes uniess designated otherwise).-ALABAMA. Colbert Co.: Gallymore Cave. 7 ₹. 4 ×. S. B. Peck. 21 Jul 1965: Morgan Co.: Laughlin Spring Cave. 2 ?. 1 \%. J. E. Cooper et al.. 4

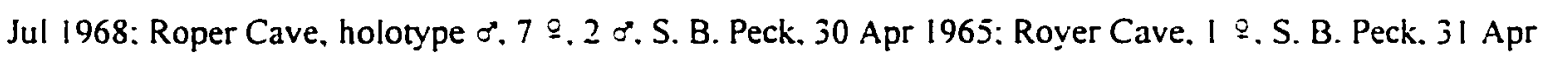
1965: Talucah Cave. I ơ. J. E. Cooper. 10 Apr 1966: Tarkington Farm Cave. 2 z. 1 I". J. E. Cooper. 20 Apr 1967.

Diagnosis.--A relatively small species morphologically similar to $C$. orientalis but distinguished easily from it by absence of comb spines on outer ramus of male uropod 2. which are replaced by thin spines (setal-like) instead. Largest male. $5.6 \mathrm{~mm}$ : largest female. $7.5 \mathrm{~mm}$.

Female.--Eye large and ovate. Antenna 1, 50-55 percent length of body. about 1.8-2.0 times longer than antenna 2: primary flagellum with about 25 segments. Antenna 2. flagellum with 7 segments.

Mandibles subequal. spine row with 4-6 spines: segment 2 of palp with 7-8 long setae. segment 3 with $1 \mathrm{~A}$ seta. $3 \mathrm{C}$ setae. $5 \mathrm{E}$ setae and a row of $\mathrm{D}$ setae. lacking $\mathrm{B}$ seta. Maxilla 1: inner plate with 8 apical plumose setae: palp with 9 slender spines on apex. Maxilla 2. inner plate with oblique row of 7-8 plumose setae on inner margin. Maxilliped: inner plate apically with 4 bladelike spines. 1 naked spine. and 5-6 plumose setae extending from inner margin to apex; outer plate with row of $3-4$ slender pectinate spines on inner margin: dactyl with 2 setae on inner margin near nail; dactyl nail very long, about $1 / 3$ length of dactyl.

Propod of gnathopod I less than 2 times longer than propod: palm slightly convex. shorter than posterior margin. with 7 spine teeth on inside. 7 on outside: defining angle with 5 notched-serrate spine teeth on inside. 1 large notched and 2 serrate small spine teeth on outside: posterior margin with 5 sets of setae singly inserted or in groups of 2-5. Ventral margin of coxa 1 with 9 setae.

Propod of gnathopod 2 less than 2 times longer than carpus: palm slightly convex. much shorter than posterior margin, with 9 spine teeth on inside, 10 on outside; defining angle with 1 large notched spine tooth and 3 weakly notched-serrate spine teeth on inside. 1 large simple spine tooth and I small (simple or notched) spine tooth on outside; superior medial setae mostly singly inserted: posterior margin with 6 sets of setae in groups of 2-6. Ventral margin of coxa 2 with 10 setae.

Coxa of pereopod 3 with 9 marginal setae. Coxa of pereopod 4 with 21 marginal setae. dactyl of pereopod 4 about 35 percent length of corresponding propod. Pereopod 5: basis with 16 shallow serrations along posterior margin: 6 short spines on anterior margin: 2 long setae on the anteroproximal margin. Pereopod 6: basis with 16 serrations along posterior margin: 5 short spines on anterior margin: 3 short setae on anteroproximal margin. Pereopod 7: coxa with 2-3 setae on the posterior margin: basis with 18 shallow serrations along posterior margin; 5 short spines on anterior margin: 3 short setae on proximal margin: dactyl about 33 percent length of corresponding propod. 


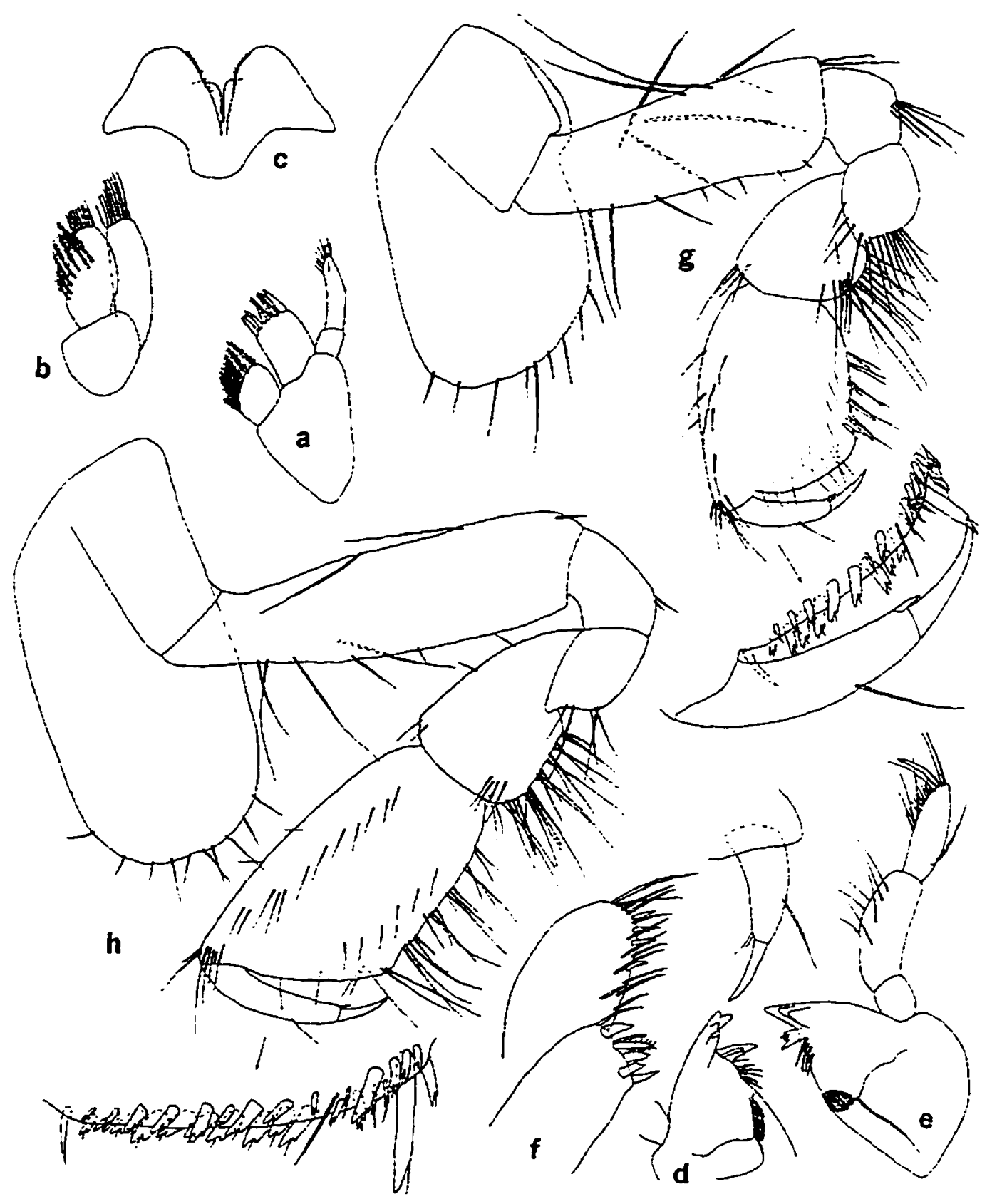

Figure 166.--Crangonyx coopert. new species. Female paratype $(7.7 \mathrm{~mm})$, Roper Cave. Morgan Co.. Alabama: a. b. maxillae 1. 2: c, lower lip: d, dentate part of left mandible: e. right mandible: $f$, inner and outer plates and palp dactyl of maxilliped (greatly enlarged): g. h. gnathopods I. 2 (palmar margins enlarged). 


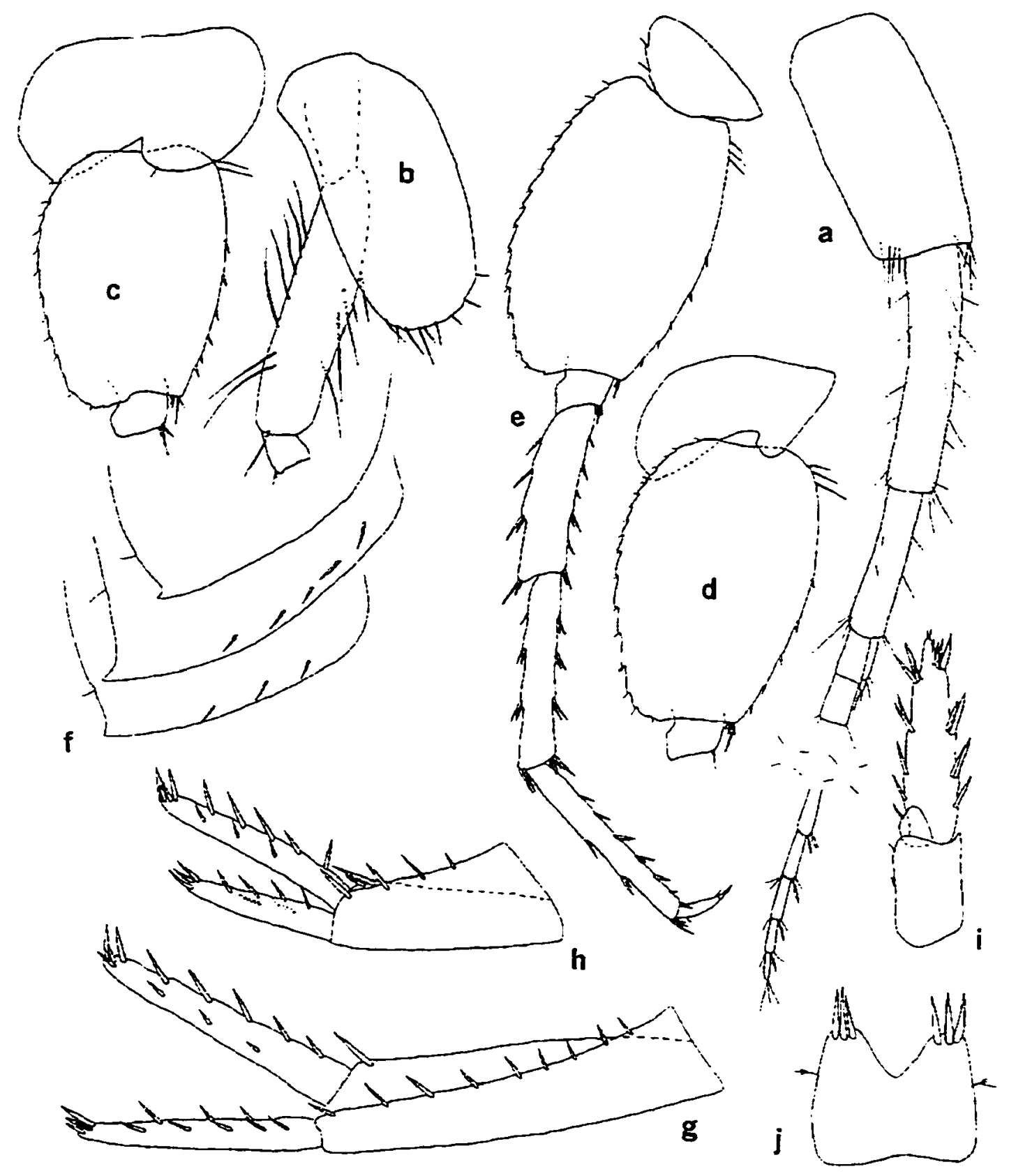

Figure 167.--Crangonyx cooperi, new species. Female paratype $(7.7 \mathrm{~mm})$. Roper Cave. Morgan Co.. Alabama: a. antenna 1: b, c, d, pereopods 3, 5, 6 (in part); e, pereopod 7: f. pleonal plates: g. h. i. uropods 1. 2. 3; j. telson. 


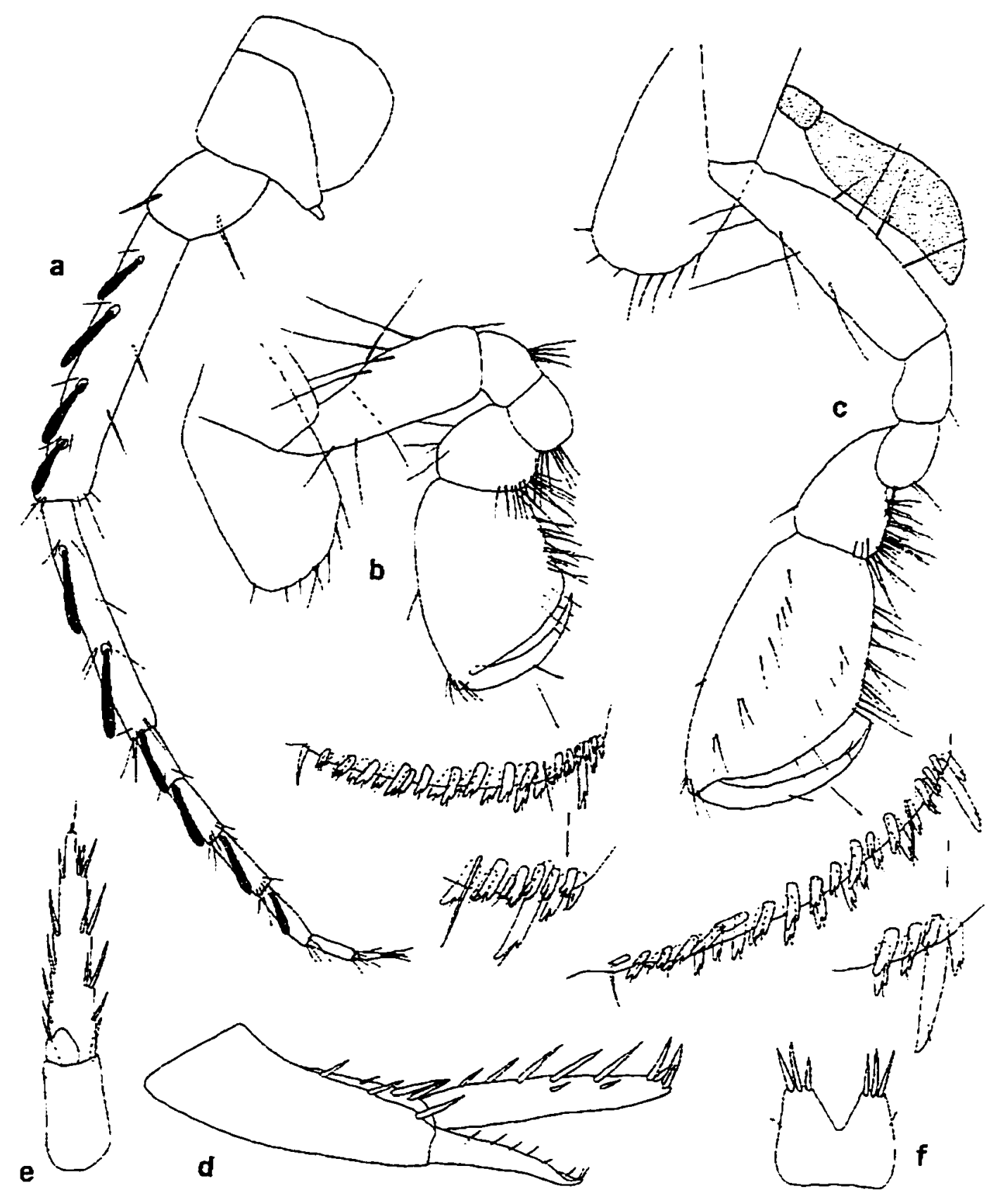

Figure 168.--Crangonvx cooperi, newv species. Male paratype (5.6 mm). Roper Cave. Morgan Co.. A labama: a, antenna 2; b, c. gnathopods 1. 2 (palmar margins enlarged): d. e. uropods 2. 3: f. telson. 
Pleonal plates: posterior margin of plate I slightly convex. with I seta. distoposterior corner produced and acute: posterior margin of plate 2 nearly straight with $1-2$ setae. distoposterior corner strongly produced and acute, ventral margin with 5 (subventral) spines; posterior margin of plate $j$ nearly straight with 1 seta, distoposterior corner weakly produced and acute, ventral margin with 3 (subventral) spines. Peduncle of pleopod I with 2 retinaculae, lacking setae on outside margin.

Uropod I: inner ramus 80 percent length of peduncle. armed with 13 spines: outer ramus with 12 spines: peduncle with 9 outer and 1 inner spines. Uropod 2: inner ramus armed with 12 spines: outer ramus with 11 spines: peduncle with 4 outer spines. and 2 inner spines. Uropod 3 : inner ramus with I spine. outer ramus about 2 times longer than peduncle. inner and outer margin each with $3-4$ sets of spines in groups 1 3. Telson slightly broader than long, notched about $1 / 3$ distance to base, each lobs with 3 apical spine: spines length less than $1 / 2$ length of telson.

Male.--Differing from females as follows. Smaller with more slender, elongate body. Antenna 2: calceoli present on peduncular segments 4 and 5 . and first 4 segments of flagellum.

Propod of gnathopod I more than 2 times longer than carpus: palm with 9 spine teeth on inside. 10 on outside. defining angle with 1 notched and 4 notched-serrate spine teeth on inside. $1-3$ small notched and 1 large notched and I serrate spine teeth on outside. Propod of gnathopod 2 more than 2 times longer than carpus: palmar margin convex and oblique. with 12 spine teeth on inside. 13 on outside: defining angle with 1 notched and 2 weakly notched-serrate spine teeth on inside and 4 notched spine teeth on outside.

Uropod 2: outer ramus slightly deflected laterally and tapering distally, with I very thin inner and 4 very thin outer spines, apex with 3 spines: peduncle with 3 outer and 3 inner spines.

Type-locality.--Roper Cave. Morgan Co., Alabama. located $14.5 \mathrm{~km} \mathrm{SW}$ of Decatur. contains a stream that flows to the surface through its mouth (Varnedoe. 1973).

Distribution and ecology.--Despite being found only in cave streams (at least to date), this species shows no obvious troglomorphic features (fig. 169). The range of the species. which lies within the Tennessee River drainage. covers a linear distance of approximately $120 \mathrm{~km}$. and extends from Colbert County east to Morgan County. The August collection from Roper Cave contained two ovigerous females. I with 7 young in the brood pouch. the other with 23 eggs.

Etymology.--It is pleasure to name this species in honor of Dr. John. E. Cooper. a biospeleologist whose extensive fieldwork in Alabama resulted in the discovery of this species.

Crangonvx lewisi. new species

Figures $170-173$

Material examined (paratypes unless designated otherwise).--INDIANA. Clark Co.: Sunset Village Cave, $1 \subsetneq .4$ juvs. J. J. Lewis, 26 Mar 1975; Jefferson Co.: Deputy Comer Cave, 69.3 ơ, J. J. Lewis. 28 


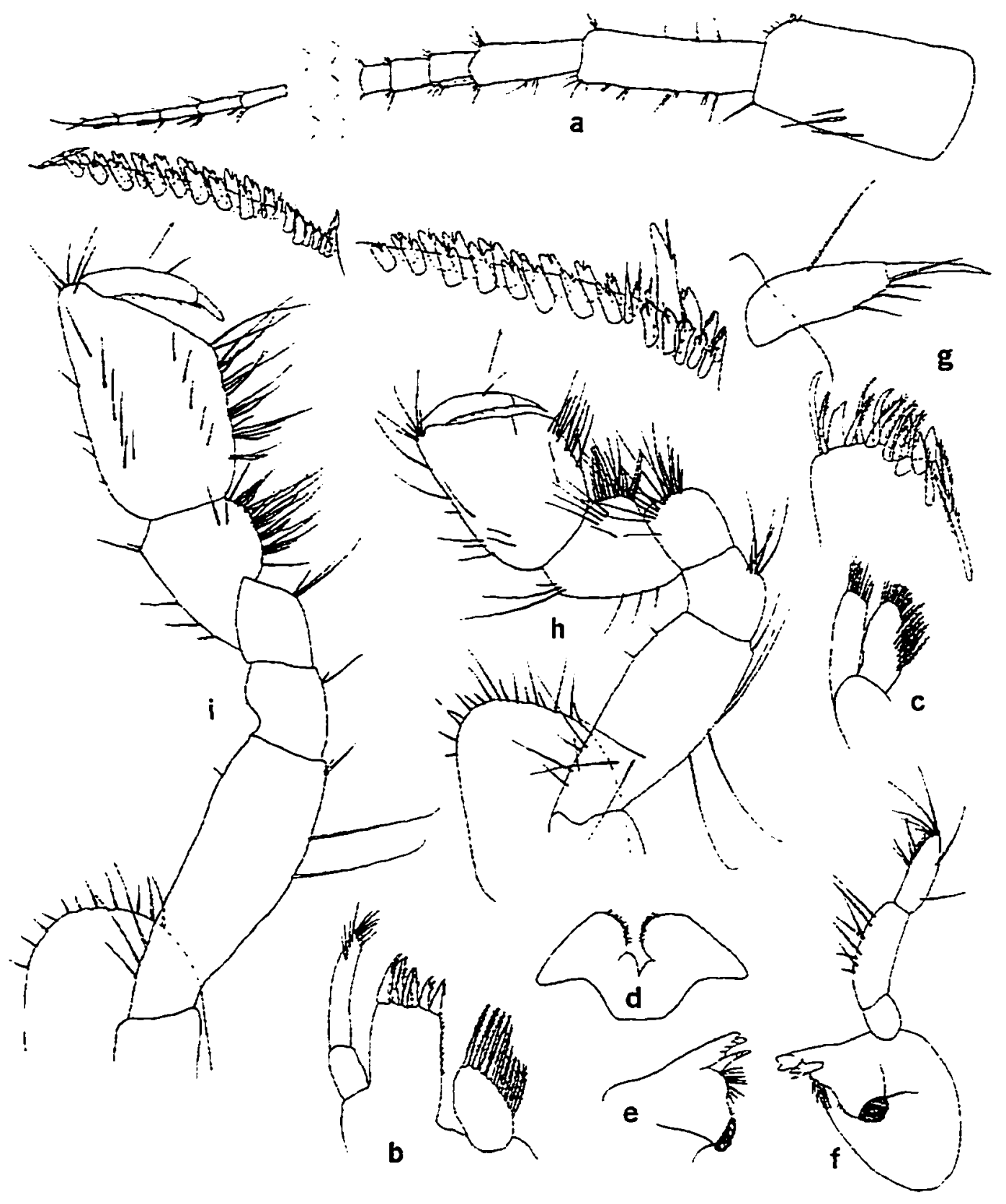

Figure 170.--Crangonyx lewisi, new species. Female paratype $(8.2 \mathrm{~mm})$. Wilsons Cave. Jefferson Co.. Indiana: a. antenna 1: b. c, maxillae 1. 2: d. lower lip: e. dentate part of left mandible: $f$, right mandible: g. inner plate and palp dactyl of maxilliped (greatly enlarged): h. i. gnathopods 1. 2 (palmar margins enlarged). 


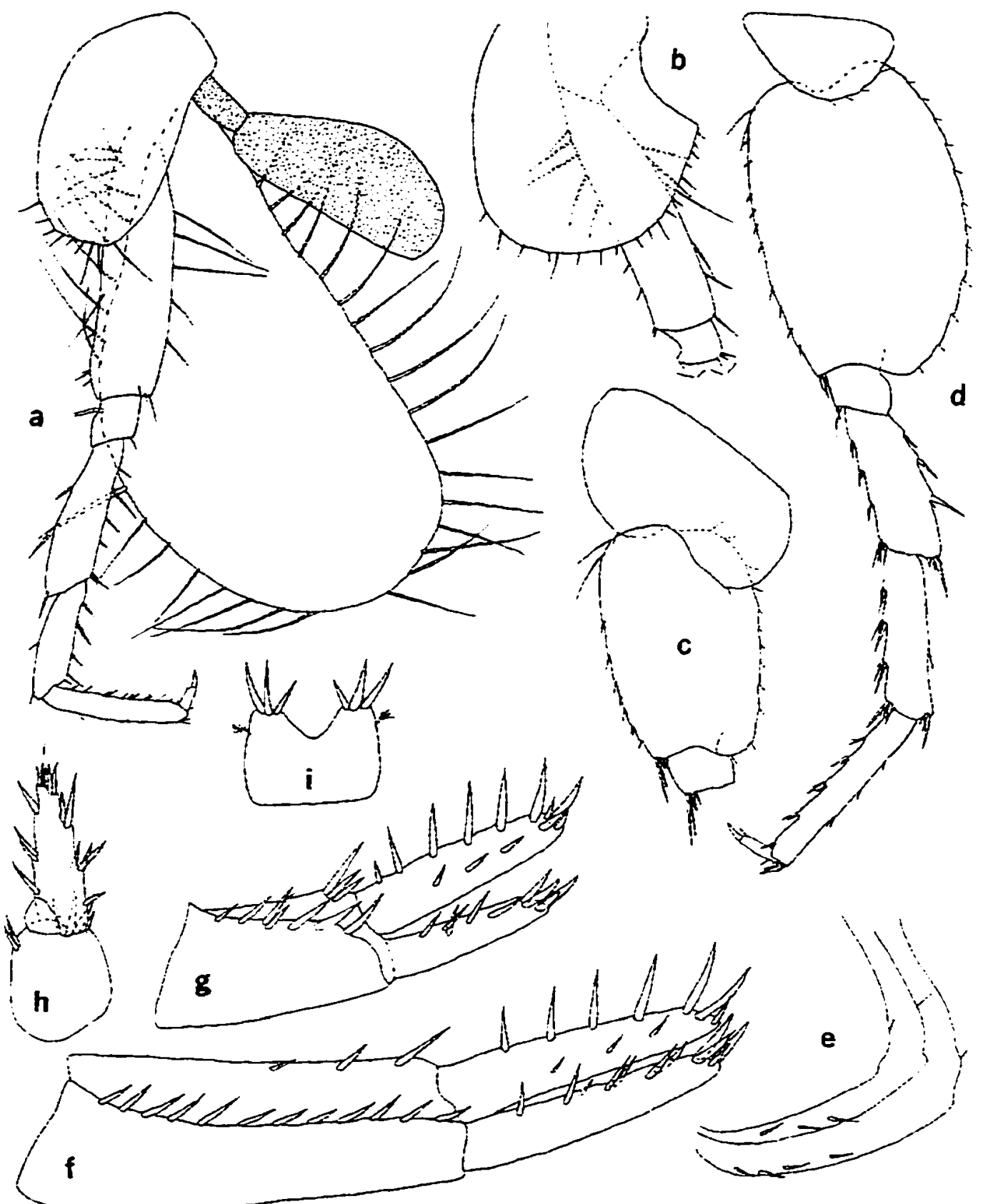

Figure 171 .--Crangonyx lewisi. new species. Female paratype $(8.2 \mathrm{~mm})$. Wilsons Cave, Jefferson Co. Indiana: a. d. pereopods 3. 7: b. c. pereopods 4, 5 (in part): e. pleonal plates: f. g. h. uropods 1. 2. 3: i. telson. 


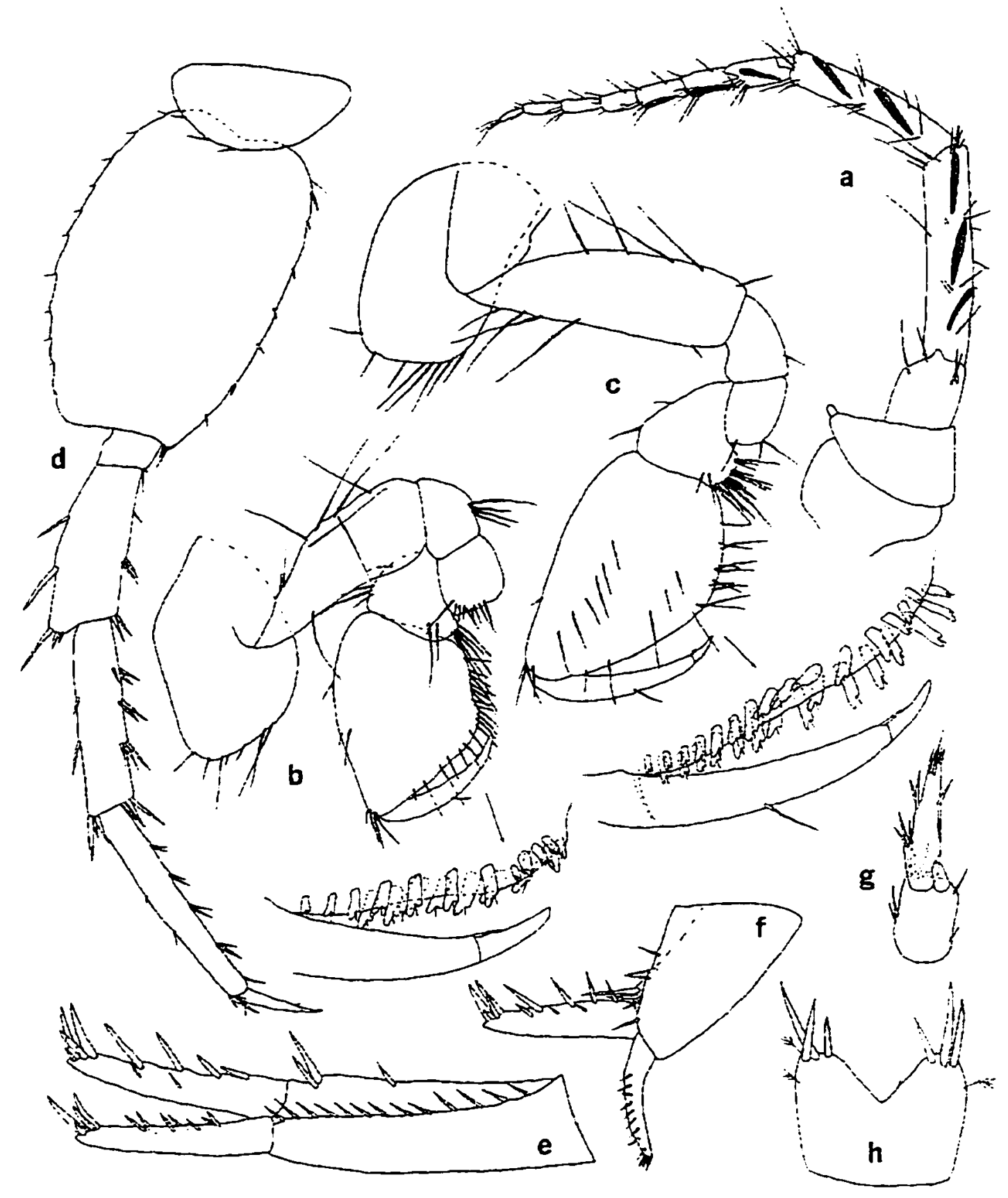

Figure 172.--Crangonvx lewisi. new species. Male paratype ( $5.3 \mathrm{~mm})$. Wilsons Cave. Jefferson Co.. Indiana: a, antenna 2; b, c. gnathopods 1, 2 (palmar margin enlarged); d. pereopod 7: e. f. g. uropods 1. 2. 3: h. telson 


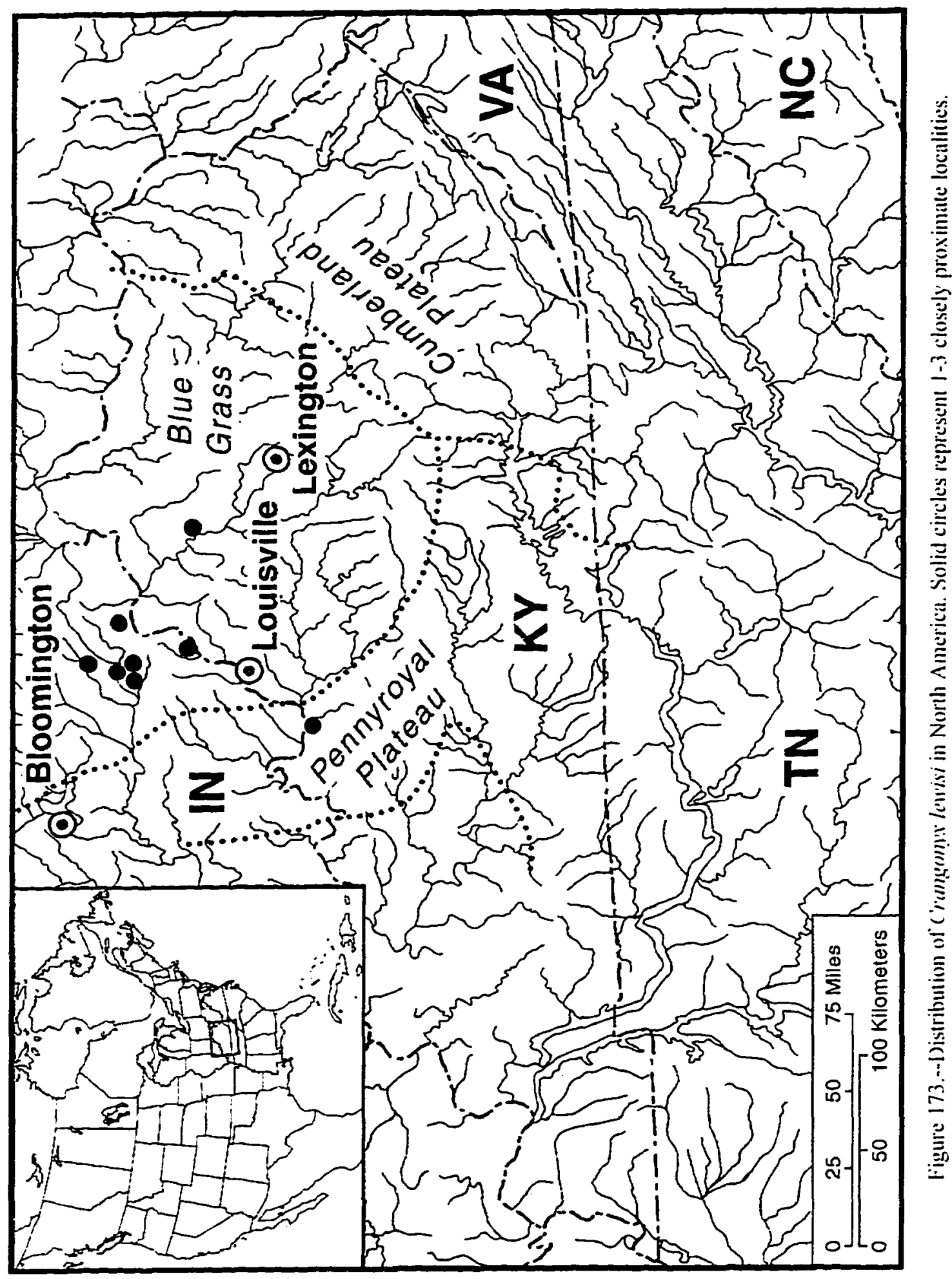


Nov. 1975: Foster Cave, $10^{\circ}$ J. J. Lewis and T. Lewis. 18 Mar 1979: Griffith Cave. 1 ๆ. 1 ¿. 1 juv. J. J. Lewis and T. Lewis, 21 Oct 1979; Waterfall Cave, 1 \%. J. J. Lewis, 28 Mar 1975: Wilsons Cave. $7 \% .1 \approx$. J. R. Holsinger, et al., 9 Aug 1964. Jennings Co.: Biehle Cave. 2 ๆ. 1 ๙0. J. J. Lewis and M. Lewis, 22 May 1994: Crosley Canyon Cave, holotype ₹. J. J. Lewis, 30 Jul 1994: Skeeter Bluff Cave. I ¿. J. J. Lewis. 18 Jun 1994. KENTUCKY. Meade Co.: spring near Ohio River. Otter Creek State Park. $1 \Xi .2 \approx$. J. J. Lewis. 24 Mar 1975: small spring. Rock Haven picnic area. Otter Creek Park. 2 . L. Hubricht 6 May 1956 (USNM): Tributary of Doe Run, 26 . $10^{*}$. W. L. Minckley. 2 Feb 1961: Owen Co.: Kemper Pit Cave. 1 Q. R. M. Norton. 17 May 1967.

Diagnosis.--A relatively small stygobiont species closely similar to $C$. bousfieldi but distinguished from it by the outer ramus of male uropod 2, which has about 8 outer and 1-2 inner (short) spines: and having only $\mathrm{I}$ seta on the posterior margins of pleonal plates. Largest male. $6.0 \mathrm{~mm}$ : largest female. 8.5 $\mathrm{mm}$.

Female.--Eye greatly reduced to several black specks. Antenna I. about 50 percent length of body. about 1.8 times longer than antenna 2: primary flagellum with 17 segments. Antenna 2. flagellum with 8-9 segments. Mandibles subequal, spine row with 4-5 spines; segment 2 of palp with 8 long setae. segment 3 with $1 \mathrm{~A}$ seta. I B seta. $4 \mathrm{C}$ setae. 4-5 $\mathrm{E}$ setae and a row of $\mathrm{D}$ setae. Maxilla 1: inner plate with 9 apical plumose setae: palp with 4 slender spines and 4 plumose setae on apex. Maxilla 2. inner plate with oblique row of 8 plumose setae on inner margin. Maxilliped: inner plate apically with 4 bladelike spines. 3 naked spines, and 7-8 plumose setae extending from inner margin to apex: outer plate with row of naked setae and $3-7$ slender pectinate spines on inner margin: 4-j setae on inner margin of dactyl: dactyl nail very long. about 2.5 length of dactyi.

Propod of gnathopod 1 less than 2 times longer than carpus: palm almost straight, shorter than posterior margin. with 8 spine teeth on inside, 8 notched and 2 simple spine teeth on the outside: defining angle with 5 notched-serrate spine teeth on inside, 1 large notched and 3 serrate spine teeth on cutside: posterior margin with 4 sets of setae inserted in groups of 2-5. Dactyl with row of short setae on inner margin: dactyl nail very long. Ventral margin of coxa 1 with 17 setae.

Propod of gnathopod 2 less 2 times longer than carpus: palm almost straight. oblique. shorter than posterior margin. with 10 spine teeth on inside, 11 on outside: defining angle with 1 notched and 5 notched. serrate spine teeth on inside. 1 very large notched and 2 serrate spine teeth on outside: superior medial setae singly inserted: posterior margin with 6 sets of setae in groups of 3-5. Dactyl with row of short setae on inferior margin. Ventral margin of coxa 2 with 11 setae.

Coxa of pereopod 3 with 9 marginal setae. Coxa of pereopod 4 with 19 marginal setae. dactyl of pereopod 4 about 33 percent length of corresponding propod. Pereopod 5: basis with 12 very shallow serrations along posterior margin; 6-8 short spines on anterior margin: 2 long setae on the anteroproximal margin. Pereopod 6: basis with 13 very shallow serrations along posterior margin: 9 short spines on anterior margin: 2 long setae on anteroproximal margin. Pereopod 7: coxa with 4 setae on the posterior 
margin: basis with 12 shallow serrations along posterior margin: 10 short spines on anterior margin: 2 long setae on anteroproximal margin; dactyl about 30 percent length of corresponding propod.

Pleonal plates: posterior margin of plate I convex, with I seta. distoposterior corner recessed. neither produced nor acute; posterior margin of plate 2 slightly convex with 1 seta. distoposterior comer weakly produced and subacute. ventral margin with 3 (subventral) spines; posterior margin of plate 3 nearly straight with I seta. distoposterior comer recessed, almost indistinct, ventral margin with 4 (subventral) spines. Peduncle of pleopod I with 2 retinaculae, lacking setae on outside margin.

Uropod 1: inner ramus about 70 percent length of peduncle, armed with 9 spines: outer ramus with 11 spines: peduncle with 12 outer and 3 inner spines. Uropod 2: inner ramus armed with 13 spines: outer ramus with 9 spines: peduncle with 7 outer spines. 3 inner spines. Uropod 3 : inner ramus lacking spines. outer ramus about 1.5 times longer than peduncle. inner and outer margins each with 2-3 set of spines in groups of $1-3$. Telson broader than long, notched about $1 / 3$ distance to base. each lobe with 3 apical spines: spines longer than $1 / 2$ length of telson.

Male.--Differing from females as follows. Smaller with more slender. elongate body. Antenna 2: calceoli present on peduncular segments 4 and 5 , and first 3 segments of flagellum.

Gnathopods 1 and 2: propod more than 2 times larger and longer than carpus. much broader. Propod of gnathopod I palm slightly concave. slightly longer than posterior margin. with 7 spine teeth on inside. 7 on outside: defining angle with 4 serrate spine teeth on inside and 2 serrate spine teeth on outside. Propod of gnathopod 2: palmar margin oblique, slightly concave. longer than posterior margin, with 11-12 spine teeth on inside. 12 on outside: defining angle with $3-4$ spine teeth on inside and 3 on outside.

Uropod 2: inner ramus with II spines; outer ramus curved laterally to nearly right angle with peduncle. with 1-2 inner spines and about 8 short outer spines. apex with 3 spines: peduncle with 4 outer and 3 inner spines.

Type-locality.--Crosley Canyon Cave, $4 \mathrm{~km} \mathrm{~S}$ of North Vernon, is located in the Crosley State Fish and Wildlife area of Jennings County, Indiana.

Distribution and ecology.--The range of this species. which is limited to southeastern Indiana and north-central Kentucky, extends from Jennings County, Indiana southwest to Meade County. Kentucky and southeast to Owen County, Kentucky (fig. 173).

Ovigerous females are recorded from May, August and November collections and brood about 20 young each. In caves this species has been found together with the amphipods C.puckardi. C. forbesi, and rarely Synurella dentata. The cave habitats include streams. stream pools, and drip pools. This species was also found in surface springs in Meade County, KY, where it was associated with the amphipods $C$. rivularis and $C$. packardi.

Etymology.--It is pleasure to name this species in honor of Dr. Julian J. Lewis. a biospeleologist whose diligent field work has led to the discovery of many records and new taxa of subterranean crustaceans in Indiana and elsewhere. 
Crangonxx orientalis. new species

Figures $174-178$

Material examined (paratypes unless designated otherwise).--MARYLAND. Dorchester Co.: swamp. $5.3 \mathrm{~km} \mathrm{~W}$ of Cambridge. $59.1 \%$. R. W. Jackson, 20 Apr 1943 (USNM): NORTH CAROLINA. Carteret Co.: Bogue Banks, ca. $0.45 \mathrm{~km}$ SE of western bridge. $49.40^{\circ}$. R. E. Ashten and E. Van Riper. 16 Mar 1978: Hertford Co.: Winton. $19.3 \%$. collector unknown, 23 Nov 1940 (USNM). VIRGINIA. Charlotte Co.: vernal pool. Keysville, I ₹.28 Mar 1967. A. Weaner: Chesapeake (Norfolk County): roadside ditch. $1.6 \mathrm{~km}$ S of Wallaceton. 20 specs (\%. ). J. R. Holsinger et al.. 18 Apr 1971: Dinwiddie Co.: Belchers pond near Petersburg, 2 ₹. $2{ }^{*}$, R. Bray, 22 Mar 1940 (USNM): Elizabeth City Co.: small stream. $6.2 \mathrm{~km}$ NW of Hampton, 12 \%. L. Hubricht. 23 Jan 1944 (USNM); Hampton (Elizabeth City County). ditch. NW of corner of NASA Langley Research Center. 1 q. R. Bedenbaugh et al.. 30 Mar 1994: small pool in forested wetland. weatern side of NASA Langley Research Center. R. L. Bedenbaugh et al.. $3 \approx$. 17 Mar 1994: Henrico Co.: stagnant pond in Maymont Park. Richmond. 9 \&. A. J. Seidenberg. 31 May 1973: seep on Sawmill Rd., ca. $5.6 \mathrm{~km}$ W of Richmond. $4 \subseteq .3 \sigma^{*}$. W. C. Sears. Nov 1977: Isle of Wight Co.: outlet of drain tile on Taylor farm. $5.6 \mathrm{~km}$ N of Chuckatuck. 1 . J. R. Holsinger. 24 Apr 1983: Mecklenburg Co.: pond at Oak Grove School. Chase city, 30.6 May 1935 (USNM) and $39.15 \approx .11$ May 1935 (USNM): Nansemond Co.: seep. $1.6 \mathrm{~km}$ S of Crittenden, $2 \%$. I c. L. Hubricht. 30 Jan 1944 (USNM): pool in Dismal Swamp near Washington Ditch. $8.0 \mathrm{~km} \mathrm{SE} \mathrm{of} \mathrm{Suffolk,} 7$ ․ L. Hubricht. $30 \mathrm{Apr} 1944$ (USNM): marsh-like pond. SW end of Dismal Swamp, $11 \%$ W. Priest. 2 Mar 1974: temporary pool in woods. 1.6 km E of Driver. 20 ๆ. $15 \delta$, L. Hubricht. 26 Mar 1944 (USNM): Parkers pond. Suffolk. I $\equiv$. R. Bray. 24 Mar 1940 (USNM): roadside ditch near Suffolk airport. 1 ? 1 juv. K. Garrett. 6 May 1971: Whaleyville. Suffolk. 5 ₹. 5 o*. R. Bray. 22 Mar 1940 (USNM); Norfolk Co.: temporary pool in Dismal Swamp. $3.2 \mathrm{~km}$ SSE of Bowers Hill. 1 \%. L. Hubricht, 14 Nov 1943 (USNM): roadside ditch, Bowers Hill, 9 . L. Hubricht. 25 Feb 1945 (USNM); stream in swamp, $3.2 \mathrm{~km} \mathrm{~S}$ of North Landing. holotype $\cong .220$ specs ( $\cong$. $\circlearrowleft$ juvs). L. Hubricht. 9 Apr 1944 (USNM): Northhampton Co.: small stream. I mi S of Capeville. $18 \subsetneq .5$ ษ. L. Hubricht. 16 Apr 1944 (USNM): Pittsylvania Co.: swale. Motley's Mill. $14 q .8$ 巳. L. Hubricht. 5 Feb 1949 (USNM): Southampton Co.: Charles Plantation. Franklin. 8 ₹. $3 \approx$ R. Bray. 22 Mar 1940 (USNM): Franklin. 6 \%. 2 ơ. R. Bray. 22 Mar 1940 (USNM); Virginia Beach (Princess Anne County): Stumpy Lake, $8.0 \mathrm{~km}$ S of Kempsville near Golf Course. 50 specs (₹. ๙*. juvs). J. R. Holsinger and C. H. Holsinger. 2 May 1971: Warwick Co.: pools in woods, $4.0 \mathrm{~km} \mathrm{NW}$ of Newport News. $139.70^{\circ}$. L. Hubricht. 23 Jan 1944 (USNM): ditch. 2.4 NE of Morrison. 3 9. 2 juvs. L. Hubricht. 23 Jan 1944 (USNM): York Co.: pond, $1.0 \mathrm{~km}$ S of Grafton Church, 2 \%, P. H. Stevenson and S. H. Holbrook. 13 Feb 1993: pond on Rt. 17 ca. I.I km S of Harris Grove, I P. J. R. Holsinger et al., 9 Mar 1994.

Diagnosis.--A small species morphologically similar to $C$. shoemakeri by possession of comb spines 


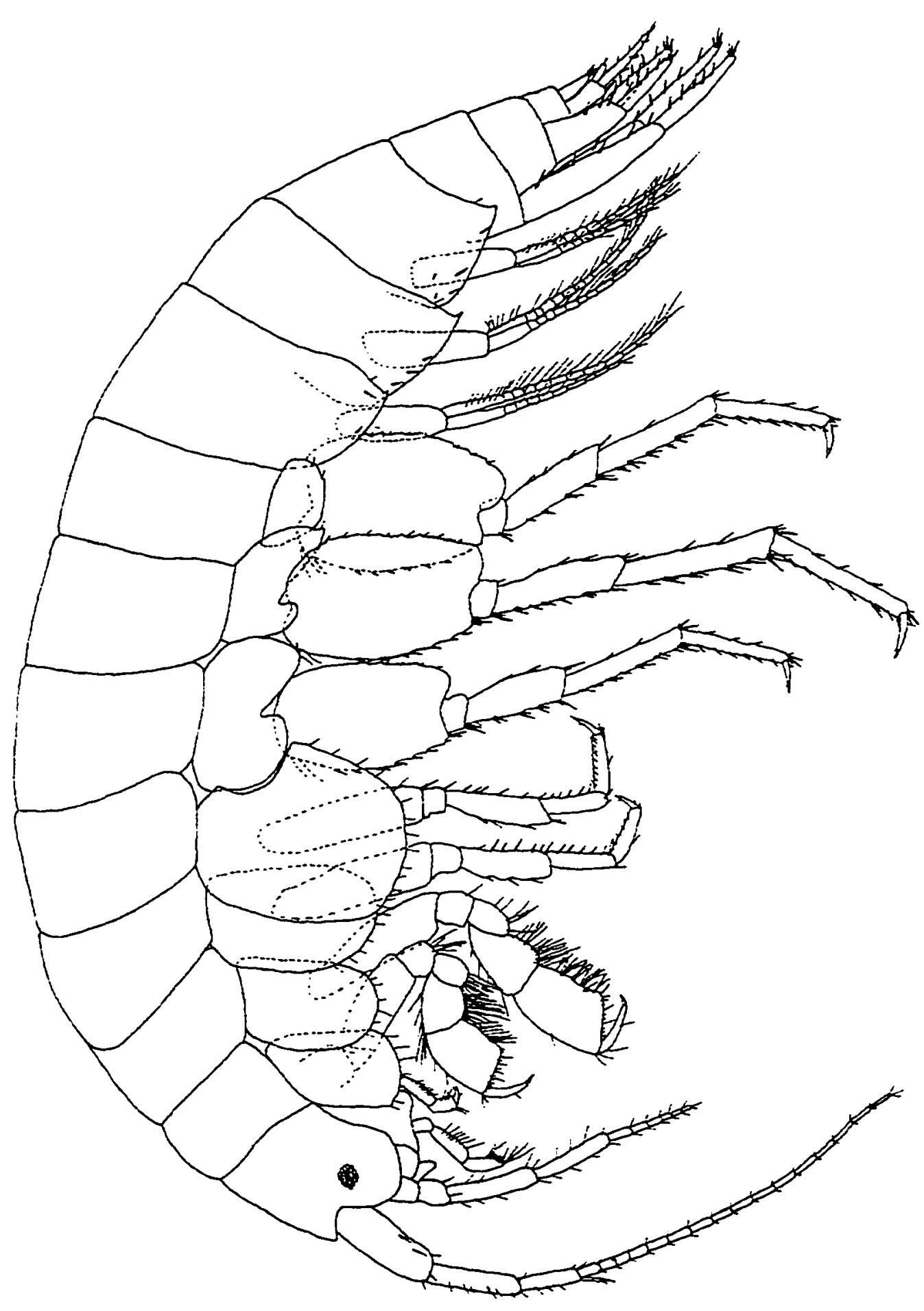

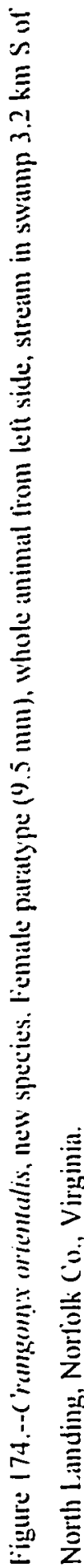




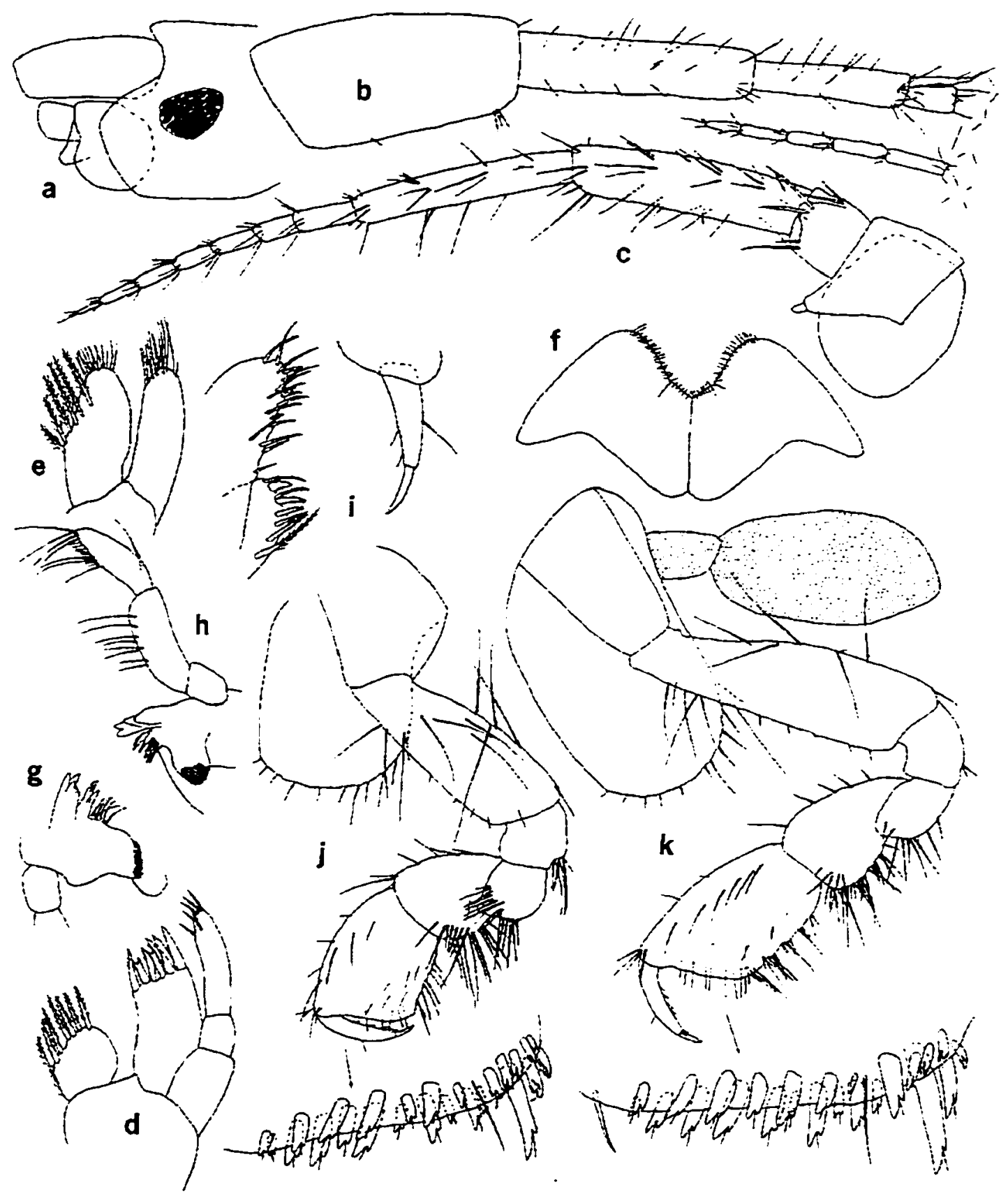

Figure 175.--Crangonyx orientalis, new species. Female paratype $(8.5 \mathrm{~mm})$. stream in swamp $3.2 \mathrm{~km}$ $S$ of North Landing. Norfolk Co., Virginia: a. head (in part): b. c. antennae 1. 2: d. e. maxillae 1. 2: $f$. lower lip: g. dentate part of left mandible: $h$, right mandible: $i$. inner and outer plates and palp dactyl of maxilliped: $j, k$, gnathopods 1.2 (palmar margins enlarged). 


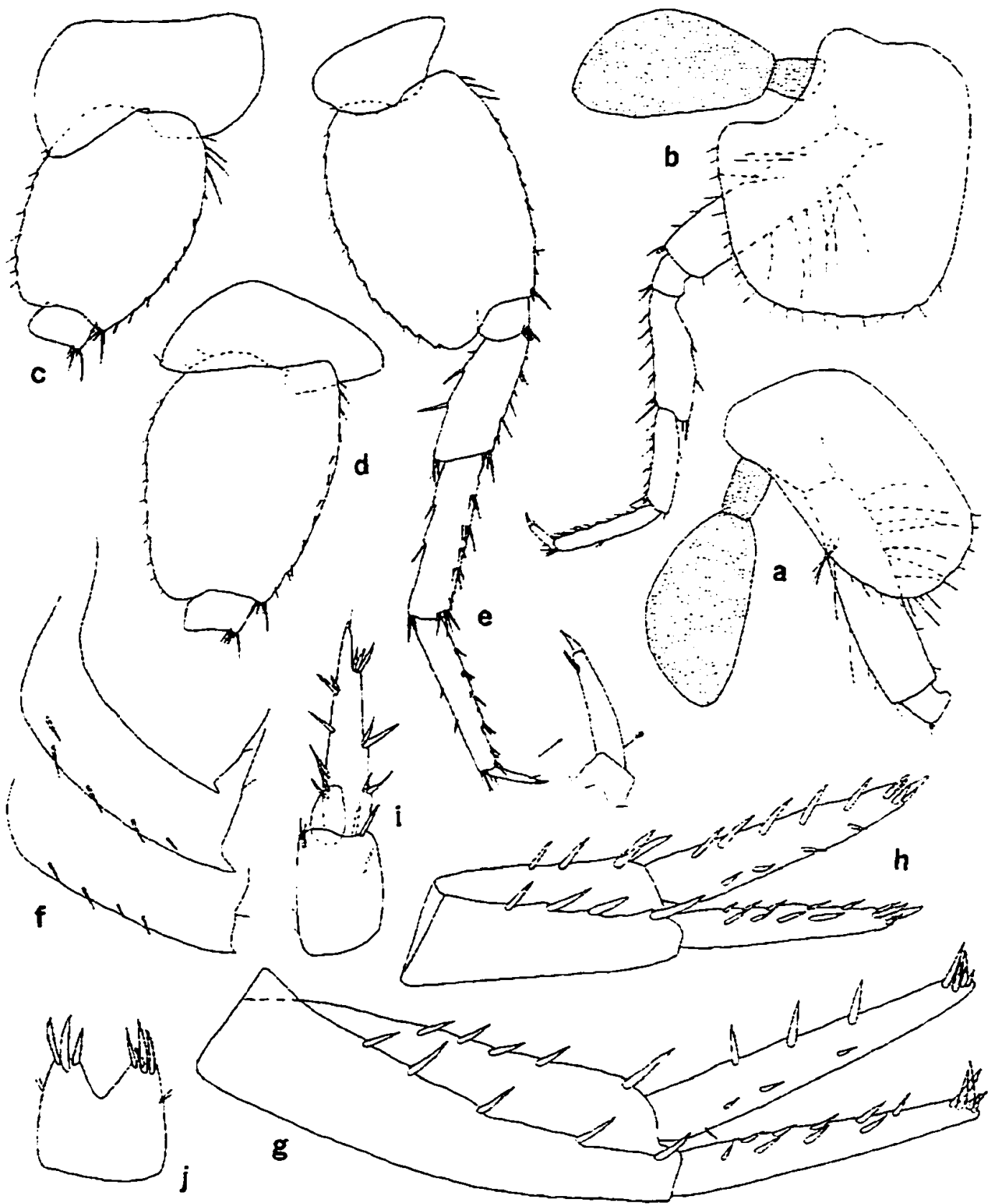

Figure 176.--Crangonyx orientalis, new species. Female paratype $(8.5 \mathrm{~mm})$, stream in swamp $3.2 \mathrm{~km}$ S of North Landing, Norfolk Co., Virginia: a, c. d, pereopods 3.5.6 (in part): b. e. pereopods 4. 7: f. pleonal plates; g. h, i. uropods $1,2,3$ : j, telson. 


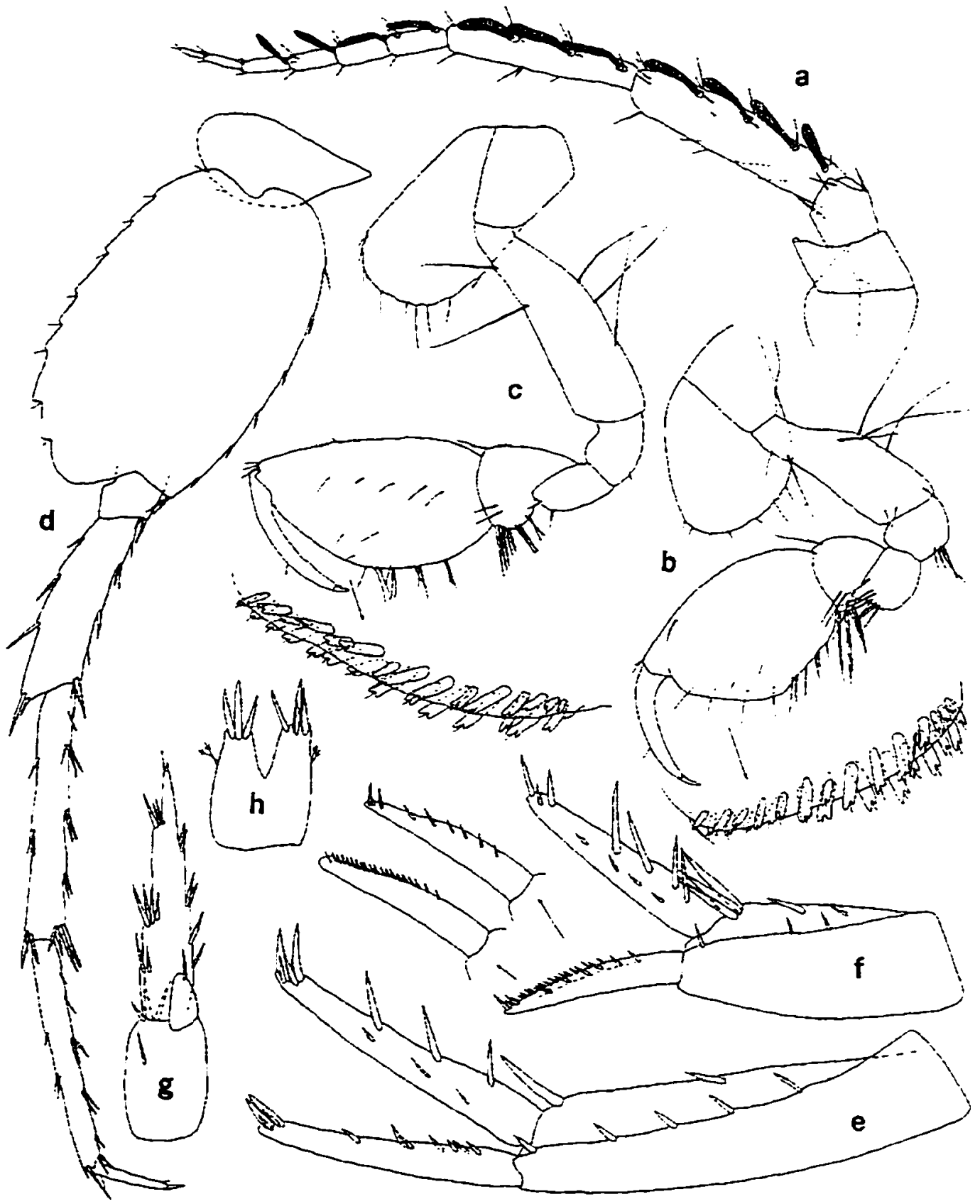

Figure 177.--Crangonyx orientalis, new species. Male paratype $(6.4 \mathrm{~mm})$. stream in swamp $3.2 \mathrm{~km} \mathrm{~S}$ of North Landing, Norfolk Co., Virginia: a, antenna 2: b, c. gnathopods 1. 2 (palmar margins enlarged): d. pereopod 7: e. g, uropods I, 2, 3: f. uropod 2 (inner and nurer rows of spines shown separately): $h$, telson. 


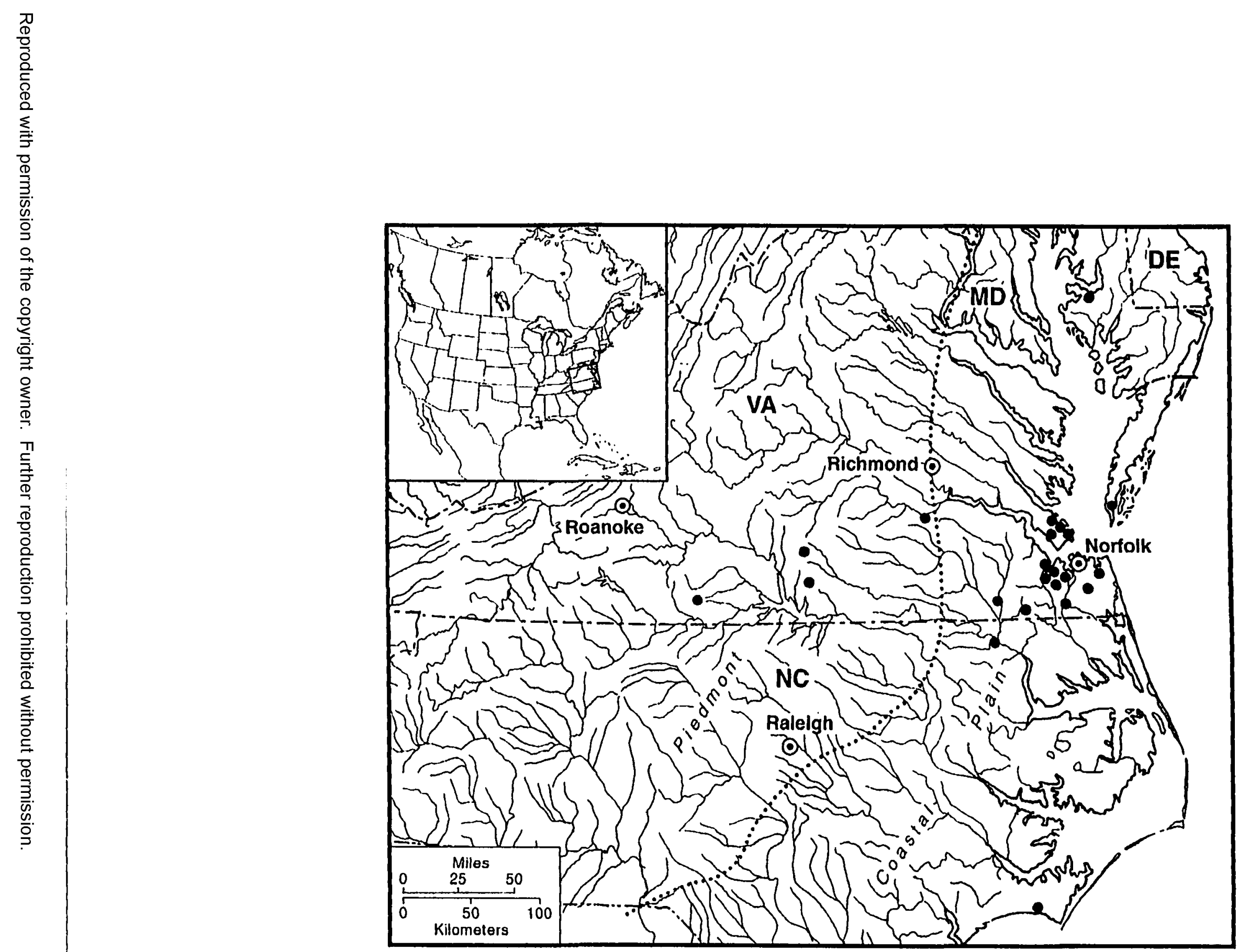

Figure 178.--Distribution of ( 'rangomyx oriennalis in North America. Solid circles represent 1-4 closely proximale localities. 
on male uropod 2. but distinguished from it by lacking spine teeth on dactyl of gnathopod propods. Largest male, $6.0 \mathrm{~mm}$ : largest female. $10.0 \mathrm{~mm}$.

Female.--Eye large and ovate. Antenna 1.55-57 percent length of body. about 1.8 times longer than antenna 2: primary flagellum with about 18 segments. Antenna 2, flagellum with 6-8 segments. Mandibles subequal. spine row with 5-6 spines: segment 2 of palp with 8-9 long setae. segment 3 with 1 A seta. $3 \mathrm{C}$ setae. $4 \mathrm{E}$ setae and a row of $\mathrm{D}$ setae, lacking $\mathrm{B}$ seta. Maxilla 1 : inner plate with $5-7$ apical plumose setae: palp with 5-6 slender spines on apex. Maxilla 2, inner plate with oblique row of 5-7 plumose setae on inner margin. Maxilliped: inner plate apically with 2 bladelike spines. I naked spine, and 5-7 plumose setae extending from inner margin to apex: outer plate with row of naked setae and $3-4$ slender pectinate spines on inner margin and $l$ apical plumose seta: dactyl with 3 setae on inner margin: dactyl nail very long. about 1,3 length of dactyl.

Propod of gnathopod I less than 2 times longer than carpus: palm straight or slightly concave. shorter than posterior margin. with 7 spine teeth on inside. 10 on the outside; defining angle with 1 large notched and 2 serrate spine teeth on inside. 3 small. notched-serrate and 2 serrate spine teeth on outside: posterior margin with 4 sets of setae singly or doubly inserted. Dactyl with row of short setae on inner margin, nail long. Ventral margin of coxa 1 with 9-11 setae.

Propod of gnathopod 2 less than 2 times longer than carpus: palm straight. shorter than posterior margin. with 6 spine teeth on inside, 10 on outside: defining angle with 2 notched and 3 notched-serrate spine teeth on inside, 2 notched and I serrate spine teeth on outside ( 1 larger): superior medial setae singly inserted: posterior margin with 5 sets of setae in groups of 2-6. Dactyl with row of short setae on inner margin. Ventral margin of coxa 2 with 9-1 I setae.

Coxa of pereopod 3 with 12-14 marginal setae. Coxa of pereopod 4 with 20-22 marginal setae. dact:1 of pereopod 4 about 35 percent length of corresponding propod. Pereopod 5: basis with 10 shallow serrations along posterior margin: 9 sets of short spines on anterior margin: $3-4$ long setae on the anteroproximal margin. Pereopod 6: basis with 12-14 siallow serrations along posterior margin. 8 sets of short spines on anterior margin; 3 short setae on anteroproximal margin. Pereopod 7: coxa with 3 setae on the posterior margin: basis with 12-15 shallow serrations along posterior margin. 9 sets of short spines on anterior margin: dactyl about 35 percent the length of corresponding propod.

Pleonal plates: posterior margin of plate 1 slightly convex, with I seta. distoposterior corner produced and acute: posterior margin of plate 2 nearly straight with 1 seta. distoposterior comer slightly produced and acute. ventral margin with 5 (subventral) spines; posterior margin of plate 3 nearly straight with I seta. distoposterior comer produced and acute. ventral margin with 4 (subventral) spines. Peduncle of pleopod 1 with 2 retinaculae: lacking setae on outside margin.

Uropod 1: inner ramus 80 percent length of peduncle, armed with II spines: outer ramus with 14 spines: peduncle with 5 outer and 5 inner spines. Uropod 2: inner ramus armed with 14 spines: outer ramus with 13 spines: peduncle with 4 outer spines, and 4 inner spines. Uropod 3 : inner ramus with I spine. outer 
ramus about 2 times longer than peduncle, inner and outer margin each with $3-4$ set of spines in groups of $1-3$. Telson broader than long, notched about $1 / 3$ distance to base. each lobe with $3-4$ apical spines: spines about $1 / 2$ length of telson.

Male.--Differing from female as follows. Smaller with more slender, elongate body. Antenna 2: flagellum with 6 segments, calceoli present on peduncular segments 4 and 5 . and first 4 segments of flagellum.

Propod of gnathopod 1 more than 2 times larger and longer than carpus, and much broader: palm convex. oblique. 10 spine teeth on inside. 9 on outside: defining angle with I notched and 3 notchedserrate spine teeth on inside. 4 on outside.

Propod of gnathopod 2 more than 2 times longer and larger than carpus: palm margin convex. oblique. with 12 spine teeth on inside. 11 on outside: defining angle with 4 spine teeth on inside and 3 on outside.

Uropod 2: inner ramus with 11 spines; outer ramus deflected laterally. with inner row of comb spines on distal $3 / 4-2 / 3.6$ short spines on outer margin, 3 spines on apex: peduncle with 3 outer and 5 inner spines.

Type-locality.--Stream in swamp. $3.2 \mathrm{~km} \mathrm{~S}$ of North Landing. Norfolk County (now in the city of Chesapeake). This locality is close to the northern margin of the Great Dismal Swamp.

Distribution and ecology.--The range of this species extends from Dorchester Co.. Maryland southward on the Coastal Plain to northeastern North Carolina and southwest into the Piedmont of southcentral Virginia (Fig. 178). It covers a linear distance of about $450 \mathrm{~km}$ north to south and about $300 \mathrm{~km}$ east to west.

The habitat of this species includes swamps. roadside ditches. small woodland ponds. seeps and outlets of drain tiles.

Crungonvx orientalis has been taken with other species of Crangonvx. including $C$. disjunctus. $C$. polustris. and $C$. serratus. It is often associated with the first two species in the Grafton "sinkhole" ponds near Hampton. VA. Ovigerous females were collected in February, March. April and May.

Etymology.--The epithet orientalis is from Latin meaning "eastern." and refers to the distribution of this species in the eastern United States.

Crangonyx bousfieldi. new species

Figures 179-182

Material examined (paratypes unless designated otherwise).-- INDIANA. Wayne Co.: spring between Dublin and Mt. Auburn, $2 q$ (not paratypes), L. Hubricht. 17 Apr 1942 (USNM). KENTUCKY. Jefferson Co.: tiny stream, $3.2 \mathrm{~km} \mathrm{~S}$ of Thixton. 30 specs $\left(q, \sigma^{\circ}\right)$, N. Hynes. 6 Jan 1985 (USNM): spring $1.6 \mathrm{~km} \mathrm{E}$ of Preston. holotype $\sigma^{*} .8$ 9. 4 o*. R. Fox. 12 Feb. 1971: Oldham Co.: tributary to Harrods Creek. 


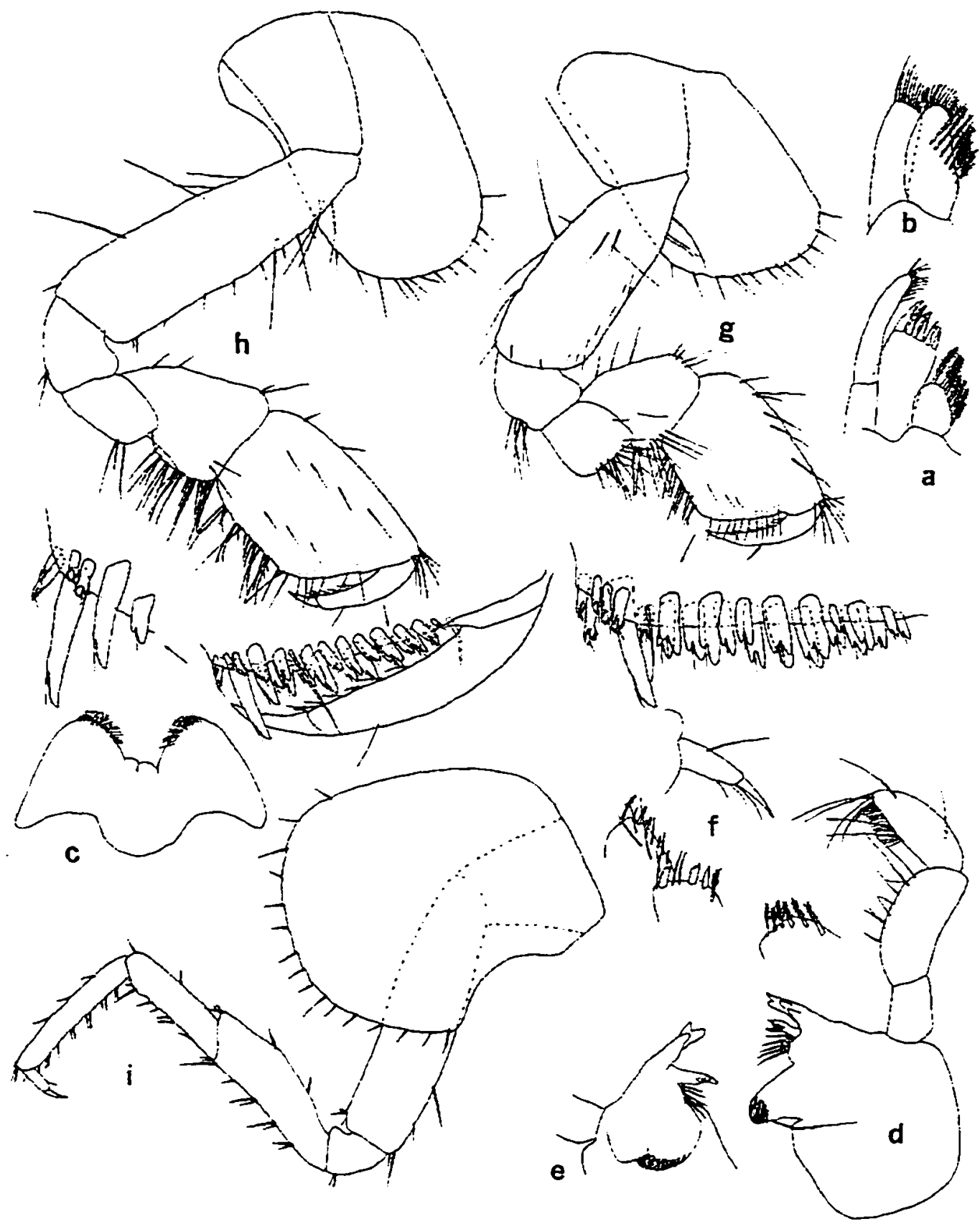

Figure 179.--Crangonyx bousfieldi, new species. Female paratype $(7.8 \mathrm{~mm})$. spring $1.6 \mathrm{~km} \mathrm{E}$ of Preston, Jefferson Co., Kentucky: a. b, maxillae 1, 2: c, lower lip; $d$. right mandible: e. dentate part of left mandible: $f$, inner and outer plates and palp dactyl of maxilliped (greatly enlarged); g. h. gnathopods 1.2 (palmar margins enlarged); i, pereopod 4. 


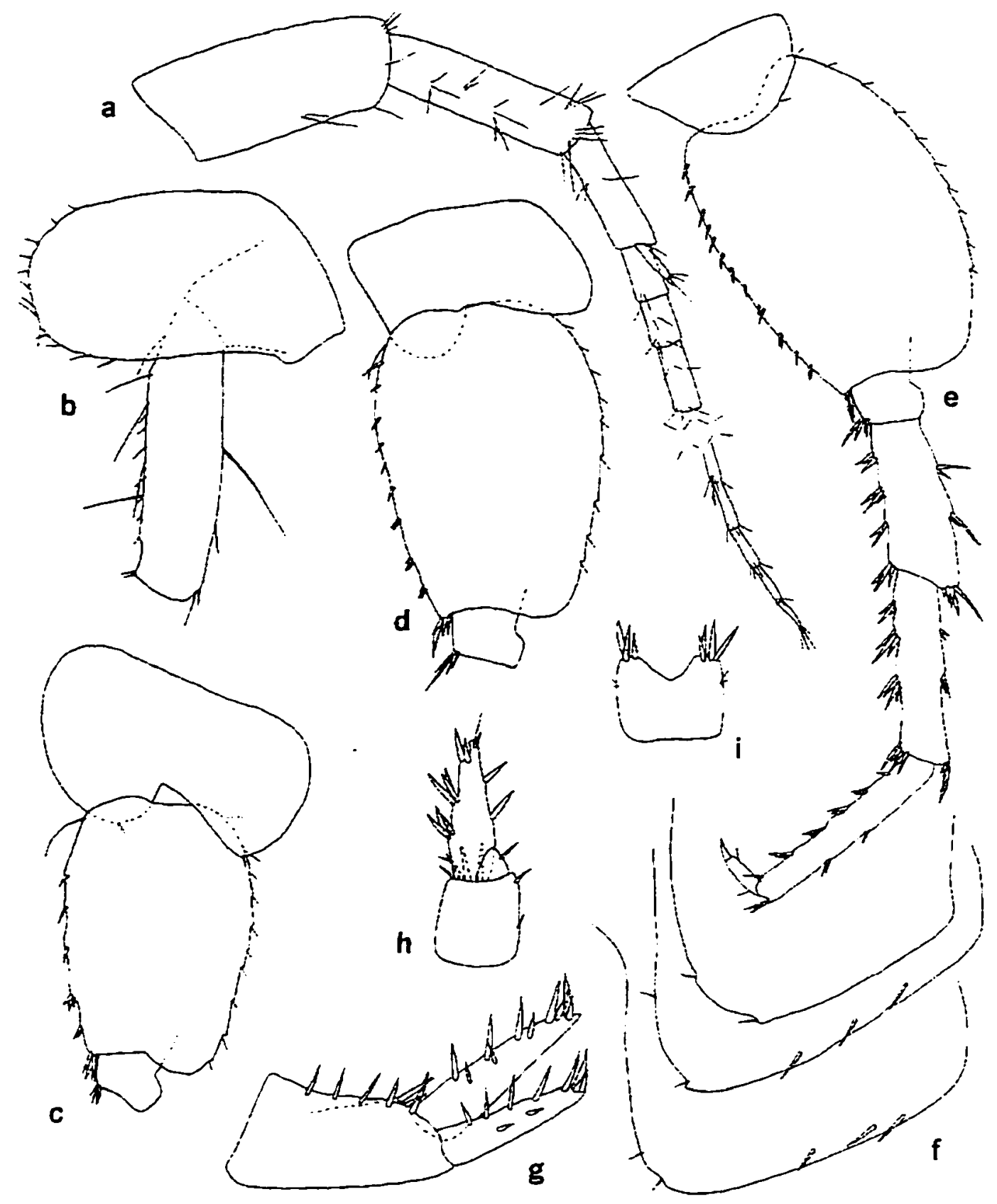

Figure 180.--Crangonyx bousfieldi. new species. Female paratype $(7.8 \mathrm{~mm})$, spring $1.6 \mathrm{~km} \mathrm{E}$ of Preston. Jefferson Co.. Kentucky: a. antenna I: b. c. d. pereopods 3.5.6 (in part): e. pereopod 7: f. pleonal plates: g, h. uropods 2. 3; i, teison. 


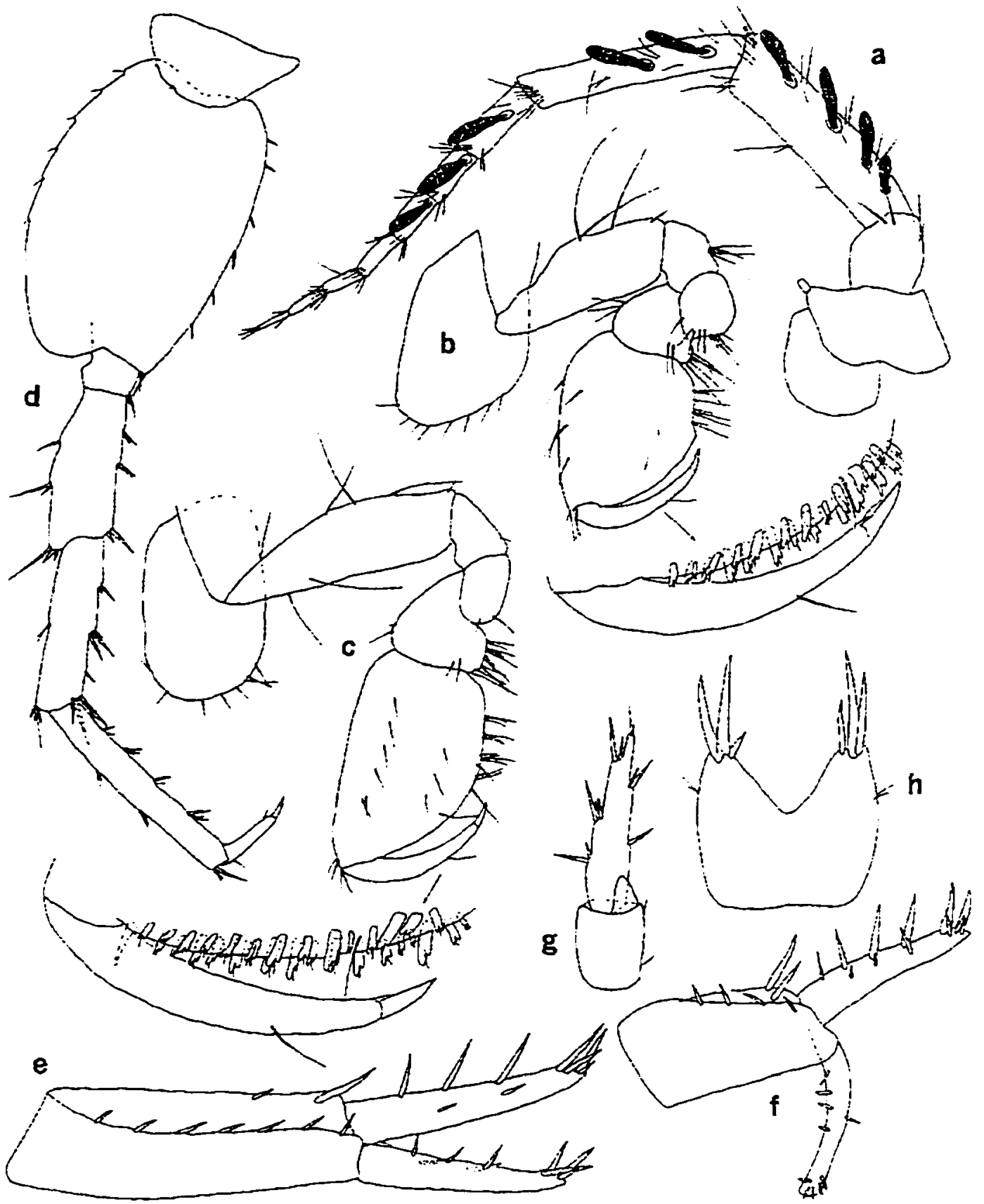

Figure 181 .--Crangonyx bousfieldi, new species. Male paratype $(4.7 \mathrm{~mm})$, spring $1.6 \mathrm{~km}$ E of Preston. Jefferson Co., Kentucky: a, antenna 2; b, c, gnathopods 1.2 (palmar margins enlarged): d. pereopod 7: e. f. g. uropods 1. 2. 3; h. telson. 
30 specs $\left(2, \sigma^{\prime}\right)$. R. Fox, 21 Nov 1970: stream beside St. Rt 329, between Harrods Creek and US Rt.48. 5 specs. R. Fox. 12 Feb. 1971 . OHIO. Highland Co.: Cave of the Springs, 3 z. $1 c^{\circ}$ H. H. Hobbs, III. 13 Dec 1983. Delaware Co.: Vergoyn's well, Delaware, 29 (not paratypes). E. L. Rice. (collection date not given) (USNM).

Diagnosis.-A small species very similar to $C$. lewisi but distinguished from it by having 2 setae on posterior margins of pleonal plates 1-3. (1 located just above the corner): well developed eyes: absence of notched-serrate spine teeth on propod of gnathopods of male; inner side of outer ramus of male uropod 2 with only I spine in the middle. Largest male. $5.1 \mathrm{~mm}$ : largest female. $8.2 \mathrm{~mm}$.

Female.--Eye large and ovate. Antenna 1, 55-57 percent length of body, about 1.8 times longer than antenna 2: primary flagellum with about 21 segments. Antenna 2, flagellum with 7-9 segments. Mandibles subequal. spine row with 5 spines: segment 2 of palp with 8-9 setae. segment 3 with I A seta. I B seta. 2 C seta. 4-5 E setae and a row of D setae. Maxilla I: inner plate with 7 apical plumose setae: palp with 10 slender spines on apex. Maxilla 2. inner plate with oblique row of 7 plumose setae on inner margin. Maxilliped: inner plate apically with 3 bladelike spines. 3 naked spines. and 5-6 plumose setae extending from inner margin to apex: outer plate with row of naked setae and 4-5 slender pectinate spines on inner margin: dactyl with 3 setae on inner margin: dactyl nail very long, $1 / 3$ length of dactyl.

Propod of gnathopod $I$ and 2 less than 2 times larger and longer than carpus: palm almost straight. shorter than posterior margin, with 8 spine teeth on inside, 12 on the outside: defining angle with 3 notched-serrate spine teeth on inside. and 1 large simple and 2 serrate spine teeth on outside: superior medial setae singly or doubly inserted: posterior margin with 6 sets of setae singly or doubly inserted. Dactyl with row of short setae on inner margin, nail long. Ventral margin of coxa 1 with 13 setae.

Propod of gnathopod 2 less than 2 times larger and longer than carpus: palm almost straight. shorter than posterior margin, with 8 spine teeth on inside, 10 on outside: defining angle with 1 notched. 1 large simple and 2 serrate spine teeth on inside, and 2 small notched and 1 large simple and 2 serrate spine teeth on outside: superior medial setae singly inserted; posterior margin with 4 sets of setae in groups of $1-5$. Dactyl with row of short setae on inner margin. Ventral margin of coxa 2 with 13 setae.

Coxa of pereopod 3 with 12 marginal setae. Coxa of pereopod 4 with 16 marginal setae. dactyl of pereopod 4 about 33 percent !ength of corresponding propod. Pereopod 5: basis with 9 shailow serrations along posterior margin: 5 sets of short spines on anterior margin: 1 long seta on the anteroproximal margin. Pereopod 6: basis with 8-9 serrations along posterior margin. 9 short spines on anterior margin: I setae on anteroproximal margin. Pereopod 7: coxa with 2 setae on the posterior margin: basis with 9 shallow serrations along posterior margin; 12 sets of short spines on anterior margin: dactyl about 33 percent length of corresponding propod.

Pleonal plates: posterior margin of plate I convex, with 2 setae, I on distoposterior corner. distoposterior corner recessed and subacute; posterior margin of plate 2 weakly convex. with 2 setae. 1 on distoposterior corner. distoposterior comer recessed, rounded, indistinct. ventral margin with 3 (subventral) 
spines: posterior margin of plate 3 nearly straight with 2 setae. I just on distoposterior corner. distoposterior comer round and not produced, ventral margin with $j$ (subventral) spines. Peduncle of pleopod I with 2 retinaculae; lacking setae on outside margin.

Uropod 1 : inner ramus 65 percent length of peduncle, armed with 10 spines; outer ramus with 12 spines: peduncle with 7 outer and 1 inner spines. Uropod 2: inner ramus armed with 10 spines: peduncle with 5 outer and 2 inner spines. Uropod 3: inner ramus with 1 spine, outer ramus about 1.5 times longer than peduncle, inner and outer margins each with 2-3 set of spines in groups of $1-3$. Telson broader than long. notched about $1 / 4$ distance to base. each lobe with $3-4$ apical spines: spines about $1 / 2$ length of telson.

Male.--Differing from female as follows. Smaller with more slender, elongate body. Antenna 2: calceoli percent on peduncular segments 4 and 5 . and first 3 segments of flagellum.

Propod of gnathopods 1 and 2 more than 2 times larger and longer than carpus and much broader. Propod of gnathopod I palm convex: defining angle with 3-4 spine teeth on inside and 4 on outside. Propod of gnathopod 2: palm oblique and convex: defining angle with 3-4 spine teeth on inside and 7 on outside.

Uropod 2: inner ramus with II spines: outer ramus deflected laterally and slightly backward. upper margin with 1 inner and 4 short outer spines, apex with 5 spines: peduncle with 4 outer spines and 2 inner spines. Uropod 3: inner ramus lacking spines; outer ramus about 2 times length of peduncle. inner and outer margin each with 2-3 set of spines in groups of $1-3$.

Type-locality.--A spring located about $46 \mathrm{~m} \mathrm{~S}$ of state road 1003, about $1.6 \mathrm{~km}$ E of Preston Highway in Jefferson County, Kentucky. The type-series consists of 13 specimens. which includes 3 ovigerous females.

Distribution and ecology.--The range of this species covers a wide area but is represented by only a few collections. It extends from middle Ohio on the north to northern Kentucky on the southwest. covering a linear distance of about $300 \mathrm{~km}$ (fig. 182). The specimens from Indiana and central Ohio differ slightly from those farther south and were not designated paratypes.

This species has been collected from small streams. one spring, one cave and one well. Ovigerous females were found in collections form the months of January, February, and November.

Etymology.---It is pleasure to name this species in honor of Dr. Edward L. Bousfield, a world authority on the systematics of the Amphipoda, who has contributed significantly to our knowledge of amphipod crustaceans. both marine and freshwater.

\section{Crangonyx species}

The following 13 populations cannot be assigned to any known species. Some of them probably represent distinct species but have not been described for lack of mature specimens or adequate material. 
All of them. however. appear to belong to the gracilis group.

I. A single ovigerous female ( $5.6 \mathrm{~mm})$, with small eyes, collected from Bear Creek at Oak Ridge. Anderson Co.. Tennessee by J. A. Payne (14 April 1963).

2. Two immature males (4.0-4.5 mm), very similar to males of $C$. gracilis. collected from a spring 4.8 $\mathrm{km}$ SE of Sulphur. DeKalb Co.. Alabama by S. B. Peck and A. Fiske ( 15 July 1967).

3. One immature male $(3.9 \mathrm{~mm})$ and 8 juveniles collected from J. E. Holt's well. $17.6 \mathrm{~km} \mathrm{E} \mathrm{of}$ Pittsboro. Chatham Co.. North Carolina by L. Hubricht (27 may 195!). The male is similar to males of $C$. stagnicolous.

4. Five immature females (largest $4.5 \mathrm{~mm}) .1$ mature male $(4.5 \mathrm{~mm}$ ) and 2 juveniles collecred from small seep draining into Red River along Kentucky Hwy 715 . about $7.2 \mathrm{~km} \mathrm{SW}$ of Pomerovton in Menifee Co.. Kentucky by J. E. \& M. R. Cooper (13 April 1968). The outer ramus of male uropod 2 has only 2 inner spines near its end.

5. Ten ovigerous female and I male collected from a seep $9 \mathrm{~km} \mathrm{E}$ of Olive Hill. Carter Co.. Kentucky by L. Hubricht (22 April 1949). The distoposterior corners of pleonal plates of the female bear 2 setae. one at the corner.

6. One female $(5.7 \mathrm{~mm}), 2$ males (largest. $5.5 \mathrm{~mm}$ ) and I juvenile collected from Croxville Cave. Pike Co.. Illinois by S. B. Peck (15 August 1968). Although these specimens are in poor condition. they are slender. eyeless and have elongate appendages and are very similar to $C$. hobbsi.

7. Six females and 6 males collected from a stream in Blount Co.. Alabama by M. R. Cooper ( 31 December (965). This species appears to be very similar to $C$. rivularis.

8. A single female $(5.3 \mathrm{~mm})$ collected from a small spring near Great Saltpeter Cave. Rockcastle Co.. Kentucky by T. C. Barr. Jr ( 18 April 1964).

9. A single mature female $(7.0 \mathrm{~mm})$ collected from Patuxent River. Maryland (county not given) by Chu-Je Tsai (collection date not given). The telson has 1 lateral spine per lobe.

10. A single female collected from a spring beside US Rt. $441 \mathrm{ca} .1 .6 \mathrm{~km} \mathrm{~S}$ of Anderson-Knox Co. line. Knox Co., Tennessee by R. Fox et al. (4 January 1971).

11. Eleven females and 2 males collected from springs $3.6 \mathrm{~km} \mathrm{NW}$ of Lancaster. Garrard Co.. Kentucky by L. Hubricht (24 April 1941).

12. Five females and 7 males collected from a spring $4.6 \mathrm{~km} \mathrm{E}$ of Castalian Springs. Trousdale Co.. Tennessee by L. Hubricht (I June 1941).

13. Three immature females (one eyeless) and 3 mature males collected from a spring $1.0 \mathrm{~km} \mathrm{E}$ of Clifton. Woodford Co.. Kentucky by J. J. Lewis et al. (19 February 1995). 


\section{CHAPTER V}

\section{FINDINGS AND INTERPRETATIONS}

\section{ORIGIN OF CRANGONYX}

As discussed by Holsinger $(1978,1986)$ the family Crangonyctidae has an ancient freshwater ancestry probably dating back at least to the Mesozoic. Two lines of evidence support this theory: (1) the crangonyctids are exclusively freshwater and have no apparent relationship with any marine forms and (2) the three largest genera (Crangonyx. Stygohromus and Synurella) of the family Crangonyctidae have a Holarctic distribution. which suggests that they were well established on the Laurasian landmass prior to continental breakup in the late Mesozoic. Putative ancestors of the genus Crangonıx probably originated as a very old freshwater lineage dating back at least to the Mesozoic and possibly earlier.

From the discussions in the Phylogenetic Analysis Section. Crangonyx and Synurella are established as sister groups. Although hypothesized cladistic relationship (figs 1-3) cannot give us a clear picture of what the ancestor of Crangonyx looked like. they all clearly support a two lineage evolutionary scheme. one lineage being gracilis-like and the other forbesi- and richmondensis-like. Most of the species of Synurella have as many ancestral characters as the forbesi and richmondensis groups. Crangony.x chebnikovi and $C$. subterraneus from Europe are morphologically more similar to species of the forhesi and richmondensis group than to the gracilis group ( $C$. chebnikov apparently belongs to the richmondensis group. at least on the basis of its description). Another European species. C. pari. is a tiny interstitial species. apparently adapted for life in microspaces of the subterranean environment and has several autapomorphic characters. The only species of the gracilis group found in Europe is C.pseudogracilis, but it has been introduced there by human transport (see discussion later). From the above discussion. I suggest that the ancestor of Crangonyx resembled $C$. forbesi and separated from the common ancestor of Synurella and Crungonyx somewhere on Laurasia. Extensive diversification occurred in North America as more and more species of the gracilis group evolved after the break up of Laurasia. In Europe the surface species :vere probably driven out by Synurella, which is more common and widespread in Eurasia than North America, and other surface amphipods such as Gammarus spp. However. a few survived in the subterranean habitats, where they now probably represent relict species. The evolution of the species groups is discussed in the Phylogenetic Analysis Section.

\section{HABITATS OF SPECIES}

Species of Crangonyx have exploited a wide variety of surface and groundwater habitats in North 
America. In an attempt to gain further insight into the biogeographic. phylogenetic. and ecological relationships among these species, I have compiled general descriptive data on the different freshwater biotopes inhabited by the species in this genus in Table 3 . The data are from observations by the collectors. wherever that can be determined. The number of different habitats recorded for some species may not be complete, because in many cases only a few collections were available.

Species have been collected from basically five different habitat types: (1) subterranean waters. (2) seepsisprings. (3) surface streams/rivers. (4) wetland waters (e.g.. bogs. swamps. ditches. etc.). and (5) large ponds/lakes. The most common habitat types are springs/seeps and wetland waters. A total of 36 species are found in one or both of these habitats. Only six species can be found in large ponds/lakes and these species are widely distributed and can be found in almost all the other habitats as well.

A total of 24 species are found in subterranean waters (primarily in caves). but only 11 of them are troglobites. An additional 16 species are found in springs/seeps habitats. Based on reduced eyes. light pigmentation and a strong association with secluded spots in cold spring waters. many of the spring seep dwellers are apparently preadpted to life in subterranean waters (Holsinger. 1994).

\section{BIOGEOGRAPHY OF CRANGONYY}

The world distribution of Crangony is shown in Figure 4 and distributions of species groups and individual species are shown in figures that accompany the species descriptions. Like the other members of the Crangonyctidae, the ranges of species of Crangomx are quite variable but fall into one of the three basic patterns as described by Holsinger (1978): (1) distribution is localized and species are known only from a few localities: (2) distribution is restricted to a single major drainage system. and (3) distribution is very broad. covering parts of two or more major drainage systems.

Of the six species groups, only the richmondensis and gracilis groups are widely distributed in North America. These two groups also contain many species. with a total of about $66 \%$ of the recognized species. This broad distribution may indicate that some species in these two groups are highly vagile and have great dispersal capability. Ranges of members of the three large species groups (shoemukeri, richmondensis. gracilis). plus the forbesi group, all overlap in the middle-western region of North America. In the Origin of Crangonyx section, I concluded that the ancestor of Crangonyx might have been more forhesi-like. Here I suggest that the ancestor might have evolved in the middle-western region of North America and subsequently all species groups (and species) evolved as the ancestral species dispersed outward from this area. The alternative hypothesis is that the ancestor may actually have been widely distributed on Laurasia and species groups later evolved in place as the result of isolation and environmental changes and subsequent genetic differentiation .

Several aspects of distribution are noted for Crangonyx in North America. The first is that almost all species of the genus are distributed in the eastern part of North America, east of the Rocky Mountains. The 
Table 3. Records of species of Crangonyx from five kinds of generalized habitats in North America.

\begin{tabular}{|c|c|c|c|c|c|}
\hline Species & subterranean waters & seeps/springs & surfaces stream & wetland waters & larger ponds/lakes \\
\hline \multicolumn{6}{|l|}{ shoemakerı group } \\
\hline shoemakert & & $\mathrm{x}$ & & $x$ & \\
\hline setodactylus & & $x$ & $x$ & $x$ & \\
\hline minor & & $x$ & $x$ & $x$ & \\
\hline aberrans & & & & $x$ & \\
\hline \multicolumn{6}{|l|}{ longicurpus group } \\
\hline longicarpus & & $r$ & $x$ & $x$ & \\
\hline \multicolumn{6}{|c|}{ richmondensis group } \\
\hline richmondensis & $x$ & $\mathbf{x}$ & $x$ & $x$ & $x$ \\
\hline anomalus & $x$ & $x$ & $x$ & $\mathrm{x}$ & $\mathrm{s}$ \\
\hline Indianensis & $x$ & $x$ & & & \\
\hline cornutus & & $r$ & & $x$ & \\
\hline obliquus & & $x$ & $\mathrm{x}$ & $\mathrm{x}$ & \\
\hline disfunctus & & $\mathrm{x}$ & $x$ & $x$ & \\
\hline dearolfi & $\mathrm{x}$ & & & & \\
\hline grandtmanus & $x$ & $x$ & & & \\
\hline mubrichnt & & $\mathrm{x}$ & $x$ & $r$ & \\
\hline \multicolumn{6}{|l|}{ forhest group } \\
\hline forbest & $x$ & $x$ & $x$ & $x$ & \\
\hline insolitus group & & & & & \\
\hline insulttus & $x$ & & & & \\
\hline \multicolumn{6}{|l|}{ gracilis group } \\
\hline gractlis & $\mathrm{x}$ & $x$ & $\mathrm{x}$ & $x$ & $x$ \\
\hline barrt & $x$ & $x$ & & & \\
\hline montanus & & $\mathrm{x}$ & & $x$ & \\
\hline rivulurts & $\mathrm{s}$ & $x$ & $x$ & $\mathrm{x}$ & $x$ \\
\hline packardt & $x$ & $\mathrm{x}$ & & & \\
\hline stagnicolouxs & & $\mathrm{x}$ & & $\mathbf{x}$ & \\
\hline$a k a$ & & $x$ & $x$ & & \\
\hline caecus & $x$ & & & & \\
\hline ohroensis & $x$ & $x$ & & & \\
\hline acicularis & & $x$ & $x$ & & \\
\hline polustits & & $x$ & $x$ & $x$ & \\
\hline
\end{tabular}


Table 3. continued

\begin{tabular}{|c|c|c|c|c|c|}
\hline Species & subterranean waters & seeps/springs & surfaces stream & wetland waters & larger ponds/akes \\
\hline castellanum & $x$ & & & & \\
\hline hohbst & $\mathbf{x}$ & $x$ & & & \\
\hline hacul:spina & & $x$ & & $x$ & \\
\hline thoridanus & $x$ & $x$ & $x$ & $x$ & $x$ \\
\hline pseiudogracelis & $x$ & $x$ & $x$ & $x$ & $x$ \\
\hline constmolis & $x$ & $\mathrm{x}$ & & $x$ & \\
\hline longudactylus & $x$ & & & $x$ & \\
\hline ungrouped spectes & & & & & \\
\hline serratus & & $x$ & $\mathrm{r}$ & $x$ & $x$ \\
\hline antennatus & $\mathrm{x}$ & $x$ & & & \\
\hline ipecus & $r$ & & & & \\
\hline fontunatus & $y$ & $x$ & & & \\
\hline coopert & $x$ & & & & \\
\hline lewtst & $x$ & $x$ & & & \\
\hline ortentalts & & & $x$ & $x$ & \\
\hline bousfiedt & & $x$ & $r$ & & \\
\hline
\end{tabular}


exceptions are $C$. pseudogracilis, $C$. floridanus, and $C$. richmondensis, which also have limited distributions in the west. The western part of North America may lack enough good habitats to support more than a few species of the genus. and the species found there, such as $C$. pseudogracilis and $C$. floridanus. were probably introduced recently by man (see Ecological Relationship section). However. as pointed out below, the very wide distribution of $C$. richmondensis needs further explanation and does not appear to have been affected by human introductions.

The second is that the species of Crangonyx with distributions in glaciated areas also have distributions south of the southern extent of Pleistocene glaciation. This is strong evidence that these species dispersed north after glaciation, expanding their ranges throughout several drainage systems. These species are all epigean forms. The subterranean species have limited dispersal ability and. in turn. the extent of subterranean drainage is generally restricted. In contrast to the large. exclusively subterranean amphipod genus Stygobromus, no surviving relicts of subglacial refugia are tound in North American Crungonyx (Holsinger. 1978).

The shoemakeri group has a disjunct distribution. with two species $(C$. setodacty/us and $C$. minor) widely distributed in middle-western North America and the other two species $(C$. shoemakeri and $C$. aherrans) distributed east of the Appalachians. These two pairs are also separated from each other by a distance of about $900 \mathrm{~km}$. One hypothesis to explain this disjunct distribution is that distributions of the two eastern species were reduced after speciation or that they moved further eastward to exploit new habitats that were developing on Coastal Plain terrains recently freed of marine/brackish transgressions in the middle to late Tertiary. The alternative hypothesis is that the shoemakeri group may not be a monophylogenetic group as I suggested in the Phylogenetic Analysis section. If we accept the possibility that the principal diagnostic (apomorphic) character of this group. which is the possession of teeth along the inner margin of the dactyl of the female gnathopod. was independently derived in the two pairs. then this group as presently recognized cannot hold. I prefer the second hypothesis, since Symurella dentata also has this "apomorphic" character. Thus, according to the latter. this complex character may have been derived independently. And although these species have similar morphology. I find that $C$. setodacr.lus and $C$. minor also share apomorphic characters with the unassigned group species $C$. lewisi and $C$. housfieldi, which are also distributed in the middle-western region. It is possible that these species may all belong to one species group. Crangonyx shoemakeri is also very similar to $C$. orientalts. except the latter does not liave teeth on the inner margin of the dactyl of the female gnathopods. These species overlap in part of their distribution. and moreover. they may be derived from a common ancestor. But as is often the case with analysis of species distributions in poorly known genera, many species distributions may not yet be completely known and only a few good characters are used in phylogenetic analysis. Thus, in the absence of contrary evidence, the shoemakeri group is at least tentatively regarded as monophyletic.

The geographic distributions of $C$. setodactylus and $C$. minor are also rather unusual. Crangomx minor has a wider distribution. which surrounds that of $C$. setodacty/us. Except in the middle of Kentucky 
and in the vicinity of their type-localities. separated by just a few kilometers. where these two species overlap, they do not come in actual contact with each other. My hypothesis is that $C$. minor has greater dispersal ability and $C$. setodactylus has greater competition ability. Thus, after glaciation. they both dispersed northward and $C$. setodactylus repiaced $C$. minor in the heart of the middle-western area.

Crungomx aberrans is the only species of the shoemakeri group that is restricted to an area north of the southern limit of Pleistocene glaciation. It might be derived from an ancestral population that moved there from an area south of the glacial boundary after glaciation.

The longicarpus group is represented by only one species on the Coastal Plain of Virginia and North Carolina. In the Phylogenetic Analysis section. I suggested that this species may be an evolutionary intermediate between the ancestor and the gracilis group. If this is the case it should have a wider distribution. One interpretation is that the longicarpus group may have been represented by many species in the past but only $C$. longicarpus has survived until the present time. The Coastal Plain of eastern North America. occupied today by $C$. longicarpus. contains geologically younger freshwater habitats than inland areas to the west. Another explanation is that the ancestor of $C$. longicarpus moved to the east after being expelled by the newly evolved gracilis group species. Populations of $C$. longicarpus are also found on the eastern shore of Virginia. which is separated from the remainder of the species range by the Chesapeake Bay. suggesting that this species occupied what is now the eastern shore before the Chesapeake Bay was formed.

The richmondensis group is the second largest group of Crangonvx and is widely distributed in North America and may also occur in eastern Europe (see above). The phylogenetic analysis indicates three species clusters in North America constituted as follows (see fig. 3): (1) C. obliquus and C. richmondensis are closely similar sister species: (2) C. anomalus. C. cornutus, C. dearolfi. C. grandimanus and $C$. hubrichti cluster together but the analysis does not reveal more about the relationship among these species: and (3) $C$. indianensis and $C$. disjunctus, which are near the group ancestor. The actual distributional relationship of these species does to some extent correspond to their phylogenetic relationship. The distributions of $C$. obliquus and $C$. richmondensis do not overlap but they are near each other geographically in the southern part of North America. Although these two species are similar morphologically, their distribution indicates that they are geographically well separated from each other. After evolving from an immediate common ancestor. $C$. obliquus has come to occupy the middle and lower Mississippi River drainage basin. while $C$. richmondensis is widely distributed over large parts of North America and is the most widely distributed species of Crangonyx. Moreover. it is the only species that is widely distributed in the western part of North America. A hypothesis to explain its western distribution is that this species was already widely distributed across North America before Pleistocene glaciation. After glaciation. the western habitats changed and some become unsuitable. But some populations of $C$. richmondensis survived as relict populations on the humid western coast in isolated packets. Crangonix richmondensis is the only species of the genus that occurs on Vancouver Island and it also occurs on two 
islands in the Alexander Archipelago of southeastern Alaska. The occurrence of this species on these islands, which are separated from the mainland and each other by marine waters, is zoogeographically very interesting. One explanation is that this species reached these islands by way of land connection during lower sea levels at glacial maxima in the Pleistocene (Bousfield. 1970b: Holsinger et al.. in press). Crangonx richmondensis is also found in Newfoundland and on Sable Island (Bousfield. 1970b) off the eastern coast of North America. According to Steele's ( 1983 ) offshore survival hypothesis. the distribution on these islands suggests this species may have survived glaciation on the continental shelf when sea level was lower and then was able to move back onto the mainland when the ice retreated.

The next clade of the richmondensis group consists of the epigean species $C$. cornutus. C. anomalus and $C$. hubrichti and subterranean species $C$. dearolf $i$ and $C$. grandimamus. Although the distributions of these five species do not overlap. the phylogenetic analysis does not resolve the relationship among them. inasmuch as their geographic relationship does not correspond with the phylogenetic analysis. Crangomx anomalus is found only in springs and spring-fed streams and also rarely in caves. Of particular interest regarding the distribution of $C$. anomalus is an approximately $250 \mathrm{~km}$ disjunction between one locality in southern Illinois and the heart of its range in northern Kentucky. This large gap may indicate that this species was once widely distributed and inhabited a wider variety of habitats, but is now reduced in distribution and largely restricted to surface springs. Morphologically it has very small eyes and therefore. combined with its apparent restriction to spring waters and caves. there is a possible indication of evolution toward life in the subterranean environment.

At present. $C$. indianensis is found only in caves of Indiana but it is not troglomorphic. Why a species found only in caves is not troglomorphic remains unclear. Apparently this species evolved very recently trom a surface ancestor. which would account for presence of eyes and some pigmentation. Crangonlx disjunctus is widely distributed on the eastern Coastal Plain of Virginia but one isolated population is found much farther west in the Appalachian mountains. This may indicate either the lack of adequate collections between these two areas or alternatively that $C$. disjunctus was widely distributed in the mountains and gradually moved to the east as Tertiary seas retreated. If this is the case. then the western mountain population(s)is a relict. Another possibility is that the Appalachian population(s) is genetically different and may not actually be the same species as the Coastal Plain $C$. disjunctus. despite morphological similarities.

The forbesi group. which contains one species. $C$. forbesi, is widely distributed in the east-central region of North America. Although its distribution is not completely continuous, this is probably due to lack of collection samples in some areas. There are many variations in certain characters. such as the telson shape. and this may indicate this species is very old. The present distribution may reflect an ancient distribution that originated by active dispersal of the ancestor. Crangonyx forbesi is morphologically similar to $C$. insolitus (of the insolitus group) and the two species may share a common ancestor (figs $1-2$ ). But the distribution of $C$. insolitus is at present limited to a single cave in southern Missouri and lies within 
the range of $C$. forbesi. If $C$. insolitus and $C$. forbesi do share a common ancestor. then the speciation of $C$. insolitus may have been sympatric or parapatric and occurred after its ancestor invaded a cave environment. But although $C$. insolitus is only found in a cave. it is not troglomorphic and has very special characters that are unique in the family Crangonyctidae (for example the short lateral spines on the peduncle of uropod 1). This species may have evolved directly from the common ancestor af the richmondensis. forbesi and insolitus groups (fig. 3). It may have once been as widely distributed as $C$. forbesi and is now limited in distribution because of inability to compete with $C$. forbesi for habitat space. However, the actual distribution of this species will probably turn out to be larger than a single locality as more areas are explored and more collections are made.

Three species. -- C. antennatus. C. serratus and C. specus --. apparently have the same common ancestor as the richmondensis. forbesi and insolitus groups. Crangomx antennatus is a cavernicolous species. distributed through a part of the Appalachians from southwest Virginia to northwestern Alabama and also found in the Sequatchie River Valley in Tennessee. It is known primarily from caves but is also found in karst springs associated with caves. It is a troglomorphic species. although the eyes of some populations are variable and in the form of a few black specks. It is clearly derived from a surface ancestor. probably originating in one area and gradually spreading through subterranean groundwater aquifers. Its large subterranean distribution is probably due to in part to active dispersal through shallow groundwater habitats (Holsinger. 1969. 1975. 1986). It is assumed that these subterranean amphipods inhabit an epikarstic zone saturated with groundwater above open cave passages and can pass downward into these passages by migration and/or accident (Holsinger. 1986). Crangonyx specus is also a cavernicolous species and to date is found only in four caves in eastern Kentucky. which is geographically not far away from the distribution of $C$. antennatus. One hypothesis for the distribution of this species is that $C$. spectus evolved from $C$. antennatus by allopatric speciation after some populations of $C$. antennatus dispersed westward into the Cumberland River drainage and became isolated. Another hypothesis is that they are both from a single common ancestor. whereas $C$. specus is a poor disperser with a limited distribution. in contrast to $C$. unlennatus which has gained a wide distribution by active dispersal in groundivater habitats.

Crangonyx serratus is widely distributed along the eastern Coastal Plain southward from Washington. $D C$ to Florida. Like $C$. untennatus and $C$. specus, its phylogenetic position is unclear (figs. $1-3$ ). Its wide distribution on the Coastal Plain. however. probably reflects a relatively recently event. After sea water recessions in the late Tertiary, the ancestor of this species moved onto the Coastal Plain from a western inland source area by dispersal. It should be noted that the distribution of $C$. serratus overlaps that of $C$. richmondensis on the Coastal Plain, but these two species are never found together in the same specific locality.

From the Phylogenetic Analysis section, five ungrouped species. including $C$. bousfieldi. C. Cooperi. C. fontinalis. C. lewist, and C. orientalis, are seen to have a close relationship with the shoemakeri and gracilis groups. but the relationships among these species are not clear (figs 1-3). Except for $C$. orientalis. 
which is known from many collections in the Piedmont and Coastal Plain from Maryland to North Carolina, the other four species are known only from a few collections, so it is difficult to explain their distributions at the present time. It is possible that they are all evolved from a single common ancestor. If this is the case, one scenario that explains the present distribution is that an ancestral species was widely distributed and then. through both physical and reproductive isolation of populations at different places. evolved into a suite of closely similar species.

The gracilis group is composed of 14 species and widely distributed in the eastern part of North America. Except for a few species such as C. hobbsi. and C. packardi. which appear to be well adapted to the subterranean environment, all of the females look alike and in some species they cannot be told apart. The males also look alike but normally there are good differential diagnostic characters on uropod 2. However, except for a well defined floridanus subgroup, the phylogenetic analysis did not tell us much about the relationship among these species. The observations discussed above support the hypothesis that the ancestor of the gracilis group was widely distributed in the eastem part of North America. The individual species that have evolved within this group are morphologically not well separated. However. many of the species of this group may not be biological species by strict definition. but this cannot be tested with our limited morphological data. It also follows that the difficulty in differentiating the species may lead to some problems with interpretations of their distribution. Another difficulty is that more than one-half of the species are known from only a few collections and therefore appear to have very limited distributions. Thus, the distributions may be incorrect due to identification error. For example. some collections contain only female specimens and/or damaged males, making it very difficult to get a correct species identification. The following is a discussion of some of the interesting species of this group.

The floridanus subgroup is well defined and separated from all other members. although the phylogenetic relationship among the subgroup members is not clear (figs. 1-3). (rungomx floridunus and C. pseudogracilis are morphologically closely similar and undoubtedly derived from a relatively recent common ancestor. Crangonyx pseudogracilis occurs primarily west of the Appalachian Mountains. In contrast, $C$. floridantus occurs generally east of the Appalachians, but several populations were also found on the western side. The distributions of $C$. floridanus and $C$. pseudogracilis overlap in the middle of the drainage basin of Mississippi River in Missouri and on the Coastal Plain in Mississippi. The overlap in these two places can be explained by dispersal of $C$. floridanus from the most western part of its range and dispersal of $C$. pseudogracilis from the most eastern part of its range. However. these species have never been found together in the same locality. The populations of $C$. floridanus found in Ohio and Kansas are very difficult to explain and the occurrences of $C$. floridanus in Colorado and Oregon may be due to accidental transports by man (discuss below).

Crangonyx gracilis is found mainly in the Great Lakes region but also found in the mountains of southeastern West Virginia and southwestern Virginia. The populations in the Virginias occur south of the southern extent of Pleistocene glaciation and may be relicts of a former continuous distribution. The Great 
Lakes populations are probably the result of dispersal to the north following the last glaciation. The above explanation can also be applied to the distribution of $C$. rivuluris.

The distribution of the stygobiont $C$. packardi is also very interesting inasmuch as its range crosses three physiographic provinces and the Mississippi River, covering a distance of about $1000 \mathrm{~km}$. This wide distribution suggests that the species might have evolved and spread before the Mississippi River developed into its present form. It might also explain some of the geographic variation of characters among different populations. However. it is very likely that genetic differences exist among some populations and some of them in turn probably represent different species. Their resolution. however. cannot be accomplished by morphological analysis alone.

Crangonyx hobbsi is one of the two subterranean species found in Florida. the other being $C$. grandimamus. These species are almost always found living together in the same caves. Additional discussion on these two species is included below in the Origin of Subterranean Species section.

\section{ORIGIN OF TROGLOBITES}

As seen from the habitat analysis. 24 species of Crangonvx have been found in caves or other subterranean habitats. Eleven are troglobites: the others may be found in cave environments but also have surface populations. Utilizing results of the biogeographic and cladistic analyses. I will attempt to draw a clear picture of the taxonomic and ecological relationships between the subterranean and surface species of Crangonyx. The principal questions are namely: how and when the surface ancestors invaded subterranean habitats and evolved into troglobites and what morphological changes were associated with these events?

From the cladograms (figs. $1-3$ ), two troglobites are found in the richmondensis group ( $C$. dearolff. and $C^{\prime}$.grandimanus), six in the gracilis group (C. packardi. C. hobbsi. C. barri. C. castellanum. C. cuecus and $C$. ohioensis) and three are unassigned to groups ( $C$. antennatus, $C$. specus and $C$. lewisi). In addition four species ( $C$. indianensis. $C$. insolitus. $C$. fontinalis and $C$. Cooperi) are almost always found in subterranean waters (caves. seeps and springs) but are not troglomorphic. Finally. eight other species are also recorded from caves but most of them are predominately epigean and. with the exception of $C$. forbesi. are far more common in surface habitats. The fact that more than half the species of Crangony $x$ have been reported from subterranean waters is a good indication that this genus has a strong association with the subterranean environment (see Holsinger, 1994). The existence of different subterranean species in different species group also indicates that cave species have evolved in different lineages at different times. and possibly under different conditions.

There are at least two acceptable theories that explain the evolution of subterranean species. One is the relict theory (Barr. 1968), which states that caves have served as refugia. Preadapted species invaded and colonized caves in order to survive harsh environmental conditions at the surface, such as glaciation. The 
relict taxa left in the caves are then isolated reproductively from their epigean relatives and become increasingly adapted to cave environments by evolving a troglomorphic facies. Mlany of the trogobitic species of Crangonyx found in the Appalachians and Interior Low Plateaus regions. which are close to areas glaciated during the Pleistocene. could be explained by this theory. The theory may also give us a fairly good idea of the time of cave invasion (obviously sometime during the Pleistocene).

The second theory considers the active role of the animals themselves. Troglobites evolved by active or passive colonization of caves by preadpted epigean taxa without the constraint of climatic changes. Under this theory the factor that triggers troglobite evolution is not dependent on harsh environmental conditions but there is a tendency for the animals to explot new nicnes with availabic fond resources (Howarth. 1987). This theory can also be used to explain the troglobites of Crangomx that are found far to the south in Florida. such as $C$. hobbsi and $C$. grandimanus. presumably in areas not drastically affected by climatic changes in the Pleistocene. But many species living in the north can also be explained by this theory. For example. C. setodactyltss, an epigean species with well developed eves is also represented by a population living in a cave in northern Ohio. It has reduced eyes. and a slight reduction in pigmentation but shows no other morphological changes for cave life. The reduction of eves suggests that populations of this species can probably change rather quickly when they move into the cave environment. Since it is usually: found in surface habitats, its occurrence in caves is best explained by active colonization. Under the second theory troglobite evolution may occur at any time. and populations that enter caves may adapt rather quickly to subterranean waters as they evolve into troglobites.

Another explanation for the evolution of troglobites in the genus Crangonvx is that some species may be evolved from ancestors that are already troglobitic. This may be the case of $C$. specus. which is morphologically similar to $C$. antennatus and distributed just beyond the range of $C$. antennutus in eastern Kentucky. These species appear to have a recent common troglobite ancestor. with $C$. specus possibly originating through peripheral isolation, possibly in subterranean waters.

In most troglobitic amphipods. the eyes are either reduced to a few black specks or are totally absent. The most striking troglomorphy can be found in C. hobbsi, which may be more specialized morohologically than any other species of Crangonyx. Eyes are lost completely, antennae are elongate. appendages are attenuate, and no calceoli are found on antennae 2 of the male. For the other troglobitic species in the gracilis group. the eyes are not completely lost, and all are morphologically similar to each other except for $C$. packardi. It is possible that $C$. packardi evolved earlier than the others, inasmuch as its range presently crosses three physiographic regions and extends both east and west of the Mississippi River. Unlike other members of the gracilis group, the gnathopods of this species lack notch-serrated spine teeth.

To date, Crangonyx is the only subterranean amphipod found in the karst regions of Florida. where it is represented by $C$. hobbsi of the gracilis group and C. grandimanus of the richmondensis group. Both 
species are almost always found living together but both are absent in the middle region of Florida: they: occur in the north, north-central and extreme south. No obvious morphological differences are found between the populations of either species in the north-central and south. An explanation for the disjunct distribution of these two species is that there may be subterranean links between the northern and southern areas. allowing species to move, at least to some extent. between these regions by subterranean dispersal. The occurrence of only two subterranean amphipods in Florida is also intriguing. Especially. since Stygobromus, the largest genus of Crangonyctidae with more than 100 species, has never been found in Florida. Holsinger (1986) suggested two possible explanations for the presence of troglobitic species of Crangonx, and absence of Sygobromus, in the karst regions of Florida: (1) the limestone region of Florida is geologically much younger than in most of the other North American regions (Ceno:nic in age) and Stygcioromus was already widely distributed in other regions prior to the relatively recent development of subterranean freshwater aquifers on the Florida peninsula. (2) there are several Crangorix spp. living or. the Coastal Plain just north of the Floridian karst. which could account for the occurrence on or near the Florida peninsula of potential ancestors of troglobitic Crangomx. Besides the two subterranean species. epigean $C$. richmondensis, $C$. floridamus and $C$. serratus are also found in Florida. From the above it seems reasonable to suggest that after karst habitats of Florida became available for amphipod colonization. species of Crangomyx were present in this region. and the ancestors of C. hobbsi and $C$. grandimanus invaded subterranean waters and evolved into troglobites. Some populations of $C$. floricanus are also tound in the same cave habitats with $C$. hobbsi and $C$. grandimamus, both in the northern and southern part of the state. They have greatly reduced eyes. but no other troglomorphic features, suggesting that populations of $C$. floridanus have recently invaded caves and are adapting rather quickly to the subterranean environments.

INTRODUCTION OF C. PSEUDOGRACILIS AND C. FLORIDANUS TO AREAS OUTSIDE THEIR

\section{ESTABLISHED RANGES}

Crangonyx pseudogracilis was first found outside North America in the London area in the 1930s (Crawford, 1937: Tattersall, 1937). It then became widely distributed in British freshwaters through migration into central and southern England and Wales, eventually spreading northward through Scotland (Gledhill et al.. 1993). It is thought that this species was brought to Europe unwittingly by man (Hynes. 1955). In 1975 it was also found in a pond in Dublin. Ireland (Holmes. 1975). In 1979 it was first found in inland waters in the Netherlands in a limited area of the province of Groningen. From here. it gradually extended its distribution in a western and southern direction (Hautus and Pinkster. 1987: Pinkster et al.. 1992). In the Dutch inland waters it inhabits all kinds of habitats such as rivers, canals, ponds, lakes. and 
reservoirs, tolerating both fresh and brackish, clean and polluted waters. The successful distribution of this species in Europe is thought to be because of its high fecundity as compared to local species (Pinkster et al.. 1992).

Crangonyx floridanus was recorded from a freshwater pond of the Tone River in Chiba Prefecture. Japan (see notes under the species description section of $C$. floridanus). The specimens were collected in [992 and the pond is not far away from sea water.

How did the populations of $C$. pseudogracilis in the Europe and the population of $C$. floriclanus in Japan get there from their original ranges in North America? One explanation of this is the ballast water theory, which is the transport of organisms in ballast water by ocean-going ships (summarized by Carlton and Geller. 1993). In their survey it was found that a total of 367 taxa were found in Japanese ballast water released in Oregon (Carlton and Geller, 1993). These studies found that the ships can take up and release ballast water in bays. estuaries. and inland waters and then release this water into similar environments elsewhere. There are also freshwater ballasts transferred to other freshwater endpoints (such as from Europe to the Laurentian Great Lakes. or vice versa).

Crangonx pseudogracilis is found near the Coastal Plain and also near the Great Lake region of North America. so it is possible that some specimens were carried in ballast water to Europe. According to Dirk Platvoet (pers. comm.) all localities where $C$. pseudogracilis was first found in the Netherlands are in connection with a canal on which several shipbuilding companies are located. He found that many ships retuming from transoceanic trips empty their ballast tanks in the shipyards. Apparently the amphipods survive in the ballast water of ships successfully and extend their distribution from there. The ballast hypothesis also explains why $C$. pseudogracilis occurs in Britain and Ireland.

The same theory also explains why $C$. floridanus is found in a freshwater pond in Japan. This pond is connected to the Tone River which flows to the sea, thus $C$. floridanus could have migrated from the ballast water inland to the ponds by following the river. There is no other good explanation for the occurrence $C$. floridanus in Japan. Both $C$. pseudogracilis and $C$. floridanus occur on the Coastal Plain of North America and could be very easily picked up by ships when they take on ballast water.

In North America, populations morphologically very similar to $C$. pseudogracilis are found in Arizona and Nevada. If they are bona fide $C$. pseudogracilis. one explanation for their occurence in western North America is that they were transported by man in tish containers or on aquarium vegetation from eastern localities. However, the population from Page Spring Fish Hatchery in Yavapai Co.. Arizona is a more likely candidate for this kind of introduction than the population in the Trukee River in Nevada. The alternative hypothesis is that these western population may be a genetically different but morphologically similar species.

In North America. C. floridanus was recorded from Colorado and Oregon far away from its distribution in eastern North America. One or more of the populations of $C$. floridanus found in Colorado 
may have also been transported by man in fish transplant containers (Pennak \& Rosine. 1976). The population of $C$. floridanus found in Oregon could. however, be explained by the ballast theory because the collections were made near Portland. which is a large inland port near the western coast of North America. Alternatively, these populations may represent new undescribed species that are morphologically similar to but genetically different from C. floridanus. 


\section{LITERATURE CITED}

Biernbaum. C. K. 1989. Distribution and seasonality of Branchiopod and Malacostracan crustaceans of the Santee National Wildlife Refuge. South Carolina. Brimleyana, 15:7-30.

Black. J. H. 1971. Cave life of Oklahoma. Oklahoma Underground (special issue), 4(1-2):2-56.

Banta. A. M. 1907. Fauna of Mayfield's Cave. The Carnegie Institution of Washington. pp. 1-114.

Barnard. J. L. 1969. The families and genera of marine gammaridean Amphipoda. Bulletin of the Cinited States National Museum. 271: 535 pages. 173 figures.

Barnard. J. L. \& C. M. Barnard. 1983. Freshwater Amphipoda of the world (Parts I \& II). Haytield Associates. Mt. Vernon, (Va.). 830pp.

Barr. T. C. Jr. 1968. Cave ecology and evolution of troglobites. Evolutionary Biolog: 2:35-102

Barton. D. R. \& Hynes. H. B. N. 1976. The distribution of Amphipoda and Isopoda on the exposed shores of the great lakes. Journal of Great Lakes Research, 2(2):207-214.

Bate. C. S. 1859. On the genus Niphargus (Schiödte). Proceedings of the Dublin Cniversity Zoological and Botanical Association. 1:237-244.

Bell. R. T. 1971. Handbook of the . Malacostraca of Vermont. Privately published. Burlington. VT. 65pp. Borutzky. E. W. Materialien über die Fauna der unterirdischen Gewässer: Crangoṇx chlebnikori sp. n. (Amphipoda) aus den Höhlen des mittleren Urals. Zoologischer tnzeiger. 77:253-259.

Bousfield. E. L. 1958. Fresh-water amphipod crustaceans of glaciated North America. Cunadian Ficld Vaturalist. 72(2):55-113.

Boustield. E. L. 1961. New records of fresh-water amphipod crustaceans from Oregon. Vatural Histor? Papers. National Museum of Canada. 12:1-7.

Bousfield. E. L. 1963a. A new fresh-water amphipod crustacean from Oregnn. Breviora. 180:1-6.

Boustield. E. L. 1963b. New fresh-water amphipod crustacean from Florida. Natural History. Papers. Vational Museum of Canuda. 18:1-9.

Bousfield. E. L. 1970a. Amphipod crustaceans of the Ottawa region. Trail and Landscape. 4(3):107-113.

Bousfield. E. L. 1970b. Amphipod and isopod crustaceans. In Fauna of Sable Island and its geographic affinities. National Museum Natural Science Publication Zoology. 4:34-37.

Bousfield. E. L. 1973. Shallow-water Gammaridean Amphipoda of New England. Cornell University Press. Ithaca. New York. $312 \mathrm{pp}$.

Cole. G. A. 1957. Some epigean isopods and amphipods from Kentucky. Transactions Kentucky .Acudemy. of Science, 18:29-39.

Cole. G. A. 1959. A summary of our knowledge of Kentucky Crustaceans. Transactions Kentuck. tcademy of Science. 20:66-81.

Craig. J. L. 1977. Invertebrate Faunas of Caves to be inundated by the Meramec Park Lake in eastern 
Missouri. National Speleological Society Bulletin. 39:80-89.

Crawford. G. I. 1937. An amphipod, Eucrangonyx gracilis S. I. Smith, new to Britain. Nature. 139:327..

Crawford.D.M.1977. A new amphipod, Crangonyx forbesi (Hubricht and Mackin), for West Virginia (Amphipoda:Gammaridae). Proceedings of the West Virginia Academy of Science. 49:18.

Dearolf. K. 1953. The invertebrates of 75 caves in the United States. Proceedings of the Pennsylvania tcudemy of Science. 27:225-241.

Derzhavin. A. N. 1927. New forms of freshwater gammarids of Ussury District. Russkii Gidrobiologicheskii Zhurnal, 6(8-10):176-179.

Ellis. T. K. 1940. A new amphipod of the genus Crangonyx from South Carolina. Charleston . I/usezm Leaflet, 13:3-8.

Embody, G. C. 1911. A new fresh-water amphipod from Virginia. with some notes on its biology:

Proceedings of the United States National Museum. 38:299-305.

Fage.L. 1951. Crustacés amphipodes et décapodes (Biospeologica LVI). Archives de Zoologie Experimentale et Generale. 71:361-374.

Farris. J. S. 1988. Hennig86. Version 1.5.

Fitzpatrick. J. F. Jr. 1983. How to know the freshwater crustacea. The pictured key nature series. W. C. Brown Company, Dubuque. lowa. 227pp.

Fleming. R. S. 1939. The larger Crustacea of the Nashville region. Journal of Tennessee Academy of Science, $14(3): 296-324$.

Franz. R. \& D. Slifer. 1971. Caves of Maryland. Educational Series No. 3. Maryland Geological Survey: pp. $1-120$.

Forbes. S. A. 1876. List of Illinois Crustacea. with description of new species. Bulletin of Illinois . Luseum of Vatural History $1: 3-24$.

Gledhill. T.. D. W. Sutcliffe \& W. D. Williams. 1993. British freshwater Crustacea Malacostraca: A key with ecological notes. Scientific Publisher Freshwater biological Association. 52:1-173.

Hay. O. P. 1882. Notes on some fresh-water Crustacea, together with descriptions of two new species. American Nuturalist. 16:143-146.241-242.

Hay. W. P. 1896. On a collection of Crustaceans from Indiana Caves. pp.206-210. In Blatchley. W. S. Indiana caves and their fauna. Indiana Dept. of Geol. and Nat. Res., 2 Ist Ann Report. pp. 121-212.

Hay, W. P. 1902. Observations on the Crustacean Fauna of Nickajack Cave. Tennessee. and Vicinity. Proceedings of United States National Museum, 25:417-439.

Hautus. T. \& S. Pinkster. 1987. Range extension in the period 1985-1986 of the alien amphipods. Gammarus tigrinus Sexton, 1939. and Crangonyx pseudogracilis Bousfield, 1958. in the Netherlands (Crustacea, Amphipoda). Bulletin Zoologisch Museum, Universiteit van .tmsterdam. 11 (6):57-64.

Hennig. W. 1966. Phylogenetic systematics. University of Illinois Press. Urbana. 263pp. 
Holsinger. J. R. 1963. Annotated checklist of the Macroscopic troglobites of Virginia with notes on their geographic distribution. Nationul Speleological Society Bulletin. 25:23-36.

Holsinger. J. R. 1967. Systematics. Speciation, and Distribution of the Subterranean Amphipod Genus Stygonectes (Gammaridae). Bulletin of the United States National Museum. 259:176 page. 36 figures.

Holsinger. J. R. 1969. Biogeography of the freshwater amphipod crustaceans (Gammaridae) of the central and southern Appalachians. Pages 19-50 in P. C. Holt (ed.). The distribution history of the biota of the southern Appalachians. Part I:Invertebrates. Virginia Polytechnic Institute Press.

Holsinger, J. R. 1972. The freshwater amphipod crustaceans (Cammaridae) of North America. In Biota of Freshwater Ecosystems, US Environmental Protection Agency Identification Manual. 5:1-89.

Holsinger. J. R. 1977. A review of the systematics of the Holarctic Amphipod Family Crangonyctidae. Crustaceana (Supplement ). 4:244-281.

Holsinger. J. R. 1978. Systematics of the subterranean amphipod genus strgohromus (Crangonyctidae). Part II: species of the eastern United States. Smithsonian Contributions to Zoologl: 266:1-144.

Holsinger. J. R. 1986. Zoogeographic patterns of North American Subterranean amphipod crustaceans. In R. H. Gore \& K. L. Heck (eds.). Crusıacean Biogeography. Balkema. Rotterdam:85-106.

Holsinger, J. R. 1994. Pattern and process in the Biogeography of subterranean Amphipod. Hy urobiologia $287: 131-145$.

Holmes. J. M. C. 1975. Crangonyx psendogracilis Bousfield. a freshwater amphipod new to Ireland. Journal of Ireland Vaturalist, 18:225-226.

Howarth. F. G. 1987. The evolution of non-relictual tropical troglobites. International Journal of Speleology.16:1-16.

Hubricht. L. 1943. Studies on the Nearctic freshwater Amphipoda. III. Notes on the freshwater Amphipod of eastern United States, with descriptions of ten new species. American .Midland Vaturalist. 29(3): $683-712$

Hubricht. L. 1950. The invertebrate Fauna of Ozark Caves. National Speleological Socien. Bulletin. 12:1617.

Hubricht. L. \& H. C. Harrison. 1941. The freshwater Amphipod of Island County. Washington. .tmerican . Widland Naturalist, 26:330-333.

Hubricht. L. \& J. G. Mackin. 1940. Descriptions of nine new species of freshwater amphipod crustacean with notes and new localities for other species. American Midland Naturalist. 23:187-218.

Humphries. C. J. and L. R. Parenti. 1986. Cladistic Biogeography. Oxford monographs on Biogeography No. 2, 98 pp. Clarendon Press. Oxford.

Huntsman. A. G. 1915. The fresh-water Malacostraca of Ontario. Contributions to Canculiun Biolog: $39 \mathrm{~b}: 145-163$.

Hynes. H. B. N. 1951. Distribution of British Freshwater Amphipoda. Nature. 167:152. 
Hynes. H. B. N. 1955a. Distribution of some freshwater Amphipoda in Britain. Proceedings of the International Association of theoretical and applied Limnology 12:620-628.

Hynes, H. B. N. 1955b. The reproductive cycle of some British freshwater Gammaridae. Journal of tnimal Ecologv, 24(2):352-387.

Jass. J \& B. Klausmeier. 1995. Amphipods (exclusive of Pontoporeiidae) of Southeastern Wisconsin. Field Station Bulletin (University of Wisconsin-Milwaukee). 28(1):1-13.

Judd. W. W. 1963. Studies of the Byron Bog in Southwestern Ontario XVI. Observations on the life cycles of two species of Crangonnx (Crustacea: Amphipoda). Natural Histony Papers, National L/usetum of Cunuda. 20:1-9.

Karaman. G. S. 1978. Contribution to the knowlwdge of the Amphipoda 97. On three interesting species. Crangonxx floridanus Bous. 1963. C. parvimanus (Hol.1903) and Orchestia tiberiadis Lor. 1883. Glasnik Republickog Zavoda =a Zustitu Prirode-Prirodnjackog . Iruseja. Titograd. 11:65-73.

Kenk. R. 1949. The animal life of temporary and permanent ponds in southern Michigan. .Miscellaneous Publications, Museum of Zoologv, University of Michigan. No. 71:1-66.

Krekeler. C. H. \& E. C. Williams. Ir. 1966. Cave Fauna. In A. A. Lindsey (ed.). Natural Features of Indicina. pp.390-400. Indiana Academy of Science. Indianapolis. Indiana.

Kunkel. B. W. 1918. The Arthrostraca of Connecticut. Bulletin of Connecticut Geologicul and Vatural History Survey, 26:1-261.

Lewis. J. J. 1983. The obligatory subterranean invertebrates of glaciated southeastern Indiana. Vational Speleological Sociery Bulletin. 45:34-40

Lindeman. D. H.. R. W. Lindeman \& M. Lindeman. 1993. A range extension for the Amphipod Crangony richmondensis laurentianus in northwestern Ontario. Canadian Field. Vaturalist 107:217-221.

Mackin. J. G. 194I. A key to the species of Amphipoda of Oklahoma. Proceedings of the Okluhoma Acculemy of Science. 21:29-30.

Maddison. W. P.. M. J. Donoghue \& D. R. Maddison. 1984. Outgroup analysis and parsimony. Systematic Zoologv. 33(1):83-103.

McDaniel. V. C. \& K L. Smith. 1976. Cave fauna of Arkansas: Selected invertebrate taxa. .trkansas Acudemy of Sciences Proceedings, 30:57-60.

Mills. E. L. 1964. No:eworthy Amphipoda (Crustacea) in the collection of the Yale Peabody Museum. Postilla. 79:1-41.

Myers. A. A. and P. S. Giller. 1988. Process, pattern and scale in biogeography. pp3-21. In . Anuliticul Biogeography. Myers. A. A and P. S. Giller, eds. London. New York. Chapman and Hall.

Nicholas. B. G. 1960. Checklist of macroscopic troglobitic organisms of the United States. American Midland Naturalist, 64(1):123-160.

Notenboom, J. 1988. Phylogenetic relationships and biogeography of the groundwater dwelling amphipod 
genus Pseudoniphargus (Crustacea), with emphasis on the Iberian species. Bijdragen tor de Dierkunde 58: $159-204$.

Packard. A. S. 1881. Crangonyx antennatus, p.880. In E. D. Cope \& A. S. Packard. The fauna of Nickajack Cave. American Naturalist. 15:877-882.

Page. L. M. 1974. Aquatic malacostraca recorded for Illinois, with notes on their distribution and habitats within the state. Transactions Illinois .Academy of Science, 67:89-104.

Pearse. A. S. 1910. A preliminary list of the Crustacea of Michigan. 12th Report of Michigan Acadenly of Sciences. pp.68-76.

Pennak. R. W. 1989. Fresh-water Invertebrates of the United States: Protozoa to Mollusca. 3rd ed. John Wiley \& Sons. Inc. 628pp.

Pennak. R. W. \& W. N. Rosine. 1976. Distribution and Ecology of Amphipoda (Crustacea) in Colorado. American Midland Naturalist, 96(2):324-331.

Pflieger. W. L. 1974. Fauna of Missouri Springs. Water Resources Report Vo. 29:31-42. Missouri Geological Survey and Water Resources.

Pinkster, S.. J. Dieleman \& D. Platvoet, 1980. The present position of Gammarus tigrinus Sexton. 1939. in the Netherlands, with the description of a newly discovered amphipod species. Crungonyx pseudogracilis Bousfield. 1958 (Crustacea. Amphipoda). Bulletin Zoologisch L/useum. Liniversiteit van .Amsterdam. 7(4):33-45.

Pinkster. S.. D. Platvoet. 1983. Further observations on the distribution and biology of two alien amphipods, Gummarus tigrinus Sexton. 1939. and Crangonvx pseudigracilis Bousfield. 1958. in the Netherlands. Bulletin Zoologisch Museum. Universiteit van Amsterdam. 9(17):153-164.

Pinkster. S., M. Scheepmaker., D. Platvoet \& N. Broodbakker. 1992. Drastic changes in the amphipod fauna (Crustacea) of Dutch inland waters during the last 25 years. Bijdragen tot de Dierkunde. $61(4): 193-204$.

Powell. R. L. 1961. Caves of Indiana. Circular No. 8. Indiana Dept. of Conservation and Geological Survey. 127pp.

Saunders. L. G. 1933. The fresh-water Amphipods of Vancouver Island. Contribution to (uncudian Biolog. and Fisheries, 19:243-251.

Schellenberg. A. 1934. Eine neue Amphipoden-Gattung aus einer belgischen Höhle. nebst Bemerkungen über die Gattung Crangonvx. Zoologischer Anzeiger, !06(9):215-21 8.

Schellenberg,A.1935. Die Höhlenfauna des Glatzer Schneeberges, 2. Höhlenamphipoden des Glatzer Schneebergs. Beitrage zur Biologie des Glat=er Schneeberg, 1:72-75.

Schellenberg, A. 1936. Die Amphipodengattungen um Crangonyx, ihre Verbreitung und ihre Arten. .Mitteilungen aus dem Zoologischen Museum in Berlin. 22:31-43.

Shoemaker, C. R. 1933. Amphipod from Florida and the West Indies. American Mhuseum Vovitiutes. 598: I- 
24.

Shoemaker. C. R. 1941. A new subterranean amphipod of the genus Crangonyx from Florida. Charleston Museum Leaflet. 16:9-14.

Shoemaker. C. R. 1942. Notes on some American fresh-water amphipod crustacean and descriptions a new genus and two new species. Smithsonian Miscellaneous Collections. 101(9):1-31.

Smith. D. G. 1977. The genus Crangonyx (Amphipod: Cammaridae) in the central Connecticut River system. Canadian Field Naturalist, $91: 256-261$.

Smith. D. G. 1981. Selected fresh-water invertebrates proposed for special concern status in .Hassachusetts. Massachusetts Department of Environmental Quality Engineering. Division of Water Pollution Control. Westborough. Massachusetts. 26pp

Smith. D. G. 1982. Range extensions of two species of gammaroidean amphipods in northeastern North America. Crustaceana. 42:315-316.

Smith. D. G. 1983. A new species of freshwater gammaroid amphipod (Crangonyctidae) from southeastern New England. Transactions of American Microscopic Socien. 102(4):355-365.

Smith. D. G. 1988. Keys to the freshwater macroimertebrates of Massachusetts: No. 3): ('rustucea .Malacostraca (cranfish, isopods, amphipods). Department of Environmental Quality Engineering. Division of Water Pollution Control. Westborough. Massachusetts. 58pp

Smith. S. I. 1871. Crangonur gracilis. In S. I. Smith \& A. E. Verrill. Notice of invertebrates dredged in lake Superior in 1871 by the United States Lake Survey under the direction of Gen. C. B. Comstock. S. I. Smith, naturalist: 452. American Journal of Science, (3), 2:448-454.

Smith. S. I. 1874. The Crustacea of the fresh waters of the United States. A Synopsis of the higher freshwater Crustacea of the northern United States. Report of the Commissioner for 1872 and 1873 . Lnited States Commission of Fish and Fisheries. 2:637-661.

Smith. S. I. 1888. Crangonyx vitreus and C. packardi. In A. S. Packard. The cave fauna of North America. with remarks on the anatomy of the brain and origin of the blind species. Memoirs of the National Accudemy of Sciences, 4:34-36.

Stebbing. T. R. R. 1899. On Amphipoda from the Copenhagen Museum and other sources. Transcactions of the Linnean Suciety of London (2. Zoology), 7(8):395-432.

Stebbing. T. R. R. 1906. Amphipoda I. Gammaridea. Das Tierreich, 21:1-806.

Steele. D. H. 1983. Fresh water Amphipod (Crustacea). pp.679-686. In South. G. R. (ed.). Bingeography. and Ecology of the Island of Newfoundland. Dr. W. Junk Publishers. The Hague.

Stewart. B. A. and C. L. Griffiths. 1995. Revision of the Family Paramelitidae (Crustacea. Amphipoda) from South African fresh waters. Annals of the South African Whuseum. 104:181-247.

Swofford. D. L. 1991. PAUP: Phylogenetic Analysis Using Parsimony. Version 3.0s. Computer program distributed by the Illinois Natural History Survey. Champaign, Illinois. 
Tattersal. W. M. 1937. Occurrence of Eucrangonyx gracilis. Nature, 139:593.

Thomas, J. D. 1976. A survey of Gammarid amphipods of the Barataria Bay. Louisiana region. Contributions in Marine Science, 20:87-100.

Van Maren. M. J. 1978. Distribution and ecology of Gammarus tigrinus Sexton. 1939 and some other amphipod crustacea near Beaufort (North Carolina. U.S.A.). Bijdrugen tot de Dierkunde. 48:45-56.

Varnedoe. W. W.. Jr. 1973. Alabama caves and caverns. I 140 pages. Published privately by author.

Warren. R. D.. 1961. The obligate cavenicoles of Florida. Special Papers of The Florida Speleological Survey. 1:1-10.

Weckel. A. L. 1907. The fresh-water Amphipoda of North America. Proceedings of the Linited States .Vational Museum. 32:25-58.

Williams. D. D. \& H. B. N. Hynes. 1976. The ecology of temporary streans: I. The faunas of two Canadian streams. International Revue der Gesamten Hydrobiologie. 61:761-787.

Williams. D. D. \& H. B. N. Hynes. 1977. The ecology of temporary streams: II. General remarks on temporary streams. Internation Revue der Gesamten Hydrobiologie. 62:53-61. 
VITA

Jun Zhang was born on September 8. 1962, in Hefei City, Capital of Anhui Province of China. He graduated from Wuhu No.I High School in 1980. He attended Sichun University in Sichun Province in 1980. received B.S. in 1984. majoring Zoology. He then attended Nanjing Normal University. began his study of insect systematics, received M.S. in 1987 by study of Ephemeroptera of China. After teaching zoology at Nanjing Normal University for two and half years, he came to United States to seek his Ph.D Degree. Jun Zhang studied in the Department of Biological Sciences. Old Dominion Univesity. He has published 6 scientific papers and described 11 new species of Ephemeroptera. He is married to Zhenhong Sun who also studied in Old Dominion University and received a MS degree in Biology in May. 1995. 